\title{
Iron Carbides and Sodium-Sulphur Promotion in Fischer-Tropsch Synthesis Catalysts
}

Pasi Petteri Paalanen 
Paalanen, Pasi Petteri

Title: $\quad$ Iron Carbides and Sodium-Sulphur Promotion in Fischer-Tropsch Synthesis Catalysts

Printed by: Off Page, Amsterdam

Cover design Huixia Yang (wiesja.yang@gmail.com) and

and artwork: Fatima García Soto (fatimagarciasoto@gmail.com)

The research work in this PhD Dissertation was carried out in the Inorganic Chemistry and Catalysis group, Debye Institute for Nanomaterials Science, Utrecht University, the Netherlands. 


\section{Iron Carbides and Sodium-Sulphur Promotion in Fischer-Tropsch Synthesis Catalysts}

Ijzercarbiden en Natrium-Zwavel Promotie in FischerTropsch Synthese Katalysatoren

(met een samenvatting in het Nederlands)

\section{Proefschrift}

ter verkrijging van de graad van doctor aan de Universiteit Utrecht op gezag van de rector magnificus, prof. dr. H. R. B. M. Kummeling, ingevolge het besluit van het college voor promoties in het openbaar te verdedigen op maandag 16 november 2020 des middags te 2.30 uur

door

Pasi Petteri Paalanen

geboren op 27 februari 1985 te Mikkeli, Finland 
Promotor: Prof. dr. ir. B. M. Weckhuysen

This PhD Dissertation received funding from de Nederlandse Organisatie voor Wetenschappelijk Onderzoek (NWO) in the framework of the TASC Technology Area "Syngas, a Switch to Flexible New feedstock for the Chemical Industry (TA-Syngas)". The Dow Chemical Company and Johnson Matthey also contributed financially on supporting this research. 
This page is intentionally left blank 


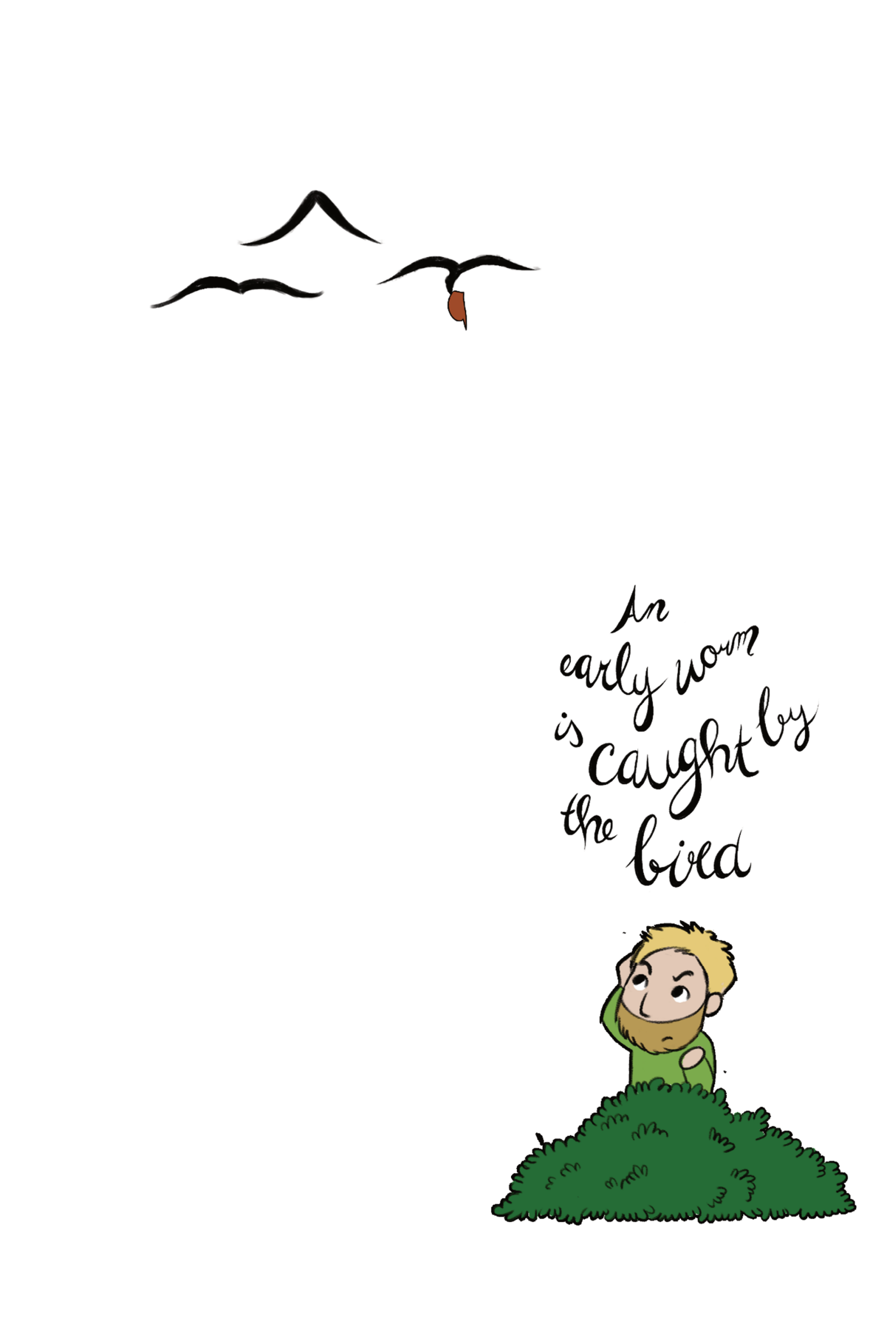




\section{Table of Contents}

\begin{tabular}{lll}
$\mathbf{1}$ & A General Introduction & $\mathbf{1 5}$ \\
\hline 1.1 & Heterogeneous Catalysis & 16 \\
1.2 & Fischer-Tropsch Synthesis in Industry & 18 \\
1.2 .1 & A Short History of the Industrial Application & 18 \\
1.2 .2 & From Feedstock to Hydrocarbons in an Industrial Process & 22 \\
1.2 .3 & Opportunities and Challenges of the Fischer-Tropsch Process & 28 \\
1.3 & Scope and Outline of this PhD Dissertation & 30 \\
1.4 & References & 32
\end{tabular}

\begin{tabular}{llr}
2 & Fischer-Tropsch Synthesis over Iron-based Catalysts: A Review & $\mathbf{3 7}$ \\
\hline 2.1 & Introduction & 38
\end{tabular}

$2.2 \quad$ Catalyst Compositions 38

2.3 Reactions, Fe Phase Changes and Catalyst Deactivation 40

2.3.1 Reactions 40

2.3.2 Fe Phase Changes $\quad 42$

2.3.3 Catalyst Deactivation 44

$2.4 \quad$ Hydrocarbon and C Pathway Selectivity 46

2.4.1 Anderson-Schulz-Flory Distribution $\quad 47$

2.4.2 Effect of Process Conditions 48

2.4.2.1 Reaction Temperature 48

2.4.2.2 Input $\mathrm{H}_{2}$ :CO Ratio 48

2.4.2.3 Gas Space Velocity $\quad 49$

2.4.2.4 Reaction Pressure $\quad 49$

2.4.3 Effect of Alkali-S Promotion 50

2.4.3.1 General $\mathrm{H}_{2}$ and CO Adsorption Principles $\quad 50$

2.4.3.2 Alkali in the Fischer-Tropsch Synthesis Reaction 52

2.4.3.3 S in the Fischer-Tropsch Synthesis Reaction 54

2.4.3.4 Alkali-S in the Fischer-Tropsch Synthesis Reaction 57

2.4.4 Comparison of $\mathrm{Fe}$ and $\mathrm{Co}$ as Fischer-Tropsch Synthesis Active $\begin{array}{ll}\text { Transition Metals } & 59\end{array}$ 
$2.5 \quad$ Fe Carbides in the Fischer-Tropsch Synthesis Reaction 60

2.5.1 “ $\varepsilon$-carbides" ( $\varepsilon$-Fe ${ }_{3} \mathrm{C}$ and $\left.\eta-\mathrm{Fe}_{2} \mathrm{C}\right) \quad 60$

2.5.2 The Hägg Carbide $\left(\chi-\mathrm{Fe}_{5} \mathrm{C}_{2}\right) \quad 64$

2.5.3 Cementite $\left(\theta-\mathrm{Fe}_{3} \mathrm{C}\right) \quad 65$

2.5.4 The Eckström-Adcock Carbide $\left(\theta-\mathrm{Fe}_{7} \mathrm{C}_{3}\right) \quad 66$

2.5.5 Fe Carbide Hyperfine Fields $\quad 67$

$\begin{array}{lll}2.5 .6 & \text { Formation of the Fe Carbides } & 70\end{array}$

2.5.6.1 Formation of $\theta-\mathrm{Fe}_{7} \mathrm{C}_{3} \quad 75$

$\begin{array}{lll}2.6 & \text { Summary } & 75\end{array}$

$\begin{array}{lll}2.7 & \text { Acknowledgements } & 78\end{array}$

$\begin{array}{lll}2.8 & \text { References } & 78\end{array}$

3 Identification of Iron Carbides in $\mathrm{Fe}(-\mathrm{Na}-\mathrm{S}) / \alpha-\mathrm{Al}_{2} \mathrm{O}_{3}$ FischerTropsch Synthesis Catalysts with X-ray Powder Diffractometry and Mössbauer Absorption Spectroscopy $\quad 89$

$\begin{array}{lll}3.1 & \text { Introduction } & 90\end{array}$

3.2 Experimental Methods 92

3.2.1 Catalyst Preparation $\quad 92$

3.2.1.1 Catalyst Synthesis $\quad 92$

3.2.1.2 Catalyst Calcining 93

3.2.2 Catalyst Reduction and Carburization 94

3.2.2.1 Catalyst Reduction $\quad 94$

3.2.2.2 Catalyst Carburization 94

3.2.2.3 Catalyst Carburization at 25 bar 94

3.2.3 Catalyst Characterization 95

3.2.3.1 Application of Transmission Electron Microscopy 95

3.2.3.2 Application of Thermogravimetric Analysis - Mass Spectrometry 95

3.2.3.3 Application of Mössbauer Absorption Spectroscopy 96

3.2.3.4 Application of X-ray Powder Diffractometry 96

3.2.4 Application of Rietveld Quantitative Phase Analysis 96

3.3 Results and Discussion 99 
3.3.1 Probing Morphological Changes and Carbon Deposition with Transmission Electron Microscopy and Thermogravimetric Analysis - Mass Spectrometry

3.3.2 Phase Quantification with X-ray Powder Diffractometry/Rietveld Quantitative Phase Analysis and Mössbauer Absorption Spectroscopy

3.3.2.1 The Effect of $\mathrm{H}_{2}$ Reduction

3.3.2.2 The Effect of the Carburization Temperature 114

3.3.2.3 The Effect of Na-S Promotion 122

3.3.2.4 Formation of the $\theta-\mathrm{Fe}_{7} \mathrm{C}_{3}$ Phase 124

3.3.2.5 Changes in the Fe Phases' Crystallite Diameters 126

3.3.3 Assignment of Fe Carbide Properties with X-ray Powder Diffractometry/Rietveld Quantitative Phase Analysis and Mössbauer Absorption Spectroscopy

3.3.3.1 Fe Carbide Lattice Parameters and Hyperfine Fields 130

3.3.3.2 Fe Carbide C Atom Order and Contents 134

$\begin{array}{lll}\text { 3.3.3.3 Fe Carbide Diffraction Patterns } & 137\end{array}$

$\begin{array}{lll}3.4 & \text { Conclusions } & 137\end{array}$

3.5 Acknowledgements 139

$\begin{array}{lll}3.6 & \text { References } & 139\end{array}$

$4 \quad$ On the Role of Iron Carbides in the Fischer-Tropsch Synthesis with $\mathrm{Fe}(-\mathrm{Na}-\mathrm{S}) / \alpha-\mathrm{Al}_{2} \mathrm{O}_{3}$ Catalysts $\quad 149$

$4.1 \quad$ Introduction 150

4.2 Experimental Methods 152

4.2.1 Catalyst Preparation 152

4.2.2 Catalyst Testing 152

4.2.3 Catalyst Characterization 153

$4.3 \quad$ Results and Discussion 154

4.3.1 Morphological Changes Probed with Transmission Electron Microscopy 155

4.3.2 Phase Quantification with X-ray Powder Diffractometry/Rietveld Quantitative Phase Analysis 158 
4.3.2.1 Fe Phase Rietveld Quantitative Phase Analysis Quantification Changes

4.3.2.2 Fe Carbide Lattice Changes

4.3.2.3 Assignment of Fe Carbide Subtypes

4.3.2.4 Changes in Fe Phases' Crystallite Diameters 175

$\begin{array}{lll}\text { 4.3.3 Catalytic Performance } & 178\end{array}$

4.3.3.1 The Effect of Na-S Promotion 179

4.3.3.2 The Effect of Used Catalyst 182

4.3.3.3 The Role of the Fe Carbides and their Subtypes in Fischer-Tropsch Synthesis Hydrocarbon Selectivity 185

4.3.3.4 Behavior of the 340 h.p. Catalysts 186

$\begin{array}{lll}4.4 & \text { Conclusions } & 188\end{array}$

$\begin{array}{lll}4.5 & 190\end{array}$

$\begin{array}{lll}4.6 & \text { References } & 190\end{array}$

$5 \quad$ On the Effect of the Reaction Pressure in Fischer-Tropsch Synthesis with $\mathrm{Fe}(-\mathrm{Na}-\mathrm{S}) / \alpha-\mathrm{Al}_{2} \mathrm{O}_{3}$ Catalysts $\quad 197$

$5.1 \quad$ Introduction 198

5.2 Experimental Methods 200

5.2.1 Catalyst Preparation 200

5.2.1.1 Catalyst Synthesis 200

5.2.1.2 Catalyst Calcining 201

5.2.1.3 Catalyst Reduction 201

5.2.2 Catalyst Testing 202

5.2.3 Catalyst Characterization 202

5.2.3.1 Quasi-In-situ Mössbauer Absorption Spectroscopy 203

$5.3 \quad$ Results and Discussion 203

5.3.1 Morphological Changes Probed with Transmission Electron Microscopy 206

5.3.2 Phase Quantification with X-ray Powder Diffractometry/Rietveld Quantitative Phase Analysis 207

5.3.2.1 Crystalline Fe Phase Quantifications 211 
5.3.2.2 Fe Phase Lattice Changes 216

$\begin{array}{ll}\text { 5.3.3 Catalytic Performance } & 219\end{array}$

5.3.3.1 Catalyst Activity 220

5.3.3.2 Catalyst Selectivity 224

$\begin{array}{lll}5.4 & \text { Conclusions } & 229\end{array}$

$5.5 \quad$ Acknowledgements 232

$\begin{array}{lll}5.6 & 232\end{array}$

6 Combined In-situ X-ray Powder Diffractometry/Raman Spectroscopy of Iron Carbide and Carbon Species Evolution in $\mathrm{Fe}(-\mathrm{Na}-\mathrm{S}) / \alpha-\mathrm{Al}_{2} \mathrm{O}_{3}$ Catalysts during Fischer-Tropsch Synthesis 239

$\begin{array}{lll}6.1 & \text { Introduction } & 240\end{array}$

6.2 Experimental Methods 242

6.2.1 Catalyst Preparation 242

6.2.2 Catalyst Characterization 242

6.2.2.1 Details of the In-situ Reactor Cell 242

6.2.2.2 Catalytic Reaction 243

6.2.2.3 In-situ X-ray Powder Diffractometry 244

6.2.2.4 In-situ Raman Spectroscopy 245

6.3 Results and Discussion 246

6.3.1 In-situ X-ray Powder Diffractometry 248

6.3.1.1 Fe Phase Rietveld Quantitative Phase Analysis Quantification Changes 252

6.3.1.2 Changes in Fe Phases' Crystallite Diameters 257

6.3.2 In-situ Raman Spectroscopy 259

6.3.2.1 Temporal Changes in the D- and G-band Intensities and Positions 263

6.3.2.2 Comparison to Carbon Reference Materials 273

$\begin{array}{lll}6.4 & 276\end{array}$

$\begin{array}{lll}6.5 & \text { Acknowledgements } & 278\end{array}$

$\begin{array}{lll}6.6 & \text { References } & 278\end{array}$ 


\begin{tabular}{llr}
$\mathbf{7}$ & Summary and Future Perspectives & $\mathbf{2 8 7}$ \\
\hline 7.1 & Summary & 288 \\
7.2 & Future Perspectives & 300 \\
7.3 & References & 301
\end{tabular}

\begin{tabular}{llr}
$\mathbf{8}$ & Nederlandse Samenvatting en Toekomstperspectieven & $\mathbf{3 0 3}$ \\
\hline 8.1 & Samenvatting & 304 \\
8.2 & Toekomstperspectieven & 316 \\
8.3 & Referenties & 318 \\
& & $\mathbf{3 1 9}$ \\
$\mathbf{\&}$ & List of Abbreviations & \\
\hline
\end{tabular}

$\begin{array}{ll}\text { \& Acknowledgements } & 323\end{array}$

\& List of Publications and Presentations 325

\begin{tabular}{ll}
$\&$ & Curriculum Vitae \\
\hline
\end{tabular} 
This page is intentionally left blank 


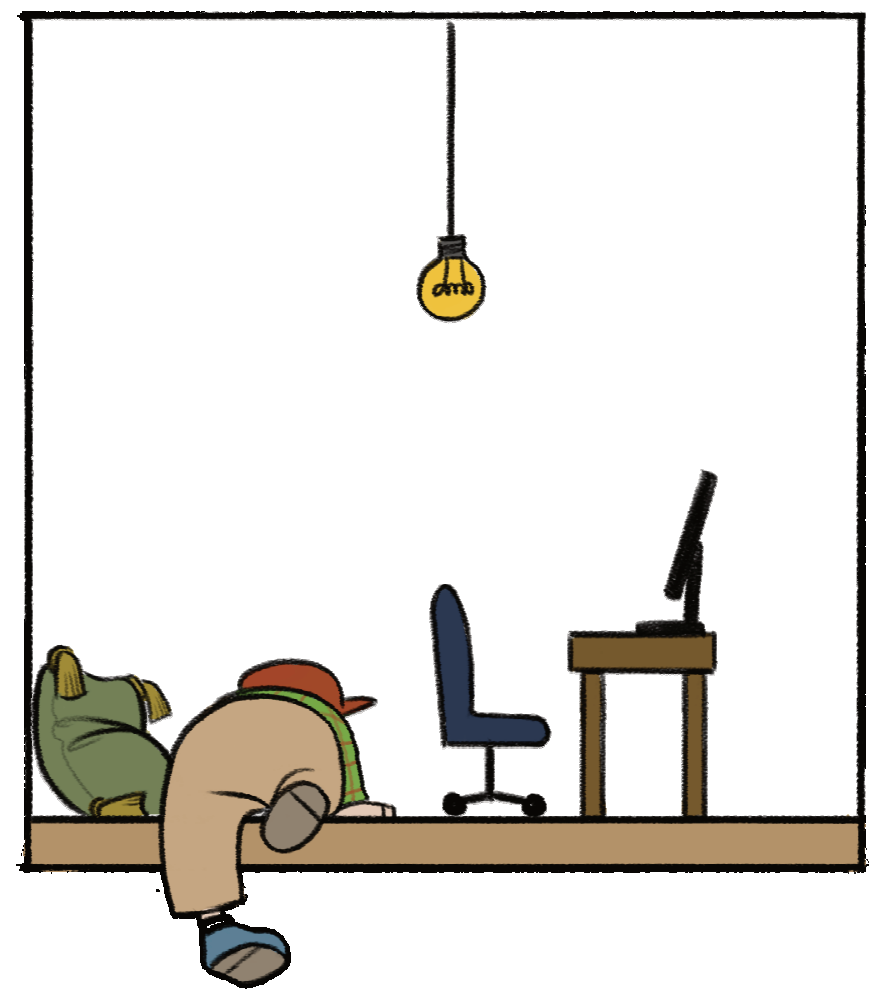




\section{Chapter 1}

\section{A General Introduction}

Heterogeneous catalysis is an industrially important field enabling a vast range of chemical reactions which otherwise would not easily take place. These catalysis reactions are utilized to produce most materials in a modern society. Many of the production processes begin with crude oil derived hydrocarbons as the feedstock. As the crude oil resources will deplete in the foreseeable future, other hydrocarbon sources are needed. Fischer-Tropsch Synthesis (FTS) reaction catalytically converts $\mathrm{CO}$ and $\mathrm{H}_{2}$ input gases into hydrocarbons over d-block transition metals, thus offering a possible future alternative source of hydrocarbons. Despite the FTS reaction already first being observed at the beginning of the $20^{\text {th }}$ century, plenty remains to be learned on this intriguing reaction. This $\mathrm{PhD}$ Dissertation concentrates on the effect of chemical promotion $\mathrm{Na}$-S on the Fe carbides in Fe-based catalysis of the FTS reaction. The Fe carbides are the catalytically active phases for the FTS reaction in the Fe-based catalyst materials. This Chapter introduces general concepts of the industrial Fischer-Tropsch Process (FTP) in which the FTS reaction is employed as part of the overall process. The aim is to relate and explain the FTS reaction's importance in a broader context. With the overall FTP, an input carbon containing feedstock is converted into refined hydrocarbon products. The history, main process steps, environmental viability and some aspects for further development of the FTP are highlighted. Finally, the scope and outline of this PhD Dissertation are presented. 


\subsection{HETEROGENEOUS CATALYSIS}

A catalyst can be defined as a chemical compound that can aid and accelerate thermodynamically feasible chemical reactions to take place. The essence of catalysis phenomenon is to lower the required activation energy for a chemical reaction, allowing it to proceed more easily than would be the case without the catalyst material (Figure 1.1). ${ }^{1}$

The major branches of the broad field of catalysis can be divided into two: heterogeneous and homogeneous catalysis. In heterogeneous catalysis the employed catalyst materials and reactants exist in distinctly different phases, e.g. as in the case of gas phase reactants being catalyzed by a solid catalyst material. With homogeneous catalysis, the catalysts do their catalyzing work in the same phase as the reactants, e.g. with both the catalyst material and the reactants indistinctively residing in a liquid phase. Essentially, for the existence of Life on Earth, enzymes perform the necessary homogenous catalysis tasks in all of the living beings. ${ }^{1}$

A vast majority of the modern industrial processes rely on heterogeneous catalysis.

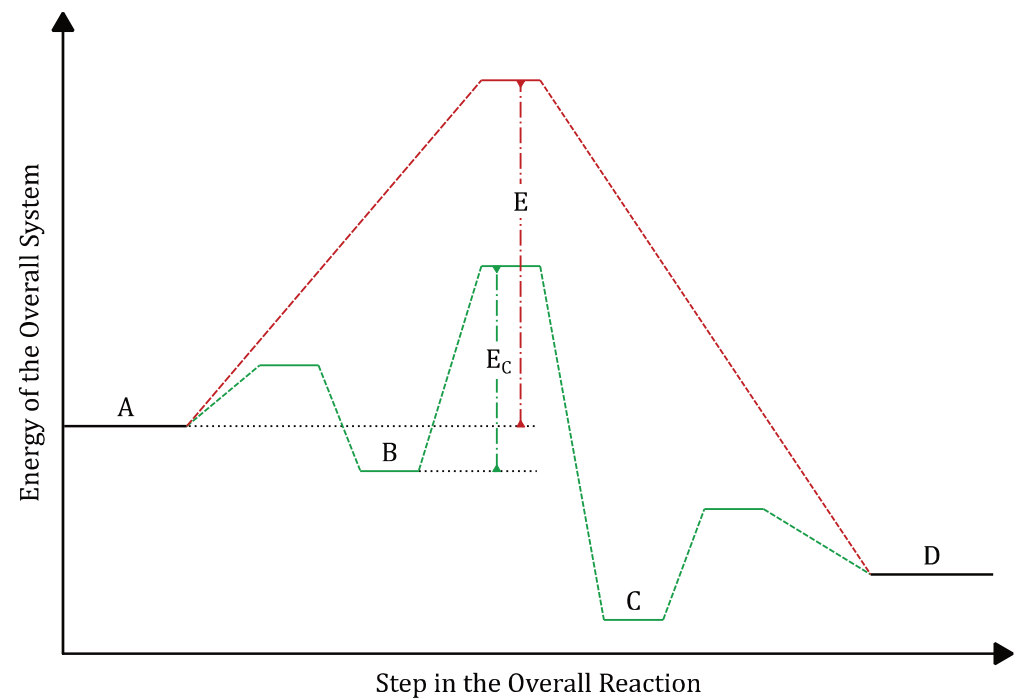

Figure 1.1

A schematic representation on the difference on the activation energies and reaction steps between uncatalysed $(\mathrm{E}, \mathrm{red})$ and catalyzed $\left(\mathrm{E}_{\mathrm{c}}\right.$, green) chemical reactions. With catalyzed chemical reactions: reactants for the chemical process (A) adsorb on the catalyst (B). The catalyst material lowers the activation energy for the chemical reaction between the adsorbed reactants so that $\mathrm{E}_{\mathrm{C}}<\mathrm{E}$ and the chemical reaction can proceed readily to form adsorbed reaction products (C) on the catalyst surface. The adsorbed products further desorb from the catalyst to give the chemical products of the overall reaction (D). Figure adapted from reference [1]. 
To name few examples: $\mathrm{NH}_{3}$ synthesis with Fe-based catalyst materials via the HaberBosch Process is the backbone in producing fertilizers for agriculture. Each modern car nowadays is equipped with catalyzators removing pollutants, such as $\mathrm{NO}_{\mathrm{x}}$ and $\mathrm{CO}$, from the engine exhaust fumes. Crude oil refineries catalytically process the input crude into ready-to-use transportation fuels for the cars and airplanes, as well as to many other petrochemical products. Furthermore, chemical building blocks used as input materials for further chemical production processes, such as preparing plastics, are derived from crude oil via catalytic refinery processes. ${ }^{1}$

The general requirements and design aspects for an industrially useful heterogeneous catalyst material can be listed, as after Le Page et al.:2

Activity - High activity in converting the reactants to reaction products, or the catalyst is able to enable the desired chemical reaction at lower reaction temperatures than without a catalyst.

Selectivity - High selectivity for the reactants to be converted only, or as much as possible, to the desired reaction products.

Stability - The catalyst should change very little during the reaction it is catalyzing. In practice, the catalyst materials degrade in time under the reaction conditions, eventually losing their catalytic capabilities.

Morphology - The external morphological features, such as the shape and size of the catalyst particle, have to suit the reaction and reactor in which it is used. Various shapes of catalyst materials as e.g. rings, beads or spherical powders, are employed in industry for matching the requirements for the reaction and the catalysis reactors they are used in.

Mechanical strength - As is especially true for the heterogeneous catalyst materials, they should maintain their shape and form without degradation in the reaction they are employed in.

Thermal characteristics - Depending on the requirements of the catalyzed reaction, the catalysts thermal properties should match the reaction. For example, for ensuring a uniform catalyst reactor bed temperature in the reactor.

Regenerability - The catalyst materials, in practice, are not indefinitely stable and eventually deactivate. The catalysts should be regenerable to their (near-)original state, making the catalyst replacement as infrequent as possible.

Reproducibility - The preparation of the catalyst materials should be reproducible from preparation batch to batch. The catalyst materials should also be reproducibly scalable from lab to the industrial scale.

Originality - For patenting and making the development of the catalyst materials economically sensible: the catalyst materials should be sufficiently original or 
improved from the previously devised catalyst materials.

Cost - The preparation of the catalyst material should be feasible on an industrial scale. This in order of having an economically competitive overall industrial process utilizing the catalyst.

Naturally, developing such catalyst materials satisfying all the required properties is not a very straightforward process. Fundamental research on understanding and further improving catalyst materials is still needed in many of the areas in which heterogeneous catalysis is applied.

One such area is the catalysis of Fischer-Tropsch Synthesis (FTS) reaction, a reaction that converts catalytically the input $\mathrm{H}_{2}$ : $\mathrm{CO}$ gas mixture (i.e. syngas) into a mixture of hydrocarbons. The FTS reaction possibly offers in its part one of the future solutions for replacing the crude oil from Earth's crust as the hydrocarbon source for industrial processes. The first observations on catalytic conversion of $\mathrm{CO}$ and $\mathrm{H}_{2}$ reactants to hydrocarbons over d-block transition metals date back to the early $20^{\text {th }}$ century. Despite of all the time that has passed and the dedicated research that has been done since the first observations, a lot remains to be learned on the complex topic of the heterogeneously catalyzed FTS reaction.

Thus, to begin with, we shall embark on a short introduction on the general industrial application of the FTS reaction, from both historic and contemporary perspectives. In its industrial application, the FTS reaction is one part of the complete Fischer-Tropsch Process (FTP) where an input carbon containing feedstock is stepwise converted into refined hydrocarbon products. The main aim of the section is to give an overview on how the catalyzed FTS reaction as part of the overall industrial FTP relates to the wider society, including discussion on its environmental and economic realities.

At the end of this Chapter, the scope and outline for the following research chapters in this PhD Dissertation are laid out.

\subsection{FISCHER-TROPSCH SYNTHESIS IN INDUSTRY}

\subsubsection{A Short History of the Industrial Application}

One of the earliest observations on the catalyzed formation of liquid hydrocarbons over d-block transition metals under a flow of $\mathrm{H}_{2}$ :CO gas mixture are by Mittasch and Schneider in their work conducted for Badische Anilin \& Soda Fabrik (BASF) around $1916 .{ }^{3,4}$ In their patent the authors describe conversion of $>5$ bar gas mixture of $\mathrm{H}_{2}$ : $\mathrm{CO}$ to various saturated and unsaturated hydrocarbons and their oxygenated derivatives. As catalysts, various d-block transition metals (e.g. Cr, Co, Mn, Mo and Os) or their 
compounds were used. It was also noted, that impregnation of their transition metal catalyst materials with a basic compound in the form of an alkali metal hydroxide or carbonate (e.g. $\mathrm{KOH}$ or $\mathrm{K}_{2} \mathrm{CO}_{3}$ ) was advantageous for the hydrocarbon conversion. ${ }^{3}$

In the beginning of the 1920's, further research on the conversion of a $\mathrm{H}_{2}$ :CO gas mixture to hydrocarbons over alkalized $\mathrm{Fe}$, and $\mathrm{Co}$ and $\mathrm{Ni}$ transition metals was performed by Fischer and Tropsch while working at the Kaiser Wilhelm Institut für Kohlenforschung at Mülheim an der Ruhr.4-6 Despite the inability of Fischer and Tropsch to reproduce some of the claims in the earlier work by Mittasch and Schneider, ${ }^{6}$ the work by Fischer and Tropsch led to the basis on the industrial application of the transition metal catalyzed $\mathrm{H}_{2}: \mathrm{CO}$ conversion into hydrocarbons. Thus, the reaction became known as the Fischer-Tropsch Synthesis (FTS).4,5,7

The first application of the FTS reaction as a part of an industrial process took place in Germany, with a demonstration plant in Mülheim commissioned in 1932. Further facilities were constructed by the beginning of the World War II with a total of nine Fischer-Tropsch facilities operational by the year 1939. The incentive for employing the FTS reaction as a part of an industrial process came for the wartime Germany from a necessity. With limited access to a crude oil supply during the War, large available German coal reserves could be transformed into liquid hydrocarbon fuels in part with utilizing the FTS reaction on an industrial scale. At 1944, $7 \%$ of fuels consumed in Germany were produced with using the FTS reaction in the process. Majority of the coal-to-liquids conversion was done via coal hydrogenation, i.e. with the Bergius Process, accounting for $40 \%$ of the consumed fuels. ${ }^{8}$

After the World War II, the Allies acquired the German FTS reaction technology. Consequently, the researchers at the United States Bureau of Mines conducted a significant amount of seminal research in the post-war years on the FTS reaction. 5,9

In 1950 in South Africa, Suid Afrikaanse Steenkool en Olie (Sasol) company was formed with the aim of converting the locally available coal into hydrocarbons, in a bid to decrease South Africa's dependency on imported crude oil. The Sasol 1 FischerTropsch facility was commissioned in 1955 using American and German technology. Initial operational issues ensued with the American (Kellogg) technology and Sasol established its own research department in 1957 to solve the issues. ${ }^{8}$ A significant amount of FTS knowledge available in the open literature has come from the Sasol research ever since.

However, mostly due to the availability of cheap crude oil, interest in utilizing the FTS reaction industrially somewhat waned in the rest of the World between the 1950's post-war period up to 1970 's oil crisis. Thus, no significant industrial investment took place beyond the Sasol 1 Fischer-Tropsch facility within the period.5,9,10

The first large scale, modern industrial utilization of the FTS reaction in an industrial 
process took place in South Africa by Sasol. Incentive for the renewed industrial application of the FTS reaction in South Africa came from both the supply scarcity of crude oil due to the global 1970's oil crisis, and the international sanctions on South Africa due to Apartheid policies up to until around 1993.5,9,11-13 The Sasol 2 and Sasol 3 facilities (nowadays collectively called Sasol Synfuels) were commissioned in 19801982 to accompany the smaller Sasol 1 facility from $1955.5,9,11$ Also, the Sasol 1 has gone through several upgrades from the 1970's up to the latest expansion project between 2009-2015, which is expected to double the facility's production capacity.8,14

Besides Sasol and conversion of coal to hydrocarbons, the South African government initiated the Mossgas project in 1984 in order to search for off-shore natural gas. By 1992, a facility converting natural gas into hydrocarbons employing the FTS reaction was commissioned. ${ }^{8}$ This facility is now operated by the Petroleum Oil and Gas Corporation of South Africa (PetroSA), after a merger of the South African state owned petroleum assets in $2002 .^{8}$

Outside South Africa, the initiative for development of industrial Fischer-Tropsch facilities came from increased discoveries of natural gas reserves. ${ }^{8}$ In the cases where natural gas reserves are far from the hydrocarbon markets, liquefaction of the gas to a more energy dense form e.g. with the use of the FTS reaction in the process, can make the transportation of the produced, dense hydrocarbon products to the market region economically viable.5,8 Thus, the fundamental driving force for the industrial FTS reaction development utilizing natural gas sites is the price difference between the process feed natural gas in comparison to the FTS produced and further refined hydrocarbons. ${ }^{5}$

Following the discoveries and utilization of natural gas fields, the Dutch Royal Shell commissioned Fischer-Tropsch facilities in both Bintulu (Malaysia) in 1993 and the Shell Pearl in Ras Laffan (Qatar) in 2011. Both facilities convert natural gas into hydrocarbons with employing the FTS reaction in the process. Explosion of the air separation unit at Bintulu facility at the end of 1997 caused the facility to shut down until 2000.8 The explosion was due to fine particles from forest fires accumulating into an air separation unit in the facility. ${ }^{15}$ Sasol and Qatar Petroleum (QP) commissioned the Oryx natural gas conversion facility using the FTS reaction, in Ras Laffan (Qatar) in 2006. The Oryx facility initially encountered issues with the fragmentation of the used Co-based FTS catalyst material, which caused clogging downstream from the FTS reactor. By 2008, the Oryx facility's production was ramped up $\approx 70 \%$ of the design capacity. ${ }^{8}$

The latest industrial scale facility utilizing the FTS reaction in the process to convert natural gas has been commissioned by Chevron and Nigerian National Petroleum Corporation (NNPC) in Escravos (Nigeria) in 2014. ${ }^{5}$ Though, at least up to 2017, the 


\section{Table 1.1}

Current operational industrial scale facilities utilizing the Fischer-Tropsch Synthesis (FTS) reaction in the process for producing hydrocarbons. Details are from references:[5][8].

\begin{tabular}{|c|c|c|c|c|c|}
\hline Operator & Site & Feedstock & $\begin{array}{l}\text { Capacitya) } \\
\text { (bbl/day) }\end{array}$ & $\begin{array}{c}\text { Commission } \\
\text { Year }\end{array}$ & $\begin{array}{c}\text { Initial investment } \\
\text { incentive }\end{array}$ \\
\hline Sasol & $\begin{array}{c}\text { Sasol 1 } \\
\text { (Sasolburg, South } \\
\text { Africa) }\end{array}$ & $\begin{array}{c}\text { Initially coal, } \\
\text { currently } \\
\text { natural gas }\end{array}$ & $7000-5500$ & $1955^{\mathrm{b})}$ & $\begin{array}{l}\text { - To decrease } \\
\text { dependency on } \\
\text { crude oil imports }\end{array}$ \\
\hline Sasol & $\begin{array}{l}\text { Sasol Synfuels } \\
\text { (formerly } \\
\text { Sasol } 2 \text { \& 3) } \\
\text { (Secunda, South } \\
\text { Africa) }\end{array}$ & $\begin{array}{l}\text { Coal, later } \\
\text { also natural } \\
\text { gas }\end{array}$ & 120000 & 1980-1982" & \multirow{2}{*}{$\begin{array}{l}\text { - 1970's oil crisis } \\
\text { - Sanctions against } \\
\text { Apartheid }\end{array}$} \\
\hline PetroSA & $\begin{array}{c}- \\
\text { (formerly Mossgas) } \\
\text { (Mossel Bay, South } \\
\text { Africa) }\end{array}$ & Natural gas & 22500 & 1992 & \\
\hline Shell & (Bintulu, Malaysia) & Natural gas & 14700 & 1993 & \multirow{4}{*}{$\begin{array}{l}\text { - Conversion of } \\
\text { natural gas to } \\
\text { higher value } \\
\text { products }\end{array}$} \\
\hline $\begin{array}{c}\text { Sasol \& } \\
\text { QP }\end{array}$ & $\begin{array}{c}\text { Oryx } \\
\text { (Ras Laffan, Qatar) }\end{array}$ & Natural gas & $\left.(34000)^{c}\right)$ & 2006 & \\
\hline Shell & $\begin{array}{c}\text { Pearl } \\
\text { (Ras Laffan, Qatar) }\end{array}$ & Natural gas & 140000 & 2011 & \\
\hline $\begin{array}{l}\text { Chevron } \\
\& \text { NNPC }\end{array}$ & $\begin{array}{c}- \\
\text { (Escravos, Nigeria) }\end{array}$ & Natural gas & $\left.(34000)^{d}\right)$ & 2014 & \\
\hline
\end{tabular}

a) Estimated Fischer-Tropsch Process (FTP) design capacity at 2013 in crude oil equivalents.

b) Since the initial commissioning in 1955 , significant upgrades have taken place. ${ }^{8,14}$

c) At least up to 2008, the production was constrained to $24000 \mathrm{bbl} /$ day.

d) At least up to 2017, the site has experienced production problems. ${ }^{16,17}$

Chevron-NNPC Escravos Fischer-Tropsch facility has experienced production issues and the plant is under continued optimization of the processes. ${ }^{16,17}$

To summarize the discussion so far, the driving force for industrial scale application of the FTS reaction in the process for producing hydrocarbon products has mainly either been:

i) to decrease dependency on the availability of the hydrocarbons from crude oil (Germany 1930-1945, South Africa 1955-1993), or

ii) economic incentive in valorizing natural gas to higher valued, more energy dense, liquid hydrocarbons (chemical companies from around 1990's onwards). 
The currently operational industrial scale Fischer-Tropsch facilities, their initial investment incentive and FTP design capacities at 2013 are summarized in Table 1.1.

From the 2010's onwards, new directions for the industrial utilization of the FTS reaction are emerging as current companies (e.g. Velocys, formerly Oxford Catalysts, CompactGTL, Greyrock Energy) pursuing "scaling down" of the Fischer-Tropsch facilities mature. ${ }^{5}$ Small-scale Fischer-Tropsch facilities may be better suited in utilizing carbon from biomass and/or municipal waste, as the FTS reaction feedstock. The first commercial small-scale FTS facility in the United States for converting municipal waste into liquid hydrocarbons via the FTS reaction is expected to be commissioned within 2020.18 The new facility employs cost effective FTS technology developed by BP and Johnson Matthey (JM). Also, stranded and small natural gas sources e.g. on off-shore oil platforms, where natural gas is normally flared, may be utilized towards hydrocarbon production with small-scale Fischer-Tropsch facilities in the future. A specific future opportunity for small-scale Fischer-Tropsch facilities could be to address the evergrowing problem with recycling municipal plastic waste, ${ }^{19-21}$ via the $\mathrm{H}_{2}: \mathrm{CO}$ gas mixture, ${ }^{21,22}$ into hydrocarbons usable in industrial processes. Significant benefits for the small-scale Fischer-Tropsch facilities are the decreased capital investment, thus decreased risk in investment, and the ability to adapt to the local availability of the carbon feedstock. ${ }^{5,23}$

\subsubsection{From Feedstock to Hydrocarbons in an Industrial Process}

In the previous section, the commissioning of several Fischer-Tropsch facilities was discussed. However, by no means is the FTS reaction the only catalytic process taking place in these manufacturing complexes, when the raw feedstock conversion into hydrocarbon products takes place.

The complete Fischer-Tropsch Process (FTP) utilizing the FTS reaction as one of the chemical process steps, from the input feedstock into refined chemical products, can be simplified to general steps of (Figure 1.2):8

i) Feedstock-to-Syngas - conversion of carbon containing feedstock into a mixture of $\mathrm{H}_{2}$ : $\mathrm{CO}$ (i.e. to synthesis gas or syngas),

ii) Syngas-to-Syncrude - conversion of the produced $\mathrm{H}_{2}$ : $\mathrm{CO}$ mixture into a mixture of hydrocarbons and hydrocarbon oxygenate derivatives (i.e. to synthetic crude or syncrude) using the catalyzed FTS reaction,

iii) Syncrude-to-Products - processing, separating and upgrading (i.e. refining) the desired hydrocarbons or oxygenates from the syncrude depending on their desired use (i.e. transportation fuels or feedstock for chemical industry). 


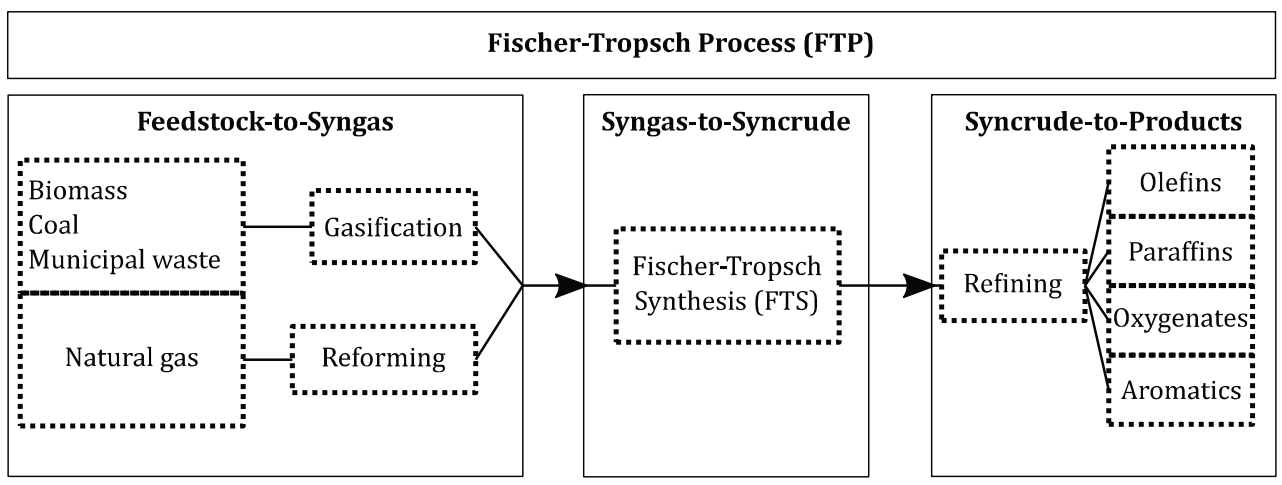

\section{Figure 1.2}

The overall Fischer-Tropsch Process (FTP) for converting carbon containing feedstocks into various product hydrocarbons utilizing the Fischer-Tropsch Synthesis (FTS) reaction in the process.

The overall FTP is similar to crude oil refining with the exception of the syncrude being "made up" from a carbon containing feedstock via syngas with the FTS reaction, instead of using crude oil from Earth's crust. In the following sections, primary contributions from each process step to the total industrial FTP are briefly considered in order to highlight some of their important aspects. The fundamentals of the FTS reaction catalysis will be discussed more in depth in the later research chapters.

Feedstock-to-Syngas. The overall FTP, from the input feedstock to refined hydrocarbon products, can be named after the feedstock: Biomass-to-Liquids (BTL), Coal-to-Liquids (CTL), Gas-to-Liquids (GTL) and Waste-to-Liquids (WTL), or collectively Anything-to-Liquids (XTL). Syngas can be produced from a solid, carbon containing material (BTL, CTL, WTL) by heating the feedstock material under $\mathrm{O}_{2}$ and $\mathrm{H}_{2} \mathrm{O}$ without burning the material (i.e. gasification) or via reforming (e.g. with Steam Methane Reforming, SMR) natural gas as in the case of GTL. ${ }^{5}$

The $\mathrm{CO}_{2}$ footprint (i.e. the measure of greenhouse gas emissions in $\mathrm{CO}_{2}$ equivalents) of the total FTP is in part depended on the syngas production and the type of the used feedstock. ${ }^{5}$ The FTS reaction can be idealized as (Reaction 1.1),

$$
\mathrm{CO}+2 \mathrm{H}_{2} \rightarrow-\left(\mathrm{CH}_{2}\right)-+\mathrm{H}_{2} \mathrm{O} \quad \text { Idealized FTS Reaction (1.1) }
$$

where the hydrocarbons are polymerized as $-\left(\mathrm{CH}_{2}\right)-$ units and $\mathrm{O}$ atom from $\mathrm{CO}$ is removed as $\mathrm{H}_{2} \mathrm{O}$. This ideal synthesis consumes input elements in ratio of 4:1 H:C (i.e. in molecular ratio $2: 1 \mathrm{H}_{2}: \mathrm{CO}$ ).

In Table 1.2, the nominal and effective $\mathrm{H}: \mathrm{C}$ ratios and $\mathrm{CO}_{2}$ footprints are given for the different FTP feedstocks. The effective $\mathrm{H}: \mathrm{C}$ ratio is the actual ratio of elemental $\mathrm{H}: \mathrm{C}$ 


\section{Table 1.2}

Estimated $\mathrm{CO}_{2}$ footprint for the total Fischer-Tropsch Process (FTP) as depending only on the process input feedstock. The table is modified from reference [5].

\begin{tabular}{|c|c|c|c|c|c|c|c|c|}
\hline \multirow[t]{2}{*}{ Feedstock } & \multicolumn{5}{|c|}{$\begin{array}{l}\text { Elemental Analysis } \\
\text { (wt-\%) }\end{array}$} & \multicolumn{2}{|c|}{$\begin{array}{c}\mathrm{H}: \mathrm{C} \\
\text { (elemental ratio) }\end{array}$} & \multirow[t]{2}{*}{$\begin{array}{l}\mathrm{CO}_{2} \text { footprint due to feed } \\
\left(\mathrm{CO}_{2} \text { :Hydrocarbons } \mathrm{kg}: \mathrm{kg}\right)\end{array}$} \\
\hline & $\mathrm{C}$ & $\mathrm{H}$ & $\mathrm{N}$ & $\mathrm{S}$ & 0 & Nominal & Effective & \\
\hline Natural gas $\left.{ }^{\mathrm{b}}\right)$ & 74.9 & 25.1 & 0 & $<0.1$ & 0 & 4.00 & 4.00 & 0.00 \\
\hline Wastec) & 53.3 & 8.2 & 4.9 & 0.1 & 33.5 & 1.83 & 0.65 & 3.96 \\
\hline Coald) $^{\text {d }}$ & 75.7 & 5.0 & 1.2 & 1.0 & 17.1 & 0.80 & 0.40 & 4.80 \\
\hline Biomasse) & 50.1 & 6.1 & 0.9 & 0.1 & 42.8 & 1.45 & 0.12 & 5.75 \\
\hline
\end{tabular}

a) Based in ideal Fischer-Tropsch Synthesis (FTS) reaction i.e. $\mathrm{CO}+2 \mathrm{H}_{2} \rightarrow-\left(\mathrm{CH}_{2}\right)-+\mathrm{H}_{2} \mathrm{O} . \mathrm{CO}_{2}$ footprint estimated from carbon efficiency using $\mathrm{CO}_{2}$ equivalent.

b) Dry natural gas with all the hydrocarbons removed.

c) Municipal waste.

d) Mean values over various coal types.

e) Switchgrass.

available for hydrocarbon formation in the FTS reaction after all of the heteroatoms $(\mathrm{N}$, $\mathrm{O}, \mathrm{S}$ ) in the feedstock are hydrogenated and subsequently removed during a syngas cleaning step. ${ }^{5,24}$ As can be seen from the Table 1.2, the $\mathrm{CO}_{2}$ footprints are in the order GTL $<$ WTL $<$ CTL $<$ BTL. The increase in the $\mathrm{CO}_{2}$ footprint due to input feedstock fundamentally comes from the necessity of adding more $\mathrm{H}$ to the system for satisfying the required FTS reaction stoichiometry. That is, from satisfying the effective elemental $\mathrm{H}: \mathrm{C}$ ratio of $\approx 4$, for the hydrocarbon synthesis via the FTS reaction.5,24

To further highlight the use of different raw carbon feedstocks, only GTL can actually be a net producer of $\mathrm{H}_{2} \mathrm{O}$ as per 1 mole of $\mathrm{CO}$ consumed 1 mole of $\mathrm{H}_{2} \mathrm{O}$ is produced in the FTS reaction without need for extra added H. ${ }^{5}$ BTL, CTL and WTL will require input of $\mathrm{H}$ atoms in a one way or another, e.g. from commonly used Water-gas Shift (WGS) reaction converting $\mathrm{H}_{2} \mathrm{O}+\mathrm{CO}$ to $\mathrm{H}_{2}+\mathrm{CO}_{2}$ or possibly from $\mathrm{H}_{2} \mathrm{O}$ electrolysis, for satisfying the need for the effective input $\mathrm{H}: \mathrm{C}$ ratio of $\approx 4$. This in practice means input of fresh water, thus making the non-GTL processes rather water intensive. ${ }^{5}$ For industrial Fischer-Tropsch facilities, the benchmark in total FTP water management is the zero fresh water input Shell Pearl GTL facility. In the facility, the acidic and corrosive water produced in the FTS reactor units is purified and reused for the needs of the facility and even for some irrigation of the plant surroundings. ${ }^{5,25}$

Although, despite the very simplified and idealized discussion presented here on the different feedstocks for the FTP, the point is: BTL and WTL processes via the FTS reaction are often mentioned as sustainable pathways for hydrocarbon production, but their low effective $\mathrm{H}: \mathrm{C}$ ratio makes them more demanding feedstocks than GTL for the purpose. To make up the $\mathrm{H}: \mathrm{C}$ deficiency, the added $\mathrm{H}$ will need to come from an 
environmentally sustainable process for the BTL and WTL processes to be able to compete against the GTL in environmental viability and in minimal $\mathrm{CO}_{2}$ footprint. Furthermore, biomass and waste need to be gathered to the Fischer-Tropsch facility in a sufficient quantity implying added transportation costs, decreasing the availability of the feedstocks with added environmental penalty. ${ }^{5}$

Naturally, the Feedstock-to-Syngas step is not the only step affecting the FischerTropsch facility's $\mathrm{CO}_{2}$ footprint. Although, the step can have an significant impact on the total footprint. ${ }^{5,26}$ Each process step, from feedstock to refined hydrocarbon products should be optimized by the Green Chemistry principles: avoiding waste, maximizing atom economy, increasing energy efficiency and designing less hazardous chemical syntheses. ${ }^{5}$ In other words, from each process input CO molecule maximum amount elemental $\mathrm{C}$ should end up to the hydrocarbon products with minimum energy used, and waste products formed.

The carbon efficiencies for the total FTP from feedstock into hydrocarbon products are $\approx 75 \%$ for $\mathrm{GTL}^{5}$ and $\approx 28-34 \%$ for CTL. ${ }^{5,24}$ For comparison, in crude oil refineries the carbon efficiency is estimated to be about $89 \% .^{5}$ The production of purified syngas accounts for around 30-50 \% of the capital costs for GTL- and 60-70\% for CTL-based processes. $^{8,27}$ The expensive syngas should be turned into hydrocarbon products as efficiently as possible also from the viewpoint of economic viability of a FischerTropsch facility.

Syngas-to-Syncrude. Within the step, the FTS reaction is used for converting the prepared $\mathrm{H}_{2}$ : $\mathrm{CO}$ syngas mixture into syncrude with various hydrocarbons. Two important parameters for the step are i) the used catalyst material and ii) the used reaction conditions. The aim with both is to steer the hydrocarbon synthesis towards the desired products with maximum $\mathrm{C}$ efficiency. General requirements for an industrially good FTS reaction catalyst material are: ${ }^{5}$

i) Activity towards FTS - the less of catalyst material is required for converting the input CO to product hydrocarbons, the lower the associated costs are. Alternatively, the catalyst can also be negligibly cheap.

ii) Selectivity - the FTS reaction inherently produces a slate of various hydrocarbons. The narrower the selectivity range towards the desired products is, the better. This potentially allows the whole FTP to be simplified (e.g. less gas looping, less separation and purification processes).

iii) Stability - the longer the catalyst stays active with a stable selectivity towards the desired hydrocarbons, the less of the catalyst material is needed. This, in turn lowers the associated costs. Also, an important point is the mechanical stability of catalyst and not just the catalytic stability. No active catalyst 
Table 1.3

General compositions of industrially produced syncrude compositions for Fischer-Tropsch Synthesis (FTS) reaction types applied in industry. The table is modified from reference [5].

\begin{tabular}{|c|c|c|c|c|}
\hline \multirow[t]{2}{*}{$\begin{array}{l}\text { Hydrocarbon Chain } \\
\text { Length }\end{array}$} & \multirow[t]{2}{*}{ Hydrocarbon Type } & \multicolumn{3}{|c|}{$\begin{array}{l}\text { Syncrude Composition } \\
(w \mathrm{w}-\%)^{\mathrm{a})}\end{array}$} \\
\hline & & Fe-HTFT & Fe-LTFT & Co-LTFT \\
\hline $\mathrm{C}_{1}$ & Methane & 12.7 & 4.3 & 5.6 \\
\hline \multirow[t]{2}{*}{$\mathrm{C}_{2}-\mathrm{C}_{4}$} & Olefin & 26.8 & 7.0 & 3.5 \\
\hline & Paraffin & 7.5 & 2.8 & 2.8 \\
\hline \multirow[t]{2}{*}{$\mathrm{C}_{5}-\mathrm{C}_{10}$} & Olefin & 25.8 & 7.7 & 7.8 \\
\hline & Paraffin & 4.3 & 3.3 & 12.0 \\
\hline \multirow[t]{2}{*}{$\mathrm{C}_{11}-\mathrm{C}_{22}$} & Olefin & 4.8 & 5.7 & 1.1 \\
\hline & Paraffin & 0.9 & 13.5 & 20.8 \\
\hline \multirow[t]{2}{*}{$>\mathrm{C}_{22}$} & Olefin & 1.6 & 0.7 & 0.0 \\
\hline & Paraffin & 0.4 & 49.2 & 44.6 \\
\hline \multirow[t]{2}{*}{$>\mathrm{C}_{5}$} & Aromatic & 3.2 & 0.0 & 0.0 \\
\hline & Oxygenate & 2.3 & 1.6 & 0.2 \\
\hline $\mathrm{C}_{1}-\mathrm{C}_{5}$ & Alcohol & 4.5 & 3.9 & 1.4 \\
\hline \multirow[t]{2}{*}{ (Aqueous products) } & Carbonyl & 3.9 & 0.0 & 0.0 \\
\hline & Carboxylic acid & 1.3 & 0.3 & 0.2 \\
\hline
\end{tabular}

Olefin = at least one unsaturated $\mathrm{C}-\mathrm{C}$ bond, paraffin = all $\mathrm{C}-\mathrm{C}$ bonds are saturated.

a) Excluding $\mathrm{CO}_{2}$.

material should leach downstream from the catalytic FTS reactor as its pieces will cause issues and also need to be separated from the hydrocarbon products.

Beyond the intrinsic properties of the used catalyst material, the reaction conditions (e.g. reaction temperature, input feed $\mathrm{H}_{2}$ : $\mathrm{CO}$ ratio and pressure) affect how the catalyst behaves and which hydrocarbon products are formed. In the currently operational Fischer-Tropsch facilities, either Low-temperature Fischer-Tropsch $\left(200-250^{\circ} \mathrm{C}\right.$, LTFT) or High-temperature Fischer-Tropsch $\left(320-375^{\circ} \mathrm{C}, \mathrm{HTFT}\right)$ have been used with reaction pressures of 15-60 bar. ${ }^{5}$

So far, only Fe and Co have been employed as the active transition metals in the FTS reaction catalysts applied in the industrial Fischer-Tropsch facilities. Fe-based catalyst materials have been used on both LTFT and HTFT, while Co has only been used for LTFT. Table 1.3 summarizes the generic compositions for the industrially produced syncrude compositions obtained from the used FTS reaction types. ${ }^{5}$ The hydrocarbons formed in the FTS reaction are primarily straight-chained, but also minor amounts of methyl-branched hydrocarbons can be observed. Low amounts of oxygenated hydrocarbons and at high temperature, aromatics, may also form in the FTS reaction. ${ }^{28}$

The most significant differences from Table 1.3 are: Fe-HTFT produces $\approx 53$ weight 
percent (wt-\%) of $\mathrm{C}_{2}-\mathrm{C}_{10}$ olefins (unsaturated hydrocarbons) in comparison to $\approx 11$ $15 \mathrm{wt}$ \% from the Fe- or Co-LTFT. Furthermore, Fe-HTFT produced syncrude contains $\approx 1 \mathrm{wt}-\%>\mathrm{C}_{11}$ paraffins (saturated hydrocarbons) against $\approx 63-65 \mathrm{wt}-\%$ obtainable from the Fe- or Co-LTFT reactions. This means, Fe-HTFT is best suited for short-chained olefin-rich syncrude while the low temperature Fe- or Co-LTFT favors long-chained, paraffin-rich syncrude. Ideally, no low economic value $\mathrm{CH}_{4}$ should be produced and its production should be suppressed. Especially with GTL, the production of $\mathrm{CH}_{4}$ is merely reversing the first FTP step of Feedstock-to-Syngas with no added value.

In terms of catalyst stabilities, Fe-based FTS catalyst materials can be stable up to $\approx 4$ months for LTFT and $\approx 1.5$ months for HTFT while Co-based LTFT catalysts can be used for up to a couple of years. Co-based catalyst material used at the Shell Bintulu facility, may have lasted with acceptable stability for 8 years without a catalyst regeneration step. ${ }^{5}$ Especially for the Fe-based catalyst materials, the stability aspects have room for further improvement. A major issue for the Fe-based FTS catalysts, especially in the HTFT application, is the fragmentation of the catalyst material into fine powders. ${ }^{5,9,27-30}$

Syncrude-to-Products. The final step for a Fischer-Tropsch facility and for the total FTP, is to process the syncrude into final hydrocarbon products. This is the step where the most of the value addition to the FTP occurs with only $\approx 10 \%$ of the facility's capital costs. ${ }^{8}$ Similar chemical processes can be applied to syncrude refining as are applied to crude oil refining e.g. fractional distillation, hydrocracking, hydrotreating and oligomerization. The most significant differences in syncrude in comparison to crude oil are, that syncrude can contain: ${ }^{8}$

i) multiple product phases: gaseous, organic liquid and aqueous. Organic solids can also be part of the product mixture in case of the LTFT,

ii) higher concentration of oxygenates, olefins and linear products, and

iii) metal carboxylates.

Crude oil does not contain significant amounts of olefins and syncrude, unlike crude oil, does not contain $\mathrm{N}$ or $\mathrm{S}$ impurities. These impurities are removed during the Feedstock-to-Syngas process step, when syngas is purified, as discussed earlier. All of the listed differences essentially make crude oil and syncrude refining facilities incompatible with each other and all of the Fischer-Tropsch facilities listed in Table 1.1 refine their syncrude on site. ${ }^{8}$

In the past, products from a Fischer-Tropsch facility were steered towards assuring energy security, i.e. by producing fuels in German (1939-1945), and in South African facilities until about 1993. The post-1993 Fischer-Tropsch facilities aim for production 
of hydrocarbons with high added value used as is or as chemical building blocks in production other chemical compounds. These developments mainly follow from the global political and economic events. $5,8,31$

The final item worth attention is that the so-called paraffinic fuels derived from the syncrude produce less tail pipe emissions and burn cleaner than the corresponding crude oil derived fuels. As the $\mathrm{S}$ and $\mathrm{N}$ heteroatoms are cleaned during the Feedstockto-Syngas step, the FTP produced fuels burn $\mathrm{NO}_{\mathrm{x}}$ and $\mathrm{SO}_{\mathrm{x}}$ free and release less particulate matter. In addition, the FTP derived fuels have several other favorable liquid fuel properties. ${ }^{26,32-34}$

\subsubsection{Opportunities and Challenges for the Fischer-Tropsch Process}

From the discussed historical developments as well as from the aspects on the industrial application of the FTP, there are some reasons in favor and against continued investment and research on the field, as well as some opportunities for improvement. ${ }^{5}$ Some of them can be summarized as follows:

\section{Arguments in favor:}

Strategic - diversification from reliance on crude oil availability either due to political incentive (short-term), or due to eventual depletion and scarcity of the non-renewable crude oil supplies from Earth's crust (long-term).

Economic - valorization of input feedstock to value-added hydrocarbon products of high-quality paraffinic fuels and various hydrocarbons for chemical industries.

Syngas-to-Syncrude directly - the FTS reaction employed in FTP is a direct step from syngas to syncrude while most of the alternative hydrocarbon synthesis routes go via an intermediate chemical species. ${ }^{35}$ Potentially, direct routes for the Syngas-to-Syncrude process step can be less complex to realize in comparison to going via an intermediate chemical species.

\section{Arguments against:}

Complexity - the more parts in the complex FTP need to function together, the more likely there are problems. Several Fischer-Tropsch facilities have experienced start-up and operational problems, the Chevron-NNPC Escravos GTL plant being the latest. ${ }^{16,17}$

High capital costs - a Fischer-Tropsch facility is a multi-billion US\$ investment. High capital costs make failures expensive and limit the amount of parties that would be interested and capable of developing and utilizing the complete FTP on industrial scale.

Price of crude oil - the economic viability of the total FTP is determined by the 
price differences and processing costs between FTP feedstocks (i.e. coal, biomass, natural gas and waste) against crude oil as the feedstock for traditional crude oil refineries. Average break even crude oil price estimates for FTP against crude oil refining per barrel are: for GTL $\approx 30$ US\$, for CTL $\approx 65$ US\$ and for BTL $\approx 100$ US\$ (in 2011 terms). ${ }^{31}$ As crude oil prices may fluctuate wildly, it can be demanding to predict the competitiveness of a Fischer-Tropsch facility.

\section{Opportunities for improvement:}

Engineering - simplify and reduce the complexity of the Fischer-Tropsch facilities by streamlining the FTP and instead of re-using old technologies, innovation to create new solutions.

Fundamental research - even after 100 years, the FTS reaction at the heart of the FTP is still relatively poorly understood. This lack of fundamental understanding has led to initial production problems in several Fischer-Tropsch facilities. Continued research in the fundamental understanding of the FTS reaction is still needed.

Syncrude flexibility - via the FTS reaction, the produced syncrude can be steered towards desired hydrocarbons. Ideally, the syncrude synthesis should be as specific hydrocarbon product selective as possible, with minimum amount of $\mathrm{CH}_{4}$ produced, leaving room for catalyst material development.

Fe catalyst stability - Fe-based catalyst is usable for LTFT $\lesssim 4$ months, and for HTFT $\approx 1.5$ months, while Co-based LTFT catalyst can be used for a few years. Thus, especially improving Fe-based catalyst materials' stability is of interest.

Small-scale facilities - can offer a possible solution for high risk/high capital investment problem. Smaller-scale Fischer-Tropsch facilities are far cheaper to construct with less penalty from failure. With less capital investment needed, more parties have the possibility to invest. Thus, the potential for disruptive innovations is increased. Potentially less technical complexity and the possibility for tailoring to the availability and amount of the feedstock and, if needed: scaling-up can be done with small units operating in parallel.

All in all, GTL-based Fischer-Tropsch facilities are at the moment the most competitive primarily due to i) cheap and abundant natural gas as feedstock, and ii) high ratio of effective $\mathrm{H}: \mathrm{C}$ (Table 1.2). The high effective $\mathrm{H}$ : $\mathrm{C}$ ratio decreases complexity in the syngas production step, decreasing associated costs. ${ }^{27}$ The future of BTL and WTL might lie in the small-scale facility integrations into the local waste management systems turning "waste into money". Otherwise, the feedstock quantities might be too small for large industrial scale facilities. CTL remains as the less attractive and more 
expensive FTP option. If natural gas for GTL is not available, CTL will probably continue to-be employed when the strategic diversification from crude oil availability is the most important aspect, as its role has been historically.

In terms of fundamental FTS catalyst material research, important aspects for improvement are to: i) improve the overall stability of the Fe-based catalyst materials, ii) increase understanding on how to steer selectivities of the FTS catalyst materials towards desired hydrocarbon products without wasted $\mathrm{C}$ atoms, and iii) how to minimize/eliminate the $\mathrm{CH}_{4}$ from the product slate.

As an alternative to the FTS reaction, the leading competing method for converting syngas to hydrocarbons in future industrial processes are the methanol intermediate based Methanol-to-Hydrocarbons (MTH) reactions. ${ }^{36-39}$ Of these, Methanol-to-Olefins (MTO) facilities are currently being implemented beyond demonstration scale for commercial hydrocarbon production. ${ }^{40}$

On the lab-scale, an interesting new method type for hydrocarbon synthesis using a bifunctional catalyst bed, dubbed as OX-ZEO (Oxide-Zeolite), has recently been demonstrated. ${ }^{41,42}$ The OX-ZEO method can be used to directly synthesize shortchained hydrocarbons from input $\mathrm{H}_{2}$ :CO gas. ${ }^{41,42}$ Whether the FTP with the FTS reaction or something else will be the future choice for hydrocarbon production, remains to be seen.

\subsection{SCOPE AND OUTLINE OF THIS PHD DISSERTATION}

Within this PhD Dissertation, we aim to further advance the understanding on the FTS reaction when Fe-based catalyst materials are used on its heterogeneous catalysis. More specifically, the main aim of this work is to probe: the effect of chemical promotion $\mathrm{Na}$-S on the Fe carbides in Fe-based catalysis of the FTS reaction. Fe carbides are the catalyst's active phases in the Fe-based FTS reaction. The PhD Dissertation is arranged as follows.

Chapter 2 - An overview of the literature on the Fe-based FTS reaction catalysis is presented. The FTS reaction related topics, such as generic Fe-based catalyst compositions, various chemical reaction pathways, and means to alter the product hydrocarbon selectivity, are reviewed. As the specific topics of this Dissertation: i) the application of alkali( $\mathrm{Na}, \mathrm{K})$-S promotion for modifying the FTS reaction hydrocarbon product selectivity is discussed in a reasonably detailed manner within the Chapter. In addition, ii) the nomenclature and identification of various Fe carbides observed in the FTS reaction, a traditionally confusing topic in the Fe-based FTS literature, is reviewed in detail. The Fe carbides relevant for the FTS research field are identified from literature as $\varepsilon-\mathrm{Fe}_{3} \mathrm{C}, \eta-\mathrm{Fe}_{2} \mathrm{C}, \chi-\mathrm{Fe}_{5} \mathrm{C}_{2}$ (the Hägg carbide), $\theta-\mathrm{Fe}{ }_{3} \mathrm{C}$ (cementite) and $\theta-\mathrm{Fe}_{7} \mathrm{C}_{3}$ 
(the Eckström-Adcock carbide). Each of these FTS-relevant Fe carbides is assigned with their precise crystal structures and Mössbauer Absorption Spectroscopy (MAS) hyperfine field parameters, in a unified manner, for use in the later Chapters.

Chapter 3 - The Fe carbides identified in Chapter 2 are prepared on $\mathrm{Fe}(-\mathrm{Na}-\mathrm{S}) / \alpha-$ $\mathrm{Al}_{2} \mathrm{O}_{3}$ FTS reaction catalyst materials, with and without Na-S promotion, via CO carburization. Both, ex-situ X-ray Powder Diffractometry (XRPD) with Rietveld Quantitative Phase Analysis (R-QPA) and ex-situ MAS are employed for verifying the unified Fe carbide nomenclature as established in Chapter 2. Furthermore, any effects arising from the Na-S promotion on the Fe carbide formation and/or properties are probed. Thermogravimetric Analysis with Mass Spectrometry (TGA-MS) is applied for determining the inactive carbon deposition on the prepared catalyst materials due to the employed CO carburizations. Transmission Electron Microscopy (TEM) is used to follow the catalyst materials' morphology.

Chapter 4 - The $\mathrm{Fe}(-\mathrm{Na}-\mathrm{S}) / \alpha-\mathrm{Al}_{2} \mathrm{O}_{3}$ catalyst materials with the preformed $\mathrm{Fe}$ carbides prepared in Chapter 3, are further tested for their $72 \mathrm{~h}$ FTS reaction $\left(340^{\circ} \mathrm{C}\right.$, 10 bar, 2:1 $\mathrm{H}_{2}: \mathrm{CO}$ ) catalysis performance. R-QPA is applied on the ex-situ collected XRPD patterns for quantifying the effects of the Na-S promotion on the Fe carbides after their utilization under 10 bar $\mathrm{H}_{2}: \mathrm{CO}$ atmosphere in the FTS reaction. The results are compared to the Fe carbide properties from Chapter 3 before the FTS reaction. Especially, with utilizing the R-QPA method, the often overlooked property of the Fe carbide lattice parameters and their changes are followed in detail. The Fe carbides are assigned into subtypes after their defining lattice parameters. Additionally, TEM is used to follow the catalysts' morphology changes after the FTS reaction.

Chapter 5 - $\mathrm{Fe}(-\mathrm{Na}-\mathrm{S}) / \alpha-\mathrm{Al}_{2} \mathrm{O}_{3}$ catalysts materials, with and without Na-S promotion, are prepared for study. These catalysts are either reduced with $\mathrm{H}_{2}$ or applied directly after a catalyst calcining step in $72 \mathrm{~h}$ FTS reactions $\left(340^{\circ} \mathrm{C}, 3,10\right.$ and 20 bar, $1: 1 \mathrm{H}_{2}: \mathrm{CO}$ ). R-QPA is used to quantify the differences in the ex-situ collected XRPD patterns. This quantification in particularly, in the terms of Fe carbide lattice parameters versus the different combinations of i) reaction pressure, ii) Na-S promotion and iii) calcined versus $\mathrm{H}_{2}$ pre-reduced catalyst materials. The catalysts' FTS reaction performance i.e. activity and selectivity, is further correlated to the Fe carbide subtypes assigned in Chapter 4.

Chapter 6 - With the use of combined XRPD/Raman Spectroscopy approach, the $\mathrm{Fe}(-\mathrm{Na}-\mathrm{S}) / \alpha-\mathrm{Al}_{2} \mathrm{O}_{3}$ catalyst materials with the preformed Fe carbides from Chapter 3 are characterized in-situ during $72 \mathrm{~h}$ FTS reaction $\left(340^{\circ} \mathrm{C}, 10 \mathrm{bar}, 2: 1 \mathrm{H}_{2}\right.$ :CO). R-QPA is applied for quantifying the Fe phases from the quasi- and in-situ collected XRPD patterns, while Raman Spectroscopy is simultaneously used to study the accompanying carbon species evolution. The Raman spectra of the formed carbon species are 
compared to those measured ex-situ for carbon reference materials in order to identify the formed carbon species during the FTS reaction catalysis.

Chapter 7 - The overall summary of the work performed as well as the main findings in this $\mathrm{PhD}$ Dissertation are given. Furthermore, some interesting future research directions are highlighted which may inspire new research directions in $\mathrm{Fe}$ based FTS reaction catalysis.

\subsection{REFERENCES}

[1] Le Page, J. F., Cosyns, J., Courty, P., Freund, E., Franck, J. P., Jacquin, Y., Juguin, B., Marcilly, C., Martino, G., Miquel, J., Montarnal, R., Sugier, A. and van Landeghem, H., "General" in Applied Heterogeneous Catalysis: Design, Manufacture, Use of Solid Catalysts (ed. Le Page, J. F.), 1-15 (Éditions Technip, Paris, 1987).

[2] Le Page, J. F., Cosyns, J., Courty, P., Freund, E., Franck, J. P., Jacquin, Y., Juguin, B., Marcilly, C., Martino, G., Miquel, J., Montarnal, R., Sugier, A. and van Landeghem, H., "Developing Industrial Catalysts" in Applied Heterogeneous Catalysis: Design, Manufacture, Use of Solid Catalysts (ed. Le Page, J. F.), 6774 (Éditions Technip, Paris, 1987).

[3] Mittasch, A. and Schneider, C., "Producing Compounds Containing Carbon and Hydrogen", Patent US1201850A (1916).

[4] Blekkan, E. A., "Fischer-Tropsch Synthesis Over Cobalt Catalysts: State of the Art and Examples from Natural Gas and Biomass Conversion to Fuels" in Kokkola Material Week (Metal Kokkola, Kokkola, 2013).

[5] Maitlis, P. M. and de Klerk, A., "Greener Fischer-Tropsch Processes for Fuels and Feedstocks", (WileyVCH, Weinheim, 2013). DOI:10.1002/9783527656837

[6] Xiang, Y. and Kruse, N., "Tuning the catalytic CO hydrogenation to straight- and long-chain aldehydes/alcohols and olefins/paraffins", Nat. Commun. 7, 13058-13064 (2016).

[7] Fischer, F. and Tropsch, H., "Verfahren zur Gewinnung mehrgliedriger Paraffinkohlenwasserstoffe aus Kohlenoxyden und Wasserstoff auf katalytischem Wege", Patent DE484337C (1929).

[8] de Klerk, A., "Fischer-Tropsch Refining", (Wiley-VCH, Weinheim, 2011). DOI:10.1002/9783527635603

[9] van de Loosdrecht, J., Botes, G. F., Ciobica, I. M., Ferreira, A. C., Gibson, P., Moodley, D. J., Saib, A. M., Visagie, J. L., Weststrate, C. J. and Niemantsverdriet, J. W., "Fischer-Tropsch Synthesis: Catalysts and Chemistry" in Comprehensive Inorganic Chemistry II (eds. Reedijk, J. \& Poeppelmeier, K.), 525-557 (Elsevier, Amsterdam, 2013). DOI:10.1016/B978-0-08-097774-4.00729-4

[10] Mitchell, T., "The Resources of Economics - Making the 1973 Oil Crisis", J. Cult. Econ. 3, 189-204 (2010).

[11] Murphy, C., "To Cope With Embargoes, S. Africa Converts Coal Into Oil", Washington Post (1979). Available at: https://www.washingtonpost.com/archive/politics/1979/04/27/to-cope-withembargoes-s-africa-converts-coal-into-oil/cd39adab-5084-4e46-a28f79de2896f75e/?utm_term=.291aeccf9cef (Accessed: January 2018).

[12] Levy, P. I., "Sanctions on South Africa: What Did They Do?", Am. Econ. Rev. 89, 415-420 (1999).

[13] Crawford, N. C., "Oil Sanctions Against Apartheid" in How Sanctions Work (eds. Crawford, N. C. \& Klotz, A.), 103-126 (Palgrave Macmillan UK, London, 1999). DOI:10.1057/9781403915917

[14] Chemicals-Technology, "Sasol Wax Expansion Project", (2017). Available at: http://www.chemicals- 
technology.com/projects/sasol-wax-expansion-project/ (Accessed: January 2018).

[15] Khelaly, M. El, "Shell restarts damaged Bintulu GTL plant", Oil \& Gas Journal (2000). Available at: http://www.ogj.com/articles/2000/06/shell-restarts-damaged-bintulu-gtl-plant.html (Accessed: January 2018).

[16] Chevron, "Nigeria", (2016). Available at: https://www.chevron.com/worldwide/nigeria (Accessed: January 2018).

[17] Wood-McKenzie, "Escravos GTL - Asset Report", (2017). Available at: https://www.woodmac.com/reports/upstream-oil-and-gas-escravos-gtl-19005114 (Accessed: January 2018).

[18] Green Car Congress, "BP and Johson Matthey License Fischer-Tropsch Technology to Waste-toBiofuels Producer Fulcrum BioEnergy for Biojet", Green Car Congress (2018). Available at: https://www.greencarcongress.com/2018/09/20180928-bp.html (Accessed: April 2019).

[19] Schlanger, Z., "The World Will Finally Have to Confront Its Massive Plastic Problem Now that China Won't Handle It", Quartz (2018). Available at: https://qz.com/1310240 (Accessed: June 2018).

[20] Doyle, A., "Plastics mines? Europe struggles as pollution piles up", Reuters (2018). Available at: https://uk.reuters.com/article/uk-environment-plastics-insight/plastics-mines-europe-strugglesas-pollution-piles-up-idUKKBN1ICOFS (Accessed: May 2018).

[21] Brems, A., Dewil, R., Baeyens, J. and Zhang, R., "Gasification of plastic waste as waste-to-energy or waste-to-syngas recovery route", Nat. Sci. 05, 695-704 (2013).

[22] Hirn, G., "Generating syngas from plastic wastes", (BINE Information Service, EggensteinLeopoldshafen Germany, 2016). Available at: http://www.bine.info/en/topics/renewable-energysources/biomass/publikation/synthesegas-aus-kunststoffabfaellen-erzeugen/ (Accessed: May 2018).

[23] Williams, M., "Smaller Scale Fischer-Tropsch Enables Biomass-to-Liquids" in Gasification \& Syngas Technologies Council (Velocys, 2014).

[24] de Klerk, A., "Indirect Liquefaction Carbon Efficiency" in Synthetic Liquids Production and Refining (eds. de Klerk, A. \& King, D.) 1084, 215-235 (American Chemical Society, Washington, DC, 2011).

[25] Shell Global, "Pearl GTL - Overview", (2017). Available at: https://www.shell.com/about-us/majorprojects/pearl-gtl/pearl-gtl-an-overview.html (Accessed: January 2018).

[26] Dry, M. E., "Fischer-Tropsch reactions and the environment", Appl. Catal. A Gen. 189, 185-190 (1999).

[27] Dry, M. E., "The Fischer-Tropsch process: 1950-2000", Catal. Today 71, 227-241 (2002).

[28] Dry, M. E., "The Fischer-Tropsch Synthesis" in Catalysis - Science and Technology (eds. Anderson, J. R. \& Boudart, M.) 1, 159-256 (Springer-Verlag, Berlin, 1981).

[29] Shultz, J. F., Hall, W. K., Seligman, B. and Anderson, R. B., "Studies of the Fischer-Tropsch Synthesis. XIV. Hägg Carbide as Catalysts", J. Am. Chem. Soc. 77, 213-221 (1955).

[30] Steynberg, A. P., Espinoza, R. L., Jager, B. and Vosloo, A. C., "High temperature Fischer-Tropsch synthesis in commercial practice", Appl. Catal. A Gen. 186, 41-54 (1999).

[31] Höök, M., Fantazzini, D., Angelantoni, A. and Snowden, S., "Hydrocarbon liquefaction: viability as a peak oil mitigation strategy", Philos. Trans. R. Soc. A Math. Phys. Eng. Sci. 372, 20120319-20120355 (2013).

[32] Shell Global, "Shell GTL Fuel - Product Benefits", (2017). Available at: https://www.shell.com/business-customers/commercial-fuels/shell-gtl-fuel/productbenefits.html (Accessed: January 2018).

[33] Parafinic Fuels for Europe (ASFE), "Air Quality of Paraffinic Fuels", (2017). Available at: http://www.synthetic-fuels.eu/paraffinic-fuels/air-quality (Accessed: January 2018). 


\section{Chapter 1}

[34] Dry, M. E., "High quality diesel via the Fischer-Tropsch process - a review",J. Chem. Technol. Biotechnol. 77, 43-50 (2001).

[35] Torres Galvis, H. M. and de Jong, K. P., "Catalysts for Production of Lower Olefins from Synthesis Gas: A Review", ACS Catal. 3, 2130-2149 (2013).

[36] Keil, F. J., "Methanol-to-hydrocarbons: process technology", Microporous Mesoporous Mater. 29, 4966 (1999).

[37] Stöcker, M., "Methanol-to-hydrocarbons: catalytic materials and their behavior", Microporous Mesoporous Mater. 29, 3-48 (1999).

[38] Yarulina, I., Chowdhury, A. D., Meirer, F., Weckhuysen, B. M. and Gascon, J., "Recent trends and fundamental insights in the methanol-to-hydrocarbons process", Nat. Catal. 1, 398-411 (2018).

[39] Olsbye, U., Svelle, S., Bjørgen, M., Beato, P., Janssens, T. V. W., Joensen, F., Bordiga, S. and Lillerud, K. P., "Conversion of Methanol to Hydrocarbons: How Zeolite Cavity and Pore Size Controls Product Selectivity", Angew. Chem., Int. Ed. 51, 5810-5831 (2012).

[40] Tian, P., Wei, Y., Ye, M. and Liu, Z., "Methanol to Olefins (MTO): From Fundamentals to Commercialization", ACS Catal. 5, 1922-1938 (2015).

[41] Jiao, F., Li, J., Pan, X., Xiao, J., Li, H., Ma, H., Wei, M., Pan, Y., Zhou, Z., Li, M., Miao, S., Li, J., Zhu, Y., Xiao, D., He, T., Yang, J., Qi, F., Fu, Q. and Bao, X., "Selective conversion of syngas to light olefins", Science 351, 1065-1068 (2016).

[42] de Jong, K. P., "Surprised by selectivity", Science 351, 1030-1031 (2016). 


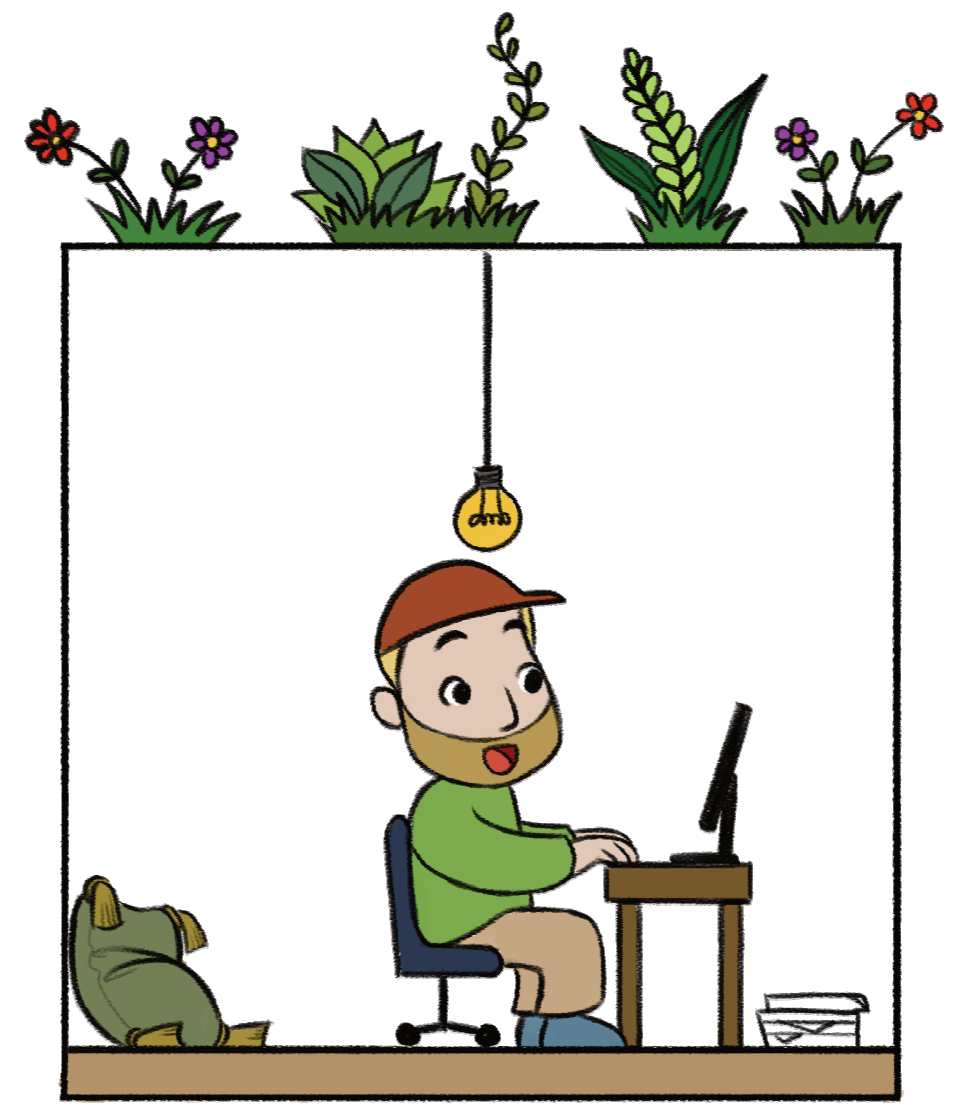




\section{Chapter 2}

\section{Fischer-Tropsch Synthesis over Iron- based Catalysts: A Review}

Fischer-Tropsch Synthesis (FTS) reaction, the synthesis of hydrocarbons over a transition metal from a $\mathrm{H}_{2}$ : $\mathrm{CO}$ gas mixture, is reviewed. This review concentrates on Fe-based FTS reaction catalysis. The overall chemical reactions taking place over the course of application of the FTS reaction are reviewed. Furthermore, the reaction pathways for the $\mathrm{C}$ originating from the input $\mathrm{CO}$ under FTS reaction conditions are defined. The process conditions and catalyst compositions for steering the $\mathrm{C}$ pathways and the FTS hydrocarbon product selectivities are overviewed. As the specific topics of this PhD Dissertation: the alkali-S chemical promotion is discussed in detail and the confusing Fe carbide nomenclature used within the FTS reaction literature is clarified. This clarification is done by assigning the Fe carbide crystal structures with their corresponding Mössbauer Absorption Spectroscopy (MAS) hyperfine fields, in a unified and precise manner, on the basis of the available literature. Furthermore, the expected formation conditions of the identified $\mathrm{Fe}$ carbides encountered in the FTS research field, namely $\varepsilon-\mathrm{Fe}_{3} \mathrm{C}, \eta-\mathrm{Fe} 2 \mathrm{C}, \chi-\mathrm{Fe}_{5} \mathrm{C}_{2}, \theta-$ $\mathrm{Fe}_{3} \mathrm{C}$ and $\theta-\mathrm{Fe}_{7} \mathrm{C}_{3}$, are reviewed. The literature review performed in this Chapter establishes the necessary background for understanding the following experimental research Chapters of this PhD Dissertation. 


\subsection{INTRODUCTION}

Within this Chapter, the fundamentals of the Fischer-Tropsch Synthesis (FTS) reaction are introduced with emphasis on the Fe-based catalyst material chemistry. Especially, i) the use of alkali-S promotion and ii) the identification of the Fe-based catalysts' FTS reaction active phases i.e. Fe carbides, are both discussed with more detail. Occasionally, few comparisons to Co-based FTS reaction chemistry are made. In the following sections:

Catalyst Compositions the components making up a FTS reaction catalyst material are introduced in general terms,

Reactions, Fe Phase Changes and Catalyst Deactivation discusses the overall reactions related to the FTS reaction and their effect on the working catalyst,

Hydrocarbon and C Pathway Selectivity introduces possible ways for altering the selectivity of the reactions taking place in the FTS reaction, by varying the process conditions and alkali, S or combined alkali-S chemical promotion.

Fe carbides in the Fischer-Tropsch Synthesis Reaction section discusses the identification of the Fe carbides $\left(\mathrm{Fe}_{\mathrm{x}} \mathrm{C}, x=2-3\right)$ which are the active phases in the Fe-based FTS reaction. A common nomenclature is established between Fe carbide crystal structures and available Mössbauer Absorption Spectroscopy (MAS) hyperfine field assignments from literature. This is done in order to clarify and unify the often unclear and ambivalent Fe carbide phase nomenclature. The established nomenclature is applied to identify the general formation conditions for each of the identified Fe carbides.

Aim for the current Chapter is not to give a comprehensive review on the Fe-based FTS reaction up-to-date. Rather, the aim is to give a solid base for understanding the following research Chapters and each Chapter's relevance in the FTS research field. This especially with regard to the topics of $\mathrm{Na}-\mathrm{S}$ chemical promotion and different $\mathrm{Fe}$ carbides in the Fe-based FTS reaction.

\subsection{CATALYST COMPOSITIONS}

A FTS reaction catalyst material is commonly composed of, at least one, transition metal and a chemical promoter and either an inert structural promoter or a support material.

A wide range of transition metals can catalyze the FTS reaction. They include $\mathrm{Ru}, \mathrm{Fe}$, $\mathrm{Ni}, \mathrm{Co}, \mathrm{Rh}, \mathrm{Pd}, \mathrm{Pt}$, Ir, Os and Mo.1-3 In practice, $\mathrm{Ru}, \mathrm{Fe}$, Co and $\mathrm{Ni}$ are the most suitable 
ones with respect to their activity and selectivity towards the FTS reaction. ${ }^{1,4-6} \mathrm{Ru}$ is active and can be well-suited for the FTS reaction, but is very expensive, and is not readily available in large quantities. Thus, $\mathrm{Ru}$ is less applied in commercial FTS reaction catalysis. ${ }^{3,4,6} \mathrm{Ni}$ has the tendency to produce larger amounts of undesired $\mathrm{CH}_{4}$ than $\mathrm{Fe}$ or Co do, and $\mathrm{Ni}$ is also susceptible for formation of volatile Ni carbonyls under its FTS reaction conditions. ${ }^{1,5,7-9} \mathrm{~A} \mathrm{Ni}$-based, $\mathrm{Ni}-\mathrm{MnO}-\mathrm{Al}_{2} \mathrm{O}_{3}$-kieselguhr catalyst was one of the first FTS reaction catalysts tested in Germany, around 1933, in a demonstration scale FTS facility. However, difficulties with the $\mathrm{Ni}$ as the active metal lead to research efforts towards the more suitable Fe- and Co-based FTS reaction catalyst materials. ${ }^{7,9}$ The Fe and Co have been, and are still, the most suitable active transition metals for catalyzing the FTS reaction, and the only ones applied on a large scale in industry. ${ }^{3,10}$

Besides the catalytically active transition metal, chemical promoters can also be added to a FTS reaction catalyst. These promoters are most often basic alkali, or sometimes earth-alkaline, compounds which are added in order to modify the catalyst's hydrocarbon product selectivities. ${ }^{2,3,11-14}$ The chemical promoters enhance the electronic properties of the FTS reaction active transition metal. ${ }^{15}$ Combining the basic chemical promoter with $S$ has shown further favorable properties towards the FTS reaction, ${ }^{16-21}$ giving an additional dimension for modifying the properties of the active transition metal in the FTS catalyst materials.

Addition of possibly alloying metals to Fe- or Co-based catalyst materials, such as e.g. Mn, Ni or Pt, can also modify the catalyst's FTS reaction properties. ${ }^{7,22-24}$ Especially in older FTS literature, also $\mathrm{Cu}$ was added to the Fe-based catalyst's composition in order to aid with the Fe oxide phases reduction. . $^{3,4,10}$

The most common catalyst preparation methods for the Fe-based FTS reaction catalyst materials have been, either i) precipitating the catalyst from a precursor solution, or ii) fusing the catalyst from input materials at high temperatures, cooling down and breaking the formed ingot.3,25 The catalyst materials prepared via these routes are commonly known as the bulk or unsupported catalysts. For the bulk catalyst materials, structural promoters as inert oxides of, e.g. $\mathrm{Si}, \mathrm{Al}$ or $\mathrm{Mg}$, can be added for mainly to prevent loss of the active metal area and to improve the catalyst's stability under the FTS reaction conditions. . $^{3,14}$

Impregnation methods are commonly used to prepare supported Fe-based FTS reaction catalyst materials. Usually, $\$ 20$ weight percent (wt-\%) of elemental Fe is impregnated on a support material with the use of a precursor solution containing the desired chemical promoters and the solvated Fe-ions. ${ }^{20,22,25}$ The major advantage from the impregnation of support materials in comparison to the bulk preparation methods is that the active Fe is in a highly dispersed form. This allows its efficient utilization in the FTS reaction catalysis. ${ }^{25}$ Some of the support materials which have been applied for 
FTS reaction catalyst materials are e.g. $\alpha-\mathrm{Al}_{2} \mathrm{O}_{3}, \gamma-\mathrm{Al}_{2} \mathrm{O}_{3}, \beta-\mathrm{SiC}, \mathrm{SiO}_{2}$ and carbon nanofibers (CNF). ${ }^{20,25-27}$

\subsection{REACTIONS, FE PHASE CHANGES AND CATALYST DEACTIVATION}

\subsubsection{Reactions}

The main chemical reaction in the FTS is the conversion of the input $\mathrm{H}_{2}$ : $\mathrm{CO}$ gas mixture into various hydrocarbons and the side-product $\mathrm{H}_{2} \mathrm{O}, 4,28$

$$
\begin{array}{ll}
\mathrm{nCO}+(2 \mathrm{n}+1) \mathrm{H}_{2} \rightleftharpoons \mathrm{C}_{\mathrm{n}} \mathrm{H}_{2 \mathrm{n}+2}+\mathrm{nH}_{2} \mathrm{O} & \text { for paraffins } \\
\mathrm{nCO}+2 \mathrm{nH}_{2} \rightleftharpoons \mathrm{C}_{\mathrm{n}} \mathrm{H}_{2 \mathrm{n}}+\mathrm{nH}_{2} \mathrm{O} & \text { for olefins }
\end{array}
$$

where Reaction 2.1 refers to formation of paraffins (i.e. saturated hydrocarbons) and Reaction 2.2 refers to formation of olefins (i.e. unsaturated hydrocarbons). The olefins formed as the FTS reaction's primary products may re-adsorb on the catalyst material and be hydrogenated into paraffins via secondary reactions. ${ }^{28,29}$

As both of the hydrocarbon forming reactions are exothermic (Reaction 2.1 and Reaction 2.2), attention needs to be paid on the isothermal operation of the FTS reactor bed. ${ }^{3,10}$ Exposing the $\mathrm{H}_{2}$ :CO mixture directly to the FTS reaction catalyst material with too high starting temperature results in non-isothermal bed operation and undesired heating of the catalyst bed. ${ }^{30}$

Both $\mathrm{CO}$ and $\mathrm{H}_{2}$ adsorb dissociatively under the FTS reaction conditions giving $\mathrm{C}, \mathrm{O}$ and $\mathrm{H}$ atoms adsorbed on the Fe phases' surface. $\mathrm{H}$ and $\mathrm{C}$ atoms then recombine to form - $\mathrm{CH}_{2}$ - units which polymerize to hydrocarbon chains, via the so-called "surface carbide mechanism". 4,28,31 Preferably, the $\mathrm{O}$ atoms from the $\mathrm{CO}$ dissociation recombine with $\mathrm{H}$ atoms and desorb as a $\mathrm{H}_{2} \mathrm{O}$ molecule. If $\mathrm{H}_{2}$ is unavailable, as can be the case with low $\mathrm{H}_{2}$ : $\mathrm{CO}$ ratio syngas, ${ }^{32}$ the adsorbed $\mathrm{O}$ atom from the $\mathrm{CO}$ dissociation may react with an another $\mathrm{CO}$ molecule and be removed as $\mathrm{CO}_{2}$, instead of $\mathrm{H}_{2} \mathrm{O}^{3,31,33}$ Several mechanisms for the FTS reaction have been proposed to explain the variety of hydrocarbon products and their derivatives. ${ }^{3,4,31,34}$ However, within this Dissertation their existence is acknowledged, but only the surface carbide mechanism is considered to be active.

The formation of oxygenate derivatives of hydrocarbons (mainly alcohols) can be taken as a side-reaction, as their contribution to the overall FTS reaction products is usually low..$^{3,10,35}$ Saturated alcohols form in the FTS reaction according to equation: ${ }^{28}$

$$
\mathrm{nCO}+2 \mathrm{nH}_{2} \rightleftharpoons \mathrm{C}_{\mathrm{n}} \mathrm{H}_{2 \mathrm{n}+1} \mathrm{OH}+(\mathrm{n}-1) \mathrm{H}_{2} \mathrm{O} \quad \text { for alcohols }
$$


Besides the Reaction 2.3 for exothermic formation of alcohol oxygenates, also some water soluble carboxylic acids and aldehydes may form in the FTS reaction. ${ }^{3,10,35}$ The generic reaction equations for the carboxylic acid and aldehyde formation are omitted here, but can be found e.g. as presented by Dry. ${ }^{3}$ Mechanism for the formation of the hydrocarbon oxygenate derivatives possibly involves a non-dissociative CO adsorption/insertion step in contrast to the main FTS reactions forming hydrocarbons via CO dissociation. $3,4,31,34$

The Water-gas shift (WGS) reaction is used industrially in the production of $\mathrm{H}_{2} \cdot{ }^{36,37}$ It follows the reaction equation:

$$
\mathrm{CO}+\mathrm{H}_{2} \mathrm{O} \rightleftharpoons \mathrm{CO}_{2}+\mathrm{H}_{2} \quad \text { Water-gas Shift }
$$

The WGS reaction is not usually active for Co-based FTS reaction catalyst materials but can be active under the FTS reaction conditions if a Fe-based catalyst is used (Reaction 2.4).4,6,11,12,28,38 With increasing reaction temperature, the WGS reaction equilibrium shifts towards the formation of the reactants $\mathrm{CO}$ and $\mathrm{H}_{2} \mathrm{O}$ (i.e. is an exothermic reaction). If the reaction is kinetically limited i.e. $\mathrm{CO}+\mathrm{H}_{2} \mathrm{O}$ partial pressures are above the equilibrium expectations, the total forward reaction may benefit from an increased reaction temperature. ${ }^{37,39}$ In the presence of sufficient amounts of $\mathrm{CO}_{2}$ and $\mathrm{H}_{2}$, the reverse WGS reaction can also take place forming $\mathrm{CO}$ and $\mathrm{H}_{2} \mathrm{O} \cdot{ }^{33,35,40-42}$ If a low $\mathrm{H}_{2}$ :CO ratio reactant feed is used; the WGS reaction may become the FTS reaction ratedetermining step. In this case all of the available $\mathrm{H}_{2}$ for the FTS reaction hydrocarbon products is coming from the WGS reaction. ${ }^{11}$

In the industrial application of the WGS reaction, a $\mathrm{Cu} / \mathrm{ZnO} / \mathrm{Al}_{2} \mathrm{O}_{3}$ catalyst material is used for $\approx 210-240^{\circ} \mathrm{C}$ and $\mathrm{a} \mathrm{Fe}_{3} \mathrm{O}_{4} / \mathrm{Cr}_{2} \mathrm{O}_{3}$ catalyst for $\approx 310-450^{\circ} \mathrm{C}$ reaction temperatures. ${ }^{36,37,39}$ For both high and low WGS reaction temperatures, the reaction proceeds with an oxidic active phase unable for CO dissociation. ${ }^{36,37}$ This is unlike in the FTS reaction where $\mathrm{CO}$ is dissociatively adsorbed by Fe carbides. That is, the WGS reaction active phase with $\mathrm{Fe}$-based FTS reaction catalysts is $\mathrm{Fe}_{3} \mathrm{O}_{4}$, not a FTS active $\mathrm{Fe}_{\mathrm{x}} \mathrm{C}$ Fe carbide phase(s), this despite the presence of Fe phases carburizing CO.

The Boudouard reaction is an unwanted reaction pathway under the FTS reaction conditions causing formation of inactive carbon species ("coke") on the catalyst:43

$$
2 \mathrm{CO} \rightleftharpoons \mathrm{C}+\mathrm{CO}_{2} \quad \text { Boudouard Reaction }
$$

The Boudouard reaction (i.e. CO disproportionation) deposits adsorbed carbon species on the catalyst, while releasing $\mathrm{CO}_{2}$ (Reaction 2.5). When raising the FTS reaction temperature from Low-temperature Fischer-Tropsch (LTFT) at $\approx 225^{\circ} \mathrm{C}$ to 
High-temperature Fischer-Tropsch (HTFT) conditions at $\approx 345^{\circ} \mathrm{C}$, the rate of carbon deposition becomes significant for Fe-based FTS catalyst materials at $\gtrsim 300^{\circ} \mathrm{C} . .^{3,14,44,45}$ The Boudouard reaction becomes a major reaction consuming $\mathrm{CO}$, if the $\mathrm{CO}$ is exposed to $\mathrm{Fe}$ at temperatures $\geq 400^{\circ} \mathrm{C} .46,47$

Small amounts of added $\mathrm{H}_{2}$, or $\mathrm{H}_{2} \mathrm{O}$ converted to $\mathrm{H}_{2}$ via the WGS reaction, can increase the carbon deposition rate from the Boudouard reaction. 3,44,48,49 Presumably, $\mathrm{H}_{2}$ removes the adsorbed and catalyst surface sites blocking $\mathrm{O}$ atoms from the $\mathrm{CO}$ dissociation as $\mathrm{H}_{2} \mathrm{O}$ more efficiently than $\mathrm{CO}$ does as $\mathrm{CO}_{2}$. The improved $\mathrm{O}$ removal probably increases the rate at which $\mathrm{CO}$ molecules can adsorb and dissociate on the surfaces of (near-)zerovalent Fe phases (i.e. $\alpha$-Fe or FexC). ${ }^{33,50}$ However, the increased carbon deposition only takes place with small amounts of added $\mathrm{H}_{2}$ to input $\mathrm{CO}$. If higher $\mathrm{H}_{2}$ :CO ratios $(\gtrsim 5: 1)$ with above $\approx 15-20$ bar reaction pressure are used, the carbon deposition can be decreased during the FTS reaction in relation to the hydrocarbon formation. 3,51

Noteworthy emphasis is that both the Boudouard reaction (Reaction 2.5), and the main FTS reactions (Reaction 2.1 and Reaction 2.2), result in an adsorbed elemental C intermediates on the FexC surface. The adsorbed $\mathrm{C}$ atoms can either react to form hydrocarbon products or inactive carbon deposits on the catalyst.3,4,34 The generic types of inactive carbon formed are either encapsulating carbon over the Fe phases if only CO is present or filamentous carbon if both $\mathrm{CO}$ and $\mathrm{H}_{2}$ are available. ${ }^{52-54}$

\subsubsection{Fe Phase Changes}

Several possible $\mathrm{Fe}$ phase changes take place on a Fe-based catalyst material when moving from the as-synthesized catalyst to catalyst under FTS reaction conditions and, possibly, to a deactivated catalyst material. The generic Fe phase changes for a Fe-based catalyst follow relation:3,4,35

\section{Initial Catalyst $\Rightarrow$ Reduced Catalyst $\Rightarrow$ Active Catalyst \\ Fe oxide $\quad$ Metallic Fe $\rightarrow \quad$ Fe carbide}

The initial, as-synthesized, FTS reaction catalyst material is commonly in a form of a $\mathrm{Fe}$ oxide, mainly either as $\alpha-\mathrm{Fe}_{2} \mathrm{O}_{3}$ (hematite), $\gamma-\mathrm{Fe}_{2} \mathrm{O}_{3}$ (maghemite) or $\mathrm{Fe}_{3} \mathrm{O}_{4}$ (magnetite). ${ }^{4}$ The Fe oxides are not active for the hydrocarbon forming FTS reactions (Reaction 2.1 and Reaction 2.2), ${ }^{55-57}$ and are commonly reduced to $\alpha$-Fe (metallic Fe) prior to starting the FTS reaction. Upon exposure to $\mathrm{H}_{2}$ :CO in the FTS reaction, the $\alpha$-Fe then readily reacts to form various $\mathrm{Fe}$ carbide phases $\left(\mathrm{Fe}_{\mathrm{x}} \mathrm{C}, x=2-3\right) \cdot{ }^{34,58-62}$ Alternatively, the Fe oxides can also be exposed directly to the FTS reaction conditions, i.e. to a $\mathrm{H}_{2}$ :CO atmosphere. In this occasion the FTS active Fe carbides form 
simultaneously with the Fe oxide reduction to $\alpha-\mathrm{Fe}^{29,47,63-67}$

The reaction equations for reducing Fe oxides to metallic $\alpha$-Fe with either $\mathrm{H}_{2}$ or $\mathrm{CO}$ are: ${ }^{4,28,68}$

$$
\begin{array}{ll}
\mathrm{Fe}_{\mathrm{x}} \mathrm{O}_{\mathrm{y}}+\mathrm{yH}_{2} \rightleftharpoons \mathrm{xFe}+\mathrm{yH}_{2} \mathrm{O} & \mathrm{H}_{2} \text { reduction } \\
\mathrm{Fe}_{\mathrm{x}} \mathrm{O}_{\mathrm{y}}+\mathrm{yCO} \rightleftharpoons \mathrm{xFe}+\mathrm{yCO}_{2} & \text { CO reduction } \gtrsim 500^{\circ} \mathrm{C} \\
\mathrm{Fe}_{\mathrm{x}} \mathrm{O}_{\mathrm{y}}+(\mathrm{y}+2) \mathrm{CO} \rightleftharpoons \mathrm{Fe}_{\mathrm{x}} \mathrm{C}+(\mathrm{y}+1) \mathrm{CO}_{2} & \text { CO reduction } \lesssim 500^{\circ} \mathrm{C}
\end{array}
$$

Fe oxide reduction with $\mathrm{H}_{2}$ leads to formation of pure metallic $\alpha$-Fe (Reaction 2.6). In general, a $\mathrm{H}_{2}$ reduction temperature of $\gtrsim 350^{\circ} \mathrm{C}$ is required, with adequate reaction time, for reducing $\mathrm{Fe}_{\mathrm{x}} \mathrm{O}_{\mathrm{y}}$ to $\alpha$-Fe. ${ }^{68}$ The most stable $\mathrm{Fe}_{\mathrm{x}} \mathrm{C}$ phase (namely $\theta-\mathrm{Fe} 3 \mathrm{C}$ ) decomposes between $500-600^{\circ} \mathrm{C}$ into $\alpha$-Fe and carbon species. ${ }^{30,69-71}$ Thus, only $\alpha$-Fe is observed as the $\mathrm{CO}$ reduction product $\gtrsim 500^{\circ} \mathrm{C}$ (Reaction 2.7). ${ }^{68,71}$ With $\mathrm{CO}$ (or $\mathrm{H}_{2}: \mathrm{CO}$ ) reduction temperatures of $\$ 500^{\circ} \mathrm{C}, \mathrm{Fe}_{\mathrm{x}} \mathrm{C}$ phases are stable and can form, i.e. the $\mathrm{Fe}$ oxides are reduced to $\alpha$-Fe and simultaneously carburized (Reaction 2.8). ${ }^{58,69,71}$

Furthermore, the $\mathrm{H}_{2}$ reduction pathway for the Fe oxides is dependent on the used temperature: 68

$$
\begin{array}{ll}
\mathrm{Fe}_{2} \mathrm{O}_{3} \rightarrow \mathrm{Fe}_{3} \mathrm{O}_{4} \rightarrow \mathrm{Fe}^{0} & \mathrm{H}_{2} \text { reduction } \lesssim 570^{\circ} \mathrm{C} \\
\mathrm{Fe}_{2} \mathrm{O}_{3} \rightarrow \mathrm{Fe}_{3} \mathrm{O}_{4} \rightarrow \mathrm{Fe}_{\mathrm{x}} \mathrm{O} \rightarrow \mathrm{Fe}^{0} & \mathrm{H}_{2} \text { reduction } \gtrsim 570^{\circ} \mathrm{C}
\end{array}
$$

With $\mathrm{H}_{2}$ reduction temperatures above $570^{\circ} \mathrm{C}$ (Reaction 2.10), an intermediate $\mathrm{Fe}_{\mathrm{x}} \mathrm{O}$ (wüstite) phase appears, which is absent if the reduction is done $\$ 570^{\circ} \mathrm{C}$ (Reaction 2.9). ${ }^{68}$ Experimentally, however, the $\mathrm{Fe}_{\mathrm{x}} \mathrm{O}$ phase can be stabilized also at lower temperatures $\$ 570^{\circ} \mathrm{C}$ by strong interaction between the Fe oxide with other oxides (e.g. $\mathrm{SiO}_{2}$ ) or by addition of alkali (e.g. K). ${ }^{68,72}$ The $\mathrm{Fe}_{\mathrm{x}} \mathrm{O}$ phase is an intermediate also if the reduction is done with $\mathrm{CO}$ instead of $\mathrm{H}_{2}$, with reduction temperature $\gtrsim 570^{\circ} \mathrm{C} .68$

At sufficiently high reduction temperatures, $\mathrm{Fe}_{3} \mathrm{O}_{4}$ may go through an undesired solid-state reaction with the support or the structural promoter inert oxide.6,73 As an example, the formation of $\mathrm{FeAl}_{2} \mathrm{O}_{4}$ ( $\mathrm{Fe}$ aluminate) with a $\mathrm{Fe} / \gamma-\mathrm{Al}_{2} \mathrm{O}_{3}$ catalyst material at temperatures around $450-470^{\circ} \mathrm{C}$, can take place. ${ }^{74}$ Such solid-state reactions are undesirable as the $\mathrm{Fe}$ in the $\mathrm{FeAl}_{2} \mathrm{O}_{3}$ compound stays in the oxidized, inactive ionic state for the FTS reaction. ${ }^{6,73}$

The FTS reaction active $\mathrm{Fe}_{\mathrm{x}} \mathrm{C}$ phases form in the presence of $\mathrm{CO}$ from reduced $\alpha$-Fe (Reaction 2.11):

$$
\mathrm{xzFe}+2 \mathrm{zCO} \rightarrow \mathrm{zFe}_{\mathrm{x}} \mathrm{C}+\mathrm{zCO}_{2} \quad \alpha-\mathrm{Fe} \text { carburization }
$$


where $x=2-3$ and $z=2 x-3$. If $\mathrm{H}_{2}$ is also present during the carburization reaction, then the $\mathrm{O}$ from $\mathrm{CO}$ dissociation may also be removed as $\mathrm{H}_{2} \mathrm{O}$ instead of $\mathrm{CO}_{2}$, as was discussed earlier.

\subsubsection{Catalyst Deactivation}

The primary pathways for Fe-based catalyst deactivation in the FTS reaction are: ${ }^{3}$

i) Fe phase changes - conversion of the FTS reaction active $\mathrm{Fe}_{\mathrm{x}} \mathrm{C}$ into inactive $\mathrm{Fe}_{3} \mathrm{O}_{4}$,

ii) Sintering - loss of active $\mathrm{Fe}_{\mathrm{x}} \mathrm{C}$ surface area due to increasing Fe particle size,

iii) Fouling - deposition of inactive carbon on the catalyst material, and

iv) Chemical poisoning - deposition of chemical compounds of e.g. $\mathrm{S}, \mathrm{Cl}, \mathrm{Br}, \mathrm{Pb}$ or $\mathrm{Sn}$, on the $\mathrm{Fe}_{\mathrm{x}} \mathrm{C}$ surface.

The conversion of $\mathrm{Fe}_{\mathrm{x}} \mathrm{C}$ phases into $\mathrm{Fe}_{3} \mathrm{O}_{4}$ takes place if either of the $\mathrm{H}_{2} \mathrm{O}: \mathrm{H}_{2}$ or $\mathrm{CO}_{2}$ :CO ratios are above critical molar ratios. ${ }^{3,36,37,57,63,75-77}$ In general, $\mathrm{H}_{2} \mathrm{O}$ is more potent Fe oxidant than $\mathrm{CO}_{2} \cdot{ }^{36,63,77}$

When moving along a fixed bed reactor towards the FTS reactor outlet, ${ }^{65,78}$ or towards the inner core of the Fe-based catalyst material particle, ${ }^{57}$ the reducing $\mathrm{H}_{2}: \mathrm{CO}$ mixture progressively converts to $\mathrm{H}_{2} \mathrm{O}$ and $\mathrm{CO}_{2}$. Due to the increasing $\mathrm{H}_{2} \mathrm{O}$ and $\mathrm{CO}_{2}$ concentrations, the oxidation potential towards forming $\mathrm{Fe}_{3} \mathrm{O}_{4}$ from $\mathrm{Fe}_{\mathrm{x}} \mathrm{C}$

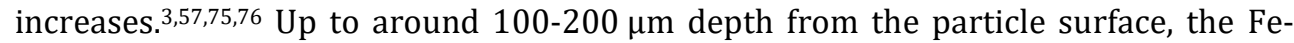
based catalyst particle might remain under reductive environment at FTS reaction conditions. For depths beyond $>200-400 \mu \mathrm{m}$ from the FTS reaction catalyst particle surface, the environment may become oxidative and $\mathrm{Fe}_{\mathrm{x}} \mathrm{C}$ oxidation to $\mathrm{Fe}_{3} \mathrm{O}_{4}$ can take over. ${ }^{57}$

$\mathrm{Fe}_{3} \mathrm{O}_{4}$ is active for the WGS but not for the FTS reaction. ${ }^{3,36,37}$ With sufficient reaction temperature $\left(>310^{\circ} \mathrm{C}\right)$, the catalyst may become a bifunctional catalyst where the $\mathrm{Fe}_{\mathrm{x}} \mathrm{C}$ catalyst part reoxidized to $\mathrm{Fe}_{3} \mathrm{O}_{4}$ works as a WGS and the $\mathrm{Fe}_{\mathrm{x}} \mathrm{C}$ part as a FTS reaction catalyst. At lower $\left(<310^{\circ} \mathrm{C}\right)$ temperatures the $\mathrm{Fe}_{3} \mathrm{O}_{4}$ phase is a less efficient WGS reaction catalyst. ${ }^{36,37}$

The loss of active Fe surface area via Fe particle growth, i.e. sintering, can cause loss in the number of available active Fe surface sites. This in turn causes lost activity towards the FTS reaction. Deposition of the inactive carbon, i.e. fouling, takes place in the presence of $\mathrm{CO}$ or $\mathrm{H}_{2}: \mathrm{CO}$, causing gradually lost FTS reaction activity as the active $\mathrm{Fe}$ sites become harder to access for the reactants. ${ }^{3}$ Excessive inactive carbon deposition via the Boudouard reaction (Reaction 2.5) and/or carbon filament growth 


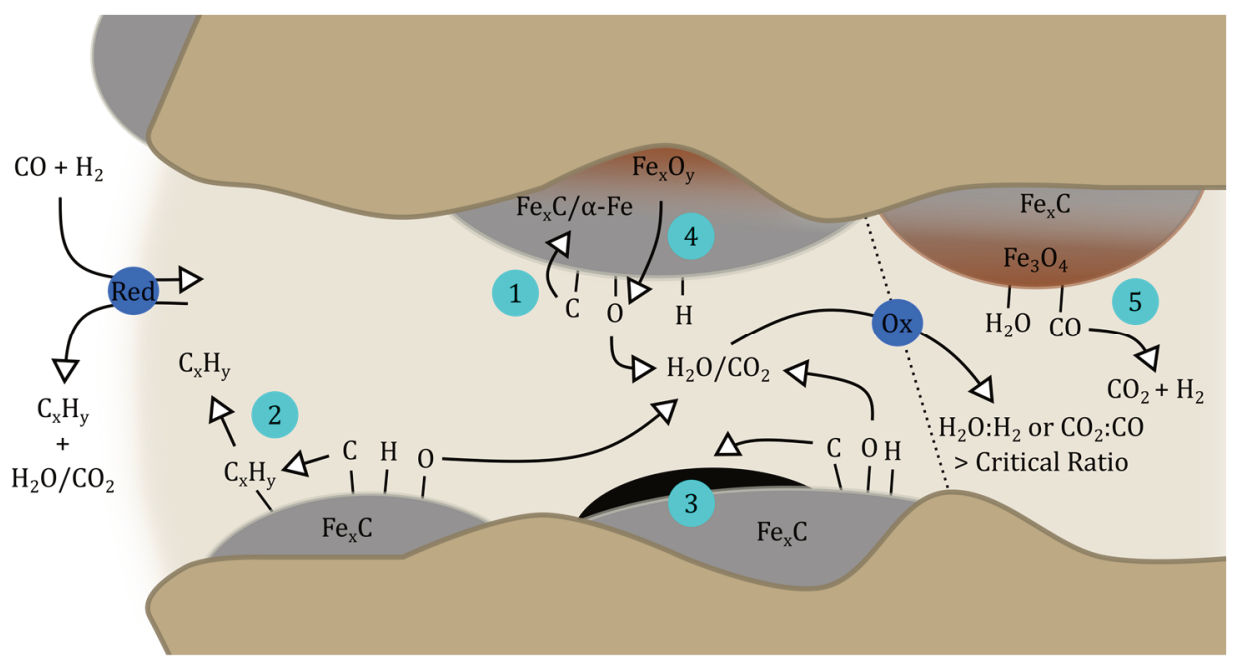

\section{Figure 2.1}

A simplified summary of reaction pathways for $\mathrm{C}$ atoms from input $\mathrm{CO}$ in a Fe-based Fischer-Tropsch Synthesis (FTS) reaction catalyst particle. The input $\mathrm{CO}+\mathrm{H}_{2}$ gives originally a reductive environment (Red). For $C$ from $C O$ dissociation the pathways to react are: (1) with $\alpha$-Fe to form $\mathrm{Fe}_{\mathrm{x}} \mathrm{C}$, (2) with $\mathrm{H}$ to form hydrocarbons, (3) with $\mathrm{C}$ to form inactive carbon. Without $\mathrm{CO}$ dissociation: $\mathrm{C}$ pathway (4) may reduce $\mathrm{Fe}_{\mathrm{x}} \mathrm{O}_{\mathrm{y}}$ to $\alpha$-Fe while releasing $\mathrm{CO}_{2}$. Removal of $\mathrm{O}$ atoms from $\mathrm{CO}$ as $\mathrm{H}_{2} \mathrm{O}$ or $\mathrm{CO}_{2}$ gradually leads to an oxidative environment $(O x)$ towards the core of the catalyst particle, and the outlet of a fixed bed reactor. If the FTS reaction active $\mathrm{Fe}_{\mathrm{x}} \mathrm{C}$ is oxidized to $\mathrm{Fe}_{3} \mathrm{O}_{4}$, pathway (5) may convert $\mathrm{H}_{2} \mathrm{O}+\mathrm{CO}$ to $\mathrm{CO}_{2}+\mathrm{H}_{2}$ via the Water-gas Shift (WGS) reaction.

causes fragmentation of the, especially bulk, Fe-based catalyst materials into a fine powder. ${ }^{3,6,10,76,79}$ The carbon deposition relates to sintering in a twofold manner: i) initially the availability of the FTS reaction active Fe sites may increase due to the catalyst fragmentation into smaller Fe particles exposing new active Fe sites. However, ii) after a longer Time-on-Stream (ToS) the Fe tends to sinter into larger Fe particles, in total resulting in loss of available $\mathrm{Fe}_{\mathrm{x}} \mathrm{C}$ surface area. ${ }^{4,80}$

The fragmentation of the Fe-based FTS reaction catalyst materials is a major issue for their industrial application. The catalyst fragments cause clogging downstream from the FTS reactor and the fragments also need to be separated from the liquid/wax hydrocarbon products.3,6,10,79 If a Fe-based FTS reaction catalyst material is deactivated by carbon deposition, it can be regenerated with $\mathrm{H}_{2}$ reduction $>350^{\circ} \mathrm{C}$ by hydrogenating and removing the carbon deposits. ${ }^{3}$ However, if the catalyst has fragmented, the resulting fine Fe powder still needs to be dealt with to avoid the related issues.3,6,10,79 In this case, the simple $\mathrm{H}_{2}$ regeneration is insufficient method for increasing the $\mathrm{Fe}$ catalyst's life-time.

Deposition of chemical compounds on the Fe phases can decrease their reactivity 
under FTS reaction conditions. Commonly, the addition of electropositive compounds as chemical promoters (e.g. compounds of $\mathrm{K}$ or $\mathrm{Na}$ ) can have a beneficial effect on the hydrocarbon forming FTS reaction.3,11-13 While deposition of electronegative compounds, most commonly $\mathrm{S}$ containing compounds, have a deactivating effect decreasing CO conversion and thus the catalyst's FTS reaction activity. 3,19,81-83

Work function describes the minimum energy that is required to remove an electron from a chemical compound's highest occupied energy level (the Fermi level). ${ }^{84}$ In general, lower the work function, the easier for a chemical phase is to donate electrons towards an adsorbate causing it to react. Within context of current discussion, the electronegative compounds increase the $\alpha-\mathrm{Fe} / \mathrm{Fe}_{\mathrm{x}} \mathrm{C}$ surface's work function, thereby lowering its reactivity and ability to adsorb and dissociate $\mathrm{H}_{2}$ and CO. ${ }^{85-88}$ Deactivation of Fe with $\mathrm{S}$ containing compounds is not only via the mentioned electronic effects but also geometric effects i.e. $\mathrm{Fe}_{\mathrm{x}} \mathrm{C}$ surface site dependent effects, can play a role.86,87,89 These electron donation and geometric effects are discussed more later on.

To conclude the current section and to summarize the main discussion points, the $C$ atoms from input $\mathrm{CO}$ has five pathways to react under FTS reaction conditions when a Fe-based catalyst material is used (Figure 2.1):

1) with $\alpha$-Fe to form $\mathrm{Fe}_{\mathrm{x}} \mathrm{C}$ phases

2) with $\mathrm{H}$ to form hydrocarbons

3) with $\mathrm{C}$ to form inactive carbon

4) as $\mathrm{CO}$ to form $\mathrm{CO}_{2}$ via $\mathrm{Fe}_{\mathrm{x}} \mathrm{O}_{\mathrm{y}}$ reduction

5) as $\mathrm{CO}$ to form $\mathrm{CO}_{2}$ via WGS reaction
(Reaction 2.11)

(Reactions 2.1 and 2.2)

(Reaction 2.5)

(Reaction 2.8)

(Reaction 2.4)

The $\mathrm{O}$ from $\mathrm{CO}$ dissociation is removed as $\mathrm{H}_{2} \mathrm{O}$ or $\mathrm{CO}_{2}$, possibly gradually transforming the FTS reaction atmosphere, from reductive/carburizing $\mathrm{H}_{2}$ : $\mathrm{CO}$ towards oxidative $\mathrm{H}_{2} \mathrm{O}$ - and/or $\mathrm{CO}_{2}$-rich conditions. Under the oxidative atmosphere, FTS reaction active $\mathrm{Fe}_{\mathrm{x}} \mathrm{C}$ may reoxidize to $\mathrm{Fe}_{3} \mathrm{O}_{4}$, a phase which can catalyze the WGS reaction. The reverse WGS reaction in turn may convert the $\mathrm{CO}_{2}$ back to $\mathrm{CO}$. Ideally, to keep up with the earlier mentioned Green Chemistry principles with minimum waste of elemental C (Chapter 1): only the pathway 2) should be active with all the 0 atoms preferably removed as $\mathrm{H}_{2} \mathrm{O}$. This in order to achieve the ideal FTS reaction carbon efficiency from the input $\mathrm{H}_{2}: \mathrm{CO}^{32}$

\subsection{HYDROCARBON AND C PATHWAY SELECTIVITY}

By varying the FTS reaction process conditions and the catalyst composition, the FTS hydrocarbon product selectivity can be steered towards the desired hydrocarbons. The 
changes can also affect the relative $C$ pathways (Figure 2.1) by modifying the pathway selectivity towards or away from the FTS reaction producing the hydrocarbons.

In the following sections, we will discuss the effects of the applied FTS reaction process conditions and the alkali-S chemical promotion, mainly as when applied in Febased FTS reaction. Within the scope of this Chapter, the FTS reaction hydrocarbon selectivity discussion is limited to the $\mathrm{CH}_{4}$ formation, to so-called $\alpha$-value and to FTS hydrocarbon products' olefinicity. Processes such as chain-branching and oxygenate formation are not discussed here. ${ }^{28}$ We shall start by introducing the Anderson-SchulzFlory (ASF) distribution that governs the hydrocarbon chain length fractions obtainable from the FTS reaction.

\subsubsection{Anderson-Schulz-Flory Distribution}

In the ASF distribution model, the earlier discussed $-\mathrm{CH}_{2}$ - entities on the FTS reaction catalyst's active metal surface may either polymerize to longer hydrocarbon chains or terminate the chain growth and desorb as the primary products. The ASF distribution in its logarithmic form can be expressed as (Equation 2.1):73

$$
\ln \left(\frac{S_{n}}{n}\right)=n \ln \alpha+\ln \frac{(1-\alpha)^{2}}{\alpha} \quad \text { ASF Distribution }
$$

where $S_{n}$ is the hydrocarbon selectivity with $n$ carbon atoms. The probability for the hydrocarbon chain growth is represented by the parameter $\alpha$. A higher $\alpha$-value gives longer chained hydrocarbons while a lower $\alpha$-value gives increasing amounts of $\mathrm{CH}_{4}$ and short-chained hydrocarbons from the FTS reaction. Plotting the $\ln \left(S_{n} / n\right)$ versus $n$, should give a linear correlation in which the term $\ln (\alpha)$ is equal to the slope of the fitted line and the value of the parameter $\alpha$ can be solved.

In practice, deviations from the predicted linearity are commonly observed. The slope of the $\ln \left(S_{n} / n\right)$ versus $n$ plot may change with higher chain length $\left(\gtrsim C_{10}\right)$ hydrocarbon selectivities, ${ }^{28,29,90}$ and averaged $\alpha$-values from separate linear fits may be needed for linearly approximating the data. ${ }^{29}$ Alternatively, more complex carbon chain length distribution models can be used. ${ }^{28,90}$ A single $\alpha$-value obtained from the fit within the region $\lesssim \mathrm{C}_{10}$ is generally lower than the $\gtrsim \mathrm{C}_{10} \alpha$-values, especially for Fe-based catalyst materials. ${ }^{28,29,90}$

Within the $\lesssim \mathrm{C}_{10}$ region, the $\mathrm{C}_{1}$ and $\mathrm{C}_{2}$ selectivities also commonly deviate from the expected linearity. The $\mathrm{C}_{1}$ selectivity (i.e. mainly $\mathrm{CH}_{4}$ ) can be higher, and the $\mathrm{C}_{2}$ selectivity is commonly lower than predicted by the ASF distribution. The deviating $\mathrm{C}_{1}$ selectivity might be e.g. due to hotspots in the FTS reactor bed or preferable reaction sites on the catalyst towards methanation instead of hydrocarbon chain growth, among 
other possible reasons. The $\mathrm{C}_{2}$ deviation is commonly attributed to the secondary reactivity of $\mathrm{C}_{2} \mathrm{H}_{4}$ (ethene) which can readsorb on the active metal surface and polymerize, participating on the hydrocarbon chain growth. $3,28,90$

Nevertheless, despite the deviations, the ASF distribution (Equation 2.1) via the $\alpha$ value is a simple method for approximating the hydrocarbon chain length selectivities between various catalyst materials applied in the FTS reaction.

\subsubsection{Effect of Process Conditions}

Pinpointing the causality effects of FTS reaction process conditions on the formed hydrocarbon products can be a challenging task, due to the intertwined nature of the FTS reaction processes.3,91 The main FTS reaction process parameters for controlling the formed hydrocarbon products and the $\mathrm{C}$ reaction pathways are i) reaction temperature, ii) input $\mathrm{H}_{2}$ :CO ratio and iii) the used gas space velocity. The effect of total reaction pressure is less straightforward for a Fe-based FTS reaction catalyst material. CO conversion level directly by itself does not significantly affect the hydrocarbon selectivities. ${ }^{11,91}$

\subsubsection{Reaction Temperature}

The FTS reaction temperature has the effect of decreasing the $\alpha$-value.3,29,91,92 The reaction temperature's effect is more complex and unclear with respect to the olefin formation. With increasing reaction temperature, the olefin content in total may not change or decreases,3,29,91,93 or even might increase for some of the Fe-based catalyst materials promoted with $\mathrm{K}^{29}$ Conversion of $\alpha$-olefins into internal olefins increases with increasing reaction temperature. ${ }^{29,91}$

On Fe-based catalyst materials, also conversion of CO via the WGS reaction, ${ }^{36,37,39}$ and via the Boudouard reaction increases with increasing reaction temperature. ${ }^{3,14,44,45}$ This increases the total CO conversion, but away from the FTS reaction hydrocarbon products. The inactive carbon deposition from the Boudouard reaction increases roughly $50 \%$ for every $10^{\circ} \mathrm{C}$ increase in the reactor bed temperature. ${ }^{3}$ How the FTS reaction hydrocarbon production $\mathrm{C}$ pathways change in relative terms in comparison to the competing WGS and inactive carbon formation $\mathrm{C}$ pathways, against increasing temperature, is not clear at the time being.

\subsubsection{Input $\mathrm{H}_{2}$ :CO Ratio}

Increasing the FTS reaction input feed $\mathrm{H}_{2}$ : $\mathrm{CO}$ ratio increases the hydrogenation ability of the applied catalyst material. The increased hydrogenation ability decreases the $\alpha$ value via increased probability for the hydrocarbon chain termination and also, the olefin content decreases as any unsaturated C-C bonds are more likely to be hydrogenated. ${ }^{3,29,91}$ Short-chained $\alpha$-olefins are affected more than longer internal 
olefins, as the short $\alpha$-olefins are more susceptible towards secondary hydrogenation reactions. ${ }^{29,91} \mathrm{Also}$, increasing input feed $\mathrm{H}_{2}$ : $\mathrm{CO}$ ratio, in general decreases the inactive carbon deposition rate.3,6,51 This, while keeping in mind the earlier mentioned exception of small amount $(\approx 1 \%)$ of $\mathrm{H}_{2}$ in $\mathrm{CO}$ increasing the inactive carbon deposition in comparison to pure CO input reactant. $3,44,48,49$

Despite the input feed $\mathrm{H}_{2}$ : $\mathrm{CO}$ ratio effect being rather clear - more input $\mathrm{H}_{2}$ in relation to $\mathrm{CO}$ results in more hydrogenation - noteworthy emphasis is that the actual $\mathrm{H}_{2}$ :CO ratio over the different catalyst sites and bed positions may vary. Conversion of $\mathrm{H}_{2}$ :CO in ratio of $\approx 2: 1$ (i.e. ideal FTS), 6,91 WGS activity in forward or reverse directions, 6,33,35,40-42,91 and the possibility of incorporating $\mathrm{H}$ and/or $\mathrm{C}$ to inactive carbon species, ${ }^{14,44,52-54}$ can all modify the local ratio of $\mathrm{H}_{2}: \mathrm{CO}$ (and $\mathrm{H}_{2} \mathrm{O}$ and $\mathrm{CO}_{2}$ content, as discussed earlier) along the catalyst particle or the reactor bed. The locally altered $\mathrm{H}_{2}$ :CO ratios can affect the hydrocarbon selectivities. $3,6,57$

\subsubsection{Gas Space Velocity}

Increasing the reactor gas space velocity has the effect of increasing FTS reaction's hydrocarbon products' olefin content, especially in the short-chained $\alpha$-olefins fraction. As the space velocity increases, the probability for olefin readsorption decreases, decreasing the probability for the secondary hydrogenation reactions.3,29,91 The $\alpha$-value remains unchanged with the changing space velocity.3,91 CO conversion decreases, while the FTS and WGS reaction rates, especially with $\mathrm{Na}$ and $\mathrm{K}$ promoted catalyst materials, increase with increasing space velocity. ${ }^{11,38}$ As the FTS reaction hydrocarbon production rate increases faster than the WGS reaction rate, ${ }^{11,38}$ relatively more $\mathrm{CO}$ is converted towards the desired hydrocarbon products at higher space velocities than is consumed by the WGS reaction.

\subsubsection{Reaction Pressure}

The effect of the total FTS reaction $\mathrm{H}_{2}$ : $\mathrm{CO}$ pressure on the hydrocarbon product selectivity is more complex than the effects of the other aforementioned FTS reaction process parameters. While for a Co-based FTS catalyst the $\mathrm{CH}_{4}$ selectivity decreases with increasing $\mathrm{H}_{2}$ : CO pressure within range $\approx 5-25$ bar, no significant change in the $\mathrm{CH}_{4}$ selectivity with changing reaction pressure is observed for a Fe-based catalyst material. ${ }^{94}$ This holds when the reactor outlet $\mathrm{H}_{2}: \mathrm{CO}$ ratio (i.e. $\approx$ reactor $\mathrm{H}_{2}: \mathrm{CO}$ ratio) is kept constant. ${ }^{94}$ With increasing $\mathrm{H}_{2}$ :CO total pressure within the range $\approx 15-25$ bar, Todic et al. observed a somewhat increased $\alpha$-value and decreased olefin content, but also the outlet $\mathrm{H}_{2}$ :CO ratio dropped, in this way affecting the hydrocarbon selectivities. ${ }^{91}$ In experiments at Sasol with Fe-based catalyst materials, both an increase in the $\alpha$-value and an increase in the olefin content, as well as no change in either of the hydrocarbon selectivity variables with increasing $\mathrm{H}_{2}$ : $\mathrm{CO}$ reaction pressure, have been observed. ${ }^{3} \mathrm{An}$ 
increase in the total $\mathrm{H}_{2}$ : $\mathrm{CO}$ pressure increases the Fe-based catalyst material's activity for the FTS reaction. ${ }^{94}$ This in turn can affect the actual reactor $\mathrm{H}_{2}$ : $\mathrm{CO}$ ratio as well as the partial pressures of $\mathrm{H}_{2} \mathrm{O}$ and $\mathrm{CO}_{2}$ via increased $\mathrm{CO}$ conversion, altering the hydrocarbon selectivities, as discussed previously. In overall, the effect of total reaction pressure on the Fe-based FTS hydrocarbon selectivities depend on the individual partial pressures of $\mathrm{H}_{2}, \mathrm{CO}, \mathrm{H}_{2} \mathrm{O}$ and $\mathrm{CO}_{2}$ present in the FTS reactor. 3,6,9,91 Their relation further depends on the used reaction temperature.6,9

With regard to the inactive carbon deposition, as previously briefly mentioned earlier, with the total reaction pressure of $\gtrsim 15$ bar with $\mathrm{H}_{2}$ : $\mathrm{CO}$ ratio $\gtrsim 5$, the inactive carbon formation can be suppressed. ${ }^{3,51}$ This, while improving the $\mathrm{C}$ pathway selectivity towards the FTS reaction hydrocarbon products. ${ }^{3,51}$ With lower input $\mathrm{H}_{2}$ : $\mathrm{CO}$ ratios of $\lesssim 1$, the carbon deposition increases with increasing reaction pressure. ${ }^{51}$ According to Todic et al., the WGS reaction extent decreases with increasing total FTS reaction pressure. ${ }^{91}$

\subsubsection{Effect of Alkali-S Promotion}

Modifying the catalyst composition by adding chemical promoters can alter the FTS reaction's hydrocarbon product and C pathway selectivities towards the desired reaction products.

The addition of combined alkali-S (alkali as Na or $\mathrm{K}$ ) as chemical promoters for Febased FTS catalyst materials has the following effects: i) a decreased $\mathrm{CH}_{4}$ selectivity to a lower value than predicted by the ASF-distribution when alkali and $S$ are present in an optimal ratio, ${ }^{19,20}$ ii) a lesser increase in the hydrocarbon chain length ( $\alpha$-value) in comparison to if only alkali is added, ${ }^{16,18,95}$ ii) an increased hydrocarbon selectivity towards olefins, ${ }^{16,17,19,20}$ and iv) an overall increased CO conversion. ${ }^{20,81,95,96}$ Suppression of the $\mathrm{CO}_{2}$ selectivity, without detrimentally affecting the hydrocarbon production, by addition of sufficient amount of $\mathrm{S}$ to a $\mathrm{K}$ containing Fe-based FTS reaction catalyst material has also been claimed. ${ }^{97}$

First, we shall discuss the general principles of the effect of individual alkali (as $\mathrm{Na}$ or $\mathrm{K}$ ) and $\mathrm{S}$ on the adsorption of $\mathrm{H}_{2}$ and $\mathrm{CO}$ over a transition metal surface. The discussion is then continued towards the experimentally observed effects of alkali, $\mathrm{S}$ and alkali-S promotion on the FTS reaction hydrocarbon and C pathway selectivities.

\subsubsection{General $\mathrm{H}_{2}$ and $\mathrm{CO}$ Adsorption Principles}

The simplified effects of chemical promoters added as electropositive (e.g. Na or K) or -negative (e.g. S) adsorbates on a transition metal (e.g. Fe) are (Figure 2.2):

i) Electropositive adsorbates donate electrons towards the transition metal 
a)

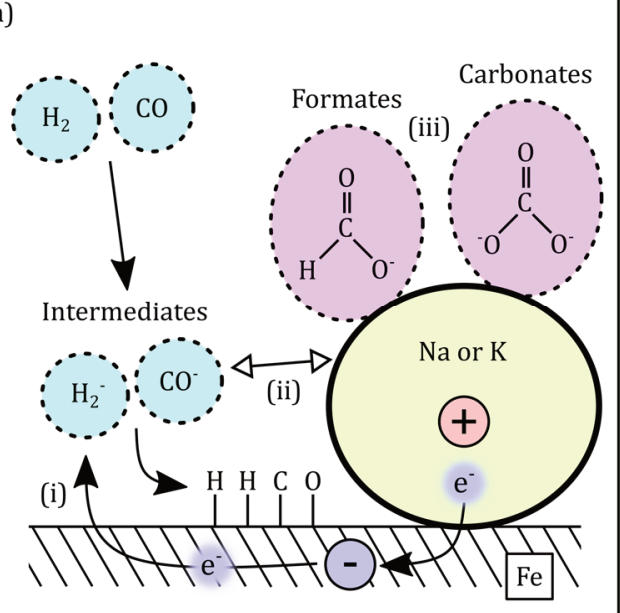

b)

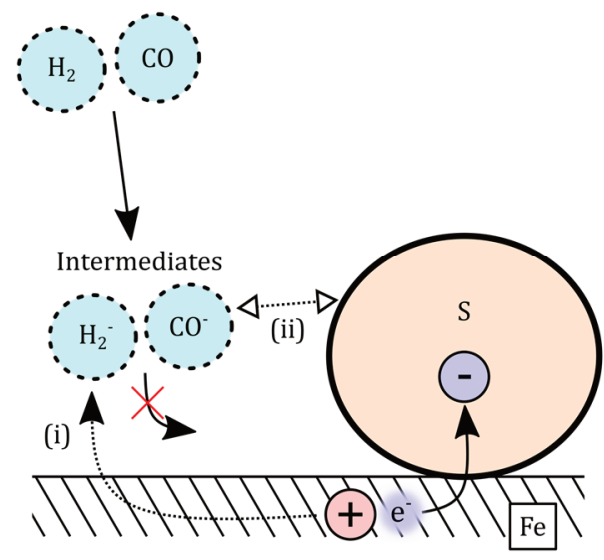

\section{Figure 2.2}

A schematic summary on the general effects of chemical promoter addition on $\mathrm{H}_{2}$ and $\mathrm{CO}$ adsorption over the (near-)zerovalent Fe surfaces (e.g. $\alpha$-Fe or $\mathrm{Fe}_{\mathrm{X}} \mathrm{C}$ ). With added electropositive alkali (as Na or K): a) (i) increased Fe surface electron density increases electron back-donation towards antibonding orbitals of Fe surface adsorbed intermediate $\mathrm{H}_{2}^{-}$and $\mathrm{CO}^{-}$states, (ii) formed alkali-Fe surface dipole moment stabilizes the intermediate $\mathrm{H}_{2}^{-}$and $\mathrm{CO}^{-}$states via electrostatic interaction, and also (iii) formation of formates and/or carbonates is observed. With alkali, both effects (i) and (ii) increase dissociative adsorption of $\mathrm{H}_{2}$ and $\mathrm{CO}$. With added electronegative S: b) (i) electron back-donation from Fe surface is weakened, and (ii) intermediate $\mathrm{H}_{2}^{-}$and $\mathrm{CO}^{-}$states are destabilized. With $\mathrm{S}$, the effects (i) and (ii) decrease dissociative adsorption of $\mathrm{H}_{2}$ and $\mathrm{CO}$.

surface, decreasing the measurable surface work function; while the electronegative adsorbates do the opposite. ${ }^{84,86,98}$

ii) To modify the electrostatic potential near the transition metal surface, due to dipole moment formed between the - partly or fully ionized - chemical promoter adsorbate and the transition metal surface. ${ }^{84,98}$

iii) To possibly offer direct adsorption or reaction sites for reactants (e.g. $\mathrm{H}_{2}$ or CO). ${ }^{87,98-100}$

Under FTS reaction relevant conditions on $\mathrm{Fe}_{\mathrm{x}} \mathrm{C}$ surface, both $\mathrm{H}_{2}$ and $\mathrm{CO}$ adsorb dissociatively due to the electron back-donation from the $\mathrm{Fe} d$-orbitals to the antibonding $\mathrm{C}-\mathrm{O} \pi^{*}$ and $\mathrm{H}-\mathrm{H} \sigma^{*}$ molecular orbitals. ${ }^{85-88,98-100}$ The dissociation of the adsorbed $\mathrm{H}_{2}$ and $\mathrm{CO}$ on $\mathrm{Fe}_{\mathrm{x}} \mathrm{C}$ surface is affected by i) the availability of electrons in the $\mathrm{Fe}$ d-orbitals for the back-donation and ii) stability of the $\mathrm{Fe}_{\mathrm{x}} \mathrm{C}$ surface adsorbed, intermediate states of the $\mathrm{H}_{2}$ and $\mathrm{CO}$ molecules. ${ }^{85,88,98,99}$

Addition of alkali in moderation increases the Fe d-orbital electron density and availability, i.e. decreases the surface work function; while the S addition does the 
opposite. ${ }^{85-88,98-100}$ Via the electrostatic interaction between the formed promotersurface dipole moment and the reactant adsorbates: electropositive alkali stabilizes the $\mathrm{H}_{2}^{-}$and $\mathrm{CO}^{-}$intermediate states on the $\mathrm{Fe}_{\mathrm{x}} \mathrm{C}$ surface, while the electronegative $\mathrm{S}$ destabilizes them. ${ }^{85,88,98,99}$ In total then, alkali as an individual chemical promoter in $\mathrm{Fe}$ based catalyst material increases the dissociative adsorption of $\mathrm{H}_{2}$ and $\mathrm{CO}$. While added $\mathrm{S}$ promoters, individually, work vice versa decreasing the dissociative adsorption of $\mathrm{H}_{2}$ and $\mathrm{CO}$. The dissociative $\mathrm{H}_{2}$ and $\mathrm{CO}$ adsorption promoting effects by electropositive alkali $\mathrm{K}$ and inhibiting effects by electronegative $\mathrm{C}, \mathrm{O}$ and $\mathrm{S}$ over a surface of metallic state Fe have been experimentally observed e.g. by Benziger and Madix. ${ }^{87}$

Furthermore, with added alkali, carbonate or formate species are commonly observed upon CO adsorption, species which are absent without alkali promotion. ${ }^{26,98,101-104}$ Also, direct interaction between the alkali and the metallic state Fe surface adsorbed $\mathrm{H}_{2}$ and $\mathrm{CO}$ has been suspected, possibly aiding in the molecules' dissociation. 87,105

Upon addition of alkali (as $\mathrm{K}$ ), the desorption temperatures for adsorption products of $\mathrm{H}_{2}$ and $\mathrm{CO}$ are increased with the expense of lower temperature desorption. ${ }^{87,99,100}$ That is, added alkali increases the overall binding strength of $\mathrm{H}_{2}$ and $\mathrm{CO}$ adsorption/desorption products. Within the FTS reaction relevant temperatures $\left(\$ 340^{\circ} \mathrm{C}\right.$ ), the $\mathrm{H}_{2}$ adsorption products are more strongly bound than the CO products with addition of alkali K. ${ }^{87}$ Addition of electronegative adsorbates (e.g. S or 0 ) has the opposite effect of lowering the desorption temperatures for the adsorbed products of $\mathrm{CO}$ and $\mathrm{H}_{2} .87,99$

The measured adsorption energy change for an alkali K promoted Fe metal surface is higher for $\mathrm{CO}(\approx+3.0 \mathrm{kcal} / \mathrm{mol})$ than for $\mathrm{H}_{2}(\approx+1.5 \mathrm{kcal} / \mathrm{mol}) .{ }^{100}$ Worth noting is that in a commonly cited study in the realm of FTS reaction research by Dry et al., ${ }^{106}$ the heat of $\mathrm{H}_{2}$ chemisorption was found to decrease due to the addition of $\mathrm{K}$ promotion. That is, added $\mathrm{K}$ would inhibit the $\mathrm{H}_{2}$ adsorption, in contradiction to the present discussion. However, as noted by Benziger and Madix, ${ }^{87}$ the results by Dry et al. were likely obtained on an oxide rather than a Fe metal (or carbide) surface and the observed $\mathrm{H}_{2}$ inhibition effect was more likely due to the presence of $\mathrm{O}$ atoms rather than $\mathrm{K} .{ }^{87}$

Besides the added chemical promoter electropositivity and -negativity arguments on the $\mathrm{H}_{2}$ and $\mathrm{CO}$ adsorption, as mainly discussed above, also the alkali or $\mathrm{S}$ adsorbate Fe surfaces' binding geometry can play a role. ${ }^{87}$ Despite 0 atom adsorbates being more electronegative and $\mathrm{C}$ atom adsorbates having similar electronegativity as $\mathrm{S}$ has, the $\mathrm{S}$ atom adsorbates on metallic Fe surface showed more pronounced inhibition effect on $\mathrm{CO}$ and $\mathrm{H}_{2}$ adsorption than either $\mathrm{O}$ or $\mathrm{C}$ did. The effect was thought to be due to different binding geometries which the $\mathrm{O}$ and $\mathrm{C}$ take versus the $\mathrm{S}$ adsorbates on the metallic Fe surface. ${ }^{87}$ The different binding of $\mathrm{O}$ and $\mathrm{S}$ elements might be a very relevant 
factor when a combined Na-S promotion is applied in the FTS reaction, as will be discussed later.

\subsubsection{Alkali in the Fischer-Tropsch Synthesis Reaction}

The most common chemical promoter added to a Fe-based FTS catalyst material is alkali as an electropositive chemical species of $\mathrm{K}$ or $\mathrm{Na} .2,3,11,13,95,107$ Other alkali and alkaline-earth elements have also been screened for the FTS reaction promotion but $\mathrm{K}$ and Na remain the most suitable, in both terms of the FTS reaction activity and selectivity. ${ }^{9,11-13}$

The addition of alkali by itself to a Fe-based catalyst material has the effect on the FTS reaction hydrocarbon selectivity of increasing i) the $\alpha$-value and ii) the hydrocarbon product slate olefin content.3,9,11,29,50,93,107 The alkali hydrocarbon selectivity effect is commonly attributed to an overall decrease in the catalyst's hydrogenation ability i.e. on the $\mathrm{Fe}_{\mathrm{x}} \mathrm{C}$ surface $\mathrm{H}: \mathrm{C}$ ratio. The decreased $\mathrm{H}$ :C ratio results in decrease in the hydrocarbon chain termination and in olefin hydrogenation via primary or secondary pathways. 3,9,29,50,107

The lowered $\mathrm{H}: \mathrm{C}$ surface ratio with alkali addition can be explained as $\mathrm{i}$ ) adsorption energy is increased more for $\mathrm{CO}(+3.0 \mathrm{~kJ} / \mathrm{mol})$ than for $\mathrm{H}_{2}(+1.5 \mathrm{~kJ} / \mathrm{mol}){ }^{100}$ Thus, $\mathrm{CO}$ is more preferably adsorbed than $\mathrm{H}_{2}$ when $\mathrm{H}_{2}$ and $\mathrm{CO}$ compete for the alkali promoted $\mathrm{Fe}_{\mathrm{x}} \mathrm{C}$ surface adsorption sites. Additionally, ii) alkali increases the elemental binding strength of $\mathrm{H}$ more than that of $\mathrm{C}$ on metallic $\mathrm{Fe}\left(/ \mathrm{Fe}_{\mathrm{x}} \mathrm{C}\right)$ surface, as has been observed via the increased desorption temperatures of the $\mathrm{H}_{2}$ and $\mathrm{CO}$ adsorption products. ${ }^{87}$ The increased binding strength of $\mathrm{H}$ to the $\mathrm{Fe}_{\mathrm{x}} \mathrm{C}$ surface results in depletion of weakly bound $\mathrm{H}$ available for the FTS reaction. ${ }^{108}$ Therefore, both of the mentioned alkali addition effects in total effectively decrease the $\mathrm{Fe}_{\mathrm{x}} \mathrm{C} \mathrm{H}: \mathrm{C}$ surface ratio available for hydrocarbon forming FTS reactions.

Increasing alkali promotion has the intrinsic tendency to increase the inactive carbon deposition $\mathrm{C}$ pathway via the Boudouard reaction, along the in overall increased CO conversion and dissociation. ${ }^{3,14,46}$ Although, a sufficiently low level of added alkali may nevertheless decrease the carbon deposition rate in comparison to a respective unpromoted Fe-based catalyst material. ${ }^{3}$ This effect with still observable alkali promotion effects in the FTS hydrocarbon selectivity. ${ }^{3}$ The alkali also increases the WGS reaction rate, $50,107,109$ consuming $\mathrm{CO}$ towards the WGS reaction products. This simultaneously decreases the C pathway selectivity towards the FTS reaction hydrocarbon production. ${ }^{11,12}$ Alkali promotes the WGS reaction even without a presence of a transition metal. ${ }^{103,104}$ That is, as undesired effects from the alkali addition: $\mathrm{C}$ pathway selectivity towards FTS reaction hydrocarbon products decreases due to CO being consumed towards the WGS and/or inactive carbon depositing 
Boudouard reactions.

Nevertheless, in the case of low input $\mathrm{H}_{2}$ :CO ratio, the WGS reaction may become the rate determining step for the FTS reaction as the hydrocarbon production becomes dependent on the $\mathrm{H}_{2}$ from the WGS reaction. ${ }^{11,38}$ In this case, the CO consuming and alkali promoted the WGS reaction becomes a possibly useful property for the applied FTS reaction catalyst material. ${ }^{28}$

\subsubsection{S in the Fischer-Tropsch Synthesis Reaction}

The effect of $\mathrm{S}$ individually in heterogeneous catalysis is a complex phenomenon. ${ }^{86,89}$ Too much of added $S$ compounds result in a catalyst poisoning. ${ }^{81,82,86,110}$ However, small amounts of added S may have beneficial FTS reaction catalysis effects. S containing impurities in the $\mathrm{CO}$ gas can also gradually enrich on the catalyst, thus altering its behaviour. ${ }^{46}$ From a review article by Madon and Shaw, some of the possible effects of the $\mathrm{S}$ addition in FTS reaction are: 86

1) avoiding reactor hotspots via decreased CO conversion,

2) selective $S$ adsorption on different reaction sites i.e. selectively affecting reaction pathways,

3) S itself may become an active site for reactions,

4) S functions as an electron acceptor i.e. is an electronegative adsorbate on the transition metals and depletes the metals from electrons, as also was discussed previously.

As mentioned earlier, the FTS reaction is in total an exothermic reaction and any hotspots created by the released heat to the reactor bed may cause the bed temperature to increase locally. This results in decreased $\alpha$-value, which most easily can be seen as increased $\mathrm{CH}_{4}$ selectivity, and/or inactive carbon deposition via the Boudouard reaction (Reaction 2.5). If a FTS reactor bed is operated non-isothermally, S poisoning decreases $\mathrm{CO}$ conversion eliminating the hotspots, which can result in increased $\alpha$ value (decreased $\mathrm{CH}_{4}$ ) and decreased carbon deposition. That is, the listed point 1) is relatively easy to understand.

Dalla Betta, Piken and Shelef observed decreased catalyst activities with added S, as well as decreased $\mathrm{CH}_{4}$ and increased $\mathrm{C}_{2}$ selectivities and also discussed signs of possible non-isothermal reactor bed operation in their data. ${ }^{111}$ Similar effects where $\mathrm{S}$ addition likely affects the initial reactor bed operating temperature, can be observed in the studies by van Dijk et al. and Zhou et al. with Fe-based FTS reaction at $350^{\circ} \mathrm{C} .112,113$ In the study by van Dijk et al., the added S prevented an initial spike of high FTS catalysis activity in comparison to the non-sulphated catalyst. The added S likely prevented any 
excessive reactor bed heating at the initiation of the FTS reaction, limiting the fast catalyst deactivation from excessive formation of inactive carbon species. ${ }^{112}$ Same comments apply to the study by Zhou et al. who exposed their pre-reduced Fe-based FTS reaction catalyst material to $\mathrm{H}_{2}$ : CO directly at $350^{\circ} \mathrm{C} .113$ This is an initial reaction temperature too high for ensuring isothermal bed operation without excessive reactor bed heating. ${ }^{30}$ The likely role of the sufficient $S$ addition in their study has been to poison the catalyst and to decrease the CO conversion, and thus to dampen the initial non-isothermal FTS reactor bed operation. This would explain the observed decrease in the carbon deposition with S addition in the study. ${ }^{113}$

Definitely pinpointing the effect of the listed points 2), 3) and 4) and their possible combinations in relation to Fe-based FTS reaction catalysis effects is a complex task. In addition, unfortunately, a variety of the S poisoning studies with FTS reaction have been conducted with alkali containing Fe-based catalyst materials. Thus, mixing both the FTS reaction promotion effects by alkali-S combination and possible poisoning by excessive $\mathrm{S}$ addition. This further complicates the task of assigning causality on the $\mathrm{S}$ effects in the FTS reaction, in relation to the listed points.

In several studies, positive $\mathrm{S}$ promotion effects in the Fe-based FTS reaction have been observed. However, in these studies alkali promoted catalysts promoted, intentionally or inadvertently, with synthesis precursors or precipitation agents containing the alkali promoters, have commonly been used.81,82,95,114-118 Washing of the prepared catalyst materials with deionized water in order to remove the alkali, ${ }^{81,117}$ or possible S-ions, ${ }^{117,119}$ is insufficient in order to have an alkali- or a S-free FTS reaction catalyst material free of promotion or poisoning effects.

From a $\mathrm{S}$ poisoning study by Bromfield and Coville, ${ }^{117}$ it can be pointed out that $\mathrm{Na}_{2} \mathrm{~S}$ was used as the $\mathrm{S}$ precursor and $\mathrm{Na}_{2} \mathrm{CO}_{3}$ as the catalyst precipitation agent. Despite the subsequent washing to remove the $\mathrm{Na}$ atoms from the precipitated Fe catalyst material, with low levels of added $S$, the catalytic FTS reaction results resemble of what would be expected for the Na-S promotion. That is, increased catalytic activity and increased olefin content. ${ }^{117}$ It seems unlikely that the $\mathrm{Na}$ atoms were removed with catalyst washing and the combined Na-S rather than S-only promotion explains the positive $\mathrm{S}$ addition effects in their study. Presumably, the work led to a later Sasol patent on Na-S promotion with Fe-based FTS reaction catalyst materials. ${ }^{18}$

Furthermore, in a study by Shultz et al., the initial S addition on $\mathrm{K}$ containing catalysts resulted in an initial promotion effect by K-S but this was followed by $S$ poisoning effects when too much of $S$ was added. ${ }^{82}$ In the study, with low level of COS added as the $S$ bearing gas compound, slight increase in hydrocarbon products olefinicity could be observed. Despite the increased reaction temperature, there was no increase in $\mathrm{CH}_{4}$ selectivity. Both effects can be explained by K-S promotion. Further 
addition of COS increased the $\mathrm{CH}_{4}$ selectivity (and seemingly decreased the $\alpha$-value), this despite the simultaneously decreased reaction temperature which should decrease the $\mathrm{CH}_{4}$ selectivity. And also simultaneously with the increasing $\mathrm{S}$ addition, the olefin selectivity decreased for the catalyst. ${ }^{82}$ The $\mathrm{S}$ poisoning effects of decreased olefin content and increased $\mathrm{CH}_{4}$ formation can also be observed when different experimental runs with the same reaction temperature are compared in the study. ${ }^{82}$ Similar observations have been made by Botes et al., i.e. excessive $\mathrm{S}$ addition on alkali containing Fe-based catalysts increases $\mathrm{CH}_{4}$ selectivity in the FTS reaction. ${ }^{19}$

In the simplest way, the "promotion effects" of increasing $\mathrm{CH}_{4}$ selectivity and decreasing olefin content by $\mathrm{S}$ poisoning of alkali containing catalyst material can be explained as S being electronegative adsorbate (i.e. with the listed point 4)). That is, functioning in an opposite way to the alkali addition (Figure 2.2). ${ }^{86}$ The $\mathrm{S}$ addition thus likely increases the overall $\mathrm{H}: \mathrm{C}$ surface ratio resulting in increased hydrocarbon hydrogenation and chain termination reactions.

It is unclear how the $\mathrm{S}$ effects which are not directly related to the $\mathrm{S}$ elements electronegativity, i.e. the points 2) and 3), affect the observed selectivities in the Febased FTS reaction. Generally, the adsorbate $\mathrm{S}$ might selectively block some geometric reaction sites, such as the active steps. This might affect different the reaction rates and pathways to different degrees in the FTS reaction. ${ }^{86,89}$ Some evidence has been observed on the effect of site-blocking for some model catalytic systems. ${ }^{89}$ However, how the data obtained from ultra-high vacuum model experiments compares to a more dynamic Fe-based FTS catalyst materials behavior at higher reaction pressures, is not clear. ${ }^{89}$

As excessive addition of $\mathrm{S}$ eventually covers the active $\mathrm{Fe}_{\mathrm{x}} \mathrm{C}$ surface decreasing its FTS reaction activity, $, 2,114,115,117,118$ and CO dissociation capability, ${ }^{46,120}$ it's unlikely that $\mathrm{S}$ itself performs as an active site for the FTS reaction. However, some cautious doubt can be expressed as observations of the $\mathrm{S}$ compounds possibly assisting in dehydrogenation reactions with Fe-based catalyst materials have been made. ${ }^{121}$

In contrast to the cited alkali-containing Fe-based catalyst material studies on the $\mathrm{S}$ effects in the FTS reaction, when catalyst precursors free of alkali have been used, the catalyst is directly poisoned by a low level of $\mathrm{S}$ addition. $\mathrm{S}$ poisoning effects of decreasing: i) $\alpha$-value, ${ }^{83}$ ii) olefin content versus paraffins, ${ }^{83,112}$ and iii) overall CO conversion, ${ }^{81,83}$ have been observed. Interestingly in the study by $\mathrm{Xu}$ et al. with alkalifree Fe-based catalyst material in the FTS reaction: the $\mathrm{CH}_{4}$ selectivity remained virtually unaffected despite the increasing $S$ content and decreasing $\alpha$-value.83 This seems to be in contrast to the Fe-based, alkali containing catalyst materials for which the $\mathrm{CH}_{4}$ selectivity increases with excessive addition of S. ${ }^{19,82}$

In terms of the various $C$ pathways, the effect of the added $S$ might be simply to 
decrease the overall $\mathrm{CO}$ and $\mathrm{H}_{2}$ dissociation, without altering the relation between the $\mathrm{C}$ pathway selectivities. Although the overall carbon deposition decreases with added $\mathrm{S},{ }^{46,120,122}$ so does the CO conversion. ${ }^{83}$ That is, whether S truly suppresses carbon deposition without decreasing the FTS reaction hydrocarbon production as a promotion effect, as is sometimes discussed, needs to be compared in relative terms to the CO converted to FTS reaction hydrocarbon products. Such studies do not seem to exist at the moment. On Fe-based WGS reaction catalysts, $\mathrm{S}$ behaves as a poison. ${ }^{37,39}$ Thus, unlike the alkali, $\mathrm{S}$ does not promote the WGS reaction. In overall, further studies would be of interest on determining - with alkali-free Fe-based catalyst materials - the effect of individually added $\mathrm{S}$ on the relative $\mathrm{C}$ pathway selectivities in FTS reaction catalysis. Moreover, in these studies, the results should be normalized to the overall CO conversion over the observation period.

\subsubsection{Alkali-S in the Fischer-Tropsch Synthesis Reaction}

In the light of individual alkali and S promotion properties with regard to the FTS reaction hydrocarbon selectivities: the combined alkali-S promotion effect resembles both. The increased olefin selectivity and CO conversion are "alkali-like", while the decreased $\alpha$-value in comparison to alkali-only addition is "sulfur-like". The unique effect of decreased $\mathrm{CH}_{4}$ selectivity to a lower value than expected from the ASFdistribution is solely an combined alkali-S effect. ${ }^{19}$ Together these effects enable increased yields towards short-chained $\mathrm{C}_{2}-\mathrm{C}_{4}$ olefins.

The unique disruption of the lowered $\mathrm{CH}_{4}$ selectivity has been proposed to be due to a changed binding geometry of the chemical promoter compound when the $S$ is added as a counter-ion for the alkali promoter. ${ }^{123}$ Commonly, the alkali is thought to be in an oxidized form under the FTS reaction conditions, although the exact chemical compound is uncertain. ${ }^{98,106}$ Benziger and Madix have observed oxidation of $\mathrm{K}$ atoms by $\mathrm{O}$ from $\mathrm{CO}$ dissociation in their metallic Fe surface studies, in support of the thought for Koxidation. ${ }^{87}$

The added $\mathrm{S}$ to $\mathrm{Na}$ promotion likely replaces the $\mathrm{O}$ as the alkali's counter-ion resulting in a Na-S compound instead of a Na-O compound under the FTS reaction conditions. ${ }^{123}$ From a combination of experimental data and theoretical calculations applying Density Functional Theory (DFT), Xie et al. concluded that the counter-ion change from $\mathrm{O}$ to $\mathrm{S}$ i) increases the $\mathrm{Na}$ electron donation towards $\mathrm{Fe}$ as $\mathrm{S}$ is less electronegative than $\mathrm{O}$, as well as ii) changes the Na-S promoter compound binding geometry on the $\mathrm{Fe}_{\mathrm{x}} \mathrm{C}$ surface. The combined effect decreases the availability of $\mathrm{H}$ for the hydrogenation reactions, resulting in especially suppressed $\mathrm{CH}_{4}$ formation. ${ }^{123}$

As mentioned before, Benziger and Madix have discussed the individual difference between $\mathrm{O}$ (and $\mathrm{C}$ ) and $\mathrm{S}$ adsorbates on the metallic Fe surface. ${ }^{87}$ Their experimental 
a)

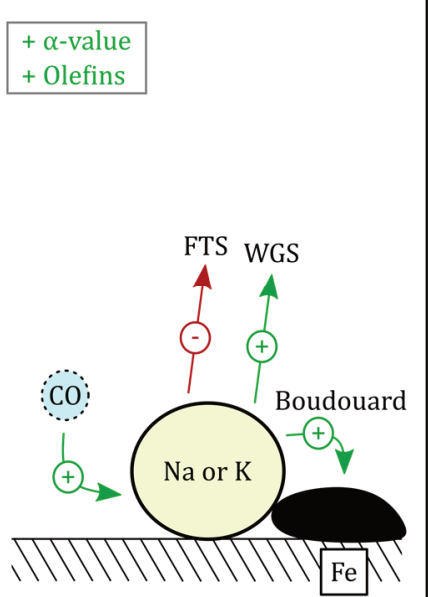

b)
$-\alpha$-value
- Olefins

c)

- Olefins

Figure 2.3

A schematic summary on the effects of chemical promoters on (near-)zerovalent Fe surfaces (e.g. $\alpha$-Fe or $\mathrm{Fe}_{\mathrm{X}} \mathrm{C}$ ). The effects of a) alkali (as Na or K), b) S and c) alkali-S in molar ratio of 2:1 in Fe-based Fischer-Tropsch Synthesis (FTS) reaction, on FTS hydrocarbon product and C pathway selectivities and on overall CO conversion. Green - increased, Red - decreased, and ? - an unknown change in the property in comparison to an unpromoted catalyst material. The combined c) alkali-S promotion increases $\mathrm{CO}_{2}$ selectivity, likely indicating increased Water-gas Shift (WGS) and/or Boudouard reaction C pathway selectivities.

results and discussion are in support of the different binding modes for $\mathrm{O}$ and $\mathrm{S}$. The $\mathrm{S}$ adsorbates bind on the metallic Fe surface in a manner which more effectively inhibits the adsorptive dissociation of $\mathrm{H}_{2}$ and $\mathrm{CO}$ than do $\mathrm{O}$ adsorbates, despite $\mathrm{O}$ being more electronegative and Fe surface electron depleting than S. ${ }^{87}$ The experimental results from Benziger and Madix thus make similar suggestions as do the results by Xie et al.. How the chemical promoter alkali-S, in comparison to alkali-O adsorbate compound, covers the $\mathrm{Fe}_{\mathrm{x}} \mathrm{C}$ surface, can have an effect on the observed FTS reaction promotion effects.

Combined alkali-S promotion affects the $\mathrm{C}$ reaction pathways for the dissociated $\mathrm{CO}$ by: increasing the overall CO conversion, ${ }^{20,81,95,96}$ and might also increase, at least the initial, carbon deposition rate. ${ }^{21,95,96}$ Although, in the cited studies regarding the carbon deposition, the vast differences in the $\mathrm{CO}$ conversion levels have not been accounted for between the different catalysts and promotion levels. The source for inactive carbon deposited on the catalysts is CO. For low CO conversion carbon deposition is necessarily low and vice versa. For meaningful comparison of carbon deposition between various alkali-S promoted catalysts: the carbon deposition needs to be normalized to the total (i.e. integral of) CO conversion over the measurement period. Otherwise, the carbon deposition results can be misleading and the conclusions misguided. 


\section{Table 2.1}

The effects of various Fischer-Tropsch Synthesis (FTS) reaction process parameters and alkali, S and alkali$\mathrm{S}$ chemical promoters on the $\mathrm{C}$ reaction pathways and on the FTS hydrocarbon selectivity in the Fe-based FTS reaction.

\begin{tabular}{|c|c|c|c|c|c|c|c|}
\hline \multirow[t]{3}{*}{ Parameter } & \multicolumn{3}{|c|}{ Hydrocarbon Selectivity } & \multirow{3}{*}{$\begin{array}{c}\text { CO } \\
\text { Conversion }\end{array}$} & \multicolumn{3}{|c|}{ C Pathway Selectivity } \\
\hline & $\mathrm{CH}_{4}$ & $\alpha$-value & Olefins & & FTS & WGS & Carbon \\
\hline & & & & & & & Deposition \\
\hline Temperature & $\uparrow$ & I & $\sim$ & $\uparrow$ & $\sim$ & $\uparrow$ & $\uparrow$ \\
\hline $\mathrm{H}_{2}: \mathrm{CO}$ & $\uparrow$ & $\downarrow$ & $\downarrow$ & $\sim$ & $\sim$ & $\sim$ & $\downarrow$ \\
\hline Space Velocity & $\sim$ & $\leftrightarrow$ & $\uparrow$ & $\downarrow$ & $\uparrow^{\mathrm{a})}$ & $\downarrow$ & $\sim$ \\
\hline Total Pressure & $\sim$ & $\sim$ & $\sim$ & $\uparrow$ & $\sim$ & 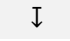 & 1b) \\
\hline Alkali & I & $\uparrow$ & $\uparrow$ & $\uparrow$ & $\beth$ & $\uparrow$ & \c) \\
\hline S & $\backsim d)$ & $\downarrow$ & $\downarrow$ & $\downarrow$ & $\sim$ & $\sim$ & $\sim e)$ \\
\hline Alkali-S $(\approx 2: 1)$ & $\left.I^{f}\right)$ & $\uparrow \mathrm{g})$ & $\uparrow$ & $\uparrow$ & $\sim$ & $\backsim$ h) & $\Upsilon^{h, i)}$ \\
\hline
\end{tabular}

Symbols: $\uparrow$ or $\downarrow$ - increasing or decreasing FTS reaction property against increasing parameter.

$\leftrightarrow \quad$ - no effect.

$\sim \quad$ - complex or unclear effect.

Alkali: $\quad \mathrm{Na}$ or $\mathrm{K}$ atoms as added promoters.

a) FTS rate increases faster than the Water-gas Shift (WGS) reaction rate with increasing space velocity.

b) With $\gtrsim 15$ bar total reaction pressure and $5 \gtrsim \mathrm{H}_{2}: \mathrm{CO}$ ratio carbon deposition can be suppressed.

c) Low addition levels of alkali may not increase the carbon deposition rate and might even lower it.

d) Excessive $\mathrm{S}$ addition increases the $\mathrm{CH}_{4}$ selectivity, if alkali is present.

e) Commonly, a decrease is observed, likely, due to the overall decrease in CO conversion.

f) Selectivity of $\mathrm{CH}_{4}$ can be suppressed to a value below the Anderson-Schulz-Flory (ASF) distribution expectations.

g) The $\alpha$-value is increased less than with only alkali addition.

h) $\mathrm{CO}_{2}$ selectivity with alkali-S promotion increases.

i) At least, the initial carbon deposition rate at the start of the FTS reaction might increase.

Nevertheless, as significant $\mathrm{CO}_{2}$ selectivity in the FTS reaction has been observed in the presence of alkali-S promotion with the Fe-based catalyst materials: ${ }^{20,21,95,96}$ either increased WGS and/or carbon depositing Boudouard reactions, both releasing $\mathrm{CO}_{2}$, can be suspected to be active. Increased $\mathrm{CO}_{2}$ selectivity with the presence of alkali-S promotion would be akin to the earlier discussed alkali-only addition of increasing WGS and carbon deposition reactions, therefore, being an "alkali-like" property. Moreover, after experiments at Sasol, Botes et al. suggest that CO conversion selectivity towards FTS reaction hydrocarbon production decreases with the added alkali-S promotion. However, the precise data was not given. ${ }^{19}$

In precisely quantified terms, how the alkali-S promotion affects the WGS reaction or the inactive carbon depositing Boudouard reaction, in relation to the FTS reaction hydrocarbon production, is unclear for the time being. More research on the effect of alkali-S promotion on the $\mathrm{C}$ pathway selectivities is warranted.

Figure 2.3 gives a schematic summary on the effects of alkali ( $\mathrm{Na}$ or $\mathrm{K}$ ), $\mathrm{S}$ and 
combined alkali-S and their effect on the FTS hydrocarbon and C pathway selectivities. Table 2.1 further summarizes the effects of all of the discussed FTS reaction process parameters together with the effects of the alkali, $\mathrm{S}$ and alkali-S chemical promoters in the Fe-based FTS reaction.

\subsubsection{Comparison of Fe and Co as Fischer-Tropsch Synthesis Active Transition Metals}

In the following, the Fe and Co transition metals are shortly compared regarding their general FTS reaction catalysis capabilities. Co is more i) active per gram of metal for hydrocarbon production, ${ }^{6,94}$ and ii) selective towards paraffin formation than $\mathrm{Fe}$ is. ${ }^{3,28}$ Co responds strongly to increased reaction temperature, producing mostly $\mathrm{CH}_{4}$ if the reaction temperature is increased from LTFT conditions $\left(200-250^{\circ} \mathrm{C}\right) \cdot{ }^{6,94}$ In general, Fe can operate over a wider range of FTS reaction conditions with an useful reaction hydrocarbon selectivity than Co can. ${ }^{94}$ Finally, Co-based catalysts generally do not show WGS reaction activity unlike the Fe-based catalyst materials do. ${ }^{28,73,94}$ Regarding the application of alkali-S promotion, both Fe-based, ${ }^{16}$ and Co-based FTS catalyst materials, ${ }^{17}$ seem to react in a same similar manner.

\subsection{FE CARBIDES IN THE FISCHER-TROPSCH SYNTHESIS REACTION}

The most common Fe carbide observed in the FTS reaction related research is the $\chi$ $\mathrm{Fe}_{5} \mathrm{C}_{2}$ phase, which is also known as the Hägg carbide. The so-called " $\varepsilon$-carbides" (or (pseudo-)hexagonal) Fe carbides are also often observed and labelled as $\varepsilon^{\prime}-\mathrm{Fe}_{2.2} \mathrm{C}, \mathrm{Fe}_{2} \mathrm{C}$, $\varepsilon-\mathrm{Fe}_{2} \mathrm{C}$ or $\varepsilon-\mathrm{Fe}_{3} \mathrm{C}$ in the literature. Less common Fe carbides encountered under FTS reaction conditions are $\theta-\mathrm{Fe}_{3} \mathrm{C}$ (cementite) and $\mathrm{Fe}_{7} \mathrm{C}_{3}$ (the Eckström-Adcock carbide). The $\mathrm{Fe}_{7} \mathrm{C}_{3}$ phase is a somewhat rare, but an interesting special case. The mentioned $\mathrm{Fe}$ carbides, while excluding the $\mathrm{Fe}_{7} \mathrm{C}_{3}$ phase, are also commonly formed as unwanted precipitates during tempering (i.e. heat treating) of martensitic steels.

Within this section, we concentrate the discussion on the proper identification and formation conditions of the different Fe carbides observable in the FTS reaction research. Especially, the nomenclature and identification of the " $\varepsilon$-carbides" in the literature is rather confusing, while the $\chi-\mathrm{Fe}_{5} \mathrm{C}_{2}$ and $\theta-\mathrm{Fe}_{3} \mathrm{C}$ phases are more consistently identified. Identification and formation conditions of the $\mathrm{Fe}_{7} \mathrm{C}_{3}$ phase are also somewhat unclear as the phase is less encountered in overall.

Thus, the aims within this section are to identify from the literature i) the expected lattice parameters (space group, unit cell) and their proper crystal structures for the Fe carbides. Furthermore, also ii) to determine the corresponding Mössbauer hyperfine field parameters for each of the identified Fe carbide crystal structure. This is done in 


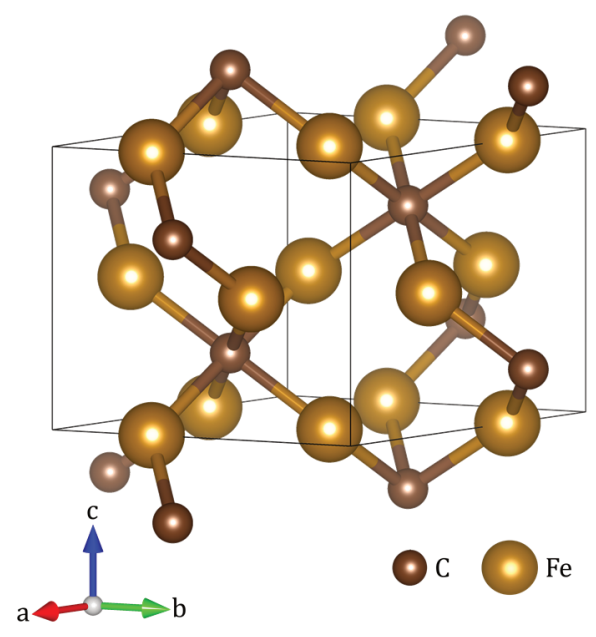

Figure 2.4

The unit cell of the hexagonal $\varepsilon-\mathrm{Fe}_{3} \mathrm{C} P 6_{3} 22$ (182). The Fe lattice is purely hexagonal and the $\mathrm{C}$ atoms occupy third of the octahedral Fe lattice interstitial spaces in a random, distorted manner.

order to establish a unified nomenclature between the precise crystal structures and Mössbauer Absorption Spectroscopy characterization technique. And lastly, the expected formation conditions from the literature for each of the identified Fe carbide are shortly reviewed using the established, unified $\mathrm{Fe}$ carbide nomenclature. The identified unit cells for each of the Fe carbides are visualized within this Chapter with the use of VESTA software. ${ }^{124}$

\subsection{1 " $\varepsilon$-carbides" $\left(\varepsilon-\mathrm{Fe}_{3} \mathrm{C}\right.$ and $\left.\eta-\mathrm{Fe}_{2} \mathrm{C}\right)$}

The so-called " $\varepsilon$-carbides" or $\mathrm{Fe}$ carbides with (pseudo-)hexagonal Fe lattices have traditionally been the most difficult Fe carbides to identify due to an unfortunate confusion with the labelling and identification of the $\varepsilon^{\prime}-\mathrm{Fe}_{2.2} \mathrm{C},(\varepsilon-) \mathrm{Fe}_{2} \mathrm{C}$ and $\varepsilon-\mathrm{Fe}_{3} \mathrm{C}$ phases. It is often unclear to which actual crystal lattice/structure the used nomenclature, as per author, refers to.

The $\varepsilon-\mathrm{Fe}_{3} \mathrm{C}$ phase has been identified by Nagakura. ${ }^{69}$ In their Electron Diffraction (ED) study on Fe thin films, the author solved the diffracting Fe carbide structure to have a space group of hexagonal $P 6_{3} 22$ (182). This Fe lattice is hexagonally ordered and the $\mathrm{C}$ atoms occupy one third of the octahedral interstitial Fe spaces in a disordered manner (Figure 2.4). The disordered $\mathrm{C}$ packing was observed from the broadening of the superstructural diffraction peaks, which for the Fe carbides originate from the interstitial $\mathrm{C}$ atoms. By decomposing the formed $\varepsilon-\mathrm{Fe}_{3} \mathrm{C}$ phase by heating it under vacuum back to $\alpha-\mathrm{Fe}$ and graphite, its stoichiometry was estimated to be around $\mathrm{Fe}_{2.8} \mathrm{C} .{ }^{69}$

As commented by Nagakura, ${ }^{69}$ the studied $\varepsilon$-Fe ${ }_{3} \mathrm{C}$ phase had the same lattice parameters, when ignoring the weak superstructural $\mathrm{C}$ diffraction, as a Fe carbide labelled as " $\mathrm{Fe}_{2} \mathrm{C}$ " with hexagonal Fe lattice observed earlier by Hofer, Cohn and Peebles. ${ }^{62}$ That is, the earlier "hexagonal $\mathrm{Fe}_{2} \mathrm{C}$ " and Nagakura's $\varepsilon$ - $\mathrm{Fe}_{3} \mathrm{C}$ were the exact same carbide.

The stoichiometry for the Hofer's $\mathrm{Fe}_{2} \mathrm{C}$ was determined by a comparison to - what at the time was thought to be - isomorphism with a "Hägg carbide $\mathrm{Fe}_{2} \mathrm{C}$ ". Experimental 
$\mathrm{C}:$ Fe ratio for the carbide was further determined by total sample weight increase upon carburization. ${ }^{62}$ Both, in retrospective, are inaccurate assumptions for determining a carbide stoichiometry. Later, after more accurate structural solutions were provided for the Hägg carbide, ${ }^{125}$ the original assumptions in the identification of the "hexagonal $\mathrm{Fe}_{2} \mathrm{C}$ " and its stoichiometry were acknowledged by the researchers at the time to be inaccurate. ${ }^{126}$ No evidence of a Fe carbide as "hexagonal $\mathrm{Fe}_{2} \mathrm{C}$ " exists as after Hofer, Cohn and Peebles. ${ }^{62}$ Unfortunately, the acknowledgement of the mistaken original labelling went unnoticed and several later studies cite a "hexagonal $\mathrm{Fe}_{2} \mathrm{C}$ " carbide originating from the study by Hofer, Cohn and Peebles. ${ }^{22,67,127}$ This has resulted in unfortunate confusion in the characterization of the actual Fe carbide phases in the FTS reaction.

Furthermore, unfortunately, in a French written study by Dirand and Afqir, ${ }^{128}$ the " $\mathrm{Fe}_{2} \mathrm{C}$ " carbide with the data from the study of Hofer, Cohn and Peebles was solved to have a space group of hexagonal $P 6_{3} / m m c$ (194). This was done with the use of newly available computerized diffraction pattern indexing (i.e. space group solving) software. Dirand and Afqir also acknowledged the existence of the $\varepsilon-\mathrm{Fe}_{3} \mathrm{C}$ phase indexed as $P 6_{3} 22$ (182) after Nagakura, but unfortunately did not acknowledge that the carbides were the same. The reason why the same carbide was solvable with different space groups is due to the originally used diffraction techniques to collect the data. The electron diffraction pattern by Nagakura contains the diffraction originating from both Fe and $C$ in the crystal lattice. The X-ray Powder Diffractometry (XRPD) data collected by Hofer, Cohn and Peebles is not sensitive to the low atomic number element $C$ and essentially only the diffraction from $\mathrm{Fe}$ was observable. Thus, the $\mathrm{P}_{3} 22$ (182) is the full, proper space group with $\mathrm{Fe}$ and $\mathrm{C}$ diffraction while the $P 6_{3} / m m c(194)$ is an incomplete space group only for the Fe diffraction, for the same hexagonal $\varepsilon$ - $\mathrm{Fe}_{3} \mathrm{C}$ phase observed by both Nagakura and Hofer, Cohn and Peebles. ${ }^{62,69}$

The International Centre for Diffraction Data Powder Diffraction File-4+ (ICDD-PDF4+) database lists the Dirand and Afqir's solution based on Hofer, Cohn and Peebles's unit cell as a Fe carbide with space group $P 6_{3} / m m c$ (194) with database number 00036-1249 (or 36-1249 in pre-2016 databases). As discussed above, Nagakura's crystal structure solution with space group $\mathrm{P}_{3} 22$ (182) and database number 04-008-9572 (or 89-3689) should be used instead. Dirand and Afqir's $P 6_{3} / m m c$ (194) incomplete lattice solution should not be used in order to avoid any unnecessary confusion for the future identification of the $\varepsilon-\mathrm{Fe}_{3} \mathrm{C}$ phase.

Nagakura's $\varepsilon-\mathrm{Fe}_{3} \mathrm{C}$ is not the only $\mathrm{Fe}$ carbide phase observed with a (pseudoJhexagonal Fe lattice. In a spent FTS reaction catalyst material, Barton and Gale observed a similar hexagonal Fe carbide to what Hofer, Cohn and Peebles observed earlier (i.e. the $\varepsilon-\mathrm{Fe}_{3} \mathrm{C}$ phase properly identified by Nagakura). However, the Fe carbide differed from the previous observations by i) having split X-ray diffraction peaks, 
suggesting that a distortion from the hexagonal Fe lattice was present, and by ii) having significantly different unit cell parameters. ${ }^{129}$ As XRPD was used, Barton and Gale could not conclusively solve the observed Fe carbide structure or the space group due to the missing superstructural diffraction from the interstitial $\mathrm{C}$ atoms. Barton and Gale assumed a stoichiometry of $\mathrm{Fe}_{2} \mathrm{C}$ after the study by Hofer, Cohn and Peebles for their carbide.

Barton and Gale, however, calculated from their XRPD data that instead of having a purely hexagonal Fe lattice with lattice parameter $\gamma=120^{\circ}$, their observed Fe carbide crystal lattice had a distortion from the Fe lattice hexagonality where $\gamma=120.92^{\circ}$. This angle distortion accounting for the observed peak splitting in the X-ray diffraction patterns. As possible space group solutions for the crystal lattice, Barton and Gale gave trigonal $P \overline{3} m 1$ (164), hexagonal $P 6_{3} / m m c$ (194) and orthorhombic Pbcn (60). ${ }^{129}$

From tempering studies on martensitic steel, Hirotsu and Nagakura solved a Fe carbide crystal structure on the basis of its electron diffraction patterns, ${ }^{131}$ which resembled the earlier hexagonal $\varepsilon$-carbide (i.e. the $\varepsilon$-Fe ${ }_{3} \mathrm{C}$ phase) observed by Hofer, Cohn and Peebles and Nagakura. ${ }^{62,69}$ However, the Fe carbide was different to Hofer, Cohn and Peeble's observations as the Fe lattice was not purely hexagonal but rather a pseudo-hexagonal one with the Fe lattice distorted from hexagonality. Hirotsu and Nagakura labelled their $\mathrm{Fe}$ carbide as $\eta-\mathrm{Fe}_{2} \mathrm{C}$ with an orthorhombic space group Pnnm (58) (Figure 2.5). ${ }^{131}$ The $\mathrm{C}$ atoms in $\eta-\mathrm{Fe}_{2} \mathrm{C}$ structure occupy half of the octahedral interstitial spaces in the Fe lattice, in a regular manner. ${ }^{131}$ Hirotsu and Nagakura have proposed orthorhombic unit cell dimensions, after their $\eta-\mathrm{Fe}_{2} \mathrm{C}$ phase, for the Fe carbide observed by Barton and Gale. ${ }^{130}$

The crystal structures of the $\varepsilon-\mathrm{Fe}_{3} \mathrm{C}$ and $\eta-\mathrm{Fe}_{2} \mathrm{C}$ phases, with the unit cell by Hirotsu and Nagakura corresponding to the Barton and Gale's Fe carbide, are depicted in the Figure 2.6. Their hexagonal (in $\varepsilon-\mathrm{Fe}_{3} \mathrm{C}$ ) and distorted hexagonal (in $\eta-\mathrm{Fe}_{2} \mathrm{C}$ ) $\mathrm{Fe}$ lattice planes are in plane with the paper. The carbides indeed have very similar hexagonal Fe planes with differences of, that for i) $\eta-\mathrm{Fe}_{2} \mathrm{C} \gamma=120.47^{\circ}$ instead of $\gamma=120.0^{\circ}$ as for the purely hexagonal

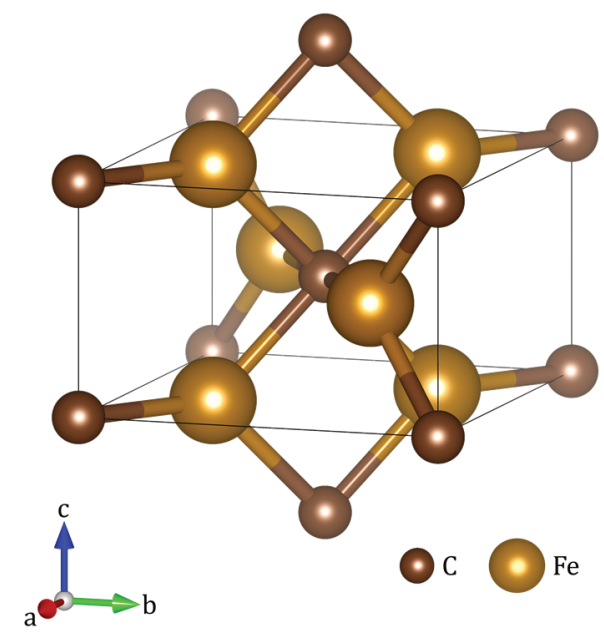

Figure 2.5

The unit cell of the orthorhombic $\eta-\mathrm{Fe}_{2} \mathrm{C}$ Pnnm (58). The Fe lattice is distorted from hexagonality and the $C$ atoms occupy half of the octahedral Fe lattice interstitial spaces in an ordered manner. 

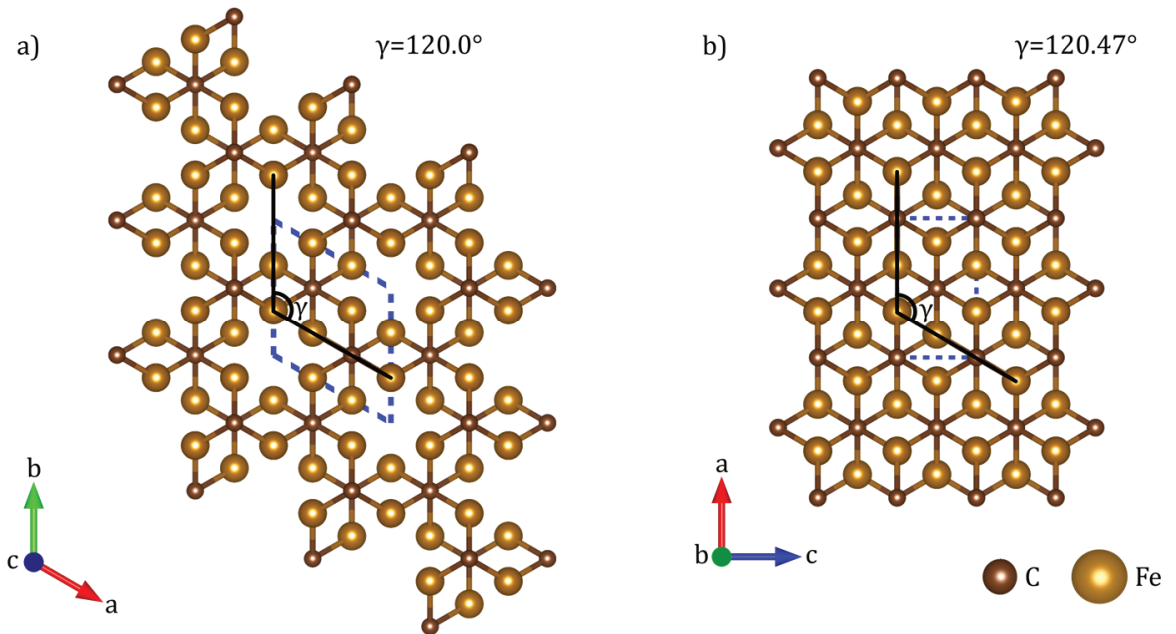

Figure 2.6

The crystal lattices of a) the hexagonal $\varepsilon-\mathrm{Fe}_{3} \mathrm{C} P 6_{3} 22$ (182) viewed along the c-axis, b) the orthorhombic $\eta$ $\mathrm{Fe}_{2} \mathrm{C}$ Pnnm (58) viewed along the b-axis, so that the (distorted-)hexagonal Fe planes are in plane with the paper. The $\eta-\mathrm{Fe}_{2} \mathrm{C}$ unit cell is after Hirotsu and Nagakura corresponding the Fe carbide by Barton and Gale. ${ }^{130}$ Blue, dashed line - the respective unit cells for each crystal lattice, Black, solid line - In plane Fe atom positions used for measuring the lattice angle parameter $\gamma$. For the $\varepsilon-\mathrm{Fe}_{3} \mathrm{C} \gamma=120.0^{\circ}$ i.e. the Fe lattice is hexagonal, while for $\eta-\mathrm{Fe}_{2} \mathrm{C} \gamma=120.47^{\circ}$ i.e. the Fe lattice is distorted from hexagonality. In the $\eta-\mathrm{Fe}_{2} \mathrm{C}$ lattice the interstitial $\mathrm{Fe}$ spaces are occupied by $\mathrm{C}$ to a higher degree than in the $\varepsilon$ - $\mathrm{Fe}_{3} \mathrm{C}$ lattice.

lattice of $\varepsilon-\mathrm{Fe}_{3} \mathrm{C}$ and ii) there are no empty octahedral interstitial Fe lattice spaces in the lattice of $\eta-\mathrm{Fe}_{2} \mathrm{C}$. The crystal structures of $\varepsilon-\mathrm{Fe}_{3} \mathrm{C}$ and $\eta-\mathrm{Fe}_{2} \mathrm{C}$ are similar and closely related.

That is, the orthorhombic structure of the $\eta-\mathrm{Fe}_{2} \mathrm{C}$ phase with the orthorhombic unit cell dimensions proposed by Hirotsu and Nakagura is a proper solution for the Fe carbide observed by Barton and Gale. It is the crystal structure for what is occasionally labelled in the literature as a pseudo-hexagonal $\left(\varepsilon^{\prime}-\mathrm{Fe}_{2.2} \mathrm{C}\right.$ or $\left.\mathrm{Fe}_{2} \mathrm{C}\right) \mathrm{Fe}$ carbide phase. ${ }^{60,132}$ The crystal structure for the $\eta-\mathrm{Fe}_{2} \mathrm{C}$ phase by Hirotsu and Nakagura is listed with database number 04-013-9510 in the ICDD-PDF-4+ 2016 database.

To summarize the discussion so far, the labelling of a Fe carbide in the FTS reaction as $(\varepsilon-) \mathrm{Fe}_{2} \mathrm{C}$ phase is improper and should not be used further as there is no evidence that such a Fe carbide exists. What can be grouped as the " $\varepsilon$-carbides" are two different carbides: $\varepsilon-\mathrm{Fe}_{3} \mathrm{C}$ with hexagonal space group $P 6_{3} 22$ (182) where the Fe lattice is purely hexagonal and $\mathrm{C}$ atoms occupy third of the octahedral $\mathrm{Fe}$ interstitial spaces in a random, disordered manner; $\eta-\mathrm{Fe}_{2} \mathrm{C}$ with orthorhombic space group Pnnm (58) where the $\mathrm{Fe}$ lattice is distorted from the hexagonal symmetry and $\mathrm{C}$ atoms occupy half of the octahedral Fe interstitial spaces in a regular, ordered manner. A "pseudo-hexagonal Fe 
carbide" either labelled as a $\mathrm{Fe}_{2} \mathrm{C}$ or $\varepsilon^{\prime}$ $\mathrm{Fe}_{2.2} \mathrm{C}$, should not be used to describe a Fe carbide with distorted Fe lattice but rather the orthorhombic $\eta-\mathrm{Fe}_{2} \mathrm{C}$ should be used instead. The crystal lattice of the $\eta-\mathrm{Fe}_{2} \mathrm{C}$ phase contains the distorted (pseudo-)hexagonal Fe lattice.

\subsubsection{The Hägg Carbide $(\chi-$ $\mathrm{Fe}_{5} \mathrm{C}_{2}$ )}

The most commonly observed $\mathrm{Fe}$ carbide in the FTS reaction is the $\chi-\mathrm{Fe}_{5} \mathrm{C}_{2}$ phase, which is mostly properly and consistently identified. The first observations of the Hägg carbide are from 1934,133 and the carbide's crystal

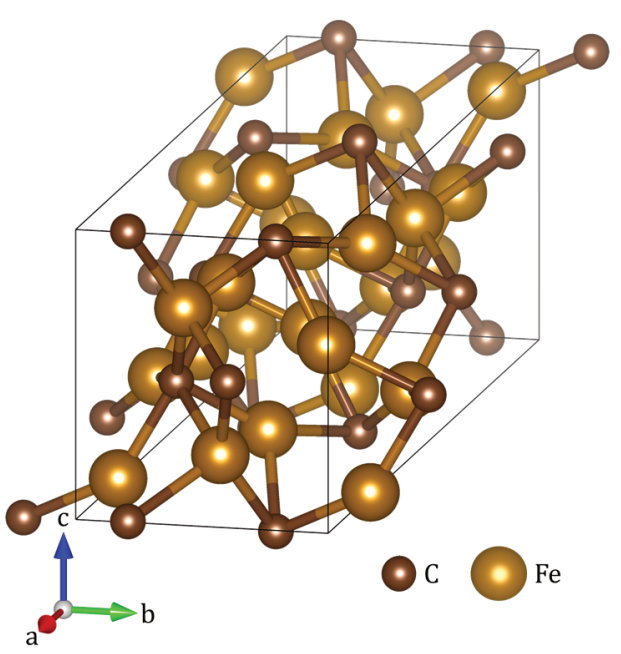

Figure 2.7

The unit cell of the monoclinic $\chi-\mathrm{Fe}_{5} \mathrm{C}_{2} C 2 / c$ (15) (the Hägg carbide). structure was later solved to be monoclinic, with a stoichiometry $\mathrm{Fe}_{5} \mathrm{C}_{2} \cdot{ }^{125}$ Retief has further improved the accuracy of the structural solution over the years for the known $\chi-\mathrm{Fe}_{5} \mathrm{C}_{2}$ phase with a monoclinic space group C2/c (15) (Figure 2.7). ${ }^{134}$ Du Plessis et al. have argued that the proper space group for the $\chi-\mathrm{Fe}_{5} \mathrm{C}_{2}$ phase is not a monoclinic $C 2 / c$ (15) but a triclinic $P \overline{1}$ (2). ${ }^{135}$ However, Leineweber et al. have explained the observations by du Plessis et al. being due to anisotropic microstrain broadening and the monoclinic $C 2 / c$ (15) space group should be regarded as the correct one for the Hägg carbide $\chi-\mathrm{Fe}_{5} \mathrm{C}_{2} .{ }^{136}$ The crystal structure solved by Retief is a proper solution for the Hägg carbide $\chi-\mathrm{Fe}_{5} \mathrm{C}_{2}$ and is listed in the ICDD-PDF-4+ 2016 database with number 00-051-0997. The crystal structure solutions provided by Leineweber et al. are also accurate for the Hägg carbide. ${ }^{136}$

\subsubsection{Cementite $\left(\theta-\mathrm{Fe}_{3} \mathrm{C}\right)$}

As being the most thermally stable $\mathrm{Fe}$ carbide, ${ }^{58,137}$ cementite $\theta-\mathrm{Fe}_{3} \mathrm{C}$ is - akin to $\chi-\mathrm{Fe}_{5} \mathrm{C}_{2}$ - a well-studied and consistently identified Fe carbide phase. Already the early studies have identified the cementite $\theta-\mathrm{Fe}_{3} \mathrm{C}$ crystal structure

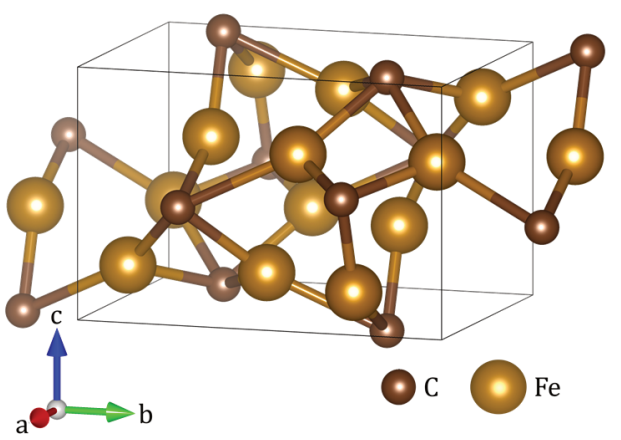

Figure 2.8

The unit cell of the orthorhombic $\theta-\mathrm{Fe}_{3} \mathrm{C}$ Pnma (62) (cementite). 
with an orthorhombic space group Pnma (62). ${ }^{138}$ Later, Wood et al. have applied Neutron Powder Diffraction (NPD) to further improve the cementite $\theta-\mathrm{Fe}_{3} \mathrm{C}$ structure solution. These experiments provide to date the most accurate crystal structural solutions for the $\theta-\mathrm{Fe}_{3} \mathrm{C}$ phase, at various temperatures. ${ }^{70}$ The crystal structure of $\theta-\mathrm{Fe}_{3} \mathrm{C}$ by Wood et al., at room temperature, is available in the Crystallography Open Database (COD) with the database number 9014027 (Figure 2.8).139

\subsubsection{The Eckström-Adcock Carbide $\left(\boldsymbol{\theta}-\mathrm{Fe}_{7} \mathrm{C}_{3}\right)$}

The Eckström-Adcock carbide is rather rarely observed in the FTS reaction but has been known to exist since the initial observations of a new carbide by Eckström and Adcock. ${ }^{140}$ It may form during the FTS reaction after extended times under industrial high-pressure reaction conditions. ${ }^{3}$

The initial identification of the carbide observed by Eckström and Adcock was done as a hexagonal $\mathrm{Fe}_{7} \mathrm{C}_{3}$ with a space group $\mathrm{P}_{3} m c(186)$ by Herbstein and Snyman. ${ }^{141}$ However, in later studies Fruchart et al. proposed the crystal structure of $\mathrm{Fe}_{7} \mathrm{C}_{3}$ to be an orthorhombic Pnma (62). ${ }^{142}$ In a single crystal ED study by Audier, Bowen and Jones, the orthorhombic space group was agreed over the hexagonal one. ${ }^{143}$ The observed diffraction intensities were more accurately predicted by using an orthorhombic rather than a hexagonal crystal lattice for the $\mathrm{Fe}_{7} \mathrm{C}_{3}$ phase. ${ }^{143}$ Therefore, the preferred crystal structure for the Eckström-Adcock carbide $\mathrm{Fe}_{7} \mathrm{C}_{3}$ has an orthorhombic space group Pnma (62) by Fruchart et al. (Figure 2.9). ${ }^{142}$ The orthorhombic crystal structure for the $\mathrm{Fe}_{7} \mathrm{C}_{3}$ phase is available in the ICDD-PDF-4+ database with the database number 04002-8165.

To make the distinction and to avoid any confusion between the $\mathrm{Fe}_{7} \mathrm{C}_{3}$ crystal lattices, the orthorhombic $\mathrm{Fe}_{7} \mathrm{C}_{3}$ Pnma (62) is arbitrarily labelled as $\theta-\mathrm{Fe}_{7} \mathrm{C}_{3}$ akin to same space group $\theta-\mathrm{Fe}_{3} \mathrm{C}$. The use of various Greek prefix letters, ${ }^{144}$ to name the $\mathrm{Fe}$ carbides is rather arbitrary and not systematic.

The crystal lattice details for the discussed Fe carbides observable in the FTS reaction research i.e. $\varepsilon-\mathrm{Fe}_{3} \mathrm{C}, \eta-\mathrm{Fe}_{2} \mathrm{C}, \chi-\mathrm{Fe}_{5} \mathrm{C} 2, \theta-\mathrm{Fe}_{3} \mathrm{C}$ and $\theta-\mathrm{Fe}_{7} \mathrm{C}_{3}$ are listed in the 


\section{Table 2.2}

The crystal lattice details of the Fe carbides encountered in Fe-based Fischer-Tropsch (FTS) reaction with author references for the most accurate crystal structure solutions up-to-date. All lattice parameters are at room temperature. It is advised that the nomenclature of $\varepsilon^{\prime}-\mathrm{Fe}_{2.2} \mathrm{C}$ or $(\varepsilon-) \mathrm{Fe}_{2} \mathrm{C}$ should not be used for describing any of the Fe carbides.

\begin{tabular}{|c|c|c|c|c|c|}
\hline \multirow{2}{*}{$\begin{array}{c}\text { Fe Carbide } \\
\text { Phase }\end{array}$} & \multirow[t]{2}{*}{ Space Group } & \multicolumn{2}{|c|}{ Lattice Parameters } & \multirow{2}{*}{$\begin{array}{c}\text { Database Number } \\
\text { ICDD-PDF-4+ (2016) }\end{array}$} & \multirow[t]{2}{*}{ Author } \\
\hline & & $(\AA)$ & $\left({ }^{\circ}\right)$ & & \\
\hline & Hexagonal, & $a=4.767$ & $\alpha=90$ & & \\
\hline \multirow[t]{3}{*}{$\varepsilon-\mathrm{Fe}_{3} \mathrm{C}$} & $\mathrm{P}_{3} 22$ (182) & $\mathrm{b}=4.767$ & $\beta=90$ & 04-008-9572 & Nagakura (1959) [69] \\
\hline & & $c=4.354$ & $\gamma=120$ & & \\
\hline & 'Orthorhombic, & $\mathrm{a}=4.704$ & $\alpha=90$ & & \\
\hline \multirow[t]{3}{*}{$\eta-\mathrm{Fe}_{2} \mathrm{C}$} & Pnnm (58) & $\mathrm{b}=4.318$ & $\beta=90$ & 04-013-9510 & Hirotsu (1972) [131] \\
\hline & & $c=2.830$ & $\gamma=90$ & & \\
\hline & Orthorhombic, & $a=4.862$ & $\alpha=90$ & & \\
\hline \multirow[t]{3}{*}{$\eta-\mathrm{Fe}_{2} \mathrm{C}^{\mathrm{a}}$} & Pnnm (58) & $\mathrm{b}=4.360$ & $\beta=90$ & - & Hirotsu (1974) $\left[{ }^{130}\right]$ \\
\hline & & $c=2.755$ & $\gamma=90$ & & \\
\hline & Monoclinic, & $a=11.588$ & $\alpha=90$ & & \\
\hline \multirow[t]{3}{*}{$\chi-\mathrm{Fe}_{5} \mathrm{C}_{2}$} & $\mathrm{C} 2 / \mathrm{c}(15)$ & $\mathrm{b}=4.579$ & $\beta=97.746$ & 00-051-0997 & Retief (1999) [134] \\
\hline & & $c=5.059$ & $\gamma=90$ & & \\
\hline & Orthorhombic, & $a=5.081$ & $\alpha=90$ & & \\
\hline \multirow[t]{3}{*}{$\theta-\mathrm{Fe}_{3} \mathrm{C}$} & Pnma (62) & $b=6.754$ & $\beta=90$ & $9014027^{b)}$ & Wood (2004) [70] \\
\hline & & $c=4.515$ & $\gamma=90$ & & \\
\hline & Orthorhombic, & $a=4.540$ & $\alpha=90$ & & \\
\hline \multirow[t]{2}{*}{$\theta-\mathrm{Fe}_{7} \mathrm{C}_{3}$} & Pnma (62) & $b=6.879$ & $\beta=90$ & 04-002-8165 & Fruchart $(1965)^{\mathrm{c})}[142]$ \\
\hline & & $\mathrm{c}=11.942$ & $\gamma=90$ & & \\
\hline
\end{tabular}

a) Lattice parameters are translated according to Hirotsu and Nagakura, ${ }^{130}$ to correspond the distorted Fe carbide observed by Barton and Gale. ${ }^{129}$

b) Crystallography Open Database number.

c) The original article written in French by Fruchart et al. is not readily available but some discussion on the crystal structure can be found in publication by Audier, Bowen and Jones. ${ }^{143}$

Table 2.2. The crystal structure solutions are available from the listed authors in Table 2.2 and from the ICDD-PDF-4+ and COD databases. As a next step, the established nomenclature for the Fe carbides is expanded to the MAS literature. This is done for connecting the Fe carbide crystal lattices to observed hyperfine fields, which are used to fingerprint the various Fe carbides with MAS.

\subsubsection{Fe Carbide Hyperfine Fields}

Just as was the case with their X-ray and electron diffraction data based crystal lattice solutions, the literature data with various Fe carbides identified in FTS research with MAS can be challenging to follow due to the unclear nomenclature, as per author.

As based on the solved crystal structures for the Fe carbides, by the authors listed 
in Table 2.2, the total count and the site ratios for the crystallographically nonequivalent Fe positions can be determined. Each non-equivalent Fe position should ideally give a distinct MAS hyperfine field component with fields' spectral contribution ratios following the non-equivalent $\mathrm{Fe}$ site ratios. ${ }^{145}$ Determining the non-equivalent $\mathrm{Fe}$ positions from the crystal structures gives the ideal $\mathrm{Fe}$ site ratios as: i) the $\varepsilon-\mathrm{Fe}_{3} \mathrm{C}$ and $\eta-\mathrm{Fe}_{2} \mathrm{C}$ phases have one equivalent $\mathrm{Fe}$ position in their respective crystal structures (i.e. all $\mathrm{Fe}$ sites are ideally equivalent), ii) the $\chi-\mathrm{Fe}_{5} \mathrm{C}_{2}$ phase has three non-equivalent $\mathrm{Fe}$ positions in ratio of $2: 2: 1$, iii) the $\theta-\mathrm{Fe}_{3} \mathrm{C}$ phase has two non-equivalent $\mathrm{Fe}$ positions in ratio $2: 1$ and iv) the $\theta-\mathrm{Fe}_{7} \mathrm{C}_{3}$ phase has five non-equivalent $\mathrm{Fe}$ positions in ratio $2: 2: 1: 1: 1$. In the following, we shall assign the room-temperature hyperfine fields from experimental MAS data in literature, for each of the non-equivalent Fe positions for the Fe carbides listed in Table 2.2.

Le Caër et al. have discussed the MAS hyperfine field assignment of the " $\varepsilon$-carbides" on the basis of experimental data gathered from literature. ${ }^{61}$ As Le Caër et al. note, the " $\varepsilon$-carbides" can be grouped around two hyperfine field values of $24.5 \pm 0.8 \mathrm{~T}$ and $17.2 \pm 0.3 \mathrm{~T}$ (at room temperature). Furthermore, increasing the amount of interstitial $\mathrm{C}$ atoms in the Fe lattice decreases the measured hyperfine field. ${ }^{61,146-148}$ Thus, the field $24.5 \pm 0.8 \mathrm{~T}$ belongs to a " $\varepsilon$-carbide" with a low $\mathrm{C}$ content and the field $17.2 \pm 0.3 \mathrm{~T}$ for a " $\varepsilon$-carbide" with a higher $\mathrm{C}$ content. Recalling from the earlier discussion on the crystal structures of the $\mathrm{Fe}$ carbides: for the $\varepsilon-\mathrm{Fe}_{3} \mathrm{C}$ phase third of the interstitial Fe lattice sites are occupied by $\mathrm{C}$ atoms while for the $\eta-\mathrm{Fe}_{2} \mathrm{C}$ phase half of the interstitial sites are occupied by $\mathrm{C}$. That is, the $\eta-\mathrm{Fe}_{2} \mathrm{C}$ phase has the higher $\mathrm{C}$ content in its crystal structure. Therefore, the hyperfine field $24.5 \pm 0.8 \mathrm{~T}$ is assigned for the one equivalent Fe site in the $\varepsilon-\mathrm{Fe}_{3} \mathrm{C}$ crystal structure and $17.2 \pm 0.3 \mathrm{~T}$ for the one $\mathrm{Fe}$ site in the $\eta-\mathrm{Fe}_{2} \mathrm{C}$ phase. Occasionally, also an additional minor intensity hyperfine field $\approx 13.0 \mathrm{~T}$ has been assigned for " $\varepsilon$-carbides". $61,149,150$ However, whether this is correct and from where the low $\approx 13.0 \mathrm{~T}$ hyperfine field arises from, is uncertain. The two assigned hyperfine fields are the primary ones for the $\varepsilon-\mathrm{Fe}_{3} \mathrm{C}$ and $\eta-\mathrm{Fe}_{2} \mathrm{C}$ phases for correctly identifying the carbides.

The experimentally assigned, averaged hyperfine fields from the cited studies, for the Hägg carbide $\chi-\mathrm{Fe}_{5} \mathrm{C}_{2}$ are the fields $21.4 \pm 0.3 \mathrm{~T}, 18.1 \pm 0.4 \mathrm{~T}$ and $10.9 \pm 0.9 \mathrm{~T}$ with respective spectral contribution ratio of $\approx 2: 2: 1 \cdot^{60,77,150,151}$ The spectral contribution ratio is in agreement with the expected non-equivalent $\mathrm{Fe}$ site ratios for the $\chi-\mathrm{Fe}_{5} \mathrm{C}_{2}$ phase. In addition to the commonly assigned hyperfine fields, Barinov, Protasov and Surikov have proposed an additional field of $\approx 16.0 \mathrm{~T}$ to account for a possible anisotropy in the $\chi-\mathrm{Fe}_{5} \mathrm{C}_{2}$ crystal structure. ${ }^{151}$ The proposal by Barinov, Protasov and Surikov perhaps could account for the earlier discussed anisotropy phenomena observed by Leineweber et al. for the $\chi-\mathrm{Fe}_{5} \mathrm{C}_{2}$ phase. ${ }^{136}$ However, whether or not such 


\section{Table 2.3}

Mössbauer Absorption Spectroscopy (MAS) hyperfine field assignments based on the experimental literature data. The hyperfine fields are compared to the theoretically calculated values for the Fe carbides observable in the Fischer-Tropsch Synthesis (FTS) reaction research.

\begin{tabular}{|c|c|c|c|c|c|}
\hline \multirow[t]{3}{*}{$\begin{array}{c}\text { Fe Carbide } \\
\text { Phase }\end{array}$} & \multirow[t]{3}{*}{ Space Group } & \multicolumn{2}{|c|}{$\begin{array}{c}\text { Non-equivalent Fe } \\
\text { Positions }{ }^{\text {a) }}\end{array}$} & \multicolumn{2}{|c|}{$\begin{array}{c}\text { Hyperfine Field } \\
\text { (at room temperature) }\end{array}$} \\
\hline & & \multirow[t]{2}{*}{ Tot. Count } & \multirow[t]{2}{*}{ Rel. Ratio } & Experimental $^{\text {b) }}$ & Theoretical $^{\mathrm{c})}$ \\
\hline & & & & $(\mathrm{T})$ & $(\mathrm{T})$ \\
\hline & Hexagonal, & & & & \\
\hline \multirow[t]{2}{*}{$\varepsilon-\mathrm{Fe}_{3} \mathrm{C}$} & $\mathrm{P}_{3} 22$ (182) & 1 & 1 & $24.5 \pm 0.8$ & $\mathrm{~N} / \mathrm{A}$ \\
\hline & 'Orthorhombic, & & & & \\
\hline \multirow[t]{2}{*}{$\eta-\mathrm{Fe}_{2} \mathrm{C}$} & Pnnm (58) & 1 & 1 & $17.2 \pm 0.3$ & $18.0^{\mathrm{d})}$ \\
\hline & Monoclinic, & & 2 & $21.4 \pm 0.3$ & 23.7 \\
\hline \multirow[t]{3}{*}{$\chi-\mathrm{Fe}_{5} \mathrm{C}_{2}$} & C2/c (15) & 3 & 2 & $18.1 \pm 0.4$ & 20.9 \\
\hline & & & 1 & $10.9 \pm 0.9$ & 14.0 \\
\hline & 'Orthorhombic, & & (2) & $20.8 \pm 0.3$ & 24.4 \\
\hline \multirow[t]{4}{*}{$\theta-\mathrm{Fe}_{3} \mathrm{C}$} & Pnma (62) & 2 & (1) & $20.0 \pm 0.3$ & 23.7 \\
\hline & & & Avg.e) & $20.8 \pm 0.5$ & \\
\hline & & & (2) & $22.9 \pm 0.1$ & 22.9 \\
\hline & Orthorhombic, & & (2) & - & 22.9 \\
\hline \multirow[t]{3}{*}{$\theta-\mathrm{Fe}_{7} \mathrm{C}_{3}$} & Pnma (62) & 5 & (1) & $18.5 \pm 0.1$ & 18.7 \\
\hline & & & (1) & $16.3 \pm 0.1$ & 17.4 \\
\hline & & & (1) & $(21.0)^{\mathrm{f}} \mathrm{f}$ & 19.9 \\
\hline
\end{tabular}

a) Determined on the basis of the crystal structures from authors in Table 2.2. The nominal relative ratios in parentheses () could not be conclusively assigned.

b) Mean hyperfine fields from the experimental literature data with their Standard Deviations (STDs).

c) Theoretical calculations by Liu et al. from reference [148]. No calculations were performed for the structure corresponding to Nagakura's $\varepsilon$ - $\mathrm{Fe}_{3} \mathrm{C}$ phase. ${ }^{69}$

d) Based on the unit cell of $\eta-\mathrm{Fe}_{2} \mathrm{C}$ (\#04-013-9510) from reference [131].

e) Commonly, a single (averaged) hyperfine field is used for the $\theta-\mathrm{Fe}_{3} \mathrm{C}$ phase in the experimental literature data.

f) The assignment of hyperfine field $21.0 \mathrm{~T}$ for the $\theta-\mathrm{Fe}_{7} \mathrm{C}_{3}$ phase is uncertain.

anisotropy is present, the three assigned hyperfine fields are sufficient for identifying the $\chi-\mathrm{Fe}_{5} \mathrm{C}_{2}$ phase.

The experimentally assigned, averaged hyperfine fields for cementite $\theta-\mathrm{Fe}_{3} \mathrm{C}$ can be obtained from studies by Bi et al. and Kniep et al. and have values of $20.8 \pm 0.3 \mathrm{~T}$ and $20.0 \pm 0.3$ T. ${ }^{152,153}$ However, as the values are very close to each other, commonly only one average hyperfine field value of $20.8 \pm 0.5 \mathrm{~T}$ is used for the MAS fitting of $\theta$ $\mathrm{Fe}_{3} \mathrm{C} .{ }^{60,127,154,155}$ Neither Bi et al. nor Kniep et al. report the MAS-based relative spectral contribution areas for the $\theta-\mathrm{Fe}_{3} \mathrm{C}$ assigned hyperfine fields. Thus, how the crystallographically expected 2:1 non-equivalent $\mathrm{Fe}$ site ratio is assigned to the 
hyperfine fields, is unclear for the time being for the $\theta-\mathrm{Fe}_{3} \mathrm{C}$ phase.

The assignment of the hyperfine fields from the experimental literature data for the $\theta-\mathrm{Fe}_{7} \mathrm{C}_{3}$ phase is problematic as the carbide is less often observed than the other carbides, and therefore less studied. For the orthorhombic crystal lattice of the $\theta-\mathrm{Fe}_{7} \mathrm{C}_{3}$ phase, five non-equivalent Fe sites are expected with corresponding hyperfine fields. However, only three fields have been experimentally assigned so far in the literature. Averaging the hyperfine fields from the studies of Bi et al. and Yamada et al. gives field assignments as $22.9 \pm 0.1 \mathrm{~T}, 18.5 \pm 0.1 \mathrm{~T}$ and $16.3 \pm 0.1 \mathrm{~T} .{ }^{152,155} \mathrm{Bi}$ et al. have also assigned a fourth field for their $(\theta-) \mathrm{Fe}_{7} \mathrm{C}_{3}$ phase as $21.0 \mathrm{~T}$ which might belong to the $\theta-\mathrm{Fe}_{3} \mathrm{C}$ impurity from the sample synthesis. ${ }^{152}$

In a theoretical MAS study, Liu et al. have predicted the hyperfine fields of $22.9 \mathrm{~T}$, $22.9 \mathrm{~T}, 19.9 \mathrm{~T}, 18.7 \mathrm{~T}$ and $17.4 \mathrm{~T}$ for the orthorhombic $\theta-\mathrm{Fe}_{7} \mathrm{C}_{3}$ phase. ${ }^{148}$ The assignments by Liu et al. are relatively close to the experimental assignments by Bi et al.. ${ }^{152}$ That is, if the hyperfine field $21.0 \mathrm{~T}$ is assumed belonging to the $\theta-\mathrm{Fe}_{7} \mathrm{C}_{3}$ phase rather than an impurity $\theta-\mathrm{Fe}_{3} \mathrm{C}$ phase and if the same field of $22.9 \mathrm{~T}$ is indeed assignable for two non-equivalent $\mathrm{Fe}$ positions. Bi et al. considered the $\mathrm{Fe}_{7} \mathrm{C}_{3}$ phase to only have three non-equivalent Fe sites, ${ }^{152}$ as based on the hexagonal $\mathrm{Fe}_{7} \mathrm{C}_{3}$ structure by Herbstein and Snyman. ${ }^{141}$ However, as discussed earlier, the hexagonal crystal lattice for the $\mathrm{Fe}_{7} \mathrm{C}_{3}$ phase is not a fully satisfactory crystal structure solution as based on the diffraction studies. The orthorhombic $\theta-\mathrm{Fe}_{7} \mathrm{C}_{3}$ crystal structure should be used instead. ${ }^{143}$ None of the cited studies assigns spectral contribution ratios for the assigned hyperfine fields for the $\theta-\mathrm{Fe}_{7} \mathrm{C}_{3}$ phase. Therefore, the hyperfine fields corresponding to the specific nonequivalent $\mathrm{Fe}$ sites cannot be definitively identified from the literature for the $\theta-\mathrm{Fe}_{7} \mathrm{C}_{3}$ phase at the moment.

To conclude, the MAS hyperfine field assignments for the $\theta-\mathrm{Fe}_{7} \mathrm{C}_{3}$ phase are uncertain at the moment. However, as based on both experimental and theoretical data, the possible field assignments for the expected five sites for the orthorhombic $\theta-\mathrm{Fe}_{7} \mathrm{C}_{3}$ phase are: $22.9 \pm 0.1 \mathrm{~T}$ twice, $18.5 \pm 0.1 \mathrm{~T}$ and $16.3 \pm 0.1 \mathrm{~T}$, with the field $21.0 \mathrm{~T}$ being uncertain. The hyperfine field spectral contribution ratios corresponding to the nonequivalent Fe site ratios are unassigned.

The assigned hyperfine fields for MAS fingerprinting of the Fe carbides encountered in the FTS reaction research, as based on the experimental literature data, are summarized in the Table 2.3. Hyperfine fields based on theoretical calculations by Liu et al., are reproduced in the table for comparison. ${ }^{148}$

\subsubsection{Formation of the Fe Carbides}

Fe carbides form by dissolution of $C$ into the interstitial spaces in the $\alpha$-Fe lattice. The formation of the various Fe carbides is primarily dependent on the temperature where 


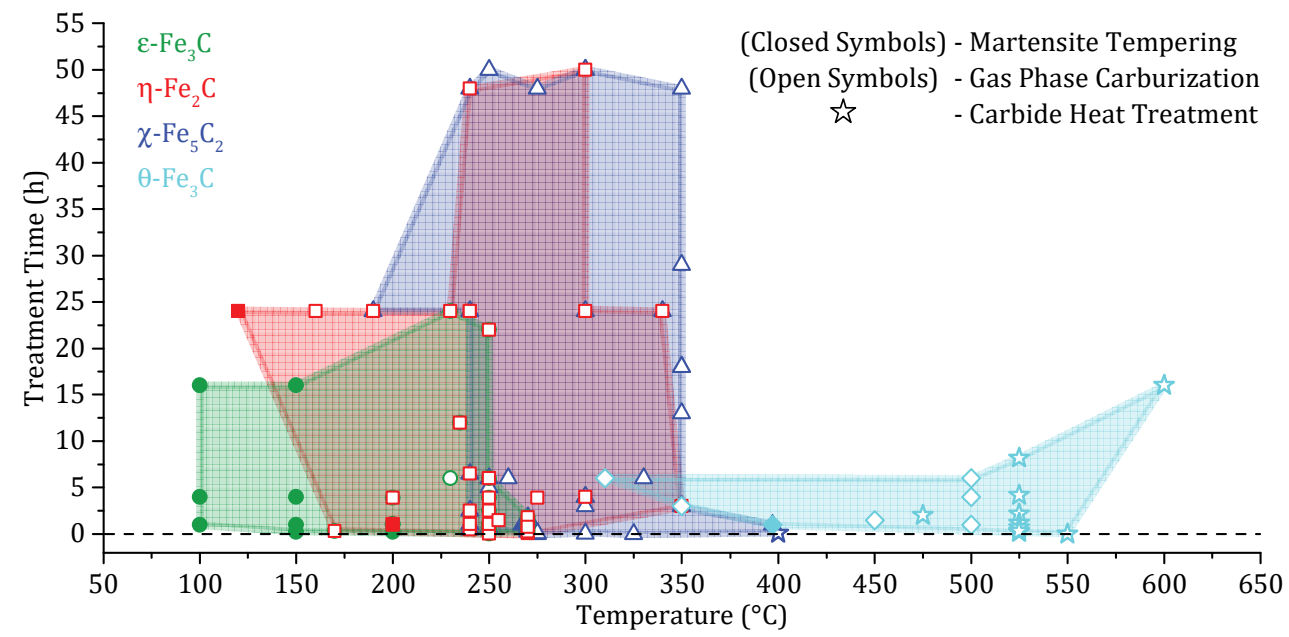

Figure 2.10

Collected observation regions (solid fill) of the Fe carbides as temperature versus treatment exposure time from experimental literature data. $\theta-\mathrm{Fe}_{7} \mathrm{C}_{3}$ data points are excluded. Data points are collected according to the criteria presented in the text.

the $\alpha$-Fe lattice is exposed to an available source of C. Fe in Fe carbides has a valency state close to zero, as for example in the $\chi-\mathrm{Fe}_{5} \mathrm{C}_{2}$ phase. ${ }^{123}$ If a $\mathrm{Fe}$ oxide is used as a $\mathrm{Fe}$ carbide precursor, it needs to be reduced prior/simultaneously to a zero Fe valency state during the Fe carbide formation.

Fe carbides can form from $\alpha$-Fe by i) gas phase carburization where e.g. the CO, either from pure $\mathrm{CO}$ or $\mathrm{H}_{2}: \mathrm{CO}$, acts as the $\mathrm{C}$ source, $58,60 \mathrm{ii}$ ) heat treating the pre-formed Fe carbides to interchange the carbide phases, ${ }^{58,69,77}$ and iii) tempering (i.e. heat treating) pre-formed martensite (or austenite) resulting in $\mathrm{Fe}$ carbide precipitation. ${ }^{137,156}$ The $\varepsilon-\mathrm{Fe}_{3} \mathrm{C}, \eta-\mathrm{Fe}_{2} \mathrm{C}, \chi-\mathrm{Fe}_{5} \mathrm{C}_{2}$ and $\theta-\mathrm{Fe}_{3} \mathrm{C}$ phases form relatively easily from an $\alpha-\mathrm{Fe} /$ martensite precursor with any of the listed methods, i.e. by varying the heat treatment or gas phase carburization temperature. The formation of the $\theta-\mathrm{Fe}_{7} \mathrm{C}_{3}$ phase seems to be a more complex phenomenon, as based on the available literature. It will be discussed separately after the formation conditions of the more common Fe carbides.

The observation temperatures for the $\varepsilon-\mathrm{Fe}_{3} \mathrm{C}, \eta-\mathrm{Fe}_{2} \mathrm{C}, \chi-\mathrm{Fe}_{5} \mathrm{C}_{2}$ and $\theta-\mathrm{Fe}_{3} \mathrm{C}$ phases from the literature are gathered with the following criteria: $\mathrm{i})$ the reaction takes place from metallic, zero valency $\alpha$-Fe, in order to decouple the Fe reduction/carburization effects, ii) reaction treatment time is reported, and iii) it is possible to re-evaluate the reported data to correspond the nomenclature defined in Table 2.2 and Table 2.3. From criteria iii) follows that only MAS- and ED-based results are included to evaluate the presence of the $\varepsilon-\mathrm{Fe}_{3} \mathrm{C}$ and $\eta-\mathrm{Fe}_{2} \mathrm{C}$ phases. XRPD and Thermomagnetic (TM) data are 


\section{Table 2.4}

The references to the experimental literature data for the Fe carbide observations, excluding the $\theta-\mathrm{Fe}_{7} \mathrm{C}_{3}$ phase, and adhering to the criteria presented in the main text. The data points from the references are collected to the Figure 2.10.

\begin{tabular}{|c|c|c|c|}
\hline $\begin{array}{c}\text { Fe Carbide } \\
\text { Phase }\end{array}$ & $\begin{array}{c}\text { Characterization } \\
\text { Technique }\end{array}$ & Experiment Type & Reference Numbers \\
\hline$\varepsilon-\mathrm{Fe}_{3} \mathrm{C}$ & ED, MAS & $\begin{array}{l}\text { - Gas phase carburization } \\
\text { - Martensite tempering }\end{array}$ & $\begin{array}{c}26,59,66,69,77, \\
127,149,150,157,158]\end{array}$ \\
\hline$\eta-\mathrm{Fe}_{2} \mathrm{C}$ & ED, MAS & $\begin{array}{l}\text { - Gas phase carburization } \\
\text { - Martensite tempering }\end{array}$ & $\begin{array}{l}{[26,27,150,158,59,60,} \\
66,77,92,127,130,149]\end{array}$ \\
\hline$\chi-\mathrm{Fe}_{5} \mathrm{C}_{2}$ & ED, MAS, XRPD & $\begin{array}{l}\text { - Gas phase carburization } \\
\text { - Martensite tempering } \\
\text { - Carbide Heat Treatment }\end{array}$ & $\begin{array}{r}{[35,58,157,159,60,66,} \\
69,76,77,92,135,150]\end{array}$ \\
\hline$\theta-\mathrm{Fe}_{3} \mathrm{C}$ & $\begin{array}{c}\text { ED, MAS, XRPD, } \\
\text { TM }\end{array}$ & $\begin{array}{l}\text { - Gas phase carburization } \\
\text { - Martensite tempering } \\
\text { - Carbide Heat Treatment }\end{array}$ & {$[60,69,160,161]$} \\
\hline
\end{tabular}

ED = Electron Diffraction, MAS = Mössbauer Absorption Spectroscopy, XRPD = X-ray Powder Diffractometry, $\mathrm{TM}=$ Thermomagnetic.

also included for evaluating the $\chi-\mathrm{Fe}_{5} \mathrm{C}_{2}$ and $\theta-\mathrm{Fe}_{3} \mathrm{C}$ phases' formation behavior. The references to the studies used to collect the data points in Figure 2.10 are collected to Table 2.4 .

The collected data points in Figure 2.10 refer to the experimental observations on the Fe carbides after respective Fe carbide forming treatment. Possibly coexisting phases of $\alpha$-Fe, martensite or Fe oxides are excluded from the figure. From the literature-based data points in Figure 2.10, the expected observation temperatures and carbide-to-carbide transition temperatures for the Fe carbides with $5 \mathrm{~h}$ treatment time can be roughly estimated as:

$\varepsilon-\mathrm{Fe}_{3} \mathrm{C}\left(100-265^{\circ} \mathrm{C}\right)<\eta-\mathrm{Fe}_{2} \mathrm{C}\left(160-350^{\circ} \mathrm{C}\right)<\chi-\mathrm{Fe}_{5} \mathrm{C}_{2}\left(240-350^{\circ} \mathrm{C}\right)<\theta-\mathrm{Fe}_{3} \mathrm{C}\left(310-600^{\circ} \mathrm{C}\right)$

These expectations on the Fe carbide thermal stabilities and the formation temperatures compare mainly well with the expectations presented by other authors. $58,69,128,137,156$ This, while bearing in mind the unclear nomenclature in the literature regarding the " $\varepsilon$-carbides" i.e. the identification of the $\varepsilon$ - $\mathrm{Fe}_{3} \mathrm{C}$ and $\eta$ - $\mathrm{Fe}_{2} \mathrm{C}$ phases.

A good example on the behavior of the $\varepsilon-\mathrm{Fe}_{3} \mathrm{C}$ and $\eta-\mathrm{Fe}_{2} \mathrm{C}$ phases is from a study by Boellaard, van der Kraan and Geus. ${ }^{26}$ With the FTS reaction experiments at reaction temperatures between $200-275^{\circ} \mathrm{C}$, either increasing the reaction time or the temperature caused $\mathrm{Fe}$ carbide formation to shift from the $\varepsilon$ - $\mathrm{Fe}_{3} \mathrm{C}$ towards $\eta-\mathrm{Fe}_{2} \mathrm{C}$ phase. Already at $\approx 200^{\circ} \mathrm{C}$ with $\approx 1 \mathrm{~h}$ reaction time, the $\eta-\mathrm{Fe}_{2} \mathrm{C}$ phase is the $\mathrm{Fe}$ carbide to 
form in larger quantities over the $\varepsilon$ - $\mathrm{Fe}_{3} \mathrm{C}$ phase. With further increasing the reaction time or temperature, the $\eta-\mathrm{Fe}_{2} \mathrm{C}$ phase content increases. ${ }^{26}$ In a martensite tempering study by Williamson, Nakazawa and Krauss, ${ }^{127}$ at $200^{\circ} \mathrm{C}$ the $\varepsilon-\mathrm{Fe}_{3} \mathrm{C}$ phase (component M2 in their study) disappears if the tempering time is increased above $1 \mathrm{~h}$. At temperatures $<150^{\circ} \mathrm{C}, \varepsilon-\mathrm{Fe}_{3} \mathrm{C}$ is stable and is able to withstand longer tempering times. ${ }^{127}$ In short, the $\varepsilon$ - $\mathrm{Fe}_{3} \mathrm{C}$ phase is the least stable $\mathrm{Fe}$ carbide that easily transforms to the $\eta-\mathrm{Fe}_{2} \mathrm{C}$ phase, with increasing treatment time or temperature. Minor quantities of the $\varepsilon-\mathrm{Fe}_{3} \mathrm{C}$ phase may nevertheless be observed with temperatures up to 250 $270^{\circ} \mathrm{C} .{ }^{26,59,66,150,157}$

Within the temperature range of $\approx 240-350^{\circ} \mathrm{C}$, both of the $\eta-\mathrm{Fe}_{2} \mathrm{C}$ and $\chi-\mathrm{Fe}_{5} \mathrm{C}_{2}$ phases may coexist. For these carbides the reaction exposure time seems to be less influential than in the case of relative formation of the $\varepsilon-\mathrm{Fe}_{3} \mathrm{C}$ and $\eta-\mathrm{Fe}_{2} \mathrm{C}$ phases. For at least up to $50 \mathrm{~h}$ in the FTS reaction at $300^{\circ} \mathrm{C}$, both the $\eta-\mathrm{Fe}_{2} \mathrm{C}$ and $\chi-\mathrm{Fe}_{5} \mathrm{C}_{2}$ phases can be observed, although at the higher temperatures the $\chi-\mathrm{Fe}_{5} \mathrm{C}_{2}$ phase becomes the Fe carbide to form in higher quantities. ${ }^{93}$ After a short $3 \mathrm{~h}$ FTS reaction, Niemantsverdriet et al. were able to observe low quantities of the $\eta-\mathrm{Fe}_{2} \mathrm{C}$ phase even at $350^{\circ} \mathrm{C}$, while the $\chi-\mathrm{Fe}_{5} \mathrm{C}_{2}$ and $\theta$ $\mathrm{Fe}_{3} \mathrm{C}$ phases were the major Fe carbide phases. ${ }^{60} \mathrm{Also}$, after a $24 \mathrm{~h}$ FTS reaction at $340^{\circ} \mathrm{C}$, Wezendonk et al. observed a significant presence of the $\eta-\mathrm{Fe}_{2} \mathrm{C}$ phase. ${ }^{77}$

Few catalyst composition properties are known to increase the stability of the $\varepsilon$ $\mathrm{Fe}_{3} \mathrm{C}$ or $\eta-\mathrm{Fe}_{2} \mathrm{C}$ phases (i.e. " $\varepsilon$-carbides") in relation to the $\chi-\mathrm{Fe}_{5} \mathrm{C}_{2}$ phase. Addition of i) alkali, as at least if added as $\mathrm{Na}$ or $\mathrm{K},{ }^{29,56,64,162}$ or ii) $\mathrm{Si}$ as an inert oxide, ${ }^{61,72,150}$ to the $\mathrm{Fe}$ catalyst composition can stabilize the $\varepsilon-\mathrm{Fe}_{3} \mathrm{C}$ and/or $\eta-\mathrm{Fe}_{2} \mathrm{C}$ phase(s). Also, iii) smaller $\mathrm{Fe}$ particle size tends to favor the formation of the $\varepsilon-\mathrm{Fe}_{3} \mathrm{C}$ and/or $\eta-\mathrm{Fe}_{2} \mathrm{C}$ phases over the $\chi$ - $\mathrm{Fe}_{5} \mathrm{C}_{2 .}{ }^{60,150,158,159}$ Unfortunately, from the literature it is not possible to conclusively determine which of the " $\varepsilon$-carbides" i.e. $\varepsilon$ - $\mathrm{Fe}_{3} \mathrm{C}$ and/or $\eta-\mathrm{Fe}_{2} \mathrm{C}$ is stabilized, in relation to the mentioned catalyst composition properties.

Above the temperature of $\approx 300-350^{\circ} \mathrm{C}$, the $\chi-\mathrm{Fe}_{5} \mathrm{C}_{2}$ and $\theta-\mathrm{Fe}_{3} \mathrm{C}$ phases are the expected main Fe-carbide phases. The highest temperature stability for the $\chi-\mathrm{Fe}_{5} \mathrm{C}_{2}$ phase included in this review, has been observed to be up to $\approx 400^{\circ} \mathrm{C}$ from a martensite tempering study. ${ }^{156}$ Also, the $\chi-\mathrm{Fe}_{5} \mathrm{C}_{2}$ phase has been observed after heat treating of preformed Fe carbides under vacuum at $400^{\circ} \mathrm{C}$ for short periods of time $(\approx 0.1 \mathrm{~h}) .69$ Above $350^{\circ} \mathrm{C}$, the preferred Fe carbide to form is $\theta-\mathrm{Fe}_{3} \mathrm{C} .60,69,160,161$

From martensite tempering studies, Nagakura et al. have reported formation of $\theta$ $\mathrm{Fe}_{3} \mathrm{C}$ after tempering martensitic steel at $267^{\circ}$ for $1 \mathrm{~h},{ }^{156}$ and Williamson, Nakazawa and Krauss after $250^{\circ} \mathrm{C}$ for 5 h..$^{127}$ Both of these temperatures seem very low for $\theta-\mathrm{Fe}_{3} \mathrm{C}$ formation when comparing to the other literature data, and are excluded from the Figure 2.10.

Decomposition of the $\theta-\mathrm{Fe}_{3} \mathrm{C}$ phase into $\alpha$-Fe and $\mathrm{C}$ under vacuum heat treatment 
may start already slowly at a temperature as low as $377^{\circ} \mathrm{C}$ and becomes rapid at $>527^{\circ} \mathrm{C}$ as has been observed by Wood et al. ${ }^{70}$ Nagakura in comparison observed that the $\theta$ $\mathrm{Fe}_{3} \mathrm{C}$ decomposition to $\alpha$-Fe only started at $\approx 600^{\circ} \mathrm{C}$ under vacuum heat treatment. ${ }^{69}$ The increase in the most thermally stable $\mathrm{Fe}$ carbide's (i.e. $\theta-\mathrm{Fe}_{3} \mathrm{C}$ ) decomposition temperature has been explained by Podgurski et al. to be due to formed Fe carbide oxidation shell. ${ }^{30}$ The shell can retard the decomposition of the $\theta-\mathrm{Fe}_{3} \mathrm{C}$ core to $\alpha$-Fe and C. The Fe carbides readily form a $\approx 4-5 \mathrm{~nm}$ oxidation layer if any $\mathrm{O}$ is available for the oxidation reaction. ${ }^{163}$

As mentioned, the main Fe carbide phase determining factor is the temperature. For gas phase carburization, factors such as $\mathrm{H}_{2}$ : $\mathrm{CO}$ ratio and reaction pressure do not seem to play a significant role when the Fe carbides are formed from a zero valency $\alpha$-Fe.

For example, with the FTS reaction at $250^{\circ} \mathrm{C}$ with $\mathrm{H}_{2}$ : $\mathrm{CO}$ ratios of $1: 1(0.5 \mathrm{~h}), 592: 1$ $(1.1 \mathrm{~h}),{ }^{26}$ and $3: 1(0.66 \mathrm{~h}),{ }^{150}$ only the $\varepsilon-\mathrm{Fe}_{3} \mathrm{C}$ (minor phase) and the $\eta-\mathrm{Fe}_{2} \mathrm{C}$ (major phase) phases are observed along with differences in the extent of $\alpha$-Fe carburization. At $270^{\circ} \mathrm{C}$ after $0.66 \mathrm{~h}$ of FTS reaction with $10: 1 \mathrm{H}_{2}: \mathrm{CO}$, Tau et al. observed formation of the $\chi-\mathrm{Fe}_{5} \mathrm{C}_{2}$ phase in addition to the $\varepsilon-\mathrm{Fe}_{3} \mathrm{C}$ and $\eta-\mathrm{Fe}_{2} \mathrm{C}$ phases from $\alpha-\mathrm{Fe},{ }^{66}$ as would be expected on the basis of the reaction temperature (Figure 2.10). In a systematic study, Jung et al. have observed that the increasing $\mathrm{H}_{2}$ : $\mathrm{CO}$ ratio decreases the rate of carburization of $\alpha$-Fe. ${ }^{159}$ Despite the CO-rich atmosphere being favored for the increased carburization rate, small amounts of $(\approx 1 \%) \mathrm{H}_{2}$ can still aid the carburization by more efficiently, than $\mathrm{CO}$, removing the surface 0 from $\mathrm{CO}$ dissociation, ${ }^{33,50}$ as was also discussed in the previous sections. All in all, despite the changing $\mathrm{H}_{2}$ :CO ratios, the reaction temperature is still the determining factor for the Fe carbide formation. Low $\mathrm{H}_{2}$ : $\mathrm{CO}$ ratios increase the $\alpha$-Fe carburization rate in comparison to the higher $\mathrm{H}_{2}: \mathrm{CO}$ ratios or pure $\mathrm{CO}$.

It is, however, noteworthy to mention that when carburization with $\mathrm{H}_{2}$ : $\mathrm{CO}$ is done with a Fe oxide as the starting material instead of $\alpha-F e$, the Fe carbide formation is dependent on the simultaneous Fe reduction behavior.64,67,78,164,165 This effect will be discussed more in the Chapter 3.

The effect of the overall reaction pressure under which the $\alpha$-Fe phase is carburized from the gas phase is somewhat unclear from the literature, as systematic studies are lacking. It seems, however, that the increased pressure does not change the expected order of the Fe carbide formation, again, when the carburization is done from the $\alpha$-Fe. Amelse et al. performed $300^{\circ} \mathrm{C}$ FTS reaction $\left(\mathrm{H}_{2}: \mathrm{CO} 3: 1\right)$ for $24 \mathrm{~h}$ at atmospheric pressure, ${ }^{157}$ and Jiang et al. $\left.300^{\circ} \mathrm{C} \mathrm{FTS} \mathrm{reaction} \mathrm{(} \mathrm{H}_{2}: \mathrm{CO} 1: 1\right)$ at 10 bar pressure for $50 \mathrm{~h} . .^{93}$ In both studies, only the $\eta-\mathrm{Fe}_{2} \mathrm{C}$ and $\chi-\mathrm{Fe}_{5} \mathrm{C}_{2}$ phases were observed as the $\mathrm{Fe}$ carbides. After 30 bar FTS reaction $\left(\mathrm{H}_{2}: \mathrm{CO} 2: 1\right)$ for $0.3 \mathrm{~h}$ at $170^{\circ} \mathrm{C}$, Xu et al. observed only the $\varepsilon$ $\mathrm{Fe}_{3} \mathrm{C}$ and $\eta-\mathrm{Fe}_{2} \mathrm{C}$ phases as the Fe carbides. ${ }^{149}$ With 23 bar FTS reaction $\left(\mathrm{H}_{2}: \mathrm{CO} 3: 2\right)$ at 
$235^{\circ} \mathrm{C}$ for $12 \mathrm{~h}$, only $\varepsilon-\mathrm{Fe}_{3} \mathrm{C}$ and $\eta-\mathrm{Fe}_{2} \mathrm{C}$ phases were present in a study by Wang et al., 158 which is a quantification result comparable to 1.03 bar FTS reaction $\left(3.9 \mathrm{~h}, 250^{\circ} \mathrm{C}\right.$, $\mathrm{H}_{2}$ :CO 2:1) by Boellaard, van der Kraan and Geus. ${ }^{26}$

In each of the mentioned studies, the Fe carbide formation follows the expectations from the reaction temperature regardless of the applied reaction pressure. As noted by $\mathrm{Xu}$ et al., the elevated pressure only seems to increase the rate of carburization from $\alpha$ Fe (see Supplementary Figure 2, in their study). ${ }^{149}$ The formed Fe carbide phases, however, are not affected.

\subsubsection{Formation of $\theta-\mathrm{Fe}_{7} \mathrm{C}_{3}$}

The formation of the $\theta-\mathrm{Fe}_{7} \mathrm{C}_{3}$ phase is a special case and does not follow the temperature formation regions in Figure 2.10. In literature, the $\theta-\mathrm{Fe}_{7} \mathrm{C}_{3}$ phase is either observed to form at extreme conditions, such as at $60 \mathrm{kbar}$ with $>1100^{\circ} \mathrm{C}$ from a mixture of graphite and $\alpha$-Fe, ${ }^{166}$ or via laser pyrolysis of $\mathrm{Fe}(\mathrm{CO})_{5}$ under $\mathrm{C}_{2} \mathrm{H}_{2 / 4} \cdot{ }^{152,155,167}$

A more interesting case for the FTS reaction related discussion in the realm of $\theta$ $\mathrm{Fe}_{7} \mathrm{C}_{3}$ formation is observed in a study by Tajima and Hirano, where Ba promoted $\mathrm{Fe}_{3} \mathrm{O}_{4}$ was carburized with pure $\mathrm{CO}$ at unspecified reaction pressure. ${ }^{168}$ Within the

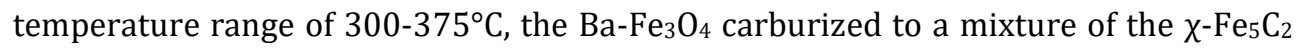
and $\theta-\mathrm{Fe}_{7} \mathrm{C}_{3}$ phases. ${ }^{168}$ Without $\mathrm{Ba}$, only the $\chi-\mathrm{Fe}_{5} \mathrm{C}_{2}$ phase was formed within the same temperature range from the unpromoted $\mathrm{Fe}_{3} \mathrm{O}_{4} \cdot{ }^{168,169}$ In a single crystal ED study of the $\theta-\mathrm{Fe}_{7} \mathrm{C}_{3}$ phase by Audier, Bowen and Jones, single crystals of the $\theta-\mathrm{Fe}_{7} \mathrm{C}_{3}$ phase were formed by carburizing the $\alpha$-Fe phase under $3: 1 \quad \mathrm{CO}: \mathrm{CO}_{2}$ at $500^{\circ} \mathrm{C} .143$ As the carburization to, at least some amounts of, the $\theta-\mathrm{Fe}_{7} \mathrm{C}_{3}$ phase took place without $\mathrm{Ba}$ or a Fe oxide, perhaps, the $\mathrm{CO}_{2}$ might play a role in facilitating the formation of the $\theta-\mathrm{Fe}_{7} \mathrm{C}_{3}$ phase. The $\theta-\mathrm{Fe}_{7} \mathrm{C}_{3}$ phase has also been observed in catalysts extracted from industrial FTS reactors after long reaction times at high-pressure. ${ }^{3}$

A mechanical synthesis route for the $\theta-\mathrm{Fe}_{7} \mathrm{C}_{3}$ phase has been attempted by Barinov, Tsurin and Surikov. ${ }^{170}$ The route has more likely to have only formed the $\chi-\mathrm{Fe}_{5} \mathrm{C}_{2}$ phase, ${ }^{151}$ rather than any observable quantities of the $\theta-\mathrm{Fe}_{7} \mathrm{C}_{3}$ phase.

Regarding the $\theta-\mathrm{Fe}_{7} \mathrm{C}_{3}$ phase thermal stability, heat treatment of the $\theta-\mathrm{Fe}_{7} \mathrm{C}_{3}$ phase under He leads to the phase's decomposition with temperature depending on the 0 atom impurity content in the $\theta-\mathrm{Fe}_{7} \mathrm{C}_{3}$ crystal lattice. ${ }^{167}$ High $\mathrm{O}$ atom impurity content causes the $\theta-\mathrm{Fe}_{7} \mathrm{C}_{3}$ phase to decompose to Fe oxides and $\alpha$-Fe between $300-400^{\circ} \mathrm{C}$. $\mathrm{O}$ atom free $\theta-\mathrm{Fe}_{7} \mathrm{C}_{3}$ phase can survive up to $\approx 400-500^{\circ} \mathrm{C}$ before decomposing directly to $\alpha-\mathrm{Fe}$ and $\mathrm{C}$ atoms. ${ }^{167}$

To conclude, the formation of the $\theta-\mathrm{Fe}_{7} \mathrm{C}_{3}$ phase does not follow the same route as for the $\varepsilon-\mathrm{Fe}_{3} \mathrm{C}, \eta-\mathrm{Fe}_{2} \mathrm{C}, \chi-\mathrm{Fe}_{5} \mathrm{C}_{2}$ or $\theta-\mathrm{Fe}_{3} \mathrm{C}$ phases but might have its own formation route. Nevertheless, formation of the $\theta-\mathrm{Fe}_{7} \mathrm{C}_{3}$ phase is possible under relatively mild and FTS 
reaction relevant conditions, although more systematic research is warranted on its formation specifics.

\subsection{SUMMARY}

The main points from the discussion in this Chapter can be summarized as follows for each of the Chapter sections:

\section{Catalyst Compositions}

A FTS reaction catalyst is most commonly comprised of the active metal (commonly as either $\mathrm{Fe}$ or $\mathrm{Co}$ ), possibly in combination with chemical (alkali, possibly combined with S) and structural (inert oxides of e.g. Al, Si or Mg) promoters. Instead of a structural promoter, as with precipitated or fused bulk catalyst materials, a support material (e.g. $\mathrm{Al}_{2} \mathrm{O}_{3}$ or $\mathrm{SiO}_{2}$ ) can be used instead. The support material is impregnated with a FTS reaction active metal in order to obtain the metal in a highly dispersed form on the ready catalyst.

\section{Reactions, Fe Phase Changes and Catalyst Deactivation}

In Fe-based FTS reaction, $\mathrm{C}$ atoms from input $\mathrm{CO}$ have five pathways to react (Figure 2.1). More specifically, $C$ from CO dissociation can react i) with $\alpha$-Fe to form Fe carbides, ii) with $\mathrm{H}$ from dissociated $\mathrm{H}_{2}$ to form hydrocarbons, iii) with another $\mathrm{C}$ to form inactive carbon. Without $\mathrm{CO}$ dissociation, $\mathrm{CO}$ may iv) reduce $\mathrm{Fe}_{\mathrm{x}} \mathrm{O}_{\mathrm{y}}$ or v) react via the WGS reaction, with both pathways converting $\mathrm{CO}$ into $\mathrm{CO}_{2}$. The phase transformations for a Fe-based FTS reaction catalyst material go from a Fe oxide in the as-synthesized catalyst, to $\alpha$-Fe in pre-reduced state, to $\mathrm{Fe}_{\mathrm{x}} \mathrm{C}$ phases under FTS reaction conditions. As deactivation pathways: the $\mathrm{Fe}_{\mathrm{x}} \mathrm{C}$ phases may reoxidize to $\mathrm{Fe}_{3} \mathrm{O}_{4}$ under FTS reaction conditions if the local $\mathrm{H}_{2} \mathrm{O}: \mathrm{H}_{2}$ or $\mathrm{CO}_{2}: \mathrm{CO}$ ratios become too high. The $\mathrm{Fe}_{3} \mathrm{O}_{4}$ phase is active for the WGS but not for the FTS reaction. The formation of inactive carbon via pathway iii) causes hindered access to the active $\mathrm{Fe}_{\mathrm{x}} \mathrm{C}$ surface and loss of catalyst's mechanical stability via fragmentation. The process decreases catalyst's life-time in the FTS reaction.

\section{Hydrocarbon and C Pathway Selectivity}

Adjustment of the process conditions and/or the catalyst material compositions can be used as tools for modifying the Fe-based FTS reaction's hydrocarbon and C pathway selectivities (Table 2.1). Input $\mathrm{H}_{2}$ : $\mathrm{CO}$ ratio, reaction temperature and reactor space velocity are the main process parameters for modifying the $\alpha$-value or olefin content of the FTS produced hydrocarbons. The reaction temperature 
greatly increases the inactive carbon deposition $\mathrm{C}$ pathway above $\approx 300^{\circ} \mathrm{C}$. Increasing temperature also increases any possible WGS reaction activity via the reaction kinetics. Addition of alkali individually increases the $\alpha$-value and olefin content, while individual addition of $S$ does the opposite. Addition of combined alkali-S in the molar ratio of $\approx 2: 1$ : increases the $\alpha$-value less than with alkali only addition, and as an unique alkali-S promotion feature, it also decreases the $\mathrm{CH}_{4}$ selectivity to a value lower than expected from the ASF distribution. The hydrocarbon products' olefin content increases with the alkali-S addition akin to alkali-only addition. Possibly, the $\mathrm{S}$ replaces $\mathrm{O}$ as the alkali counter-ion changing the chemical promoter compound binding on the $\mathrm{Fe}_{\mathrm{x}} \mathrm{C}$ surface and affecting the promotion effects with alkali-S. On the $C$ pathways: added alkali increases the inactive carbon deposition and WGS reactions, decreasing CO conversion towards FTS reaction hydrocarbon products. Effect of individual S on C pathways is unclear. Increased $\mathrm{CO}_{2}$ selectivity suggests increased carbon formation and/or WGS reaction $\mathrm{C}$ pathways for alkali-S promoted catalysts, effects that are akin to alkalionly addition.

\section{Fe carbides in the Fischer-Tropsch Synthesis Reaction}

Fe carbides ( $\left.\mathrm{Fe}_{\mathrm{x}} \mathrm{C}, x=2-3\right)$ are the active phases in the Fe-based FTS reaction. The Fe carbides, which may form in the FTS reaction, with their respective crystal lattice space groups, are: hexagonal $\varepsilon-\mathrm{Fe}_{3} \mathrm{C} \mathrm{P}_{3} 22$ (182), orthorhombic $\eta-\mathrm{Fe}_{2} \mathrm{C}$ Pnnm (58), monoclinic $\chi-\mathrm{Fe}_{5} \mathrm{C}_{2} \mathrm{C} 2 / c$ (15) (the Hägg carbide), orthorhombic $\theta-\mathrm{Fe}_{3} \mathrm{C}$ Pnma (62) (cementite) and orthorhombic $\theta-\mathrm{Fe}_{7} \mathrm{C}_{3}$ Pnma (62) (the Eckström-Adcock carbide) (Table 2.2). The $\varepsilon-\mathrm{Fe}_{3} \mathrm{C}$ phase has a purely hexagonal $\mathrm{Fe}$ lattice with $\mathrm{C}$ atoms occupying a third of the octahedral interstitial Fe lattice spaces in a random manner. The $\eta-\mathrm{Fe}_{2} \mathrm{C}$ phase has a distorted (pseudo-)hexagonal Fe lattice where $\mathrm{C}$ atoms occupy half of the octahedral interstitial Fe lattice spaces, in an ordered manner. Nomenclature of $(\varepsilon-) \mathrm{Fe}_{2} \mathrm{C}$ or $\varepsilon^{\prime}-\mathrm{Fe}_{2.2} \mathrm{C}$ should not be used to label the $\mathrm{Fe}$ " $\varepsilon$ carbides". The hexagonal $\mathrm{Fe}_{7} \mathrm{C}_{3}$ is should not be used to identify the $\theta-\mathrm{Fe}_{7} \mathrm{C}_{3}$ phase as the correct structure is orthorhombic rather than a hexagonal $\mathrm{Fe}_{7} \mathrm{C}_{3}$. The MAS hyperfine fields are assigned in the Table 2.3 from the literature following the established nomenclature.

The observed temperature regions for the identified Fe carbides from a zero valency $\alpha$-Fe precursor are plotted in Figure 2.10 by using the experimental data collected from the literature. The literature-based thermal stabilities of the $\mathrm{Fe}$ carbides are in order $\varepsilon-\mathrm{Fe}_{3} \mathrm{C}\left(100-265^{\circ} \mathrm{C}\right)<\eta-\mathrm{Fe}_{2} \mathrm{C}\left(160-340^{\circ} \mathrm{C}\right)<\chi-\mathrm{Fe}_{5} \mathrm{C}_{2}(240-$ $\left.350^{\circ} \mathrm{C}\right)<\theta-\mathrm{Fe}_{3} \mathrm{C}\left(310-600^{\circ} \mathrm{C}\right)$. The temperature range in parenthesis refers to the estimated observation temperature limits for the carbides at $5 \mathrm{~h}$ treatment 
exposure time (Figure 2.10). The $\theta-\mathrm{Fe}_{7} \mathrm{C}_{3}$ phase has a different formation route in comparison to the other Fe carbides and it may form under relatively mild, FTS reaction relevant conditions. Such as, if a $\mathrm{Ba}-\mathrm{Fe}_{3} \mathrm{O}_{4}$ catalyst is carburized with $\mathrm{CO}$ between $300-375^{\circ} \mathrm{C}$, or $\alpha-\mathrm{Fe}$ is carburized with $\mathrm{CO}: \mathrm{CO}_{2}$ at $500^{\circ} \mathrm{C}$. Other formation pathways for the $\theta-\mathrm{Fe}_{7} \mathrm{C}_{3}$ phase require extreme conditions (laser pyrolysis or 60 kbar with $>1100^{\circ} \mathrm{C}$ ).

\subsection{ACKNOWLEDGEMENTS}

A. I. Dugulan (Technische Universiteit Delft, TU Delft) is acknowledged for discussion and help on the MAS literature used in the Chapter, and P. F. Vittoz (Universiteit Utrecht, UU) for translation and discussion on the reference [128].

\subsection{REFERENCES}

[1] Vannice, M. A., "The catalytic synthesis of hydrocarbons from $\mathrm{H}_{2} / \mathrm{CO}$ mixtures over the group VIII metals I. The specific activities and product distributions of supported metals", J. Catal. 37, 449-461 (1975).

[2] Mittasch, A. and Schneider, C., "Producing Compounds Containing Carbon and Hydrogen", Patent US1201850A (1916).

[3] Dry, M. E., "The Fischer-Tropsch Synthesis" in Catalysis - Science and Technology (eds. Anderson, J. R. \& Boudart, M.) 1, 159-256 (Springer-Verlag, Berlin, 1981).

[4] de Smit, E. and Weckhuysen, B. M., "The renaissance of iron-based Fischer-Tropsch synthesis: on the multifaceted catalyst deactivation behaviour", Chem. Soc. Rev. 37, 2758-2781 (2008).

[5] Dry, M. E., "High quality diesel via the Fischer-Tropsch process - a review",J. Chem. Technol. Biotechnol. 77, 43-50 (2001).

[6] Dry, M. E., "The Fischer-Tropsch process: 1950-2000", Catal. Today 71, 227-241 (2002).

[7] Enger, B. C. and Holmen, A., "Nickel and Fischer-Tropsch Synthesis", Catal. Rev. 54, 437-488 (2012).

[8] Brynestad, J., "Iron and nickel carbonyl formation in steel pipes and its prevention: literature survey", (Oak Ridge, TN (United States), 1976). DOI:10.2172/7349456 Available at: http://www.osti.gov/bridge/product.biblio.jsp?osti_id=7349456 (Accessed: September 2015).

[9] Dry, M. E., "Chemical concepts used for engineering purposes" in Studies in Surface Science and Catalysis - Fischer-Tropsch Technology (eds. Steynberg, A. P. \& Dry, M. E.) 152, 196-257 (Elsevier B.V., Amsterdam, 2004).

[10] Maitlis, P. M. and de Klerk, A., "Greener Fischer-Tropsch Processes for Fuels and Feedstocks", (WileyVCH, Weinheim, 2013). DOI:10.1002/9783527656837

[11] Ngantsoue-Hoc, W., Zhang, Y., O’Brien, R. J., Luo, M. and Davis, B. H., "Fischer-Tropsch synthesis: activity and selectivity for Group I alkali promoted iron-based catalysts", Appl. Catal. A Gen. 236, 7789 (2002).

[12] Luo, M. and Davis, B. H., "Fischer-Tropsch synthesis: Group II alkali-earth metal promoted catalysts", Appl. Catal. A Gen. 246, 171-181 (2003).

[13] Dry, M. E. and Oosthuizen, G. J., "The correlation between catalyst surface basicity and hydrocarbon 
selectivity in the Fischer-Tropsch synthesis", J. Catal. 11, 18-24 (1968).

[14] Dry, M. E., Shingles, T. and van H. Botha, C. S., "Factors influencing the formation of carbon on iron Fischer-Tropsch catalysts I. The influence of promoters", J. Catal. 17, 341-346 (1970).

[15] Hutchings, G. J., "Promotion in Heterogeneous Catalysis: A Topic Requiring a New Approach?", Catal. Lett. 75, 1-12 (2001).

[16] Johnson, J. Y., "Improvements in the Manufacture and Production of Unsaturated Hydrocarbons of Low Boiling Point", Patent GB322284A (1929).

[17] Myddleton, W. W., "Improvements Relating to the Catalytic Synthesis of Hydrocarbon Oils from Gaseous Mixtures of Carbon Monoxide and Hydrogen", Patent GB509325A (1939).

[18] Crous, R., Bromfield, T. C. and Booyens, S., "Olefin Selective FT Catalyst Composition and Preparation Thereof", Patent W02010066386A1 (2010).

[19] Botes, G. F., Bromfield, T. C., Coetzer, R. L. J., Crous, R., Gibson, P. and Ferreira, A. C., "Development of a chemical selective iron Fischer Tropsch catalyst", Catal. Today 275, 40-48 (2016).

[20] Torres Galvis, H. M., Bitter, J. H., Khare, C. B., Ruitenbeek, M., Dugulan, A. I. and de Jong, K. P., "Supported Iron Nanoparticles as Catalysts for Sustainable Production of Lower Olefins", Science 335, 835-838 (2012).

[21] Xie, J., Torres Galvis, H. M., Koeken, A. C. J., Kirilin, A., Dugulan, A. I., Ruitenbeek, M. and de Jong, K. P., "Size and Promoter Effects on Stability of Carbon-Nanofiber-Supported Iron-Based Fischer-Tropsch Catalysts", ACS Catal. 6, 4017-4024 (2016).

[22] Butt, J. B., "Carbide phases on iron-based Fischer-Tropsch synthesis catalysts part I: Characterization studies", Catal. Lett. 7, 61-82 (1990).

[23] Butt, J. B., "Carbide phases on iron-based Fischer-Tropsch synthesis catalysts part II: Some reaction studies", Catal. Lett. 7, 83-106 (1990).

[24] Falkenhagen, J. P., Maisonneuve, L., Paalanen, P. P., Coste, N., Malicki, N. and Weckhuysen, B. M., "Cobalt-Iron-Manganese Catalysts for the Conversion of End-of-Life-Tire-Derived Syngas into Light Terminal Olefins", Chem. Eur. J. 24, 4597-4606 (2018).

[25] Torres Galvis, H. M. and de Jong, K. P., "Catalysts for Production of Lower Olefins from Synthesis Gas: A Review", ACS Catal. 3, 2130-2149 (2013).

[26] Boellaard, E., van der Kraan, A. M. and Geus, J. W., "Behaviour of a cyanide-derived $\mathrm{Fe} / \mathrm{Al}_{2} \mathrm{O}_{3}$ catalyst during Fischer-Tropsch synthesis", Appl. Catal. A Gen. 147, 229-245 (1996).

[27] Amelse, J. A., Butt, J. B. and Schwartz, L. H., "Carburization of supported iron synthesis catalysts", J. Phys. Chem. 82, 558-563 (1978).

[28] van der Laan, G. P. and Beenackers, A. A. C. M., "Kinetics and Selectivity of the Fischer-Tropsch Synthesis: A Literature Review", Catal. Rev. 41, 255-318 (1999).

[29] Dictor, R. and Bell, A. T., "Fischer-Tropsch synthesis over reduced and unreduced iron oxide catalysts", J. Catal. 97, 121-136 (1986).

[30] Podgurski, H. H., Kummer, J. T., DeWitt, T. W. and Emmett, P. H., "Preparation, Stability and Adsorptive Properties of the Carbides of Iron", J. Am. Chem. Soc. 72, 5382-5388 (1950).

[31] Davis, B. H., "Fischer-Tropsch Synthesis: Reaction mechanisms for iron catalysts", Catal. Today 141, 25-33 (2009).

[32] de Klerk, A., "Indirect Liquefaction Carbon Efficiency" in Synthetic Liquids Production and Refining (eds. de Klerk, A. \& King, D.) 1084, 215-235 (American Chemical Society, Washington, DC, 2011).

[33] Krishnamoorthy, S., Li, A. and Iglesia, E., "Pathways for $\mathrm{CO}_{2}$ Formation and Conversion During FischerTropsch Synthesis on Iron-Based Catalysts", Catal. Lett. 80, 77-86 (2002). 
[34] Niemantsverdriet, J. W. and van der Kraan, A. M., "On the time-dependent behavior of iron catalysts in Fischer-Tropsch synthesis", J. Catal. 72, 385-388 (1981).

[35] Riedel, T., Schulz, H., Schaub, G., Jun, K.-W., Hwang, J.-S. and Lee, K.-W., "Fischer-Tropsch on Iron with $\mathrm{H}_{2} / \mathrm{CO}$ and $\mathrm{H}_{2} / \mathrm{CO}_{2}$ as Synthesis Gases: The Episodes of Formation of the Fischer-Tropsch Regime and Construction of the Catalyst", Top. Catal. 26, 41-54 (2003).

[36] Rhodes, C., Hutchings, G. J. and Ward, A. M., "Water-gas shift reaction: finding the mechanistic boundary", Catal. Today 23, 43-58 (1995).

[37] Ratnasamy, C. and Wagner, J. P., "Water Gas Shift Catalysis", Catal. Rev. 51, 325-440 (2009).

[38] Raje, A. P., O’Brien, R. J. and Davis, B. H., "Effect of Potassium Promotion on Iron-Based Catalysts for Fischer-Tropsch Synthesis", J. Catal. 180, 36-43 (1998).

[39] Chianese, S., Loipersböck, J., Malits, M., Rauch, R., Hofbauer, H., Molino, A. and Musmarra, D., "Hydrogen from the high temperature water gas shift reaction with an industrial Fe/Cr catalyst using biomass gasification tar rich synthesis gas", Fuel Process. Technol. 132, 39-48 (2015).

[40] Fischer, N., Henkel, R., Hettel, B., Iglesias, M., Schaub, G. and Claeys, M., "Hydrocarbons via $\mathrm{CO}_{2}$ Hydrogenation Over Iron Catalysts: The Effect of Potassium on Structure and Performance", Catal. Lett. 146, 509-517 (2016).

[41] Ning, W., Koizumi, N. and Yamada, M., "Researching Fe catalyst suitable for $\mathrm{CO}_{2}$-containing syngas for Fischer-Tropsch synthesis", Energy and Fuels 23, 4696-4700 (2009).

[42] Martinelli, M., Visconti, C. G., Lietti, L., Forzatti, P., Bassano, C. and Deiana, P., "CO ${ }_{2}$ reactivity on Fe$\mathrm{Zn}-\mathrm{Cu}-\mathrm{K}$ Fischer-Tropsch synthesis catalysts with different K-loadings", Catal. Today 228, 77-88 (2014).

[43] Bartholomew, C. H., "Mechanisms of catalyst deactivation", Appl. Catal. A Gen. 212, 17-60 (2001).

[44] Dry, M. E., Shingles, T., Boshoff, L. J. and van H. Botha, C. S., "Factors influencing the formation of carbon on iron Fischer-Tropsch catalysts. II. The effect of temperature and of gases and vapors present during Fischer-Tropsch synthesis", J. Catal. 17, 347-354 (1970).

[45] Cohn, E. M., Bean, E. H., Mentser, M., Hofer, L. J. E., Pontello, A., Peebles, W. C. and Jack, K. H., "The carburization of iron oxide with carbon monoxide: Modifications of Hägg iron carbide", J. Appl. Chem. 5, 418-425 (1955).

[46] Berry, T. F., Ames, R. N. and Snow, R. B., "Influence of Impurities and Role of Iron Carbides in Deposition of Carbon from Carbon Monoxide", J. Am. Ceram. Soc. 39, 308-318 (1956).

[47] Herranz, T., Rojas, S., Pérez-Alonso, F. J., Ojeda, M., Terreros, P. and Fierro, J. L. G., "Genesis of iron carbides and their role in the synthesis of hydrocarbons from synthesis gas", J. Catal. 243, 199-211 (2006).

[48] Turkdogan, E. T. and Vinters, J. V., "Catalytic effect of iron on decomposition of carbon monoxide: I. carbon deposition in $\mathrm{H}_{2}$-CO Mixtures", Metall. Trans. 5, 11-19 (1974).

[49] Olsson, R. G. and Turkdogan, E. T., "Catalytic effect of iron on decomposition of carbon monoxide: II. Effect of additions of $\mathrm{H}_{2}, \mathrm{H}_{2} \mathrm{O}, \mathrm{CO}_{2}, \mathrm{SO}_{2}$ and $\mathrm{H}_{2} \mathrm{~S}^{\prime \prime}$, Metall. Trans. 5, 21-26 (1974).

[50] Arakawa, H. and Bell, A. T., "Effects of potassium promotion on the activity and selectivity of iron Fischer-Tropsch catalysts", Ind. Eng. Chem. Process Des. Dev. 22, 97-103 (1983).

[51] Koeken, A. C. J., Torres Galvis, H. M., Davidian, T., Ruitenbeek, M. and de Jong, K. P., "Suppression of Carbon Deposition in the Iron-Catalyzed Production of Lower Olefins from Synthesis Gas", Angew. Chem., Int. Ed. 51, 7190-7193 (2012).

[52] Alstrup, I., Tavares, M. T., Bernardo, C. A., Sørensen, O. and Rostrup-Nielsen, J. R., "Carbon formation on nickel and nickel-copper alloy catalysts", Mater. Corros. 49, 367-372 (1998).

[53] Nolan, P. E., Lynch, D. C. and Cutler, A. H., "Catalytic disproportionation of CO in the absence of 
hydrogen: Encapsulating shell carbon formation", Carbon 32, 477-483 (1994).

[54] Mazzucco, S., Wang, Y., Tanase, M., Picher, M., Li, K., Wu, Z., Irle, S. and Sharma, R., "Direct evidence of active and inactive phases of Fe catalyst nanoparticles for carbon nanotube formation", J. Catal. 319, 54-60 (2014).

[55] Pendyala, V. R. R., Graham, U. M., Jacobs, G., Hamdeh, H. H. and Davis, B. H., "Fischer-Tropsch Synthesis: Morphology, Phase Transformation, and Carbon-Layer Growth of Iron-Based Catalysts", ChemCatChem 6, 1952-1960 (2014).

[56] Pendyala, V. R. R., Graham, U. M., Jacobs, G., Hamdeh, H. H. and Davis, B. H., "Fischer-Tropsch Synthesis: Deactivation as a Function of Potassium Promoter Loading for Precipitated Iron Catalyst", Catal. Lett. 144, 1704-1716 (2014).

[57] Shultz, J. F., Abelson, M., Stein, K. C. and Anderson, R. B., "Studies of the Fischer-Tropsch Synthesis. XVIII. Influence of Catalyst Geometry on Synthesis on Iron Catalysts", J. Phys. Chem. 63, 496-500 (1959).

[58] Hofer, L. J. E., "Nature of the carbides of iron", U.S. Bureau of Mines Bulletin 631 (1966). Available at: http://www.onemine.org/document/abstract.cfm?docid=186163\&title=Nature-Of-The-CarbidesOf-Iron (Accessed: February 2014).

[59] Pijolat, M., Perrichon, V. and Bussière, P., "Study of the carburization of an iron catalyst during the Fischer-Tropsch synthesis: Influence on its catalytic activity", J. Catal. 107, 82-91 (1987).

[60] Niemantsverdriet, J. W., van der Kraan, A. M., van Dijk, W. L. and van der Baan, H. S., "Behavior of Metallic Iron Catalysts during Fischer-Tropsch Synthesis Studied with Mössbauer Spectroscopy, X-ray Diffraction, Carbon Content Determination, and Reaction Kinetic Measurements", J. Phys. Chem. 84, 3363-3370 (1980).

[61] Le Caër, G., Dubois, J. M., Pijolat, M., Perrichon, V. and Bussière, P., "Characterization by Moessbauer Spectroscopy of Iron Carbides Formed by Fischer-Tropsch Synthesis", J. Phys. Chem. 86, 4799-4808 (1982).

[62] Hofer, L. J. E., Cohn, E. M. and Peebles, W. C., "The Modifications of the Carbide, $\mathrm{Fe}_{2} \mathrm{C}$; Their Properties and Identification", J. Am. Chem. Soc. 71, 189-195 (1949).

[63] O’Brien, R. J., Xu, L., Spicer, R. L. and Davis, B. H., "Activation Study of Precipitated Iron Fischer-Tropsch Catalysts", Energy \& Fuels 10, 921-926 (1996).

[64] O’Brien, R. J., Xu, L., Milburn, D. R., Li, Y.-X., Klabunde, K. J. and Davis, B. H., "Fischer-Tropsch synthesis: Impact of potassium and zirconium promoters on the activity and structure of an ultrafine iron oxide catalyst", Top. Catal. 2, 1-15 (1995).

[65] Ning, W., Koizumi, N., Chang, H., Mochizuki, T., Itoh, T. and Yamada, M., "Phase transformation of unpromoted and promoted Fe catalysts and the formation of carbonaceous compounds during Fischer-Tropsch synthesis reaction", Appl. Catal. A Gen. 312, 35-44 (2006).

[66] Tau, L. M., Borcar, S., Bianchi, D. and Bennett, C. O., "Investigation of supported iron Fischer-Tropsch synthesis catalysts by Mössbauer spectroscopy", J. Catal. 87, 36-54 (1984).

[67] de Smit, E., Cinquini, F., Beale, A. M., Safonova, O. V., van Beek, W., Sautet, P. and Weckhuysen, B. M., "Stability and Reactivity of $\epsilon-\chi-\theta$ Iron Carbide Catalyst Phases in Fischer-Tropsch Synthesis: Controlling $\mu_{C}$ ", J. Am. Chem. Soc. 132, 14928-14941 (2010).

[68] Jozwiak, W. K., Kaczmarek, E., Maniecki, T. P., Ignaczak, W. and Maniukiewicz, W., "Reduction behavior of iron oxides in hydrogen and carbon monoxide atmospheres", Appl. Catal. A Gen. 326, 1727 (2007).

[69] Nagakura, S., "Study of Metallic Carbides by Electron Diffraction Part III. Iron Carbides", J. Phys. Soc. Japan 14, 186-195 (1959).

[70] Wood, I. G., Vočadlo, L., Knight, K. S., Dobson, D. P., Marshall, W. G., Price, G. D. and Brodholt, J., 
"Thermal expansion and crystal structure of cementite, $\mathrm{Fe}_{3} \mathrm{C}$, between 4 and $600 \mathrm{~K}$ determined by timeof-flight neutron powder diffraction", J. Appl. Crystallogr. 37, 82-90 (2004).

[71] Jin, Y. and Datye, A. K., "Phase Transformations in Iron Fischer-Tropsch Catalysts during Temperature-Programmed Reduction", J. Catal. 196, 8-17 (2000).

[72] Mogorosi, R. P., Fischer, N., Claeys, M. and van Steen, E., "Strong-metal-support interaction by molecular design: Fe-silicate interactions in Fischer-Tropsch catalysts", J. Catal. 289, 140-150 (2012).

[73] van de Loosdrecht, J., Botes, G. F., Ciobica, I. M., Ferreira, A. C., Gibson, P., Moodley, D. J., Saib, A. M., Visagie, J. L., Weststrate, C. J. and Niemantsverdriet, J. W., "Fischer-Tropsch Synthesis: Catalysts and Chemistry" in Comprehensive Inorganic Chemistry II (eds. Reedijk, J. \& Poeppelmeier, K.), 525-557 (Elsevier, Amsterdam, 2013). DOI:10.1016/B978-0-08-097774-4.00729-4

[74] Ren-Yuan, T., Su, Z., Chengyu, W., Dongbai, L. and Liwu, L., "An in situ combined temperatureprogrammed reduction-Mössbauer spectroscopy of alumina-supported iron catalysts", J. Catal. 106, 440-448 (1987).

[75] Anderson, R. B., Hofer, L. J. E., Cohn, E. M. and Seligman, B., "Studies of the Fischer-Tropsch Synthesis. IX. Phase Changes of Iron Catalysts in the Synthesis", J. Am. Chem. Soc. 73, 944-946 (1951).

[76] Shultz, J. F., Hall, W. K., Seligman, B. and Anderson, R. B., "Studies of the Fischer-Tropsch Synthesis. XIV. Hägg Carbide as Catalysts", J. Am. Chem. Soc. 77, 213-221 (1955).

[77] Wezendonk, T. A., Sun, X., Dugulan, A. I., van Hoof, A. J. F., Hensen, E. J. M., Kapteijn, F. and Gascon, J., "Controlled formation of iron carbides and their performance in Fischer-Tropsch synthesis", J. Catal. 362, 106-117 (2018)

[78] Bukur, D. B., Okabe, K., Rosynek, M. P., Li, C. P., Wang, D. J., Rao, K. R. P. M. and Huffman, G. P., "Activation Studies with a Precipitated Iron Catalyst for Fischer-Tropsch Synthesis. I. Characterization Studies", Journal of Catalysis 155, 353-365 (1995).

[79] Steynberg, A. P., Espinoza, R. L., Jager, B. and Vosloo, A. C., "High temperature Fischer-Tropsch synthesis in commercial practice", Appl. Catal. A Gen. 186, 41-54 (1999).

[80] Sarkar, A., Seth, D., Dozier, A. K., Neathery, J. K., Hamdeh, H. H. and Davis, B. H., "Fischer-Tropsch Synthesis: Morphology, Phase Transformation and Particle Size Growth of Nano-scale Particles", Catal. Lett. 117, 1-17 (2007).

[81] Anderson, R. B., Karn, F. S. and Shultz, J. F., "Factors in sulfur poisoning of iron catalysts in FischerTropsch synthesis", J. Catal. 4, 56-63 (1965).

[82] Shultz, J. F., Hofer, L. J. E., Karn, F. S. and Anderson, R. B., "Studies of the Fischer-Tropsch Synthesis; Prepoisoning of Iron Catalysts by Sulfur Compounds", J. Phys. Chem. 66, 501-506 (1962).

[83] Xu, J.-D., Chang, Z.-Y., Zhu, K.-T., Weng, X.-F., Weng, W.-Z., Zheng, Y.-P., Huang, C.-J. and Wan, H.-L., "Effect of sulfur on $\alpha-\mathrm{Al}_{2} \mathrm{O}_{3}$-supported iron catalyst for Fischer-Tropsch synthesis", Appl. Catal. A Gen. 514, 103-113 (2016).

[84] San-Miguel, M. A., Oviedo, J. and Sanz, J. F., "Cluster-Substrate Interaction" in Handbook of Nanophysics: Clusters and Fulleres (ed. Sattler, K. D.), 328-344 (CRC Press, Boca Raton, 2017).

[85] Nørskov, J. K., Holloway, S. and Lang, N. D., "Microscopic model for the poisoning and promotion of adsorption rates by electronegative and electropositive atoms", Surf. Sci. 137, 65-78 (1984).

[86] Madon, R. J. and Shaw, H., "Effect of Sulfur on the Fischer-Tropsch Synthesis", Catal. Rev. 15, 69-106 (1977).

[87] Benziger, J. and Madix, R. J., "The effects of carbon, oxygen, sulfur and potassium adlayers on CO and $\mathrm{H}_{2}$ adsorption on $\mathrm{Fe}(100) "$, Surf. Sci. 94, 119-153 (1980).

[88] Nørskov, J. K. and Besenbacher, F., "Theory of hydrogen interaction with metals", J. Less Common Met. 
130, 475-490 (1987).

[89] Nørskov, J. K., Bligaard, T., Hvolbæk, B., Abild-Pedersen, F., Chorkendorff, I. and Christensen, C. H., "The nature of the active site in heterogeneous metal catalysis", Chem. Soc. Rev. 37, 2163 (2008).

[90] Förtsch, D., Pabst, K. and Groß-Hardt, E., "The product distribution in Fischer-Tropsch synthesis: An extension of the ASF model to describe common deviations", Chem. Eng. Sci. 138, 333-346 (2015).

[91] Todic, B., Nowicki, L., Nikacevic, N. and Bukur, D. B., "Fischer-Tropsch synthesis product selectivity over an industrial iron-based catalyst: Effect of process conditions", Catal. Today 261, 28-39 (2016).

[92] Dry, M. E., "Commercial conversion of carbon monoxide to fuels and chemicals", J. Organomet. Chem. 372, 117-127 (1989).

[93] Jiang, F., Zhang, M., Liu, B., Xu, Y. and Liu, X., "Insights into the influence of support and potassium or sulfur promoter on iron-based Fischer-Tropsch synthesis: understanding the control of catalytic activity, selectivity to lower olefins, and catalyst deactivation", Catal. Sci. Technol. 7, 1245-1265 (2017).

[94] Botes, G. F., Niemantsverdriet, J. W. and van de Loosdrecht, J., "A comparison of cobalt and iron based slurry phase Fischer-Tropsch synthesis", Catal. Today 215, 112-120 (2013).

[95] Torres Galvis, H. M., Koeken, A. C. J., Bitter, J. H., Davidian, T., Ruitenbeek, M., Dugulan, A. I. and de Jong, K. P., "Effects of sodium and sulfur on catalytic performance of supported iron catalysts for the Fischer-Tropsch synthesis of lower olefins", J. Catal. 303, 22-30 (2013).

[96] Torres Galvis, H. M., Koeken, A. C. J., Bitter, J. H., Davidian, T., Ruitenbeek, M., Dugulan, A. I. and De Jong, K. P., "Effect of precursor on the catalytic performance of supported iron catalysts for the FischerTropsch synthesis of lower olefins", Catal. Today 215, 95-102 (2013).

[97] Layng, E. T., "Synthesis of hydrocarbons with sulfur containing catalyst", Patent US2446426A (1948).

[98] Bonzel, H. P., "Alkali-metal-affected adsorption of molecules on metal surfaces", Surf. Sci. Rep. 8, 43125 (1988).

[99] Kiskinova, M., "Interaction of CO with Single Crystal Metal Surfaces" in New Trends in CO Activation (ed. Guczi, L.) 64, 37-86 (Elsevier, Amsterdam, 1991).

[100] Ertl, G., Lee, S. B. and Weiss, M., "The influence of potassium on the adsorption of hydrogen on iron", Surf. Sci. 111, L711-L715 (1981).

[101] Ponec, V., "Selectivity in the Syngas Reactions" in New Trends in CO Activation (ed. Guczi, L.) 64, 117157 (Elsevier, Amsterdam, 1991).

[102] Zhang, H.-B. and Schrader, G. L., "Characterization of a fused iron catalyst for Fischer-Tropsch synthesis by in situ laser Raman spectroscopy", J. Catal. 95, 325-332 (1985).

[103] Krupay, B. W. and Amenomiya, Y., "Alkali-promoted Alumina Catalysts I. Chemisorption and oxygen exchange of carbon monoxide and carbon dioxide on potassium-promoted alumina catalysts", J. Catal. 67, 362-370 (1981).

[104] Amenomiya, Y. and Pleizier, G., "Alkali-promoted Alumina Catalysts II. Water-Gas Shift Reaction", J. Catal. 76, 345-353 (1982).

[105] Paul, J., "CO dissociation on potassium-promoted aluminium", Nature 323, 701-703 (1986).

[106] Dry, M. E., Shingles, T., Boshoff, L. J. and Oosthuizen, G. J., "Heats of chemisorption on promoted iron surfaces and the role of alkali in Fischer-Tropsch synthesis", J. Catal. 15, 190-199 (1969).

[107] Bukur, D. B., Mukesh, D. and Patel, S. A., "Promoter effects on precipitated iron catalysts for FischerTropsch synthesis", Ind. Eng. Chem. Res. 29, 194-204 (1990).

[108] Guczi, L., "Effect of Hydrogen in Controlling CO Hydrogenation", New Trends in CO Activation 64, 350380 (1991). 
[109] Hwang, K.-R., Lee, C.-B. and Park, J.-S., "Advanced nickel metal catalyst for water-gas shift reaction", J. Power Sources 196, 1349-1352 (2011).

[110] Bartholomew, C. H., Agrawal, P. K. and Katzer, J. R., "Sulfur Poisoning of Metals" in Advances in Catalysis (eds. Eley, D. D., Pines, H. \& Weisz, P. B.) 31, 135-242 (Academic Press, New York, 1982).

[111] Dalla Betta, R. A., Piken, A. G. and Shelef, M., "Heterogeneous methanation: Steady-state rate of CO hydrogenation on supported ruthenium, nickel and rhenium", J. Catal. 40, 173-183 (1975).

[112] van Dijk, W. L., Niemantsverdriet, J. W., van dar Kraan, A. M. and van der Baan, H. S., "Effects of Manganese Oxide and Sulphate on the Olefin Selectivity of Iron Catalysts in the Fischer Tropsch Reaction", Appl. Catal. 2, 273-288 (1982).

[113] Zhou, X., Ji, J., Wang, D., Duan, X., Qian, G., Chen, D. and Zhou, X., "Hierarchical structured $\alpha$-Al203 supported S-promoted Fe catalysts for direct conversion of syngas to lower olefins", Chem. Commun. 51, 8853-8856 (2015).

[114] Li, T., Yang, Y., Tao, Z., Wan, H., An, X., Zhang, C., Xiang, H. and Li, Y., "Effect of Sulfate on an Iron Manganese Catalyst for Fischer-Tropsch Synthesis", J. Nat. Gas Chem. 16, 354-362 (2007).

[115] Stenger, H. G. and Satterfield, C. N., "Effects of sulfur poisoning of a reduced fused magnetite catalyst in the Fischer-Tropsch synthesis", Ind. Eng. Chem. Process Des. Dev. 24, 415-420 (1985).

[116] Wu, B., Bai, L., Xiang, H., Li, Y.-W., Zhang, Z. and Zhong, B., "An active iron catalyst containing sulfur for Fischer-Tropsch synthesis", Fuel 83, 205-212 (2004).

[117] Bromfield, T. C. and Coville, N. J., "The effect of sulfide ions on a precipitated iron Fischer-Tropsch catalyst", Appl. Catal. A Gen. 186, 297-307 (1999).

[118] Karn, F. S., Schultz, J. F., Kelly, R. E. and Anderson, R. B., "Fischer-Tropsch Synthesis. Poisoning of Iron Catalysts by $\mathrm{H}_{2} \mathrm{~S}$ in Synthesis Gas", Ind. Eng. Chem. Prod. Res. Dev. 2, 43-47 (1963).

[119] Sarkar, A., Dozier, A. K., Graham, U. M., Thomas, G., O’Brien, R. J. and Davis, B. H., "Precipitated iron Fischer-Tropsch catalyst: Effect of carbidization on the morphology of iron oxyhydroxide nanoneedles", Appl. Catal. A Gen. 326, 55-64 (2007).

[120] Arabczyk, W., Moszyński, D., Narkiewicz, U., Pelka, R. and Podsiadły, M., "Poisoning of iron catalyst by sulfur", Catal. Today 124, 43-48 (2007).

[121] Sun, Y., Tao, L., You, T., Li, C. and Shan, H., "Effect of sulfation on the performance of $\mathrm{Fe}_{2} \mathrm{O}_{3} / \mathrm{Al}_{2} \mathrm{O}_{3}$ catalyst in catalytic dehydrogenation of propane to propylene", Chem. Eng. J. 244, 145-151 (2014).

[122] Moszyński, D., Grabke, H. J. and Schneider, A., "Effect of sulphur on the formation of graphite at the surface of carburized iron", Surf. Interface Anal. 34, 380-383 (2002).

[123] Xie, J., Yang, J., Dugulan, A. I., Holmen, A., Chen, D., De Jong, K. P. and Louwerse, M. J., "Size and Promoter Effects in Supported Iron Fischer-Tropsch Catalysts: Insights from Experiment and Theory", ACS Catal. 6, 3147-3157 (2016).

[124] Momma, K. and Izumi, F., "VESTA 3 for three-dimensional visualization of crystal, volumetric and morphology data", J. Appl. Crystallogr. 44, 1272-1276 (2011).

[125] Duggin, M. J. and Hofer, L. J. E., "Nature of $\chi$-Iron Carbide", Nature 212, 248-248 (1966).

[126] Jack, K. H. and Wild, S., "Nature of $\chi$-Carbide and Its Possible Occurrence in Steels", Nature 212, 248250 (1966).

[127] Williamson, D. L., Nakazawa, K. and Krauss, G., "A study of the early stages of tempering in an Fe-1.2 Pct alloy", Metall. Trans. A 10, 1351-1363 (1979).

[128] Dirand, M. and Afqir, L., "Identification structurale precise des carbures precipites dans les aciers faiblement allies aux divers stades du revenu, mecanismes de precipitation", Acta Metall. 31, 10891107 (1983). 
[129] Barton, G. H. and Gale, B., "The structure of a pseudo-hexagonal iron carbide", Acta Crystallogr. 17, 1460-1462 (1964).

[130] Hirotsu, Y. and Nagakura, S., "Electron Microscopy and Diffraction Study of the Carbide Precipitated at the First Stage of Tempering of Martensitic Medium Carbon Steel", Trans. Japan Inst. Met. 15, 129134 (1974).

[131] Hirotsu, Y. and Nagakura, S., "Crystal structure and morphology of the carbide precipitated from martensitic high carbon steel during the first stage of tempering", Acta Metall. 20, 645-655 (1972).

[132] Stanfield, R. M. and Delgass, W. N., "Mössbauer spectroscopy of supported Fe-Co alloy catalysts for Fischer-Tropsch synthesis", J. Catal. 72, 37-50 (1981).

[133] Hägg, G., "Pulverphotogramme eines neuen Eisencarbides", Zeitschrift für Krist. - Cryst. Mater. 89, 9294 (1934).

[134] Retief, J. J., "Powder diffraction data and Rietveld refinement of Hägg-carbide, $\chi$-Fe ${ }_{5} \mathrm{C}_{2}$ ", Powder Diffr. 14, 130-132 (1999).

[135] du Plessis, H. E., de Villiers, J. P. R., Kruger, G. J., Steuwer, A. and Brunelli, M., "Rietveld and pair distribution function study of Hägg carbide using synchrotron X-ray diffraction", J. Synchrotron Radiat. 18, 266-271 (2011).

[136] Leineweber, A., Shang, S., Liu, Z.-K., Widenmeyer, M. and Niewa, R., "Crystal structure determination of Hägg carbide, $\chi-\mathrm{Fe}_{5} \mathrm{C}_{2}$ by first-principles calculations and Rietveld refinement", Zeitschrift für Krist. 227, 207-220 (2012).

[137] Hofer, L. J. E. and Cohn, E. M., "Some Reactions in the Iron-Carbon System: Application to the Tempering of Martensite", Nature 167, 977-978 (1951).

[138] Fasiska, E. J. and Jeffrey, G. A., "On the cementite structure", Acta Crystallogr. 19, 463-471 (1965).

[139] Crystallography Open Database, "Open-access collection of crystal structures", (2018). Available at: http://www.crystallography.net/cod/ (Accessed: February 2018).

[140] Eckstrom, H. C. and Adcock, W. A., "A New Iron Carbide in Hydrocarbon Synthesis Catalysts", J. Am. Chem. Soc. 72, 1042-1043 (1950).

[141] Herbstein, F. H. and Snyman, J. A., "Identification of Eckstrom-Adcock Iron Carbide as $\mathrm{Fe}_{7} \mathrm{C}_{3}$ ", Inorg. Chem. 3, 894-896 (1964).

[142] Fruchart, R., Senateur, J. P., Bouchaud, J. P. and Michel, A., "A propos de la structure exacte du carbure de fer $\mathrm{Fe}_{7} \mathrm{C}_{3} "$, Bull. Soc. Chim. Fr. 2, 392 (1965).

[143] Audier, M., Bowen, P. and Jones, W., "Transmission electron microscopic study of single crystals of $\mathrm{Fe}_{7} \mathrm{C}_{3}{ }^{\prime \prime}$, J. Cryst. Growth 63, 125-134 (1983).

[144] Kattner, U. R., "What's in a Name?", J. Phase Equilibria Diffus. 34, 437-437 (2013).

[145] Pringle, O. A. and Long, G. J., "Mössbauer Effects Studies of Hard Magnetic Materials" in Mössbauer Spectroscopy Applied to Inorganic Chemistry (eds. Long, G. J. \& Grandjean, F.) 3, 644 (Springer, Boston, 1989).

[146] Dubois, J. M. and Le Caër, G., "Electron diffraction and Mossbauer studies of the $\epsilon$ phase retained in splat-quenched Fe-C and Fe-C-Si alloys", Acta Metall. 25, 609-618 (1977).

[147] Foct, J., Senateur, J. P., Dubois, J. M. and Le Caër, G., "Mössbauer Spectroscopy of Different Interstitial Compounds and Solid Solutions Containing ${ }^{57} \mathrm{Fe} "$, Le J. Phys. Colloq. 40, 647-649 (1979).

[148] Liu, X.-W., Zhao, S., Meng, Y., Peng, Q., Dearden, A. K., Huo, C.-F., Yang, Y., Li, Y.-W. and Wen, X.-D., "Mössbauer Spectroscopy of Iron Carbides: From Prediction to Experimental Confirmation", Sci. Rep. 6, 26184 (2016).

[149] Xu, K., Sun, B., Lin, J., Wen, W., Pei, Y., Yan, S., Qiao, M., Zhang, X. and Zong, B., "E-Iron carbide as a low- 
temperature Fischer-Tropsch synthesis catalyst", Nat. Commun. 5, 5783 (2014).

[150] Raupp, G. B. and Delgass, W. N., "Mössbauer Investigation of Supported Fe and FeNi Catalysts II. Carbides Formed by Fischer-Tropsch Synthesis", J. Catal. 58, 348-360 (1979).

[151] Barinov, V. A., Protasov, A. V. and Surikov, V. T., "Studying mechanosynthesized Hägg carbide ( $\chi$ $\mathrm{Fe}_{5} \mathrm{C}_{2}$ )", Phys. Met. Metallogr. 116, 791-801 (2015).

[152] Bi, X.-X., Ganguly, B., Huffman, G. P., Huggins, F. E., Endo, M. and Eklund, P. C., "Nanocrystalline $\alpha-F e$, $\mathrm{Fe}_{3} \mathrm{C}$, and $\mathrm{Fe}_{7} \mathrm{C}_{3}$ produced by $\mathrm{CO}_{2}$ laser pyrolysis", J. Mater. Res. 8, 1666-1674 (1993).

[153] Kniep, B., Constantinescu, A., Niemeier, D. and Becker, K. D., "An In-situ Mössbauer Study of the Formation of Cementite, $\mathrm{Fe}_{3} \mathrm{C}^{\prime \prime}$, Zeitschrift für Anorg. und Allg. Chemie 629, 1795-1804 (2003).

[154] Ron, M., Shechter, H., Hirsch, A. A. and Niedzwiedz, S., "On the Mössbauer study of cementite", Phys. Lett. 20, 481-483 (1966).

[155] Yamada, Y., Yoshida, H., Kouno, K. and Kobayashi, Y., "Iron carbide films produced by laser deposition", J. Phys. Conf. Ser. 217, 012096 (2010).

[156] Nagakura, S., Hirotsu, Y., Kusunoki, M., Suzuki, T. and Nakamura, Y., "Crystallographic study of the tempering of martensitic carbon steel by electron microscopy and diffraction", Metall. Trans. A 14, 1025-1031 (1983).

[157] Amelse, J. A., Grynkewich, G., Butt, J. B. and Schwartz, L. H., "Moessbauer spectroscopic study of passivated small particles of iron and iron carbide", J. Phys. Chem. 85, 2484-2488 (1981).

[158] Wang, P., Chen, W., Chiang, F., Dugulan, A. I., Song, Y., Pestman, R., Zhang, K., Yao, J., Feng, B., Miao, P., $\mathrm{Xu}, \mathrm{W}$. and Hensen, E. J. M., "Synthesis of stable and low- $\mathrm{CO}_{2}$ selective $\varepsilon$-iron carbide Fischer-Tropsch catalysts", Sci. Adv. 4, eaau2947 (2018).

[159] Jung, H. and Thomson, W. J., "Dynamic X-ray diffraction study of an unsupported iron catalyst in Fischer-Tropsch synthesis", J. Catal. 134, 654-667 (1992).

[160] Cohn, E. M. and Hofer, L. J. E., "Mode of Transition from Hägg Iron Carbide to Cementite", J. Am. Chem. Soc. 72, 4662-4664 (1950).

[161] Shultz, J. F., Hall, W. K., Dubs, T. A. and Anderson, R. B., "Studies of the Fischer-Tropsch Synthesis. XV. Cementite as Catalysts", J. Am. Chem. Soc. 78, 282-285 (1956).

[162] An, X., Wu, B., Wan, H.-J., Li, T.-Z., Tao, Z.-C., Xiang, H.-W. and Li, Y.-W., "Comparative study of ironbased Fischer-Tropsch synthesis catalyst promoted with potassium or sodium", Catal. Commun. 8, 1957-1962 (2007).

[163] Janbroers, S., Louwen, J. N., Zandbergen, H. W. and Kooyman, P. J., "Insights into the nature of ironbased Fischer-Tropsch catalysts from quasi in situ TEM-EELS and XRD", J. Catal. 268, 235-242 (2009).

[164] Berry, F. J. and Smith, M. R., "A Mössbauer investigation of iron-containing catalysts prepared at low temperatures and active for carbon monoxide hydrogenation", J. Chem. Soc. Faraday Trans. 1 Phys. Chem. Condens. Phases 85, 467-477 (1989).

[165] Ribeiro, M. C., Jacobs, G., Davis, B. H., Cronauer, D. C., Kropf, A. J. and Marshall, C. L., "Fischer-Tropsch Synthesis: An In-Situ TPR-EXAFS/XANES Investigation of the Influence of Group I Alkali Promoters on the Local Atomic and Electronic Structure of Carburized Iron/Silica Catalysts", J. Phys. Chem. C 114, 7895-7903 (2010).

[166] Tsuzuki, A., Sago, S., Hirano, S.-I. and Naka, S., "High temperature and pressure preparation and properties of iron carbides $\mathrm{Fe}_{7} \mathrm{C}_{3}$ and $\mathrm{Fe}_{3} \mathrm{C}^{\prime \prime}$, J. Mater. Sci. 19, 2513-2518 (1984).

[167] Sethuraman, A. R., "In-situ high temparature X-ray diffraction studies of nanocrystalline iron carbides", Nanostructured Mater. 4, 79-92 (1994).

[168] Tajima, S. and Hirano, S., "Synthesis and Magnetic Properties of $\mathrm{Fe}_{7} \mathrm{C}_{3}$ Particles with High Saturation 
Magnetization", Jpn. J. Appl. Phys. 29, 662-668 (1990).

[169] Hirano, S.-I. and Tajima, S., "Synthesis and magnetic properties of $\mathrm{Fe}_{5} \mathrm{C}_{2}$ by reaction of iron oxide and carbon monoxide", J. Mater. Sci. 25, 4457-4461 (1990).

[170] Barinov, V. A., Tsurin, V. A. and Surikov, V. T., "Study of mechanically synthesized carbide $\mathrm{Fe}_{7} \mathrm{C}_{3}$ ", $\mathrm{Phys}_{\mathrm{s}}$ Met. Metallogr. 110, 474-484 (2010). 


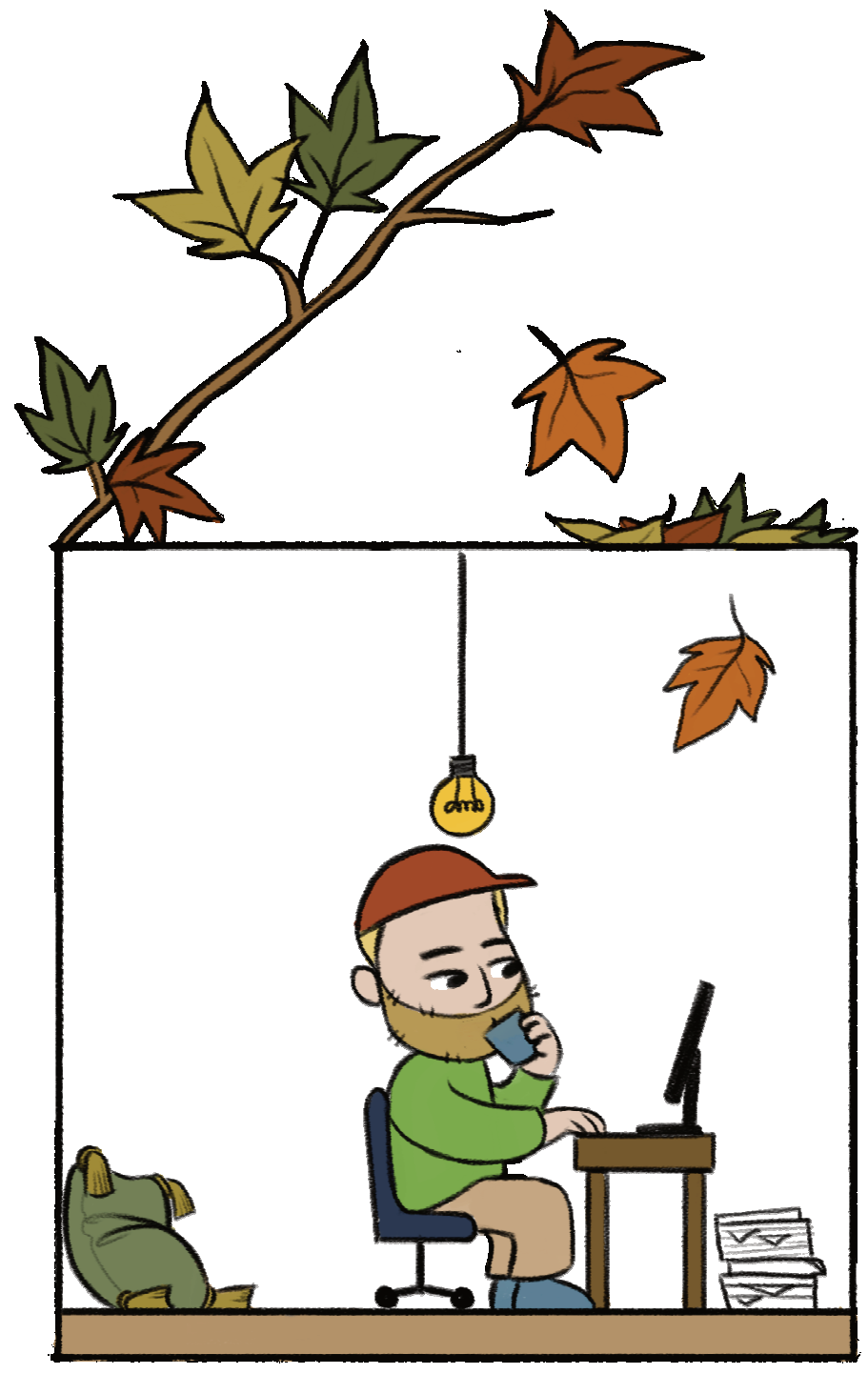




\section{Chapter 3}

\section{Identification of Iron Carbides in $\mathrm{Fe}$ (-Na- S) $/ \alpha-\mathrm{Al}_{2} \mathrm{O}_{3}$ Fischer-Tropsch Synthesis Catalysts with X-ray Powder Diffractometry and Mössbauer Absorption Spectroscopy}

In Fe-based Fischer-Tropsch Synthesis (FTS), the Fe carbides form under the carburizing $\mathrm{H}_{2}$ :CO reaction atmosphere providing the active phases for hydrocarbon synthesis. Identification of the Fe carbides can be challenging, as studies with the commonly applied techniques of X-ray Powder Diffractometry (XRPD) and Mössbauer Absorption Spectroscopy (MAS) apply inconsistent nomenclature for the $\mathrm{Fe}$ carbides in between the techniques. $\mathrm{H}_{2}$ reduced $\mathrm{Fe}(-\mathrm{Na}-\mathrm{S}) / \alpha-\mathrm{Al}_{2} \mathrm{O}_{3}$ catalyst materials, with and without Na-S promotion, have been carburized under $\mathrm{CO}$ at 240$440^{\circ} \mathrm{C}$ to form Fe carbides observed in the FTS reaction. Rietveld Quantitative Phase Analysis (R-QPA) was used to quantify Fe phases from the measured XRPD patterns and the results were compared with MAS. Carbon deposition and morphological changes on the catalysts were also followed. The Fe carbides formed in order of increasing temperature are $\varepsilon-\mathrm{Fe}_{3} \mathrm{C}, \eta-\mathrm{Fe}_{2} \mathrm{C}, \chi-\mathrm{Fe}_{5} \mathrm{C}_{2}$ and $\theta-\mathrm{Fe}_{3} \mathrm{C}$. Furthermore, $\theta-\mathrm{Fe}_{7} \mathrm{C}_{3}$ and a distorted $\chi-\mathrm{Fe}_{5} \mathrm{C}_{2}$ phase formed at 25 bar $\mathrm{CO}\left(340^{\circ} \mathrm{C}\right)$ from a Fe oxide precursor. Crystal structures, lattice parameters, hyperfine fields and relative $\mathrm{C}$ contents were assigned for the carbides. Fe carbide formation was unaffected by Na-S addition, but it had temperature dependent effects of increased Fe oxidation $\left(\leq 290^{\circ} \mathrm{C}\right)$, carbon deposition $\left(340-390^{\circ} \mathrm{C}\right)$ and decreased $\theta-\mathrm{Fe}_{3} \mathrm{C}$ crystallite diameters $\left(440^{\circ} \mathrm{C}\right)$. The results unify the $\mathrm{Fe}$ carbide nomenclature and specify the role of $\mathrm{Na}-\mathrm{S}$ in the carburization process.

This PhD chapter is based on: Pasi P. Paalanen, Sophie H. van Vreeswijk, A. Iulian Dugulan and Bert M. Weckhuysen, "Identification of Iron Carbides in $\mathrm{Fe}(-\mathrm{Na}-\mathrm{S}) / \alpha-\mathrm{Al}_{2} \mathrm{O}_{3}$ Fischer-Tropsch Synthesis Catalysts with X-ray Powder Diffractometry and Mössbauer Absorption Spectroscopy", ChemCatChem, submitted. 


\subsection{INTRODUCTION}

In Fe-based Fischer-Tropsch Synthesis (FTS) reaction, Fe phases present under the carburizing $\mathrm{H}_{2}$ : $\mathrm{CO}$ atmosphere are Fe carbides that form via dissolution of $\mathrm{C}$ atoms to zerovalent $\alpha$-Fe lattice. The Fe carbides form the reactive part for the Fe-based FTS reaction dissociating $\mathrm{CO}$ and $\mathrm{H}_{2}$. The $\mathrm{C}$ atoms may then further react to either form hydrocarbons with $\mathrm{H}$ atoms or react with other $\mathrm{C}$ atoms to form inactive carbon deposits on the catalyst material.

In the FTS reaction literature, the Fe carbide phases are commonly identified as (pseudo-)hexagonal or as the " $\varepsilon$-carbides" $\left(\varepsilon^{\prime}-\mathrm{Fe}_{2.2} \mathrm{C}, \mathrm{Fe}_{2} \mathrm{C}, \varepsilon-\mathrm{Fe}_{2} \mathrm{C}\right.$ or $\left.\varepsilon-\mathrm{Fe}_{3} \mathrm{C}\right),{ }^{1-9} \chi-\mathrm{Fe}_{5} \mathrm{C}_{2}$ (the Hägg carbide), ${ }^{1-3,10-13} \theta-\mathrm{Fe}_{3} \mathrm{C}$ (cementite), ${ }^{1-3,14,15}$ and $\mathrm{Fe}_{7} \mathrm{C}_{3}$ (the Eckström-Adcock carbide). ${ }^{1,3,16-18}$ While " $\varepsilon$-carbides", $\chi$ - $\mathrm{Fe}_{5} \mathrm{C}_{2}$ and $\theta-\mathrm{Fe}_{3} \mathrm{C}$ are often observed, the $\mathrm{Fe}_{7} \mathrm{C}_{3}$ phase is less common in FTS reaction literature and seems to form under more severe, industrial FTS reaction conditions, than the rest of the carbide phases. ${ }^{16}$ From tempering studies on martensitic steel, a $\mathrm{Fe}$ carbide labelled as $\eta-\mathrm{Fe}_{2} \mathrm{C}$ has been observed. ${ }^{19}$ The $\eta-\mathrm{Fe}_{2} \mathrm{C}$ phase has been suggested to be closely related to the " $\varepsilon$ carbides" encountered in the FTS reaction research field. 2,9,20

The most common and suitable characterization techniques applied for identifying the Fe carbide phases present in Fe-based FTS reaction catalyst materials are X-ray Powder Diffractometry (XRPD) and Mössbauer Absorption Spectroscopy (MAS). From the obtained XRPD patterns, the $\chi-\mathrm{Fe}_{5} \mathrm{C}_{2}, \theta-\mathrm{Fe}_{3} \mathrm{C}$ and $\mathrm{Fe}_{7} \mathrm{C}_{3}$ phases can readily be identified,1,3,17,18,21-24 while differentiation between the " $\varepsilon$-carbides" is more challenging with XRPD. In FTS literature, when using XRPD as the characterization method, the precise crystal structures for Fe carbides identified as the " $\varepsilon$-carbides" are often left ambiguous. ${ }^{1,3,6,21,25-29}$ With MAS, on the basis of the different hyperfine fields, the Fe carbides can be fingerprinted also including the Fe carbide phases which are referred to as the " $\varepsilon$-carbides". $2,5,12,13,30-34$ Unfortunately, the connection between the experimentally observed Fe carbides and their precise crystal structures from XRPD, in comparison to the assigned hyperfine fields from MAS, is often unclear. This being most evident in the case of the " $\varepsilon$-carbides". Which " $\varepsilon$-carbides" truly exist and with what crystal structures, can be a challenging task to interpret from the literature due to the lack of unified nomenclature between the XRPD and MAS studies.

In the literature review Chapter 2, we have argued on the basis of our interpretation of the literature, that the Fe carbides with their crystal lattices to form in within FTS reaction research field are: $\varepsilon-\mathrm{Fe}_{3} \mathrm{C} P 6_{3} 22$ (182), $\eta-\mathrm{Fe}_{2} \mathrm{C}$ Pnnm (58), $\chi-\mathrm{Fe}_{5} \mathrm{C}_{2} C 2 / c$ (15), $\theta$ $\mathrm{Fe}_{3} \mathrm{C}$ Pnma (62) and $\theta-\mathrm{Fe}_{7} \mathrm{C}_{3} P n m a$ (62). Of these carbides, we have discussed that the $\varepsilon-\mathrm{Fe}_{3} \mathrm{C}$ and $\eta-\mathrm{Fe}_{2} \mathrm{C}$ phases are the $\mathrm{Fe}$ carbides cited in the literature as the " $\varepsilon$-carbides" or (pseudo-)hexagonal Fe carbides. For each of the identified Fe carbide phases, we also 


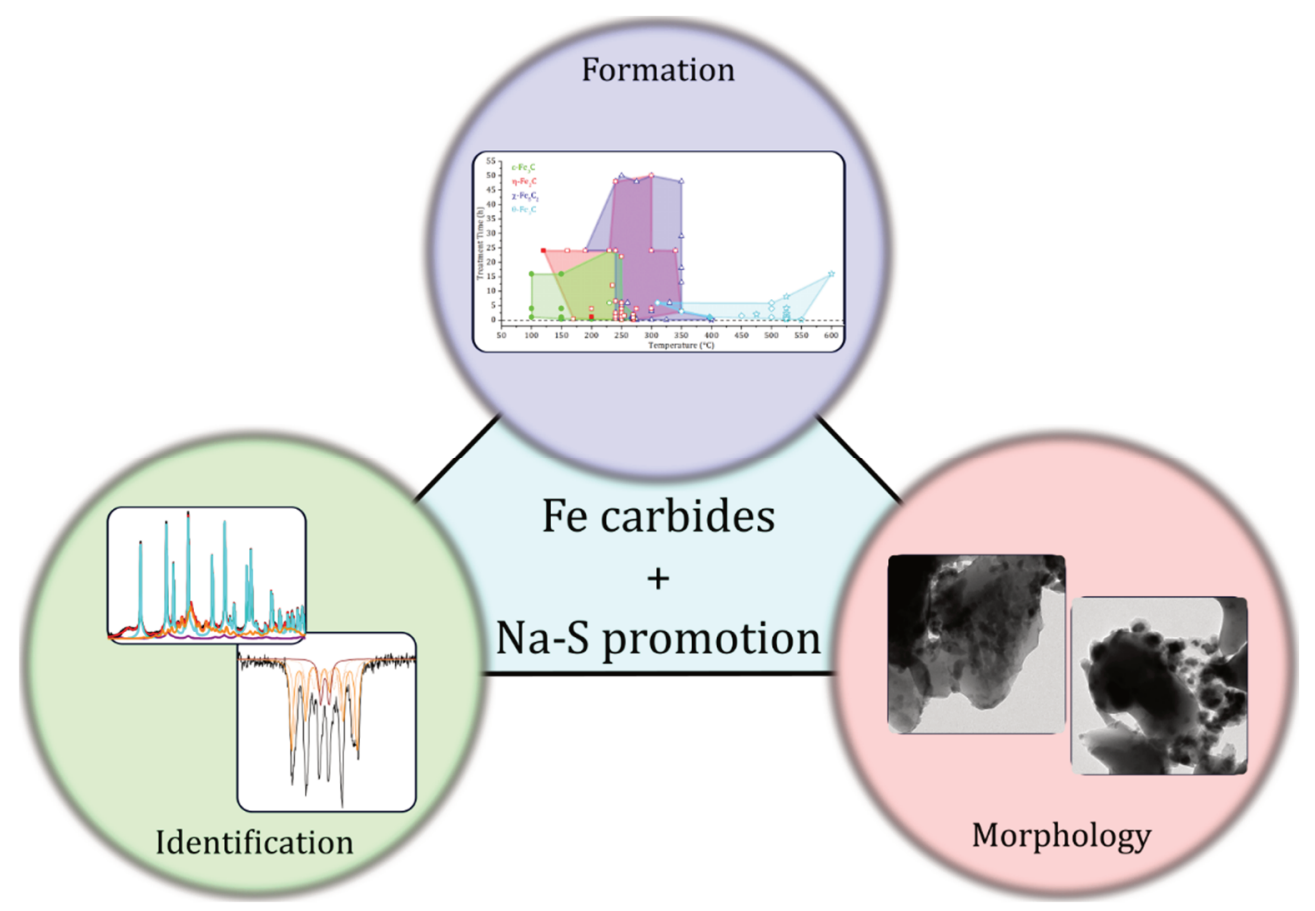

Figure 3.1

A schematic of the approach taken in this Chapter. Unpromoted and Na-S promoted $\mathrm{Fe}(-\mathrm{Na}-\mathrm{S}) / \alpha-\mathrm{Al}_{2} \mathrm{O}_{3}$ catalyst materials are used to follow the formation of various Fe carbides after $\mathrm{CO}$ carburization treatments between $240-440^{\circ} \mathrm{C}$. The formed Fe carbides are identified with both X-ray Powder Diffractometry (XRPD) and Mössbauer Absorption Spectroscopy (MAS) techniques, and the corresponding changes in catalyst materials morphology is followed with Transmission Electron Microscopy (TEM). Carbon deposition associated with changes in catalyst materials morphology is quantified with the use of Thermogravimetric Analysis combined with Mass Spectrometry (TGA-MS).

assigned hyperfine fields for MAS fingerprinting based on the established Fe carbide nomenclature.

The addition of Na-S as chemical promoters on Fe-based FTS reaction catalyst materials increases the $\mathrm{CO}$ conversion, $\alpha$-value, hydrocarbon products' olefin content and suppresses $\mathrm{CH}_{4}$ selectivity below expectations from the Anderson-Schulz-Flory (ASF) Distribution. ${ }^{35-38}$ The addition of the alkali (as Na or K) individually has been observed to stabilize the " $\varepsilon$-carbides",25,26,39,40 and to decrease the Fe catalyst reoxidation during the FTS reaction. ${ }^{39,40}$

However, how the combined Na-S promotion affects the formation of the aforementioned Fe carbides is not known. Also unknown is whether the combined Na$\mathrm{S}$ promotion alters the properties of the individual Fe carbides, providing possible explanations for the observed FTS reaction hydrocarbon selectivity promotion effects. 
Within this Chapter, we combine both: ex-situ XRPD with Rietveld Quantitative Phase Analysis (R-QPA) and ex-situ MAS. The Fe carbide formation and properties are studied via $\mathrm{CO}$ carburization of supported $\mathrm{Fe}(-\mathrm{Na}-\mathrm{S}) / \alpha-\mathrm{Al}_{2} \mathrm{O}_{3}$ FTS reaction catalyst materials, with and without Na-S promotion. This scientific approach is summarized in Figure 3.1. In more detail, the primary objectives of this study are i) to experimentally verify the interpretation of the literature, as proposed in Chapter 2, concerning the Fe carbide phases and their formation temperatures. In addition, ii) also to establish an experimental, precise connection between the hyperfine fields and the Fe carbide crystal structures. Furthermore, we aim iii) to follow the effect of Na-S promotion on the formation of the Fe carbides via $\mathrm{CO}$ carburization in terms of phase quantities, crystallite diameters and possible effects on the crystal lattice parameters. Additionally, the effect of $\mathrm{Na}-\mathrm{S}$ promotion on morphological changes and carbon deposition on the $\mathrm{CO}$ carburized $\mathrm{Fe}(-\mathrm{Na}-\mathrm{S}) / \alpha-\mathrm{Al}_{2} \mathrm{O}_{3}$ catalyst materials is studied. Both, Transmission Electron Microscopy (TEM) and Thermogravimetric Analysis with combined Mass Spectrometry (TGA-MS) are employed for the purpose.

\subsection{EXPERIMENTAL METHODS}

\subsubsection{Catalyst Preparation}

\subsubsection{Catalyst Synthesis}

Supported catalyst materials with a nominal 7 weight percent (wt-\%) Fe loading, with and without $\mathrm{Na}-\mathrm{S}$ promotion, were prepared via Incipient Wetness Impregnation (IWI) of an alpha-alumina $\left(\alpha-\mathrm{Al}_{2} \mathrm{O}_{3}\right)$ support (BASF, $7 \mathrm{~m}^{2} / \mathrm{g}, 212-425 \mu \mathrm{m}$ particle size). Ammonium Fe(III) citrate (Acros, 211840010) was used as the Fe precursor, Fe(II) sulfate heptahydrate (Sigma-Aldrich, 215422) as the S precursor and Na tribasic citrate dihydrate (Sigma-Aldrich, S4641) as the Na precursor. The elemental analysis of the ammonium Fe(III) citrate Fe precursor was performed using Inductively Coupled Plasma Optical Emission Spectrophotometry (ICP-OES) (Table 3.1).

The precursor solution for preparing the Na-S promoted catalyst material was readied into a glass vial by individually solvating $0.011 \mathrm{~g}$ of the Fe sulfate and $0.040 \mathrm{~g}$ of the Na citrate precursors into $0.5 \mathrm{ml}$ of demineralized water. After this, $1.281 \mathrm{~g}$ of the Fe citrate precursor was added to the glass vial. The closed glass vial was gently heated to $40-50^{\circ} \mathrm{C}$ for solvating all of the Fe citrate precursor and refluxing the glass vial walls clean of the added Fe citrate. The nominal promoter loadings are $0.29 \mathrm{wt}-\%$ for Na and $0.04 \mathrm{wt}-\%$ for $\mathrm{S}$ in the Na-S promoted catalyst material. The unpromoted catalyst material was prepared in the same manner as the promoted one, while using $1.290 \mathrm{~g}$ of the Fe citrate precursor but without adding the Fe sulfate or the Na citrate precursors. 
Identification of Iron Carbides in Fe(-Na-S)/ $\alpha$-Al203 Fischer-Tropsch Synthesis Catalysts with X-ray Powder Diffractometry and Mössbauer Absorption Spectroscopy

\section{Table 3.1}

Inductively Coupled Plasma Optical Emission Spectrophotometry (ICP-OES) results of elemental analysis for ammonium $\mathrm{Fe}(\mathrm{III})$ citrate precursor $\mathrm{xFe}\left(\mathrm{C}_{6} \mathrm{H}_{8} \mathrm{O}_{7}\right) \cdot \mathrm{yNH}_{3}$ (Acros, 211840010) and the calcined unpromoted (UP) and Na-S promoted (Na-S) catalyst materials. The measurements for the same citrate precursor were done performed both at Mikrolab Kolbe and Universiteit Utrecht (UU) GeoLab.

\begin{tabular}{cccccccc}
\hline & \multicolumn{7}{c}{ Elemental Analysis (weight-ppm) } \\
\cline { 2 - 7 } & $\mathrm{Co}$ & $\mathrm{Cr}$ & $\mathrm{Mn}$ & $\mathrm{Fe}$ & $\mathrm{K}$ & $\mathrm{Na}$ & $\mathrm{S}$ \\
\hline $\mathrm{xFe}\left(\mathrm{C}_{6} \mathrm{H}_{8} \mathrm{O}_{7}\right) \cdot \mathrm{yNH}_{3}$ & 16 & 28 & 44 & 153600 & $<\mathrm{dl}$ & $<\mathrm{dl}$ & $<\mathrm{dl}$ \\
$($ citrate precursor) & - & - & - & 162500 & 57 & 121 & 9 \\
$\mathrm{Fe} / \alpha-\mathrm{Al}_{2} \mathrm{O}_{3}(\mathrm{UP})$ & - & - & - & 65787 & - & $<132$ & $<91$ \\
$\mathrm{Fe}-\mathrm{Na}-\mathrm{S} / \alpha-\mathrm{Al}_{2} \mathrm{O}_{3}(\mathrm{Na}-\mathrm{S})$ & - & - & - & 66315 & - & 2883 & $400^{\mathrm{a})}$ \\
\hline
\end{tabular}

a) - Analysis error can be $>10 \%$.

$<\mathrm{dl}$ - Below detection limit.

(-) - Not determined.

$3.00 \mathrm{~g}$ of the $\alpha-\mathrm{Al}_{2} \mathrm{O}_{3}$ support was placed into a round-bottom boiling flask along with a magnetic stirring rod. The impregnation of the $\alpha-\mathrm{Al}_{2} \mathrm{O}_{3}$ support was performed with two sequential drying steps. First, $0.5 \mathrm{ml}$ and $0.4 \mathrm{ml}$ of the prepared precursor solution was dropwise added on the $\alpha-\mathrm{Al}_{2} \mathrm{O}_{3}$ support with constant stirring using the magnetic rod. $0.2 \mathrm{ml}$ of demineralized water was added to the precursor solution for washing the glass vial walls and diluting the precursor solution in order to avoid precursor recrystallization. $\approx 0.05 \mathrm{ml}$ of the diluted precursor solution was further dropwise added on the $\alpha-\mathrm{Al}_{2} \mathrm{O}_{3}$ support. Some dry and sticky agglomerates were formed from the impregnated support material, which were gently broken apart with a glass rod. The impregnated catalyst material was then set to oven to dry for $2 \mathrm{~h}$ at $120^{\circ} \mathrm{C}$ under static air. After drying, the singly impregnated catalyst material was further impregnated with $0.15 \mathrm{ml}$ of the precursor solution. $0.1 \mathrm{ml}$ of demineralized water was added to the precursor solution glass vial for washing the vial walls. The remaining precursor solution was then added on the $\alpha-\mathrm{Al}_{2} \mathrm{O}_{3}$ support and the impregnated catalyst material was set to oven to dry overnight at $120^{\circ} \mathrm{C}$ under static air.

The impregnation procedure was separately repeated three times for both, the unpromoted and Na-S promoted catalyst materials. After the impregnations, the three batches of catalyst material were mixed together after their respective final drying step.

\subsubsection{Catalyst Calcining}

Catalyst material calcining was done under flowing air in an open quartz vessel by heating $\left(10^{\circ} \mathrm{C} / \mathrm{min}\right)$ the catalyst from room temperature to $500^{\circ} \mathrm{C}$, and holding the temperature for $6 \mathrm{~h}$. In order to avoid gas diffusion limitations, the catalyst materials were calcined in batches of $\approx 2.8 \mathrm{~g}$. Each batch lost $\approx 21 \%$ of its weight during the calcining step. The calcined catalyst materials were combined for each of the 
unpromoted and $\mathrm{Na}-\mathrm{S}$ promoted catalyst batches and re-sieved to the catalyst particle fraction of $212-425 \mu \mathrm{m}$ in order to obtain the ready, calcined catalyst materials. The results for the elemental contents of $\mathrm{Fe}, \mathrm{Na}$ and $\mathrm{S}$ using ICP-OES analysis on the two sets of the calcined catalyst materials are given in Table 3.1.

\subsubsection{Catalyst Reduction and Carburization}

\subsubsection{Catalyst Reduction}

The calcined catalyst materials were reduced into metallic, zerovalent $\alpha$-Fe on the $\alpha$ $\mathrm{Al}_{2} \mathrm{O}_{3}$ support prior to carburization with $\mathrm{CO}$. For each reduction or carburization treatment, a $\approx 600 \mathrm{mg}$ of calcined, unpromoted or Na-S promoted catalyst material was loaded into a plug flow quartz reactor. The reactor was heated up $\left(10^{\circ} \mathrm{C} / \mathrm{min}\right)$ from room temperature to $400^{\circ} \mathrm{C}$ under $\mathrm{N}_{2}: \mathrm{H}_{2}$ flow $(20: 12 \mathrm{ml} / \mathrm{min}$, at atmospheric pressure) and held at $400^{\circ} \mathrm{C}$ for $5 \mathrm{~h}$. After the $5 \mathrm{~h}$ reduction step, the reactor was cooled down to $200^{\circ} \mathrm{C}$ under the set $\mathrm{N}_{2}: \mathrm{H}_{2}$ flow and let to stabilize for $15 \mathrm{~min}$. For the catalysts only reduced with $\mathrm{H}_{2}$, without any subsequent $\mathrm{CO}$ carburization step, the reactor was cooled down to room temperature under the $\mathrm{N}_{2}: \mathrm{H}_{2}$ flow and flushed with $\mathrm{N}_{2}$ for several hours.

\subsubsection{Catalyst Carburization}

The $\mathrm{H}_{2}$ reduced catalyst materials, either with or without Na-S promotion, were subsequently carburized with $\mathrm{CO}$ without removing the catalyst from the reactor after the $\mathrm{H}_{2}$ reduction step. Under the set $\mathrm{N}_{2}: \mathrm{H}_{2}$ flow, the reduced catalyst was heated up $\left(10^{\circ} \mathrm{C} / \mathrm{min}\right)$ from $200^{\circ} \mathrm{C}$ to a desired carburization temperature. Upon reaching the desired carburization temperature, the $\mathrm{N}_{2}: \mathrm{H}_{2}$ flow was switched to pure CO flow $(20 \mathrm{ml} / \mathrm{min}$, at atmospheric pressure). The carburization temperatures and times used were: $240^{\circ} \mathrm{C}(2 \mathrm{~h}), 290^{\circ} \mathrm{C}(3 \mathrm{~h}), 340^{\circ} \mathrm{C}(16 \mathrm{~h}), 390^{\circ} \mathrm{C}(16 \mathrm{~h})$ and $440^{\circ} \mathrm{C}(16 \mathrm{~h})$. After the desired carburization period, the $\mathrm{CO}$ flow was switched to pure $\mathrm{N}_{2}$ and the reactor was cooled down to room temperature and flushed with $\mathrm{N}_{2}$ for several hours.

\subsubsection{Catalyst Carburization at 25 bar}

Calcined, unpromoted and Na-S promoted catalyst materials were carburized directly without the $\mathrm{H}_{2}$ reduction step at an elevated pressure. $\approx 600 \mathrm{mg}$ of calcined, unpromoted or Na-S promoted, catalyst material was loaded into a plug flow quartz reactor. Under a flow of $\mathrm{He}(5 \mathrm{ml} / \mathrm{min})$, the reactor was heated to $\left(10^{\circ} \mathrm{C} / \mathrm{min}\right) 120^{\circ} \mathrm{C}$ from the room temperature. The reactor was pressurized at $120^{\circ} \mathrm{C}$ to 25 bar total pressure with CO:He flow $(40: 5 \mathrm{ml} / \mathrm{min})$ over a period of $2 \mathrm{~h}$. The reactor was then heated up $\left(10^{\circ} \mathrm{C} / \mathrm{min}\right)$ to $340^{\circ} \mathrm{C}$. The unpromoted catalyst material was carburized for $120 \mathrm{~h}$ and the Na-S promoted catalyst for $168 \mathrm{~h}$ at $340^{\circ} \mathrm{C}$ at the 25 bar total CO:He pressure. After the carburization period, the reactor was first cooled down to room temperature after 
Identification of Iron Carbides in Fe(-Na-S)/ $\alpha$-Al203 Fischer-Tropsch Synthesis Catalysts with X-ray Powder Diffractometry and Mössbauer Absorption Spectroscopy

which the gas flow was switched to $\operatorname{Ar}(12 \mathrm{ml} / \mathrm{min})$ and the pressure reduced from 25 bar to atmospheric pressure.

All of the gases used in this study $\left(\mathrm{Ar}, \mathrm{He}, \mathrm{N}_{2}, \mathrm{H}_{2}\right)$ were grade 5.0 purity $(99.999 \%$ pure), with the exception of the CO gas which had the purity grade of 2.5 (99.5\% pure). All of the used gases were supplied by Linde Gas Benelux.

\subsubsection{Catalyst Characterization}

\subsubsection{Application of Transmission Electron Microscopy}

The catalyst materials were prepared on carbon polymer layered $\mathrm{Cu}$ grids for performing bright field TEM imaging on the catalysts. The catalyst materials were ground with a mortar and mixed to ethanol. Droplets from the prepared catalyst/ethanol mixture were dropped on the $\mathrm{Cu}$ grids using a syringe piston and the $\mathrm{Cu}$ grids were let to dry under room temperature. The TEM imaging measurements were performed using Philips Tecnai 12 (120 kV) Field Emission Gun (FEG). From the obtained TEM images, the Fe particle diameters were manually counted by their longest diameter. The obtained Fe particle counts $(\mathrm{N} \gtrsim 500)$ were binned to $4 \mathrm{~nm}$ count frequencies and fitted with a lognormal distribution function. The Fe particle (numberweighted) mean diameter and Fe particle size distribution Standard Deviations (STDs) were derived from the fitted lognormal distribution.

\subsubsection{Application of Thermogravimetric Analysis - Mass Spectrometry}

The amount of carbon deposited on the carburized catalyst materials was determined with Thermogravimetric Analysis with online analysis of the exhaust gases using Mass Spectrometry (TGA-MS). Perkin Elmer 1 TGA and Pfeiffer Vacuum OmniStar MS instruments were employed in the TGA-MS measurements. The catalyst materials were first flushed with $\mathrm{N}_{2}(20 \mathrm{ml} / \mathrm{min})$ and then heated up and held at $50^{\circ} \mathrm{C}$ for $10 \mathrm{~min}$ under $\mathrm{O}_{2}(10 \mathrm{ml} / \mathrm{min})$. The carbon burn-off was performed by heating the catalysts from $50^{\circ} \mathrm{C}$ to $800^{\circ} \mathrm{C}$ with $10^{\circ} \mathrm{C} / \mathrm{min}$ ramp under the $\mathrm{O}_{2}$ flow. Carbon-free, $\mathrm{H}_{2}$ reduced only, parent unpromoted and Na-S promoted catalyst materials were also analyzed in order to account for the catalysts' weight gain from the Fe reoxidation during the TGA measurements. Also, any possible Fe weight loading differences between the parent unpromoted and Na-S promoted catalyst materials could be accounted for this way. The carbon wt- $\%$ on the carburized catalyst materials was determined as the weight change difference between the parent, reduced only and the corresponding carburized catalyst material at the end of the TGA-MS program. The carbon wt-\%s were normalized to the total CO exposure time, in order to obtain the mean carbon deposition rate during the $\mathrm{CO}$ carburization treatments. Total $\mathrm{CO}_{2}$ peak areas $(\mathrm{m} / \mathrm{z} 44)$ recorded with the MS were determined by integration and normalized to the initial catalyst material amounts 
loaded for the TGA-MS measurements.

\subsubsection{Application of Mössbauer Absorption Spectroscopy}

Ex-situ transmission ${ }^{57} \mathrm{Fe}$ MAS was performed at room temperature (300 K) with a conventional constant-acceleration spectrometer using a ${ }^{57} \mathrm{Co}(\mathrm{Rh})$ source. Velocity calibration was carried out using an $\alpha$-Fe foil. The MAS spectra were fitted using the Mosswinn 4.0 software. ${ }^{41}$ Non-equivalent Fe position ratios were determined from the fitted relation of spectral contributions for each of the Fe carbide phases' hyperfine fields.

\subsubsection{Application of X-ray Powder Diffractometry}

The XRPD patterns for the prepared catalyst materials were measured ex-situ using a Bruker D8 Discover instrument in Debye-Scherrer transmission (capillary) geometry with a Mo ( $\mathrm{K}_{\alpha 1} 0.709 \AA$ ) source. The use of high energy Mo radiation in capillary geometry is especially suitable for phase quantification purposes. ${ }^{42} \mathrm{~A}$ Göbel-mirror was used to focus a near-parallel $\approx 600 \times 15000 \mu \mathrm{m}$ (height $\mathrm{x}$ width) X-ray beam on a static i.e. non-rotating $1000 \mu \mathrm{m}$ (outer diameter, wall thickness $10 \mu \mathrm{m}$ ) borosilicate capillary loaded with the catalyst material. An energy dispersive LynxEye XE Position Sensitive Detector (PSD) was used, only accepting the diffracted X-ray photons originating from the Mo Ka emission lines. $2.5^{\circ}$ Soller slits were used on source and detector sides.

$2 \theta$ range of $6-39^{\circ}$ with step size $0.015^{\circ}$ and exposure time of $30 \mathrm{~s}$ per step, were used for each sample measurement. Prior to each measurement, the sample capillary was manually rotated and shortly test measured in order to verify the powder diffraction conditions. No changes in diffraction intensities were observed following the capillary rotation, thus, all of the measured samples contained a sufficient amount of diffracting Fe crystallites for proper powder diffraction measurements. Throughout this Dissertation, the term X-ray Powder Diffractometry (XRPD) is used to refer to X-ray diffraction phenomena based characterization experiments that are done under powder diffraction conditions. ${ }^{43}$

For each measurement, the sample capillary was focused to the incident X-ray beam for maximum diffraction intensity. Air scattering contribution to the low angle background in the diffraction pattern was reduced by installing a stainless steel plate on top of the sample capillary during the measurements.

\subsubsection{Application of Rietveld Quantitative Phase Analysis}

Bruker TOPAS v5.0 software was used to apply the R-QPA method on the measured XRPD diffractograms. ${ }^{44,45}$ The major advantage of the Rietveld method is that it can be used for diffraction patterns with overlapping peaks. ${ }^{44-46}$

In the TOPAS software, a diffraction pattern convolution based approach is 
Identification of Iron Carbides in Fe(-Na-S)/ $\alpha$-Al203 Fischer-Tropsch Synthesis Catalysts with X-ray Powder Diffractometry and Mössbauer Absorption Spectroscopy

applied, ${ }^{47}$ which can be presented as:

$$
\mathrm{F}(\theta)=\mathrm{I}(\theta) \times \mathrm{S}(\theta) \quad \text { XRPD Pattern Convolution }
$$

In the Equation 3.1, $F(\theta)$ is the observed diffraction pattern with diffraction angle dependency $\theta, I(\theta)$ is the instrument and $S(\theta)$ is the sample contribution to the observed diffraction pattern. The instrument term $I(\theta)$ is a convolution of the instrument emission profile $(W)$ and the geometrical and instrumental aberrations $(G(\theta)){ }^{47}$

The goal in the application of the Rietveld method is to solve a theoretical term $I(\theta) \times S(\theta)$, with the use of (non-linear) least squares fitting, which reproduces the observed term $F(\theta)$ as closely as possible. For separating the contributions originating from $I(\theta)$ and $S(\theta)$, the instrumental contribution from $I(\theta)$ needs to be solved separately. ${ }^{48,49} I(\theta)$ can be solved by using known geometric relations and a reference material with a known defect-free crystal structure, for which the peak broadening from the term $S(\theta)$ is negligible. The separately solved $I(\theta)$ is then kept constant while refining parameters for the diffraction contribution from the $S(\theta)$ in a measured diffraction pattern. From the best fitting calculated sample contribution $S(\theta)$, quantifiable properties of the measured sample, such as phase quantification when using predetermined crystalline structures (i.e. R-QPA), ${ }^{45}$ and the phase crystallite sizestrain values, ${ }^{48,50,51}$ can be obtained.

Within this Dissertation, the instrumental contribution term $I(\theta)$ was solved using National Bureau of Standards - Standard Reference Material 640 (NBS-SRM) (Si, $\mathrm{a}=5.43088 \AA) .{ }^{52}$ For calculating a solution for the $I(\theta)$, the Fundamental Parameters Approach (FPA) implemented in TOPAS, ${ }^{49}$ and (Le Bail) powder pattern decomposition with the Si standard unit cell without fitting sample size-strain peak broadening, ${ }^{53}$ were applied. Shifts in the natural Mo K-emission lines introduced by the used Göbelmirror, ${ }^{49}$ were corrected by fixing the $\mathrm{K}_{\alpha 1}$ emission to its natural value of $0.709300 \AA$ and refining the $\mathrm{K}_{\alpha 2}$ line to an arbitrary value of $0.713467 \AA$ (natural Mo $\mathrm{K}_{\alpha 2}$ $0.713607 \AA$ ) for the best peak position fit on the used Si reference standard. Two broad, low intensity $\mathrm{K}_{\alpha}$ 's were added to the emission profile to account for the asymmetry from the multiplet K-emission lines. ${ }^{49}$ High $2 \theta$ diffraction angle peak splitting due to dense $\mathrm{Si}$ reference material was minimized by focusing the X-ray beam in the top part of the measurement capillary. The parameters for instrumental contribution $I(\theta)$ were refined to the best fit (lowest R-value) on the measured Si reference diffraction pattern over $2 \theta$ range $11.5-60^{\circ}$ using non-linear least squares fitting (Levenberg-Marquardt).

The sample contributions $S(\theta)$ were calculated as based on known crystal structures. Sample fit parameters were refined to achieve the best calculated pattern fit on the measured diffractograms over $2 \theta$ range $6-39^{\circ}$. This while keeping the previously 
solved $I(\theta)$ constant. The crystal structures for the phases used in the Rietveld fitting were obtained from the International Centre for Diffraction Data Powder Diffraction File-4+ 2016 (ICDD-PDF-4+) and Crystallography Open Databases (COD). The ICDDPDF-4+ and COD database numbers for Fe carbide structures used for the R-QPA fitting are given in Chapter 2. Crystal structures from ICDD-PDF-4+ 2016 database, namely: $\alpha-\mathrm{Al}_{2} \mathrm{O}_{3}$ (\#04-004-2852), $\alpha-\mathrm{Fe}_{2} \mathrm{O}_{3}$ (\#04-006-6579), $\mathrm{Fe}_{3} \mathrm{O}_{4}$ (\#04-012-7038), $\mathrm{Fe}_{0.974} \mathrm{O}$ (\#01-073-2143) and $\alpha$-Fe (\#04-007-9753) were also used.

Fit parameters of sample displacement, cylindrical $2 \theta$ correction, ${ }^{54}$ Fe structures' lattice parameters, background ( $8^{\text {th }}$ order Chebyshev polynomial) and diffraction peak size-strain broadening, ${ }^{48,50,51}$ and crystal structure scale factors, ${ }^{45}$ were all refined simultaneously. The possible angular unit cell lattice parameters $(\alpha, \beta, \gamma)$ were not refined in order to avoid any lattice parameter correlation. Lattice parameters for the $\alpha-\mathrm{Al}_{2} \mathrm{O}_{3}$ support material were not refined in order to avoid correlation between the sample displacement, the cylindrical $2 \theta$ correction and any of the Fe structures' lattice parameters. Parameter constrains were used to achieve the visually most representative fits on the measured diffractograms, when needed.

For fitting of the size-strain diffraction peak broadening on the Fe phases, only a single Lorentzian peak shape component was used to estimate the size broadening while ignoring the Gaussian peak shape component for the strain broadening. ${ }^{51,55}$ The strain broadening is more prominent at the high $2 \theta$ diffraction where the measured Fe carbides gave low diffraction intensities, with significant diffraction peak overlap between the carbide phases. This resulted in unrealistic values for both the size and strain and thus prevented the reliable determination of the Gaussian strain broadening component. The crystallite mean diameter (i.e. volume-weighted mean column length or domain size) was estimated from the Lorentzian peak's Integral Breadth with the Scherrer Equation. When the Integral Breadth is used, the Scherrer Equation shape factor $k$ can be taken as $k=1.51,55-57$ The lack of the fitted Gaussian strain component introduces an error to the determined crystallite mean diameters, ${ }^{55}$ making the diameters only comparable in relative terms within this Dissertation. The size-strain peak broadening for the $\alpha-\mathrm{Al}_{2} \mathrm{O}_{3}$ support material was fitted with both, Lorentzian size and Gaussian strain peak components for more accurate overall fitting.

Contribution from the amorphous diffraction/scattering, a broad peak with low intensity within range $\approx 8-12^{\circ} 2 \theta$, was estimated with two or three individual Lorentzian peaks in order to aid the background fitting.

The crystalline phase quantification was performed using the refined scale factors, ${ }^{45}$ with the database structures without any structure refinement. The obtained wt-\%s for each crystalline Fe phase were normalized to the total sum of the Fe phase wt-\%s for achieving comparable crystalline $\mathrm{Fe}$ phase percentages to the $\mathrm{Fe}$ phase spectral 
Identification of Iron Carbides in Fe(-Na-S)/ $\alpha$-Al203 Fischer-Tropsch Synthesis Catalysts with X-ray Powder Diffractometry and Mössbauer Absorption Spectroscopy

Table 3.2

Overview of the catalyst treatments and the used shorthand notation in this Chapter. The treatments were performed on supported $\mathrm{Fe}(-\mathrm{Na}-\mathrm{S}) / \alpha-\mathrm{Al}_{2} \mathrm{O}_{3}$ catalyst materials with or without the Na-S promotion.

\begin{tabular}{cl}
\hline Shorthand Notation & \multicolumn{1}{c}{ Catalyst Material Description } \\
\hline Calcin. & Calcining in air at $500^{\circ} \mathrm{C}$ for $6 \mathrm{~h}$ \\
$\mathrm{H}_{2}$ & $\mathrm{H}_{2}$ reduction at $400^{\circ} \mathrm{C}$ for $5 \mathrm{~h}$, after the calcining step \\
$240,290,340,390,440$ & $\mathrm{CO}$ carburization (at $1 \mathrm{~atm}$ ) temperature in ${ }^{\circ} \mathrm{C}$, after the reduction step \\
340 h.p. & $\mathrm{CO}$ carburization at $25 \mathrm{bar}$ at $340^{\circ} \mathrm{C}$, after the calcining step \\
UP & Unpromoted $\mathrm{Fe} / \alpha-\mathrm{Al}_{2} \mathrm{O}_{3}$ catalyst \\
Na-S & Na-S promoted $\mathrm{Fe}-\mathrm{Na}-\mathrm{S} / \alpha-\mathrm{Al}_{2} \mathrm{O}_{3}$ catalyst \\
\hline
\end{tabular}

contributions from the MAS quantifications. Elemental Fe contents for the catalyst materials were calculated on the basis of the nominal molar amounts of Fe per fitted crystalline Fe phase.

For presenting the measured diffractograms and their calculated patterns, all of the diffraction patterns were aligned in $2 \theta$ to $\alpha-\mathrm{Al}_{2} \mathrm{O}_{3}$ (\#04-004-2852) peak $\left(\begin{array}{ll}0 & 2\end{array}\right)$ at $23.515^{\circ} 2 \theta$ (at Mo $\mathrm{K}_{\alpha 1}$ ) for correcting the sample displacement. Furthermore, the calculated background polynomials were subtracted and the diffractograms were normalized in counts to the maximum counts of the $\alpha-\mathrm{Al}_{2} \mathrm{O}_{3}\left(\begin{array}{lll}0 & 2\end{array}\right)$ peak for each diffractogram.

\subsection{RESULTS AND DISCUSSION}

In this Chapter, we have used ex-situ XRPD with R-QPA applied for the diffraction pattern Fe phase quantification on a set of Fe-based FTS reaction catalyst materials. The $\mathrm{R}-\mathrm{QPA}$ results were compared to the respective ex-situ MAS quantifications. This was done in order to follow the formation of Fe carbides from $\alpha$-Fe with $\mathrm{CO}$ carburization within the temperature range of $240-440^{\circ} \mathrm{C}$. Furthermore, the effect of Na-S promotion on the Fe oxide and carbide formation was followed by exposing unpromoted $\mathrm{Fe} / \alpha$ $\mathrm{Al}_{2} \mathrm{O}_{3}$ (shorthand UP) and Na-S promoted Fe-Na-S/ $\alpha-\mathrm{Al}_{2} \mathrm{O}_{3}(\mathrm{Na}-\mathrm{S})$ catalyst materials to identical $\mathrm{CO}$ carburization treatments. Additionally, morphological changes in the catalyst materials were followed with TEM while carbon deposition on the catalyst materials was quantified with the use of TGA-MS.

The shorthand notation of $240,290,340,390$ or 440 is used to refer to the applied CO carburization temperature of the $\mathrm{H}_{2}$ reduced catalyst material, at atmospheric pressure. Catalyst materials $\mathrm{CO}$ carburized directly after the calcining step at 25 bar CO pressure at $340^{\circ} \mathrm{C}$ are noted in shorthand as 340 h.p.. Shorthand notation Calcin. is used for catalyst materials characterized directly after catalysts' calcining step. Notation $\mathrm{H}_{2}$ is used for the only $\mathrm{H}_{2}$ reduced catalyst materials. The overview of the shorthand notation used within this Chapter is given Table 3.2. 

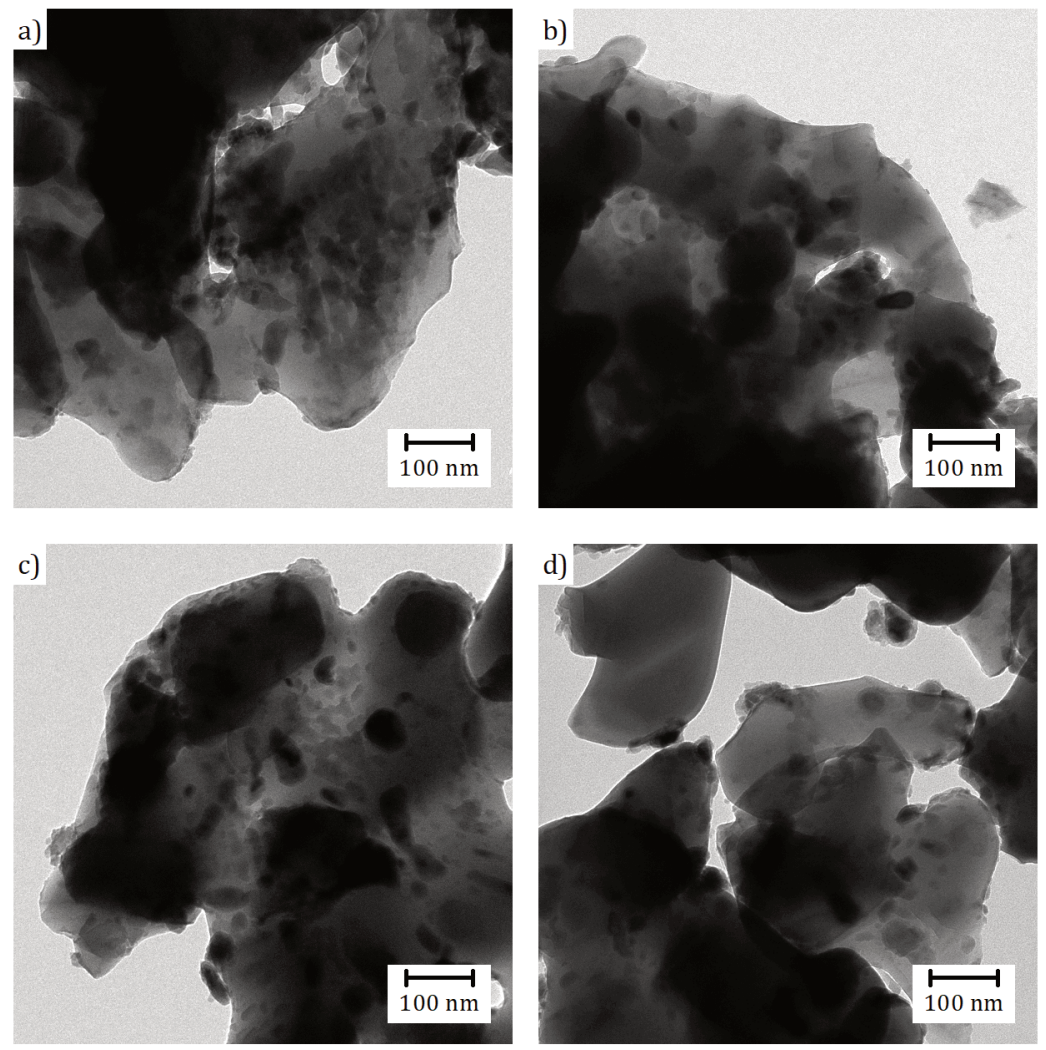

Figure 3.2

Transmission Electron Microscopy (TEM) images for the Na-S (Fe-Na-S $/ \alpha-\mathrm{Al}_{2} \mathrm{O}_{3}$ ) catalyst materials after the applied catalyst treatments. Images after a) Calcin. and b) $\mathrm{H}_{2}$ treatments and after $\mathrm{CO}$ carburization treatments c) 240 , and d) 290 . The TEM images measured for the corresponding UP catalyst materials were similar and are not shown.

\subsubsection{Probing Morphological Changes and Carbon Deposition with Transmission Electron Microscopy and Thermogravimetric Analysis - Mass Spectrometry}

TEM images taken after the calcining, $\mathrm{H}_{2}$ reduction and the carburizing 240 and 290 treatments are shown in Figure 3.2 for the Na-S catalyst materials. TEM images taken after the higher temperature carburization treatments (340, 390, 440 and 340 h.p.) for the Na-S catalyst materials are shown in Figure 3.3. The TEM images for the UP catalyst materials after their respective treatments were very similar to their $\mathrm{Na}-\mathrm{S}$ counterparts and are not shown. The number-weighted Fe particle mean diameters and the size 

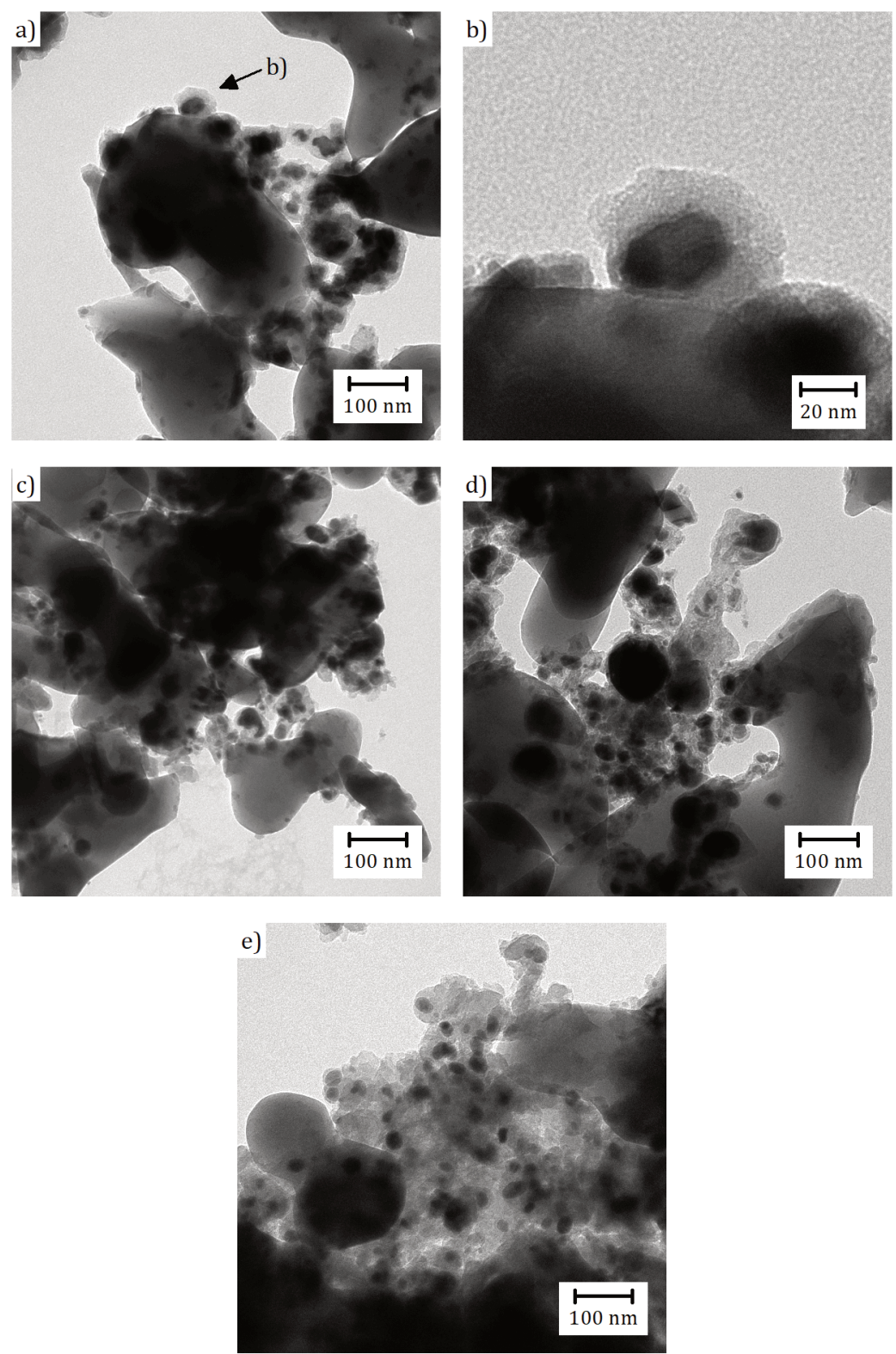

\section{Figure 3.3}

Transmission Electron Microscopy (TEM) images for Na-S (Fe-Na-S $\left./ \alpha-\mathrm{Al}_{2} \mathrm{O}_{3}\right)$ catalyst materials after the applied catalyst treatments. Images after CO carburization treatments a) 340 , b) zoom to the region pointed with an arrow in (a), c) 390, d) 440, and e) 340 h.p.. The TEM images measured for the corresponding UP catalyst materials were similar and are not shown. 

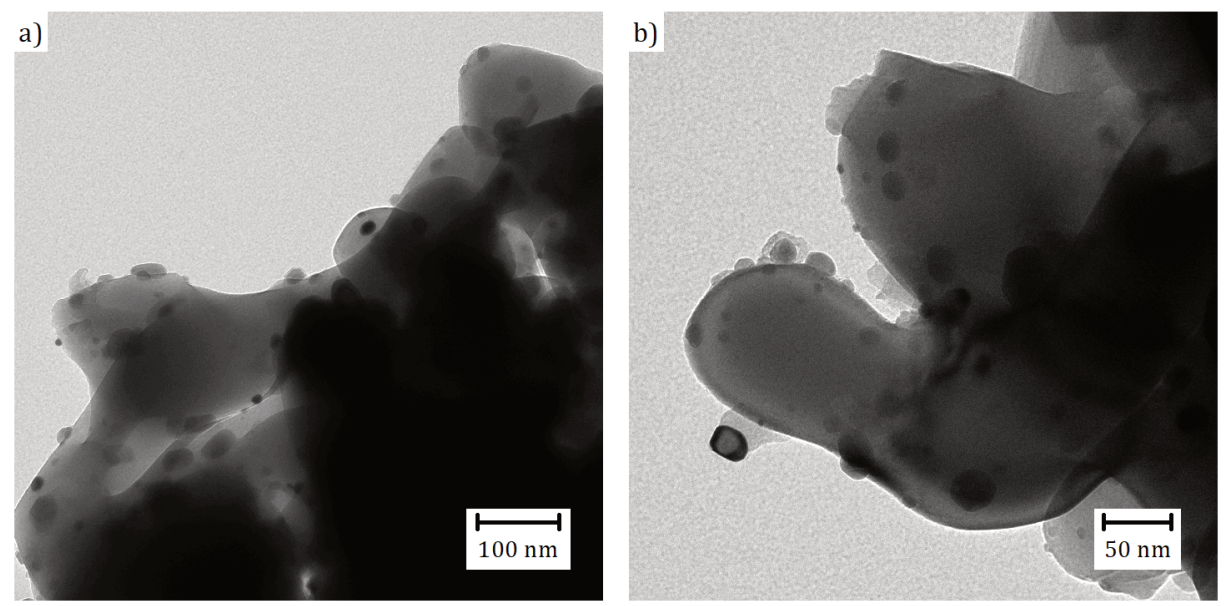

Figure 3.4

Transmission Electron Microscopy (TEM) images for the $\mathrm{Na}-\mathrm{S} \mathrm{H}_{2}\left(\mathrm{Fe}-\mathrm{Na}-\mathrm{S} / \alpha-\mathrm{Al}_{2} \mathrm{O}_{3}\right)$ catalysts materials on different locations. Despite a vast majority of Fe particles on the catalyst being clustered and poorly separated (Figure 3.2b), in some areas a) and b), the Fe particles were well separated.

distribution STDs obtained on the basis of the measured TEM images, are shown in Figure 3.5.

After the Calcin. (Figure 3.2a) and $\mathrm{H}_{2}$ (Figure 3.2b) catalyst treatments, $\mathrm{Fe}$ is present on the $\alpha-\mathrm{Al}_{2} \mathrm{O}_{3}$ support as clusters, rather than as individual Fe particles. Each Fe cluster was counted as a "Fe particle" by its longest diameter. In some, occasional regions the $\mathrm{Na}-\mathrm{S} \mathrm{H}_{2}$ catalyst material however did show vastly different $\mathrm{Fe}$ distributions in where the Fe particles were individually separated (Figure 3.4). At $500^{\circ} \mathrm{C}$, a shorter calcining time $(\approx 1 \mathrm{~h})$ might lessen the Fe clustering. ${ }^{58,59}$

After applying the mildest CO carburization treatments 240 and 290 (Figure 3.2cd), the Fe particles start to separate and resemble single Fe particles more than "a cluster of Fe particles". This is reflected in the Fe particle size distribution values in Figure 3.5: from treatment Calcin. to 290, the Fe particle mean diameters and the STD values both decrease. That is, the Fe particles become smaller, with more homogenous size distribution. Both UP and Na-S catalysts show the same overall trend.

With CO carburization treatments with temperatures above $340^{\circ} \mathrm{C}$, the Fe particles become encapsulated in carbon and invariably are not attached to the $\alpha-\mathrm{Al}_{2} \mathrm{O}_{3}$ support (Figure 3.3). This is unlike with the Fe particles after the $\mathrm{CO}$ carburization treatments 240 and 290 , which are still attached to the $\alpha-\mathrm{Al}_{2} \mathrm{O}_{3}$ support. The catalyst materials after the treatments $340,390,440$, and 340 h.p. resemble more of a composite system where the carbon-encapsulated $\mathrm{Fe}$ is incorporated to the $\alpha-\mathrm{Al}_{2} \mathrm{O}_{3}$ support than a pure, metal 
supported catalyst system.

From the treatment 340 to the higher temperature 440 treatment, a somewhat increasing trend in the Fe particle mean diameters and STD's can be observed for the Na-S catalyst materials. The Fe particle mean diameter growth with increasing carburization temperature seems to be significant for only the Na-S promoted materials (Figure 3.5). This is akin to a literature observation on the Na-S promotion effect increasing Fe particle diameters. ${ }^{60}$

Furthermore, it is worth noting that it can be expected that the relatively small Fe particles of $\lesssim 60 \mathrm{~nm}$ within the catalyst materials described in this Chapter (Figure 3.5), after all catalyst treatments, prevent any $\mathrm{C}$ atom diffusion problems to the $\alpha$-Fe lattice. This allows complete, unrestricted Fe carburization. If bulk Fe catalyst materials were used instead, $\mathrm{C}$ atom diffusion problems might arise thus decreasing the degree of $\mathrm{Fe}$ carburization.5,6,15

The TGA measured carbon deposition as wt-\%s and the average carbon deposition rates after each applied carburization treatment are shown in Figure 3.6. The simultaneously measured MS data for the total $\mathrm{CO}_{2}$ released and the catalyst material weight loss fractions during the TGA runs are given in the Figure 3.7. The quantification results of the $\mathrm{CO}_{2}$ released in the TGA measurements (Figure 3.7c) are in perfect agreement with the deposited carbon wt-\% results (Figure 3.6a). That is, the TGA observed weight decrease on the catalyst materials is due to carbon deposition during
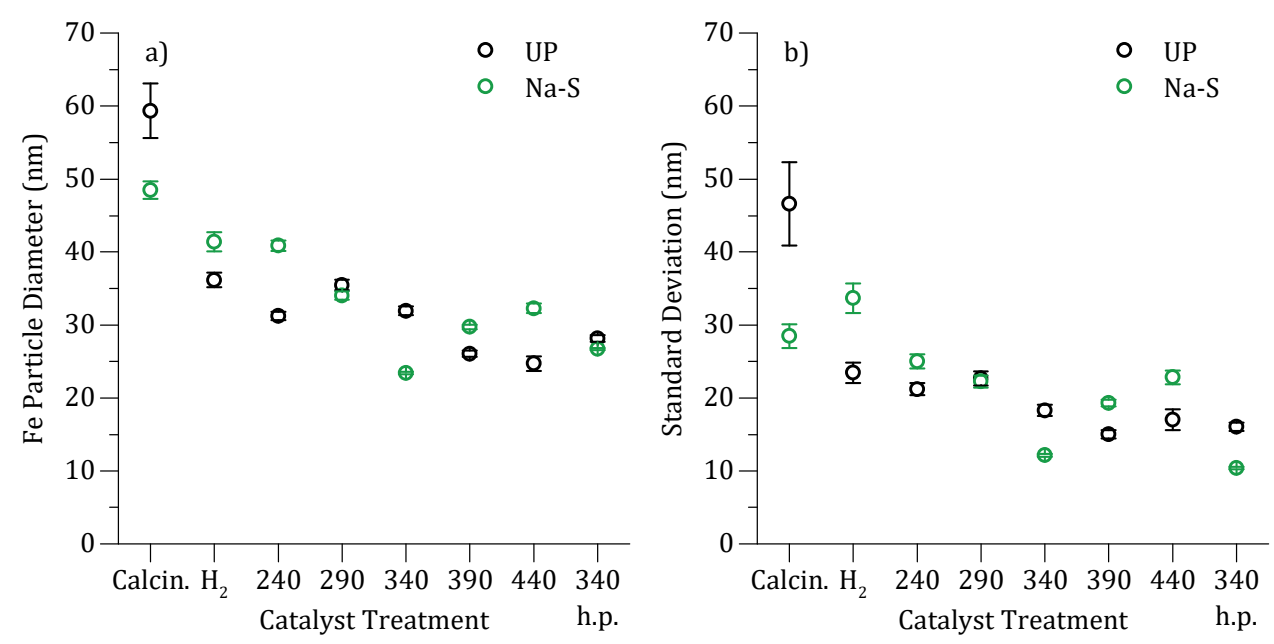

Figure 3.5

Transmission Electron Microscopy (TEM) image based, Fe particle a) (number-weighted) mean diameters and b) size distribution Standard Deviations (STDs) after the respective catalyst treatments of the UP and Na$\mathrm{S}\left(\mathrm{Fe}(-\mathrm{Na}-\mathrm{S}) / \alpha-\mathrm{Al}_{2} \mathrm{O}_{3}\right)$ catalyst materials. 

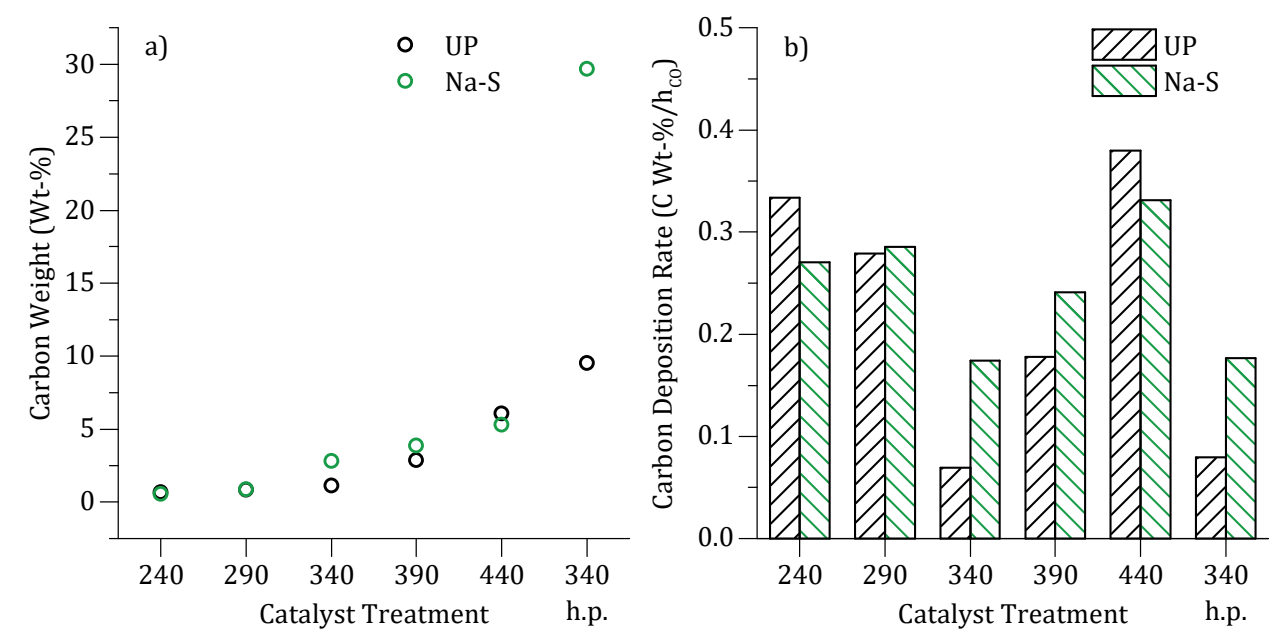

Figure 3.6

Thermogravimetric Analysis (TGA) derived carbon deposition with UP and Na-S (Fe(-Na-S) $/ \alpha-\mathrm{Al}_{2} \mathrm{O}_{3}$ ) catalyst materials, after the $\mathrm{CO}$ carburization catalyst treatments. The results for a) total wt- $\%$ of carbon deposited and b) carbon deposition rate.

the carburization treatments.

Some trends in the carbon deposition can be observed as (Figure 3.6): i) treatments 240 and 290 show small total amounts of deposited carbon but with high rates of carbon deposition, and ii) from the treatment 340 to 440 the carbon deposition rate increases with increasing carburization temperature and iii) the effect of Na-S promotion is to increase the carbon deposition rate within the temperature range of $340-390^{\circ} \mathrm{C}$. The Na-S promotion effect however diminishes with increasing carburization treatment temperature. In addition, iv) the high-pressure treatment 340 h.p. at 25 bar CO has around the same carbon deposition rates as the corresponding treatment 340 with atmospheric CO pressure.

With the treatments 240 and 290, the "carbon deposition" is due to carbide formation and dissolution of $\mathrm{C}$ atoms into the Fe lattice, a process which is very fast with $\alpha$-Fe. ${ }^{15,61}$ The dissolution of $\mathrm{C}$ to the Fe lattice is not affected by the Na-S promotion (Figure 3.6b).

From the treatment 340 to 440 , the Boudouard reaction (i.e. CO disproportionation into $\mathrm{C}$ and $\mathrm{CO}_{2}$ ) takes over and starts to deposit carbon on the catalyst materials, in agreement with both the TGA (Figure 3.6) and TEM results (Figure 3.3). Carbon deposition via the Boudouard reaction becomes significant with Fe-based catalyst materials at $\gtrsim 300^{\circ} \mathrm{C} .16,62-65$ From the TGA-MS observations it can be devised that the $\mathrm{Na}-\mathrm{S}$ promotion increases the carbon deposition rates at $\approx 340^{\circ} \mathrm{C}$ and with increasing 


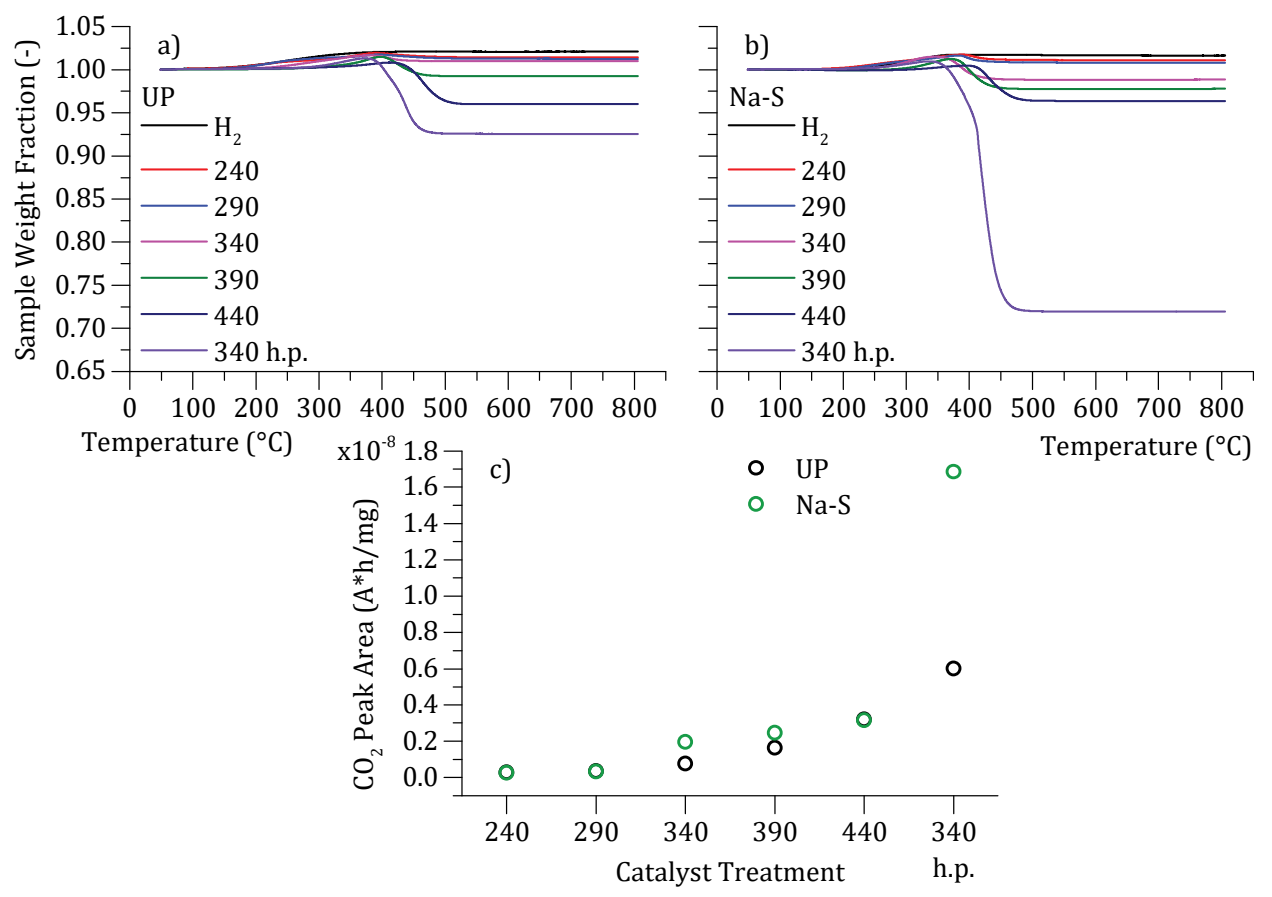

Figure 3.7

Thermogravimetric Analysis - Mass Spectrometry (TGA-MS) measurements for UP and Na-S (Fe(-Na-S)/ $\alpha-$ $\mathrm{Al}_{2} \mathrm{O}_{3}$ ) catalyst materials after each respective catalyst treatment. TGA weight loss fractions for a) UP and $b$ ) $\mathrm{Na}-\mathrm{S}$ catalyst materials, and the corresponding c) MS-derived $\mathrm{CO}_{2}$ peak areas $(\mathrm{m} / \mathrm{z} 44)$ on the total $\mathrm{CO}_{2}$ released during the TGA measurements, as normalized to the initial catalyst material amount.

temperature $\left(\gtrsim 390-440^{\circ} \mathrm{C}\right)$ the Na-S promotion effect diminishes and disappears (Figure 3.6b). From literature, the addition of alkali elements has been observed intrinsically to increase the carbon deposition. ${ }^{16,62,66}$ Therefore, the Na-S promotion behaves akin to alkali-only addition within the temperature range of $340-390^{\circ} \mathrm{C}$, increasing the carbon deposition with $\mathrm{CO}$ carburization via the Boudouard reaction.

By comparing the treatments 340 and 340 h.p., the average carbon deposition rates are the same i.e. the higher 25 bar CO pressure does not seem to increase the rate of the carbon deposition. However, under $\mathrm{H}_{2}$ : $\mathrm{CO}$ atmosphere i.e. in the FTS reaction, increased reaction pressure does increase, at least the initial, carbon deposition rate on the Febased catalyst materials. ${ }^{16,67}$

For further research on the effect of Na-S promotion on the Boudouard reaction during the FTS reaction, the amount of deposited carbon should be compared in CO conversion normalized terms to unpromoted catalysts. Otherwise, the carbon deposition rates and amounts will merely follow the CO conversion levels in the FTS reaction, not the intrinsic Boudouard reaction rate. 

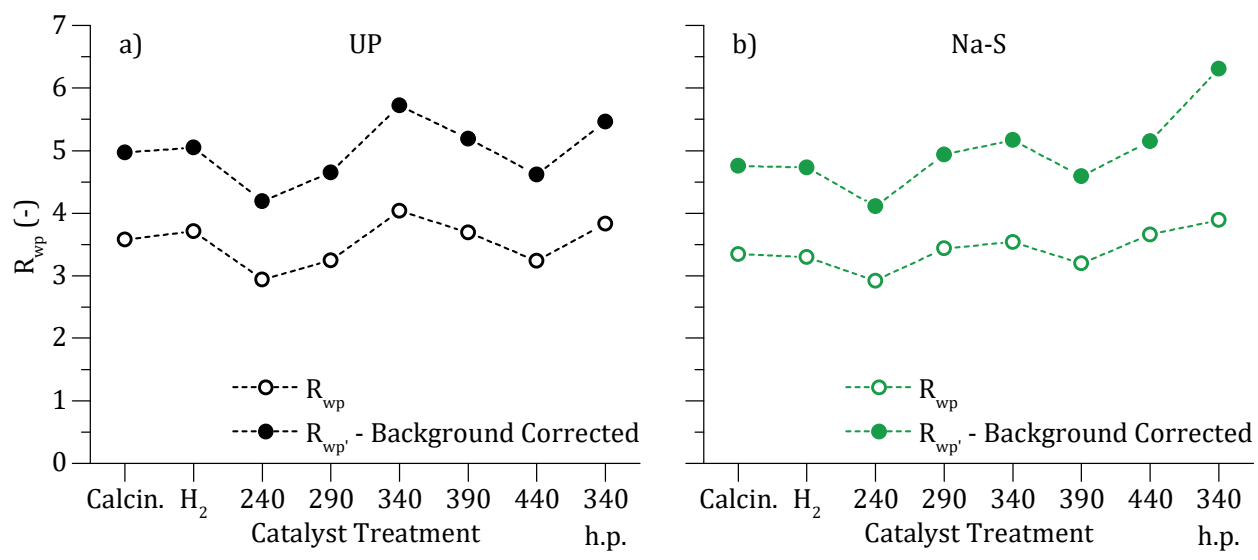

Figure 3.8

$\mathrm{R}_{\mathrm{wp}}$-values for the differences between the X-ray Powder Diffractometry (XRPD) measured and Rietveld Quantitative Phase Analysis (R-QPA) calculated diffractograms. $\mathrm{R}_{w p}$ - and $\mathrm{R}_{w p}$-values for a) UP and b) Na-S $\left(\mathrm{Fe}(-\mathrm{Na}-\mathrm{S}) / \alpha-\mathrm{Al}_{2} \mathrm{O}_{3}\right)$ catalyst materials, after the respective catalyst treatment.

\subsubsection{Phase Quantification with X-ray Powder Diffractometry/Rietveld Quantitative Phase Analysis and Mössbauer Absorption Spectroscopy}

Background corrected, ex-situ XRPD measured and the Rietveld method calculated diffraction patterns with their difference to the experimentally measured XRPD patterns, for the Calcin. and $\mathrm{H}_{2}$ catalyst materials are shown in Figure 3.9. The diffraction patterns after the CO carburization treatments are given in Figure $\mathbf{3 . 1 0}$ for the UP and in Figure 3.11 for the Na-S catalyst materials. For sake of clarity, only the range $13-29^{\circ} 2 \theta$ Mo $\mathrm{K}_{\alpha}$, where the Fe carbides show their most intense diffraction peaks, is shown in the figures. R-weighted pattern ( $\left.\mathrm{R}_{w p}\right)$ values for the Rietveld fits are given in the Figure 3.8.68 The MAS spectra with fitted phase peaks for the UP catalyst materials after $\mathrm{H}_{2}$ and carburizing 240-440 and 340 h.p. catalyst treatments are given in Figure 3.12. The MAS spectra for the Na-S catalyst materials are omitted.

The crystalline Fe phase quantifications with R-QPA and the corresponding quantification results as based on the MAS measurements are presented in Figure 3.13. The used MAS fit parameters are given in Table 3.3 for the UP and in Table 3.4 for the Na-S catalyst materials. The R-QPA and MAS quantifications are in good agreement with each other.

The R-QPA quantification results for the Fe phases in the Calcin. catalyst materials, which only quantified $\alpha-\mathrm{Fe}_{2} \mathrm{O}_{3}$ (hematite) for both UP and Na-S catalysts, are excluded from the Figure 3.13b. These quantification results were nevertheless used for 


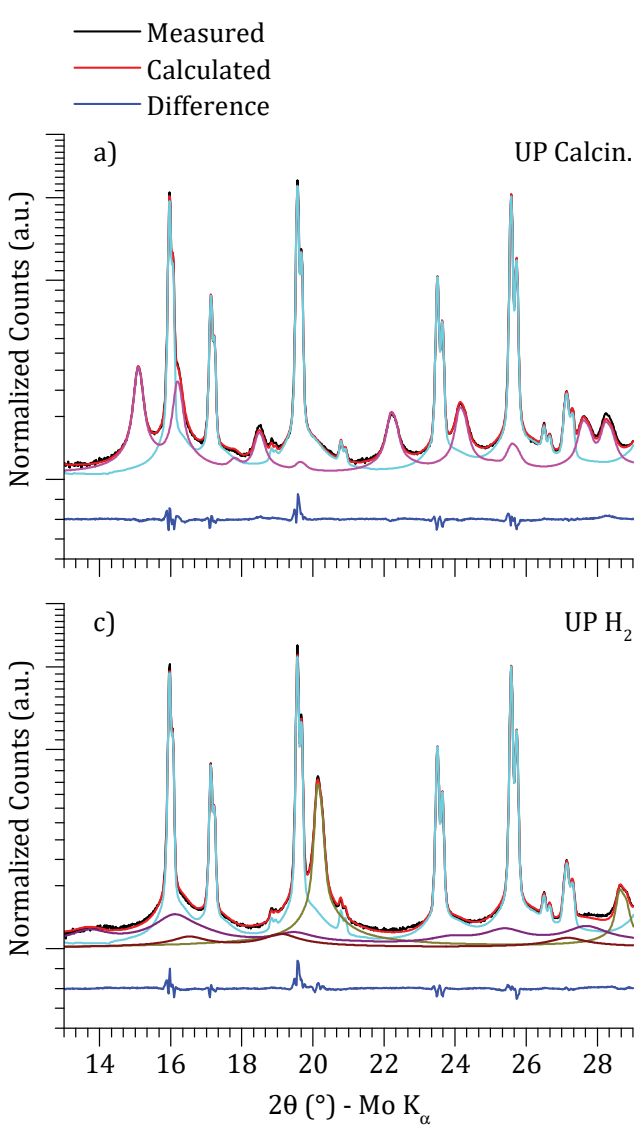

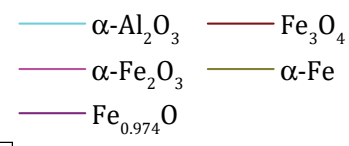
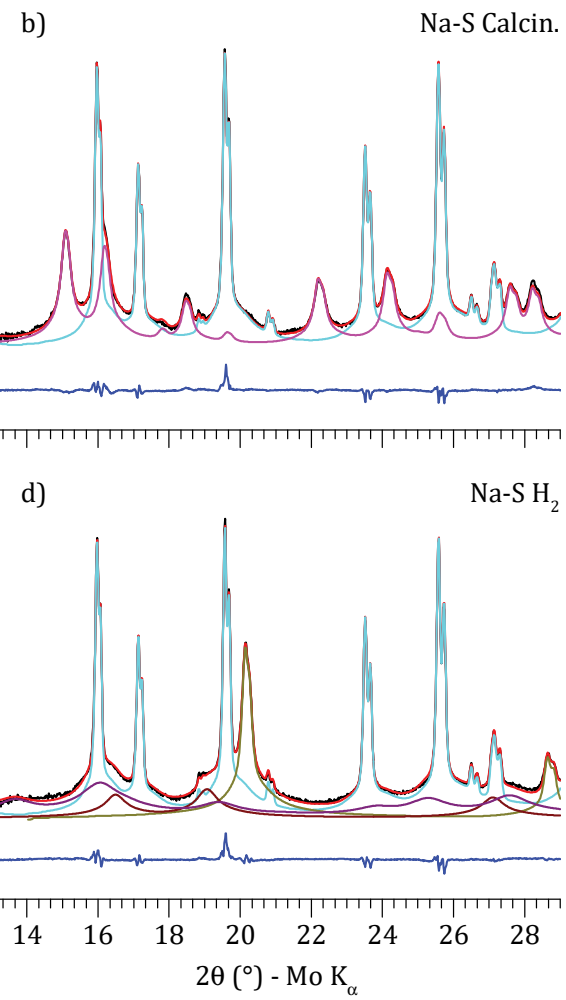

Figure 3.9

Background corrected, ex-situ measured X-ray Powder Diffractometry (XRPD) and Rietveld method calculated diffraction patterns over a range 13-29 $2 \theta$ for UP a) Calcin. and c) $\mathrm{H}_{2}$; and for Na-S b) Calcin. and d) $\mathrm{H}_{2}$ treated catalyst materials $\left(\mathrm{Fe}(-\mathrm{Na}-\mathrm{S}) / \alpha-\mathrm{Al}_{2} \mathrm{O}_{3}\right)$. Normalized counts are on a square-root scale.

calculating the elemental Fe contributions for the UP and Na-S Calcin. catalyst materials in the Figure 3.13c.

The elemental Fe contributions do not change significantly for the studied catalyst materials. The mean elemental Fe wt- $\%$ s are for UP catalyst materials $6.4 \pm 0.5 \mathrm{wt}-\%$ and for Na-S materials $6.7 \pm 0.5 \mathrm{wt}-\%$, in comparison between all of the applied treatments within the promotion groups (Figure 3.13c). This can be taken as an indication that all of the Fe phases present in the XRPD patterns have been fitted consistently with a calculated Fe crystal structure. ICP-OES quantification results indicated the presence of $\approx 6.6 \mathrm{wt}-\%$ of elemental Fe for both the parent UP and Na-S Calcin. catalyst materials (Table 3.1). 

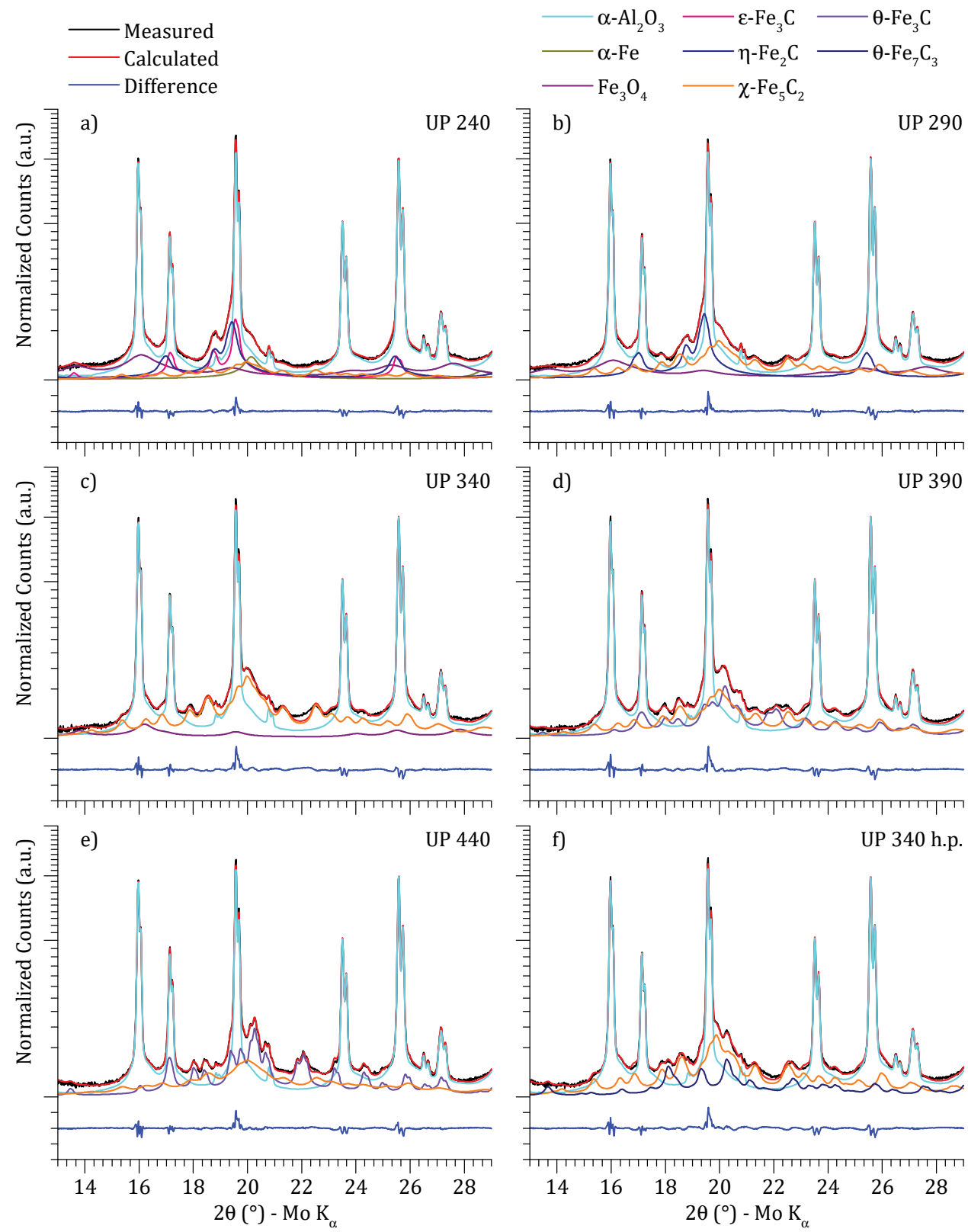

Figure 3.10

Background corrected, ex-situ measured X-ray Powder Diffractometry (XRPD) and Rietveld method calculated diffraction patterns over a range $13-29^{\circ} 2 \theta$ for the UP $\left(\mathrm{Fe} / \alpha-\mathrm{Al}_{2} \mathrm{O}_{3}\right)$ catalyst materials after the respective catalyst treatment. The diffraction patterns as after catalyst treatment a) 240 , b) 290, c) 340, d) 390, e) 440 and f) 340 h.p.. Normalized counts are on a square-root scale. 
Identification of Iron Carbides in Fe(-Na-S)/ $\alpha$-Al203 Fischer-Tropsch Synthesis Catalysts with X-ray Powder Diffractometry and Mössbauer Absorption Spectroscopy
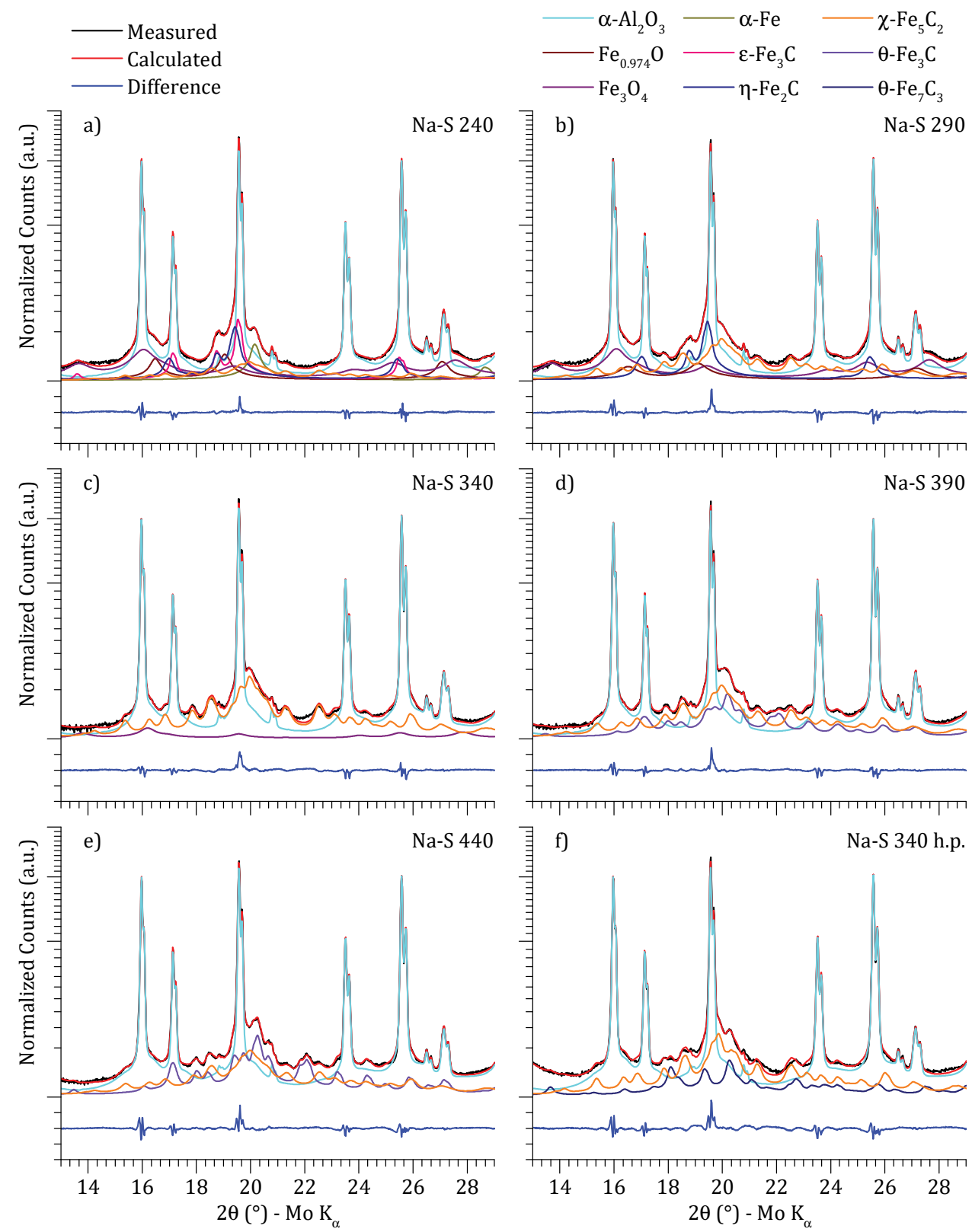

\section{Figure 3.11}

Background corrected, ex-situ measured X-ray Powder Diffractometry (XRPD) and Rietveld method calculated diffraction patterns over a range $13-29^{\circ} 2 \theta$ for the $\mathrm{Na}-\mathrm{S}\left(\mathrm{Fe}-\mathrm{Na}-\mathrm{S} / \alpha-\mathrm{Al}_{2} \mathrm{O}_{3}\right)$ catalyst materials after the respective catalyst treatment. The diffraction patterns as after catalyst treatment a) 240, b) 290, c) 340, d) 390 , e) 440 and f) 340 h.p.. Normalized counts are on a square-root scale. 


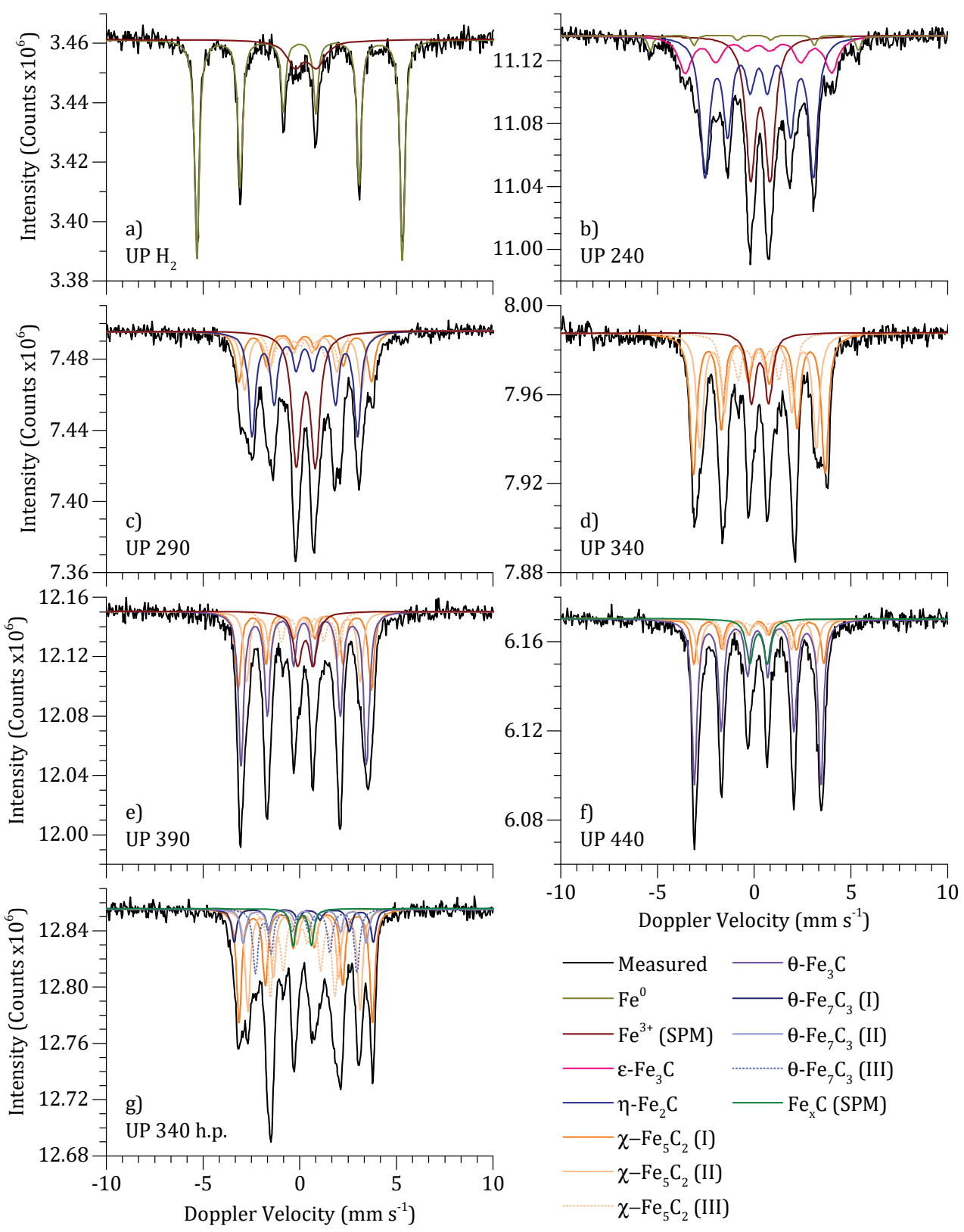

Figure 3.12

Ex-situ measured Mössbauer Absorption Spectroscopy (MAS) spectra with fitted patterns for the Fe phases for the UP $\left(\mathrm{Fe} / \alpha-\mathrm{Al}_{2} \mathrm{O}_{3}\right)$ catalyst materials. The MAS spectra as measured at room temperature $(300 \mathrm{~K})$ after catalyst treatment a) $\mathrm{H}_{2}$, b) 240, c) 290, d) 340, e) 390, f) 440 and g) 340 h.p.. The Roman numbers (I, II, III) mark the different non-equal Fe positions fitted for the Fe phases. SPM = superparamagnetic. 
Identification of Iron Carbides in Fe(-Na-S)/ $\alpha$-Al203 Fischer-Tropsch Synthesis Catalysts with X-ray Powder Diffractometry and Mössbauer Absorption Spectroscopy

By comparing the elemental Fe content after each catalyst treatment (Figure 3.13c) to the trends on deposited carbon (Figure 3.6a), it can be concluded that no significant amount of $\mathrm{Fe}$ is lost, despite the carbon deposition and $\mathrm{Fe}$ growing off the $\alpha-\mathrm{Al}_{2} \mathrm{O}_{3}$ support material with CO treatments $\geq 340^{\circ} \mathrm{C}$ (Figure 3.3).

As a general note on performing bulk characterization studies on zerovalent Febased materials: $\mathrm{a} \approx 4-5 \mathrm{~nm}$ Fe reoxidation shell layer is expected to form upon exposure of the catalyst material to air or other source of 0 atoms. ${ }^{69,70}$ Such layer on Fe is indeed observable in e.g. Figure 3.3b. This effectively decreases the crystalline content of the Fe carbides from what they were during the application of each catalyst treatment. That is, as a general recommendation: at least $\mathrm{a} \approx 20 \mathrm{~nm}$ Fe particle diameter is needed in order to preserve sufficient quantities of the Fe carbides for a bulk technique study, as is done here (Figure 3.5a). Fe particles smaller than $\approx 10 \mathrm{~nm}$ by diameter would be increasingly or solely measured as Fe oxides in bulk quantification studies due to the reoxidation by 0 atoms, skewing the quantification results. ${ }^{13,71}$

In the following discussion, the effects of each catalyst treatment on the Fe phases present in the studied catalyst materials are discussed based on the R-QPA and MAS quantification results. A discussion on the results for the Calcin. catalyst materials is omitted. First, catalyst materials after the applied $\mathrm{H}_{2}$ reduction treatment are discussed. This discussion is followed with remarks on the effect of the CO carburization treatments 240-440.

The carburizing treatments are first discussed, i) regarding the effect of increasing CO carburization temperature, and after which ii) the effect of Na-S promotion in the CO carburization process is treated. The formation of $\theta-\mathrm{Fe}_{7} \mathrm{C}_{3}$ with the catalyst treatment 340 h.p. is discussed separately after the other CO carburization treatments.

The discussion is concluded with collective comments on the Fe crystallite diameter evolution due to the applied treatments (Figure 3.16), in relation to the Fe particle diameter trends derived from the TEM images (Figure 3.6).

\subsubsection{The Effect of $\mathrm{H}_{2}$ Reduction}

After the $\mathrm{H}_{2}$ reduction catalyst treatment, both the UP and Na-S catalyst materials are well reduced into $\alpha$-Fe. This observation is valid regardless of the reduction retarding effect of alkali in the Na-S catalysts, ${ }^{72}$ and possible catalyst material reoxidation upon exposure of the zerovalent Fe to air.69,70,73

All of the catalysts under study were cooled down under $\mathrm{N}_{2}$ or $\mathrm{N}_{2}: \mathrm{H}_{2}$ flow and flushed with $\mathrm{N}_{2}$ at room temperature for a period of time, without a dedicated passivation step. ${ }^{70}$ No detrimental reoxidation of $\alpha$ - $\mathrm{Fe}$ is observed in the $\mathrm{H}_{2}$ treated catalyst materials, which implies that the $\mathrm{O}$ atom containing impurities in the input $\mathrm{N}_{2}$ gas sufficiently passivate the catalysts for the present bulk Fe study purposes. 


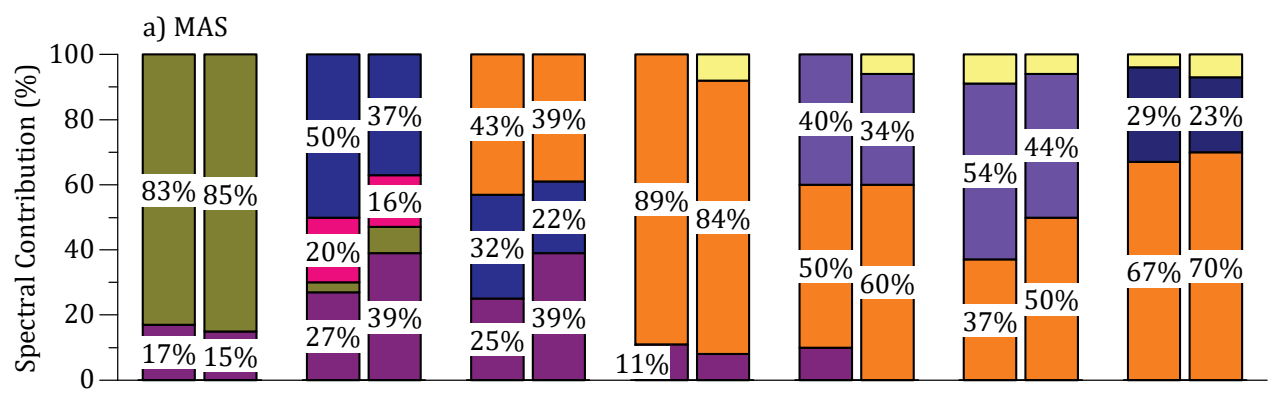

b) R-QPA
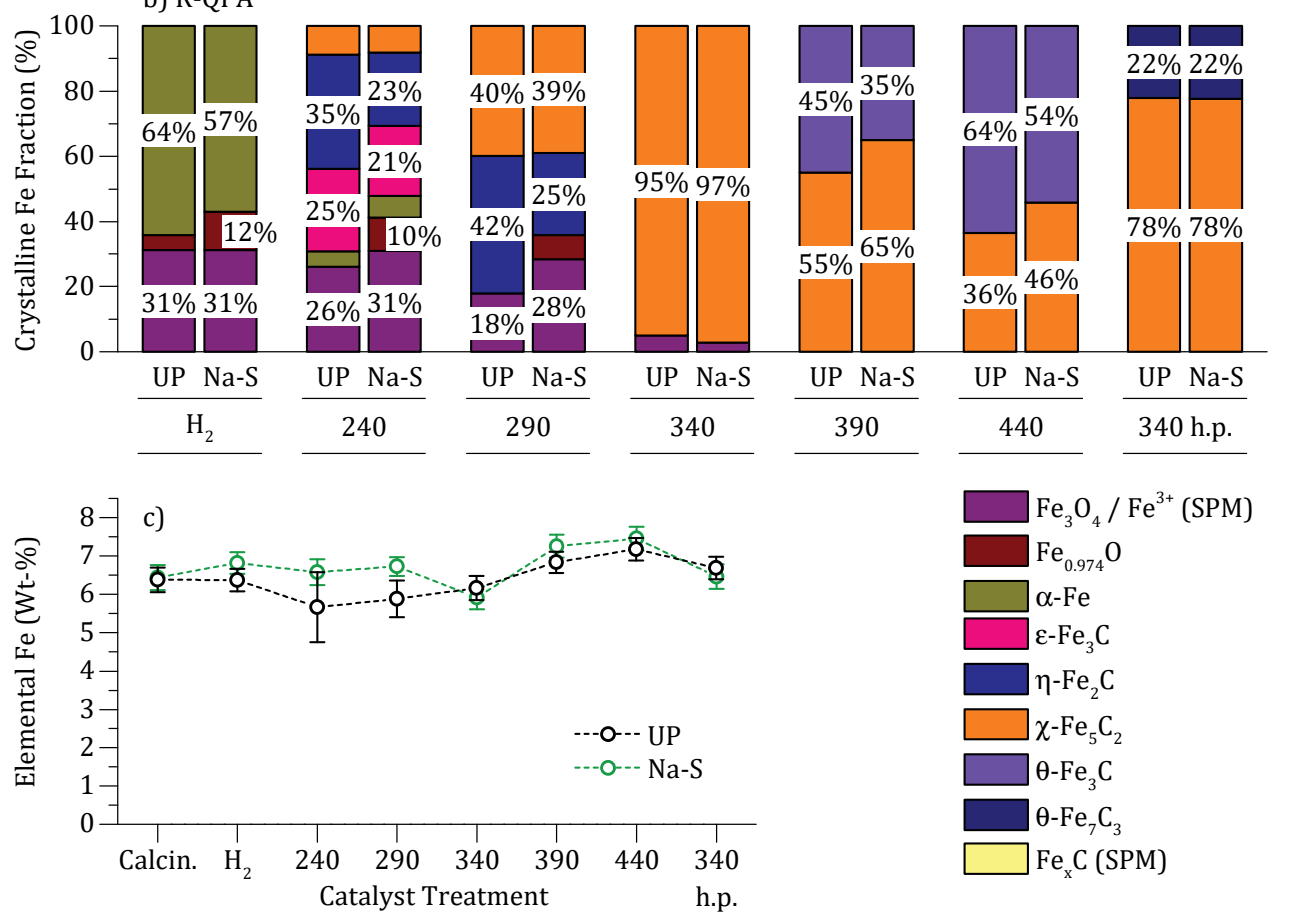

Figure 3.13

The Fe phase quantifications for UP and Na-S $\left(\mathrm{Fe}(-\mathrm{Na}-\mathrm{S}) / \alpha-\mathrm{Al}_{2} \mathrm{O}_{3}\right)$ catalyst materials after the applied catalyst treatments. Quantification results for a) room temperature (300 K) Mössbauer Absorption Spectroscopy (MAS), and b) Rietveld Quantitative Phase Analysis (R-QPA). R-QPA results were used to derive c) the elemental Fe wt-\% loadings from the crystalline Fe phases. Percentage labels cut-off is set to $<10 \%$. SPM = superparamagnetic.

Furthermore, the $\mathrm{Fe}$ oxide content quantifications of $31 \%$ of $\mathrm{Fe}_{3} \mathrm{O}_{4}$ (magnetite) with R-QPA and 15-17\% of $\mathrm{Fe}^{3+}$ (Superparamagnetic, SPM) with MAS are in discrepancy with each other. This Fe oxide content is likely due to the formed passivation layer, ${ }^{69,70}$ which is hard to quantify with the bulk characterization techniques applied in this 
Identification of Iron Carbides in Fe(-Na-S)/ $\alpha$-Al203 Fischer-Tropsch Synthesis Catalysts with X-ray Powder Diffractometry and Mössbauer Absorption Spectroscopy

Table 3.3

Ex-situ Mössbauer Absorption Spectroscopy (MAS) fitted parameters for the UP (Fe/ $\left.\alpha-\mathrm{Al}_{2} \mathrm{O}_{3}\right)$ catalyst materials after the respective catalyst treatment. Measurements were done at room temperature $(300 \mathrm{~K})$.

\begin{tabular}{|c|c|c|c|c|c|c|}
\hline \multirow[t]{2}{*}{ Treatment } & Isomer Shift & $\begin{array}{c}\text { Quadrupole } \\
\text { Splitting }\end{array}$ & $\begin{array}{l}\text { Hyperfine } \\
\text { Field }\end{array}$ & Line Width & Phase & $\begin{array}{c}\text { Spectral } \\
\text { Contribution }\end{array}$ \\
\hline & $\left(\mathrm{mm} \mathrm{s}^{-1}\right)$ & $\left(\mathrm{mm} \mathrm{s}^{-1}\right)$ & $(\mathrm{T})$ & $\left(\mathrm{mm} \mathrm{s}^{-1}\right)$ & & $(\%)$ \\
\hline \multirow[t]{2}{*}{ UP $\mathrm{H}_{2}$} & 0.00 & - & 33.0 & 0.29 & $\mathrm{Fe}^{0}$ & 83 \\
\hline & 0.32 & 1.11 & - & 0.98 & $\mathrm{Fe}^{3+}(\mathrm{SPM})$ & 17 \\
\hline \multirow[t]{4}{*}{ UP 240} & 0.00 & - & '33.4" & 0.30 & $\mathrm{Fe}^{0}$ & 3 \\
\hline & 0.20 & - & 23.6 & 0.82 & $\varepsilon-\mathrm{Fe}_{3} \mathrm{C}$ & 20 \\
\hline & 0.24 & - & 17.4 & 0.55 & $\eta-\mathrm{Fe}_{2} \mathrm{C}$ & 50 \\
\hline & 0.31 & 0.99 & - & 0.60 & $\mathrm{Fe}^{3+}(\mathrm{SPM})$ & 27 \\
\hline \multirow[t]{5}{*}{ UP 290} & 0.26 & - & 21.3 & 0.43 & $\chi-\mathrm{Fe}_{5} \mathrm{C}_{2}(\mathrm{I})$ & 16 \\
\hline & 0.16 & - & 18.8 & 0.43 & $\chi-\mathrm{Fe}_{5} \mathrm{C}_{2}(\mathrm{II})$ & 17 \\
\hline & 0.28 & - & 10.9 & 0.43 & $\chi-\mathrm{Fe}_{5} \mathrm{C}_{2}$ (III) & 10 \\
\hline & 0.25 & - & 16.9 & 0.43 & $\eta-\mathrm{Fe}_{2} \mathrm{C}$ & 32 \\
\hline & 0.30 & 0.98 & - & 0.55 & $\mathrm{Fe}^{3+}(\mathrm{SPM})$ & 25 \\
\hline \multirow[t]{4}{*}{ UP 340} & 0.24 & - & 21.2 & 0.42 & $\chi-\mathrm{Fe}_{5} \mathrm{C}_{2}(\mathrm{I})$ & 40 \\
\hline & 0.18 & - & 18.7 & 0.42 & $\chi-\mathrm{Fe}_{5} \mathrm{C}_{2}(\mathrm{II})$ & 32 \\
\hline & 0.21 & - & 11.2 & 0.42 & $\chi-\mathrm{Fe}_{5} \mathrm{C}_{2}$ (III) & 17 \\
\hline & 0.30 & 0.89 & - & 0.47 & $\mathrm{Fe}^{3+}(\mathrm{SPM})$ & 11 \\
\hline \multirow[t]{5}{*}{ UP 390} & 0.24 & - & 21.4 & 0.33 & $\chi-\mathrm{Fe}_{5} \mathrm{C}_{2}(\mathrm{I})$ & 21 \\
\hline & 0.17 & - & 18.3 & 0.33 & $\chi-\mathrm{Fe}_{5} \mathrm{C}_{2}(\mathrm{II})$ & 18 \\
\hline & 0.15 & - & 11.7 & 0.33 & $\chi-\mathrm{Fe}_{5} \mathrm{C}_{2}$ (III) & 11 \\
\hline & 0.20 & - & 20.2 & 0.33 & $\theta-\mathrm{Fe}_{3} \mathrm{C}$ & 40 \\
\hline & 0.30 & 0.81 & - & 0.51 & $\mathrm{Fe}^{3+}(\mathrm{SPM})$ & 10 \\
\hline \multirow[t]{5}{*}{ UP 440} & 0.24 & - & 20.9 & 0.32 & $\chi-\mathrm{Fe}_{5} \mathrm{C}_{2}(\mathrm{I})$ & 15 \\
\hline & 0.17 & - & 18.8 & 0.32 & $\chi-\mathrm{Fe}_{5} \mathrm{C}_{2}(\mathrm{II})$ & 15 \\
\hline & 0.20 & - & 11.0 & 0.32 & $\chi-\mathrm{Fe}_{5} \mathrm{C}_{2}(\mathrm{III})$ & 7 \\
\hline & 0.18 & - & 20.3 & 0.32 & $\theta-\mathrm{Fe}_{3} \mathrm{C}$ & 54 \\
\hline & 0.23 & 0.90 & - & 0.41 & $\mathrm{Fe}_{\mathrm{x}} \mathrm{C}(\mathrm{SPM})$ & 9 \\
\hline UP 340 & 0.25 & 0.08 & 21.4 & 0.32 & $\chi-\mathrm{Fe}_{5} \mathrm{C}_{2}(\mathrm{I})$ & 25 \\
\hline \multirow[t]{6}{*}{ h.p. } & 0.26 & -0.05 & 18.1 & 0.32 & $\chi-\mathrm{Fe}_{5} \mathrm{C}_{2}(\mathrm{II})$ & 23 \\
\hline & 0.14 & 0.03 & 10.4 & 0.32 & $\chi-\mathrm{Fe}_{5} \mathrm{C}_{2}(\mathrm{III})$ & 19 \\
\hline & 0.32 & -0.28 & 22.4 & 0.32 & $\theta-\mathrm{Fe}_{7} \mathrm{C}_{3}(\mathrm{I})$ & 7 \\
\hline & 0.19 & 0.29 & 16.3 & 0.32 & $\theta-\mathrm{Fe}_{7} \mathrm{C}_{3}(\mathrm{II})$ & 14 \\
\hline & 0.25 & 0.03 & 19.8 & 0.32 & $\theta-\mathrm{Fe}_{7} \mathrm{C}_{3}$ (III) & 8 \\
\hline & 0.15 & 0.89 & - & 0.30 & $\mathrm{Fe}_{\mathrm{x}} \mathrm{C}(\mathrm{SPM})$ & 4 \\
\hline
\end{tabular}

Experimental uncertainties: Isomer Shift $\pm 0.01 \mathrm{~mm} \mathrm{~s}^{-1}$, Quadrupole Splitting $\pm 0.01 \mathrm{~mm} \mathrm{~s}^{-1}$, Line Width $\pm 0.01 \mathrm{~mm} \mathrm{~s}^{-1}$, Hyperfine Field $\pm 0.1 \mathrm{~T}$, Spectral Contribution $\pm 3 \%$. SPM = Superparamagnetic.

Chapter. As an example, for the Na-S 340 catalyst material, the $\approx 4 \mathrm{~nm}$ passivation layer can be seen in Figure 3.3b around the carbon encapsulated Fe particle.

MAS measurements at liquid He temperature at $4.2 \mathrm{~K}$ would improve the quantification accuracy of the Fe oxide/hydroxide surface layer with respect to the 
room temperature MAS applied in this Chapter. ${ }^{73,74}$ XRPD-based data is naturally less accurate with poorly crystalline, ${ }^{42,75}$ and/or with small $\lesssim 4-5 \mathrm{~nm}$ crystallite diameter phases. The R-QPA fitted $\mathrm{Fe}_{3} \mathrm{O}_{4}$ phase (Figure 3.9c-d), gives very weak and broad diffraction peaks with very small estimated crystallite diameters of $\approx 3 \mathrm{~nm}$ (Figure 3.16).

It is more likely that the MAS measurements quantify the Fe oxides in the $\mathrm{H}_{2}$ treated catalyst materials more reliably in the presented results, despite the measurements at room temperature. Although $\mathrm{Fe}_{3} \mathrm{O}_{4}$ crystal structure is used in the R-QPA fits, ferrihydrite structures also gave similar overall R-QPA fits (the fits are not shown). ${ }^{76}$

In the $\mathrm{Na}-\mathrm{S} \mathrm{H}_{2}$ catalyst materials, the presence of $12 \%$ of $\mathrm{Fe}_{0.974} \mathrm{O}$ (wüstite) phase is enhanced in the R-QPA phase quantification as a difference to the UP $\mathrm{H}_{2}$ catalyst materials with only $5 \%$ of the $\mathrm{Fe}_{0.974} \mathrm{O}$ phase present (Figure 3.13b). Various chemical interactions between the Fe and support materials or other added compounds, such as alkali K, can stabilize the wüstite phase. ${ }^{77}$ Thus, the Na-S promotion somewhat stabilizes the $\mathrm{Fe}_{0.974} \mathrm{O}$ phase. The $\alpha-\mathrm{Al}_{2} \mathrm{O}_{3}$ support does not play a role in the stabilization as the UP $\mathrm{H}_{2}$ catalyst material does not have a significant $\mathrm{Fe}_{0.974} \mathrm{O}$ phase content. No clear presence of the $\mathrm{Fe}_{0.974} \mathrm{O}$ phase was detected with any of the MAS quantifications (Figure 3.13a).

\subsubsection{The Effect of the Carburization Temperature}

The effect of increasing $\mathrm{CO}$ carburization temperature is to shift the Fe carbide phase formation from the $\alpha$-Fe starting material in order of $\varepsilon-\mathrm{Fe}_{3} \mathrm{C}<\eta-\mathrm{Fe}_{2} \mathrm{C}<\chi-\mathrm{Fe}_{5} \mathrm{C}_{2}<\theta-\mathrm{Fe}_{3} \mathrm{C}$, as can be observed in the Fe phase quantification data (Figure 3.13a-b). This order is in agreement with the Fe carbide temperature evolution interpreted from the literature, following the nomenclature proposed for the Fe carbides in Chapter 2. With a short CO carburization time $\leq 3 \mathrm{~h}$ at low temperature $\leq 290^{\circ} \mathrm{C}$, also Fe oxide phases are present in the catalyst materials, in addition to the Fe carbides.

\section{A. Effect on Fe Oxides}

When moving from the catalyst treatment $\mathrm{H}_{2}$ to the treatments 240 and 290, the Fe oxide ( $\mathrm{Fe}_{3} \mathrm{O}_{4} / \mathrm{Fe}^{3+} \mathrm{SMP}$ ) content in the catalyst materials increase, according to the MAS quantification results (Figure 3.13a). When comparing the XRPD patterns for the catalyst treatments $\mathrm{H}_{2}$ UP and Na-S (Figure 3.9c-d), 240 and 290 UP (Figure 3.10a-b) and 240 and $290 \mathrm{Na}-\mathrm{S}$ (Figure 3.11a-b), the diffraction originating from the fitted $\mathrm{Fe}_{3} \mathrm{O}_{4}$ phase (e.g. at $27.62^{\circ} 2 \theta$ ) is increased after the catalyst treatments 240 and 290 . The total MAS $\left(\mathrm{Fe}^{3+}\right)$ and $\mathrm{R}-\mathrm{QPA}\left(\mathrm{Fe}_{3} \mathrm{O}_{4}+\mathrm{Fe} 0.974 \mathrm{O}\right)$ quantifications of the $\mathrm{Fe}$ oxide phases are in fair agreement for the catalyst treatments 240 and 290. Thus, the increased crystallinity of the $\mathrm{Fe}$ oxide phases $\left(\mathrm{Fe}_{3} \mathrm{O}_{4}+\mathrm{Fe}_{0.974} \mathrm{O}\right)$ in respect to the $\mathrm{H}_{2}$ treated catalyst materials improves the R-QPA accuracy for the catalyst treatments 240 and 290 
Identification of Iron Carbides in Fe(-Na-S)/ $\alpha$-Al203 Fischer-Tropsch Synthesis Catalysts with X-ray Powder Diffractometry and Mössbauer Absorption Spectroscopy

\section{Table 3.4}

Ex-situ Mössbauer Absorption Spectroscopy (MAS) fitted parameters for the Na-S (Fe-Na-S $\left./ \alpha-\mathrm{Al}_{2} \mathrm{O}_{3}\right)$ catalyst materials after the respective catalyst treatment. Measurements were done at room temperature (300 K).

\begin{tabular}{|c|c|c|c|c|c|c|}
\hline \multirow[t]{2}{*}{ Treatment } & Isomer Shift & $\begin{array}{c}\text { Quadrupole } \\
\text { Splitting }\end{array}$ & $\begin{array}{l}\text { Hyperfine } \\
\text { Field }\end{array}$ & Line Width & Phase & $\begin{array}{c}\text { Spectral } \\
\text { Contribution }\end{array}$ \\
\hline & $\left(\mathrm{mm} \mathrm{s}^{-1}\right)$ & $\left(\mathrm{mm} \mathrm{s}^{-1}\right)$ & $(\mathrm{T})$ & $\left(\mathrm{mm} \mathrm{s}^{-1}\right)$ & & $(\%)$ \\
\hline \multirow[t]{2}{*}{ Na-S H 2} & 0.00 & - & 33.5 & 0.30 & $\mathrm{Fe}^{0}$ & 85 \\
\hline & 0.32 & 1.22 & - & 0.98 & $\mathrm{Fe}^{3+}(\mathrm{SPM})$ & 15 \\
\hline \multirow[t]{5}{*}{ Na-S 240} & 0.00 & - & 33.2 & 0.30 & $\mathrm{Fe}^{0}$ & 8 \\
\hline & 0.18 & - & 23.2 & 0.68 & $\varepsilon-\mathrm{Fe}_{3} \mathrm{C}$ & 16 \\
\hline & 0.23 & - & 17.5 & 0.53 & $\eta-\mathrm{Fe}_{2} \mathrm{C}$ & 37 \\
\hline & 0.38 & 1.01 & - & 0.89 & $\mathrm{Fe}^{3+}(\mathrm{SPM})$ & 31 \\
\hline & 0.38 & 0.39 & 45.1 & 1.08 & $\mathrm{Fe}^{3+}$ & 8 \\
\hline \multirow[t]{6}{*}{ Na-S 290} & 0.23 & - & 21.8 & 0.43 & $\chi-\mathrm{Fe}_{5} \mathrm{C}_{2}(\mathrm{I})$ & 15 \\
\hline & 0.17 & - & 19.3 & 0.43 & $\chi-\mathrm{Fe}_{5} \mathrm{C}_{2}(\mathrm{II})$ & 16 \\
\hline & 0.22 & - & 11.4 & 0.43 & $\chi-\mathrm{Fe}_{5} \mathrm{C}_{2}$ (III) & 8 \\
\hline & 0.23 & - & 17.2 & 0.43 & $\eta-\mathrm{Fe}_{2} \mathrm{C}$ & 22 \\
\hline & 0.34 & 1.02 & - & 0.68 & $\mathrm{Fe}^{3+}(\mathrm{SPM})$ & 25 \\
\hline & 0.38 & 0.17 & 44.9 & 1.44 & $\mathrm{Fe}^{3+}$ & 14 \\
\hline \multirow[t]{5}{*}{ Na-S 340} & 0.27 & - & 21.6 & 0.44 & $\chi-\mathrm{Fe}_{5} \mathrm{C}_{2}(\mathrm{I})$ & 35 \\
\hline & 0.20 & - & 18.8 & 0.44 & $\chi-\mathrm{Fe}_{5} \mathrm{C}_{2}$ (II) & 30 \\
\hline & 0.18 & - & 11.5 & 0.44 & $\chi-\mathrm{Fe}_{5} \mathrm{C}_{2}$ (III) & 19 \\
\hline & 0.19 & 1.03 & - & 0.39 & $\mathrm{Fe}_{\mathrm{x}} \mathrm{C}(\mathrm{SPM})$ & 8 \\
\hline & 0.39 & -0.03 & 48.0 & 1.07 & $\mathrm{Fe}^{3+}$ & 8 \\
\hline \multirow[t]{5}{*}{ Na-S 390} & 0.27 & - & 21.3 & 0.38 & $\chi-\mathrm{Fe}_{5} \mathrm{C}_{2}(\mathrm{I})$ & 22 \\
\hline & 0.20 & - & 18.4 & 0.38 & $\chi-\mathrm{Fe}_{5} \mathrm{C}_{2}(\mathrm{II})$ & 22 \\
\hline & 0.18 & - & 11.7 & 0.38 & $\chi-\mathrm{Fe}_{5} \mathrm{C}_{2}$ (III) & 16 \\
\hline & 0.16 & - & 20.9 & 0.38 & $\theta-\mathrm{Fe}_{3} \mathrm{C}$ & 34 \\
\hline & 0.17 & 1.02 & - & 0.30 & $\mathrm{Fe}_{\mathrm{x}} \mathrm{C}(\mathrm{SPM})$ & 6 \\
\hline \multirow[t]{5}{*}{ Na-S 440} & 0.23 & - & 21.7 & 0.35 & $\chi-\mathrm{Fe}_{5} \mathrm{C}_{2}(\mathrm{I})$ & 20 \\
\hline & 0.18 & - & 18.7 & 0.35 & $\chi-\mathrm{Fe}_{5} \mathrm{C}_{2}(\mathrm{II})$ & 20 \\
\hline & 0.15 & - & 11.8 & 0.35 & $\chi-\mathrm{Fe}_{5} \mathrm{C}_{2}$ (III) & 10 \\
\hline & 0.20 & - & 20.7 & 0.36 & $\theta-\mathrm{Fe}_{3} \mathrm{C}$ & 44 \\
\hline & 0.15 & 1.02 & - & 0.45 & $\mathrm{Fe}_{\mathrm{x}} \mathrm{C}(\mathrm{SPM})$ & 6 \\
\hline Na-S 340 & 0.27 & - & 21.6 & 0.35 & $\chi-\mathrm{Fe}_{5} \mathrm{C}_{2}(\mathrm{I})$ & 26 \\
\hline \multirow[t]{6}{*}{ h.p. } & 0.23 & - & 17.9 & 0.35 & $\chi-\mathrm{Fe}_{5} \mathrm{C}_{2}(\mathrm{II})$ & 24 \\
\hline & 0.16 & - & 10.2 & 0.35 & $\chi-\mathrm{Fe}_{5} \mathrm{C}_{2}$ (III) & 20 \\
\hline & 0.32 & -0.25 & 22.3 & 0.35 & $\theta-\mathrm{Fe}_{7} \mathrm{C}_{3}(\mathrm{I})$ & 7 \\
\hline & 0.21 & 0.30 & 16.2 & 0.35 & $\theta-\mathrm{Fe}_{7} \mathrm{C}_{3}(\mathrm{II})$ & 11 \\
\hline & 0.30 & 0.01 & 19.2 & 0.35 & $\theta-\mathrm{Fe}_{7} \mathrm{C}_{3}$ (III) & 5 \\
\hline & 0.23 & 1.12 & - & 0.45 & $\mathrm{Fe}_{\mathrm{x}} \mathrm{C}(\mathrm{SPM})$ & 7 \\
\hline
\end{tabular}

Experimental uncertainties: Isomer Shift $\pm 0.01 \mathrm{~mm} \mathrm{~s}^{-1}$, Quadrupole Splitting $\pm 0.01 \mathrm{~mm} \mathrm{~s}^{-1}$, Line Width $\pm 0.01 \mathrm{~mm} \mathrm{~s}^{-1}$, Hyperfine Field $\pm 0.1 \mathrm{~T}$, Spectral Contribution $\pm 3 \%$. SPM = Superparamagnetic.

quantification results. 
As discussed with the $\mathrm{H}_{2}$ treated catalyst materials, reoxidation of the (near)zerovalent Fe i.e. $\alpha$-Fe or carbides, is expected to take place upon air exposure after the respective carburization treatments. ${ }^{69,70}$ The more accurate MAS quantification results with regard to the Fe oxides show roughly a doubling in the quantified Fe oxide content from $15-17 \%$ to $25-39 \%$ (Figure 3.13a), when comparing the $\mathrm{H}_{2}$ to 240 and 290 treated catalyst materials. As the Fe oxide content increases quite a bit in comparison to the "reoxidation baseline case" of the $\mathrm{H}_{2}$ treated catalyst materials, other phenomena than simply a Fe reoxidation by air exposure, are also likely to play a role.

The increased Fe oxidation can possibly be due to the $\mathrm{O}$ from $\mathrm{CO}$ dissociation during the $\mathrm{CO}$ carburization behaving as an oxidation agent. ${ }^{78}$ As possible explanations: i) the $\mathrm{O}$ dissociated from $\mathrm{CO}$ is poorly removed as $\mathrm{CO}_{2},{ }^{79,80}$ with short applied $\mathrm{CO}$ treatment time of $<3 \mathrm{~h}$ and/or due to low reaction temperature of $<290^{\circ} \mathrm{C}$, and/or ii) the 0 removal product $\mathrm{CO}_{2}$ causes oxidation of $\alpha$-Fe downstream in the catalyst bed.16,27,81-83

Any reoxidized $\mathrm{Fe}$ is expected to reduce poorly back to $\alpha$-Fe for recarburization by CO, with the applied reduction temperatures $\leq 290^{\circ} \mathrm{C} .7^{77}$ Low amounts of $(\approx 1 \%) \mathrm{H}_{2}$ in the $\mathrm{CO}$ stream increase the $\mathrm{CO}$ dissociation rate over $\alpha-\mathrm{Fe} .84,85$ This effect as $\mathrm{H}_{2}$ possibly removes surface $\mathrm{O}$ from dissociated $\mathrm{CO}$ as $\mathrm{H}_{2} \mathrm{O}$ more efficiently than $\mathrm{CO}$ does as $\mathrm{CO}_{2}$. When low temperature $\left(\lesssim 235^{\circ} \mathrm{C}\right)$ carburization is done with a $\mathrm{H}_{2}$ :CO mixture, instead of pure $\mathrm{CO}$, the resulting Fe oxidization degree in the Fe carburization process might be decreased.12 Or alternatively, 0 -free hydrocarbons can also be used for the $\alpha$-Fe carburization to decrease Fe reoxidation. ${ }^{78}$

Thus, indirectly, the quantification results for 240 and 290 catalyst materials can be interpreted to support the thought of the Fe oxidation via poor removal of $\mathrm{O}$ from $\mathrm{CO}$ dissociation by $\mathrm{CO}$ only. In addition, the oxidative effect by $\mathrm{CO}_{2}$ can participate to the $\mathrm{Fe}$ reoxidation process but this effect is harder to determine based on the results. Worth noting is that the addition of Na-S promoters increases the Fe oxide formation after the catalyst treatments 240 and 290, from 25-27\% with UP to $39 \%$ with Na-S catalyst materials, on the basis of the MAS quantifications (Figure 3.13a). This oxidation effect will be discussed more later on in the Chapter along with rest of the Na-S promotion effects on Fe carburization.

The $\mathrm{Fe}$ oxide content decreases in respect to the $\mathrm{H}_{2}$ catalyst materials with the catalyst treatments with longer $\mathrm{CO}$ exposure times of $\geq 16 \mathrm{~h}$ and above temperature of $340^{\circ} \mathrm{C}$, i.e. with the treatments $340,390,440$. This is observed with both the R-QPA and MAS quantifications (Figure 3.13a-b). That is, the catalyst materials' oxidation by 0 from the $\mathrm{CO}$ dissociation does not play a role, and the catalyst materials' reoxidation by air exposure is also decreased. Both the higher carburization temperature and the increased $\mathrm{CO}$ exposure time are expected to improve the $\mathrm{O}$ removal as $\mathrm{CO}_{2}$, and to increase the re-reduction of any reoxidized $\alpha-F e .{ }^{77}$ Thus the overall carburization 
Identification of Iron Carbides in Fe(-Na-S)/ $\alpha$-Al203 Fischer-Tropsch Synthesis Catalysts with X-ray Powder Diffractometry and Mössbauer Absorption Spectroscopy

extent increases as observed. Also, the observed increase in the carbon deposition by the Boudouard reaction (Figure 3.6), 16,62-64,66 likely decreases the Fe phases propensity for reoxidation upon exposure to air.

\section{B. Fe Carbide Formation below $\approx 300^{\circ} \mathrm{C}$}

After the mildest $\mathrm{CO}$ carburization treatment $240, \varepsilon-\mathrm{Fe}_{3} \mathrm{C}$ and $\eta-\mathrm{Fe}_{2} \mathrm{C}$ are the major $\mathrm{Fe}$ carbide phases formed on the catalyst materials (Figure 3.13a-b). Small amounts of residual $\alpha$-Fe can be detected as a sign of incomplete carburization for both UP and NaS 240 catalyst materials. With R-QPA, also small amounts of $\chi-\mathrm{Fe}_{5} \mathrm{C}_{2}$ (8-9\%) after the catalyst treatment 240 could be quantified (Figure 3.13b). However, MAS did not quantify any of the $\chi-\mathrm{Fe}_{5} \mathrm{C}_{2}$ phase for either the UP or the Na-S catalyst materials (Figure 3.13a). In the XRPD patterns for the 240 catalyst materials, weak diffraction peaks originating from the $\chi-\mathrm{Fe}_{5} \mathrm{C}_{2}$ phase can be seen, perhaps best, from the two small peaks at $\approx 21.3^{\circ}$ and $\approx 22.5^{\circ} 2 \theta$ (Figure 3.10a and Figure 3.11a).

With further increasing the catalyst $\mathrm{CO}$ carburization time and temperature with the catalyst treatment 290 , the contribution from the $\chi-\mathrm{Fe}_{5} \mathrm{C}_{2}$ phase increases. At the same time, the $\eta-\mathrm{Fe}_{2} \mathrm{C}$ phase is still present, while the $\varepsilon-\mathrm{Fe}_{3} \mathrm{C}$ phase is no longer observed with either R-QPA or MAS (Figure 3.13a-b).

The $\varepsilon-\mathrm{Fe}_{3} \mathrm{C}$ phase is expected to form within a carburization the temperature range of $100-265^{\circ} \mathrm{C}, 30,31,34,73,86-89$ and the $\eta-\mathrm{Fe}_{2} \mathrm{C}$ phase between $160-300^{\circ} \mathrm{C} .2,19,30-32,34,87-90$ Thus, the R-QPA and MAS quantification results with regard to the $\varepsilon-\mathrm{Fe}_{3} \mathrm{C}$ and $\eta-\mathrm{Fe}_{2} \mathrm{C}$ phases are in good agreement with the literature.

Furthermore, in literature, the formation of the $\chi-\mathrm{Fe}_{5} \mathrm{C}_{2}$ phase has been observed to start, with $\alpha$-Fe as the starting material, from temperatures of $\approx 250-260^{\circ} \mathrm{C} .86,87,91 \mathrm{As}$ a notable discrepancy, Niemantsverdriet et al. have observed the formation of the $\chi-\mathrm{Fe}_{5} \mathrm{C}_{2}$ phase to already start from a temperature of $190^{\circ} \mathrm{C} .{ }^{2}$ This temperature is a lot lower than expected, on the basis of both the R-QPA and MAS quantifications (Figure 3.13ab) and the literature.

With the use of MAS, Niemantsverdriet et al. observed formation of Fe carbides from $\alpha$-Fe under $\mathrm{H}_{2}$ :CO $1: 1$ at 1 bar as (when following our nomenclature): at $190^{\circ} \mathrm{C}(24 \mathrm{~h}$ ) unknown quantity of $\eta-\mathrm{Fe}_{2} \mathrm{C}$ and $36 \% \chi-\mathrm{Fe}_{5} \mathrm{C}_{2}$, at $240^{\circ} \mathrm{C}(24 \mathrm{~h}) 35 \% \eta-\mathrm{Fe}_{2} \mathrm{C}$ and $65 \%$ $\chi-\mathrm{Fe}_{5} \mathrm{C}_{2}$, at $350^{\circ} \mathrm{C}(3 \mathrm{~h}) 12 \% \eta-\mathrm{Fe}_{2} \mathrm{C}, 47 \% \chi-\mathrm{Fe}_{5} \mathrm{C}_{2}$ and $41 \% \theta-\mathrm{Fe}_{3} \mathrm{C}$ and at $450^{\circ} \mathrm{C}(1.5 \mathrm{~h})$ $100 \% \theta-\mathrm{Fe}_{3} \mathrm{C} .{ }^{2}$ When comparing the results by Niemantsverdriet et al. to the quantification results for the Fe carbides in Figure 3.13a-b, it can observed that the results by Niemantsverdriet et al. would compare more reasonably, if a constant temperature shift of $\approx+50^{\circ} \mathrm{C}$ is applied to their data.

With FTS reaction under $\mathrm{H}_{2}$ : CO 2:1 at 1.03 bar at $200^{\circ} \mathrm{C}(3.9 \mathrm{~h})$ Boellaard, van der Kraan and Geus, ${ }^{30}$ quantified Fe carbides with MAS as $18 \% \varepsilon-\mathrm{Fe}_{3} \mathrm{C}$ and $54 \% \eta$-Fe ${ }_{2} \mathrm{C}$. No observation of the $\chi-\mathrm{Fe}_{5} \mathrm{C}_{2}$ phase was made. In a similar manner, with MAS 
quantification, Amelse et al. observed only the $\varepsilon-\mathrm{Fe}_{3} \mathrm{C}$ and $\eta-\mathrm{Fe}_{2} \mathrm{C}$ phases after exposing $\alpha$-Fe to $\mathrm{H}_{2}: \mathrm{CO} 3: 1$ at $250^{\circ} \mathrm{C}(2.5 \mathrm{~h})$ at atmospheric pressure, without observing any presence of the $\chi-\mathrm{Fe}_{5} \mathrm{C}_{2}$ phase. ${ }^{73}$ At higher carburization temperatures, Jiang et al. have quantified with MAS $14 \% \mathrm{Fe}_{3} \mathrm{O}_{4}, 25 \% \eta-\mathrm{Fe}_{2} \mathrm{C}$ and $61 \% \chi-\mathrm{Fe}_{5} \mathrm{C}_{2}$ after $\mathrm{H}_{2}: \mathrm{CO} 1: 1$ at 10 bar at $300^{\circ} \mathrm{C}(50 \mathrm{~h})$ with pre-reduced $\mathrm{K}$ promoted Fe catalyst materials..$^{90}$ The results at $240^{\circ} \mathrm{C}(24 \mathrm{~h})$ by Niemantsverdriet et al. seem very similar to the results by Jiang et al. at $300^{\circ} \mathrm{C}(50 \mathrm{~h})$, and are the best match to the R-QPA and MAS results with the catalyst treatment $290\left(\mathrm{CO}, 290^{\circ} \mathrm{C}, 3 \mathrm{~h}\right)$ (Figure 3.13a-b). Again, all the above literature examples were interpreted to follow our nomenclature for the Fe carbide phases.

Thus, we shall presume a temperature offset of $+50^{\circ} \mathrm{C}$ to all of the results by Niemantsverdriet et al., ${ }^{2}$ after which their quantifications are in a good agreement with the R-QPA and MAS results in Figure 3.13a-b, and also with the above literature examples.

The exception in the results by Niemantsverdriet et al. in comparison to the R-QPA and MAS quantifications in Figure 3.13a-b, is that even with the $+50^{\circ} \mathrm{C}$ shift some $\eta$ $\mathrm{Fe}_{2} \mathrm{C}(12 \%)$ should be present even after carburizing reaction at $400^{\circ} \mathrm{C}(3 \mathrm{~h})$. Furthermore, Wezendonk et al. have observed the $\eta-\mathrm{Fe}_{2} \mathrm{C}$ phase at $340^{\circ} \mathrm{C}(\geq 24 \mathrm{~h})$ in their Fe carburization and FTS reaction studies. ${ }^{13}$ We could not observe any $\eta-\mathrm{Fe}_{2} \mathrm{C}$ with $\geq 340^{\circ} \mathrm{C} \mathrm{CO}$ carburization temperature, i.e. with the catalyst treatments $340-440$. The common denominator for both of the cited studies is that the $\eta-\mathrm{Fe}_{2} \mathrm{C}$ phase is preformed first at a lower carburization temperature and then heated subsequently to a higher reaction temperature. Thus, if a $\eta-\mathrm{Fe}_{2} \mathrm{C}$ phase is present a priori to the FTS reaction, some of it may remain stable i.e. its carbide-to-carbide transition is hindered with reaction temperatures higher than $300^{\circ} \mathrm{C}$. However, on the basis of the quantification results in Figure 3.13a-b: $\eta$-Fe $e_{2} \mathrm{C}$ does not directly form at $340^{\circ} \mathrm{C}$ when $\alpha$-Fe is used as the starting phase for the carburization.

Thus, we assign $160-300^{\circ} \mathrm{C}$ as formation temperature for the $\eta-\mathrm{Fe}_{2} \mathrm{C}$ phase from $\alpha$ Fe. Nonetheless, a maximum thermal stability of the phase can reach at least $\approx 340^{\circ} \mathrm{C}$ if the $\eta-\mathrm{Fe}_{2} \mathrm{C}$ phase is preformed prior to the temperature increase. We acknowledge but exclude the rather high $400^{\circ} \mathrm{C}$ maximum thermal stability for the $\eta-\mathrm{Fe}_{2} \mathrm{C}$ phase as observed by Niemantsverdriet et al. for now, due to possible uncertainty in the reaction temperature.

\section{Fe Carbide Formation above $\approx 300^{\circ} \mathrm{C}$}

With increasing severity of the CO carburization with treatments 340,390 , and 440, only $\chi-\mathrm{Fe}_{5} \mathrm{C}_{2}$ and $\theta-\mathrm{Fe}_{3} \mathrm{C}$ are observed as the Fe carbide phases with both R-QPA and MAS quantifications (Figure 3.13a-b). Some SPM, poorly crystalline $\mathrm{Fe}_{\mathrm{x}} \mathrm{C}$ phases are also observed with MAS quantifications after the higher temperature catalyst carburization treatments (Figure 3.13a). These phases are not observable with XRPD- 
Identification of Iron Carbides in Fe(-Na-S)/ $\alpha$-Al203 Fischer-Tropsch Synthesis Catalysts with X-ray Powder Diffractometry and Mössbauer Absorption Spectroscopy

based R-QPA (Figure 3.13b). The poorly crystalline $\mathrm{Fe}_{\mathrm{x}} \mathrm{C}$ phases could perhaps be related to intergrowth structures of the $\chi-\mathrm{Fe}_{5} \mathrm{C}_{2}$ and $\theta-\mathrm{Fe}_{3} \mathrm{C}$ phases. ${ }^{92-94}$

After the catalyst treatment 340 , only the $\chi-\mathrm{Fe}_{5} \mathrm{C}_{2}$ phase is observed as the sole carbide phase and the treatment 340 is the only one to form a near pure, single Fe carbide phase catalyst. Both R-QPA and MAS agree on the quantification (Figure 3.13ab), and the XRPD patterns do not show any unassigned diffraction peaks (Figure 3.10c and Figure 3.11c). The expected formation temperature range for the $\chi-\mathrm{Fe}_{5} \mathrm{C}_{2}$ phase is about $250-400^{\circ} \mathrm{C}$ as based on the experimental literature observations. ${ }^{6,15,22,73,86-}$ $88,90,91,95$

With both R-QPA and MAS quantifications, formation of the $\chi-\mathrm{Fe}_{5} \mathrm{C}_{2}$ phase from $\alpha$-Fe is still observed with the catalyst treatment 440 . That is, the $\chi-\mathrm{Fe}_{5} \mathrm{C}_{2}$ phase forms at as high as the temperature of $440^{\circ} \mathrm{C}$ with $16 \mathrm{~h} \mathrm{CO}$ exposure time (Figure 3.13a-b). This temperature is $\approx+40^{\circ} \mathrm{C}$ higher than the highest observations in literature for the $\chi-\mathrm{Fe}_{5} \mathrm{C}_{2}$ phase's high temperature formation. Nagakura observed preservation of the $\chi-\mathrm{Fe}_{5} \mathrm{C}_{2}$ phase after heating their precursor carbide at $400^{\circ} \mathrm{C}$ for $0.1 \mathrm{~h} .{ }^{86}$ With the assumption of the previously discussed $+50^{\circ} \mathrm{C}$ temperature shift, $400^{\circ} \mathrm{C}$ is also the highest observation temperature for the $\chi-\mathrm{Fe}_{5} \mathrm{C}_{2}$ phase by Niemantsverdriet et al. ${ }^{2}$

As viewing from the diffraction patterns in Figure 3.10c-d and Figure 3.11c-d, the increasing amount of the $\theta-\mathrm{Fe}_{3} \mathrm{C}$ phase overlaps with the diffraction from the most intense peaks of the $\chi-\mathrm{Fe}_{5} \mathrm{C}_{2}$ phase, obfuscating its presence. Moreover, with the increasing carburization temperature the crystallite diameters of the $\chi-\mathrm{Fe}_{5} \mathrm{C}_{2}$ phase also decrease (Figure 3.16), further complicating its identification from the XRPD patterns. Nevertheless, the weak diffraction peaks at $\approx 21.3^{\circ} 2 \theta$ and $\approx 22.5^{\circ} 2 \theta$ originating from the $\chi-\mathrm{Fe}_{5} \mathrm{C}_{2}$ phase, make the presence of the phase clear in the XRPD patterns even after the catalyst treatment 440 (Figure 3.10e and Figure 3.11e). The mentioned $\chi-\mathrm{Fe}_{5} \mathrm{C}_{2}$ phase diffraction peak obfuscating effects in relation to the presence of the $\theta-\mathrm{Fe}_{3} \mathrm{C}$ phase might explain the lack of literature observations for $\chi-\mathrm{Fe}_{5} \mathrm{C}_{2}$ up to temperature of $440^{\circ} \mathrm{C}$, at least for the diffraction based methods.

Thus, together with the quantification of the $\chi-\mathrm{Fe}_{5} \mathrm{C}_{2}$ phase after the low temperature catalyst treatment 240 , we shall assign the formation temperature range for $\chi-\mathrm{Fe}_{5} \mathrm{C}_{2}$ as about $240-440^{\circ} \mathrm{C}$ (Figure 3.13a-b). This is somewhat higher but close to the temperature range expected from the literature. However, we did not test for highend thermal stability for the $\chi-\mathrm{Fe}_{5} \mathrm{C}_{2}$ phase by heating the phase up until decomposition. That is, the $\chi-\mathrm{Fe}_{5} \mathrm{C}_{2}$ phase is stable and forms at least up to $440^{\circ} \mathrm{C}$ reaction temperature, although even higher formation and stability temperature cannot be excluded.

The presence of $\theta-\mathrm{Fe}_{3} \mathrm{C}$ is expected within the temperature range of $310-600^{\circ} \mathrm{C}$ on the basis of literature. ${ }^{2,23,86,96}$ However, we could not observe any presence of the $\theta-\mathrm{Fe}_{3} \mathrm{C}$ phase in the catalyst materials with carburization treatment temperatures below 
$390^{\circ} \mathrm{C}$.

The lowest observation temperatures for the $\theta-\mathrm{Fe}_{3} \mathrm{C}$ phase from gas phase carburizations are by Nagakura with $350^{\circ} \mathrm{C},{ }^{86}$ and Shultz et al. with $310^{\circ} \mathrm{C} . .^{23}$ Furthermore, in martensite tempering studies, formation of the $\theta-\mathrm{Fe}_{3} \mathrm{C}$ phase as low as $250-267^{\circ} \mathrm{C}$ has been observed..$^{34,92}$ These formation temperatures for the $\theta-\mathrm{Fe}_{3} \mathrm{C}$ phase in the martensite tempering studies seem very low in comparison to other literature, and disagree with the R-QPA and MAS quantification results in Figure 3.13a-b. They are thus excluded from further discussion.

Observations on the formation of the $\theta-\mathrm{Fe}_{3} \mathrm{C}$ phase after carburizing polycrystalline $\alpha$-Fe film with $\mathrm{CO}$ at $350^{\circ} \mathrm{C}(3 \mathrm{~h})$ have been made by Nagakura, with the use of Electron Diffraction (ED). ${ }^{86}$ The closest carburization conditions corresponding to Nagakura's reaction conditions are with the catalyst treatment 340 , with which we could not form any observable amounts of $\theta-\mathrm{Fe}_{3} \mathrm{C}$ using the applied bulk characterization techniques (Figure 3.13a-b). That is, Nagakura's observations at $350^{\circ} \mathrm{C}$ could be very close to the precise lower temperature limit for the $\theta-\mathrm{Fe}_{3} \mathrm{C}$ formation.

With regard to the observations with XRPD by Shultz et al. on formation of $\theta-\mathrm{Fe}_{3} \mathrm{C}$ from $\alpha$-Fe under $\mathrm{H}_{2}$ : $\mathrm{CO} 2: 1$ at $310^{\circ} \mathrm{C}(6 \mathrm{~h}),{ }^{23}$ no imminently clear explanation can be given for the deviation to the R-QPA and MAS Fe quantification results. However, possibly, in the study by Shultz et al., the $\alpha$-Fe was exposed to the carburizing $\mathrm{H}_{2}$ : $\mathrm{CO}$ gas mixture directly at $310^{\circ} \mathrm{C}$, which might have caused significant reactor bed heating as the exothermic FTS reaction is initiated. This idea would be supported by an another experiment by Shultz et al. in the same publication, where by step-wise increasing the CO exposure temperature from $150^{\circ} \mathrm{C}$ to $350^{\circ} \mathrm{C}$ on the $\alpha$-Fe starting material, only $\chi$ $\mathrm{Fe}_{5} \mathrm{C}_{2}$ was formed. ${ }^{23}$ This result by Shultz et al. would be in close agreement with the results in Figure 3.13a-b. Thus, we shall presume that the actual, efficient reactor bed temperature has been a lot higher than $310^{\circ} \mathrm{C}$ in the discussed experiment by Shultz et al.. ${ }^{23}$ Therefore, the $310^{\circ} \mathrm{C}$ data point is ignored from the expected low-end formation temperature for the $\theta-\mathrm{Fe}_{3} \mathrm{C}$ phase.

We did not test for the high-end temperature limit for the $\theta-\mathrm{Fe}_{3} \mathrm{C}$ phase formation or stability. With gas phase carburizations at $500^{\circ} \mathrm{C}$ : Shultz et al. observed $\theta-\mathrm{Fe}_{3} \mathrm{C}$ with $6 \mathrm{~h}$ carburization of $\alpha$-Fe with $\mathrm{CH}_{4},{ }^{23}$ while also under $\mathrm{H}_{2}: \mathrm{CO}(1.5 \mathrm{~h}),{ }^{2}$ and $\mathrm{CO}(1 \mathrm{~h}),{ }^{86}$ the $\theta-\mathrm{Fe}_{3} \mathrm{C}$ phase has been detected. Jozwiak et al. reduced $\alpha-\mathrm{Fe}_{2} \mathrm{O}_{3}$ with $\mathrm{CO}$ to $\alpha$ - $\mathrm{Fe}$ at $500^{\circ} \mathrm{C}$ without formation of the $\theta-\mathrm{Fe}_{3} \mathrm{C}$ phase. ${ }^{77}$

By heat treating the preformed $\theta-\mathrm{Fe}_{3} \mathrm{C}$ phase Wood et al. have observed initial slow, at $377^{\circ} \mathrm{C}$, and rapid decomposition of $\theta-\mathrm{Fe}_{3} \mathrm{C}$ to $\alpha$-Fe above $527^{\circ} \mathrm{C} . .^{97}$ Other authors have still observed the $\theta-\mathrm{Fe}_{3} \mathrm{C}$ phase after heating their catalyst materials between 525 $550^{\circ} \mathrm{C} .86,96$ The highest stability temperature for the $\theta-\mathrm{Fe}_{3} \mathrm{C}$ phase has been observed by Nagakura, who observed the $\theta-\mathrm{Fe}_{3} \mathrm{C}$ phase even after heating a preformed Fe carbide 
Identification of Iron Carbides in Fe(-Na-S)/ $\alpha$-Al203 Fischer-Tropsch Synthesis Catalysts with X-ray Powder Diffractometry and Mössbauer Absorption Spectroscopy

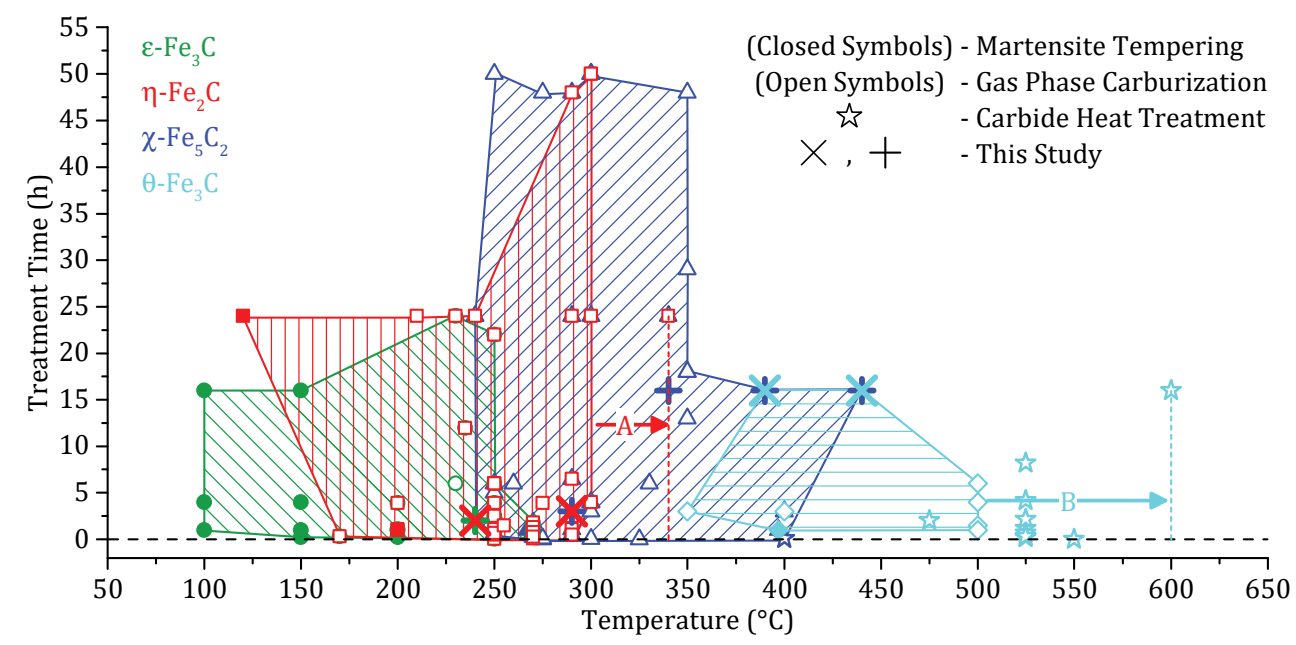

\section{Figure 3.14}

Fe carbide formation regions (hatched fill) as temperature versus treatment exposure time as based on the available experimental literature data and this Chapter. Arrows: Maximum thermal stability with preformed Fe carbides for $(A) \eta-\mathrm{Fe}_{2} \mathrm{C}$ and $(\mathrm{B}) \theta-\mathrm{Fe}_{3} \mathrm{C}$. Formation temperatures for the $\theta-\mathrm{Fe}_{7} \mathrm{C}_{3}$ phase are excluded. Figure is modified from Chapter 2.

for $16 \mathrm{~h}$ in vacuum at $600^{\circ} \mathrm{C} .86$

Podgurski et al. have noted that decomposition of a high temperature carbide, most likely $\theta-\mathrm{Fe}_{3} \mathrm{C}$, to $\alpha$-Fe takes place at $500^{\circ} \mathrm{C}$. If a Fe particle surface oxide was formed e.g. possibly when $\mathrm{CO}$ is used as a carburizing agent in comparison to 0 -free hydrocarbons, the decomposition temperature of the carbide to $\alpha$-Fe was increased. ${ }^{78}$ Thus, the higher-end stability temperature of the $\theta-\mathrm{Fe}_{3} \mathrm{C}$ phase is dependent on how easily the $\mathrm{C}$ atoms can be expulsed from the $\theta-\mathrm{Fe}_{3} \mathrm{C}$ lattice, allowing the carbide phase to decompose to $\alpha$-Fe and $\mathrm{C}$ atoms. Thus, the presence or absence of carbide decomposition inhibiting shell oxide layers on $\theta-\mathrm{Fe}_{3} \mathrm{C}$ phase can be used to explain the discussed differences in the $\theta-\mathrm{Fe}_{3} \mathrm{C}$ phase stability between $500-600^{\circ} \mathrm{C}$.

Finally, as based on the R-QPA and MAS quantifications (Figure 3.13a-b) and the discussion on the literature observations: we assign the formation temperature range for the $\theta-\mathrm{Fe}_{3} \mathrm{C}$ phase as $350-500^{\circ} \mathrm{C}$. A preformed $\theta-\mathrm{Fe}_{3} \mathrm{C}$ phase can be stable up to $600^{\circ} \mathrm{C}$ if $\mathrm{C}$ atom expulsion from the $\theta-\mathrm{Fe}_{3} \mathrm{C}$ lattice is hindered due to a $\mathrm{Fe}$ oxide layer. ${ }^{78}$ Without the $\mathrm{Fe}$ oxide layer, $\theta-\mathrm{Fe}_{3} \mathrm{C}$ decomposes readily to $\alpha$ - $\mathrm{Fe}$ and $\mathrm{C}$ species above $527^{\circ} \mathrm{C} .97$

The Fe carbide formation temperature regions have been updated from the purely literature based observations of noninterpreted data points in Chapter 2 to the Figure 3.14 as based on the presented discussion. The updated Figure 3.14 also includes the R-QPA and MAS quantification based observations in this Chapter for the Fe carbides (Figure 3.13a-b). The rejected data points and the $+50^{\circ} \mathrm{C}$ shift to the 


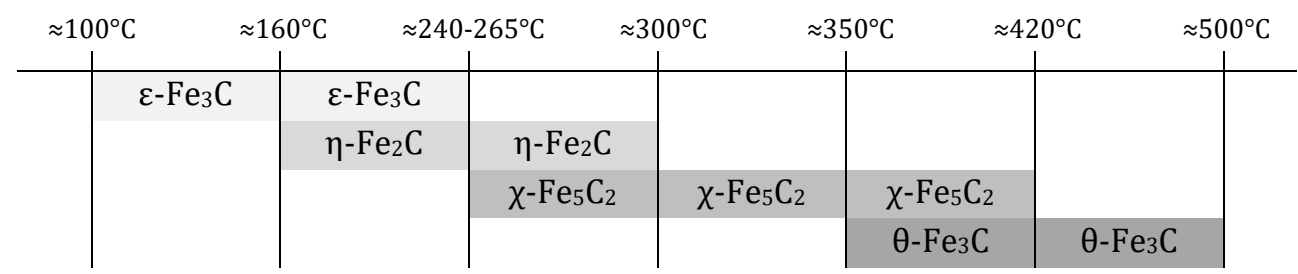

Figure 3.15

The Fe carbide formation temperature ranges as estimated from the Figure $\mathbf{3 . 1 4}$ at $5 \mathrm{~h}$ treatment time. The $\theta-\mathrm{Fe}_{7} \mathrm{C}_{3}$ phase is excluded as its formation is a more complex, not a solely temperature dependent, phenomenon.

reaction temperatures in the results by Niemantsverdriet et al., ${ }^{2}$ have also been updated to the Figure 3.14. The complete list of source publications for the literature data points in Figure 3.14 is given in Chapter 2.

With the applied modifications, the Fe carbide formation temperature regions are (Figure 3.15): $\varepsilon-\mathrm{Fe}_{3} \mathrm{C}\left(100-265^{\circ} \mathrm{C}\right), \eta-\mathrm{Fe}_{2} \mathrm{C}\left(160-300^{\circ} \mathrm{C}\right), \chi-\mathrm{Fe}_{5} \mathrm{C}_{2}\left(240-420^{\circ} \mathrm{C}\right)$ and $\theta-$ $\mathrm{Fe}_{3} \mathrm{C}\left(350-500^{\circ} \mathrm{C}\right)$, when compared at $5 \mathrm{~h}$ treatment time in the Figure 3.14. Furthermore, the $\chi-\mathrm{Fe}_{5} \mathrm{C}_{2}$ high-end formation temperature can be as high as $440^{\circ} \mathrm{C}$ after a longer, $16 \mathrm{~h} \mathrm{CO}$ carburization time. The preformed $\eta-\mathrm{Fe}_{2} \mathrm{C}$ and $\theta-\mathrm{Fe}_{3} \mathrm{C}$ phases can be stable up to $\approx 340^{\circ} \mathrm{C}$ and $\approx 600^{\circ} \mathrm{C}$, respectively, but do not directly form at these temperatures from $\alpha$-Fe.

As a final note on the Fe carbide formation temperatures: for the formation of the $\varepsilon$ $\mathrm{Fe}_{3} \mathrm{C}$ phase within the temperature range of $\approx 100-160^{\circ} \mathrm{C}$, the synthesis route will likely need to go via martensite tempering (Figure 3.14). At these very low carburization temperatures $\mathrm{CO}$ adsorption/dissociation products do not necessarily desorb, ${ }^{98}$ likely inhibiting $\alpha$-Fe carburization via a gas phase C0.12,99

\subsubsection{The Effect of Na-S Promotion}

When comparing the UP and Na-S catalyst materials for the formation of various Fe carbides with the catalyst treatments 240-440: the relative formation quantities for the different Fe carbides from $\alpha$-Fe are not affected by the added Na-S promotion in any major way (Figure 3.13a-b).

Significant effects from the Na-S promotion on $\alpha$-Fe carburization are (Figure 3.13): i) the increased Fe oxide contribution with treatments 240 and 290 and ii) the added $\mathrm{Na}-\mathrm{S}$ promotion seems to increase the $\chi-\mathrm{Fe}_{5} \mathrm{C}_{2}$ phase content with expense of the $\theta-\mathrm{Fe}_{3} \mathrm{C}$ phase with treatments 390 and 440. In MAS observations, a non-SPM (i.e. larger crystallite diameter) Fe phase with $\mathrm{Fe}^{3+}$ appeared after catalyst treatments 240 and 290 with the addition of the Na-S promotion (Table 3.4).

The increased Fe oxide contribution with Na-S 240 and Na-S 290 catalyst materials in relation to the respective UP catalyst materials is in agreement with both R-QPA and 
Identification of Iron Carbides in Fe(-Na-S)/ $\alpha$-Al203 Fischer-Tropsch Synthesis Catalysts with X-ray Powder Diffractometry and Mössbauer Absorption Spectroscopy

MAS quantifications (Figure 3.13a-b). Addition of alkali in general decreases the reducibility of Fe oxides by $\mathrm{H}_{2}{ }^{26,72,100}$ possibly by stabilizing the oxides. ${ }^{72}$ Together with the earlier discussion on the possible oxidative effects due to the $\mathrm{O}$ atoms from $\mathrm{CO}$ dissociation, we thus assign the increased Fe oxidation with Na-S 240 and Na-S 290 catalyst materials to the increased stability for Fe oxide formation. From the treatment 340 upwards towards higher carburization temperatures and times, the Na-S effect is absent as the gas environment becomes more reducing with $\mathrm{CO}$ (and $\mathrm{H}_{2}$ ). ${ }^{77}$ The increased carburization/reduction temperature likely overcomes the possibly increased Fe oxide stability.

Regarding the relative stabilities of the $\chi-\mathrm{Fe}_{5} \mathrm{C}_{2}$ and $\theta-\mathrm{Fe}_{3} \mathrm{C}$ phases, the $\mathrm{Na}-\mathrm{S}$ promotion stabilizes the $\chi-\mathrm{Fe}_{5} \mathrm{C}_{2}$ while decreasing the presence of the $\theta-\mathrm{Fe}_{3} \mathrm{C}$ phase with the high temperature catalyst treatments 390 and 440 (Figure 3.13a-b). In the R-QPA quantifications: the $\chi-\mathrm{Fe}_{5} \mathrm{C}_{2}$ phase amount is increased $\approx+10 \%$ in the $\mathrm{Na}-\mathrm{S}$ catalyst materials after the treatments 390 and 440 , with the expense of the $\theta-\mathrm{Fe}_{3} \mathrm{C}$ phase. The MAS quantifications also agree on the trend of the decreased $\theta-\mathrm{Fe}_{3} \mathrm{C}$ phase content after the catalyst treatments.

As further effects on the Na-S promotion in Fe carburization it can be mentioned that occasionally in literature the addition of alkali has been cited to increase the carburization of Fe catalysts, but the R-QPA and MAS results do not agree with this (Figure 3.13a-b). The observed differences can be explained with the starting Fe phase for the carburization, i.e. whether the carburization process is started with $\alpha$-Fe or a Fe oxide.

Berry and Smith have observed, with MAS, that the added alkali K promotion aids in carburization of the starting $\alpha-\mathrm{Fe}_{2} \mathrm{O}_{3}$ material. ${ }^{101}$ Under pure $\mathrm{CO}$ at $270^{\circ} \mathrm{C}$ after $12 \mathrm{~h}$ of CO exposure time, the added alkali K enhanced the Fe carbide formation from the $\alpha$ $\mathrm{Fe}_{2} \mathrm{O}_{3}$ starting material in comparison to the unpromoted catalyst. Interestingly, with a short $\mathrm{CO}$ exposure time of $2 \mathrm{~h}$ at $270^{\circ} \mathrm{C}$ regardless of the $\mathrm{K}$ promotion, the authors observed that only $\mathrm{Fe}_{3} \mathrm{O}_{4}$ formed without any Fe carbides. The dependency of the extent of carburization on the $\mathrm{CO}$ exposure time would also explain the previously discussed increased Fe oxidation with $\mathrm{Na}-\mathrm{S} 240$ and Na-S 290 catalyst materials with the short exposure times ( $\leq 3 \mathrm{~h}$ ) (Figure 3.13a-b). The increased CO carburization rate of $\alpha-\mathrm{Fe}_{2} \mathrm{O}_{3}$ starting materials due to the alkali promotion has been also observed by Ribeiro et al., ${ }^{102}$ and also can be seen in MAS quantification results by both O'Brien et al. and Bukur et al. 26,83

The addition of alkali (as $\mathrm{Na}$ or $\mathrm{K}$ ) promotes $\mathrm{CO}_{2}$ releasing Water-gas Shift (WGS) reaction with $\mathrm{Fe}$ catalyst materials. ${ }^{79,103,104}$ The addition of alkali has been observed to increase the exchangeability of surface $\mathrm{O}$ atoms with $\mathrm{CO}$ on $\mathrm{K}$ promoted $\mathrm{Al}_{2} \mathrm{O}_{3}$, especially at lower temperatures of $\approx 300^{\circ} \mathrm{C} .{ }^{105}$ Also, increase in the rate of the WGS reaction with 
the same transition metal-free $\mathrm{K} / \mathrm{Al}_{2} \mathrm{O}_{3}$ catalyst material has been observed. ${ }^{106}$ That is, the addition of alkali seems to enhance the interaction of $\mathrm{CO}$ with the catalyst surface $\mathrm{O}$ atoms, while also increasing the WGS reaction rate. The enhanced $\mathrm{O} / \mathrm{CO}$ interaction could also suggest a possibility for improved Fe oxide reduction by $\mathrm{CO}$ in presence of alkali.

Effectively then, according to the presented discussion, with the added alkali to a Fe oxide: the Fe oxide's reducibility to zerovalent $\mathrm{Fe}$ should be i) increased at low temperatures $\left(\approx 300^{\circ} \mathrm{C}\right)$ with $\mathrm{CO}$ as the reductant when sufficient $\mathrm{CO}$ exposure time ( $>3 \mathrm{~h}$ ) is applied, ${ }^{26,101,105}$ and ii) decreased with $\mathrm{H}_{2},{ }^{26,72,100}$ due to Fe oxide stabilization by the alkali.

Indeed, in a XRPD study where a precursor $\alpha-\mathrm{Fe}_{2} \mathrm{O}_{3}$ was carburized directly with i) pure $\mathrm{CO}$ or, ii) a $\mathrm{H}_{2}: \mathrm{CO} 100: 1$ mixture, the Fe reduction steps for the $\alpha-\mathrm{Fe}_{2} \mathrm{O}_{3}$ were modified. ${ }^{3}$ As reading from the XRPD patterns in the study; the Fe reduction steps can be seen to shift for $\alpha-\mathrm{Fe}_{2} \mathrm{O}_{3} \rightarrow \mathrm{Fe}_{3} \mathrm{O}_{4}\left(\approx+35^{\circ} \mathrm{C}\right), \mathrm{Fe}_{3} \mathrm{O}_{4} \rightarrow \mathrm{Fe}_{x} \mathrm{O}$ (wüstite) $\left(\approx+65^{\circ} \mathrm{C}\right)$ and $\mathrm{Fe}_{\mathrm{x}} \mathrm{O}->\mathrm{Fe}_{\mathrm{x}} \mathrm{C}$ (i.e. $\left.\alpha-\mathrm{Fe}\right)\left(\approx+93^{\circ} \mathrm{C}\right)$ when switching the carburizing gas from pure $\mathrm{CO}$ to $\mathrm{a}$ 100:1 $\mathrm{H}_{2}: \mathrm{CO}$ mixture. That is, the $\alpha-\mathrm{Fe}_{2} \mathrm{O}_{3}$ was more poorly reducible with the $100: 1$ $\mathrm{H}_{2}$ :CO mixture than with pure CO. Noteworthy is that in the study, the used $\alpha-\mathrm{Fe}_{2} \mathrm{O}_{3}$ catalyst materials were prepared using $\mathrm{Na}_{2} \mathrm{CO}_{3}$ as the precipitation agent which, despite a washing, ${ }^{107}$ is likely to leave residual $\mathrm{Na}$ in the catalyst with sufficient concentration to act as a promoter. Furthermore, X-ray Fluorescence (XRF) spectrometer was used to evaluate the catalyst material purity, a technique which detects the $\mathrm{Na}$ elements poorly. ${ }^{108}$ The inadvertent $\mathrm{Na}$ promotion would explain both: the more efficient reduction of the $\mathrm{Fe}$ oxides at lower temperatures by $\mathrm{CO}$ as discussed, and the observations on the $\mathrm{Fe}_{\mathrm{x}} \mathrm{O}$ (wüstite) phase during Fe reduction step. ${ }^{72,77}$ The $\mathrm{Fe}_{0.974} \mathrm{O}$ phase is also seen to stabilize for the $\mathrm{Na}-\mathrm{S} \mathrm{H}_{2}$ catalyst material (Figure 3.13b).

Therefore, in total, the increased carburization of alkali promoted Fe oxides can be explained with a more efficient $\mathrm{Fe}$ oxide reduction to $\alpha$-Fe with $\mathrm{CO}$. $\alpha$-Fe then subsequently carburizes to Fe carbides with the available CO. That is, the Fe reduction step to $\alpha$-Fe is enhanced by alkali, not the carburization step when starting with a Fe oxide material under a carburizing atmosphere. This explains the lack of significant $\mathrm{Na}(-\mathrm{S})$ effect in the results on the $\alpha$-Fe carburization with the applied CO treatments 240-440 (Figure 3.13a-b).

\subsubsection{Formation of the $\mathrm{\theta}-\mathrm{Fe}_{7} \mathrm{C}_{3}$ Phase}

Low yet clearly observable amounts of $22-29 \%$ of the $\theta-\mathrm{Fe}_{7} \mathrm{C}_{3}$ phase were formed after the catalyst treatments 340 h.p. (Figure 3.13a-b). With these treatments, the $\alpha-\mathrm{Fe}_{2} \mathrm{O}_{3}$ phase was used as the carburization starting Fe phase, directly for carburization reaction at 25 bar $\mathrm{CO}$ pressure at $340^{\circ} \mathrm{C}$. The addition of the Na-S promotion did not 
Identification of Iron Carbides in Fe(-Na-S)/ $\alpha$-Al203 Fischer-Tropsch Synthesis Catalysts with X-ray Powder Diffractometry and Mössbauer Absorption Spectroscopy

affect the formation of the $\theta-\mathrm{Fe}_{7} \mathrm{C}_{3}$ phase. This while minding for the differences in the applied CO exposure times of $120 \mathrm{~h}$ for the UP and $168 \mathrm{~h}$ for the Na-S 340 h.p. catalyst materials.

The $\theta-\mathrm{Fe}_{7} \mathrm{C}_{3}$ phase can be formed under rather extreme conditions, ${ }^{109-112}$ but may also form under milder FTS reaction conditions which are more relevant to the present discussion. ${ }^{16,24,113}$ In order to form the $\theta-\mathrm{Fe}_{7} \mathrm{C}_{3}$ phase under mild conditions: Tajima and Hirano used $\mathrm{Ba}$ promoted $\mathrm{Fe}_{3} \mathrm{O}_{4}$ carburized with pure $\mathrm{CO}$ under unspecified pressure at $300-375^{\circ} \mathrm{C} .{ }^{24}$ Audier, Bowen and Jones were able to prepare single crystals of $\theta-\mathrm{Fe}_{7} \mathrm{C}_{3}$ from $\alpha$-Fe under $\mathrm{CO}: \mathrm{CO}_{2} \quad 3: 1$ at $500^{\circ} \mathrm{C}, 113$ with undefined, yet presumably, at atmospheric pressure.

As Audier, Bowen and Jones were able to prepare the $\theta-\mathrm{Fe}_{7} \mathrm{C}_{3}$ phase without a $\mathrm{Fe}$ oxide, Ba promotion or high $\mathrm{CO}$ pressure: the results suggests that the common required factor for preparing the $\theta-\mathrm{Fe}_{7} \mathrm{C}_{3}$ phase is none of the mentioned parameters. $\mathrm{A}$ common factor for mild condition preparation of the $\theta-\mathrm{Fe}_{7} \mathrm{C}_{3}$ phase might be the $\mathrm{CO}_{2}$, as Audier, Bowen and Jones used $\mathrm{CO}_{2}$ in their carburizing gas mixture. ${ }^{113}$

In the study by Tajima and Hirano, ${ }^{24}$ perhaps, the role of $\mathrm{Ba}$ has been to aid the Fe oxide reduction by $\mathrm{CO}$, akin to the earlier discussed alkali effect, releasing $\mathrm{CO}_{2}$ in the reduction process. Ba promotes $\mathrm{Fe}$ oxides towards the WGS reaction, ${ }^{104,114,115}$ suggesting similar expected behavior for Ba promotion as for the earlier discussed alkali WGS promotion effect.

At Sasol, formation of the $\theta-\mathrm{Fe}_{7} \mathrm{C}_{3}$ phase has been observed at higher pressure (>60 bar) under industrial FTS reaction conditions in end-of-life catalysts. ${ }^{16}$ The Febased FTS reaction catalysts have the tendency to reoxidize to WGS catalyzing $\mathrm{Fe}_{3} \mathrm{O}_{4}$ over time. ${ }^{16,22,81,82,104,116,117}$ The catalyst reoxidation could explain the formation of the $\theta-\mathrm{Fe}_{7} \mathrm{C}_{3}$ phase at later stages of the catalyst life-time at Sasol along the WGS catalyzing $\mathrm{Fe}_{3} \mathrm{O}_{4},{ }^{16}$ as the WGS reaction releases $\mathrm{CO}_{2} .{ }^{104,116}$

The formation of the $\theta-\mathrm{Fe}_{7} \mathrm{C}_{3}$ phase with catalyst treatment 340 h.p. might then be explained with the increased concentration of $\mathrm{CO}_{2}$ in the carburizing gas stream, due to the reduction of the Fe oxide with C0.77 Perhaps, with the elevated CO pressure, the rate of the initial Fe oxide reduction is momentaneously sufficiently high for increasing the $\mathrm{CO}_{2}$ concentration and thus allowing the $\theta-\mathrm{Fe}_{7} \mathrm{C}_{3}$ formation, irrespective of the added Na-S promotion. The formed quantity of the $\theta-\mathrm{Fe}_{7} \mathrm{C}_{3}$ phase was not affected by the different carburization times (Figure 3.13a-b), i.e. $168 \mathrm{~h}$ for UP as opposed to $120 \mathrm{~h}$ for Na-S catalysts with the catalyst treatment 340 h.p.. This would suggest that the formation of $\theta-\mathrm{Fe}_{7} \mathrm{C}_{3}$ does not take place during the steady state of the applied treatments, but perhaps, during the temperature ramp or early period of the catalyst treatment. This is also when then $\mathrm{CO}_{2}$ concentration in the carburizing gas stream would be expected to be highest due to the Fe oxide reduction. As the carbon deposition 
rates between the catalyst treatments 340 and 340 h.p. are very similar (Figure 3.6b), the deposited carbon from the Boudouard reaction probably does not play a major role in the formation of the $\theta-\mathrm{Fe}_{7} \mathrm{C}_{3}$ phase.

Thus, we speculate that the $\theta-\mathrm{Fe}_{7} \mathrm{C}_{3}$ formation is dependent on the presence of $\mathrm{CO}_{2}$ in the gas mixture during the gas phase carburization. However, more research on the topic should be performed in the future by systematically carburizing $\alpha$-Fe and Fe oxides with $\mathrm{CO}: \mathrm{CO}_{2}$ mixtures. This might reveal whether a $\mathrm{CO}_{2}$ correlation to the $\theta-\mathrm{Fe}_{7} \mathrm{C}_{3}$ formation exists as proposed.

Solely based on the R-QPA and MAS quantifications in Figure 3.13a-b, the formation temperature ranges for the $\theta-\mathrm{Fe}_{7} \mathrm{C}_{3}$ phase cannot be devised. However, according to Tajima and Hirano: the $\theta-\mathrm{Fe}_{7} \mathrm{C}_{3}$ phase is only stable within the temperature range 300 $375^{\circ} \mathrm{C}^{24}$ This temperature range is in agreement with the observation of the $\theta-\mathrm{Fe}_{7} \mathrm{C}_{3}$ phase forming with the catalyst treatment 340 h.p. at $340^{\circ} \mathrm{C}$ (Figure 3.13a-b). Although, the higher end temperature stability of the $\theta-\mathrm{Fe}_{7} \mathrm{C}_{3}$ phase might be dependent in the carbide phase's purity. ${ }^{112} \mathrm{O}$ atom impurity free $\theta-\mathrm{Fe}_{7} \mathrm{C}_{3}$ phase has been witnessed to survive temperatures up to $\approx 400-500^{\circ} \mathrm{C}$ before decomposing to $\alpha$-Fe and $\mathrm{C}$ atoms. ${ }^{112}$

\subsubsection{Changes in Fe Phases' Crystallite Diameters}

The Fe phase (volume-weighted) crystallite mean diameters obtained via the R-QPA, after each applied catalyst treatment, are shown in Figure 3.16.

Few observations are worth a short discussion. The addition of the Na-S promotion has the effect of (Figure 3.16): i) increasing the crystallite diameters for both $\alpha-\mathrm{Fe}_{2} \mathrm{O}_{3}$ (Calcin.) and $\alpha-\mathrm{Fe}\left(\mathrm{H}_{2}\right)$ and ii) decreasing the crystallite diameters for the $\theta-\mathrm{Fe}_{3} \mathrm{C}$ phase (catalyst treatments 390 and 440). Especially with the catalyst treatment 440, the crystallite diameter of the $\theta-\mathrm{Fe}_{3} \mathrm{C}$ phase is decreased from $28.1 \pm 0.9 \mathrm{~nm}$ to $17.2 \pm 0.8 \mathrm{~nm}$ with the added $\mathrm{Na}-\mathrm{S}$ promotion. Only the $\theta-\mathrm{Fe}_{3} \mathrm{C}$ phase seems to be affected by the Na$\mathrm{S}$ promotion, as the rest of the Fe carbides are nearly identical in their crystallite diameters when UP catalyst materials are contrasted with the Na-S materials. At the moment, no precise explanation for the observed crystallite diameter effects, as due to the Na-S promotion addition, can be given. Additional, fundamental research is warranted.

The $\varepsilon-\mathrm{Fe}_{3} \mathrm{C}$ phase possesses the largest $\mathrm{Fe}$ carbide crystallite mean diameters, while excluding the $\theta-\mathrm{Fe}_{3} \mathrm{C}$ phase in the UP 440 catalyst material. The $\varepsilon-\mathrm{Fe}_{3} \mathrm{C}$ phase's crystallite diameters are close to those of $\alpha$-Fe in its UP or Na-S $\mathrm{H}_{2}$ carburization starting materials (Figure 3.16). A similar observation has earlier been made by Nagakura with $\mathrm{ED}: 86$ the $\varepsilon$ - $\mathrm{Fe}_{3} \mathrm{C}$ phase forms from $\alpha$-Fe as single crystals i.e. one $\alpha$-Fe crystallite transforms into one $\varepsilon-\mathrm{Fe}_{3} \mathrm{C}$ crystallite. This explains the similarity between the $\alpha$-Fe and $\varepsilon-\mathrm{Fe}_{3} \mathrm{C}$ crystallite diameters. 


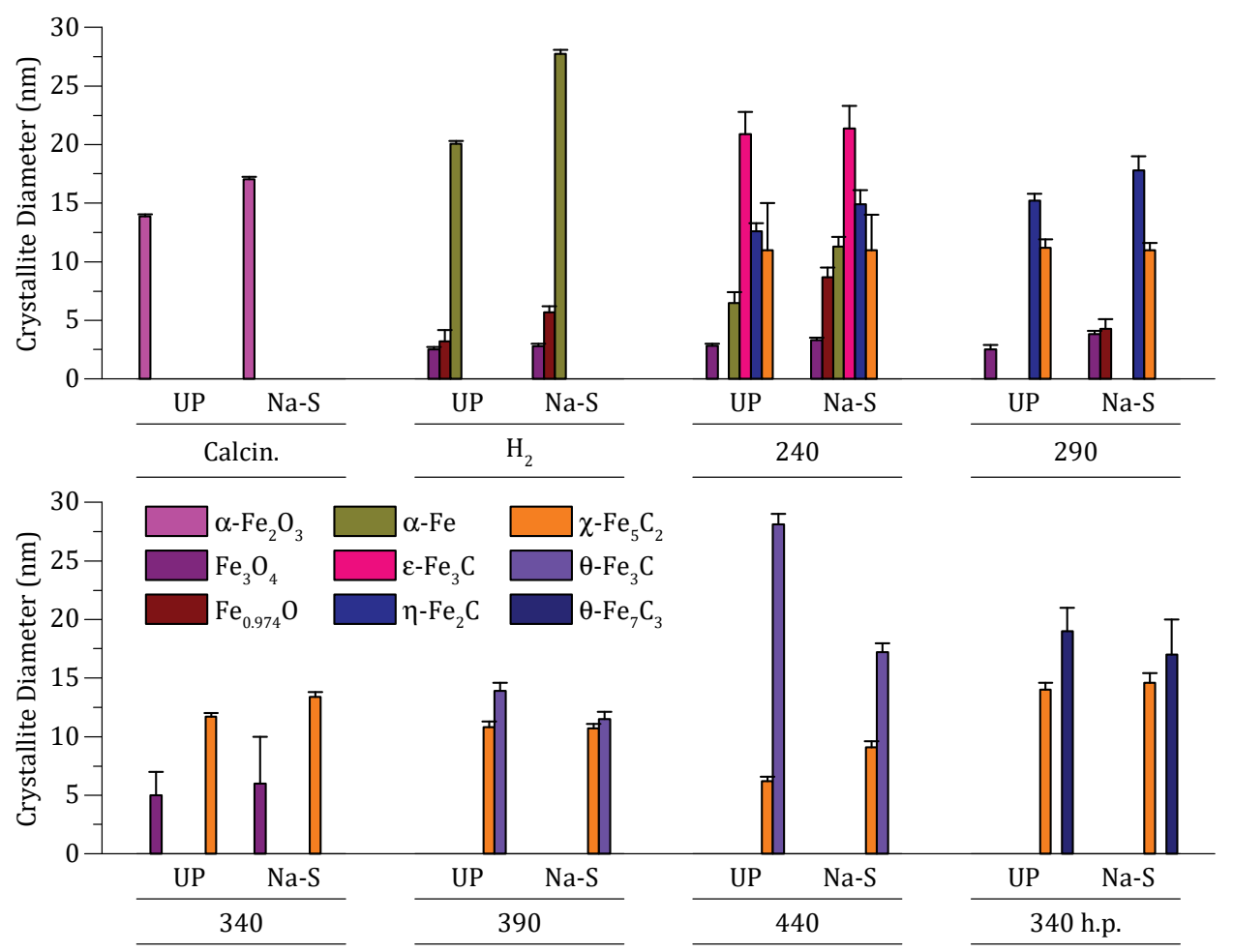

Figure 3.16

The Rietveld Quantitative Phase Analysis (R-QPA) derived Fe phase volume-weighted crystallite mean diameters, after each applied catalyst treatment for UP and $\mathrm{Na}-\mathrm{S}\left(\mathrm{Fe}(-\mathrm{Na}-\mathrm{S}) / \alpha-\mathrm{Al}_{2} \mathrm{O}_{3}\right)$ catalyst materials.

The $\chi-\mathrm{Fe}_{5} \mathrm{C}_{2}$ phase seems to have rather uniform crystallite diameters after the applied catalyst treatments with the smallest mean diameters of all of the Fe carbides. This suggests that the $\chi-\mathrm{Fe}_{5} \mathrm{C}_{2}$ phase forms by dividing its precursor Fe crystallites or particles into smaller fractions. An effect akin to this has been observed with Environmental-TEM (ETEM) where the $\chi-\mathrm{Fe}_{5} \mathrm{C}_{2}$ phase formed as a belt-like phase, rather than uniformly over a single crystal or particle of the carburized $\alpha$-Fe. ${ }^{118}$

Comparing the Fe particle mean diameters (i.e. from TEM) to the crystallite mean diameters (i.e. from R-QPA) is not straightforward as: i) a single Fe particle may contain several Fe crystallites (or "coherent scattering regions"),57,118,119 ii) Fe particle mean diameters are number-weighted while Fe crystallite mean diameters are volumeweighted values, ${ }^{57,119}$ iii) the Gaussian strain component for the diffraction peak sizestrain broadening could not be determined with the R-QPA (see Experimental Methods), ${ }^{55}$ and iv) the formed reoxidation layer (Figure $3.3 \mathbf{b}$ ) reduces the possible Fe crystallite diameters by $\approx 8-10 \mathrm{~nm}$ in comparison to the Fe particle diameters. 
Nevertheless, a few comments can be made when comparing the TEM-based Fe particle diameters to R-QPA originating crystallite diameters. The main trend together in the mean Fe particle (Figure 3.5) and Fe crystallite diameters (Figure 3.16) is to decrease towards the catalyst treatment 340 . With the treatment 340 , the decreasing diameters can be assigned to the Fe fragmentation effect due to the carbon deposition, ${ }^{120,121}$ as agreed by the TGA-MS (Figure 3.6) and TEM (Figure 3.3) results. With treatments $\leq 290$ the initial "Fe clusters" transform into more clearly individually separated Fe particles. This process decreases the measured mean Fe particle and crystallite diameters. The particle separation takes place without any significant carbon deposition.

The TEM derived Fe particle mean diameters suggest that only the Na-S promoted catalyst materials show Fe particle growth when moving from the catalyst treatment 340 towards 440 (Figure 3.5). However, the R-QPA-based Fe crystallite diameter values disagree with this (Figure 3.16). With increasing the treatment temperature, the $\theta-\mathrm{Fe}_{3} \mathrm{C}$ phase crystallite diameter growth against $\chi-\mathrm{Fe}_{5} \mathrm{C}_{2}$ can be observed for both the UP and Na-S 440 catalyst materials (Figure 3.16).

The largest Fe carbide crystallites of $28.1 \pm 0.9 \mathrm{~nm}$ over all catalyst materials are the $\theta-\mathrm{Fe}_{3} \mathrm{C}$ crystallites in the UP 440 catalyst material. This crystallite diameter is larger than the Fe particle mean diameter of $24.2 \mathrm{~nm}$ from TEM-based results for the same catalyst material; a seemingly odd physical result. The discrepancy is due to the volumeweighting of the R-QPA crystallite mean diameter values, as the relatively few large volume Fe particles (e.g. in Figure 3.3d) with large crystallites contribute far more to the volume-weighted Fe crystallite mean diameters than to the number-weighted Fe particle mean diameters. Also, R-QPA derived values rely on far larger sample size and are less prone to sampling errors than are the TEM derived Fe particle mean diameter values. ${ }^{57}$

Thus, the R-QPA crystallite diameters do also imply Fe particle growth for the UP 440 catalyst materials, and not only for the Na-S 440, as the TEM derived values would suggest (Figure 3.5). Then, when considering both the TEM and R-QPA results for Fe particle or crystallite diameters in total: catalyst treatment 440 causes increasing Fe particle or crystallite growth for both the UP and Na-S catalyst materials.

\subsubsection{Assignment of Fe Carbide Properties with X-ray Powder Diffractometry/Rietveld Quantitative Phase Analysis and Mössbauer Absorption Spectroscopy}

In the literature review done in Chapter 2, we have assigned the Fe carbide crystal structures and the corresponding MAS hyperfine fields, as based on our interpretation of the literature. The assignments establish a common Fe carbide nomenclature 

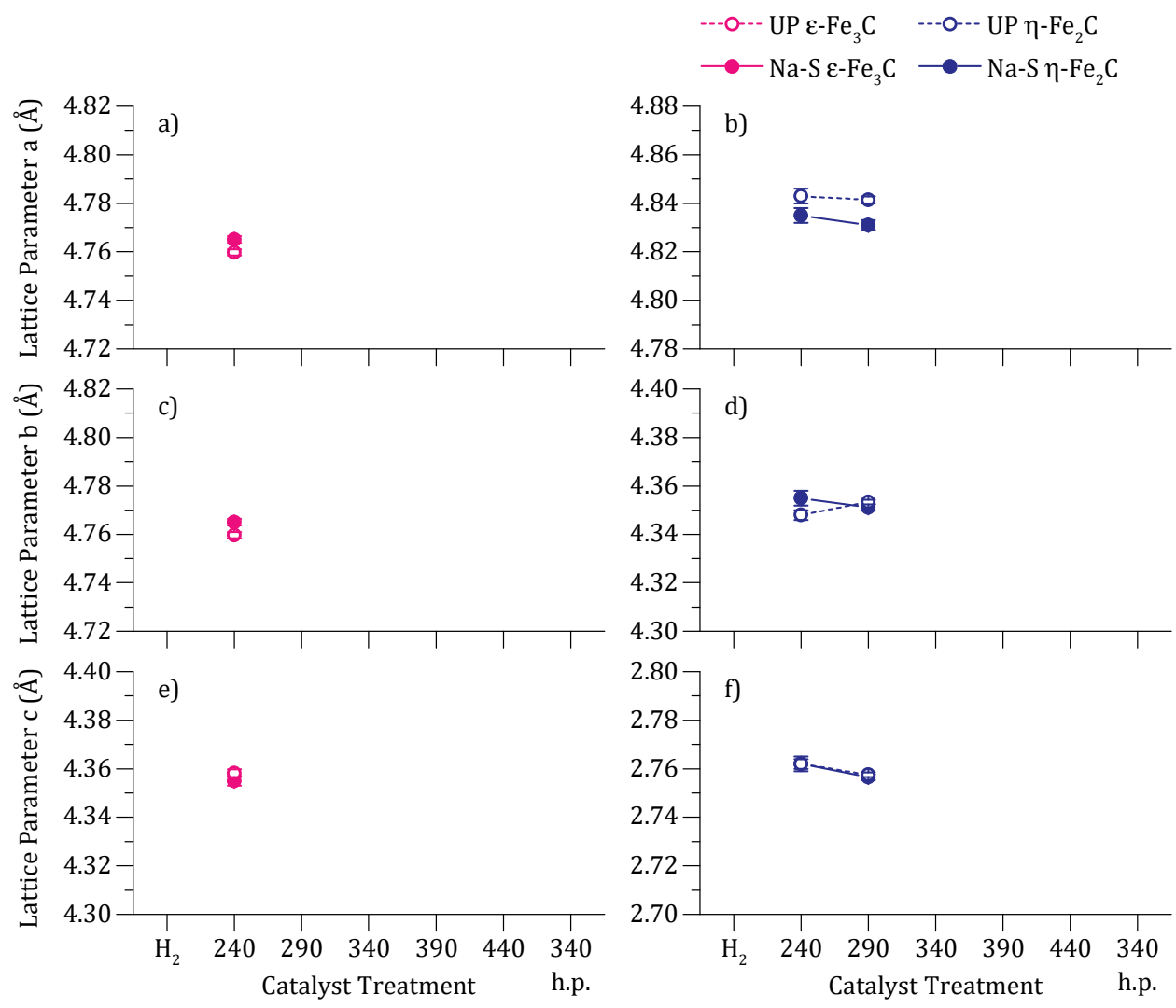

\section{Figure 3.17}

The lattice parameters used in Rietveld Quantitative Phase Analysis (R-QPA) for the $\varepsilon-\mathrm{Fe}_{3} \mathrm{C}$ and $\eta-\mathrm{Fe}_{2} \mathrm{C}$ phases in the UP and $\mathrm{Na}-\mathrm{S}\left(\mathrm{Fe}(-\mathrm{Na}-\mathrm{S}) / \alpha-\mathrm{Al}_{2} \mathrm{O}_{3}\right)$ catalyst materials after the respective catalyst treatment. Lattice parameters $a, b$, and $c$ for $\varepsilon-\mathrm{Fe}_{3} \mathrm{C}$ are in a), c) and e); and for $\eta-\mathrm{Fe}_{2} \mathrm{C}$ in b), d) and f), respectively.

between the XRPD and MAS techniques. The experimental MAS and XRPD based R-QPA quantifications are very much in line with each other (Figure 3.13a-b), and the R-QPA fittings to the measured diffractograms are very good with the employed Fe carbide crystal structures, as can be seen in Figure 3.10 for UP and in Figure 3.11 for Na-S catalyst materials. It is therefore safe to conclude that our original interpretation of the literature is a reasonable one.

That is, the Fe carbides encountered in the FTS research can be identified as $\varepsilon-\mathrm{Fe}_{3} \mathrm{C}$, $\eta-\mathrm{Fe}_{2} \mathrm{C}, \chi-\mathrm{Fe}_{5} \mathrm{C}_{2}, \theta-\mathrm{Fe}_{3} \mathrm{C}$ and $\theta-\mathrm{Fe}_{7} \mathrm{C}_{3}$, with the lattice parameters and the crystal structures by the listed authors in Table 3.5. The use of inaccurate nomenclature of assigning any of the Fe carbides as pseudo-hexagonal, " $\varepsilon$-carbides", ( $\varepsilon$-) $\mathrm{Fe}_{2} \mathrm{C}$ or $\varepsilon^{\prime}-\mathrm{Fe}_{2.2} \mathrm{C}$ is discouraged. 


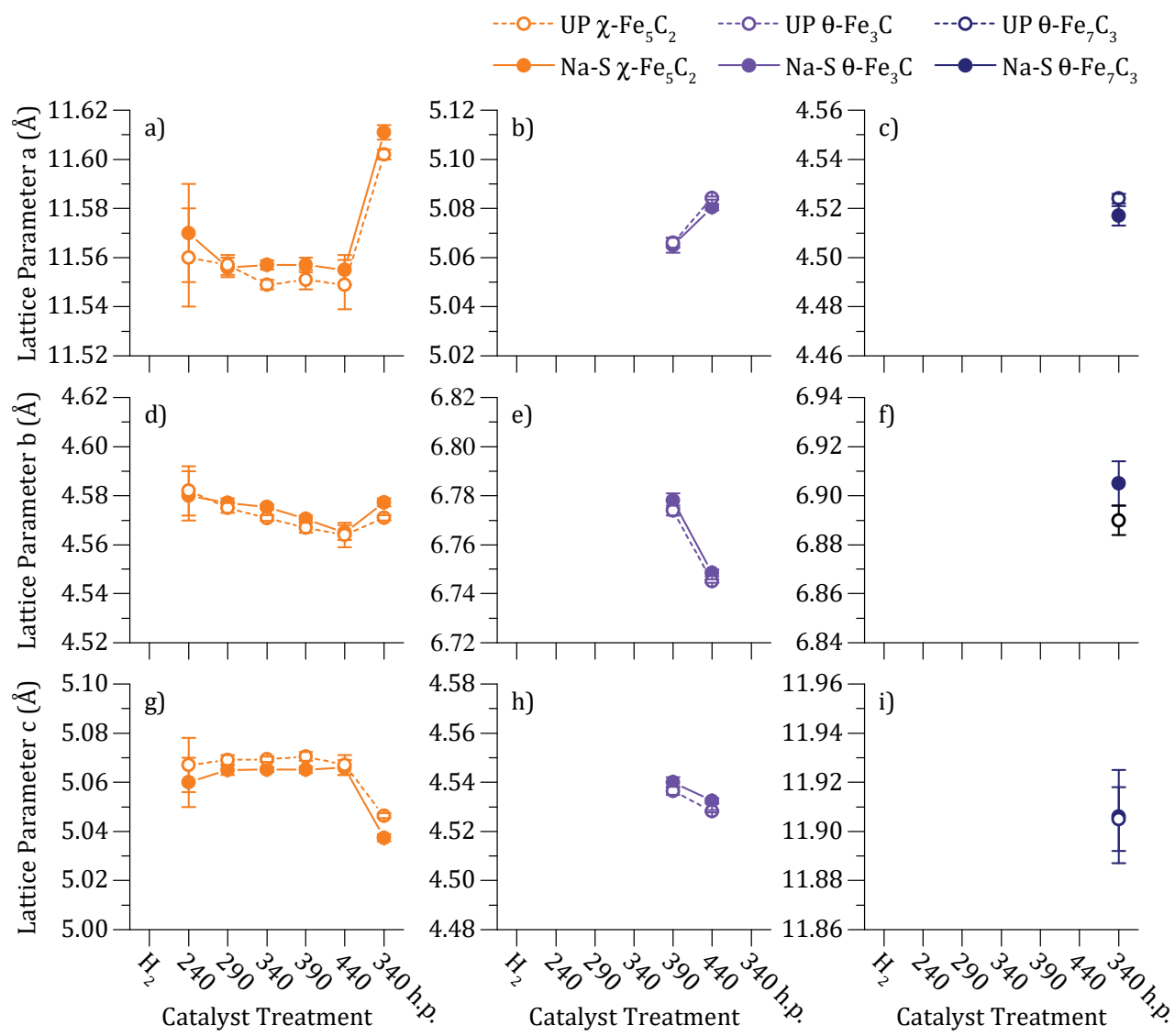

Figure 3.18

The lattice parameters used in Rietveld Quantitative Phase Analysis (R-QPA) for the $\chi-\mathrm{Fe}_{5} \mathrm{C}_{2}, \theta-\mathrm{Fe}_{3} \mathrm{C}$ and $\theta$ $\mathrm{Fe}_{7} \mathrm{C}_{3}$ phases in the UP and $\mathrm{Na}-\mathrm{S}\left(\mathrm{Fe}(-\mathrm{Na}-\mathrm{S}) / \alpha-\mathrm{Al}_{2} \mathrm{O}_{3}\right)$ catalyst materials after the respective catalyst treatment. Lattice parameters $a, b$, and $c$ for $\chi-\mathrm{Fe}_{5} \mathrm{C}_{2}$ are in a), d) and g); for $\theta-\mathrm{Fe} \mathrm{e}_{3} \mathrm{C}$ in b), e) and h); and for $\theta-\mathrm{Fe}_{7} \mathrm{C}_{3}$ in c), f) and i), respectively.

\subsubsection{Fe Carbide Lattice Parameters and Hyperfine Fields}

The R-QPA fitted lattice parameters comparing the effect of the Na-S promotion on the different $\mathrm{Fe}$ carbide phases are given in Figure 3.17 for the $\varepsilon-\mathrm{Fe}_{3} \mathrm{C}$ and $\eta-\mathrm{Fe}_{2} \mathrm{C}$ and in Figure 3.18 for the $\chi-\mathrm{Fe}_{5} \mathrm{C}_{2}, \theta-\mathrm{Fe}_{3} \mathrm{C}$ and $\theta-\mathrm{Fe}_{7} \mathrm{C}_{3}$ phases. The R-QPA fitted and averaged Fe carbide lattice parameters, over all the observations per Fe carbide phase, are given in the Table 3.5.

The averaged MAS-based Fe hyperfine fields and relative fitted spectral contribution derived non-equivalent Fe site ratios are given in Table 3.6 for the Fe carbides. Used MAS fit parameters are given in the Table 3.3 for UP and in Table 3.4 for Na-S catalyst 
Identification of Iron Carbides in Fe(-Na-S)/ $\alpha$-Al203 Fischer-Tropsch Synthesis Catalysts with X-ray Powder Diffractometry and Mössbauer Absorption Spectroscopy

\section{Table 3.5}

The averaged Fe carbide lattice parameters and crystal structures used in the Rietveld Quantitative Phase Analysis (R-QPA) with comparisons to the lattice parameters by the crystal structures' authors.

\begin{tabular}{|c|c|c|c|c|c|c|}
\hline \multirow{2}{*}{$\begin{array}{c}\text { Fe Carbide } \\
\text { Phase }\end{array}$} & \multirow[t]{2}{*}{ Space Group } & & \multicolumn{2}{|c|}{ Lattice Parameters } & \multirow{2}{*}{$\begin{array}{c}\text { Database Number } \\
\text { ICDD-PDF-4+ } \\
(2016)\end{array}$} & \multirow[t]{2}{*}{ Author } \\
\hline & & & $\begin{array}{c}\text { Author } \\
(\AA)\end{array}$ & $\begin{array}{c}\text { This Studya) } \\
(\AA)\end{array}$ & & \\
\hline & Hexagonal, & $\mathrm{a}$ & 4.767 & $4.76 \pm 0.00$ & & \\
\hline \multirow[t]{3}{*}{$\varepsilon-\mathrm{Fe}_{3} \mathrm{C}$} & $\mathrm{P}_{3} 22(182)$ & $\mathrm{b}$ & 4.767 & $4.76 \pm 0.00$ & 04-008-9572 & Nagakura (1959) [86] \\
\hline & & c & 4.354 & $4.36 \pm 0.00$ & & \\
\hline & '"Orthorhombic, & $a$ & 4.862 & $4.84 \pm 0.01$ & & \\
\hline \multirow[t]{3}{*}{$\eta-\mathrm{Fe}_{2} \mathrm{C}^{\mathrm{b}}$} & Pnnm (58) & $\mathrm{b}$ & 4.360 & $4.35 \pm 0.00$ & 04-013-9510 & Hirotsu (1972) [19] \\
\hline & & c & 2.755 & $2.76 \pm 0.00$ & & \\
\hline & Monoclinic, & $\mathrm{a}$ & 11.588 & $11.56 \pm 0.01$ & & \\
\hline \multirow[t]{3}{*}{$\chi-\mathrm{Fe}_{5} \mathrm{C}_{2}$} & $\mathrm{C} 2 / \mathrm{c}(15)$ & $\mathrm{b}$ & 4.579 & $4.57 \pm 0.01$ & 00-051-0997 & Retief (1999) [11] \\
\hline & & c & 5.059 & $5.07 \pm 0.00$ & & \\
\hline & Monoclinic, & $\mathrm{a}$ & - & $11.61 \pm 0.01$ & & \\
\hline$\chi-\mathrm{Fe}_{5} \mathrm{C}_{2}$ & $\mathrm{C} 2 / \mathrm{c}(15)$ & $\mathrm{b}$ & - & $4.57 \pm 0.00$ & - & - \\
\hline \multirow[t]{2}{*}{ (h.p.) ${ }^{c)}$} & & c & - & $5.04 \pm 0.00$ & & \\
\hline & Orthorhombic, & $\mathrm{a}$ & 5.081 & $5.07 \pm 0.01$ & & \\
\hline \multirow[t]{3}{*}{$\left.\theta-\mathrm{Fe}_{3} \mathrm{Cd}\right)$} & Pnma (62) & $\mathrm{b}$ & 6.754 & $6.76 \pm 0.02$ & $9014027 \mathrm{e})$ & Wood (2004) [97] \\
\hline & & c & 4.515 & $4.53 \pm 0.01$ & & \\
\hline & 'Orthorhombic, & $\mathrm{a}$ & 4.540 & $4.52 \pm 0.00$ & & \\
\hline \multirow[t]{2}{*}{$\left.\theta-\mathrm{Fe}_{7} \mathrm{C}_{3} \mathrm{~d}\right)$} & Pnma (62) & $\mathrm{b}$ & 6.879 & $6.90 \pm 0.01$ & $04-002-8165$ & Fruchart $(1965)^{\mathrm{ff}}[122]$ \\
\hline & & c & 11.942 & $11.91 \pm 0.00$ & & \\
\hline
\end{tabular}

a) The mean values over all observations of the Fe carbide phase with Standard Deviations (STDs).

b) The author's lattice parameters are translated, according to Hirotsu and Nagakura, ${ }^{20}$ to correspond the Fe carbide observed by Barton and Gale. ${ }^{9}$

c) Formed from $\mathrm{Fe}$ oxide precursor $\left(\alpha-\mathrm{Fe}_{2} \mathrm{O}_{3} / \alpha-\mathrm{Al}_{2} \mathrm{O}_{3}\right)$ with the catalyst treatment 340 h.p.

d) Diffraction peak overlap with $\chi-\mathrm{Fe}_{5} \mathrm{C}_{2}$ or $\chi-\mathrm{Fe}_{5} \mathrm{C}_{2}$ (h.p.) causes inaccuracy on the lattice parameters.

e) Crystallography Open Database (COD) number.

f) The original article written in French by Fruchart et al. is not readily available but some discussion on the crystal lattice can be found in publication by Audier, Bowen and Jones. ${ }^{113}$

materials. The reference MAS values from experimental literature studies in Table 3.6 are reproduced from Chapter 2 . The addition of Na-S promotion did not have any significant effect, on either the fitted Fe carbide lattice parameters or the hyperfine fields.

The mean values of the lattice parameters used for the R-QPA fits in Table 3.5 are in general in good agreement with the lattice parameters by the authors of the crystal structures. The crystal structure of $\eta-\mathrm{Fe}_{2} \mathrm{C}$ by Hirotsu and Nagakura, ${ }^{19}$ with the unit cell lattice translation by the same authors, ${ }^{20}$ to correspond the Fe carbide observed by Barton and Gale, ${ }^{9}$ is in near agreement to the fitted unit cell for the $\eta-\mathrm{Fe}_{2} \mathrm{C}$ phase.

The most significant deviations from the authors' unit cells are with the $\chi-\mathrm{Fe}_{5} \mathrm{C}_{2}$ and 


\section{Table 3.6}

The averaged Mössbauer Absorption Spectroscopy (MAS) spectral contribution derived relative ratios for non-equivalent Fe positions and hyperfine fields per Fe carbide. Averaged, experimental literature values are also provided for comparison.

\begin{tabular}{|c|c|c|c|c|c|c|}
\hline \multirow[t]{3}{*}{$\begin{array}{l}\text { Fe Carbide } \\
\text { Phase }\end{array}$} & \multirow[t]{3}{*}{ Space Group } & \multicolumn{3}{|c|}{ Non-equivalent Fe Positions } & \multicolumn{2}{|c|}{$\begin{array}{c}\text { Hyperfine Field } \\
\text { (at room temperature) }\end{array}$} \\
\hline & & \multirow{2}{*}{$\begin{array}{c}\text { Tot. } \\
\text { Count }{ }^{\mathrm{b}}\end{array}$} & \multicolumn{2}{|c|}{ Rel. Ratio } & \multirow{2}{*}{$\begin{array}{c}\text { Literature } \\
(\mathrm{T})\end{array}$} & \multirow{2}{*}{$\begin{array}{c}\text { This Study }^{\mathrm{a})} \\
(\mathrm{T}) \\
\end{array}$} \\
\hline & & & Nomin.c) & Fittedd) & & \\
\hline & Hexagonal, & & & & & \\
\hline \multirow[t]{2}{*}{$\varepsilon-\mathrm{Fe}_{3} \mathrm{C}$} & $\mathrm{P}_{3} 22(182)$ & 1 & 1 & 1 & $24.5 \pm 0.8$ & $23.4 \pm 0.3$ \\
\hline & Orthorhombic, & & & & & \\
\hline \multirow[t]{2}{*}{$\eta-\mathrm{Fe}_{2} \mathrm{C}$} & Pnnm (58) & 1 & 1 & 1 & $17.2 \pm 0.3$ & $17.3 \pm 0.3$ \\
\hline & Monoclinic, & & 2 & $1.9 \pm 0.3$ & $21.4 \pm 0.3$ & $21.4 \pm 0.3$ \\
\hline \multirow[t]{3}{*}{$\chi-\mathrm{Fe}_{5} \mathrm{C}_{2}$} & C2/c (15) & 3 & 2 & $1.8 \pm 0.3$ & $18.1 \pm 0.4$ & $18.7 \pm 0.3$ \\
\hline & & & 1 & $1.0 \pm 0.0$ & $10.9 \pm 0.9$ & $11.4 \pm 0.3$ \\
\hline & Monoclinic, & & 2 & $1.3 \pm 0.0$ & - & $21.5 \pm 0.1$ \\
\hline$\chi-\mathrm{Fe}_{5} \mathrm{C}_{2}$ & $\mathrm{C} 2 / \mathrm{c}(15)$ & 3 & 2 & $1.2 \pm 0.0$ & - & $18.0 \pm 0.1$ \\
\hline \multirow[t]{2}{*}{ (h.p.) ${ }^{\text {e) }}$} & & & 1 & $1.0 \pm 0.0$ & - & $10.3 \pm 0.1$ \\
\hline & Orthorhombic, & & $(2)$ & - & $20.8 \pm 0.3$ & - \\
\hline \multirow[t]{4}{*}{$\theta-\mathrm{Fe}_{3} \mathrm{C}$} & Pnma (62) & 2 & (1) & - & $20.0 \pm 0.3$ & - \\
\hline & & & Avg.f) & 1 & $20.8 \pm 0.5$ & $20.5 \pm 0.3$ \\
\hline & & & (1) & $1.0 \pm 0.0$ & $22.9 \pm 0.1$ & $22.4 \pm 0.1$ \\
\hline & Orthorhombic, & & (2) & - & $\left.(-)^{g}\right)$ & - \\
\hline \multirow[t]{3}{*}{$\theta-\mathrm{Fe}_{7} \mathrm{C}_{3}$} & Pnma (62) & 5 & (1) & $0.9 \pm 0.3$ & $18.5 \pm 0.1$ & $19.5 \pm 0.4$ \\
\hline & & & (2) & $1.8 \pm 0.3$ & $16.3 \pm 0.1$ & $16.3 \pm 0.1$ \\
\hline & & & (1) & - & $\left.(21.0)^{\mathrm{g}}\right)$ & - \\
\hline
\end{tabular}

a) Mean values over all observations of the phase with the Standard Deviations (STDs).

b) Determined based on the crystal structures from authors in Table 3.5.

c) Nominal ratios assigned per experimental MAS data, where assignments in parenthesis () are uncertain.

d) Averaged ratios derived from the spectral contributions per fitted hyperfine field.

e) Formed from $\mathrm{Fe}$ oxide precursor $\left(\alpha-\mathrm{Fe}_{2} \mathrm{O}_{3} / \alpha-\mathrm{Al}_{2} \mathrm{O}_{3}\right)$ with the treatment 340 h.p.

f) Commonly, an averaged hyperfine field is fitted for the $\theta-\mathrm{Fe}_{3} \mathrm{C}$ in the experimental literature data.

g) Uncertain assignments values.

$\theta-\mathrm{Fe}_{7} \mathrm{C}_{3}$ phases. Especially noteworthy is the distinctively distorted $\chi-\mathrm{Fe}_{5} \mathrm{C}_{2}$ (h.p.) phase formed with the catalyst treatment 340 h.p., in both the UP and Na-S catalyst materials. The $\theta-\mathrm{Fe}_{3} \mathrm{C}$ phase's mean lattice parameters have large STDs complicating discussion on the phase's parameters (Table 3.5).

The MAS hyperfine fields for the $\varepsilon-\mathrm{Fe}_{3} \mathrm{C}, \eta-\mathrm{Fe}_{2} \mathrm{C}, \chi-\mathrm{Fe}_{5} \mathrm{C} 2$ and $\theta-\mathrm{Fe}_{3} \mathrm{C}$ phases are in good agreement with the expected experimental values interpreted from literature (Table 3.6). For the $\theta-\mathrm{Fe}_{3} \mathrm{C}$ phase, two Fe hyperfine fields should be assigned for each of the non-equivalent Fe positions. However, as the hyperfine fields are close to each 
Identification of Iron Carbides in Fe(-Na-S)/ $\alpha$-Al203 Fischer-Tropsch Synthesis Catalysts with X-ray Powder Diffractometry and Mössbauer Absorption Spectroscopy

other, therefore commonly a single averaged field is used.

The distinctively distorted unit cell for the $\chi-\mathrm{Fe}_{5} \mathrm{C}_{2}$ (h.p.) phase is formed after the catalyst treatment 340 h.p.. By comparing the Rietveld method calculated $\chi-\mathrm{Fe}_{5} \mathrm{C}_{2}$ phase diffraction patterns (Figure 3.10b-e and Figure 3.11b-e) to the $\chi-\mathrm{Fe}_{5} \mathrm{C}_{2}$ (h.p.)'s calculated phase pattern after the high-pressure treatment (Figure 3.10f and Figure 3.11f), a shift towards lower $2 \theta$ in the most intense diffraction peak can be observed. The shift towards lower $2 \theta$ signifies increase in, at least one, unit cell lattice parameter for the diffracting phase. Indeed, the $\chi-\mathrm{Fe}_{5} \mathrm{C}_{2}$ (h.p.) phase's lattice parameter $a$ is noticeably larger than the literature reference value by Retief (Table 3.5).

The formation of a distorted or modified $\chi-\mathrm{Fe}_{5} \mathrm{C}_{2}$ phase from a $\mathrm{Fe}$ oxide as the carburization precursor has been previously observed in distorted XRPD patterns and in changes in the Hägg carbide's Curie Temperature by Cohn et al..64 Cohn et al. suggested that some 0 atoms could be retained in the Hägg carbide lattice when the carburization was performed on a Fe oxide instead of a pre-reduced $\alpha$-Fe. ${ }^{64}$

Both, R-QPA and MAS quantifications, are in agreement that the formed crystal lattice for the $\chi-\mathrm{Fe}_{5} \mathrm{C}_{2}$ phase is dependent on whether $\alpha$-Fe e.g. with catalyst treatment 340 , or $\alpha-\mathrm{Fe}_{2} \mathrm{O}_{3}$ with treatment 340 h.p. is used as the $\mathrm{CO}$ carburization starting material (Table 3.5 and Table 3.6). In comparison to the $\chi-\mathrm{Fe}_{5} \mathrm{C}_{2}$, the $\chi-\mathrm{Fe}_{5} \mathrm{C}_{2}$ (h.p.) phase has i) shifted lattice parameters $a$, from $11.56 \pm 0.01 \AA$ to $11.61 \pm 0.01 \AA$ and $c$, from $5.07 \pm 0.00 \AA$ to $5.04 \pm 0.00 \AA$ as read from the Table 3.5. Furthermore, ii) the non-equivalent Fe position ratios are distorted from 1.9:1.8:1.0 to 1.3:1.2:1.0 and iii) the hyperfine field corresponding to the Fe position with fraction 1.0 drops from $11.4 \pm 0.3 \mathrm{~T}$ to $10.3 \pm 0.1 \mathrm{~T}$ (Table 3.6). No precise explanation for these observations can be given this time. That is, whether the differences in the lattices and hyperfine fields are due to retained 0 atoms in the lattice as proposed by Cohn et al., or due to something else. ${ }^{64}$

Additionally, a lowered lattice parameter $a$ can be observed for the $\chi-\mathrm{Fe}_{5} \mathrm{C}_{2}$ phase formed from $\alpha$-Fe with catalyst treatments 240-440, in comparison to Retief's lattice parameters for $\chi-\mathrm{Fe}_{5} \mathrm{C}_{2}$ (Table 3.5). This can be tentatively explained with the used carburization conditions: with the use of pure CO carburization as opposed to Retief's $\mathrm{H}_{2}$ :CO carburization during the FTS reaction. ${ }^{11}$

With respect to the $\theta-\mathrm{Fe}_{7} \mathrm{C}_{3}$ discrepancies, the orthorhombic crystal structure by Fruchart et al., ${ }^{122}$ gives a good fit for the diffraction patterns of the $\theta-\mathrm{Fe}_{7} \mathrm{C}_{3}$ phase, in both peak intensity and positions. However, with different unit cell lattices than the original ones from the crystal structure's authors (Table 3.5).

The deviations on the lattice parameters for the $\theta-\mathrm{Fe}_{7} \mathrm{C}_{3}$ phase could be, in part, due to inaccurate peak position determination. The $\theta-\mathrm{Fe}_{7} \mathrm{C}_{3}$ phase contents are low (Figure 3.13a-b), and the $\theta-\mathrm{Fe}_{7} \mathrm{C}_{3}$ peaks also overlap with the more abundant $\chi-\mathrm{Fe}_{5} \mathrm{C}_{2}$ (h.p.) phase (Figure 3.10f and Figure 3.11f). 
Using the hexagonal crystal structure by Herbstein and Snyman, ${ }^{18}$ instead of the orthorhombic structure by Fruchart et al., ${ }^{122}$ for the $\mathrm{Fe}_{7} \mathrm{C}_{3}$ phase gave a clearly worse fit to the measured XRPD patterns with R-QPA (fits are not shown). That is, the EckströmAdcock carbide $\theta-\mathrm{Fe}_{7} \mathrm{C}_{3}$ has an orthorhombic rather than a hexagonal crystal structure. ${ }^{113}$

As with the $\theta-\mathrm{Fe}_{7} \mathrm{C}_{3}$ phase, the diffraction peak overlap issues can also explain the large STD values for the lattice parameters of $\theta-\mathrm{Fe}_{3} \mathrm{C}$. For the UP and $\mathrm{Na}-\mathrm{S} 390$ catalyst materials, the $\theta-\mathrm{Fe}_{3} \mathrm{C}$ phase diffraction peaks are poorly defined and overlap with the $\chi$ $\mathrm{Fe}_{5} \mathrm{C}_{2}$ phase. This as can be seen in Figure 3.10d for the phases in UP and in Figure 3.11d for phases in Na-S catalyst materials. The overlap, therefore, results in large STDs in the determined lattice parameters (Figure 3.18b, $\boldsymbol{e}, \boldsymbol{h}$ and Table 3.5).

Only three of the five expected non-equivalent $\mathrm{Fe}$ positions could be definitely assigned for the $\theta-\mathrm{Fe}_{7} \mathrm{C}_{3}$ phase on the basis of the MAS quantifications (Table 3.6). We assign the hyperfine fields $22.4 \pm 0.1 \mathrm{~T}, 19.5 \pm 0.4 \mathrm{~T}$ and $16.3 \pm 0.1 \mathrm{~T}$ for spectral contributions in ratios $\approx 1.0: 0.9: 1.8$, respectively. As based on the nominal nonequivalent Fe position ratios derived from the crystal structures, this is plausible, as three positions can be assigned in ideal ratios of 1:1:2 with the used orthorhombic crystal structure for the $\theta-\mathrm{Fe}_{7} \mathrm{C}_{3}$ phase. For the hexagonal crystal lattice for $\mathrm{Fe}_{7} \mathrm{C}_{3}$ by Herbstein and Snyman, ${ }^{18}$ three non-equivalent Fe positions should be assigned in ratio $3: 3: 1$. This is a further sign that the hexagonal lattice is not a correct one for the $\mathrm{Fe}_{7} \mathrm{C}_{3}$ phase.

The leftover two hyperfine fields, corresponding to the unassigned non-equivalent Fe positions, should be assigned in ratio of $2: 1$ for the $\theta-\mathrm{Fe}_{7} \mathrm{C}_{3}$ phase with orthorhombic structure. However, we could not conclusively assign these two missing hyperfine fields. A catalyst material with larger relative quantity of the $\theta-\mathrm{Fe}_{7} \mathrm{C}_{3}$ phase would be needed for assigning the remaining hyperfine fields for the phase.

\subsubsection{Fe Carbide C Atom Order and Contents}

Earlier in Chapter 2, as based on the crystal structures from literature, the $\varepsilon$ - $\mathrm{Fe}_{3} \mathrm{C}$ phase was discussed to have $\mathrm{C}$ atoms occupying the Fe lattice interstitial spaces in a random, disordered manner. For the $\eta-\mathrm{Fe}_{2} \mathrm{C}$ phase the $\mathrm{C}$ atoms are in the interstitial spaces in a regular, ordered manner. According to discussion by Gatte and Phillips, ${ }^{123}$ this interstitial $\mathrm{C}$ atom disorder should cause spectral broadening in the measured MAS spectra.

Indeed, in the fitted MAS results the expected spectral broadening can be seen from Table 3.3 for UP and Table 3.4 for Na-S catalyst materials: the $\varepsilon-\mathrm{Fe}_{3} \mathrm{C}$ phase has a mean line width of $0.75 \pm 0.07 \mathrm{~mm} \mathrm{~s}^{-1}$ versus a mean line width of $0.49 \pm 0.06 \mathrm{~mm} \mathrm{~s}^{-1}$ for the $\eta$ $\mathrm{Fe}_{2} \mathrm{C}$ phase over all of the catalysts. The MAS results are thus in agreement with the 
expectations: in the $\varepsilon-\mathrm{Fe}_{3} \mathrm{C}$ phase the $\mathrm{C}$ atoms occupy the interstitial spaces in a random, disordered manner, ${ }^{86}$ and in the $\eta-\mathrm{Fe}_{2} \mathrm{C}$ phase the $\mathrm{C}$ atoms occupy the interstitial spaces in a regular, ordered manner. ${ }^{19}$

The $\mathrm{C}$ atom content in the Fe carbide lattice can be indirectly determined from the fitted MAS hyperfine fields. Increasing amounts of $\mathrm{C}$ atoms in the Fe lattice interstitial spaces decrease the observed hyperfine fields for each non-equivalent $\mathrm{Fe}$ position.5,31,33,124,125 In Figure 3.19 are depicted the MAS spectral contribution weighted mean hyperfine fields for each Fe carbide phase versus the unit cell volume normalized to the Fe atoms in the phase's unit cell. By adding $C$ atoms to the $\alpha$-Fe lattice via the carburization process, the cell volume per Fe atom increases while the hyperfine fields decrease (Figure 3.19), as would be expected.

The $\mathrm{C}$ atom content in the $\mathrm{Fe}$ carbide lattices follows increasing order, with decreasing hyperfine fields:

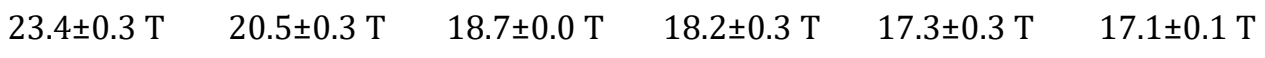

$$
\begin{aligned}
& \varepsilon-\mathrm{Fe}_{3} \mathrm{C}<\theta-\mathrm{Fe}_{3} \mathrm{C}<\theta-\mathrm{Fe}_{7} \mathrm{C}_{3}<\chi-\mathrm{Fe}_{5} \mathrm{C}_{2}<\eta-\mathrm{Fe}_{2} \mathrm{C} \lesssim \chi-\mathrm{Fe}_{5} \mathrm{C}_{2} \\
& \text { (h.p.) }
\end{aligned}
$$

as devised from the order of the MAS fitted, spectral contribution weighted mean hyperfine

\section{(Figure 3.19).}

Interestingly, the distorted $\chi-\mathrm{Fe}_{5} \mathrm{C}_{2}$ (h.p.) phase has the lowest averaged and weighted hyperfine field suggesting the highest $\mathrm{C}$ atom content in the $\mathrm{Fe}$ carbide lattice. Whether the observed lattice distortion effects for the $\chi-\mathrm{Fe}_{5} \mathrm{C}_{2}$ (h.p.) phase with decreased hyperfine fields are truly due to increased $\mathrm{C}$ atom content and/or, e.g. due to the retained $\mathrm{O}$ atoms in the $\mathrm{Fe}$ carbide lattice as suggested by Cohn et al., 64 cannot be

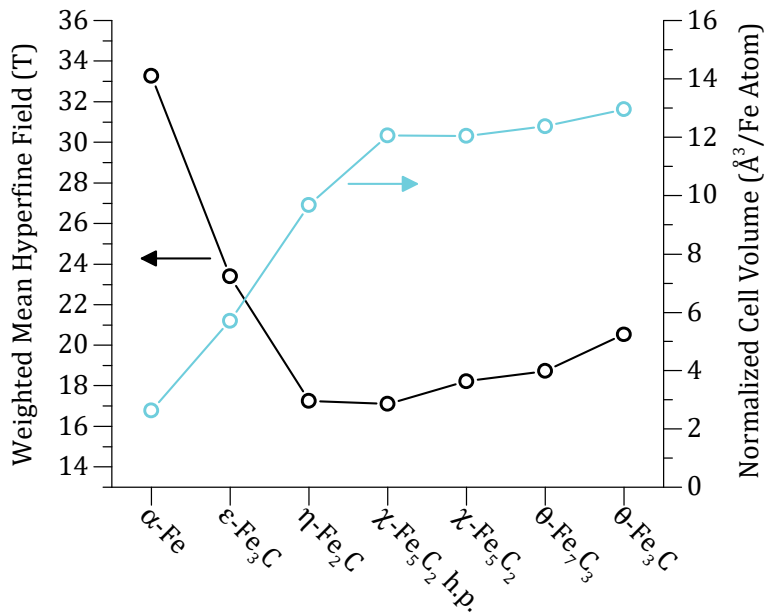

Figure 3.19

Mössbauer Absorption Spectroscopy (MAS)-based spectral contribution weighted mean hyperfine fields versus Rietveld Quantitative Phase Analysis (R-QPA)-based fitted unit cell volumes normalized to Fe atoms per unit cell for each Fe carbide phase and $\alpha$-Fe. Standard Deviations (STDs) for the averaged data points are smaller than the data markers, and are omitted. 

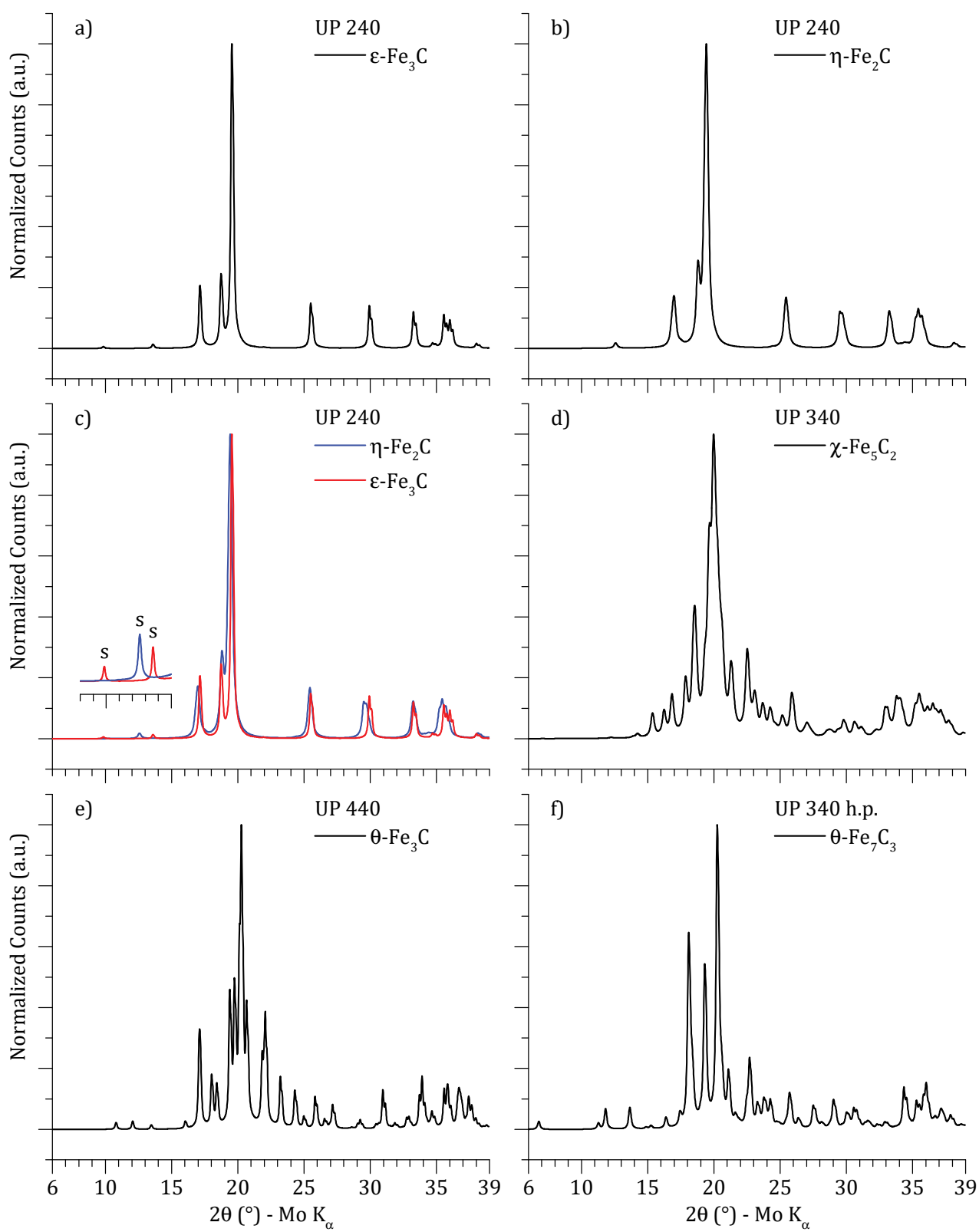

Figure 3.20

Rietveld method calculated Fe carbide phase diffraction patterns compared for UP ( $\mathrm{Fe} / \alpha-\mathrm{Al}_{2} \mathrm{O}_{3}$ ) catalyst materials. The Fe carbides as after catalyst treatment a) $\varepsilon-\mathrm{Fe}_{3} \mathrm{C} 240$, b) $\eta-\mathrm{Fe}_{2} \mathrm{C} 240$, c) $\varepsilon-\mathrm{Fe}_{3} \mathrm{C}$ and $\eta-\mathrm{Fe}_{2} \mathrm{C} 240$, d) $\chi-\mathrm{Fe}_{5} \mathrm{C}_{2} 340$, e) $\theta-\mathrm{Fe}_{3} \mathrm{C} 440$ and f) $\theta-\mathrm{Fe}_{7} \mathrm{C}_{3} 340$ h.p.. Normalization to the most intense diffraction peak. 
Identification of Iron Carbides in Fe(-Na-S)/ $\alpha$-Al203 Fischer-Tropsch Synthesis Catalysts with X-ray Powder Diffractometry and Mössbauer Absorption Spectroscopy

conclusively determined as based on the work in this Chapter.

\subsubsection{Fe Carbide Diffraction Patterns}

Examples of the Rietveld method calculated Fe carbide diffraction patterns used in the R-QPA fitting are shown in Figure 3.20 for the UP catalyst materials. The very similar $\mathrm{R}_{\mathrm{wp}}$-values for the UP and Na-S catalyst materials in Figure 3.8 suggests that the Fe carbide crystal structures, i.e. the relative peak intensities, are little perturbed by the added Na-S promotion after the applied CO carburization treatments.

The R-QPA fitted, calculated diffraction patterns for the $\varepsilon-\mathrm{Fe}_{3} \mathrm{C}$ and $\eta-\mathrm{Fe}_{2} \mathrm{C}$ phases are compared in the Figure 3.20c. The differences between the diffraction patterns for the phases are rather small. Thus, when only XRPD is used for the phase identification of the $\varepsilon$ - $\mathrm{Fe}_{3} \mathrm{C}$ and $\eta-\mathrm{Fe}_{2} \mathrm{C}$ phases, care needs to be taken for correctly identifying the carbides. In the inset graph in Figure 3.20c the weak superstructural diffraction peaks originating from the $\mathrm{C}$ atoms are marked with $s .{ }^{86}$ With XRPD, the superstructural peaks are very weak, only about $1-2 \%$ of the intensity of the most intense diffraction peak. These peaks are more pronounced with $\mathrm{ED},{ }^{86}$ which is more sensitive to low atomic number elements (i.e. C atoms).

In summary, the calculated diffraction patterns reproduced in the Figure 3.20 also hopefully serve as an aid for identifying the Fe carbide phases from XRPD patterns on past and future studies.

As a final remark, the behavior, crystal structures and diffraction patterns of the $\varepsilon$ $\mathrm{Fe}_{3} \mathrm{C}$ and $\eta-\mathrm{Fe}_{2} \mathrm{C}$ phases are very similar to their close, yet more stable, cousins of $\varepsilon-\mathrm{Fe}_{3} \mathrm{~N}$ and $\zeta-\mathrm{Fe}_{2} \mathrm{~N}$ Fe nitrides. ${ }^{126-132} \mathrm{~A}$ comparative study of the $\varepsilon-\mathrm{Fe}_{3} \mathrm{C}$ and $\eta-\mathrm{Fe}_{2} \mathrm{C}$ crystal structures in contrast to the structures of $\varepsilon-\mathrm{Fe}_{3} \mathrm{~N}$ and $\zeta-\mathrm{Fe}_{2} \mathrm{~N}$ phases and phase properties would be an intriguing topic for a future study.

\subsection{CONCLUSIONS}

The XRPD patterns for $\mathrm{H}_{2}$ reduced, CO carburized, supported $\mathrm{Fe}(-\mathrm{Na}-\mathrm{S}) / \alpha-\mathrm{Al}_{2} \mathrm{O}_{3}$ catalyst materials were quantified with R-QPA for crystalline Fe phases. The R-QPA results were compared to the catalysts' corresponding MAS quantifications. The quantification results between the two characterization techniques were in a very good agreement. As a result, a common nomenclature between the Fe carbide crystalline structures, relative $\mathrm{C}$ atom contents, non-equivalent $\mathrm{Fe}$ position ratios and their corresponding MAS hyperfine fields could be established. As based on the experimental results, the Fe carbides observable in the FTS reaction research, with their crystal lattices, are as follows: 


$\begin{array}{ccccc}\varepsilon-\mathrm{Fe}_{3} \mathrm{C} & \eta-\mathrm{Fe}_{2} \mathrm{C} & \chi-\mathrm{Fe}_{5} \mathrm{C}_{2} & \theta-\mathrm{Fe}_{3} \mathrm{C} & \theta-\mathrm{Fe}_{7} \mathrm{C}_{3} \\ \text { Hexagonal, } & \text { Orthorhombic, } & \text { Monoclinic, } & \text { Orthorhombic, } & \text { Orthorhombic, } \\ P 6_{3} 22(182) & \text { Pnnm (58) } & C 2 / c(15) & \text { Pnma (62) } & \text { Pnma (62) }\end{array}$

The experimental result-based assignment of the Fe carbides is in agreement with our interpretation of the literature data, which was discussed in Chapter 2. The use of nomenclature such as pseudo-hexagonal, " $\varepsilon$-carbides", $(\varepsilon-) \mathrm{Fe}_{2} \mathrm{C}$ or $\varepsilon^{\prime}-\mathrm{Fe}_{2.2} \mathrm{C}$ for describing the Fe carbides should not be used as it is inaccurate.

The formation of the observed Fe carbides follows an increasing $\alpha$-Fe carburization temperature. The estimated carbide thermal formation regions from literature with comparison to the results in this Chapter are established as:

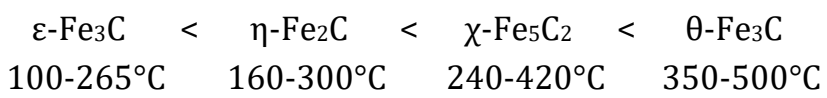

while the formation of the $\theta-\mathrm{Fe}_{7} \mathrm{C}_{3}$ phase is not solely temperature dependent.

As explained on the basis of literature observations: a preformed (alkali-free) $\eta-\mathrm{Fe}_{2} \mathrm{C}$ phase may remain stable up to $340^{\circ} \mathrm{C}$, and decomposition of $\theta-\mathrm{Fe}_{3} \mathrm{C}$ to $\alpha$ - $\mathrm{Fe}$ and $\mathrm{C}$ atoms becomes significant at $>527^{\circ} \mathrm{C}$ in the absence of a carbide oxidation shell. A shell that can increase the $\theta-\mathrm{Fe}_{3} \mathrm{C}$ phase thermal stability up to $600^{\circ} \mathrm{C}$.

The formation of the $\theta-\mathrm{Fe}_{7} \mathrm{C}_{3}$ phase is speculated to be related to the presence of $\mathrm{CO}_{2}$. The $\theta-\mathrm{Fe}_{7} \mathrm{C}_{3}$ phase could only be formed with a high-pressure carburization $\left(340^{\circ} \mathrm{C}\right.$, 25 bar, $\mathrm{CO})$ with $\mathrm{Fe}$ oxide $\left(\alpha-\mathrm{Fe}_{2} \mathrm{O}_{3} / \alpha-\mathrm{Al}_{2} \mathrm{O}_{3}\right)$ as the carburization starting material. Additionally, a distinctly distorted $\chi-\mathrm{Fe}_{5} \mathrm{C}_{2}$ (h.p.) Hägg carbide phase could be observed with both the R-QPA and MAS techniques after the high-pressure treatment, in addition to the presence of the $\theta-\mathrm{Fe}_{7} \mathrm{C}_{3}$ phase. The formation temperature of $340^{\circ} \mathrm{C}$ for the $\theta$ $\mathrm{Fe}_{7} \mathrm{C}_{3}$ phase matches literature observations.

With CO carburization of $\alpha-\mathrm{Fe}$, the added Na-S promotion did not affect the lattice parameters, the hyperfine fields or the formed phase quantities of any of the $\mathrm{Fe}$ carbides. In addition, the Fe carbide crystallite diameters remained largely unaffected by the Na-S promotion. In literature, added alkali is occasionally cited to improve the Fe carbide formation, however, the results in this Chapter do not agree with this. The literature observations were explained to be due to enhanced reduction of Fe oxides with $\mathrm{CO}$ when alkali is present. That is, alkali improves the Fe oxide reduction step with CO not the Fe carburization step. As the carburization treatments were performed with $\alpha$-Fe as the starting material - with the exception of the high-pressure treatment - the lack of observable Na-S promotion effect on the Fe carburization can be accounted for.

However, few effects due to the Na-S promotion could be observed on the Fe phases 
in the carburized catalyst materials. The added Na-S promotion i) increased the $\mathrm{Fe}$ oxidation, presumably by $\mathrm{O}$ from the $\mathrm{CO}$ dissociation acting as oxidation agent, with short, low temperature CO carburization treatments $\left(\leq 290^{\circ} \mathrm{C}, \leq 3 \mathrm{~h}\right)$ and ii) decreased the crystallite diameter of $\theta-\mathrm{Fe}_{3} \mathrm{C}$ formed after $\mathrm{CO}$ treatment at $440^{\circ} \mathrm{C}$.

The deposition and encapsulation of the Fe phases by carbon during the $\mathrm{CO}$ carburization treatments has the effect of i) fragmenting the Fe phases into smaller $\mathrm{Fe}$ particles (TEM) and crystallite diameters (R-QPA) and ii) detaching the Fe particles from the $\alpha-\mathrm{Al}_{2} \mathrm{O}_{3}$ support. The carbon deposition becomes significant with catalyst treatments $\geq 340^{\circ} \mathrm{C}(\geq 16 \mathrm{~h})$. With the treatments $\leq 290^{\circ} \mathrm{C}(\leq 3 \mathrm{~h})$, the Fe phases are not significantly encapsulated by carbon and are attached to the $\alpha-\mathrm{Al}_{2} \mathrm{O}_{3}$ support. The addition of the Na-S promotion increased the rate of carbon deposition from $\mathrm{CO}$ at $\geq 340^{\circ} \mathrm{C}$, but the effect was carburization temperature dependent. The increased carbon deposition rate diminished at $390^{\circ} \mathrm{C}$ and disappeared when reaching the CO carburization temperature of $440^{\circ} \mathrm{C}$. With the highest temperature carburization treatment at $440^{\circ} \mathrm{C}$, growth of both larger and smaller Fe particles as well as $\theta-\mathrm{Fe}_{3} \mathrm{C}$ crystallite growth, could be observed.

For further study, the formation of the $\theta-\mathrm{Fe}_{7} \mathrm{C}_{3}$ phase in relation to presence of $\mathrm{CO}_{2}$ should be more systematically investigated. This in order to synthesize larger relative quantities of the Fe carbide for more detailed characterization studies. Although, we could identify the hyperfine fields for three of the five expected non-equivalent $\mathrm{Fe}$ positions in the $\theta-\mathrm{Fe}_{7} \mathrm{C}_{3}$ crystal lattice; a purer, $\theta-\mathrm{Fe}_{7} \mathrm{C}_{3}$ phase only catalyst materials would be needed for assigning the remaining two Fe positions with hyperfine fields.

\subsection{ACKNOWLEDGEMENTS}

H. C. de Waard and C. Mulder (GeoLab at Universiteit Utrecht, UU) are acknowledged for the ICP-OES, and M. Versluijs-Helder (UU) for the TGA-MS measurements. J. D. (Hans) Meeldijk and C. W. T. M. Schneijdenberg (UU) are acknowledged for their guidance in taking the TEM images.

\subsection{REFERENCES}

[1] de Smit, E. and Weckhuysen, B. M., "The renaissance of iron-based Fischer-Tropsch synthesis: on the multifaceted catalyst deactivation behaviour", Chem. Soc. Rev. 37, 2758-2781 (2008).

[2] Niemantsverdriet, J. W., van der Kraan, A. M., van Dijk, W. L. and van der Baan, H. S., "Behavior of Metallic Iron Catalysts during Fischer-Tropsch Synthesis Studied with Mössbauer Spectroscopy, X-ray Diffraction, Carbon Content Determination, and Reaction Kinetic Measurements", J. Phys. Chem. 84, 3363-3370 (1980).

[3] de Smit, E., Cinquini, F., Beale, A. M., Safonova, O. V., van Beek, W., Sautet, P. and Weckhuysen, B. M., "Stability and Reactivity of $\epsilon-\chi-\theta$ Iron Carbide Catalyst Phases in Fischer-Tropsch Synthesis: 
Controlling $\mu c^{\prime \prime}$, J. Am. Chem. Soc. 132, 14928-14941 (2010).

[4] Stanfield, R. M. and Delgass, W. N., "Mössbauer spectroscopy of supported Fe-Co alloy catalysts for Fischer-Tropsch synthesis", J. Catal. 72, 37-50 (1981).

[5] Le Caër, G., Dubois, J. M., Pijolat, M., Perrichon, V. and Bussière, P., "Characterization by Moessbauer Spectroscopy of Iron Carbides Formed by Fischer-Tropsch Synthesis", J. Phys. Chem. 86, 4799-4808 (1982).

[6] Jung, H. and Thomson, W. J., "Dynamic X-ray diffraction study of an unsupported iron catalyst in Fischer-Tropsch synthesis", J. Catal. 134, 654-667 (1992).

[7] Liu, X.-W., Cao, Z., Zhao, S., Gao, R., Meng, Y., Zhu, J.-X., Rogers, C., Huo, C.-F., Yang, Y., Li, Y.-W. and Wen, X.-D., "Iron Carbides in Fischer-Tropsch Synthesis: Theoretical and Experimental Understanding in Epsilon-Iron Carbide Phase Assignment", J. Phys. Chem. C 121, 21390-21396 (2017).

[8] Hofer, L. J. E., Cohn, E. M. and Peebles, W. C., "The Modifications of the Carbide, $\mathrm{Fe}_{2} \mathrm{C}$; Their Properties and Identification", J. Am. Chem. Soc. 71, 189-195 (1949).

[9] Barton, G. H. and Gale, B., "The structure of a pseudo-hexagonal iron carbide", Acta Crystallogr. 17, 1460-1462 (1964).

[10] Duggin, M. J. and Hofer, L. J. E., "Nature of $\chi$-Iron Carbide", Nature 212, 248-248 (1966).

[11] Retief, J. J., "Powder diffraction data and Rietveld refinement of Hägg-carbide, $\chi$-Fe ${ }_{5} \mathrm{C}_{2}$ ", Powder Diffr. 14, 130-132 (1999).

[12] Wang, P., Chen, W., Chiang, F., Dugulan, A. I., Song, Y., Pestman, R., Zhang, K., Yao, J., Feng, B., Miao, P., $\mathrm{Xu}, \mathrm{W}$. and Hensen, E. J. M., "Synthesis of stable and low- $\mathrm{CO}_{2}$ selective $\varepsilon$-iron carbide Fischer-Tropsch catalysts", Sci. Adv. 4, eaau2947 (2018).

[13] Wezendonk, T. A., Sun, X., Dugulan, A. I., van Hoof, A. J. F., Hensen, E. J. M., Kapteijn, F. and Gascon, J., "Controlled formation of iron carbides and their performance in Fischer-Tropsch synthesis", J. Catal. 362, 106-117 (2018).

[14] Hofer, L. J. E. and Cohn, E. M., "Some Reactions in the Iron-Carbon System: Application to the Tempering of Martensite", Nature 167, 977-978 (1951).

[15] Hofer, L. J. E., "Nature of the carbides of iron", U.S. Bureau of Mines Bulletin 631 (1966). Available at: http://www.onemine.org/document/abstract.cfm?docid=186163\&title=Nature-Of-The-CarbidesOf-Iron (Accessed: February 2014).

[16] Dry, M. E., "The Fischer-Tropsch Synthesis" in Catalysis - Science and Technology (eds. Anderson, J. R. \& Boudart, M.) 1, 159-256 (Springer-Verlag, Berlin, 1981).

[17] Eckstrom, H. C. and Adcock, W. A., "A New Iron Carbide in Hydrocarbon Synthesis Catalysts", J. Am. Chem. Soc. 72, 1042-1043 (1950).

[18] Herbstein, F. H. and Snyman, J. A., "Identification of Eckstrom-Adcock Iron Carbide as $\mathrm{Fe}_{7} \mathrm{C}_{3}$ ", Inorg. Chem. 3, 894-896 (1964).

[19] Hirotsu, Y. and Nagakura, S., "Crystal structure and morphology of the carbide precipitated from martensitic high carbon steel during the first stage of tempering", Acta Metall. 20, 645-655 (1972).

[20] Hirotsu, Y. and Nagakura, S., "Electron Microscopy and Diffraction Study of the Carbide Precipitated at the First Stage of Tempering of Martensitic Medium Carbon Steel", Trans. Japan Inst. Met. 15, 129134 (1974).

[21] de Smit, E., Beale, A. M., Nikitenko, S. and Weckhuysen, B. M., "Local and long range order in promoted iron-based Fischer-Tropsch catalysts: A combined in situ X-ray absorption spectroscopy/wide angle Xray scattering study", J. Catal. 262, 244-256 (2009).

[22] Shultz, J. F., Hall, W. K., Seligman, B. and Anderson, R. B., "Studies of the Fischer-Tropsch Synthesis. XIV. Hägg Carbide as Catalysts", J. Am. Chem. Soc. 77, 213-221 (1955). 
[23] Shultz, J. F., Hall, W. K., Dubs, T. A. and Anderson, R. B., "Studies of the Fischer-Tropsch Synthesis. XV. Cementite as Catalysts", J. Am. Chem. Soc. 78, 282-285 (1956).

[24] Tajima, S. and Hirano, S., "Synthesis and Magnetic Properties of $\mathrm{Fe}_{7} \mathrm{C}_{3}$ Particles with High Saturation Magnetization", Jpn. J. Appl. Phys. 29, 662-668 (1990).

[25] Dictor, R. and Bell, A. T., "Fischer-Tropsch synthesis over reduced and unreduced iron oxide catalysts", J. Catal. 97, 121-136 (1986).

[26] O’Brien, R. J., Xu, L., Milburn, D. R., Li, Y.-X., Klabunde, K. J. and Davis, B. H., "Fischer-Tropsch synthesis: Impact of potassium and zirconium promoters on the activity and structure of an ultrafine iron oxide catalyst", Top. Catal. 2, 1-15 (1995).

[27] Ning, W., Koizumi, N., Chang, H., Mochizuki, T., Itoh, T. and Yamada, M., "Phase transformation of unpromoted and promoted Fe catalysts and the formation of carbonaceous compounds during Fischer-Tropsch synthesis reaction", Appl. Catal. A Gen. 312, 35-44 (2006).

[28] Mogorosi, R. P., Fischer, N., Claeys, M. and van Steen, E., "Strong-metal-support interaction by molecular design: Fe-silicate interactions in Fischer-Tropsch catalysts", J. Catal. 289, 140-150 (2012).

[29] Königer, A., Hammerl, C., Zeitler, M. and Rauschenbach, B., "Formation of metastable iron carbide phases after high-fluence carbon ion implantation into iron at low temperatures", Phys. Rev. B 55, 8143-8147 (1997).

[30] Boellaard, E., van der Kraan, A. M. and Geus, J. W., "Behaviour of a cyanide-derived $\mathrm{Fe} / \mathrm{Al}_{2} \mathrm{O}_{3}$ catalyst during Fischer-Tropsch synthesis", Appl. Catal. A Gen. 147, 229-245 (1996).

[31] Pijolat, M., Perrichon, V. and Bussière, P., "Study of the carburization of an iron catalyst during the Fischer-Tropsch synthesis: Influence on its catalytic activity", J. Catal. 107, 82-91 (1987).

[32] Amelse, J. A., Butt, J. B. and Schwartz, L. H., "Carburization of supported iron synthesis catalysts", J. Phys. Chem. 82, 558-563 (1978).

[33] Liu, X.-W., Zhao, S., Meng, Y., Peng, Q., Dearden, A. K., Huo, C.-F., Yang, Y., Li, Y.-W. and Wen, X.-D., "Mössbauer Spectroscopy of Iron Carbides: From Prediction to Experimental Confirmation", Sci. Rep. 6, 26184 (2016).

[34] Williamson, D. L., Nakazawa, K. and Krauss, G., "A study of the early stages of tempering in an Fe-1.2 Pct alloy", Metall. Trans. A 10, 1351-1363 (1979).

[35] Botes, G. F., Bromfield, T. C., Coetzer, R. L. J., Crous, R., Gibson, P. and Ferreira, A. C., "Development of a chemical selective iron Fischer Tropsch catalyst", Catal. Today 275, 40-48 (2016).

[36] Crous, R., Bromfield, T. C. and Booyens, S., "Olefin Selective FT Catalyst Composition and Preparation Thereof", Patent W02010066386A1 (2010).

[37] Johnson, J. Y., "Improvements in the Manufacture and Production of Unsaturated Hydrocarbons of Low Boiling Point", Patent GB322284A (1929).

[38] Torres Galvis, H. M., Bitter, J. H., Khare, C. B., Ruitenbeek, M., Dugulan, A. I. and de Jong, K. P., "Supported Iron Nanoparticles as Catalysts for Sustainable Production of Lower Olefins", Science 335, 835-838 (2012).

[39] Pendyala, V. R. R., Graham, U. M., Jacobs, G., Hamdeh, H. H. and Davis, B. H., "Fischer-Tropsch Synthesis: Deactivation as a Function of Potassium Promoter Loading for Precipitated Iron Catalyst", Catal. Lett. 144, 1704-1716 (2014).

[40] An, X., Wu, B., Wan, H.-J., Li, T.-Z., Tao, Z.-C., Xiang, H.-W. and Li, Y.-W., "Comparative study of ironbased Fischer-Tropsch synthesis catalyst promoted with potassium or sodium", Catal. Commun. 8, 1957-1962 (2007).

[41] Klencsár, Z., Kuzmann, E. and Vértes, A., "User-friendly software for Mössbauer spectrum analysis", J. 
Radioanal. Nucl. Chem. Artic. 210, 105-118 (1996).

[42] León-Reina, L., García-Maté, M., Álvarez-Pinazo, G., Santacruz, I., Vallcorba, O., De la Torre, A. G. and Aranda, M. A. G., "Accuracy in Rietveld quantitative phase analysis: a comparative study of strictly monochromatic Mo and Cu radiations", J. Appl. Crystallogr. 49, 722-735 (2016).

[43] Jenkins, R. and Snyder, R. L., "Introduction to X-ray Powder Diffractometry", Introduction to X-ray Powder Diffractometry 138 (John Wiley \& Sons, Inc., Hoboken, NJ, USA, 1996).

[44] Rietveld, H. M., "A profile refinement method for nuclear and magnetic structures", J. Appl. Crystallogr. 2, 65-71 (1969).

[45] Hill, R. J. and Howard, C. J., "Quantitative phase analysis from neutron powder diffraction data using the Rietveld method", J. Appl. Crystallogr. 20, 467-474 (1987).

[46] Bish, D. L. and Post, J. E., "Quantitative Mineralogical Analysis Using the Rietveld Full- Pattern Fitting Method", Am. Mineral. 78, 932-940 (1993).

[47] Taupin, D., "Automatic peak determination in X-ray powder patterns", J. Appl. Crystallogr. 6, 266-273 (1973).

[48] Balzar, D., "Voigt-function model in diffraction line-broadening analysis" in Microstructure Analysis from Diffraction (eds. Snyder, R. L., Bunge, H. J. \& Fiala, J.), 808 (International Union of Crystallography, 1999).

[49] Cheary, R. W., Coelho, A. A. and Cline, J. P., "Fundamental parameters line profile fitting in laboratory diffractometers", J. Res. Natl. Inst. Stand. Technol. 109, 1 (2004).

[50] Langford, J. I., Delhez, R., de Keijser, T. H. and Mittemeijer, E. J., "Profile Analysis for Microcrystalline Properties by the Fourier and Other Methods", Aust. J. Phys. 41, 173 (1988).

[51] Mittemeijer, E. J. and Welzel, U., "The "state of the art" of the diffraction analysis of crystallite size and lattice strain", Zeitschrift für Krist. 223, 552-560 (2008).

[52] Hubbard, C. R., Swanson, H. E. and Mauer, F. A., "NIST Certificate - Standard Reference Material 640", (1974).

[53] Le Bail, A., "Whole powder pattern decomposition methods and applications: A retrospection", Powder Diffr. 20, 316-326 (2005).

[54] Sabine, T. M., Hunter, B. A., Sabine, W. R. and Ball, C. J., "Analytical Expressions for the Transmission Factor and Peak Shift in Absorbing Cylindrical Specimens", J. Appl. Crystallogr. 31, 47-51 (1998).

[55] Balzar, D., Audebrand, N., Daymond, M. R., Fitch, A., Hewat, A., Langford, J. I., Le Bail, A., Louër, D., Masson, O., McCowan, C. N., Popa, N. C., Stephens, P. W. and Toby, B. H., "Size-strain line-broadening analysis of the ceria round-robin sample", J. Appl. Crystallogr. 37, 911-924 (2004).

[56] Stokes, A. R. and Wilson, A. J. C., "The diffraction of X rays by distorted crystal aggregates - I", Proc. Phys. Soc. 56, 174-181 (1944).

[57] Matyi, R. J., Schwartz, L. H. and Butt, J. B., "Particle Size, Particle Size Distribution, and Related Measurements of Supported Metal Catalysts", Catal. Rev. 29, 41-99 (1987).

[58] Butt, J. B., "Carbide phases on iron-based Fischer-Tropsch synthesis catalysts part I: Characterization studies", Catal. Lett. 7, 61-82 (1990).

[59] Zhou, X., Ji, J., Wang, D., Duan, X., Qian, G., Chen, D. and Zhou, X., "Hierarchical structured $\alpha$-Al203 supported S-promoted Fe catalysts for direct conversion of syngas to lower olefins", Chem. Commun. 51, 8853-8856 (2015).

[60] Xie, J., Yang, J., Dugulan, A. I., Holmen, A., Chen, D., De Jong, K. P. and Louwerse, M. J., "Size and Promoter Effects in Supported Iron Fischer-Tropsch Catalysts: Insights from Experiment and Theory", ACS Catal. 6, 3147-3157 (2016). 
[61] Butt, J. B., "Carbide phases on iron-based Fischer-Tropsch synthesis catalysts part II: Some reaction studies", Catal. Lett. 7, 83-106 (1990).

[62] Dry, M. E., Shingles, T. and van H. Botha, C. S., "Factors influencing the formation of carbon on iron Fischer-Tropsch catalysts I. The influence of promoters", J. Catal. 17, 341-346 (1970).

[63] Dry, M. E., Shingles, T., Boshoff, L. J. and van H. Botha, C. S., "Factors influencing the formation of carbon on iron Fischer-Tropsch catalysts. II. The effect of temperature and of gases and vapors present during Fischer-Tropsch synthesis", J. Catal. 17, 347-354 (1970).

[64] Cohn, E. M., Bean, E. H., Mentser, M., Hofer, L. J. E., Pontello, A., Peebles, W. C. and Jack, K. H., "The carburization of iron oxide with carbon monoxide: Modifications of Hägg iron carbide", J. Appl. Chem. 5, 418-425 (1955).

[65] Sancier, K. M., Isakson, W. E. and Wise, H., "Carburization Studies of Iron Fischer-Tropsch Catalysts" in Hydrocarbon Synthesis from Carbon Monoxide and Hydrogen (eds. Kugler, E. L. \& Steffgen, F. W.) 178, 129-145 (American Chemical Society, Washington, 1979).

[66] Berry, T. F., Ames, R. N. and Snow, R. B., "Influence of Impurities and Role of Iron Carbides in Deposition of Carbon from Carbon Monoxide", J. Am. Ceram. Soc. 39, 308-318 (1956).

[67] Koeken, A. C. J., Torres Galvis, H. M., Davidian, T., Ruitenbeek, M. and de Jong, K. P., "Suppression of Carbon Deposition in the Iron-Catalyzed Production of Lower Olefins from Synthesis Gas", Angew. Chem., Int. Ed. 51, 7190-7193 (2012).

[68] Toby, B. H., "R factors in Rietveld analysis: How good is good enough?", Powder Diffr. 21, 67-70 (2006).

[69] Janbroers, S., Louwen, J. N., Zandbergen, H. W. and Kooyman, P. J., "Insights into the nature of ironbased Fischer-Tropsch catalysts from quasi in situ TEM-EELS and XRD", J. Catal. 268, 235-242 (2009).

[70] Shroff, M. D. and Datye, A. K., "The importance of passivation in the study of iron Fischer-Tropsch catalysts", Catal. Lett. 37, 101-106 (1996).

[71] Xie, J., Torres Galvis, H. M., Koeken, A. C. J., Kirilin, A., Dugulan, A. I., Ruitenbeek, M. and de Jong, K. P., "Size and Promoter Effects on Stability of Carbon-Nanofiber-Supported Iron-Based Fischer-Tropsch Catalysts", ACS Catal. 6, 4017-4024 (2016).

[72] Li, J., Cheng, X., Zhang, C., Chang, Q., Wang, J., Wang, X., Lv, Z., Dong, W., Yang, Y. and Li, Y., "Effect of alkalis on iron-based Fischer-Tropsch synthesis catalysts: Alkali-FeO $\mathrm{O}_{x}$ interaction, reduction, and catalytic performance", Appl. Catal. A Gen. 528, 131-141 (2016).

[73] Amelse, J. A., Grynkewich, G., Butt, J. B. and Schwartz, L. H., "Moessbauer spectroscopic study of passivated small particles of iron and iron carbide", J. Phys. Chem. 85, 2484-2488 (1981).

[74] Sirimanothan, N., Hamdeh, H. H., Zhang, Y. and Davis, B. H., "Fischer-Trospch Synthesis; Changes in Phase and Activity during Use", Catal. Lett. 82,181-191 (2002).

[75] León-Reina, L., De la Torre, A. G., Porras-Vázquez, J. M., Cruz, M., Ordonez, L. M., Alcobé, X., GispertGuirado, F., Larrañaga-Varga, A., Paul, M., Fuellmann, T., Schmidt, R. and Aranda, M. A. G., "Round robin on Rietveld quantitative phase analysis of Portland cements", J. Appl. Crystallogr. 42, 906-916 (2009).

[76] Michel, F. M., Ehm, L., Antao, S. M., Lee, P. L., Chupas, P. J., Liu, G., Strongin, D. R., Schoonen, M. A. A., Phillips, B. L. and Parise, J. B., "The Structure of Ferrihydrite, a Nanocrystalline Material", Science 316, 1726-1729 (2007).

[77] Jozwiak, W. K., Kaczmarek, E., Maniecki, T. P., Ignaczak, W. and Maniukiewicz, W., "Reduction behavior of iron oxides in hydrogen and carbon monoxide atmospheres", Appl. Catal. A Gen. 326, 1727 (2007).

[78] Podgurski, H. H., Kummer, J. T., DeWitt, T. W. and Emmett, P. H., "Preparation, Stability and 
Adsorptive Properties of the Carbides of Iron", J. Am. Chem. Soc. 72, 5382-5388 (1950).

[79] Krishnamoorthy, S., Li, A. and Iglesia, E., "Pathways for $\mathrm{CO}_{2}$ Formation and Conversion During FischerTropsch Synthesis on Iron-Based Catalysts", Catal. Lett. 80, 77-86 (2002).

[80] Davis, B. H., "Fischer-Tropsch Synthesis: Reaction mechanisms for iron catalysts", Catal. Today 141, 25-33 (2009).

[81] Shultz, J. F., Abelson, M., Stein, K. C. and Anderson, R. B., "Studies of the Fischer-Tropsch Synthesis. XVIII. Influence of Catalyst Geometry on Synthesis on Iron Catalysts", J. Phys. Chem. 63, 496-500 (1959).

[82] O’Brien, R. J., Xu, L., Spicer, R. L. and Davis, B. H., "Activation Study of Precipitated Iron Fischer-Tropsch Catalysts", Energy \& Fuels 10, 921-926 (1996).

[83] Bukur, D. B., Okabe, K., Rosynek, M. P., Li, C. P., Wang, D. J., Rao, K. R. P. M. and Huffman, G. P., "Activation Studies with a Precipitated Iron Catalyst for Fischer-Tropsch Synthesis. I. Characterization Studies", Journal of Catalysis 155, 353-365 (1995).

[84] Arakawa, H. and Bell, A. T., "Effects of potassium promotion on the activity and selectivity of iron Fischer-Tropsch catalysts", Ind. Eng. Chem. Process Des. Dev. 22, 97-103 (1983).

[85] Olsson, R. G. and Turkdogan, E. T., "Catalytic effect of iron on decomposition of carbon monoxide: II. Effect of additions of $\mathrm{H}_{2}, \mathrm{H}_{2} \mathrm{O}, \mathrm{CO}_{2}, \mathrm{SO}_{2}$ and $\mathrm{H}_{2} \mathrm{~S}^{\prime \prime}$, Metall. Trans. 5, 21-26 (1974).

[86] Nagakura, S., "Study of Metallic Carbides by Electron Diffraction Part III. Iron Carbides", J. Phys. Soc. Japan 14, 186-195 (1959).

[87] Raupp, G. B. and Delgass, W. N., "Mössbauer Investigation of Supported Fe and FeNi Catalysts II. Carbides Formed by Fischer-Tropsch Synthesis", J. Catal. 58, 348-360 (1979).

[88] Tau, L. M., Borcar, S., Bianchi, D. and Bennett, C. O., "Investigation of supported iron Fischer-Tropsch synthesis catalysts by Mössbauer spectroscopy", J. Catal. 87, 36-54 (1984).

[89] Xu, K., Sun, B., Lin, J., Wen, W., Pei, Y., Yan, S., Qiao, M., Zhang, X. and Zong, B., "ع-Iron carbide as a lowtemperature Fischer-Tropsch synthesis catalyst", Nat. Commun. 5, 5783 (2014).

[90] Jiang, F., Zhang, M., Liu, B., Xu, Y. and Liu, X., "Insights into the influence of support and potassium or sulfur promoter on iron-based Fischer-Tropsch synthesis: understanding the control of catalytic activity, selectivity to lower olefins, and catalyst deactivation", Catal. Sci. Technol. 7, 1245-1265 (2017).

[91] Riedel, T., Schulz, H., Schaub, G., Jun, K.-W., Hwang, J.-S. and Lee, K.-W., "Fischer-Tropsch on Iron with $\mathrm{H}_{2} / \mathrm{CO}$ and $\mathrm{H}_{2} / \mathrm{CO}_{2}$ as Synthesis Gases: The Episodes of Formation of the Fischer-Tropsch Regime and Construction of the Catalyst", Top. Catal. 26, 41-54 (2003).

[92] Nagakura, S., Hirotsu, Y., Kusunoki, M., Suzuki, T. and Nakamura, Y., "Crystallographic study of the tempering of martensitic carbon steel by electron microscopy and diffraction", Metall. Trans. A 14, 1025-1031 (1983).

[93] Bauer-Grosse, E. and Le Caër, G., "Crystallisation of amorphous $\mathrm{Fe}_{1-x} C_{x}$ alloys $(0.30 \leq x \leq 0.32)$ and Chemical Twinning", J. Phys. F Met. Phys. 16, 399-406 (1986).

[94] Nagakura, S., Suzuki, T. and Kusunoki, M., "Structure of the Precipitated Particles at the Third Stage of Tempering of Martensitic Iron-Carbon Steel Studied by High Resolution Electron Microscopy", Trans. Japan Inst. Met. 22, 699-709 (1981).

[95] du Plessis, H. E., de Villiers, J. P. R., Kruger, G. J., Steuwer, A. and Brunelli, M., "Rietveld and pair distribution function study of Hägg carbide using synchrotron X-ray diffraction", J. Synchrotron Radiat. 18, 266-271 (2011).

[96] Cohn, E. M. and Hofer, L. J. E., "Mode of Transition from Hägg Iron Carbide to Cementite", J. Am. Chem. Soc. 72, 4662-4664 (1950). 
[97] Wood, I. G., Vočadlo, L., Knight, K. S., Dobson, D. P., Marshall, W. G., Price, G. D. and Brodholt, J., "Thermal expansion and crystal structure of cementite, $\mathrm{Fe}_{3} \mathrm{C}$, between 4 and $600 \mathrm{~K}$ determined by timeof-flight neutron powder diffraction", J. Appl. Crystallogr. 37, 82-90 (2004).

[98] de Smit, E., van Schooneveld, M. M., Cinquini, F., Bluhm, H., Sautet, P., de Groot, F. M. F. and Weckhuysen, B. M., "On the Surface Chemistry of Iron Oxides in Reactive Gas Atmospheres", Angew. Chem., Int. Ed. 50, 1584-1588 (2011).

[99] Bordet, A., Soulantika, A. and Chaudret, B., "Iron carbide nanoparticles, method for preparing same and use thereof for heat generation", Patent WO 2017/103492 A2 (2017).

[100] Li, J., Cheng, X., Zhang, C., Yang, Y. and Li, Y., "Effects of alkali on iron-based catalysts for FischerTropsch synthesis: CO chemisorptions study", J. Mol. Catal. A Chem. 396, 174-180 (2015).

[101] Berry, F. J. and Smith, M. R., "A Mössbauer investigation of iron-containing catalysts prepared at low temperatures and active for carbon monoxide hydrogenation", J. Chem. Soc. Faraday Trans. 1 Phys. Chem. Condens. Phases 85, 467-477 (1989).

[102] Ribeiro, M. C., Jacobs, G., Davis, B. H., Cronauer, D. C., Kropf, A. J. and Marshall, C. L., "Fischer-Tropsch Synthesis: An In-Situ TPR-EXAFS/XANES Investigation of the Influence of Group I Alkali Promoters on the Local Atomic and Electronic Structure of Carburized Iron/Silica Catalysts", J. Phys. Chem. C 114, 7895-7903 (2010).

[103] Ngantsoue-Hoc, W., Zhang, Y., O’Brien, R. J., Luo, M. and Davis, B. H., "Fischer-Tropsch synthesis: activity and selectivity for Group I alkali promoted iron-based catalysts", Appl. Catal. A Gen. 236, 7789 (2002).

[104] Ratnasamy, C. and Wagner, J. P., "Water Gas Shift Catalysis", Catal. Rev. 51, 325-440 (2009).

[105] Krupay, B. W. and Amenomiya, Y., "Alkali-promoted Alumina Catalysts I. Chemisorption and oxygen exchange of carbon monoxide and carbon dioxide on potassium-promoted alumina catalysts", J. Catal. 67, 362-370 (1981)

[106] Amenomiya, Y. and Pleizier, G., "Alkali-promoted Alumina Catalysts II. Water-Gas Shift Reaction", J. Catal. 76, 345-353 (1982).

[107] Anderson, R. B., Karn, F. S. and Shultz, J. F., "Factors in sulfur poisoning of iron catalysts in FischerTropsch synthesis", J. Catal. 4, 56-63 (1965).

[108] Towett, E. K., Shepherd, K. D. and Cadisch, G., "Quantification of total element concentrations in soils using total X-ray fluorescence spectroscopy (TXRF)", Sci. Total Environ. 463-464, 374-388 (2013).

[109] Tsuzuki, A., Sago, S., Hirano, S.-I. and Naka, S., "High temperature and pressure preparation and properties of iron carbides $\mathrm{Fe}_{7} \mathrm{C}_{3}$ and $\mathrm{Fe}_{3} \mathrm{C}^{\prime \prime}$, J. Mater. Sci. 19, 2513-2518 (1984).

[110] Bi, X.-X., Ganguly, B., Huffman, G. P., Huggins, F. E., Endo, M. and Eklund, P. C., "Nanocrystalline $\alpha-F e$, $\mathrm{Fe}_{3} \mathrm{C}$, and $\mathrm{Fe}_{7} \mathrm{C}_{3}$ produced by $\mathrm{CO}_{2}$ laser pyrolysis", J. Mater. Res. 8, 1666-1674 (1993).

[111] Yamada, Y., Yoshida, H., Kouno, K. and Kobayashi, Y., "Iron carbide films produced by laser deposition", J. Phys. Conf. Ser. 217, 012096 (2010).

[112] Sethuraman, A. R., "In-situ high temparature X-ray diffraction studies of nanocrystalline iron carbides", Nanostructured Mater. 4, 79-92 (1994).

[113] Audier, M., Bowen, P. and Jones, W., "Transmission electron microscopic study of single crystals of $\mathrm{Fe}_{7} \mathrm{C}_{3}{ }^{\prime \prime}$, J. Cryst. Growth 63, 125-134 (1983).

[114] Lywood, W. J. and Twigg, M. V., "Hydrogen production including a shift reaction process", Patent EP0361648A1 (1990).

[115] Luo, M. and Davis, B. H., "Fischer-Tropsch synthesis: Group II alkali-earth metal promoted catalysts", Appl. Catal. A Gen. 246, 171-181 (2003).

[116] Rhodes, C., Hutchings, G. J. and Ward, A. M., "Water-gas shift reaction: finding the mechanistic 
boundary", Catal. Today 23, 43-58 (1995).

[117] Anderson, R. B., Hofer, L. J. E., Cohn, E. M. and Seligman, B., "Studies of the Fischer-Tropsch Synthesis. IX. Phase Changes of Iron Catalysts in the Synthesis", J. Am. Chem. Soc. 73, 944-946 (1951).

[118] Liu, X., Zhang, C., Li, Y., Niemantsverdriet, J. W., Wagner, J. B. and Hansen, T. W., "Environmental Transmission Electron Microscopy (ETEM) Studies of Single Iron Nanoparticle Carburization in Synthesis Gas", ACS Catal. 7, 4867-4875 (2017).

[119] Kril, C. E. and Birringer, R., "Estimating grain-size distributions in nanocrystalline materials from Xray diffraction profile analysis", Philos. Mag. A 77, 621-640 (1998).

[120] Steynberg, A. P., Espinoza, R. L., Jager, B. and Vosloo, A. C., "High temperature Fischer-Tropsch synthesis in commercial practice", Appl. Catal. A Gen. 186, 41-54 (1999).

[121] Sarkar, A., Seth, D., Dozier, A. K., Neathery, J. K., Hamdeh, H. H. and Davis, B. H., "Fischer-Tropsch Synthesis: Morphology, Phase Transformation and Particle Size Growth of Nano-scale Particles", Catal. Lett. 117, 1-17 (2007).

[122] Fruchart, R., Senateur, J. P., Bouchaud, J. P. and Michel, A., "A propos de la structure exacte du carbure de fer $\mathrm{Fe}_{7} \mathrm{C}_{3} "$, Bull. Soc. Chim. Fr. 2, 392 (1965).

[123] Gatte, R. R. and Phillips, J., "The influence of particle size and structure on the Mössbauer spectra of iron carbides formed during Fischer-Tropsch synthesis", J. Catal. 104, 365-374 (1987).

[124] Foct, J., Senateur, J. P., Dubois, J. M. and Le Caër, G., "Mössbauer Spectroscopy of Different Interstitial Compounds and Solid Solutions Containing ${ }^{57} \mathrm{Fe}^{\prime}$, Le J. Phys. Colloq. 40, 647-649 (1979).

[125] Dubois, J. M. and Le Caër, G., "Electron diffraction and Mossbauer studies of the $\epsilon$ phase retained in splat-quenched Fe-C and Fe-C-Si alloys", Acta Metall. 25, 609-618 (1977).

[126] Shultz, J. F., Abelson, M., Shaw, L. and Anderson, R. B., "Fischer-Tropsch Synthesis. Nitrides and Carbonitrides of Iron as Catalysts", Ind. Eng. Chem. 49, 2055-2060 (1957).

[127] Niewa, R., Rau, D., Wosylus, A., Meier, K., Hanfland, M., Wessel, M., Dronskowski, R., Dzivenko, D. A., Riedel, R. and Schwarz, U., "High-Pressure, High-Temperature Single-Crystal Growth, Ab initio Electronic Structure Calculations, and Equation of State of $\varepsilon-\mathrm{Fe}_{3} \mathrm{~N}_{1+x}$ ", Chem. Mater. 21, 392-398 (2009).

[128] Leineweber, A., Shang, S., Liu, Z.-K., Widenmeyer, M. and Niewa, R., "Crystal structure determination of Hägg carbide, $\chi-\mathrm{Fe}_{5} \mathrm{C}_{2}$ by first-principles calculations and Rietveld refinement", Zeitschrift für Krist. 227, 207-220 (2012).

[129] Jack, K. H., "The iron-nitrogen system: the crystal structures of e-phase iron nitrides", Acta Crystallogr. 5, 404-411 (1952).

[130] Rechenbach, D. and Jacobs, H., "Structure determination of $\zeta-\mathrm{Fe}_{2} \mathrm{~N}$ by neutron and synchrotron powder diffraction", J. Alloys Compd. 235, 15-22 (1996).

[131] Jacobs, H., Rechenbach, D. and Zachwieja, U., "Structure determination of $\gamma^{\prime}-\mathrm{Fe}_{4} \mathrm{~N}$ and $\epsilon-\mathrm{Fe}_{3} \mathrm{~N}^{\prime}$, J. Alloys Compd. 227, 10-17 (1995).

[132] Nagakura, S. and Tanehashi, K., "Electronic Structure of Iron Nitrides Studied by Electron Diffraction. II. $\varepsilon-\mathrm{Fe}_{2} \mathrm{~N}$ and $\zeta-\mathrm{Fe}_{2} \mathrm{~N}^{\prime \prime}$, J. Phys. Soc. Japan 25, 840-846 (1968). 
This page is intentionally left blank 


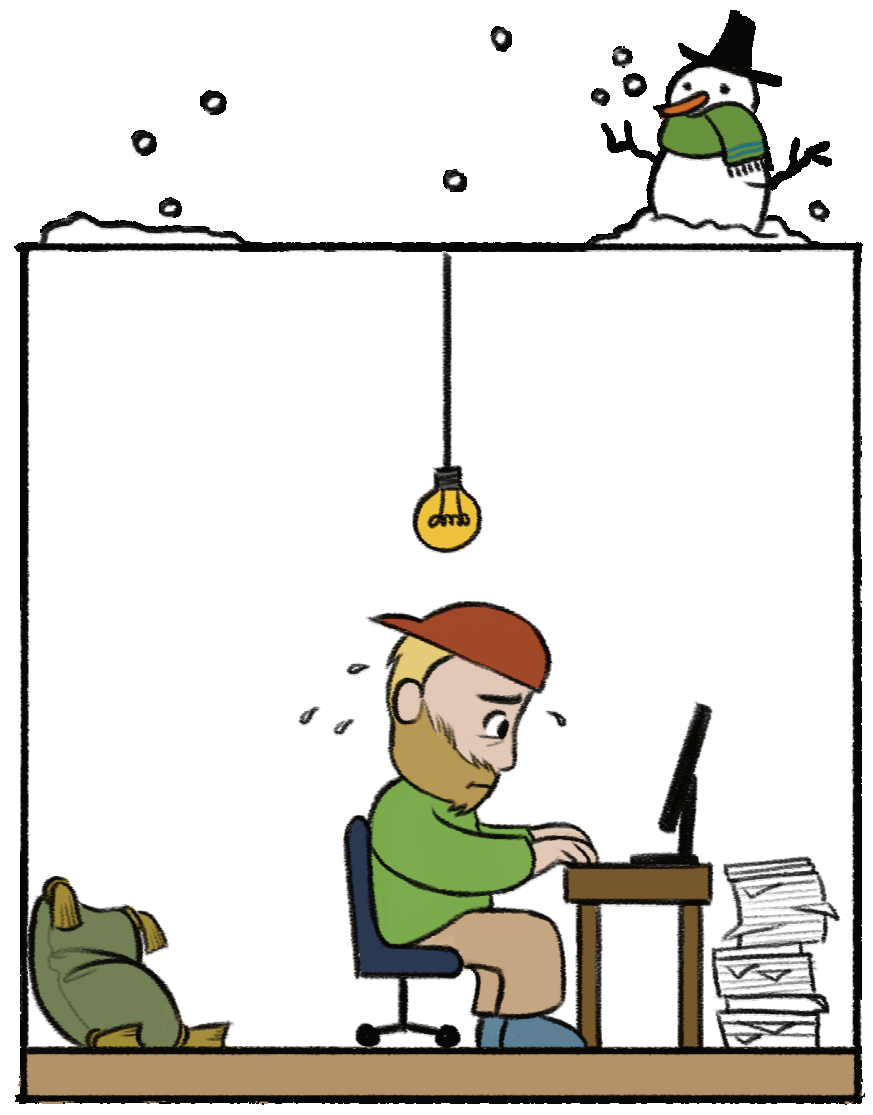




\section{Chapter 4}

\section{On the Role of Iron Carbides in the Fischer-Tropsch Synthesis with Fe(-Na- $\mathrm{S}) / \alpha-\mathrm{Al}_{2} \mathrm{O}_{3}$ Catalysts}

The Fischer-Tropsch Synthesis (FTS) reaction converts a $\mathrm{H}_{2}: \mathrm{CO}$ gas mixture with e.g. Fe-based catalysts into hydrocarbons. Fe carbides, $\varepsilon-\mathrm{Fe}_{3} \mathrm{C}, \eta-\mathrm{Fe}_{2} \mathrm{C}, \chi-\mathrm{Fe}_{5} \mathrm{C} 2, \theta-\mathrm{Fe}_{3} \mathrm{C}$ and/or $\theta-\mathrm{Fe}_{7} \mathrm{C}_{3}$, form the active phases in Fe-based FTS reaction catalysts. We aim to probe in to each of the Fe carbides' catalysis properties, in combination to the effect of $\mathrm{Na}-\mathrm{S}$ promotion on the Fe carbide phases in 10 bar FTS reaction at $340^{\circ} \mathrm{C}$. Supported $\mathrm{Fe}(-\mathrm{Na}-\mathrm{S}) / \alpha-\mathrm{Al}_{2} \mathrm{O}_{3}$ catalyst materials with preformed Fe carbides were tested for their FTS reaction performance. The catalysts were characterized ex-situ after the FTS reaction by using X-ray Powder Diffractometry (XRPD) with Rietveld Quantitative Phase Analysis (R-QPA) and Transmission Electron Microscopy (TEM). In the FTS reaction, $\mathrm{Na}-\mathrm{S}$ promotion stabilizes the $\eta-\mathrm{Fe}_{2} \mathrm{C}$ and destabilizes the $\theta-\mathrm{Fe}_{3} \mathrm{C}$ phase. Regardless of the Na-S promotion, the $\chi-\mathrm{Fe}_{5} \mathrm{C}_{2}$ and $\theta-\mathrm{Fe}_{7} \mathrm{C}_{3}$ phases were stable and the $\varepsilon-\mathrm{Fe}_{3} \mathrm{C}$ phase unstable. Any specific Fe carbide did not directly imply enhanced FTS reaction activity. This property was primarily enhanced by the added $\mathrm{Na}-\mathrm{S}$ promotion. By the differences in the observed lattice parameters, the $\eta-\mathrm{Fe}_{2} \mathrm{C}$ and $\chi-\mathrm{Fe}_{5} \mathrm{C}_{2}$ phases were divided into subtypes and the subtypes primary formation requirements were assigned. Indications on the FTS reaction hydrocarbon selectivity dependency on Fe carbide subtypes and/or phases could be observed, warranting further research. 


\subsection{INTRODUCTION}

Fe carbides form the active phases for the Fe-based Fischer-Tropsch Synthesis (FTS) reaction polymerizing a $\mathrm{H}_{2}$ : $\mathrm{CO}$ mixture (i.e. syngas) into various hydrocarbons. The $\mathrm{Fe}$ carbides observable in the FTS reaction research field have been identified with their crystal lattices as: $\varepsilon-\mathrm{Fe}_{3} \mathrm{C} P 6_{3} 22$ (182), $\eta-\mathrm{Fe}_{2} \mathrm{C} \mathrm{Pnnm} \mathrm{(58),} \chi-\mathrm{Fe}_{5} \mathrm{C}_{2} C 2 / c$ (15), $\theta-\mathrm{Fe}_{3} \mathrm{C} \mathrm{Pnma}$ (62) and $\theta-\mathrm{Fe}_{7} \mathrm{C}_{3}$ Pnma (62) on the basis of the literature (Chapter 2) and performed CO carburization experiments at various reaction temperatures (Chapter 3).

Different FTS reaction activities for the various Fe carbides have been proposed in the past. That is, that not all of the Fe carbides perform equally in the FTS reaction. The most commonly observed Fe carbide phase in the FTS reaction is the $\chi-\mathrm{Fe}_{5} \mathrm{C}_{2}$ phase (the Hägg carbide),1-6 which is usually taken as a FTS reaction active carbide phase. The " $\varepsilon$ carbides" (i.e. $\varepsilon-\mathrm{Fe}_{3} \mathrm{C}$ and/or $\eta-\mathrm{Fe}_{2} \mathrm{C}$ ) have been proposed to be more FTS active than the $\chi-\mathrm{Fe}_{5} \mathrm{C}_{2}$ phase, ${ }^{7}$ or can be present in a deactivated catalyst material. ${ }^{8} \theta-\mathrm{Fe}_{3} \mathrm{C}$ phase (cementite) has indirectly been associated to the deactivation of the Fe-based FTS catalyst materials, ${ }^{9}$ or possesses somewhat a lower FTS activity than the $\chi-\mathrm{Fe}_{5} \mathrm{C}_{2}$ phase, yet still being active for the FTS reaction. ${ }^{10}$ The less studied and more rare $\mathrm{Fe}_{7} \mathrm{C}_{3}$ (i.e. $\theta$ $\mathrm{Fe}_{7} \mathrm{C}_{3}$ ) phase has also been associated to the FTS active catalyst materials. ${ }^{11,12}$ In contrast to this, the $\mathrm{Fe}_{7} \mathrm{C}_{3}$ phase has also been encountered in the industrially applied Fe-based FTS reaction catalyst materials near their end-of-life. ${ }^{13}$

However, whether a single, bulk Fe carbide phase or a Fe phase mixture can simply be associated to a catalyst material's FTS reaction performance has been questioned before. ${ }^{8,14-16}$ Intertwined deactivation processes, e.g. carbon deposition, , ${ }^{8,13,17-19} \mathrm{Fe}$ particle sintering, ${ }^{20,21}$ and/or Fe reoxidation into inactive oxides can simultaneously take place for Fe-based FTS reaction catalyst materials.3,4,8,13,21-25 The variety of the possible deactivation processes complicate the interpretation of the effect of the Fe carbide phases' individual performance in the FTS reaction.

The hydrocarbon product selectivity in the FTS reaction can be altered, besides changing the reaction conditions: ${ }^{26,27}$ with the addition of promoters, $, 822,28-33$ or to some extent with different Fe phases as the FTS reaction's starting materials (i.e. with catalyst pretreatment or conditioning).4,34-36 Especially, Na-S promotion has been observed as an effective way for increasing the FTS reaction's hydrocarbon products' olefin content while suppressing the undesired $\mathrm{CH}_{4}$ product selectivity. ${ }^{28-30,37-40}$

In this Chapter, we aim to systematically probe further to the effect that the initial Fe carbide phase(s), with and without the Na-S promotion, have on the catalyst materials' FTS reaction catalysis performance. The changes in the initial Fe carbide phase(s) after the FTS reaction are followed and further evaluated in respect to their FTS reaction performance (Figure 4.1). Especially, the Fe carbides' lattice parameters 
and their changes, under the FTS reaction or CO carburization conditions are often an overlooked Fe carbide property. This, despite the X-ray Powder Diffractometry (XRPD), a technique capable of probing to the Fe carbide lattice changes, has been widely applied in the FTS research field. $1,4,9,11,12,15,19,26,34,41,42$ As an example, various and significantly different lattice parameters have been observed for the $\chi-\mathrm{Fe}_{5} \mathrm{C}_{2}$ phase. ${ }^{43-46}$ That is, more than one type of the $\chi-\mathrm{Fe}_{5} \mathrm{C}_{2}$ phase may exist, with an unclear relation to their FTS reaction performance.

Therefore, with the use of ex-situ XRPD with Rietveld Quantitative Phase Analysis (R-QPA), we explore the effect of Na-S promotion on the preformed Fe carbides (i.e. $\varepsilon$ $\mathrm{Fe}_{3} \mathrm{C}, \eta-\mathrm{Fe}_{2} \mathrm{C}, \chi-\mathrm{Fe}_{5} \mathrm{C}_{2}, \theta-\mathrm{Fe}_{3} \mathrm{C}$ and $\left.\theta-\mathrm{Fe}_{7} \mathrm{C}_{3}\right)$ on $\mathrm{CO}$ carburized $\mathrm{Fe}(-\mathrm{Na}-\mathrm{S}) / \alpha-\mathrm{Al}_{2} \mathrm{O}_{3}$ catalyst materials applied in the FTS reaction at $340^{\circ} \mathrm{C}$. For reference, Fe carbide free, $\mathrm{H}_{2}$ reduced only catalyst materials are also studied. The R-QPA is used to obtain the Fe

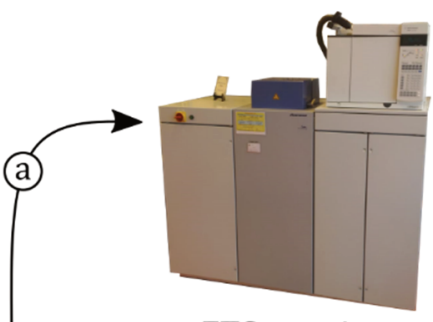

FTS reaction
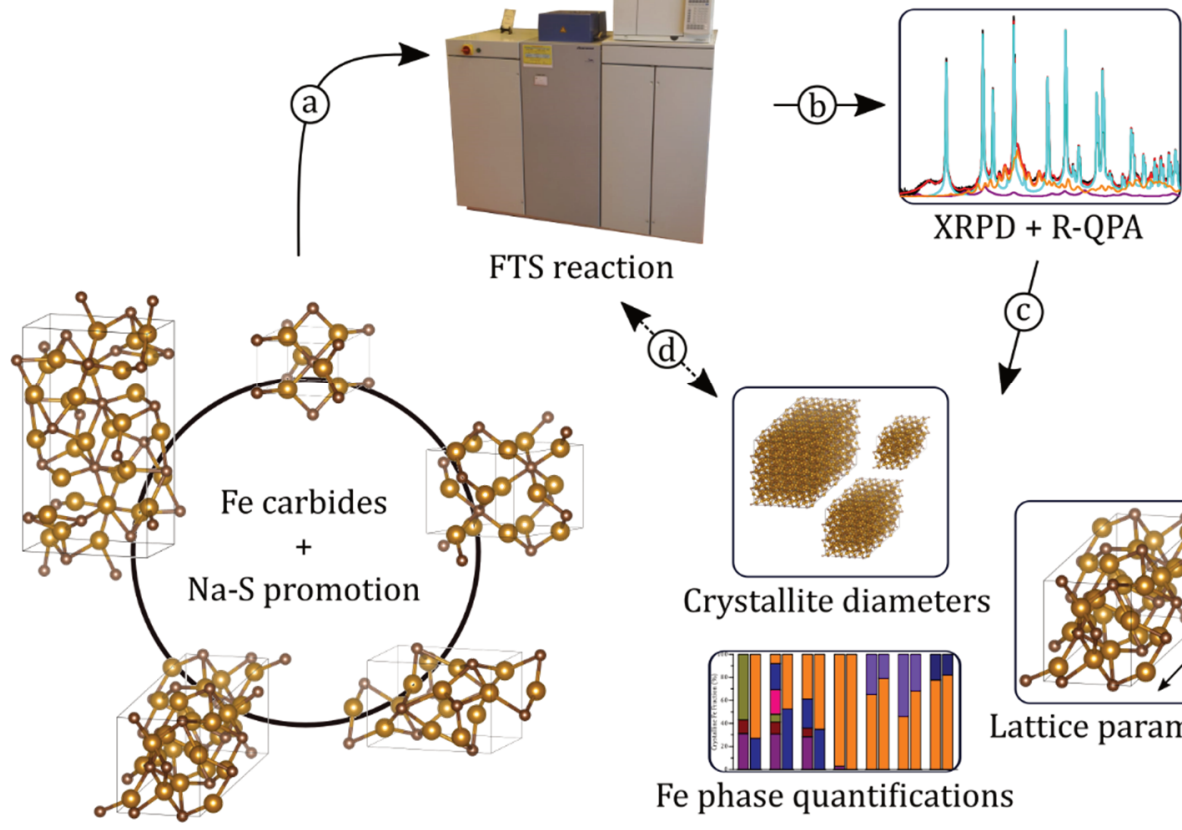

Crystallite diameters
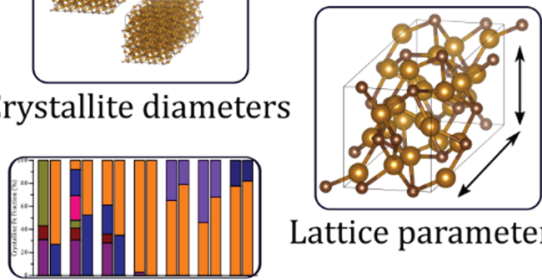

Lattice parameters

Fe phase quantifications

Figure 4.1

A schematic of the approach applied in this Chapter. Various Fe carbide phases were preformed on unpromoted and Na-S promoted $\mathrm{Fe}(-\mathrm{Na}-\mathrm{S}) / \alpha-\mathrm{Al}_{2} \mathrm{O}_{3}$ catalyst materials. The catalysts are (a) tested for their catalytic performance in the Fischer-Tropsch Synthesis (FTS) reaction and (b) subsequently characterized ex-situ with X-ray Powder Diffractometry (XRPD). Rietveld Quantitative Phase Analysis (R-QPA) is applied to the XRPD patterns in order to (c) quantify the Fe carbide phases and their crystallite diameters and lattice parameters in the catalyst materials after their application in the FTS reaction. The quantification results are then (d) compared to the catalyst materials FTS reaction catalysis performances. 
phase quantities, crystallite diameters and lattice parameters. As a complementary technique, Fe particle diameters and catalyst morphology are followed for the selected FTS reaction catalyst materials using Transmission Electron Microscopy (TEM). Both R-QPA and TEM obtained results are compared to the results for the catalyst materials before the FTS reaction, as reported in Chapter 3. These characterization results for the catalyst materials are then related to their FTS reaction catalysis performance in terms of both activity and selectivity.

\subsection{EXPERIMENTAL METHODS}

\subsubsection{Catalyst Preparation}

The details of the synthesis, $\mathrm{CO}$ carburization (within $240-440^{\circ} \mathrm{C}$ ) or only $\mathrm{H}_{2}$ reduction of the nominal 7 weight percent (wt-\%) Fe on alpha alumina supported (Fe-(Na-S) $/ \alpha-$ $\mathrm{Al}_{2} \mathrm{O}_{3}$ ) catalyst materials, with or without Na-S promotion, are described in Chapter 3. The $\mathrm{CO}$ carburized or $\mathrm{H}_{2}$ reduced FTS reaction catalyst materials within this Chapter are from the same catalyst material synthesis batches as studied in Chapter 3 .

\subsubsection{Catalyst Testing}

A high-throughput Avantium Flowrence catalyst testing set-up with 16 parallel fixed bed reactors was used for testing the prepared $\mathrm{CO}$ carburized or $\mathrm{H}_{2}$ reduced catalyst materials in the FTS reaction.

The catalyst materials ( $\approx 25 \mathrm{mg}, 212-425 \mu \mathrm{m}$ particle size) were diluted with $\mathrm{SiC}$ ( $\approx 125 \mathrm{mg}, 212-425 \mu \mathrm{m}$ ). The resulting catalyst material/SiC mixtures were loaded into stainless steel reactor tubes with an inner diameter of $2 \mathrm{~mm}$, giving catalyst beds with heights within $\approx 33-34 \mathrm{~mm}$. Each catalyst material/SiC mixture was loaded into two reactors for testing the data reproducibility. Additionally, two reactor tubes were loaded with SiC only and used as blank reactors.

The FTS reaction was performed at $340^{\circ} \mathrm{C}$ at 10 bar $\mathrm{H}_{2}: \mathrm{CO}: \mathrm{He}$ (2.0:1.0:0.33 volume ratio) total pressure. The nominal input gas flows for each reactor were $5.28 \mathrm{ml} / \mathrm{min}$ $\mathrm{H}_{2}, 2.64 \mathrm{ml} / \mathrm{min} \mathrm{CO}$ and $0.88 \mathrm{ml} / \mathrm{min}$ He giving gas hourly space velocity (GHSV, as total input gas flow per total catalyst bed volume) of $\approx 5000 \mathrm{~h}^{-1}$. The reactor pressurizing was done with the $\mathrm{H}_{2}: \mathrm{CO}: \mathrm{He}$ gas mixture at $120^{\circ} \mathrm{C}$ up to the desired 10 bar total reaction pressure. Upon reaching the 10 bar pressure, the reactor temperature was increased from $120^{\circ} \mathrm{C}$ to $340^{\circ} \mathrm{C}\left(2^{\circ} \mathrm{C} / \mathrm{min}\right)$ to start the FTS reaction test run. The FTS reaction was considered to start when the $340^{\circ} \mathrm{C}$ reaction temperature was reached. After $72 \mathrm{~h}$ of the FTS reaction, the input gas flow was switched to pure He and the reactor temperature and pressure were lowered to ambient conditions. The spent catalyst material/SiC mixtures were removed from the reactors and exposed to air. The catalysts were 
separated from the $\mathrm{SiC}$ diluent using a magnet. The $\mathrm{H}_{2}$ and $\mathrm{He}$ gas purities were grade 5.0 (99.999\% pure) and for CO grade 2.5 (99.5\% pure).

On-line gas chromatography (GC) with Thermal Conductivity (TCD) and Flame Ionization Detectors (FIDs) were used for determining the $\mathrm{H}_{2}$ and $\mathrm{CO}$ conversions, $\mathrm{CO}_{2}$ and hydrocarbon (+methanol) (mol C-\%) selectivities for each of the tested catalyst materials. $\mathrm{H}_{2}$ and $\mathrm{CO}$ conversion percentages were calculated from the difference of the outlet $\mathrm{H}_{2}$ and $\mathrm{CO}$ TCD peak areas between the average of the blank reactors and the reactors with loaded catalyst materials. Fe Time Yield (FTY) was calculated as based on the nominal converted input $\mathrm{CO}$ flow and the elemental Fe loadings of the parent (Chapter 3), as-synthesized calcined catalyst materials. Usage Ratio $\mathrm{H}_{2}: \mathrm{CO}$ was calculated as the ratio of the converted $\mathrm{H}_{2}$ and $\mathrm{CO}$. The $\mathrm{CO}$ conversion towards hydrocarbons was estimated by normalizing the cumulative total chromatogram FID peak areas per GC injection to the respective converted CO area as per TCD data.

TCD data was used to obtain the $\mathrm{CO}_{2}$ selectivities. The reported, $\mathrm{CO}_{2}$-free, methanol and $\mathrm{C}_{1}-\mathrm{C}_{4}$ hydrocarbon selectivities were obtained as identified chromatogram peak area per chromatogram cumulative total peak area using the FID data. The $\mathrm{C}_{5+}$ hydrocarbon selectivity was taken as $100-\left(\mathrm{C}_{1}-\mathrm{C}_{4}+\right.$ methanol selectivities $)$. Furthermore, individual $\mathrm{C}_{1}-\mathrm{C}_{8}$ hydrocarbon selectivities were calculated from the FID data and used for obtaining the Anderson-Schulz-Flory (ASF) distribution $\alpha$-value via the Equation 2.1 by using a linear fit to the $\mathrm{C}_{3}-\mathrm{C}_{7}$ range.

All of the TCD and FID peak areas were normalized to the variable GC injection volumes using the He peak area. Bad GC injections (i.e. outlying data points) were filtered out with taking 5-point median values over the FTS reaction time. Data points with distance $\geq 2 \mathrm{x}$ Standard Deviation (STD) of the median value were rejected.

\subsubsection{Catalyst Characterization}

The catalyst materials collected after the $72 \mathrm{~h}$ FTS reaction were analyzed ex-situ with bright field TEM and XRPD. The term X-ray Powder Diffractometry (XRPD) is used to refer to X-ray diffraction phenomena based characterization experiments that are done under powder diffraction conditions. ${ }^{47}$ R-QPA was applied on the obtained XRPD patterns, for both crystalline Fe phase quantifications and determining the crystallite diameters for the Fe phases. The Fe particles counted from the TEM images were used for determining the Fe particle size distributions. The experimental details on the application of TEM, XRPD and R-QPA have been explained earlier within Chapter 3 and the techniques are applied in an identical manner within this Chapter.

With R-QPA, in addition to the technique application details in Chapter 3, residual $\mathrm{SiC}$ phases from the catalyst material dilution for the FTS reaction gave occasional, random (non-powder diffraction), diffraction peaks. These SiC diffraction peaks were 


\section{Table 4.1}

Overview of the used shorthand notation for the catalyst materials used in this Chapter. The catalyst materials after the applied $\mathrm{H}_{2}$ reduction or $\mathrm{CO}$ carburization treatments are discussed more in detail in Chapter 3.

\begin{tabular}{cl}
\hline Shorthand Notation & \multicolumn{1}{c}{ Catalyst Material Description } \\
\hline $\mathrm{H}_{2}$ & $\mathrm{H}_{2}$ reduction at $400^{\circ} \mathrm{C}$ for $5 \mathrm{~h}$ \\
$240,290,340,390,440$ & $\mathrm{CO}$ carburization $($ at $1 \mathrm{~atm})$ temperature in ${ }^{\circ} \mathrm{C}$, after $\mathrm{H}_{2}$ reduction \\
340 h.p. & $\mathrm{CO}$ carburization at 25 bar at $340^{\circ} \mathrm{C}$, after calcining \\
$\mathrm{UP}$ & Unpromoted $\mathrm{Fe} / \alpha-\mathrm{Al}_{2} \mathrm{O}_{3}$ catalyst \\
$\mathrm{Na}-\mathrm{S}$ & $\mathrm{Na-S}$ promoted $\mathrm{Fe}-\mathrm{Na}-\mathrm{S} / \alpha-\mathrm{Al}_{2} \mathrm{O}_{3}$ catalyst \\
Carb. & Catalyst material after $\mathrm{H} 2$ reduction or CO carburization ("fresh catalyst") \\
FTS & Catalyst material after $72 \mathrm{~h} 340^{\circ} \mathrm{C}, 10$ bar FTS reaction ("spent catalyst") \\
\hline
\end{tabular}

fitted with individual Lorentzian peaks i.e. without a crystal structure or space group constrains.

\subsection{RESULTS AND DISCUSSION}

In this Chapter, we have used ex-situ XRPD for collecting X-ray diffraction patterns on Fe-based catalyst materials after their catalytic performance testing in the FTS reaction $\left(340^{\circ} \mathrm{C}, 10\right.$ bar, $\mathrm{H}_{2}: \mathrm{CO}: \mathrm{He}$ 2.0:1.0:0.33 v/v, GHSV $\left.5000 \mathrm{~h}^{-1}\right)$. R-QPA was employed on the XRPD patters for quantifying the Fe phases present in the catalyst materials applied in the FTS reaction. Fe particle diameter changes have been followed with TEM. The results have been compared to the R-QPA and TEM results for the respective catalyst materials before the FTS reaction reported in Chapter 3. This approach has been applied on both unpromoted $\mathrm{Fe} / \alpha-\mathrm{Al}_{2} \mathrm{O}_{3}$ (shorthand UP) and the Na-S promoted $\mathrm{Fe}-\mathrm{Na}$ $\mathrm{S} / \alpha-\mathrm{Al}_{2} \mathrm{O}_{3}(\mathrm{Na}-\mathrm{S})$ catalyst materials. These catalyst materials have been $\mathrm{CO}$ carburized in Chapter 3 to contain various Fe carbide phases, or only reduced with $\mathrm{H}_{2}$ to noncarbide $\alpha-\mathrm{Fe} / \mathrm{Fe}_{\mathrm{x}} \mathrm{O}_{\mathrm{y}}$ phases for comparison purposes.

The shorthand notation of $240,290,340,390$ or 440 is used to refer to catalyst material with the $\mathrm{CO}$ carburization done at the implied temperature in ${ }^{\circ} \mathrm{C}$, at atmospheric pressure. Catalyst materials $\mathrm{CO}$ carburized directly after a catalyst calcining step at 25 bar CO pressure at $340^{\circ} \mathrm{C}$ are noted in shorthand as 340 h.p.. The notation $\mathrm{H}_{2}$ is used for the only $\mathrm{H}_{2}$ reduced catalyst materials.

For the catalyst material characterizations done after the $72 \mathrm{~h}$ FTS reaction run, shorthand notation FTS (i.e. "spent catalyst") is used. For the $\mathrm{CO}$ carburized or $\mathrm{H}_{2}$ reduced catalyst materials before the FTS reaction shorthand Carb. (i.e. "fresh catalyst") is used collectively. The used shorthand notations for the catalyst materials under study are summarized in Table 4.1 


\subsubsection{Morphological Changes Probed with Transmission Electron Microscopy}

Examples of the measured TEM images for the selected catalyst materials $\left(\mathrm{H}_{2}, 240,340\right.$, 440) are compared for the UP FTS in Figure 4.2 and in Figure 4.3 for the Na-S FTS catalysts. The Fe particle (number-weighted) mean diameters for the FTS catalyst materials, as derived from the measured TEM images, are given in Figure 4.4. The values in Figure 4.4 are compared to the respective values for the Carb. catalyst materials from Chapter 3 before the FTS reaction.

The catalyst morphology, for both the UP and Na-S FTS catalyst materials, behaves as would be expected based on the discussion in Chapter 3. At $340^{\circ} \mathrm{C}$ (the FTS reaction temperature) the Fe particles are encapsulated by carbon and detached from the $\alpha$ $\mathrm{Al}_{2} \mathrm{O}_{3}$ support, ${ }^{48}$ for all the UP (Figure 4.2) and Na-S catalyst materials (Figure 4.3)
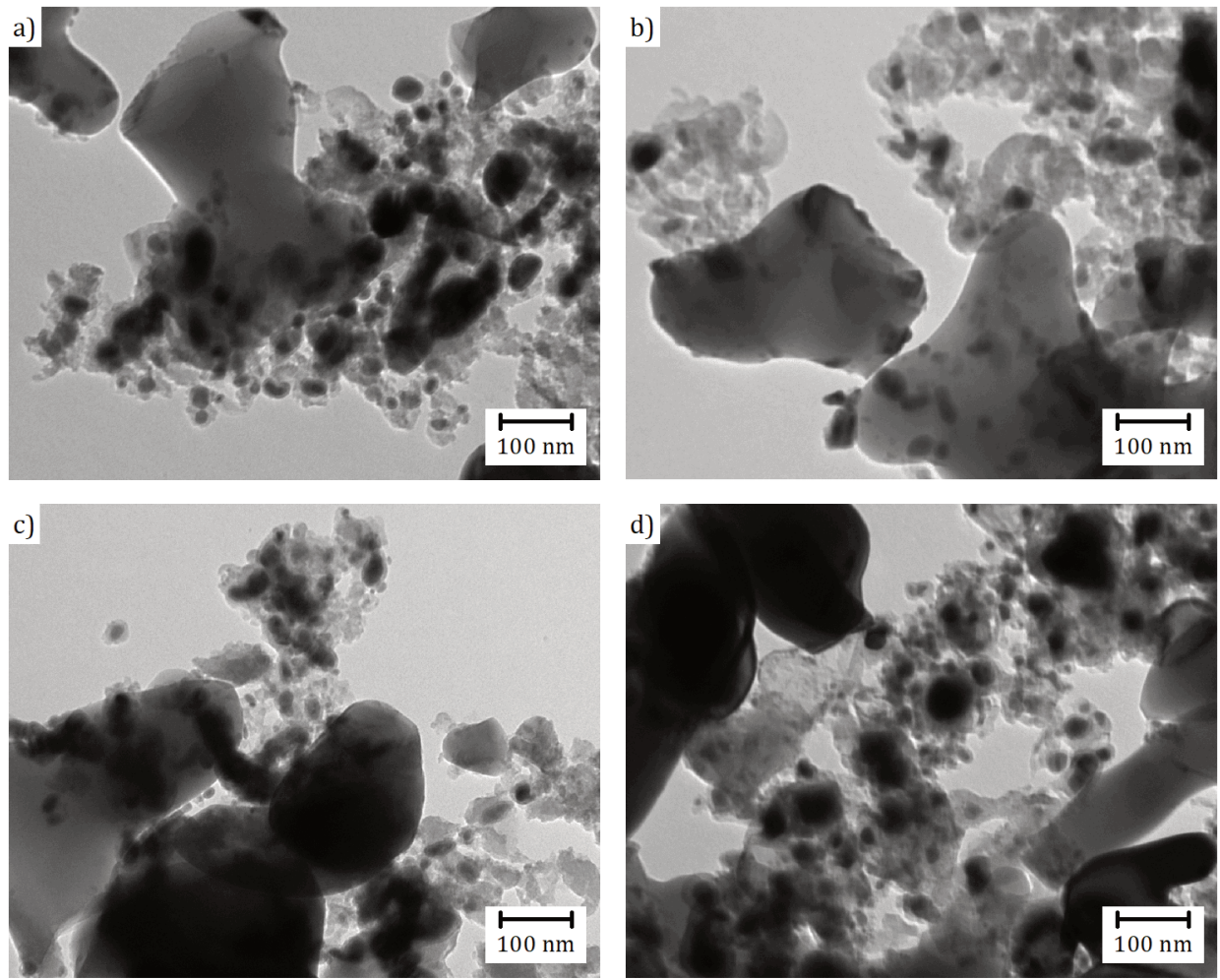

Figure 4.2

Transmission Electron Microscopy (TEM) images for the selected UP FTS ( $\left.\mathrm{Fe} / \alpha-\mathrm{Al}_{2} \mathrm{O}_{3}\right)$ catalyst materials after $72 \mathrm{~h}$ of the Fischer-Tropsch Synthesis (FTS) reaction. Images for the catalyst materials a) $\mathrm{H}_{2}$, b) 240 , c) 340 and d) 440 . 

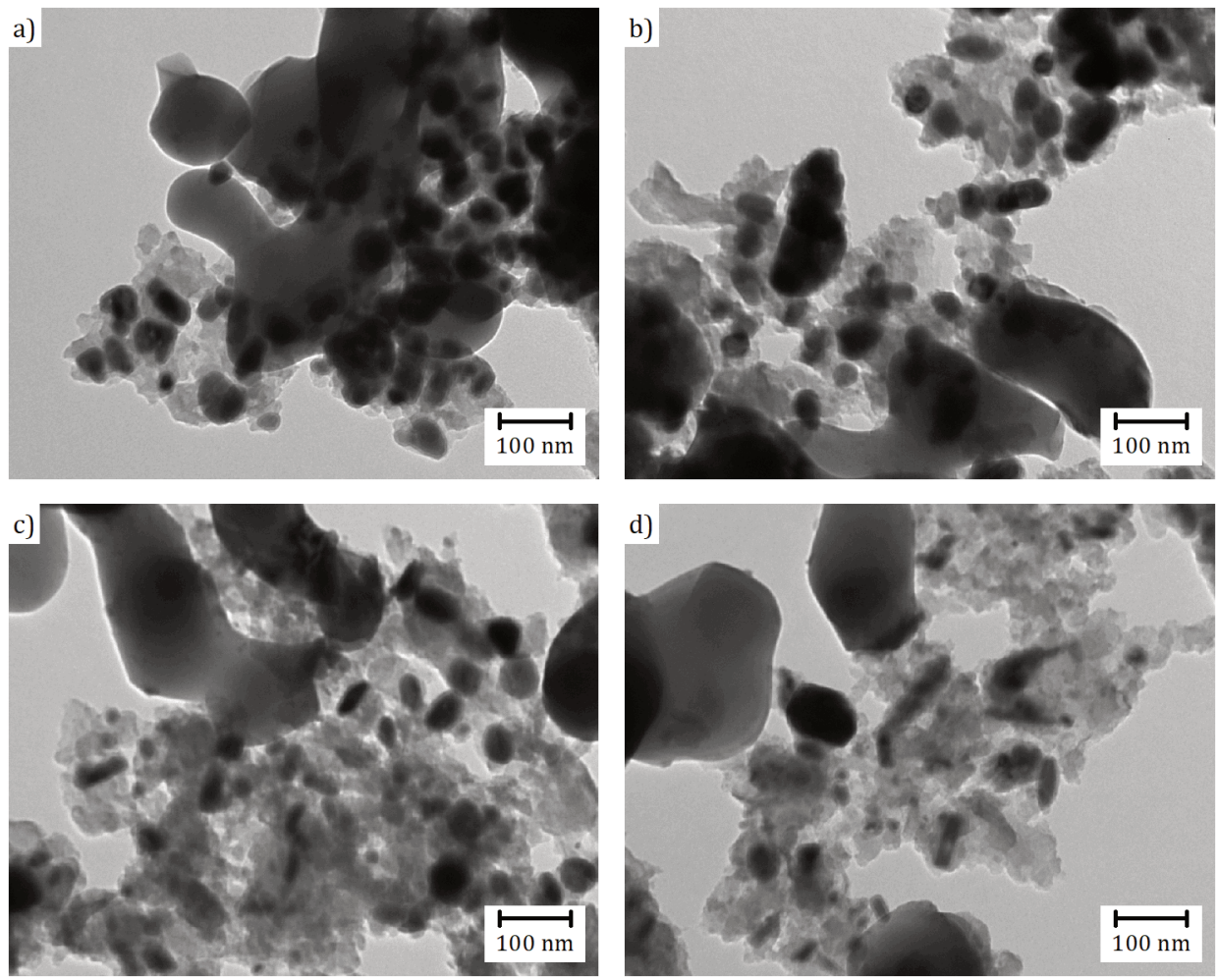

Figure 4.3

Transmission Electron Microscopy (TEM) images for selected Na-S FTS (Fe-Na-S $/ \alpha-\mathrm{Al}_{2} \mathrm{O}_{3}$ ) catalyst materials after $72 \mathrm{~h}$ of the Fischer-Tropsch Synthesis (FTS) reaction. Images for the catalyst materials a) $\mathrm{H}_{2}$, b) 240, c) 340 and d) 440 .

applied in the FTS reaction. Especially with the UP $\mathrm{H}_{2}$ and 240 (Figure 4.2a-b) and Na$\mathrm{S} \mathrm{H}_{2}$ and 240 Carb. catalyst materials (Figure 4.3a-b), for which the Fe particles were attached to the support $\alpha-\mathrm{Al}_{2} \mathrm{O}_{3}$ without carbon encapsulation before the FTS reaction (Chapter 3), the change is significant. Likewise, as with the Carb. catalyst materials, the FTS catalyst materials show $\approx 4 \mathrm{~nm}$ Fe oxidation layer due to the catalyst exposure to air. ${ }^{49,50}$

Some elongated, cylindrical Fe particles and filamentous carbon can be observed in the TEM images for the FTS catalyst materials (e.g. Figure 4.3c-d), which were not present in any of the Carb. catalysts. Under pure CO, i.e. with Carb. catalyst materials, carbon forms as a Fe encapsulating overlayer. For the FTS catalyst materials in the presence of both $\mathrm{C}$ and $\mathrm{H}$ under $\mathrm{H}_{2}: \mathrm{CO}$ (or $\mathrm{C}_{2} \mathrm{H}_{2}$ ) atmosphere, filamentous carbon growth can take place. ${ }^{51-53}$ As has been visualized with use of Environmental TEM 

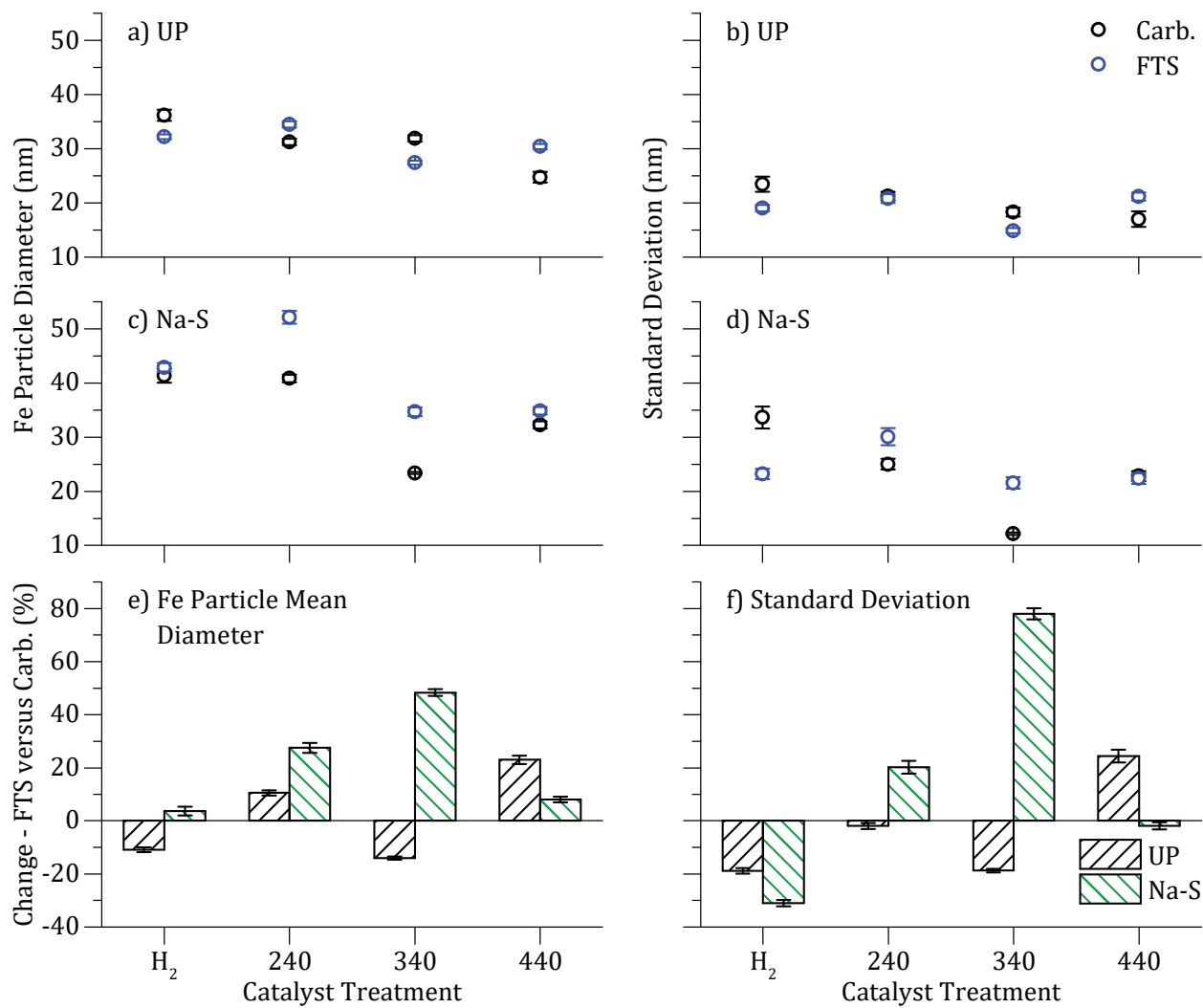

\section{Figure 4.4}

Transmission Electron Microscopy (TEM)-based, Fe particle (number-weighted) mean diameters compared for selected UP and Na-S ( $\left.\mathrm{Fe}(-\mathrm{Na}-\mathrm{S}) / \alpha-\mathrm{Al}_{2} \mathrm{O}_{3}\right)$ catalyst materials before (Carb.) and after $72 \mathrm{~h}$ Fischer-Tropsch Synthesis (FTS) reaction for a) UP and c) Na-S catalysts. The respective size distribution Standard Deviations (STDs) are given for b) UP and d) Na-S catalyst materials. The percentage changes for UP and Na-S catalysts as FTS versus Carb. for e) Fe particle mean diameters and the respective f) size distribution STDs.

(ETEM) by Mazzucco et al., the filamentous carbon growth causes elongation in the Fe particles. ${ }^{53}$ This could explain the observations of more cylindrical Fe particles in the FTS catalyst materials.

The size distribution derived Fe particle mean diameters (Figure 4.4a) or Fe particle size distribution STDs (Figure 4.4b) do not change for the UP FTS catalyst materials in any significant manner in comparison to the respective Carb. catalysts (Figure 4.4e-f). Neither, no significant change is observed for the $\mathrm{Na}-\mathrm{S} \mathrm{H}_{2}$ and $\mathrm{Na}-\mathrm{S} 440$ catalyst materials after the FTS reaction (Figure 4.4c-f). But, for the Na-S 240 FTS and Na-S 340 FTS catalyst materials the Fe particle mean diameters increase by $\approx+28 \%$ and $\approx+48 \%$ (Figure 4.4e), respectively, in comparison to the respective Carb. catalysts. 
Furthermore, the Fe particle size distribution STD for Na-S 340 FTS catalyst material increases by $\approx+78 \%$ in comparison to the Na-S 340 Carb. catalyst (Figure 4.4f). The increase in the STD signifies increased variation in the Fe particle diameters in the catalyst material.

The addition of Na-S promotion has previously been observed to increase the Fe particle mean diameters for catalyst materials applied in the FTS reaction. ${ }^{20}$ This observation is akin to the results for the FTS catalyst materials Na-S 240 and Na-S 340. However, for the Na-S $\mathrm{H}_{2}$ and Na-S 440 catalysts, only negligible changes in both mean Fe particle diameters (Figure 4.4c,e) and size distribution STDs (Figure 4.4d, $f$ ) can be observed despite the Na-S promotion.

As the catalytic performances (Figure 4.20), as will be discussed in a later section, are reasonably comparable for the selected Na-S $\left(\mathrm{H}_{2}, 240,340\right.$ and 440) FTS catalyst materials: the differences e.g. in catalytic activity do not explain the variability in the Fe particle growth. The causality when and why the Na-S promotion causes Fe particle growth is unclear from the results but may or may not occur if Na-S promotion is applied. Thus, possibly: Fe particle growth may not be an intrinsic property of the Na-S promotion.

\subsubsection{Phase Quantification with X-ray Powder Diffractometry/Rietveld Quantitative Phase Analysis}

The background corrected, ex-situ measured XRPD and Rietveld method (i.e. R-QPA) calculated diffraction and difference patterns, for the catalyst materials are given in Figure 4.6 (UP and Na-S H2 FTS), Figure 4.7 (UP FTS) and Figure 4.8 (Na-S FTS) over the range $13-29^{\circ} 2 \theta$ with Mo $\mathrm{K}_{\alpha}$. The R-QPA refinements were done over the measured $\mathrm{X}$-ray diffraction pattern range of $6-39^{\circ} 2 \theta$ for all of the catalyst materials under study.

The Fe carbide phases encountered in FTS research field have been identified to be $\varepsilon-\mathrm{Fe}_{3} \mathrm{C} \mathrm{P}_{3} 22$ (182), $\eta-\mathrm{Fe}_{2} \mathrm{C}$ Pnnm (58), $\chi-\mathrm{Fe}_{5} \mathrm{C}_{2} \mathrm{C} 2 / c$ (15) (the Hägg carbide), $\theta-\mathrm{Fe}_{3} \mathrm{C}$ Pnma (62) (cementite) and $\theta-\mathrm{Fe}_{7} \mathrm{C}_{3}$ Pnma (62) (Eckström-Adcock Carbide) on basis of the literature survey in Chapter 2 and with experimental validation in Chapter 3.

The Rietveld method calculated diffraction patterns resulted in a good agreement with the experimentally measured patterns, with fitting mismatches mainly originating from the support material $\alpha-\mathrm{Al}_{2} \mathrm{O}_{3}$ phase. Thus, the named Fe carbides represent the $\mathrm{Fe}$ phases formed in the FTS reaction accurately. R-weighted pattern $\left(R_{w p}\right)$ values for the Rietveld fits are given in Figure 4.5.54 The R-QPA quantification results for the crystalline Fe phase contents are given in the Figure 4.9. Crystallite diameters for each Fe phase are given for UP catalyst materials in Figure 4.17, and for Na-S catalysts in Figure 4.18. The respective R-QPA quantification results for the catalysts before the FTS reaction, i.e. for the Carb. catalyst materials, are reproduced from Chapter 3 for 
comparison into Figure 4.9.

\subsubsection{Fe Phase Rietveld Quantitative Phase Analysis Quantification}

\section{Changes}

The most prominent $\mathrm{Fe}$ carbide to form at $\approx 340^{\circ} \mathrm{C}$ reaction temperature under a carburizing gas atmosphere is the $\chi-\mathrm{Fe}_{5} \mathrm{C}_{2}$ phase. ${ }^{4,6,41,55-61}$ This is reflected in the R-QPA Fe phase quantification results when comparing the observed Fe phases in the Carb. and FTS catalyst materials in Figure 4.9.

After the $72 \mathrm{~h}$ FTS reaction at $340^{\circ} \mathrm{C}$ with the UP catalyst materials, the $\alpha-\mathrm{Fe} / \mathrm{Fe}_{\mathrm{x}} \mathrm{O}_{\mathrm{y}}$ phases preferentially carburize to the $\chi-\mathrm{Fe}_{5} \mathrm{C}_{2}$ phase, as can most prominently be seen for the catalyst UP $\mathrm{H}_{2}$ FTS in Figure 4.9a. The $\varepsilon-\mathrm{Fe}_{3} \mathrm{C}$ and $\eta-\mathrm{Fe}_{2} \mathrm{C}$ phases are generally unstable above $\approx 265^{\circ} \mathrm{C}$ and $\approx 300^{\circ} \mathrm{C}$, respectively, and preferentially transform to the $\chi$ $\mathrm{Fe}_{5} \mathrm{C}_{2}$ phase at $340^{\circ} \mathrm{C}$, as can be observed for the UP $240 \mathrm{FTS}$ and UP 290 FTS catalysts (Figure 4.9a).

The $\theta-\mathrm{Fe}_{3} \mathrm{C}$ phase in the UP 390 FTS and UP 440 FTS catalyst materials does not transform in the FTS reaction to the $\chi-\mathrm{Fe}_{5} \mathrm{C}_{2}$ phase as it is thermally more stable than the $\chi-\mathrm{Fe}_{5} \mathrm{C}_{2}$ phase at $340^{\circ} \mathrm{C}$ (Chapter 3). The UP 390 FTS and UP 440 FTS catalysts' $\theta$ $\mathrm{Fe}_{3} \mathrm{C}$ phase quantities thus remain unchanged in the FTS reaction. However, as will be discussed later, the $\theta-\mathrm{Fe}_{3} \mathrm{C}$ crystallite diameters do decrease in UP 390 FTS and UP 440 FTS catalyst materials (Figure 4.18). This, perhaps signaling at least some $\theta-\mathrm{Fe}_{3} \mathrm{C}$ phase instability under the applied FTS reaction conditions.

Although, the UP catalyst materials follow the expected thermal stabilities of the Fe carbides, the Na-S promoter addition changes the situation. With the Na-S catalyst
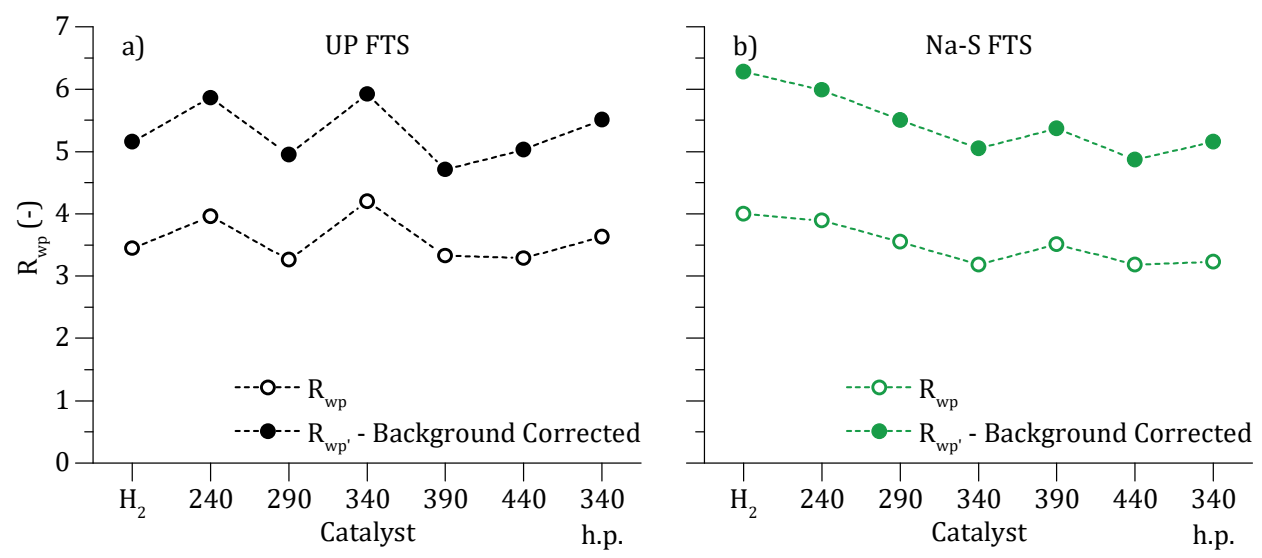

Figure 4.5

$\mathrm{R}_{\mathrm{wp}}$-values for the differences between the measured and Rietveld method calculated diffractograms, for the catalysts after $72 \mathrm{~h}$ Fischer-Tropsch Synthesis (FTS) reaction. $\mathrm{R}_{\mathrm{wp}}$ - and $\mathrm{R}_{\mathrm{wp}}$ - (background corrected) values for a) UP $\left(\mathrm{Fe} / \alpha-\mathrm{Al}_{2} \mathrm{O}_{3}\right)$ and b) Na-S $\left(\mathrm{Fe}-\mathrm{Na}-\mathrm{S} / \alpha-\mathrm{Al}_{2} \mathrm{O}_{3}\right)$ catalyst materials. 
materials, after the $72 \mathrm{~h}$ FTS reaction at $340^{\circ} \mathrm{C}$, two interesting differences in comparison to the UP catalysts can be observed: the Na-S promotion i) stabilizes the $\eta$ $\mathrm{Fe}_{2} \mathrm{C}$ phase and ii) destabilizes the $\theta-\mathrm{Fe}_{3} \mathrm{C}$ phase. These effects regardless of the expectations on the thermal stability of the Fe carbides (Chapter 3).

For the catalyst materials Na-S 240 FTS and Na-S 290 FTS, the $\eta-\mathrm{Fe}_{2} \mathrm{C}$ content is significantly higher (53\% and $35 \%$ ) after the FTS reaction than for the respective UP catalysts (21\% and $13 \%)$. This despite the UP Carb. catalyst materials having higher $\eta-\mathrm{Fe}_{2} \mathrm{C}$ contents before the FTS reaction (Figure 4.9a-b). Only the $\eta-\mathrm{Fe}_{2} \mathrm{C}$ but not the $\varepsilon$ $\mathrm{Fe}_{3} \mathrm{C}$ phase is stabilized with the Na-S promotion under the used FTS reaction conditions. None of the Na-S or UP FTS catalyst materials shows any presence of the $\varepsilon$ $\mathrm{Fe}_{3} \mathrm{C}$ phase. This, albeit the $\varepsilon-\mathrm{Fe}_{3} \mathrm{C}$ phase being present in the $240 \mathrm{Na}-\mathrm{S}$ and UP Carb. catalyst materials at the start of the FTS reaction.

The alkali stabilizing effect on " $\varepsilon$-carbides" has also been previously observed in-line with the R-QPA results in Figure 4.9a-b. $8,15,22$ Moreover, by inspecting the XRPD results by Dictor and Bell, the $\eta-\mathrm{Fe}_{2} \mathrm{C}$ phase can be identified in their $\mathrm{K} / \mathrm{Fe}_{2} \mathrm{O}_{3}$ catalyst material after it has been applied in the FTS reaction. ${ }^{26}$ In the results by Dictor and Bell, peak position $2.18 \AA(\mathrm{d} / \mathrm{n})$ (Fig 17. in their publication) belongs to the $\eta-\mathrm{Fe}_{2} \mathrm{C}$ plane $\left(\begin{array}{lll}0 & 2 & 0\end{array}\right)$ (2.18 $\AA$, or $18.74^{\circ} 2 \theta$ in $M_{0} K_{\alpha}$ ), not to the Hägg carbide as after their assignment. If no K promotion was used, in their study, the XRPD peak $2.18 \AA$ (d/n) i.e. the $\eta-\mathrm{Fe}_{2} \mathrm{C}$ phase

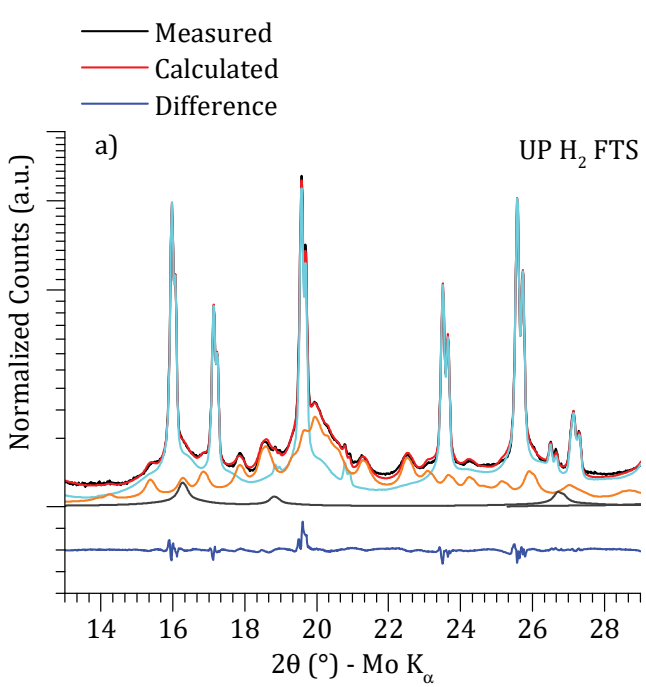

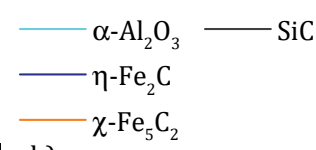

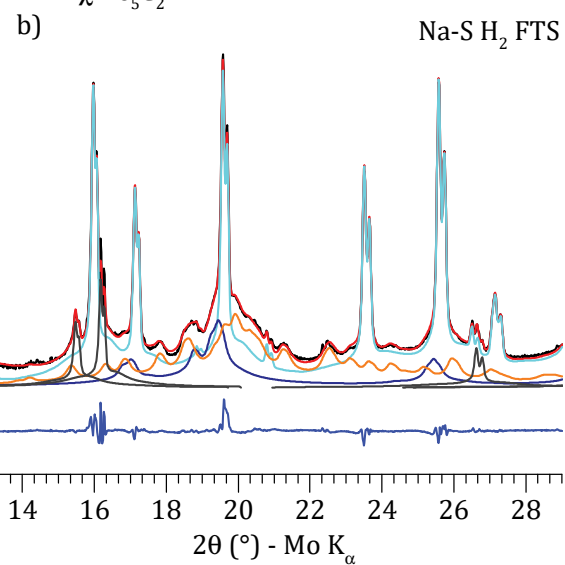

Figure 4.6

Background corrected, ex-situ measured X-ray Powder Diffractometry (XRPD) and Rietveld method calculated diffraction patterns over a range $13-29^{\circ} 2 \theta$ for the a) $\mathrm{UP} \mathrm{H}_{2}$ and b) $\mathrm{Na}-\mathrm{S} \mathrm{H}_{2}\left(\mathrm{Fe}(-\mathrm{Na}-\mathrm{S}) / \alpha-\mathrm{Al}_{2} \mathrm{O}_{3}\right.$ ) catalyst materials after $72 \mathrm{~h}$ Fischer-Tropsch Synthesis (FTS) reaction. Normalized counts on a square-root scale. 

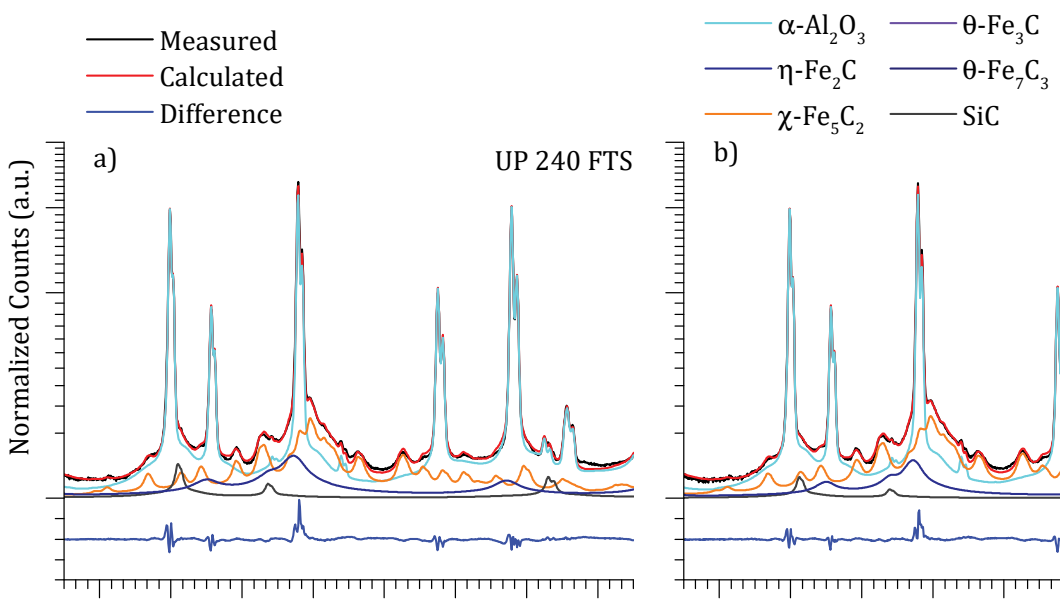

b)

UP 290 FTS
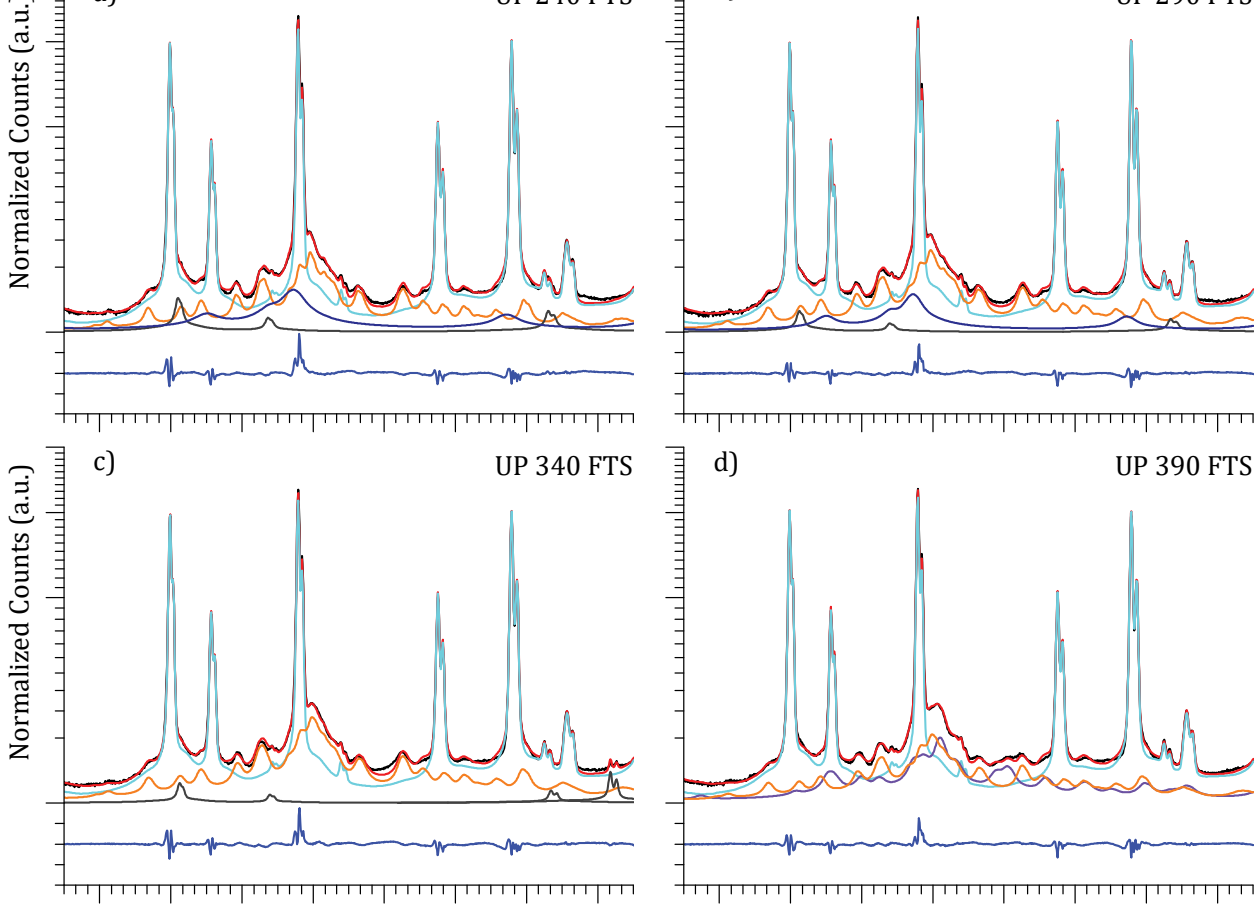

d)

UP 390 FTS
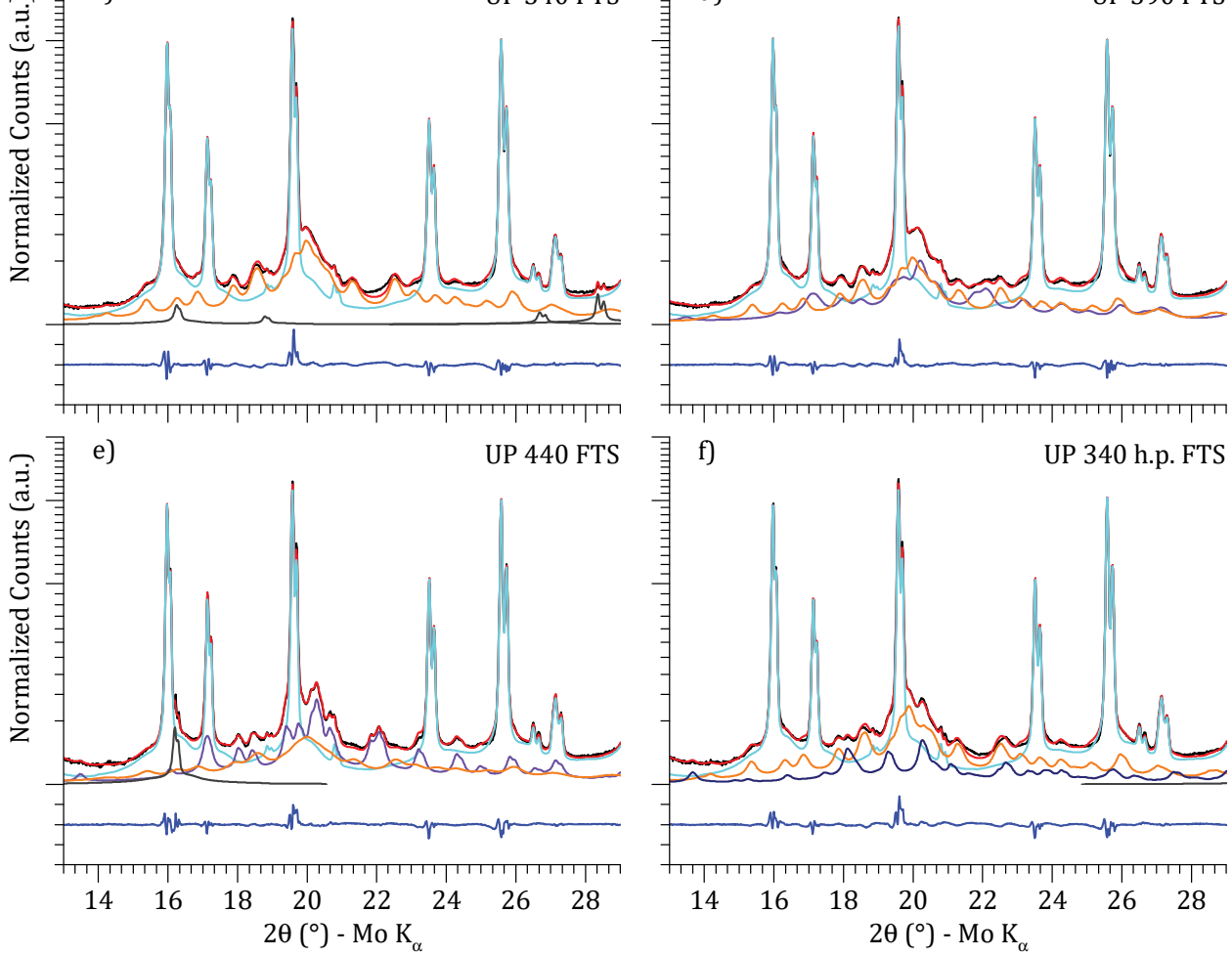

Figure 4.7

Background corrected, ex-situ measured X-ray Powder Diffractometry (XRPD) and Rietveld method calculated diffraction patterns over a range $13-29^{\circ} 2 \theta$ for the UP FTS $\left(\mathrm{Fe} / \alpha-\mathrm{Al}_{2} \mathrm{O}_{3}\right)$ catalyst materials after 72 h Fischer-Tropsch Synthesis (FTS) reaction: a) 240, b) 290, c) 340, d) 390, e) 440 and f) 340 h.p.. Normalized counts on a square-root scale. 

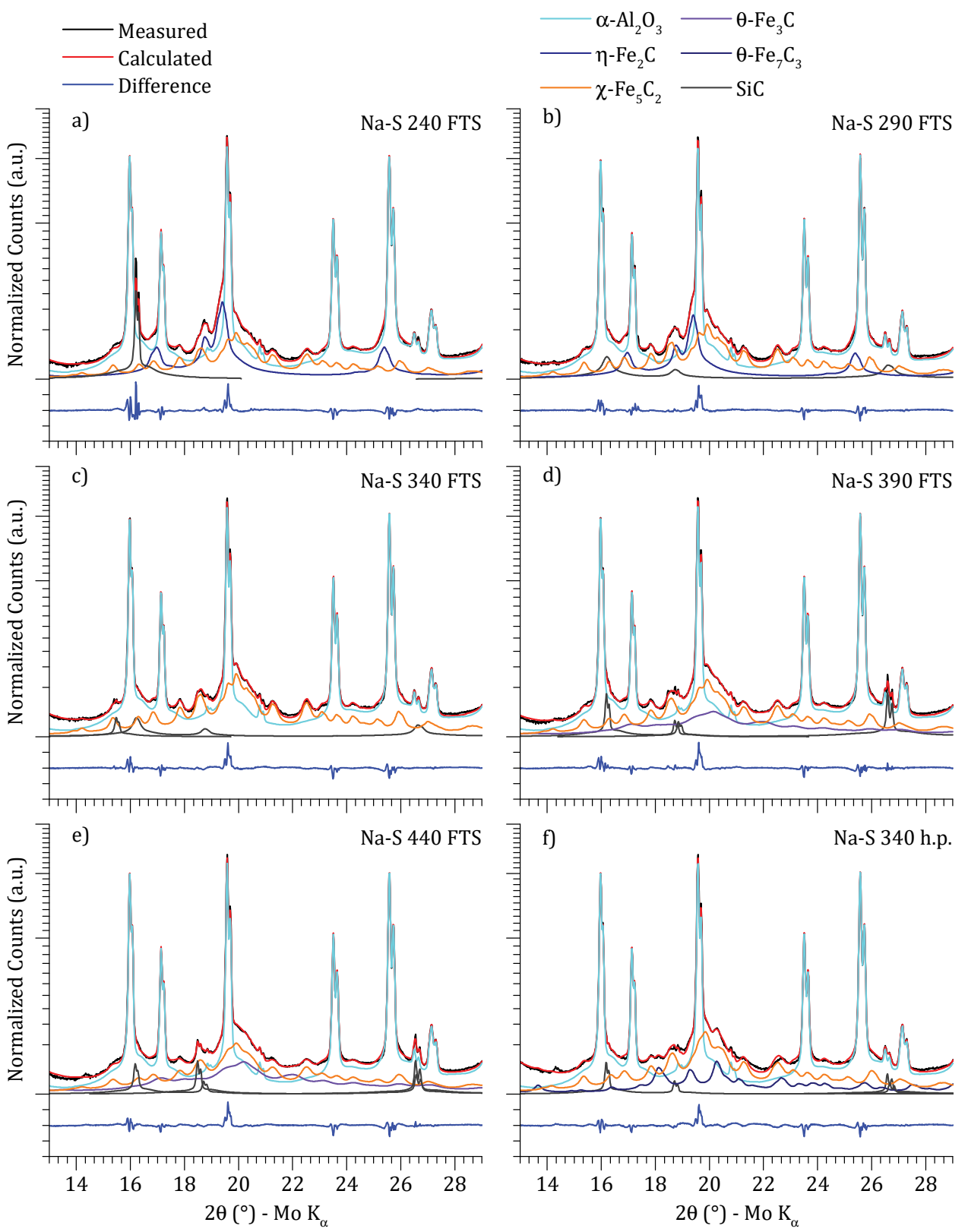

\section{Figure 4.8}

Background corrected, ex-situ measured X-ray Powder Diffractometry (XRPD) and Rietveld method calculated diffraction patterns over a range $13-29^{\circ} 2 \theta$ for the Na-S FTS $\left(\mathrm{Fe}-\mathrm{Na}-\mathrm{S} / \alpha-\mathrm{Al}_{2} \mathrm{O}_{3}\right)$ catalyst materials after 72 h Fischer-Tropsch Synthesis (FTS) reaction: a) 240, b) 290, c) 340, d) 390, e) 440 and f) 340 h.p.. Normalized counts on a square-root scale. 


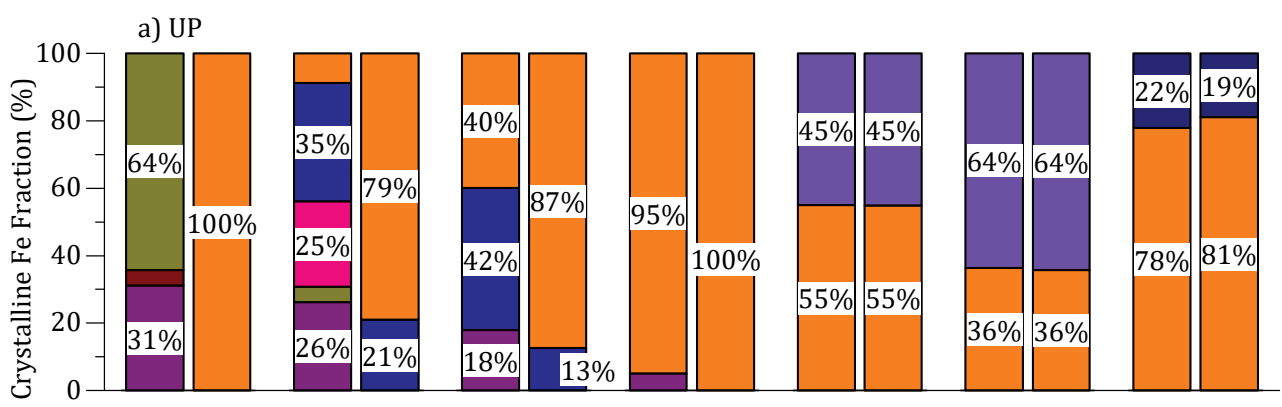

b) Na-S
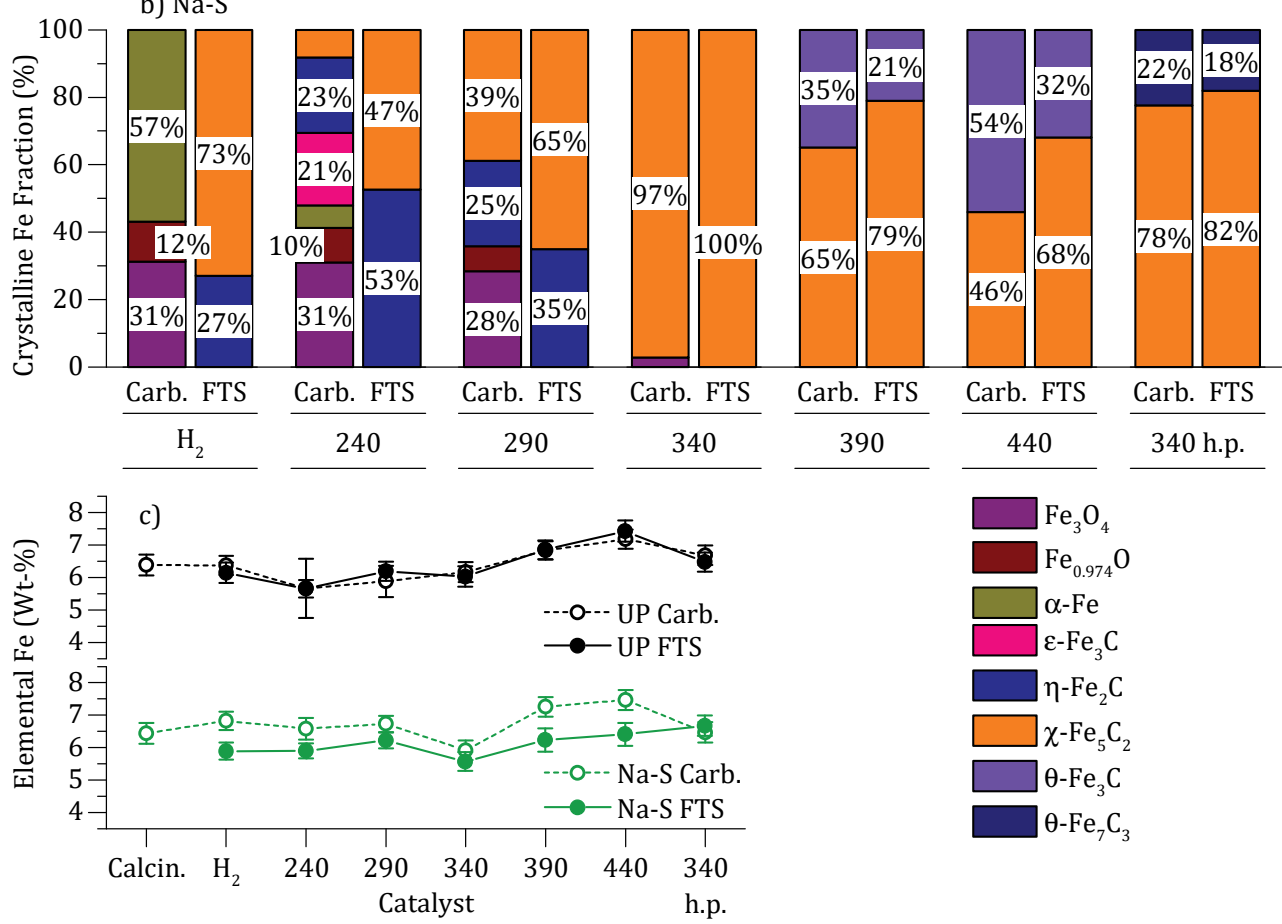

Figure 4.9

The Rietveld Quantitative Phase Analysis (R-QPA) Fe phase quantifications before (Carb.) and after $72 \mathrm{~h}$ Fischer-Tropsch Synthesis (FTS) reaction. Crystalline Fe phase fractions for a) UP and b) Na-S (Fe(-Na-S) $/ \alpha-$ $\mathrm{Al}_{2} \mathrm{O}_{3}$ ) catalyst materials, and c) the elemental Fe wt-\% loadings on basis of the crystalline Fe phases. Data for the Carb. catalyst materials, prior to FTS reaction is reproduced from Chapter 3. Percentage labels cut-off is set to $<10 \%$.

is missing after the applied FTS reaction. ${ }^{26}$

The other interesting case on the basis of the R-QPA results is the Na-S promotion imposed $\theta-\mathrm{Fe}_{3} \mathrm{C}$ instability in the FTS reaction. For both, the Na-S 390 and Na-S 440 FTS catalyst materials the $\theta-\mathrm{Fe}_{3} \mathrm{C}$ quantity has decreased relative $\approx-40 \%$ from the original 
quantity of the $\theta-\mathrm{Fe}_{3} \mathrm{C}$ phase before the FTS reaction.

That is, the $\theta-\mathrm{Fe}_{3} \mathrm{C}$ phase fractions decreased from $35 \%$ to $21 \%$ for Na-S 390 FTS, and from $54 \%$ to $32 \%$ for Na-S 440 FTS catalysts in comparison to the respective Na$\mathrm{S}$ Carb. catalyst materials. While at the same time, the $\chi-\mathrm{Fe}_{5} \mathrm{C}_{2}$ phase content increased (Figure 4.9b).

Thus, the Na-S promotion promotes the $\theta-\mathrm{Fe}_{3} \mathrm{C}$ transformation to $\chi-\mathrm{Fe}_{5} \mathrm{C}_{2}$ at $340^{\circ} \mathrm{C}$ while this conversion does not take place without the Na-S promotion. That is, not at least with a noticeable rate within the applied $72 \mathrm{~h}$ FTS reaction runs.

No Fe oxides were present in any detectable quantities in the FTS catalyst materials (Figure 4.9). The measured XRPD patterns are well explained with the Fe phases used for the R-QPA fitting. This is equally valid for the UP and Na-S $\mathrm{H}_{2}$ (Figure 4.6) and for all of the carburized UP (Figure 4.7) and Na-S catalyst materials (Figure 4.8). The CO conversion with the UP catalyst materials (2-5\%, Table 4.4) was too low during the FTS reaction for increasing the $\mathrm{H}_{2} \mathrm{O}: \mathrm{H}_{2}$ or $\mathrm{CO}_{2}$ : $\mathrm{CO}$ ratios above levels sufficient for the Fe carbide oxidation. ${ }^{3,4,13,23,24,62}$ The Na-S catalyst materials exhibited higher CO conversion levels (33-55 \% Table 4.4) i.e. higher $\mathrm{H}_{2} \mathrm{O}$ concentrations, and also higher $\mathrm{CO}_{2}$ selectivities (Table 4.5). However, added alkali $\mathrm{Na}(-\mathrm{S})$ decreases the Fe carbide oxidation tendency under the FTS reaction conditions. ${ }^{8,22}$ Therefore, no Fe oxides are observed neither on any of the Na-S FTS catalyst materials.

As compared in the Figure 4.9c, the elemental Fe content remains unchanged for the UP FTS but decreases for the Na-S FTS catalyst materials in comparison to their respective Carb. catalyst materials before the FTS reaction. For the Na-S FTS catalyst materials (excluding the 340 h.p.), the elemental Fe content is decreased by a mean value of $0.76 \pm 0.30$ wt- $\%$ of $\mathrm{Fe}$.

The unchanged elemental Fe loading for the UP FTS catalyst materials is a good sign that all the Fe phases present in the catalyst materials have been correctly accounted for in the R-QPA fittings. The systematic decrease in the elemental Fe loading with the Na-S FTS catalyst materials hints of either: i) lost Fe during the FTS reaction, ii) Fe is in phase(s) with too small crystallites to give clear Bragg diffraction or, iii) a possibly inaccurate or a missing Fe phase structure(s) used for the Rietveld fitting. Which of the options is the most correct answer, cannot be straightforwardly be devised from the RQPA results.

Nevertheless, as was mentioned, as the Rietveld calculated diffraction patterns represent the measured XRPD patterns well: a Fe phase missing from the R-QPA fits can be excluded as a reason for the decreased elemental Fe loading with the Na-S FTS catalyst materials. 
On the Role of Iron Carbides in the FischerTropsch Synthesis with Fe(-Na-S)/ $\alpha-\mathrm{Al} 203$ Catalysts

\section{Table 4.2}

Overview of the Fe carbide phases with their space groups and mean lattice parameters used for the Rietveld Quantitative Phase Analysis (R-QPA). In bold are highlighted the lattice parameters for which definite distortion by Na-S promotion could be observed.

\begin{tabular}{|c|c|c|c|c|c|c|}
\hline \multirow{2}{*}{$\begin{array}{c}\text { Fe Carbide } \\
\text { Phase }^{\text {a) }}\end{array}$} & \multirow[t]{2}{*}{ Space Group } & & \multicolumn{4}{|c|}{ Lattice Parameters ${ }^{\text {b) }}$} \\
\hline & & & $\begin{array}{l}\text { UP Carb. } \\
(\AA)\end{array}$ & $\begin{array}{c}\text { Na-S Carb. } \\
(\AA)\end{array}$ & $\begin{array}{c}\text { UP FTS } \\
(\AA)\end{array}$ & $\begin{array}{l}\text { Na-S FTS } \\
(\AA)\end{array}$ \\
\hline & Orthorhombic, & $\mathrm{a}$ & $4.84 \pm 0.00$ & $4.83 \pm 0.00$ & $4.84 \pm 0.00$ & $4.86 \pm 0.01$ \\
\hline \multirow[t]{3}{*}{$\eta-\mathrm{Fe}_{2} \mathrm{C}$} & Pnnm (58) & b & $4.35 \pm 0.00$ & $4.35 \pm 0.00$ & $4.35 \pm 0.00$ & $4.35 \pm 0.00$ \\
\hline & & c & $2.76 \pm 0.00$ & $2.76 \pm 0.00$ & $2.75 \pm 0.00$ & $2.76 \pm 0.01$ \\
\hline & Monoclinic, & $\mathrm{a}$ & $11.55 \pm 0.01$ & $11.56 \pm 0.01$ & $11.56 \pm 0.01$ & $11.58 \pm 0.00$ \\
\hline \multirow[t]{3}{*}{$\chi-\mathrm{Fe}_{5} \mathrm{C}_{2}$} & $\mathrm{C} 2 / \mathrm{c}(15)$ & b & $4.57 \pm 0.01$ & $4.57 \pm 0.01$ & $4.57 \pm 0.01$ & $4.58 \pm 0.00$ \\
\hline & & c & $5.07 \pm 0.00$ & $5.06 \pm 0.00$ & $5.06 \pm 0.01$ & $5.05 \pm 0.00$ \\
\hline & Monoclinic, & $\mathrm{a}$ & 11.60 & 11.61 & 11.61 & 11.62 \\
\hline$\chi-\mathrm{Fe}_{5} \mathrm{C}_{2}$ & C2/c (15) & $\mathrm{b}$ & 4.57 & 4.58 & 4.58 & 4.58 \\
\hline \multirow[t]{2}{*}{ (h.p.) } & & c & 5.05 & 5.04 & 5.05 & 5.04 \\
\hline & Orthorhombic, & $\mathrm{a}$ & $5.08 \pm 0.01$ & $5.07 \pm 0.01$ & $5.07 \pm 0.02$ & $5.07 \pm 0.00$ \\
\hline \multirow[t]{3}{*}{$\theta-\mathrm{Fe}_{3} \mathrm{C}$} & Pnma (62) & b & $6.76 \pm 0.02$ & $6.76 \pm 0.02$ & $6.76 \pm 0.03$ & $6.79 \pm 0.01$ \\
\hline & & c & $4.53 \pm 0.01$ & $4.54 \pm 0.01$ & $4.54 \pm 0.01$ & $4.55 \pm 0.00$ \\
\hline & Orthorhombic, & a & 4.52 & 4.52 & 4.53 & 4.54 \\
\hline \multirow[t]{2}{*}{$\left.\theta-\mathrm{Fe}_{7} \mathrm{C}_{3} \mathrm{c}\right)$} & Pnma (62) & b & 6.89 & 6.91 & 6.89 & 6.90 \\
\hline & & c & 11.91 & 11.91 & 11.89 & 11.89 \\
\hline
\end{tabular}

a) The authors for the used crystal structures are listed in Chapter 3.

b) Mean values over all observations within the catalyst material group of the phase with STDs. Values for Carb. catalyst materials reproduced from the results obtained in Chapter 3.

c) Low phase quantities and peak overlap with the $\chi-\mathrm{Fe}_{5} \mathrm{C}_{2}$ (h.p.) phase results in inaccuracy on the measured lattice parameters.

\subsubsection{Fe Carbide Lattice Changes}

The Fe carbide mean crystal lattice parameters with their STDs used in the R-QPA for are listed in Table 4.2 for both the Carb. (with the results from Chapter 3) and the FTS catalyst materials. As the most significant lattice parameter phenomenon, the Na-S promotion caused expansion in the lattice parameters for the $\eta-\mathrm{Fe}_{2} \mathrm{C}$ and $\chi-\mathrm{Fe}_{5} \mathrm{C}_{2}$ phases in the Na-S FTS catalyst materials, as is marked with bold in the Table 4.2. As exceptions, the $\chi-\mathrm{Fe}_{5} \mathrm{C}_{2}$ phase in UP $240 \mathrm{FTS}$ and UP $290 \mathrm{FTS}$ catalyst materials showed similar lattice expansion effects as did the Na-S promoted $\chi-\mathrm{Fe}_{5} \mathrm{C}_{2}$ phase in the Na-S FTS catalyst materials (Figure $\mathbf{4 . 1 3 b}, \boldsymbol{d}, \boldsymbol{f}$ ). This peculiarity will be commented more later on. The distinctively distorted $\chi-\mathrm{Fe}_{5} \mathrm{C}_{2}$ (h.p.) phase in the 340 h.p. catalyst materials did not show any significant modification trends in the lattice parameters between the UP or Na-S Carb. and the UP or Na-S FTS catalyst materials (Table 4.2).

Due to the relatively low phase amounts (18-22\%, Figure 4.9a-b), and the 


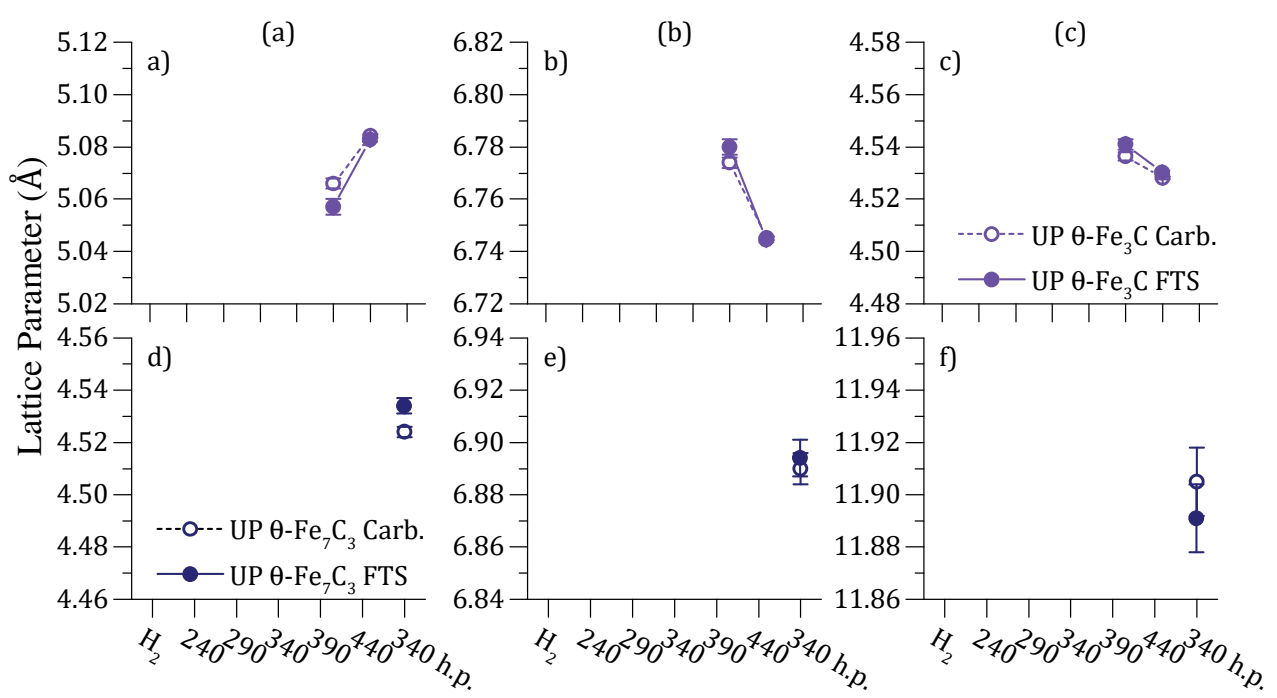

Figure 4.10

The lattice parameters used in the Rietveld Quantitative Phase Analysis (R-QPA) fitting of the $\theta-\mathrm{Fe}_{3} \mathrm{C}$ and $\theta$ $\mathrm{Fe}_{7} \mathrm{C}_{3}$ phases before (Carb.) and after $72 \mathrm{~h}$ Fischer-Tropsch Synthesis (FTS) reaction for UP $\left(\mathrm{Fe} / \alpha-\mathrm{Al}_{2} \mathrm{O}_{3}\right)$ catalyst materials. Lattice parameters for UP $\theta-\mathrm{Fe}_{3} \mathrm{C}$ are in a) $a$, b) $b$ and c) $c$ and for UP $\theta-\mathrm{Fe}_{7} \mathrm{C}_{3}$ in d) $a$, e) $b$ and f) $c$.

overlapping diffraction peaks with the $\chi-\mathrm{Fe}_{5} \mathrm{C}_{2}$ (h.p.) phase UP (Figure 4.7f) and Na-S catalysts (Figure 4.8f): discussion on the $\theta-\mathrm{Fe}_{7} \mathrm{C}_{3}$ lattice shifts is omitted due to the uncertainty in determining the its precise diffraction peak positions and therefore the $\theta-\mathrm{Fe}_{7} \mathrm{C}_{3}$ phase lattice parameters.

In a similar manner, the $\theta-\mathrm{Fe}_{3} \mathrm{C}$ phase diffraction peaks largely overlap with the $\chi$ $\mathrm{Fe}_{5} \mathrm{C}_{2}$ phase in all UP (Figure 4.7d) and Na-S catalyst materials (Figure 4.8d-e), with the $\theta-\mathrm{Fe}_{3} \mathrm{C}$ phase in the UP $440 \mathrm{FTS}$ catalyst material being an exception (Figure 4.7e). The peak overlapping results in significant STDs for the mean $\theta-\mathrm{Fe}_{3} \mathrm{C}$ lattice parameters (Table 4.2). This makes the determination of the relative lattice parameter changes for the $\theta-\mathrm{Fe}_{3} \mathrm{C}$ phase inaccurate. Therefore, the discussion on the $\theta-\mathrm{Fe}_{3} \mathrm{C}$ phase's lattice parameter changes is also omitted.

The comparison of individual lattice parameters used in the R-QPA fitting of the $\theta$ $\mathrm{Fe}_{3} \mathrm{C}$ and $\theta-\mathrm{Fe}_{7} \mathrm{C}_{3}$ phases in UP and Na-S Carb. and FTS catalyst materials is given in Figure 4.10 (for UP) and in Figure 4.11 (for Na-S catalyst materials).

As examples of the observed lattice shifts in relation to the diffraction peak shifts, in Figure 4.12 are compared the Rietveld fitted, calculated diffraction patterns for the $\eta$ $\mathrm{Fe}_{2} \mathrm{C}$ and $\chi-\mathrm{Fe}_{5} \mathrm{C}_{2}$ phases in the UP 240 and UP 340, and Na-S 240 and Na-S 340, Carb. and FTS catalyst material. The respective measured XRPD diffraction patterns for the catalyst materials are also given in the figure. 


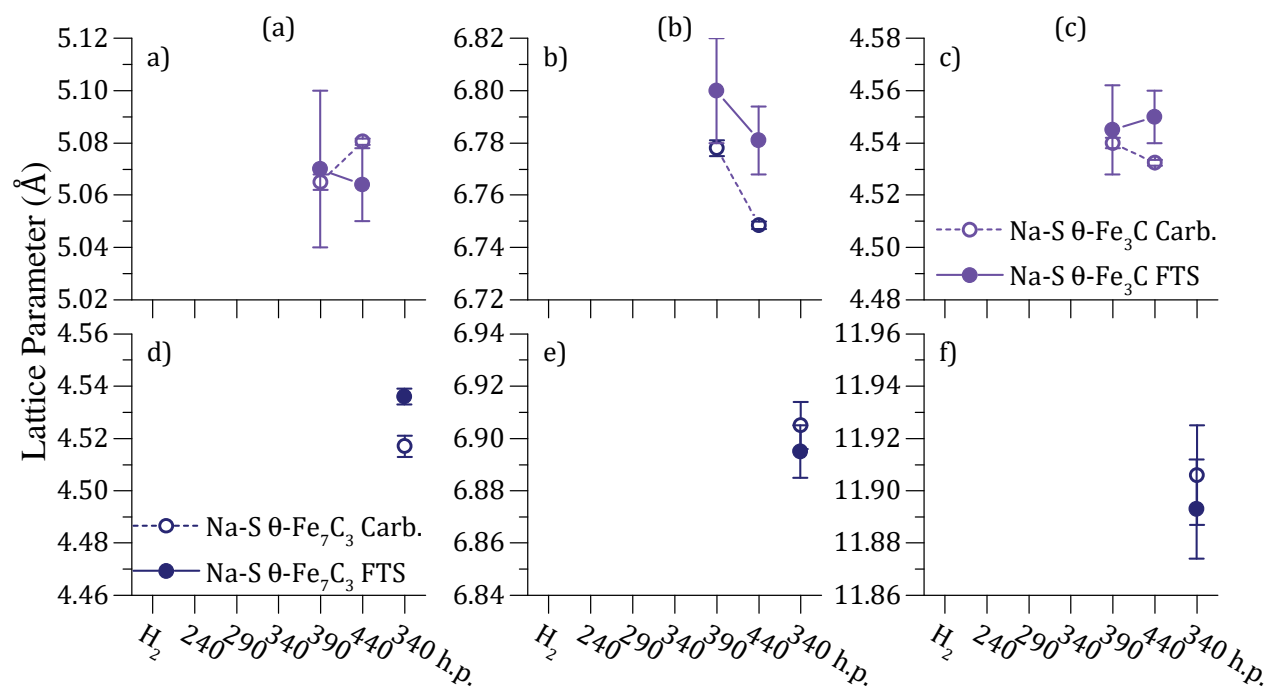

Figure 4.11

The lattice parameters used in the Rietveld Quantitative Phase Analysis (R-QPA) fitting for the $\theta-\mathrm{Fe}_{3} \mathrm{C}$ and $\theta-$ $\mathrm{Fe}_{7} \mathrm{C}_{3}$ phases before (Carb.) and after $72 \mathrm{~h}$ Fischer-Tropsch Synthesis (FTS) reaction for Na-S (Fe-Na-S/ $\alpha-$ $\mathrm{Al}_{2} \mathrm{O}_{3}$ ) catalyst materials. Lattice parameters for $\mathrm{Na}-\mathrm{S} \theta-\mathrm{Fe}_{3} \mathrm{C}$ are in a) $a$, b) $b$ and c) $c$ and for $\mathrm{Na}-\mathrm{S} \theta-\mathrm{Fe}_{7} \mathrm{C}_{3}$ in d) $a$, e) $b$ and f) $c$.

Before the FTS reaction, the $\eta-\mathrm{Fe}_{2} \mathrm{C}$ phase in the 240 Carb. (Figure 4.12a) and the $\chi$ $\mathrm{Fe}_{5} \mathrm{C}_{2}$ phase in the 340 Carb. (Figure 4.12b), in both UP and Na-S catalyst materials, gave nearly identical Rietveld calculated diffraction patterns. After the FTS reaction, however, the phases' peak positions have shifted as depending on the addition of the Na-S promotion. For the $\eta-\mathrm{Fe}_{2} \mathrm{C}$ phase the most intense diffraction peak shifts towards a lower $2 \theta\left(19.42^{\circ}\right.$ Carb. $\left.\rightarrow 19.38^{\circ} \mathrm{FTS}\right)$ after the FTS reaction when Na-S promotion is applied (Figure 4.12a, ). For the $\chi-\mathrm{Fe}_{5} \mathrm{C}_{2}$ phase, a similar shift for two peaks (marked with \#, Figure 4.12d) towards a lower $2 \theta\left(19.95^{\circ}\right.$ Carb. $\rightarrow 19.92^{\circ}$ FTS and $17.86^{\circ}$ Carb. $\rightarrow 17.83^{\circ} \mathrm{FTS}$ ) takes place (Figure $\mathbf{4 . 1 2 b}, \boldsymbol{d}$ ).

In Figure 4.13 are compared the individual R-QPA fitted lattice parameters with their R-QPA fit error estimates for the $\eta-\mathrm{Fe}_{2} \mathrm{C}$ and $\chi-\mathrm{Fe}_{5} \mathrm{C}_{2}$ phases in the UP Carb. and FTS, and in Figure 4.14 for the Na-S Carb. and FTS catalysts materials. For both the $\eta$ $\mathrm{Fe}_{2} \mathrm{C}$ and $\chi-\mathrm{Fe}_{5} \mathrm{C}_{2}$ phases, anisotropic lattice parameter changes can be observed which are assignable to be due to the $\mathrm{Na}-\mathrm{S}$ promotion. These lattice changes reflect the examples of the diffraction peak position changes in the diffraction patterns in Figure 4.12.

The added Na-S promotion increases the lattice parameter $a$ by $\approx 0.02 \AA$ for both the $\eta-\mathrm{Fe}_{2} \mathrm{C}$ and $\chi-\mathrm{Fe}_{5} \mathrm{C}_{2}$ phases in the FTS catalyst materials, in comparison to the respective phases in the Carb. catalyst materials (Table 4.2 and Figure 4.14a-b). For the $\chi-\mathrm{Fe}_{5} \mathrm{C}_{2}$ 

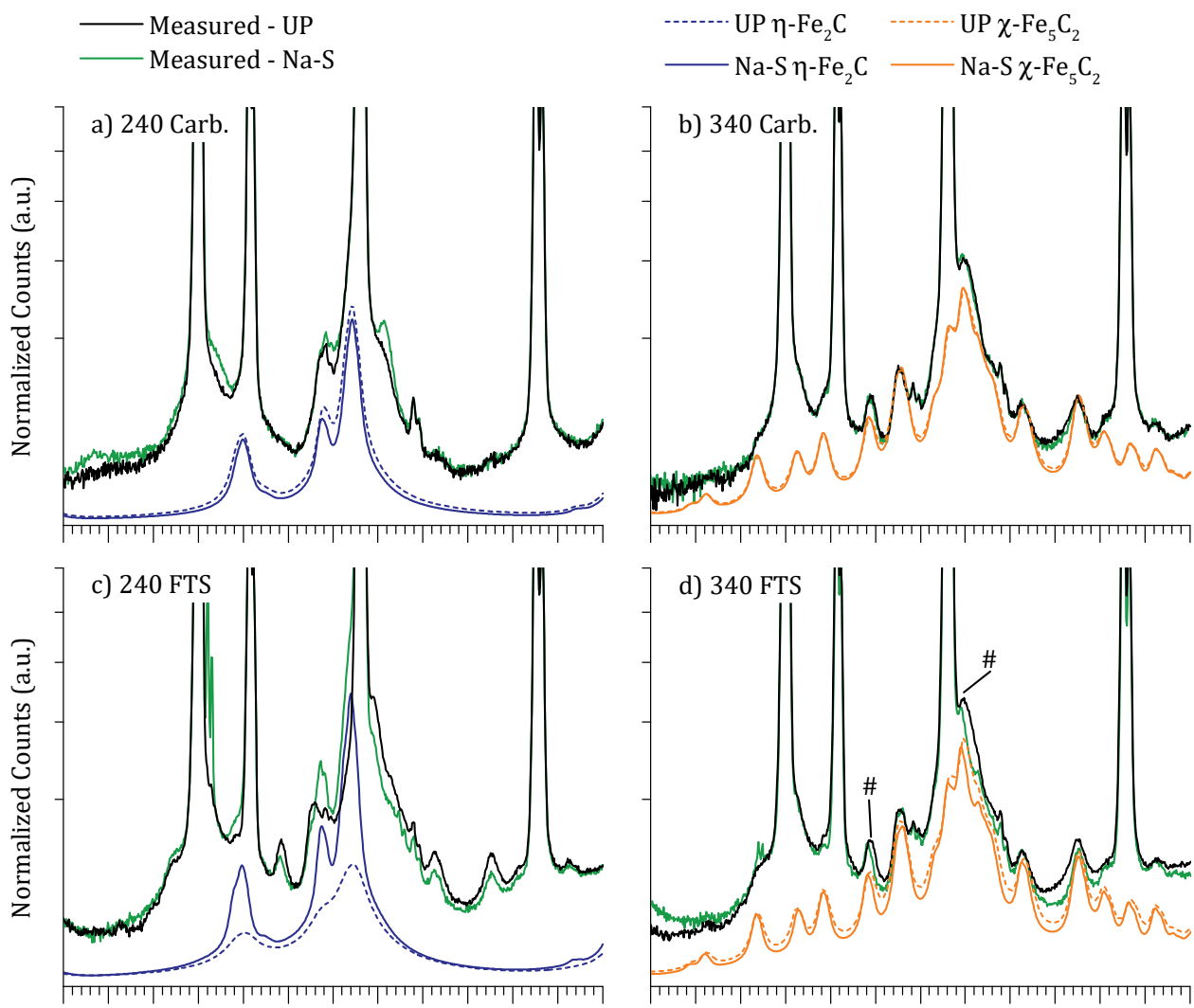

$\begin{array}{lllllllllllll}13 & 14 & 15 & 16 & 17 & 18 & 19 & 20 & 21 & 22 & 23 & 24 & 25\end{array}$

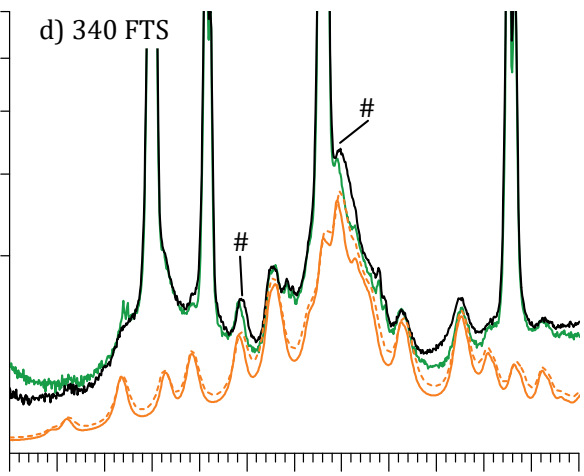

$2 \theta\left(^{\circ}\right)-\mathrm{Mo} \mathrm{K}_{\alpha}$

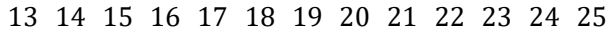

Figure 4.12

Background corrected, ex-situ measured X-ray Powder Diffractometry (XRPD) patterns with Rietveld method calculated $\eta-\mathrm{Fe}_{2} \mathrm{C}$ and $\chi-\mathrm{Fe}_{5} \mathrm{C}_{2}$ phase patterns over a range $13-25^{\circ} 2 \theta$ for both UP and $\mathrm{Na}-\mathrm{S}(\mathrm{Fe}(-\mathrm{Na}-\mathrm{S}) / \alpha-$ $\mathrm{Al}_{2} \mathrm{O}_{3}$ ) catalyst materials before (Carb.) and after $72 \mathrm{~h}$ Fischer-Tropsch Synthesis (FTS) reaction. The diffraction patterns for the $\eta-\mathrm{Fe}_{2} \mathrm{C}$ phase in UP and Na-S 240 catalysts compared a) before and c) after $72 \mathrm{~h}$ FTS reaction, and for $\chi-\mathrm{Fe}_{5} \mathrm{C}_{2}$ phase in UP and Na-S 340 catalysts compared b) before and d) after $72 \mathrm{~h}$ FTS reaction. Normalized counts on a square-root scale.

phase, the lattice parameter $c$ decreases by $\approx 0.01 \AA$ with Na-S catalyst materials while the lattice parameter $b$ experiences lesser changes (Table 4.2 and Figure 4.14d, $f$ ). The lattice parameters $c$ and $b$ for the $\eta-\mathrm{Fe}_{2} \mathrm{C}$ phase do not change consistently (Table 4.2 and Figure 4.14c, e).

The $\eta-\mathrm{Fe}_{2} \mathrm{C}$ phase does not show any decisive crystal lattice expansion or contraction in comparison between the UP Carb. and FTS catalyst materials. Although, the R-QPA fit error estimates for the lattice parameters for the $\eta-\mathrm{Fe}_{2} \mathrm{C}$ phases in the UP FTS catalyst materials are rather high (Figure 4.13a, $\boldsymbol{c}, \boldsymbol{e}$ ). This is due the $\eta-\mathrm{Fe}_{2} \mathrm{C}$ phase 

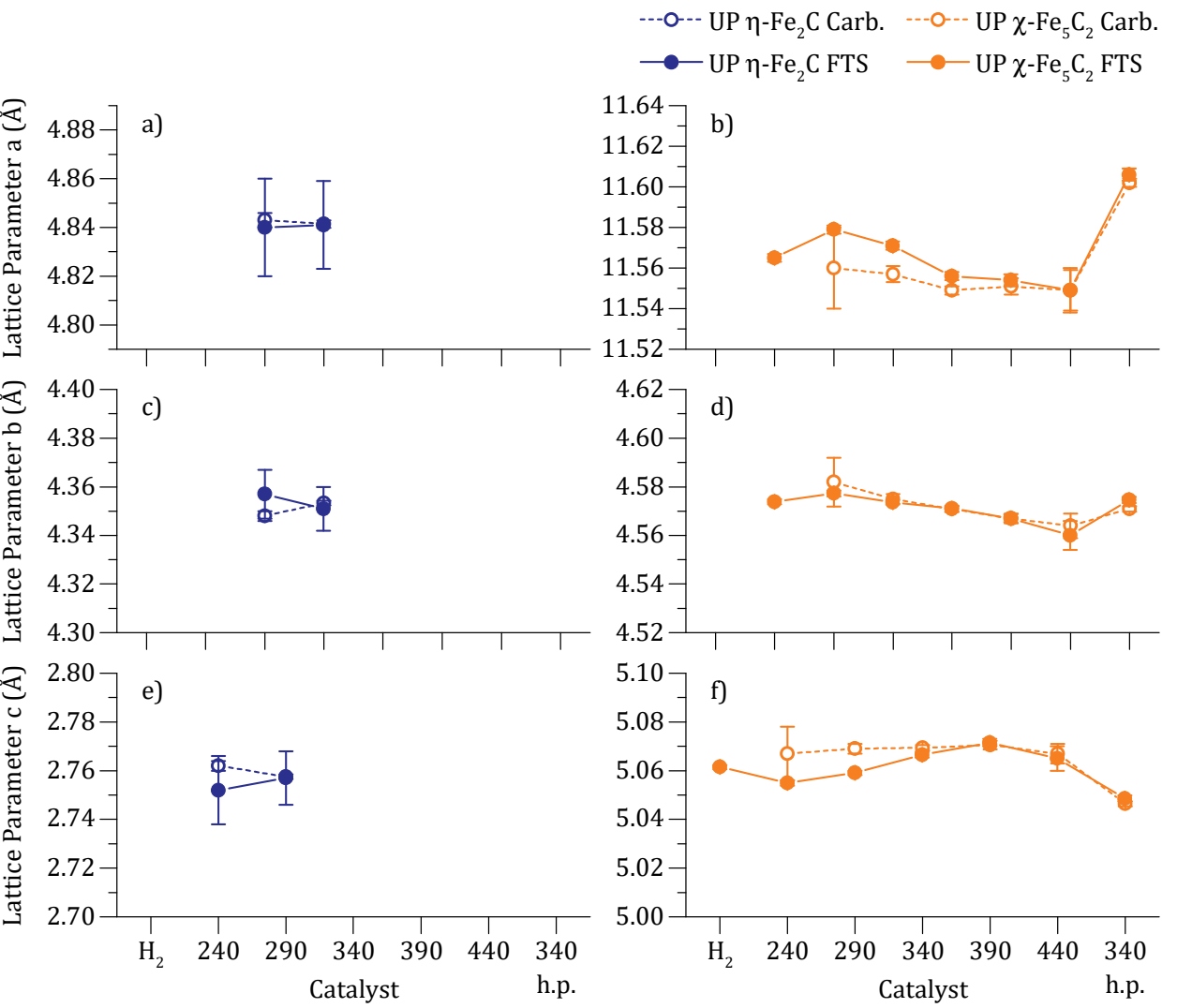

\section{Figure 4.13}

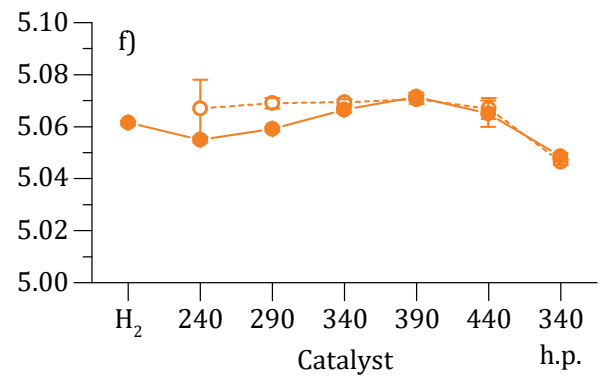

The lattice parameters used in Rietveld Quantitative Phase Analysis (R-QPA) for $\eta-\mathrm{Fe}_{2} \mathrm{C}$ and $\chi-\mathrm{Fe}_{5} \mathrm{C}_{2}$ phases compared before (Carb.) and after $72 \mathrm{~h}$ Fischer-Tropsch Synthesis (FTS) reaction for UP $\left(\mathrm{Fe} / \alpha-\mathrm{Al}_{2} \mathrm{O}_{3}\right)$ catalyst materials. Lattice parameters $a, b$, and $c$ for $\eta-\mathrm{Fe}_{2} \mathrm{C}$ in a), c) and e), and for $\chi-\mathrm{Fe}_{5} \mathrm{C}_{2}$ in b), d) and f), respectively.

content being low (Figure 4.9a), as the $\eta-\mathrm{Fe}_{2} \mathrm{C}$ phase is unstable without Na-S promotion at $340^{\circ} \mathrm{C}$ in the FTS reaction. Thus, some uncertainty remains in the exact crystal lattice behavior for the $\eta-\mathrm{Fe}_{2} \mathrm{C}$ phase in the UP FTS catalyst materials.

In an earlier study, Dry and Ferreira determined the lattice expansion for (cubic) $\mathrm{Fe}_{3} \mathrm{O}_{4}$ due to $\mathrm{Na}$ promotion being $\lesssim 0.03 \AA$ with the amount of the lattice expansion depending on the used $\mathrm{Na}$ concentration. ${ }^{63}$ The $\mathrm{Fe}_{3} \mathrm{O}_{4}$ lattice expansion was interpreted to be due to $\mathrm{Na}$ forming a solid solution with the $\mathrm{Fe}_{3} \mathrm{O}_{4}$ phase. Thus, the observed $\approx 0.01$ $0.02 \AA$ changes in the lattice parameters for the $\eta-\mathrm{Fe}_{2} \mathrm{C}$ and $\chi-\mathrm{Fe}_{5} \mathrm{C}_{2}$ phases are within an expected range, if the $\mathrm{Na}$ in the added $\mathrm{Na}-\mathrm{S}$ promotion enters the Fe lattice interstitial spaces. Granting that the literature reference value is for a $\mathrm{Fe}_{3} \mathrm{O}_{4}$ lattice, not for a $\mathrm{Fe}$ carbide. 

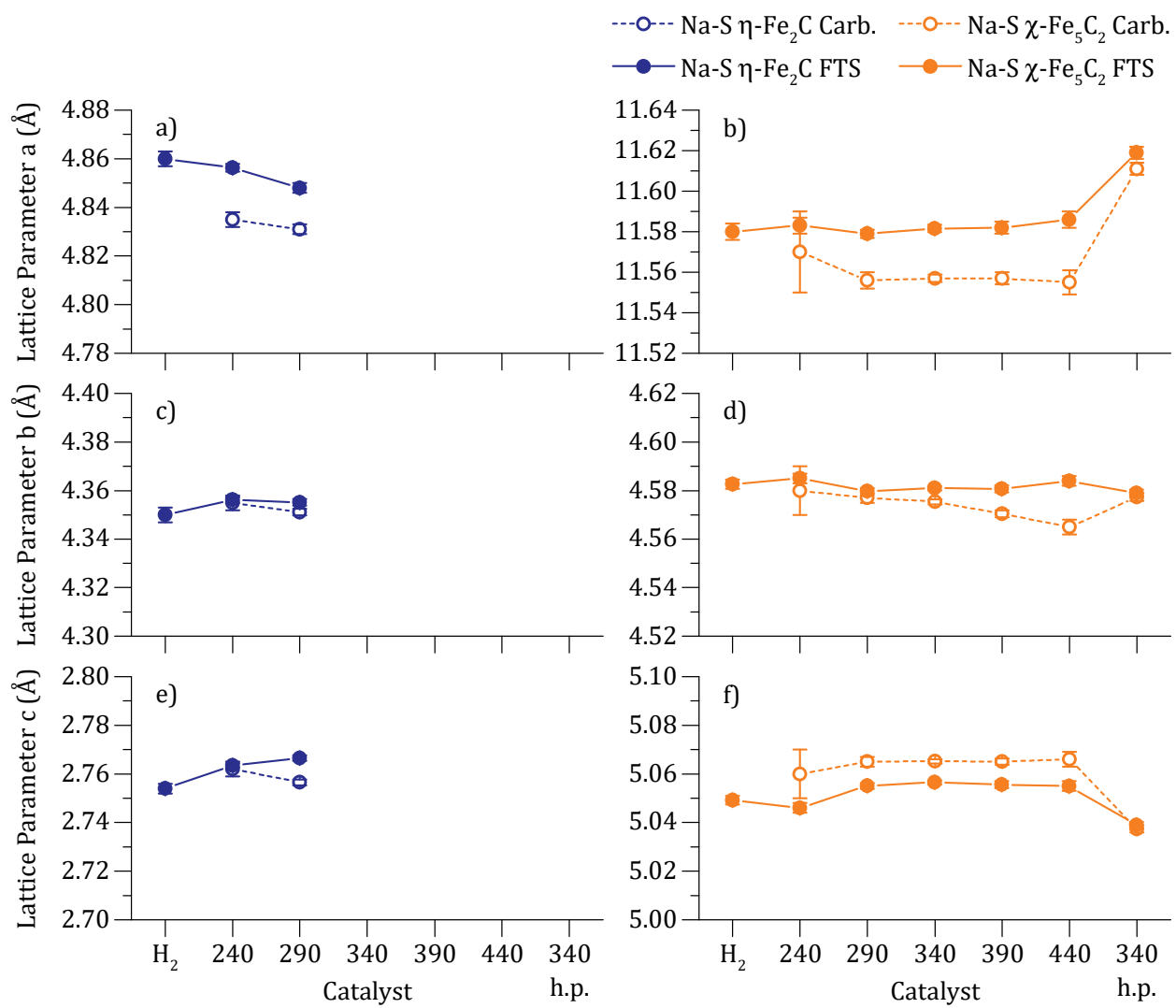

\section{Figure 4.14}

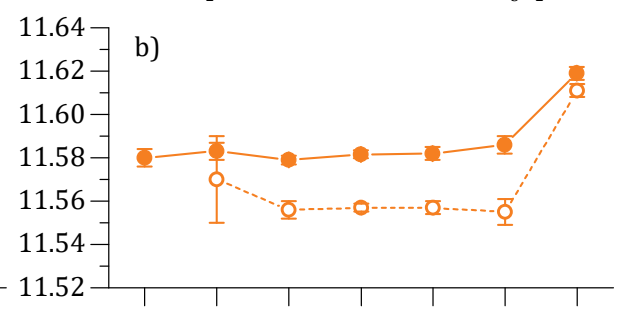

The lattice parameters used in Rietveld Quantitative Phase Analysis (R-QPA) for $\eta-\mathrm{Fe}_{2} \mathrm{C}$ and $\chi-\mathrm{Fe}_{5} \mathrm{C}_{2}$ phases compared before (Carb.) and after $72 \mathrm{~h}$ Fischer-Tropsch Synthesis (FTS) reaction for Na-S (Fe-Na-S/ $\alpha-\mathrm{Al}_{2} \mathrm{O}_{3}$ ) catalyst materials. Lattice parameters $a, b$, and $c$ for $\eta-\mathrm{Fe}_{2} \mathrm{C}$ in a), c) and e), and for $\chi-\mathrm{Fe}_{5} \mathrm{C}_{2}$ in b), d) and f), respectively.

However, as a peculiarity, also the $\chi-\mathrm{Fe}_{5} \mathrm{C}_{2}$ phase in the UP 240 FTS and UP 290 FTS catalyst materials show similar changes in its lattice parameters as the $\chi-\mathrm{Fe}_{5} \mathrm{C}_{2}$ phase with Na-S promotion does. That is, lattice parameters $a$ : increase $(\approx 0.02 \AA), b$ : unchanged, $c$ : decrease $(\approx 0.01 \AA$ ) (Figure 4.13b, $\boldsymbol{d}, f)$. The Rietveld calculated diffraction patterns for the $\chi-\mathrm{Fe}_{5} \mathrm{C}_{2}$ phase in the UP 240 FTS and Na-S 340 FTS catalyst materials are compared in Figure 4.15.

The patterns are near identical with each other, i.e. a R-QPA fitting error does not explain the lattice shifts. With the increasing $\mathrm{CO}$ treatment temperature $\left(\geq 340^{\circ} \mathrm{C}\right)$ applied in the initial catalyst carburization, the lattice expansion effect disappears for the UP FTS catalyst materials. The $\chi-\mathrm{Fe}_{5} \mathrm{C}_{2}$ phase lattice parameters then become nearly identical between the UP 340-440 Carb. and FTS catalyst materials (Figure 4.13b, $\boldsymbol{d}, \boldsymbol{f}$ ). 


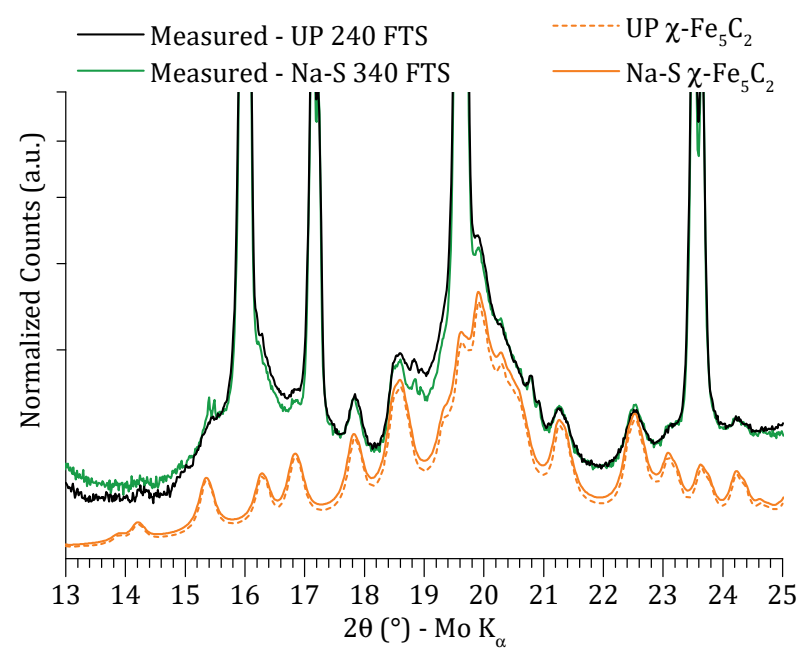

\section{Figure 4.15}

Background corrected, ex-situ measured X-ray Powder Diffractometry (XRPD) patterns with Rietveld method calculated $\eta-\mathrm{Fe}_{2} \mathrm{C}$ and $\chi-\mathrm{Fe}_{5} \mathrm{C}_{2}$ phase patterns over the range $13-25^{\circ} 2 \theta$ for UP 240 and Na-S $340(\mathrm{Fe}(-\mathrm{Na}-\mathrm{S}) / \alpha-$ $\mathrm{Al}_{2} \mathrm{O}_{3}$ ) catalyst materials after $72 \mathrm{~h}$ Fischer-Tropsch Synthesis (FTS) reaction. Normalized counts on a squareroot scale.

Why the $\chi-\mathrm{Fe}_{5} \mathrm{C}_{2}$ phase crystal lattice parameters in the UP 240 FTS and UP 290 FTS catalyst materials behaves akin to the Na-S promoted catalyst materials, is not clear from the results. $\mathrm{Na}(-\mathrm{S})$ contamination can be excluded, as the catalyst materials in question do not show any alkali or Na-S promotion effects in their FTS reaction catalysis performance, as will be discussed later on (Table 4.4 and Table 4.5).

\subsubsection{Assignment of Fe Carbide Subtypes}

As based on the differences in the $\eta-\mathrm{Fe}_{2} \mathrm{C}, \chi-\mathrm{Fe}_{5} \mathrm{C}_{2}$ and $\chi-\mathrm{Fe}_{5} \mathrm{C}_{2}$ h.p. phases lattice parameter $a$ 's (Table 4.2), the carbides can be divided to subtypes and assigned with their primary formation conditions (Table 4.3).

The $\chi-\mathrm{Fe}_{5} \mathrm{C}_{2}$ subtypes defined by lattice parameter $a$ can be divided as: Type I: $\approx 11.56 \AA$ which is observed either after CO carburization of $\alpha$-Fe (UP and Na-S Carb.) or after FTS reaction without Na-S promotion (UP FTS), Type II: $\approx 11.58 \AA$ is observed in Na-S FTS catalyst materials, and Type III: $\approx 11.61 \AA$ if a Fe oxide $\left(\alpha-\mathrm{Fe}_{2} \mathrm{O}_{3}\right)$ is carburized with $\mathrm{CO}$ at high-pressure (340 h.p.). The Type III is also stable in the FTS reaction regardless of the Na-S promotion.

The lattice parameters ( $a: 11.562 \AA, b: 4.573 \AA$, $c: 5.060 \AA$ ) corresponding to the $\chi$ $\mathrm{Fe}_{5} \mathrm{C}_{2}$ Type I have earlier been reported by Jack and Wild. ${ }^{46}$ Similar values have also been reported by Duggin and Hofer, ${ }^{45}$ on the basis of $\chi-\mathrm{Fe}_{5} \mathrm{C}_{2}$ phase prepared from $\alpha$-Fe via CO carburization. ${ }^{64}$ 


\section{Table 4.3}

Overview of the different subtypes $(I, I I, I I I)$ of $\eta-\mathrm{Fe}_{2} \mathrm{C}$ and $\chi-\mathrm{Fe}_{5} \mathrm{C}_{2}$ phases as divided by their observed lattice parameters in the UP and Na-S Carb. and FTS catalyst materials. The lattice parameters are taken as the mean value over all observations of the subtype. Value after $( \pm)$ is the Standard Deviation (STD) of the mean value.

\begin{tabular}{|c|c|c|c|c|c|}
\hline \multirow{2}{*}{$\begin{array}{c}\text { Fe Carbide } \\
\text { Phase }\end{array}$} & \multirow[t]{2}{*}{ Space Group } & & \multicolumn{3}{|c|}{ Lattice Parameters } \\
\hline & & & $\begin{array}{c}\text { Type I } \\
(\AA)\end{array}$ & $\begin{array}{c}\text { Type II } \\
(\AA)\end{array}$ & $\begin{array}{c}\text { Type III } \\
(\AA)\end{array}$ \\
\hline \multirow{3}{*}{$\eta-\mathrm{Fe}_{2} \mathrm{C}$} & Orthorhombic, & $\mathrm{a}$ & $4.84 \pm 0.01$ & $4.86 \pm 0.01$ & 4.704 \\
\hline & Pnnm (58) & $\mathrm{b}$ & $4.35 \pm 0.00$ & $4.35 \pm 0.00$ & 4.318 \\
\hline & & c & $2.76 \pm 0.00$ & $2.76 \pm 0.01$ & 2.830 \\
\hline \multirow{4}{*}{ 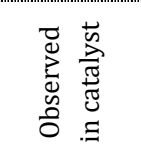 } & UP Carb. & & $\mathrm{x}$ & & \multirow{4}{*}{$\begin{array}{c}\text { Tempering of } \\
\text { martensitic steela) }\end{array}$} \\
\hline & Na-S Carb. & & $\mathrm{x}$ & & \\
\hline & UP FTS & & $\mathrm{x}$ & & \\
\hline & Na-S FTS & & & $\mathrm{x}$ & \\
\hline \multirow{3}{*}{$\chi-\mathrm{Fe}_{5} \mathrm{C}_{2}$} & Monoclinic, & $\mathrm{a}$ & $11.56 \pm 0.01$ & $11.58 \pm 0.00$ & $11.61 \pm 0.01$ \\
\hline & $\mathrm{C} 2 / \mathrm{c}(15)$ & $\mathrm{b}$ & $4.57 \pm 0.01$ & $4.58 \pm 0.00$ & $4.58 \pm 0.00$ \\
\hline & & c & $5.06 \pm 0.00$ & $5.05 \pm 0.00$ & $5.05 \pm 0.01$ \\
\hline \multirow{4}{*}{ 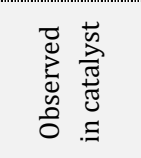 } & UP Carb. & & $\mathrm{x}$ & & $\mathrm{X}^{\mathrm{c})}$ \\
\hline & Na-S Carb. & & $\mathrm{x}$ & & $\mathrm{x}^{\mathrm{c})}$ \\
\hline & UP FTS & & $\mathrm{x}$ & $x^{b)}$ & $\mathrm{x}^{\mathrm{c})}$ \\
\hline & Na-S FTS & & & $\mathrm{x}$ & $X^{c)}$ \\
\hline
\end{tabular}

a) The $\eta-\mathrm{Fe}_{2} \mathrm{C}$ Type III carbide has been observed as a precipitate in tempering of martensitic steel at $120^{\circ} \mathrm{C},{ }^{65,66}$ and is included here for completeness.

b) With UP FTS catalyst materials, the $\chi-\mathrm{Fe}_{5} \mathrm{C}_{2}$ Type II could be observed only with catalysts 240 and 290.

c) The $\chi-\mathrm{Fe}_{5} \mathrm{C}_{2}$ Type III was only observed in catalyst materials 340 h.p.. The phase is also labelled as $\chi-\mathrm{Fe}_{5} \mathrm{C}_{2}$ (h.p.) in Table 4.2 and in Chapter 3.

Lattice parameters ( $a$ : $11.588 \AA$, $b: 4.579 \AA$, $c: 5.059 \AA$ and $\beta=97.75^{\circ}$ ) close to the $\chi$ $\mathrm{Fe}_{5} \mathrm{C}_{2}$ Type II have been determined by Retief with a proprietary Fe-based catalyst material extracted from a Sasol pilot plant, after FTS reaction $\left(320^{\circ} \mathrm{C}, 20\right.$ bar, $\mathrm{H}_{2}: \mathrm{CO}$ 1:2.3). ${ }^{44}$ Although, even as $\mathrm{Fe}_{3} \mathrm{O}_{4}$ was used as the FTS reaction starting material by Retief, the resulting $\chi-\mathrm{Fe}_{5} \mathrm{C}_{2}$ phase gave lattice parameters more akin to $\chi-\mathrm{Fe}_{5} \mathrm{C}_{2}$ Type II than to the Type III. The Type III would be expected based on our results.

By following the catalyst material Curie Temperature and XRPD patterns, a distorted $\chi-\mathrm{Fe}_{5} \mathrm{C}_{2}$ phase was observed to form from a $\mathrm{Fe}_{3} \mathrm{O}_{4}$ starting material with $\mathrm{CO}$ carburization by Cohn et al.. ${ }^{19}$ The observation by Cohn et al. is alike to the formation of the distorted $\chi-\mathrm{Fe}_{5} \mathrm{C}_{2}$ h.p. phase with the UP and Na-S 340 h.p. catalyst materials, which were not reduced with $\mathrm{H}_{2}$ to $\alpha$-Fe a priori to the $\mathrm{CO}$ carburization. Rather, the $\alpha-\mathrm{Fe}_{2} \mathrm{O}_{3}$ on the calcined catalyst materials was carburized directly (Table 4.1). Cohn et al. did not report lattice parameters on the basis of their XRPD patterns. We shall presume, nevertheless, that the distorted $\chi-\mathrm{Fe}_{5} \mathrm{C}_{2}$ h.p. phase is the same phase as the Cohn et al.'s 
distorted $\chi-\mathrm{Fe}_{5} \mathrm{C}_{2}$ phase. This distorted phase corresponds to the $\chi-\mathrm{Fe}_{5} \mathrm{C}_{2}$ Type III subtype with mean lattice parameters defined as: $a$ : $11.61 \pm 0.01 \AA, b: 4.58 \pm 0.00 \AA$, $c$ : $5.05 \pm 0.01 \AA$ and $\beta=97.75^{\circ}$.

Cohn et al. presumably used atmospheric CO pressure for forming the distorted $\chi$ $\mathrm{Fe}_{5} \mathrm{C}_{2}$ phase, ${ }^{19}$ not 25 bar $\mathrm{CO}$ as used in the carburization of the 340 h.p. Carb. catalyst materials. That is, a high $\mathrm{CO}$ pressure may not be required for the formation of the $\chi$ $\mathrm{Fe}_{5} \mathrm{C}_{2}$ Type III (i.e. the $\chi-\mathrm{Fe}_{5} \mathrm{C}_{2}$ h.p. phase) from a Fe oxide. Directly carburizing a Fe oxide with $\mathrm{CO}$ might be the sole requirement for the formation of the $\chi-\mathrm{Fe}_{5} \mathrm{C}_{2}$ Type III subtype.

As the distorted $\chi-\mathrm{Fe}_{5} \mathrm{C}_{2}$ phase by Cohn et al. would form a more crystalline $\chi-\mathrm{Fe}_{5} \mathrm{C}_{2}$ phase after an extended CO exposure period or increased carburization treatment temperature. After these observations, Cohn et al. speculated, among other possibilities, that the distortion could be due to $\mathrm{O}$ atoms being retained in the distorted $\chi-\mathrm{Fe}_{5} \mathrm{C}_{2}$ lattice. ${ }^{19}$ The recrystallization behavior also could explain why the $\chi-\mathrm{Fe}_{5} \mathrm{C}_{2}$ phase observed by Retief resembles the Type II and not the Type III $\chi-\mathrm{Fe}_{5} \mathrm{C}_{2}$ subtype, ${ }^{44}$ despite Retief having started the FTS reaction with a $\mathrm{Fe}_{3} \mathrm{O}_{4}$ phase.

Likewise to the $\chi-\mathrm{Fe}_{5} \mathrm{C}_{2}$, the $\eta-\mathrm{Fe}_{2} \mathrm{C}$ phase can be divided to carbide subtypes following the distinct ranges of lattice parameter $a$ 's as: Type I: $\approx 4.83 \AA$ with UP and NaS Carb. and UP FTS catalyst materials, and Type II: $\approx 4.86 \AA$ with Na-S FTS catalyst materials. The $\eta-\mathrm{Fe}_{2} \mathrm{C}$ phase Type III precipitates from martensitic steel with lattice parameter $a$ of $4.704 \AA$ have been observed in tempering studies at $120^{\circ} \mathrm{C} .65,66$ The $\eta$ $\mathrm{Fe}_{2} \mathrm{C}$ phase Type III has not been observed in the FTS reaction related studies - for best of the author's knowledge - but is included here for sake of completeness (Table 4.3).

The $\eta-\mathrm{Fe}_{2} \mathrm{C}$ Type II matches well with the "pseudo-hexagonal Fe carbide", detected by Barton and Gale in a K-promoted Fe-based catalyst after the FTS reaction. ${ }^{67}$ With the orthorhombic unit cell translation for the $\eta-\mathrm{Fe}_{2} \mathrm{C}$ phase by Hirotsu and Nagakura, ${ }^{68}$ the lattice parameters corresponding Barton and Gale's Fe carbide are: $a$ : $4.862 \AA, b$ : $4.360 \AA$, and $c: 2.755 \AA$. These lattices parameters are a good match to the $\eta-\mathrm{Fe}_{2} \mathrm{C}$ Type II subtype formed with the Na-S FTS catalyst materials (Table 4.2).

For the $\eta-\mathrm{Fe}_{2} \mathrm{C}$ Type $\mathrm{I}$, no matching lattice parameters could be found from the literature, despite the author's best efforts. Thus, we shall assign the $\eta-\mathrm{Fe}_{2} \mathrm{C}$ Type I in the UP and Na-S 240 and 290 Carb. and in UP 240 and 290 FTS catalyst materials with mean lattice parameters as: $a: 4.84 \pm 0.01 \AA, b: 4.35 \pm 0.00 \AA$ and $c: 2.76 \pm 0.00 \AA$. That is, the $\eta-\mathrm{Fe}_{2} \mathrm{C}$ Type I forms regardless of the Na-S promotion with $\mathrm{CO}$ carburization $\leq 290^{\circ} \mathrm{C}$.

Thus, under FTS reaction conditions: without $\mathrm{Na}-\mathrm{S}$ promotion the $\eta-\mathrm{Fe}_{2} \mathrm{C}$ Type I prevails (UP FTS), while with $\mathrm{Na}$-S promotion transformation to the $\eta-\mathrm{Fe}_{2} \mathrm{C}$ Type II takes over (Na-S FTS). These conclusions, however, are drawn while acknowledging the large lattice parameter fit errors for the $\eta-\mathrm{Fe}_{2} \mathrm{C}$ phases in the UP FTS catalyst materials (Figure 4.13a, $c, e$ ). 


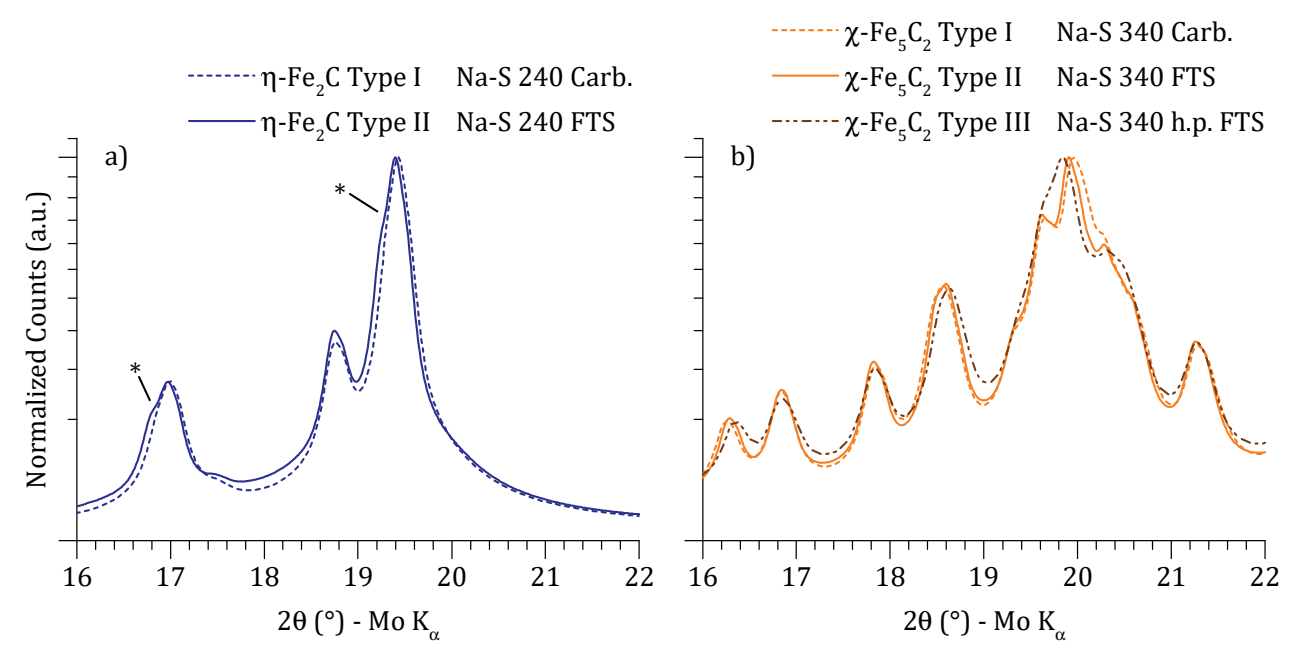

Figure 4.16

Rietveld method calculated phase diffraction pattern examples of the $\eta-\mathrm{Fe}_{2} \mathrm{C}$ and $\chi-\mathrm{Fe}_{5} \mathrm{C}_{2}$ phase subtypes over a range of $16-22^{\circ} 2 \theta$ fitted for $\mathrm{Na}-\mathrm{S}\left(\mathrm{Fe}-\mathrm{Na}-\mathrm{S} / \alpha-\mathrm{Al}_{2} \mathrm{O}_{3}\right)$ catalyst materials before (Carb.) and after $72 \mathrm{~h}$ FischerTropsch Synthesis (FTS) reaction. The calculated phase diffraction patterns for a) $\eta$ - $\mathrm{Fe}_{2} \mathrm{C}$ Type I-II and b) $\chi$ $\mathrm{Fe}_{5} \mathrm{C}_{2}$ Type I-III, as following the nomenclature in Table 4.3. The calculated patterns are normalized to the carbide subtype's most intense diffraction peak. Normalized counts on a square-root scale.

In Figure 4.16 are overlaid examples of the Rietveld calculated phase diffraction patterns for the $\eta-\mathrm{Fe}_{2} \mathrm{C}$ Types I-II and $\chi-\mathrm{Fe}_{5} \mathrm{C}_{2}$ Types I-III, as based on the R-QPA fits on the indicated $\mathrm{Na}-\mathrm{S}$ catalyst materials. When comparing the calculated phase diffraction patterns between the $\eta-\mathrm{Fe}_{2} \mathrm{C}$ Type I versus Type II: a shoulder (marked with *) appears to the diffraction pattern of the $\eta-\mathrm{Fe}_{2} \mathrm{C}$ Type II (Figure 4.16a). This shoulder is likely the origins of the "double peak" observations by Barton and Gale on their "pseudohexagonal Fe carbide", 67 i.e. on the $\eta-\mathrm{Fe}_{2} \mathrm{C}$ phase. Barton and Gale obtained their X-ray diffraction patterns with $\mathrm{Co}_{\alpha}$ radiation which provides significantly better diffraction peak separation than the Mo $\mathrm{K}_{\alpha}$ in capillary geometry applied in our study does. Thus, the shoulder in the Figure 4.16a would be a well separated diffraction peak in Barton and Gale's diffraction patterns.

For the $\chi-\mathrm{Fe}_{5} \mathrm{C}_{2}$ Types I-III the calculated diffraction patterns show clear differences, especially, in the most intense diffraction peaks (Figure 4.16b). The fundamental origins of these differences, however, are unclear at the time being.

In total, a monochromatic synchrotron radiation based XRPD study on the crystal structural details on the origins of the differences in the $\eta-\mathrm{Fe}_{2} \mathrm{C}$ and the $\chi-\mathrm{Fe}_{5} \mathrm{C}_{2}$ phases subtypes would be of future interested. 


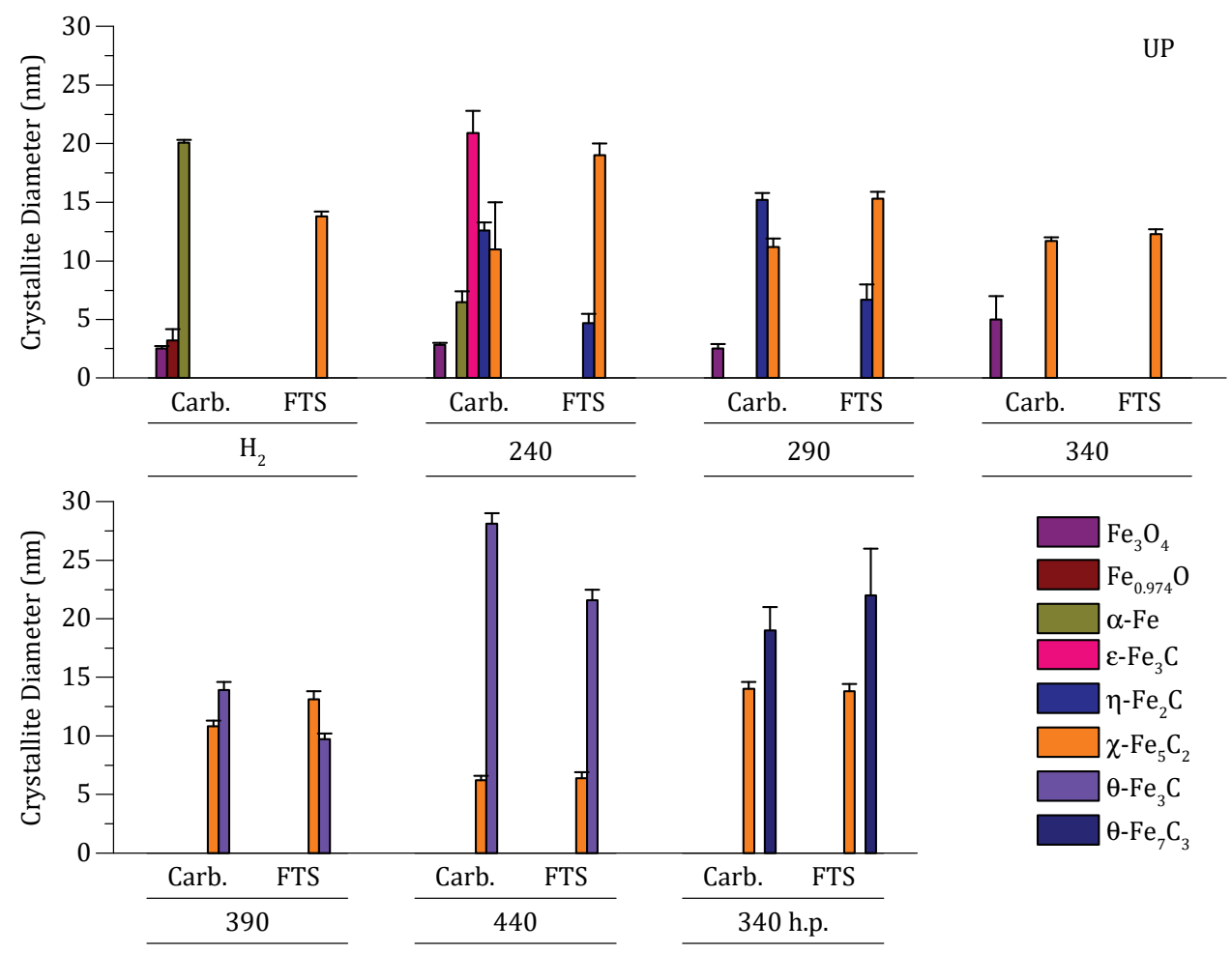

\section{Figure 4.17}

Rietveld Quantitative Phase Analysis (R-QPA) determined Fe phase (volume-weighted) crystallite mean diameters derived from the X-ray Powder Diffractometry (XRPD) patterns for UP ( $\left.\mathrm{Fe} / \alpha-\mathrm{Al}_{2} \mathrm{O}_{3}\right)$ catalyst materials after $72 \mathrm{~h}$ Fischer-Tropsch Synthesis (FTS) reaction. The respective mean Fe crystallite diameters before the FTS reaction (Carb.) are reproduced from Chapter 3 for comparison.

\subsubsection{Changes in Fe Phases' Crystallite Diameters}

The R-QPA determined Fe phase (volume-weighted) crystallite mean diameters are given in Figure 4.17 for the UP FTS catalyst materials with the respective UP Carb. results reproduced from Chapter 3 for comparison. Likewise, crystallite mean diameters for the Na-S FTS catalyst materials are given in Figure 4.18. As a general observation: no clear, straightforward trends seem to exist in the crystallite mean diameters when comparison is done between the UP or Na-S, Carb. and FTS catalyst materials.

However, few comments can be made when comparing the Fe phases crystallite diameters in the Carb. and FTS catalyst materials to each other: i) in general, the $\chi-\mathrm{Fe}_{5} \mathrm{C}_{2}$ phase crystallite mean diameter increases in the FTS reaction for the UP (Figure 4.17) and Na-S catalyst materials (Figure 4.18), ii) for UP FTS catalyst materials, the 


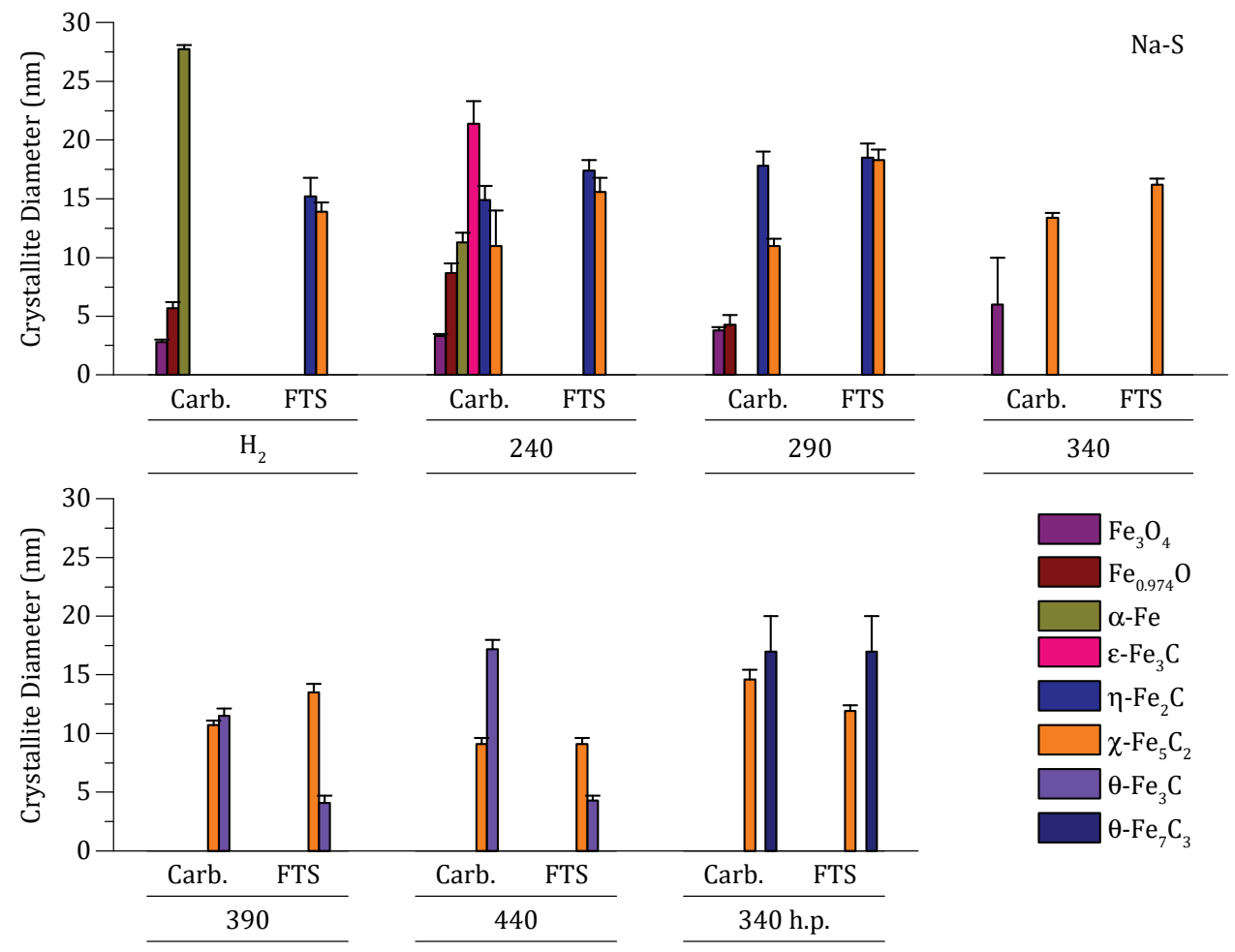

\section{Figure 4.18}

Rietveld Quantitative Phase Analysis (R-QPA) determined Fe phase (volume-weighted) crystallite mean diameters derived from the X-ray Powder Diffractometry (XRPD) patterns for Na-S (Fe-Na-S/ $\alpha-\mathrm{Al}_{2} \mathrm{O}_{3}$ ) catalyst materials after $72 \mathrm{~h}$ Fischer-Tropsch Synthesis (FTS) reaction. The respective mean Fe crystallite diameters before the FTS reaction (Carb.) are reproduced from Chapter 3 for comparison.

crystallite mean diameters decrease for both $\eta-\mathrm{Fe}_{2} \mathrm{C}$ and $\theta-\mathrm{Fe}_{3} \mathrm{C}$ phases (Figure 4.17), and iii) for Na-S FTS catalyst materials, the $\eta-\mathrm{Fe}_{2} \mathrm{C}$ phase crystallite mean diameters remain largely unchanged while the $\theta-\mathrm{Fe}_{3} \mathrm{C}$ diameters decrease significantly (Figure 4.18).

The general increase in the $\chi-\mathrm{Fe}_{5} \mathrm{C}_{2}$ crystallite mean diameters, i.e. the point i), is due to the $\chi-\mathrm{Fe}_{5} \mathrm{C}_{2}$ phase being the most thermally stable carbide phase at the used FTS reaction temperature of $340^{\circ} \mathrm{C}$ (Chapter 3$)$. The $\chi-\mathrm{Fe}_{5} \mathrm{C}_{2}$ phase is present in both the UP and Na-S FTS catalyst materials in larger relative quantities than in the respective Carb. catalysts (Figure 4.9a-b). Therefore, this is resulting in larger crystallite diameters for the $\chi-\mathrm{Fe}_{5} \mathrm{C}_{2}$ phase.

Both, the points ii) and iii), also follow the development of the Fe phase quantities in Figure 4.9a-b. As the phase $\eta-\mathrm{Fe}_{2} \mathrm{C}$ is unstable in the FTS reaction with the UP catalyst materials, the phase's quantity decreases, and so does the phase's crystallite 
mean diameter (Figure 4.17). In the Na-S FTS catalyst materials the $\eta-\mathrm{Fe}_{2} \mathrm{C}$ phase is stable, thus the crystallite mean diameters do not decrease nor notably increase (Figure 4.18), despite the increased $\eta-\mathrm{Fe}_{2} \mathrm{C}$ phase content (Figure 4.9b).

With regard to the $\theta-\mathrm{Fe}_{3} \mathrm{C}$ phase crystallite mean diameters, the Na-S promotion destabilizes and decreases the $\theta-\mathrm{Fe}_{3} \mathrm{C}$ phase content in the Na-S FTS catalyst material resulting in severely decreased crystallite mean diameters (Figure 4.18). Interestingly, also in the UP FTS catalysts the $\theta-\mathrm{Fe}_{3} \mathrm{C}$ crystallite mean diameters decrease in comparison to the Carb. catalysts, albeit less than in the Na-S catalyst materials. In relative terms, the $\theta-\mathrm{Fe}_{3} \mathrm{C}$ phase crystallite diameters decrease: $-30 \%$ for UP 390 and $23 \%$ for UP 440; and $-64 \%$ for Na-S 390 and $-75 \%$ for Na-S 440 catalysts when comparing the respective Carb. to the FTS catalyst materials. That is, the decreasing $\theta$ $\mathrm{Fe}_{3} \mathrm{C}$ crystallite mean diameters also without the $\mathrm{Na}-\mathrm{S}$ promotion might suggest that under the used reaction conditions, the $\theta-\mathrm{Fe}_{3} \mathrm{C}$ phase is unstable. However, its demise is greatly hastened by the presence of Na-S promotion.

The Na-S 240 FTS and Na-S 340 FTS catalyst materials showed significant increases in the TEM-based Fe particle mean diameters in comparison to the respective Carb. catalysts (Figure 4.4e). However, the R-QPA-based Fe crystallite mean diameters do not show any comparable increase for any of the Fe phases in the same catalyst materials (Figure 4.18).

Furthermore, the TEM-based Fe particle mean diameters for the UP and Na-S 440 FTS catalysts show an increase of $+8 \%$ and $+23 \%$, respectively, in the Fe particle mean diameters in comparison to the respective Carb. catalyst materials (Figure 4.4e). The R-QPA-based Fe crystallite mean diameters show an opposite trend: for both UP and $\mathrm{Na}-\mathrm{S} 440 \mathrm{FTS}$ catalyst materials the $\theta-\mathrm{Fe}_{3} \mathrm{C}$ phase crystallite diameters decrease $(-23 \%$, and $-75 \%$, respectively, see earlier paragraph) while the $\chi-\mathrm{Fe}_{5} \mathrm{C}_{2}$ phase crystallite diameters remain relatively unchanged. Likewise, no connection between the Fe particle (Figure 4.4e) and crystallite mean diameters exist for the UP (Figure 4.17) and $\mathrm{Na}-\mathrm{S} \mathrm{H}_{2}$ catalyst materials (Figure 4.18).

That is, the Fe particle and crystallite mean diameters give opposing trends for the mentioned catalyst materials. The lack of common trends could be interpreted to suggest that a single Fe particle is not a single Fe carbide crystallite. In literature, examples of this have been observed. ${ }^{43,69-71}$

As a final comment in the current section, comparing the TEM Fe particle based and R-QPA (i.e. XRPD-based) Fe crystallite diameters data is not straightforward, ${ }^{69,72,73}$ as is shortly yet more in detail discussed in Chapter 3. Thus, drawing any further conclusions on the Fe particle (TEM), in comparison to the Fe crystallite diameter (R$\mathrm{QPA}$ ) results is not attempted within the current context. 


\section{Table 4.4}

Median Fischer-Tropsch Synthesis (FTS) reaction activity data over Time-on-Stream (ToS) 33-72 h for UP and $\mathrm{Na}-\mathrm{S}\left(\mathrm{Fe}(-\mathrm{Na}-\mathrm{S}) / \alpha-\mathrm{Al}_{2} \mathrm{O}_{3}\right)$ catalyst materials. Value after $( \pm)$ is the Standard Deviation (STD) of the median value.

\begin{tabular}{|c|c|c|c|c|c|}
\hline \multirow[t]{2}{*}{$\begin{array}{l}\text { Promotion } \\
\text { Group }\end{array}$} & Catalyst & CO Conversion & $\begin{array}{l}\text { Fe Time Yield } \\
\text { (FTY) }\end{array}$ & Usage Ratio $\mathrm{H}_{2}: \mathrm{CO}$ & $\begin{array}{l}\mathrm{CO} \text { to Hydrocarbons } \\
\mathrm{Na}-\mathrm{S} \text { versus UPa) }\end{array}$ \\
\hline & & $(\%)$ & $\left(10^{-4} \mathrm{~mol}{ }_{\mathrm{Co}} / \mathrm{g}_{\mathrm{Fe}} \mathrm{s}\right)$ & $(-)$ & $(-)$ \\
\hline \multirow[t]{7}{*}{ UP } & $\mathrm{H}_{2}$ & $3 \pm 0$ & $0.4 \pm 0.1$ & $2.1 \pm 0.4$ & $\mathrm{~N} / \mathrm{A}$ \\
\hline & 240 & $5 \pm 0$ & $0.6 \pm 0.1$ & $1.8 \pm 0.2$ & N/A \\
\hline & 290 & $3 \pm 1$ & $0.4 \pm 0.1$ & $2.3 \pm 0.4$ & $\mathrm{~N} / \mathrm{A}$ \\
\hline & 340 & $2 \pm 0$ & $0.3 \pm 0.0$ & $1.9 \pm 0.5$ & N/A \\
\hline & 390 & $4 \pm 0$ & $0.5 \pm 0.1$ & $2.1 \pm 0.3$ & $\mathrm{~N} / \mathrm{A}$ \\
\hline & 440 & $3 \pm 0$ & $0.3 \pm 0.1$ & $2.2 \pm 0.7$ & $\mathrm{~N} / \mathrm{A}$ \\
\hline & 340 h.p. & $2 \pm 1$ & $0.3 \pm 0.2$ & $2.1 \pm 1.1$ & $\mathrm{~N} / \mathrm{A}$ \\
\hline \multirow[t]{7}{*}{$\mathrm{Na}-\mathrm{S}$} & $\mathrm{H}_{2}$ & $53 \pm 3$ & $5.9 \pm 0.3$ & $0.7 \pm 0.1$ & $0.45 \pm 0.22$ \\
\hline & 240 & $40 \pm 0$ & $4.8 \pm 0.0$ & $0.7 \pm 0.0$ & $0.47 \pm 0.11$ \\
\hline & 290 & $34 \pm 3$ & $3.7 \pm 0.3$ & $0.7 \pm 0.1$ & $0.45 \pm 0.16$ \\
\hline & 340 & $45 \pm 5$ & $5.3 \pm 0.5$ & $0.7 \pm 0.1$ & $0.42 \pm 0.20$ \\
\hline & 390 & $24 \pm 2$ & $2.8 \pm 0.3$ & $0.8 \pm 0.1$ & $0.43 \pm 0.15$ \\
\hline & 440 & $33 \pm 1$ & $4.0 \pm 0.1$ & $0.8 \pm 0.0$ & $0.42 \pm 0.14$ \\
\hline & 340 h.p. & $3 \pm 0$ & $0.4 \pm 0.0$ & $1.1 \pm 0.5$ & $0.61 \pm 0.49$ \\
\hline
\end{tabular}

FTS conditions: $340^{\circ} \mathrm{C}, 10$ bar, $\mathrm{H}_{2}: \mathrm{CO}: \mathrm{He} 2.0: 1.0: 0.33 \mathrm{v} / \mathrm{v}, \approx 5000 \mathrm{~h}^{-1}$.

a) Change in fraction of $\mathrm{CO}$ converted to hydrocarbons with addition of Na-S promotion (Na-S) versus the respective unpromoted (UP) catalyst material.

\subsubsection{Catalytic Performance}

The catalytic activity profiles as FTYs for each of the catalyst materials over the $72 \mathrm{~h}$ FTS reaction are given in Figure 4.20. The median CO conversions, FTYs and Usage Ratios $\mathrm{H}_{2}$ :CO over Time-on-Stream (ToS) 33-72 h, over relatively steady FTYs, for each catalyst material are tabulated in Table 4.4. In addition, for comparing the effect of Na$\mathrm{S}$ promotion to the relative FTS reaction C pathways (Chapter 2), the changes in CO converted towards hydrocarbons between the Na-S and the corresponding UP catalyst materials are reported in the Table 4.4. The median $\mathrm{CO}_{2}$ and $\mathrm{CO}_{2}$-free hydrocarbon selectivities over ToS 33-72 h are given in the Table 4.5.

For each catalyst material, each of the duplicate reactors used for the FTS reaction runs gave identical results with the exceptions of the catalyst materials $\mathrm{Na}-\mathrm{S} \mathrm{H}_{2}$ and $\mathrm{Na}-$ S 440. For these catalyst materials the FTY profiles differed in respect to their duplicate reactors (Figure 4.19). For the catalysts with differing duplicate reactor results the catalyst reactors with higher overall FTY are discussed. Moreover, the catalyst $\mathrm{Na}-\mathrm{S}_{2}$ also gave a differing $\mathrm{C}_{2} \mathrm{H}_{4} / \mathrm{C}_{2} \mathrm{H}_{6}$ ratio in comparison to its duplicate FTS reactor (Table 4.5). 
On the Role of Iron Carbides in the FischerTropsch Synthesis with Fe(-Na-S)/ $\alpha-\mathrm{Al} 203$ Catalysts

\section{Table 4.5}

Median Fischer-Tropsch Synthesis (FTS) reaction selectivity data over Time-on-Stream (ToS) 33-72 h for UP and Na-S (Fe(-Na-S) $\left./ \alpha-\mathrm{Al}_{2} \mathrm{O}_{3}\right)$ catalyst materials. Value after $( \pm)$ is the Standard Deviation (STD) of the median value.

\begin{tabular}{|c|c|c|c|c|c|c|c|c|}
\hline \multirow[t]{2}{*}{$\begin{array}{l}\text { Promotion } \\
\text { Group }\end{array}$} & Catalyst & $\mathrm{CO}_{2}$ & $\mathrm{CH}_{4}$ & $\begin{array}{l}\mathrm{C}_{2}-\mathrm{C}_{4} \\
\text { Olefin }\end{array}$ & $\begin{array}{c}\mathrm{C}_{2}-\mathrm{C}_{4} \\
\text { Paraffin }\end{array}$ & $\mathrm{C}_{5+}$ & $\begin{array}{l}\mathrm{C}_{2} \mathrm{H}_{4} / \\
\mathrm{C}_{2} \mathrm{H}_{6}\end{array}$ & $\begin{array}{c}\text { ASF } \\
\alpha \text {-value }\end{array}$ \\
\hline & & $(\%)$ & $(\%)$ & $(\%)$ & $(\%)$ & $(\%)$ & $(-)$ & (a.u) \\
\hline \multirow[t]{7}{*}{ UP } & $\mathrm{H}_{2}$ & $<\mathrm{dl}$ & $56 \pm 2$ & $33 \pm 1$ & $7 \pm 0$ & $4 \pm 3$ & $2.8 \pm 0.1$ & $0.36 \pm 0.05$ \\
\hline & 240 & $11 \pm 4$ & $52 \pm 1$ & $34 \pm 1$ & $8 \pm 0$ & $5 \pm 2$ & $2.4 \pm 0.0$ & $0.29 \pm 0.04$ \\
\hline & 290 & $16 \pm 2$ & $53 \pm 1$ & $35 \pm 1$ & $7 \pm 0$ & $5 \pm 2$ & $2.9 \pm 0.1$ & $0.34 \pm 0.07$ \\
\hline & 340 & $<\mathrm{dl}$ & $58 \pm 3$ & $30 \pm 1$ & $9 \pm 0$ & $4 \pm 5$ & $2.2 \pm 0.1$ & $0.30 \pm 0.02$ \\
\hline & 390 & $9 \pm 3$ & $55 \pm 0$ & $28 \pm 0$ & $14 \pm 1$ & $3 \pm 1$ & $1.0 \pm 0.1$ & $0.25 \pm 0.03$ \\
\hline & 440 & $14 \pm 0$ & $55 \pm 0$ & $28 \pm 0$ & $13 \pm 0$ & $3 \pm 1$ & $1.2 \pm 0.1$ & $0.28 \pm 0.01$ \\
\hline & 340 h.p. & $<\mathrm{dl}$ & $69 \pm 1$ & $19 \pm 1$ & $10 \pm 0$ & $2 \pm 2$ & $1.2 \pm 0.1$ & $0.18 \pm 0.05$ \\
\hline \multirow[t]{7}{*}{$\mathrm{Na}-\mathrm{S}$ ) } & $\mathrm{H}_{2}$ & $43 \pm 6$ & $10 \pm 0$ & $44 \pm 0$ & $4 \pm 0$ & $41 \pm 0$ & $\left.(6.5 \pm 0.3)^{b}\right)$ & $0.77 \pm 0.00$ \\
\hline & 240 & $43 \pm 2$ & $9 \pm 0$ & $46 \pm 1$ & $4 \pm 0$ & $41 \pm 1$ & $9.1 \pm 0.3$ & $0.76 \pm 0.01$ \\
\hline & 290 & $42 \pm 3$ & $8 \pm 0$ & $47 \pm 0$ & $4 \pm 0$ & $41 \pm 0$ & $10.5 \pm 0.6$ & $0.76 \pm 0.01$ \\
\hline & 340 & $41 \pm 8$ & $9 \pm 0$ & $45 \pm 1$ & $4 \pm 0$ & $42 \pm 1$ & $9.3 \pm 0.1$ & $0.78 \pm 0.01$ \\
\hline & 390 & $38 \pm 2$ & $9 \pm 0$ & $48 \pm 1$ & $4 \pm 0$ & $39 \pm 2$ & $10.4 \pm 0.1$ & $0.75 \pm 0.01$ \\
\hline & 440 & $40 \pm 2$ & $8 \pm 0$ & $46 \pm 1$ & $4 \pm 0$ & $41 \pm 1$ & $10.0 \pm 0.2$ & $0.77 \pm 0.01$ \\
\hline & 340 h.p. & $15 \pm 4$ & $18 \pm 0$ & $56 \pm 4$ & $3 \pm 0$ & $23 \pm 4$ & $11.3 \pm 0.2$ & $0.52 \pm 0.01$ \\
\hline
\end{tabular}

FTS conditions: $340^{\circ} \mathrm{C}, 10 \mathrm{bar}, \mathrm{H}_{2}: \mathrm{CO}: \mathrm{He} 2.0: 1.0: 0.33 \mathrm{v} / \mathrm{v}, \approx 5000 \mathrm{~h}^{-1}$.

$<\mathrm{dl}$ - Below detection limit.

a) For all of the $\mathrm{Na}-\mathrm{S}$ catalysts the $\mathrm{MeOH}$ (methanol) selectivity was $\approx 0.4 \%$, and for all of the UP catalysts the $\mathrm{MeOH}$ selectivity was below detection limit.

b) For the duplicate reactor the value is $9.6 \pm 1.0$.

In the following, first the effect of $\mathrm{Na}-\mathrm{S}$ addition on the catalytic performance of the materials under study is discussed. This is followed with remarks on the dependency of the catalytic performance on the catalyst materials themselves i.e. on the FTS reaction starting Fe phase(s). The observed catalytic performance trends are then related to the characterization results.

\subsubsection{The Effect of Na-S Promotion}

In terms of the catalytic activity in the FTS reaction (Table 4.4) and the hydrocarbon product selectivities (Table 4.5), the addition of the combined $\mathrm{Na}-\mathrm{S}$ promotion acts as expected on the basis of the literature. That is, i) the overall CO conversion (and FTY) is increased, ${ }^{29,30,74,75}$ ii) $\mathrm{CH}_{4}$ selectivity is decreased below expectations from the ASF distribution, ${ }^{28,29}$ iii) the olefin content of the hydrocarbon product slate is increased. ${ }^{28,29,37,39}$ The hydrocarbon chain length (i.e. the $\alpha$-value) is increased with the $\mathrm{Na}-\mathrm{S}$ promotion addition, but less than it would be if only $\mathrm{Na}$ was added. ${ }^{30,37,38}$

For the Na-S 340 h.p. catalyst, the Na-S addition shows a mixed effect: increased olefin selectivity but with far lower CO conversion than for the other Na-S catalyst 

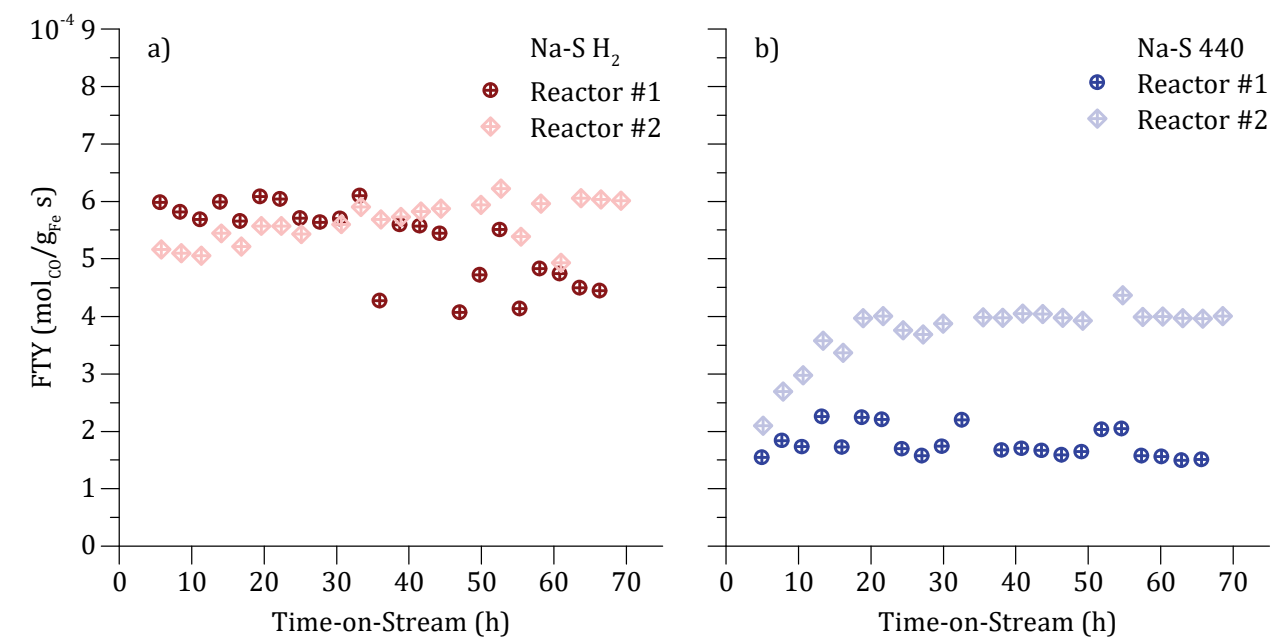

\section{Figure 4.19}

Fe Time Yield (FTY) profiles for two catalyst materials that gave differing catalytic activity profiles between the duplicate reactors. In a) Na-S H , and b) Na-S 440 (Fe-Na-S $/ \alpha-\mathrm{Al}_{2} \mathrm{O}_{3}$ ) catalyst materials. For both, FTY profiles and the associated Fischer-Tropsch Synthesis (FTS) performance data from the Reactor \#2 are discussed in the main text.

materials. Moreover, the $\alpha$-value is lower than for the other catalysts within the Na-S promotion group. The UP 340 h.p. catalyst material also behaves differently in comparison to other catalysts within the UP promotion group. The trend breaking 340 h.p. catalyst materials, for both Na-S and UP catalysts, will be discussed more later on in the section, and ignored for now.

For rest of the Na-S catalyst materials, the $8-10 \% \mathrm{CH}_{4}$ and $48-52 \%$ total $\mathrm{C}_{2}-\mathrm{C}_{4}$ selectivities match well with the "High Na - Low S" promotion $\left(\approx 8 \% \mathrm{CH}_{4}, \approx 50 \% \mathrm{C}_{2}-\mathrm{C}_{4}\right)$ by Botes et al. with experiments done at Sasol. ${ }^{28}$ The total $\mathrm{C}_{2}$ - $\mathrm{C}_{4}$ selectivity is $\approx 10 \%$ higher than is predicted by the ASF distribution with the measured $\mathrm{CH}_{4}$ selectivity. This is the unique feature of the combined Na-S promotion. ${ }^{28}$

The Na-S catalysts also show a marked decrease in Usage Ratios $\mathrm{H}_{2}$ : $\mathrm{CO}$ : from $\approx 1.8$ 2.3 to $\approx 0.7-0.8$ in comparison to the UP catalyst materials (Table 4.4). The $\mathrm{CO}_{2}$ selectivity increases to $38-43 \%$ with the Na-S addition, which is a significant $\mathrm{CO}_{2}$ selectivity increase in comparison to the UP catalysts with $\lesssim 16 \% \mathrm{CO}_{2}$ selectivity (Table 4.5). The decreased Usage Ratios $\mathrm{H}_{2}: \mathrm{CO}$ from the ideal $\approx 2,27,76$ in the FTS reaction are an indicator for additional reactions consuming CO besides the ideal FTS hydrocarbon chain polymerization.

Water-gas Shift (WGS) reaction converts $\mathrm{CO}+\mathrm{H}_{2} \mathrm{O}$ to $\mathrm{H}_{2}+\mathrm{CO}_{2}{ }^{2,32,76-78}$ Above $300^{\circ} \mathrm{C}$, the Boudouard reaction converts $\mathrm{CO}$ to inactive carbon deposits releasing $\mathrm{CO}_{2.13,17-19}$

With the pure CO only carburization in Chapter 3, the rate of carbon deposition on 
the catalyst materials at $340^{\circ} \mathrm{C}$ increased with $\mathrm{Na}-\mathrm{S}$ promotion. Thus, increase in the Boudouard reaction by the Na-S promotion is also possible under the FTS reaction conditions. Solely based on the FTS performance results in this Chapter, the definite Boudouard reaction rate changes due to the $\mathrm{Na}-\mathrm{S}$ promotion cannot be determined.

For the WGS reaction at $340^{\circ} \mathrm{C}$, the $\mathrm{Fe}_{3} \mathrm{O}_{4}$ is the active phase and not the $\mathrm{Fe}$ carbides. ${ }^{62,78-80}$ We cannot observe any $\mathrm{Fe}_{3} \mathrm{O}_{4}$ or other Fe oxides in the FTS catalysts materials (Figure 4.9). Therefore, another phase/site needs to be active for the WGS reaction in the Na-S catalyst materials in order for the WGS reaction to be contributing on the decreased Usage Ratios $\mathrm{H}_{2}$ :CO. Previously, alkali added as $\mathrm{K}_{\text {on }} \mathrm{Al}_{2} \mathrm{O}_{3}$ (ALON), has been observed to provide WGS activity via a proposed potassium formate/carbonate intermediate $\gtrsim 300^{\circ} \mathrm{C}$ mechanism..$^{81,82}$ Noteworthy is that this pathway does not require a presence of Fe or any other transition metal. The presence of alkali is sufficient for the WGS activity.

Formate-like species with K-promoted bulk Fe FTS reaction catalyst materials using in-situ Raman Spectroscopy $\left(200^{\circ} \mathrm{C}, \mathrm{H}_{2}: \mathrm{CO}\right.$ 5:1) have been observed by Zhang and Schrader.83 Zhang and Schrader do not assign their formate-like species directly to a Ksite adsorption, but do observe Raman Shifts within a region 1586-1600 cm-1. Raman Shifts which are possibly assignable to a potassium formate/carbonate phases. Alkalifree Fe-based catalysts produce less $\mathrm{CO}_{2}$ via the WGS reaction, ${ }^{36,84,85}$ than alkali containing Fe-based catalysts. ${ }^{32,85}$ Also, an alkali effect promoting the WGS reaction is present with Ni-based catalysts. ${ }^{86}$ The possibility of K-sites as the directly WGS active sites has been earlier discussed in publication by Arakawa and Bell. ${ }^{87} \mathrm{Na}$ and $\mathrm{K}$ behave in a similar manner regarding their WGS reaction activity promotion. ${ }^{32}$

Thus, for the Na-S catalyst materials the Na-S promotion most likely induces WGS activity, where alkali-Na could provide direct WGS reaction sites. Therefore, the WGS reaction may contribute to the lowered Usage Ratio $\mathrm{H}_{2}$ : $\mathrm{CO}$ with the Na-S promoted catalyst in comparison to the UP catalyst materials (Table 4.4).

The addition of Na-S promotion decreases the CO conversion to hydrocarbons to a fraction of $\approx 0.42-0.47$ in comparison to the respective UP catalysts (Table 4.4). This is in agreement if the Boudouard and/or WGS reactions are more active for the Na-S than UP catalysts converting CO to non-hydrocarbon products. However, precise changes due to the Na-S promotion on the relative FTS/WGS/Boudouard reaction rates cannot be determined from the results in this Chapter.

Nevertheless, despite the decreased selectivity and thus in overall decreased $\mathrm{C}$ efficiency towards the FTS reaction: still, the increased FTY for the Na-S catalyst materials increases the total production of hydrocarbons per unit of time. When comparing the catalysts with the highest median FTY's, i.e. UP 240 versus $\mathrm{Na}-\mathrm{S} \mathrm{H}_{2}$, in their respective promotion groups (Table 4.4), correcting the Na-S $\mathrm{H}_{2}$ FTY value with 

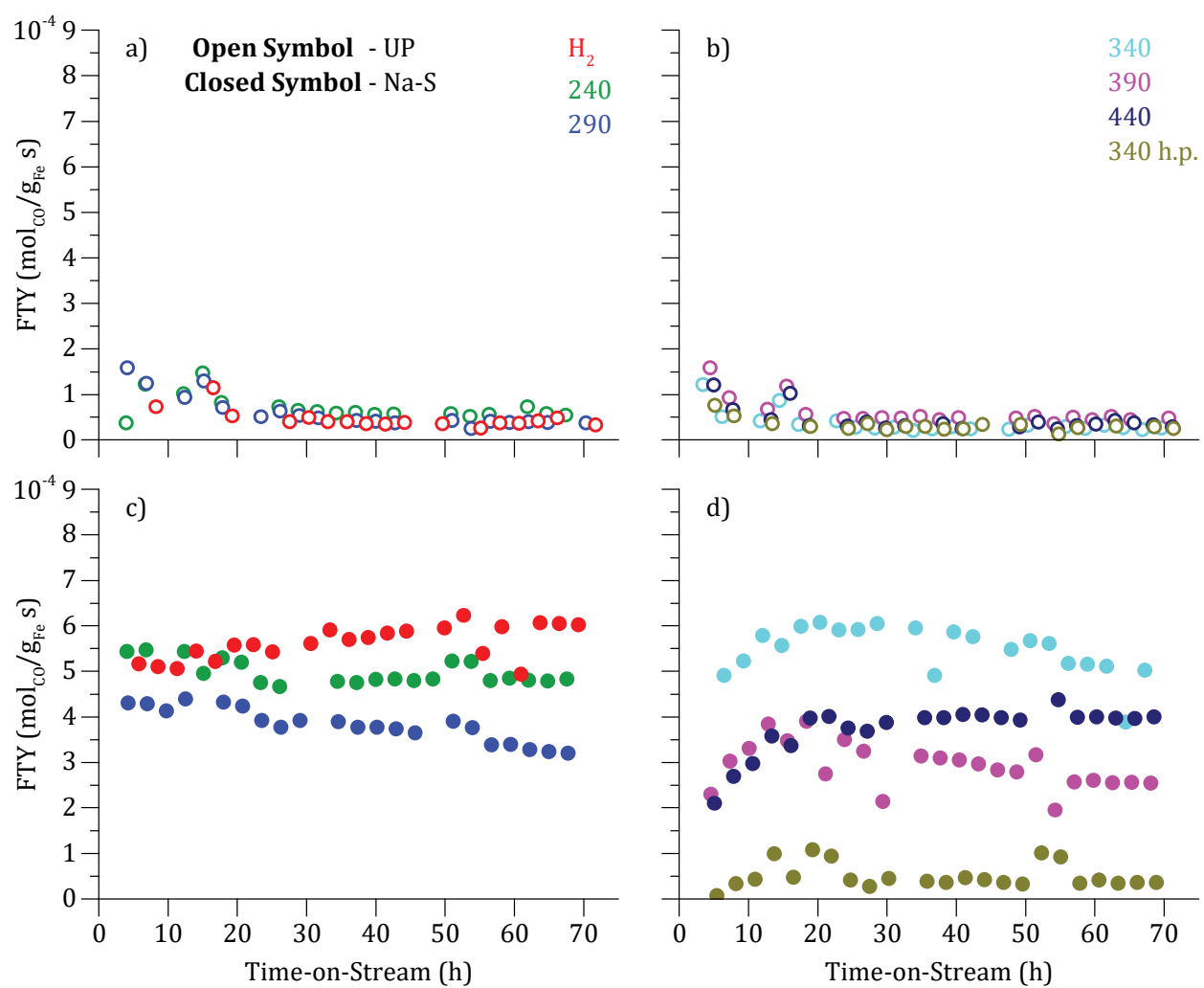

Figure 4.20

The catalytic activity profiles as Fe Time Yields (FTYs) for the UP and Na-S (Fe(-Na-S) $/ \alpha-\mathrm{Al}_{2} \mathrm{O}_{3}$ ) catalyst materials over the $72 \mathrm{~h}$ Fischer-Tropsch Synthesis (FTS) reaction. FTY profiles for a) UP and c) Na-S $\mathrm{H}_{2}, 240$ and 290 catalysts, and for b) UP and d) Na-S 340, 390, 440 and 340 h.p. catalysts. FTS conditions: $340^{\circ} \mathrm{C}, 10$ bar $\mathrm{H}_{2}: \mathrm{CO}: \mathrm{He} 2.0: 1.0: 0.33 \mathrm{v} / \mathrm{v}, \approx 5000 \mathrm{~h}^{-1}$.

the CO to hydrocarbons conversion factor of 0.45 versus the UP 230 catalyst, gives a FTY value of $\approx 2.7 \times 10^{-4} \mathrm{~mol}_{\mathrm{Co}} / \mathrm{g}_{\mathrm{Fe}} \mathrm{S}$. This is $\approx 4.4$ times the FTY of the UP 240 catalyst material.

Consequently, in total, for the production of hydrocarbons per unit of time the Na-S promotion addition is still advantageous with respect to the $\mathrm{CO}$ conversion. That is, if the lost $\mathrm{CO}$ towards non-FTS reaction $\mathrm{C}$ pathways i.e. Boudouard and/or WGS reactions and the associated decrease in the FTS reaction $\mathrm{C}$ efficiency can be tolerated.

\subsubsection{The Effect of Used Catalyst}

The catalytic performance of the tested catalysts is mainly governed by the $\mathrm{Na}-\mathrm{S}$ promotion, regarding both the CO conversion and hydrocarbon selectivities. The applied catalyst material i.e. the combination of Fe phases (Fe carbides $/ \alpha-\mathrm{Fe} / \mathrm{Fe}$ oxides) 
at the start of the catalytic run has a lesser effect on catalyst activity (Table 4.4) and selectivity (Table 4.5). Nevertheless, especially if the Na-S promotion is applied, the FTY profiles can be affected as depending on the used catalyst and its preparation method (Figure 4.20).

First, if the catalyst materials UP and Na-S 340 h.p. with heavy carbon deposits are ignored, for the UP catalysts the median FTY values over ToS 33-72 $\mathrm{h}$ are related as:

$$
\mathrm{UP} 340 \lesssim \mathrm{UPH}_{2} \approx \mathrm{UP} 290 \approx \mathrm{UP} 440 \lesssim \mathrm{UP} 390 \lesssim \mathrm{UP} 240
$$

while for the Na-S catalyst materials, the relation follows:

$$
\text { Na-S } 390<\text { Na-S } 440 \lesssim \text { Na-S } 290<\text { Na-S } 240<\text { Na-S } 340<\text { Na-S H2. }
$$

No clear trends emergence of which of the used catalysts with different starting Fe phases leads to the highest FTY values over the near steady ToS range of 33-72 $\mathrm{h}$. That is, no clear correlation between the initial Fe phase(s) and steady FTY activity exists for the studied catalyst materials.

Mogorosi et al. have estimated for a " $\varepsilon$-carbide" phase (" $\varepsilon-\mathrm{Fe}_{2} \mathrm{C}$ ") being about $+25 \%$ more active per carbide surface area in the FTS reaction $\left(250^{\circ} \mathrm{C}, 20\right.$ bar, $\left.\mathrm{H}_{2}: \mathrm{CO} 2: 1\right)$ than the $\chi-\mathrm{Fe}_{5} \mathrm{C}_{2}$ phase. $^{7}$ The Na-S 240 FTS catalyst material contains the highest amount of the $\eta-\mathrm{Fe}_{2} \mathrm{C}$ phase (a " $\varepsilon$-carbide", Chapter 2) with $53 \%$ of $\eta-\mathrm{Fe}_{2} \mathrm{C}$ (Figure 4.9b). However, lower median FTY value than for the Na-S 340 FTS and Na-S H2 FTS catalysts with $100 \%$ and $73 \%$ of the $\chi-\mathrm{Fe}_{5} \mathrm{C}_{2}$ phase, respectively, is obtained. Thus, for the studied catalyst materials: a high $\eta-\mathrm{Fe}_{2} \mathrm{C}$ content does not imply a high FTY value or a higher value than for $\chi-\mathrm{Fe}_{5} \mathrm{C}_{2}$-rich catalysts.

In an opposing view, a " $\varepsilon$-carbide" phase (" $\mathrm{Fe}_{2.2} \mathrm{C}$ ") has also been associated to a deactivating FTS reaction catalyst with lower intrinsic FTS reaction activity than a catalyst with higher $\chi-\mathrm{Fe}_{5} \mathrm{C}_{2}$ phase content. ${ }^{8}$ For the Na-S 240 FTS catalyst material, the presence of the $\eta-\mathrm{Fe}_{2} \mathrm{C}$ phase does not either lead to deactivation (Figure 4.20c). As Pendyala et al. also discuss, simply associating a bulk Fe carbide phase to the FTS reaction's CO conversion activity can be misleading. In Pendyala et al.'s case the lowering CO conversion and bulk phase transformation to a " $\varepsilon$-carbide" phase (" $F e_{2.2} \mathrm{C}$ ") also coincided with heavy carbon deposition, which likely caused the catalyst's deactivation in the FTS reaction.

The $\theta-\mathrm{Fe}_{3} \mathrm{C}$ phase has been proposed to be related to the FTS catalyst deactivation. ${ }^{9}$ For the UP 440 FTS catalyst material the presence of $64 \%$ of the $\theta-\mathrm{Fe}_{3} \mathrm{C}$ phase does not lead to a lower FTY than for e.g. UP H2 FTS catalyst material (Table 4.4) with $100 \% \chi$ $\mathrm{Fe}_{5} \mathrm{C}_{2}$ phase content (Figure 4.9a). That is, the presence of $\theta-\mathrm{Fe}_{3} \mathrm{C}$ does not imply 
catalyst deactivation or low FTY for the studied catalyst materials.

Thus, according to the results obtained in this Chapter and as noted also in literature:8,14-16 a single bulk Fe carbide phase in the FTS reaction does not correlate with the FTY. However, as will be discussed shortly, the different Fe carbide phases and their subtypes can cautiously be associated to differences in hydrocarbon selectivities (Table 4.3).

A noteworthy detail to highlight is that despite the Fe being detached from the support material $\alpha-\mathrm{Al}_{2} \mathrm{O}_{3}$, either before; as for the 340,390 and 440 catalysts (Chapter 3), or during the FTS reaction; as for the $\mathrm{H}_{2}, 240$ and 290 catalyst materials (Figure 4.2 and Figure 4.3). Neither were significant quantities of elemental Fe lost from the catalyst materials in the FTS reaction (Figure 4.9c). Furthermore, the FTY profiles during the FTS reaction for the UP and Na-S FTS catalyst materials are relatively stable (Figure 4.20). Thus, direct Fe interaction with the $\alpha-\mathrm{Al}_{2} \mathrm{O}_{3}$ support in the FTS reaction is not a prerequisite for a FTS stable catalyst. For comparison, Fe encapsulated to carbon via Metal Organic Framework (MOF) pyrolysis has also been shown to be a stable and active Fe-based FTS reaction catalyst. ${ }^{36,88}$

Some further effects warranting a discussion on the catalytic performance due to the used catalyst material can be mentioned as: i) within the UP promotion group the catalysts start active but lose activity within the first $\approx 20 \mathrm{~h}$ ToS (Figure 4.20a-b), ii) within the Na-S promotion group the catalysts either show steady (Figure 4.20c) or iii) increasing FTY within the first $\approx 20 \mathrm{~h}$ ToS (Figure 4.20d). With regard to the product selectivities (Table 4.5): iv) within the UP promotion group catalysts 390 and 440 show a modest $\approx+5 \%$ increase towards $\mathrm{C}_{2}-\mathrm{C}_{4}$ paraffin formation with decreased $\mathrm{C}_{2} \mathrm{H}_{4} / \mathrm{C}_{2} \mathrm{H}_{6}$ ratio and v) the 340 h.p. catalysts have differing product selectivities within the UP and $\mathrm{Na}-\mathrm{S}$ promotion groups in comparison to the other catalysts in the respective promotion groups.

No definite, clear reasoning for the UP catalyst materials' FTY profiles, for starting initially active and followed by deactivation, can be given here. In general, variable FTY profiles depending on the initial state of the catalysts at the start of the FTS reaction, have been observed. ${ }^{35,89}$ Assigning clear cut reasons for the FTY profiles, is in general challenging due to the intertwined processes in the FTS reaction. ${ }^{2,77}$

Nevertheless, few comments can be made. Regardless of the UP starting catalysts i.e. the initial Fe phases (Figure 4.9a), amount of initial carbon deposited on the catalyst or the initial Fe particle diameters (Chapter 3): all of the UP catalyst materials have decreasing FTY profiles with $<20 \mathrm{~h}$ ToS. The FTYs' are roughly halved from the start before reaching a steady state $>20 \mathrm{~h}$ ToS in the FTS reaction.

Fe particle diameter growth has been given as a one possible reason for the initially decreasing FTY profiles. ${ }^{20}$ That is, the gradual sintering of the Fe particles results in 
decreased active Fe surface area available for catalyzing the FTS reaction, thus decreasing the FTY's gradually. For longer reaction $(\approx 600 \mathrm{~h} \mathrm{ToS})$ times, continuous Fe particle diameter growth has been observed by Sarkar et al. along with catalyst deactivation. However, the catalyst deactivation was associated by Sarkar et al. to be due to $\mathrm{Fe}$ carbide reoxidation to $\mathrm{Fe}_{3} \mathrm{O}_{4}$, not to the $\mathrm{Fe}$ sintering. ${ }^{21}$

For the UP catalyst materials, the Fe particle diameters do not significantly change in the FTS reaction (Figure 4.4e-f). Therefore, the Fe particle sintering is not a plausible explanation for the initially decreased FTY's for the studied UP catalyst materials. Furthermore, no elemental Fe is lost during the FTS reaction (Figure 4.9c) i.e. loss of the FTS active Fe does not either explain the decreasing FTYs within the first $\approx 20 \mathrm{~h} \mathrm{ToS}$. Neither, no Fe reoxidation to $\mathrm{Fe}_{3} \mathrm{O}_{4}$ is observed (Figure 4.9a). The possibility for the catalyst deactivation due to carbon deposition cannot be devised from the results in this Chapter.

For the Na-S catalyst materials the FTY profiles either stay nearly steady; as for the $\mathrm{H}_{2}, 240$ and 290 catalysts (Figure 4.20c), or; increase for the 340, 390 and 440 catalysts within the first $\$ 20 \mathrm{~h}$ ToS (Figure 4.20d). This behavior could be associated on the carbon deposited on the catalysts during the initial $\mathrm{CO}$ carburization for preparing the catalyst materials (Chapter 3). The Na-S H2, 240 and 290 catalysts do not, but the Na-S 340,390 and 440 catalyst materials do have initial deposits of carbon from the CO carburization. We therefore associate the initially increasing FTY profiles for the Na-S 340,390 and 440 catalyst materials to the initial changes in the carbon deposit overlayer. Perhaps, the carbon overlayer gradually exposes more of the Fe carbide surface for the FTS reaction. $\mathrm{H}_{2}$ may hydrogenate and remove carbon deposits $>350^{\circ} \mathrm{C} .{ }^{13}$ Very high amount of initial carbon deposits can also be used to explain the low FTY value for the Na-S 340 h.p. catalyst material in comparison to the rest of the catalysts in the Na-S promotion group (Table 4.4).

Despite the increased Fe particle mean diameters after the $72 \mathrm{~h}$ FTS reaction (Figure 4.4), the Na-S 240 (increase of $+28 \%$ ) and Na-S 340 ( $+48 \%$ ) catalyst materials do not show significant catalyst deactivation in their FTY profiles (Figure 4.20c-d). The result is unlike as has been observed for the Fe-Na-S/CNT (Carbon Nanotube) catalyst materials. ${ }^{20}$ In the study it was concluded that the Na-S promotion lead to a significant Fe particle growth and to decreasing FTY's and thus to catalyst deactivation. The results obtained within this study do not agree with this conclusion.

\subsubsection{The Role of the Fe Carbides and their Subtypes in Fischer-Tropsch Synthesis Hydrocarbon Selectivity}

With regard to the roles of the different Fe carbides (Figure 4.9) and their subtypes (Table 4.3) in the FTS reaction's hydrocarbon selectivity, some conclusions can be 
made. Such as: i) the presence of the $\theta-\mathrm{Fe}_{3} \mathrm{C}$ phase can be associated to increased hydrocarbon paraffin selectivity (UP 390 FTS and UP 440 FTS catalysts), ii) the $\eta-\mathrm{Fe}_{2} \mathrm{C}$ and $\chi-\mathrm{Fe}_{5} \mathrm{C}_{2}$ Type II's with Na-S promotion can be associated to increased hydrocarbon olefin selectivity and decreased $\mathrm{CH}_{4}$ with increased $\alpha$-value (Na-S FTS) and, iii) possibly, $\chi-\mathrm{Fe}_{5} \mathrm{C}_{2}$ Type III can be associated to decreased $\alpha$-value (UP and Na-S 340 h.p. FTS), and with Na-S promotion also to increased olefin selectivity (Na-S 340 h.p. FTS).

The first conclusion is rather straightforward, as for the UP 390 FTS and UP 440 FTS catalysts the $\theta-\mathrm{Fe}_{3} \mathrm{C}$ phase is relatively stable and is present in significant quantities in comparison to the other UP catalysts (Figure $4.9 a$ ). That is, the $\approx+5 \%$ increased $\mathrm{C}_{2}-\mathrm{C}_{4}$ paraffin selectivity and the decreased $\mathrm{C}_{2} \mathrm{H}_{4} / \mathrm{C}_{2} \mathrm{H}_{6}$ ratio can be associated to the $\theta-\mathrm{Fe}_{3} \mathrm{C}$ phase (Table 4.5). The Na-S 390 and Na-S 440 catalysts, for which the $\theta-\mathrm{Fe}_{3} \mathrm{C}$ phase is not stable and is converted to $\chi-\mathrm{Fe}_{5} \mathrm{C}_{2}$ Type II during the FTS reaction (Figure 4.9b), do not show any deviations in the paraffin selectivities within the Na-S promotion group.

The result for the $\theta-\mathrm{Fe}_{3} \mathrm{C}$ phase is in disagreement with earlier data by Shultz et al, ${ }^{4,10}$ where Hägg carbide $\left(\chi-\mathrm{Fe}_{5} \mathrm{C}_{2}\right)$ and cementite $\left(\theta-\mathrm{Fe}_{3} \mathrm{C}\right)$ were the initial phases in the used FTS reaction catalyst materials. No increase in the paraffin selectivity (i.e. decrease in bromine number) can be observed in their selectivity data for the $\theta-\mathrm{Fe}_{3} \mathrm{C}$ phase as the catalyst. ${ }^{10}$ As a bulk Fe catalyst (Fe: $\mathrm{MgO}: \mathrm{K}_{2} \mathrm{O}: \mathrm{SiO}_{2}: \mathrm{Cr}_{2} \mathrm{O}_{3}$ ) containing $\mathrm{K}$ was used for the studies, it might be suspected that their $\theta-\mathrm{Fe}_{3} \mathrm{C}$ phase was converted towards $\chi-\mathrm{Fe}_{5} \mathrm{C}_{2}$ during the FTS reaction, akin to the results for the Na-S FTS catalyst materials. This would explain the lack of selectivity effect from the $\theta-\mathrm{Fe}_{3} \mathrm{C}$ phase in the results by Shultz et al..

Both $\eta-\mathrm{Fe}_{2} \mathrm{C}$ and $\chi-\mathrm{Fe}_{5} \mathrm{C}_{2}$ Type II's in their respective Na-S FTS catalyst materials can be associated to increased olefin and decreased $\mathrm{CH}_{4}$ selectivity, as well as to increased $\alpha$-value. Interestingly, also the UP 240 FTS and UP 290 FTS catalysts showed the presence of $\eta-\mathrm{Fe}_{2} \mathrm{C}$ and $\chi-\mathrm{Fe}_{5} \mathrm{C}_{2}$ Type II's. However, without the mentioned changes in the hydrocarbon selectivities in respect to the other UP FTS catalysts with the $\chi-\mathrm{Fe}_{5} \mathrm{C}_{2}$ Type I. That is, without the Na-S promotion the $\chi-\mathrm{Fe}_{5} \mathrm{C}_{2}$ Type I and $\chi-\mathrm{Fe}_{5} \mathrm{C}_{2}$ Type II do not seem to differ in their FTS reaction hydrocarbon selectivity. Thus, the $\chi-\mathrm{Fe}_{5} \mathrm{C}_{2}$ Type II lattice may not by itself lead to differences in the hydrocarbon selectivities in comparison to the Type I, but the Type II might be the favored lattice in the presence of Na-S promotion. Further studies, e.g. akin to the Density Functional Theory (DFT) calculations by Xie et al., ${ }^{40}$ on how the Na-S promotion occupies the surface and the bulk positions of the different $\chi-\mathrm{Fe}_{5} \mathrm{C}_{2}$ sublattice types would be of interest.

\subsubsection{Behavior of the 340 h.p. Catalysts}

The final item of interest is the catalytic behavior of the UP and Na-S 340 h.p. catalyst materials in the FTS reaction, as they break the general hydrocarbon selectivity trends 
in their respective promotion groups (Table 4.5). Both show a decreased $\alpha$-value; the UP 340 h.p. catalyst shows a $\mathrm{C}_{2} \mathrm{H}_{4} / \mathrm{C}_{2} \mathrm{H}_{6}$ ratio in-line with the UP 390 and UP 440 catalysts; and the Na-S 340 h.p. catalysts shows slightly increased $\mathrm{C}_{2} \mathrm{H}_{4} / \mathrm{C}_{2} \mathrm{H}_{6}$ ratio in comparison to the other Na-S FTS catalysts (Table 4.5).

Bukur et al. have observed increased olefin selectivity with a bulk Rührchemie (Fe:Cu:K:SiO 2 ) FTS catalyst material pretreated with $\mathrm{CO}\left(\right.$ at $280^{\circ} \mathrm{C}$ ) or $\mathrm{H}_{2}: \mathrm{CO}\left(\right.$ at $310^{\circ} \mathrm{C}$ ) without a prior $\mathrm{H}_{2}$ reduction step, in comparison to the catalysts reduced with $\mathrm{H}_{2}$ prior to the FTS reaction. Also, Bukur et al.'s selectivity data suggests increased $\alpha$-value (decreased $\mathrm{CH}_{4}$, increased $\mathrm{C}_{12}$ ) for the $\mathrm{CO}$ and $\mathrm{H}_{2}$ : $\mathrm{CO}$ pretreated catalysts. ${ }^{35}$

The increased olefin selectivity observed by Bukur et al. matches the results with the alkali containing catalyst Na-S 340 h.p., which has been directly carburized with CO without first reducing the as-synthesized Fe oxide $\left(\alpha-\mathrm{Fe}_{2} \mathrm{O}_{3}\right)$ with $\mathrm{H}_{2}$ (Table 4.1). For all of the 340 h.p. catalyst materials, the $\chi-\mathrm{Fe}_{5} \mathrm{C}_{2}$ Type III is majority Fe carbide (Figure 4.9a-b and Table 4.3). Thus, when the $\chi-\mathrm{Fe}_{5} \mathrm{C}_{2}$ Type III is combined with the $\mathrm{Na}-\mathrm{S}$ promotion, the hydrocarbon selectivity might be steered towards olefin formation more effectively than with the Na-S promotion in combination with the $\chi-\mathrm{Fe}_{5} \mathrm{C}_{2}$ Type II. Especially, the highest $\mathrm{C}_{2} \mathrm{H}_{4} / \mathrm{C}_{2} \mathrm{H}_{6}$ ratio of $11.3 \pm 0.2$ is achieved with the Na-S promoted $\chi-\mathrm{Fe}_{5} \mathrm{C}_{2}$ Type III in the Na-S 340 h.p. catalysts. This value is higher than with any of the Na-S promoted $\chi-\mathrm{Fe}_{5} \mathrm{C}_{2}$ Type II's in the other Na-S catalyst materials (Table 4.5).

Although, this conclusion needs to be taken with care for the time being. In comparison to the results by Bukur et al.: the subtype of the $\chi-\mathrm{Fe}_{5} \mathrm{C}_{2}$ phase cannot be deduced from the characterization results on the Bukur et al's FTS catalyst material,34 nor do the Bukur et al's $\alpha$-value changes match the changes for UP or Na-S 340 h.p. catalysts. $^{35}$ Also, CO conversions are very much higher for Bukur et al's catalyst (52-

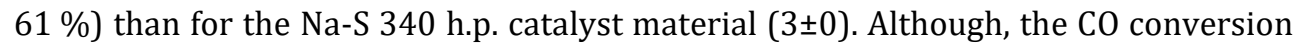
levels should not affect the hydrocarbon selectivities directly. ${ }^{27,32}$ Furthermore, it would be expected that the heavy initial carbon deposits in the Na-S 340 h.p. catalyst (Chapter 3) block the available Fe surface, reducing possibility for secondary reactions, and thus possibly increasing the olefin content. ${ }^{26,27}$ However, the UP 340 h.p. catalyst shows decreased olefin selectivity within the UP promotion group. This, thus, going against the blocking of surface via secondary reactions argument.

For the UP and Na-S FTS 340 h.p. catalyst materials the minority $\theta-\mathrm{Fe}_{7} \mathrm{C}_{3}$ phase (UP $19 \%$, Na-S $18 \%$ ) (Figure 4.9a-b), is unlikely to any play major a role in the FTS reaction catalysis performance results, and therefore is left uncommented. The results, nevertheless, can be taken as a demonstration, that the $\theta-\mathrm{Fe}_{7} \mathrm{C}_{3}$ phase may form under relatively mild conditions and is stable in the FTS reaction, without a need for industrial FTS reactors. ${ }^{13}$ More studies on the rather understudied $\theta-\mathrm{Fe}_{7} \mathrm{C}_{3}$ phase as the major FTS catalysis phase, with and without $\mathrm{Na}(-\mathrm{S})$ promotion, should be conducted in the future. 


\subsection{CONCLUSIONS}

Supported $\mathrm{Fe}(-\mathrm{Na}-\mathrm{S}) / \alpha-\mathrm{Al}_{2} \mathrm{O}_{3}$ catalyst materials, with or without $\mathrm{Na}-\mathrm{S}$ promotion, prepared via CO carburization at various temperatures $\left(240-440^{\circ} \mathrm{C}\right)$ or via $\mathrm{H}_{2}$ reduction in Chapter 3 were tested in $72 \mathrm{~h}$ FTS reaction $\left(340^{\circ} \mathrm{C}, 10\right.$ bar $\mathrm{H}_{2}: \mathrm{CO}: \mathrm{He}$ 2.0:1.0:0.33 v/v, $\mathrm{GHSV} \approx 5000 \mathrm{~h}^{-1}$ ). Ex-situ measured XRPD patterns collected after the FTS reaction runs were quantified using R-QPA. The Fe carbide phases of, as identified in Chapter 2, $\eta$ $\mathrm{Fe}_{2} \mathrm{C}$ Pnnm (58), $\chi-\mathrm{Fe}_{5} \mathrm{C}_{2} C 2 / c$ (15), $\theta-\mathrm{Fe}_{3} \mathrm{C}$ Pnma (62) and $\theta-\mathrm{Fe}_{7} \mathrm{C}_{3}$ Pnma (62) were observed in the studied catalyst materials. The phase $\varepsilon-\mathrm{Fe}_{3} \mathrm{C} \mathrm{P}_{3} 22$ (182) was not stable in the FTS reaction. No other Fe phases besides the Fe carbides, such as Fe oxides, were observed in the catalyst materials after the FTS reaction.

In relation to the formation of the Fe carbides, addition of $\mathrm{Na}-\mathrm{S}$ promotion: i) stabilizes the $\eta-\mathrm{Fe}_{2} \mathrm{C}$ phase, and ii) destabilizes the $\theta-\mathrm{Fe}_{3} \mathrm{C}$ phase, under the used FTS reaction conditions.

Furthermore, the Na-S promotion addition causes a maximum $\approx 0.02 \AA$ anisotropic changes in the crystal lattice parameters for the $\eta-\mathrm{Fe}_{2} \mathrm{C}$ and $\chi-\mathrm{Fe}_{5} \mathrm{C}_{2}$ phases after the FTS reaction. Observed lattice parameter changes are within the limits what might be expected if $\mathrm{Na}$ forms a solid solution with the Fe carbides. However, as exceptions: unpromoted catalyst materials prepared by $\mathrm{CO}$ carburization at $240^{\circ} \mathrm{C}$ or $290^{\circ} \mathrm{C}$, showed similar lattice shifts for the $\chi-\mathrm{Fe}_{5} \mathrm{C}_{2}$ phase after the FTS reaction, as would be expected with the Na-S promotion. Possible $\mathrm{Na}(-\mathrm{S})$ contamination could be ruled out based on the catalyst materials' catalytic performance. Whether similar lattice distortions due to the $\mathrm{Na}-\mathrm{S}$ promotion exists for the $\theta-\mathrm{Fe}_{3} \mathrm{C}$ and $\theta-\mathrm{Fe}_{7} \mathrm{C}_{3}$ phases, could not be conclusively determined. The distinctively distorted $\chi-\mathrm{Fe}_{5} \mathrm{C}_{2}$ (h.p.) phase (i.e. Type III) prepared via $\mathrm{CO}$ carburization of Fe oxide $\left(\alpha-\mathrm{Fe}_{2} \mathrm{O}_{3}\right)$ at high-pressure, prevailed unchanged in the FTS reaction.

As based on the observed lattice parameters, the $\eta-\mathrm{Fe}_{2} \mathrm{C}$ and $\chi-\mathrm{Fe}_{5} \mathrm{C}_{2}$ phases can be divided into subtypes with their typical formation conditions as:

$\chi-\mathrm{Fe}_{5} \mathrm{C}_{2}$

Type I After CO carburization, or FTS without Na-S promotion,

Type II After FTS with Na-S promotion,

Type III After CO carburization of Fe oxide $\left(\alpha-\mathrm{Fe}_{2} \mathrm{O}_{3}\right)$, the subtype is stable in FTS regardless of the Na-S promotion,

$\eta-\mathrm{Fe}_{2} \mathrm{C}$

Type I After CO carburization, or FTS without Na-S promotion,

Type II After FTS with Na-S promotion,

Type III After martensite tempering at $120^{\circ} \mathrm{C}$. Not observed in FTS. 
The $\eta-\mathrm{Fe}_{2} \mathrm{C}$ Type III is not observed in this study but is included from literature for completeness.

In the terms of FTS reaction performance, the Na-S promotion has the general effects of i) increased $\mathrm{CO}$ conversion, ii) $\approx+10 \%$ increased $\mathrm{C}_{2}-\mathrm{C}_{4}$ selectivity with significantly suppressed $\mathrm{CH}_{4}$ selectivity below the ASF expectations, iii) increased olefin content and iv) increased $\alpha$-value, in comparison to unpromoted catalyst materials. As adverse effects for the FTS reaction, with the Na-S promotion: i) $\mathrm{CO}_{2}$ selectivity increases from $\lesssim 16 \%$ to $\approx 38-43 \%$ and ii) fraction of $\mathrm{CO}$ converted to hydrocarbons drops to $\approx 0.42$ 0.47 in relation to the unpromoted catalyst materials. Also, the Usage Ratios $\mathrm{H}_{2}: \mathrm{CO}$ decrease from $\approx 1.8-2.3$ to $\approx 0.7-0.8$ with the added Na-S promotion as a sign of increased, $\mathrm{CO}_{2}$ releasing, Boudouard and/or WGS reactions consuming the input $\mathrm{CO}$. Despite the $\mathrm{CO}$ diverted to non-FTS reaction $\mathrm{C}$ pathways, $\approx 4.4$ times more hydrocarbons are still converted from $\mathrm{CO}$ per unit of time, when comparing the catalysts with the highest FTY's with and without Na-S promotion. This, while taking into account the decreased fraction of $\mathrm{CO}$ to hydrocarbons conversion for the Na-S promoted catalysts.

No WGS reaction active $\mathrm{Fe}_{3} \mathrm{O}_{4}$, or other $\mathrm{Fe}$ oxides, were observed in any of the catalyst materials after the FTS reaction. The WGS reaction may contribute to the observed decrease in the Usage Ratio $\mathrm{H}_{2}$ : $\mathrm{CO}$ by $\mathrm{Na}(-\mathrm{S})$ promotion directly providing active sites for the WGS reaction. This was explained with observations from literature in combination with the catalytic performance data.

Under the applied FTS reaction conditions, for all of the studied catalyst materials the $\mathrm{Fe}$ is encapsulated in carbon and detached from the support $\alpha-\mathrm{Al}_{2} \mathrm{O}_{3}$, as observed with TEM. This, however, did not affect the catalyst materials' FTY profiles versus ToS in the FTS reaction. No significant amounts of elemental Fe was lost during the $72 \mathrm{~h}$ FTS reaction, as quantified with R-QPA. Neither, no connection between the Fe particle mean diameter changes and FTY profiles were observed e.g. increasing Fe particle mean diameters did not correlate with decreases in the catalyst materials FTY profiles.

Fe crystallite mean diameters for each phase (R-QPA) in comparison to the Fe particle mean diameters (TEM) did not show any common trends. This suggests that a single Fe particle is not a single Fe phase crystallite.

No direct correlation between any of the bulk Fe carbide phases and the catalyst materials' FTYs' could be observed. However, hydrocarbon selectivity-wise cautious associations can be made as: i) the $\theta-\mathrm{Fe}_{3} \mathrm{C}$ phase mildly $(\approx+5 \%)$ increases hydrocarbon product slate paraffin content, ii) with $\mathrm{Na}-\mathrm{S}$ promotion, $\eta-\mathrm{Fe}_{2} \mathrm{C}$ Type II and $\chi-\mathrm{Fe}_{5} \mathrm{C}_{2}$ Type II are present in high olefin and low $\mathrm{CH}_{4}$ selective FTS reaction catalyst materials, and iii) $\chi-\mathrm{Fe}_{5} \mathrm{C}_{2}$ Type III, when combined with $\mathrm{Na}-\mathrm{S}$ promotion, might increase the olefin selectivity more than the $\chi-\mathrm{Fe}_{5} \mathrm{C}_{2}$ Type II with Na-S promotion does. No conclusions on the role of the $\theta-\mathrm{Fe}_{7} \mathrm{C}_{3}$ phase in the FTS reaction could be drawn, due to the minor phase 
quantities present in the studied FTS catalyst materials.

For further research: i) the general connection between, but especially for the $\chi$ $\mathrm{Fe}_{5} \mathrm{C}_{2}$ phase, crystal lattice subtypes and Na-S promotion to hydrocarbon selectivities, and also ii) investigating the understudied role of the $\theta-\mathrm{Fe}_{7} \mathrm{C}_{3}$ phase as a major phase for the FTS reaction catalysis, with and without Na-S promotion, are interesting topics for further research.

\subsection{ACKNOWLEDGEMENTS}

J. D. (Hans) Meeldijk and C. W. T. M. Schneijdenberg (Universiteit Utrecht, UU) are thanked for their guidance in taking the TEM images.

\subsection{REFERENCES}

[1] de Smit, E., Cinquini, F., Beale, A. M., Safonova, O. V., van Beek, W., Sautet, P. and Weckhuysen, B. M., "Stability and Reactivity of $\epsilon-\chi-\theta$ Iron Carbide Catalyst Phases in Fischer-Tropsch Synthesis: Controlling $\mu_{c}$ ", J. Am. Chem. Soc. 132, 14928-14941 (2010).

[2] de Smit, E. and Weckhuysen, B. M., "The renaissance of iron-based Fischer-Tropsch synthesis: on the multifaceted catalyst deactivation behaviour", Chem. Soc. Rev. 37, 2758-2781 (2008).

[3] Anderson, R. B., Hofer, L. J. E., Cohn, E. M. and Seligman, B., "Studies of the Fischer-Tropsch Synthesis. IX. Phase Changes of Iron Catalysts in the Synthesis", J. Am. Chem. Soc. 73, 944-946 (1951).

[4] Shultz, J. F., Hall, W. K., Seligman, B. and Anderson, R. B., "Studies of the Fischer-Tropsch Synthesis. XIV. Hägg Carbide as Catalysts", J. Am. Chem. Soc. 77, 213-221 (1955).

[5] Le Caër, G., Dubois, J. M., Pijolat, M., Perrichon, V. and Bussière, P., "Characterization by Moessbauer Spectroscopy of Iron Carbides Formed by Fischer-Tropsch Synthesis", J. Phys. Chem. 86, 4799-4808 (1982).

[6] Niemantsverdriet, J. W., van der Kraan, A. M., van Dijk, W. L. and van der Baan, H. S., "Behavior of Metallic Iron Catalysts during Fischer-Tropsch Synthesis Studied with Mössbauer Spectroscopy, X-ray Diffraction, Carbon Content Determination, and Reaction Kinetic Measurements", J. Phys. Chem. 84, 3363-3370 (1980).

[7] Mogorosi, R. P., Fischer, N., Claeys, M. and van Steen, E., "Strong-metal-support interaction by molecular design: Fe-silicate interactions in Fischer-Tropsch catalysts", J. Catal. 289, 140-150 (2012).

[8] Pendyala, V. R. R., Graham, U. M., Jacobs, G., Hamdeh, H. H. and Davis, B. H., "Fischer-Tropsch Synthesis: Deactivation as a Function of Potassium Promoter Loading for Precipitated Iron Catalyst", Catal. Lett. 144, 1704-1716 (2014).

[9] de Smit, E., Beale, A. M., Nikitenko, S. and Weckhuysen, B. M., "Local and long range order in promoted iron-based Fischer-Tropsch catalysts: A combined in situ X-ray absorption spectroscopy/wide angle Xray scattering study", J. Catal. 262, 244-256 (2009).

[10] Shultz, J. F., Hall, W. K., Dubs, T. A. and Anderson, R. B., "Studies of the Fischer-Tropsch Synthesis. XV. Cementite as Catalysts", J. Am. Chem. Soc. 78, 282-285 (1956).

[11] Datye, A. K., Jin, Y., Mansker, L. D., Motjope, R. T., Dlamini, T. H. and Coville, N. J., "The nature of the active phase in iron Fischer-Tropsch catalysts" in Studies in Surface Science and Catalysis 130,11391144 (2000). 
[12] Mansker, L. D., Jin, Y., Bukur, D. B. and Datye, A. K., "Characterization of slurry phase iron catalysts for Fischer-Tropsch synthesis", Appl. Catal. A Gen. 186, 277-296 (1999).

[13] Dry, M. E., "The Fischer-Tropsch Synthesis" in Catalysis - Science and Technology (eds. Anderson, J. R. \& Boudart, M.) 1, 159-256 (Springer-Verlag, Berlin, 1981).

[14] Sirimanothan, N., Hamdeh, H. H., Zhang, Y. and Davis, B. H., "Fischer-Trospch Synthesis; Changes in Phase and Activity during Use", Catal. Lett. 82, 181-191 (2002).

[15] O’Brien, R. J., Xu, L., Milburn, D. R., Li, Y.-X., Klabunde, K. J. and Davis, B. H., "Fischer-Tropsch synthesis: Impact of potassium and zirconium promoters on the activity and structure of an ultrafine iron oxide catalyst", Top. Catal. 2, 1-15 (1995).

[16] Rao, K. R. P. M., Huggins, F. E., Huffman, G. P., Gormley, R. J., O’Brien, R. J. and Davis, B. H., "Mössbauer Study of Iron Fischer-Tropsch Catalysts during Activation and Synthesis", Energy \& Fuels 10, 546-551 (1996).

[17] Dry, M. E., Shingles, T. and van H. Botha, C. S., "Factors influencing the formation of carbon on iron Fischer-Tropsch catalysts I. The influence of promoters", J. Catal. 17, 341-346 (1970).

[18] Dry, M. E., Shingles, T., Boshoff, L. J. and van H. Botha, C. S., "Factors influencing the formation of carbon on iron Fischer-Tropsch catalysts. II. The effect of temperature and of gases and vapors present during Fischer-Tropsch synthesis", J. Catal. 17, 347-354 (1970).

[19] Cohn, E. M., Bean, E. H., Mentser, M., Hofer, L. J. E., Pontello, A., Peebles, W. C. and Jack, K. H., "The carburization of iron oxide with carbon monoxide: Modifications of Hägg iron carbide", J. Appl. Chem. 5, 418-425 (1955).

[20] Xie, J., Torres Galvis, H. M., Koeken, A. C. J., Kirilin, A., Dugulan, A. I., Ruitenbeek, M. and de Jong, K. P., "Size and Promoter Effects on Stability of Carbon-Nanofiber-Supported Iron-Based Fischer-Tropsch Catalysts", ACS Catal. 6, 4017-4024 (2016).

[21] Sarkar, A., Seth, D., Dozier, A. K., Neathery, J. K., Hamdeh, H. H. and Davis, B. H., "Fischer-Tropsch Synthesis: Morphology, Phase Transformation and Particle Size Growth of Nano-scale Particles", Catal. Lett. 117, 1-17 (2007).

[22] An, X., Wu, B., Wan, H.-J., Li, T.-Z., Tao, Z.-C., Xiang, H.-W. and Li, Y.-W., "Comparative study of ironbased Fischer-Tropsch synthesis catalyst promoted with potassium or sodium", Catal. Commun. 8, 1957-1962 (2007).

[23] O’Brien, R. J., Xu, L., Spicer, R. L. and Davis, B. H., "Activation Study of Precipitated Iron Fischer-Tropsch Catalysts", Energy \& Fuels 10, 921-926 (1996).

[24] Shultz, J. F., Abelson, M., Stein, K. C. and Anderson, R. B., "Studies of the Fischer-Tropsch Synthesis. XVIII. Influence of Catalyst Geometry on Synthesis on Iron Catalysts", J. Phys. Chem. 63, 496-500 (1959).

[25] Ning, W., Koizumi, N., Chang, H., Mochizuki, T., Itoh, T. and Yamada, M., "Phase transformation of unpromoted and promoted Fe catalysts and the formation of carbonaceous compounds during Fischer-Tropsch synthesis reaction", Appl. Catal. A Gen. 312, 35-44 (2006).

[26] Dictor, R. and Bell, A. T., "Fischer-Tropsch synthesis over reduced and unreduced iron oxide catalysts", J. Catal. 97, 121-136 (1986).

[27] Todic, B., Nowicki, L., Nikacevic, N. and Bukur, D. B., "Fischer-Tropsch synthesis product selectivity over an industrial iron-based catalyst: Effect of process conditions", Catal. Today 261, 28-39 (2016).

[28] Botes, G. F., Bromfield, T. C., Coetzer, R. L. J., Crous, R., Gibson, P. and Ferreira, A. C., "Development of a chemical selective iron Fischer Tropsch catalyst", Catal. Today 275, 40-48 (2016).

[29] Torres Galvis, H. M., Bitter, J. H., Khare, C. B., Ruitenbeek, M., Dugulan, A. I. and de Jong, K. P., "Supported Iron Nanoparticles as Catalysts for Sustainable Production of Lower Olefins", Science 335, 835-838 (2012). 
[30] Torres Galvis, H. M., Koeken, A. C. J., Bitter, J. H., Davidian, T., Ruitenbeek, M., Dugulan, A. I. and de Jong, K. P., "Effects of sodium and sulfur on catalytic performance of supported iron catalysts for the Fischer-Tropsch synthesis of lower olefins", J. Catal. 303, 22-30 (2013).

[31] Dry, M. E. and Oosthuizen, G. J., "The correlation between catalyst surface basicity and hydrocarbon selectivity in the Fischer-Tropsch synthesis", J. Catal. 11, 18-24 (1968).

[32] Ngantsoue-Hoc, W., Zhang, Y., O’Brien, R. J., Luo, M. and Davis, B. H., "Fischer-Tropsch synthesis: activity and selectivity for Group I alkali promoted iron-based catalysts", Appl. Catal. A Gen. 236, 7789 (2002).

[33] Luo, M. and Davis, B. H., "Fischer-Tropsch synthesis: Group II alkali-earth metal promoted catalysts", Appl. Catal. A Gen. 246, 171-181 (2003).

[34] Bukur, D. B., Okabe, K., Rosynek, M. P., Li, C. P., Wang, D. J., Rao, K. R. P. M. and Huffman, G. P., "Activation Studies with a Precipitated Iron Catalyst for Fischer-Tropsch Synthesis. I. Characterization Studies", Journal of Catalysis 155, 353-365 (1995).

[35] Bukur, D. B., Nowicki, L., Manne, R. K. and Lang, X. S., "Activation Studies with a Precipitated Iron Catalyst for Fischer-Tropsch Synthesis II. Reaction Studies", J. Catal. 155, 366-375 (1995).

[36] Wezendonk, T. A., Sun, X., Dugulan, A. I., van Hoof, A. J. F., Hensen, E. J. M., Kapteijn, F. and Gascon, J., "Controlled formation of iron carbides and their performance in Fischer-Tropsch synthesis", J. Catal. 362, 106-117 (2018).

[37] Johnson, J. Y., "Improvements in the Manufacture and Production of Unsaturated Hydrocarbons of Low Boiling Point", Patent GB322284A (1929).

[38] Crous, R., Bromfield, T. C. and Booyens, S., "Olefin Selective FT Catalyst Composition and Preparation Thereof", Patent W02010066386A1 (2010).

[39] Myddleton, W. W., "Improvements Relating to the Catalytic Synthesis of Hydrocarbon Oils from Gaseous Mixtures of Carbon Monoxide and Hydrogen", Patent GB509325A (1939).

[40] Xie, J., Yang, J., Dugulan, A. I., Holmen, A., Chen, D., De Jong, K. P. and Louwerse, M. J., "Size and Promoter Effects in Supported Iron Fischer-Tropsch Catalysts: Insights from Experiment and Theory", ACS Catal. 6, 3147-3157 (2016).

[41] Jung, H. and Thomson, W. J., "Dynamic X-ray diffraction study of an unsupported iron catalyst in Fischer-Tropsch synthesis", J. Catal. 134, 654-667 (1992).

[42] McCartney, J. T., Hofer, L. J. E., Seligman, B., Lecky, J. A., Peebles, W. C. and Anderson, R. B., "Electron and X-Ray Diffraction Studies of Iron Fischer-Tropsch Catalysts", J. Phys. Chem. 57, 730-736 (1953).

[43] Leineweber, A., Shang, S., Liu, Z.-K., Widenmeyer, M. and Niewa, R., "Crystal structure determination of Hägg carbide, $\chi$ - $\mathrm{Fe}_{5} \mathrm{C}_{2}$ by first-principles calculations and Rietveld refinement", Zeitschrift für Krist. 227, 207-220 (2012).

[44] Retief, J. J., "Powder diffraction data and Rietveld refinement of Hägg-carbide, $\chi$-Fe $\mathrm{F}_{2}$ ", Powder Diffr. 14, 130-132 (1999).

[45] Duggin, M. J. and Hofer, L. J. E., "Nature of $\chi$-Iron Carbide", Nature 212, 248-248 (1966).

[46] Jack, K. H. and Wild, S., "Nature of $\chi$-Carbide and Its Possible Occurrence in Steels", Nature 212, 248250 (1966).

[47] Jenkins, R. and Snyder, R. L., "Introduction to X-ray Powder Diffractometry", Introduction to X-ray Powder Diffractometry 138 (John Wiley \& Sons, Inc., Hoboken, NJ, USA, 1996).

[48] Koeken, A. C. J., Torres Galvis, H. M., Davidian, T., Ruitenbeek, M. and de Jong, K. P., "Suppression of Carbon Deposition in the Iron-Catalyzed Production of Lower Olefins from Synthesis Gas", Angew. Chem., Int. Ed. 51, 7190-7193 (2012).

[49] Janbroers, S., Louwen, J. N., Zandbergen, H. W. and Kooyman, P. J., "Insights into the nature of iron- 
based Fischer-Tropsch catalysts from quasi in situ TEM-EELS and XRD", J. Catal. 268, 235-242 (2009).

[50] Shroff, M. D. and Datye, A. K., "The importance of passivation in the study of iron Fischer-Tropsch catalysts", Catal. Lett. 37, 101-106 (1996).

[51] Alstrup, I., Tavares, M. T., Bernardo, C. A., Sørensen, O. and Rostrup-Nielsen, J. R., "Carbon formation on nickel and nickel-copper alloy catalysts", Mater. Corros. 49, 367-372 (1998).

[52] Nolan, P. E., Lynch, D. C. and Cutler, A. H., "Catalytic disproportionation of CO in the absence of hydrogen: Encapsulating shell carbon formation", Carbon 32, 477-483 (1994).

[53] Mazzucco, S., Wang, Y., Tanase, M., Picher, M., Li, K., Wu, Z., Irle, S. and Sharma, R., "Direct evidence of active and inactive phases of Fe catalyst nanoparticles for carbon nanotube formation", J. Catal. 319, 54-60 (2014).

[54] Toby, B. H., "R factors in Rietveld analysis: How good is good enough?", Powder Diffr. 21, 67-70 (2006)

[55] Nagakura, S., "Study of Metallic Carbides by Electron Diffraction Part III. Iron Carbides", J. Phys. Soc. Japan 14, 186-195 (1959).

[56] Amelse, J. A., Grynkewich, G., Butt, J. B. and Schwartz, L. H., "Moessbauer spectroscopic study of passivated small particles of iron and iron carbide", J. Phys. Chem. 85, 2484-2488 (1981).

[57] Jiang, F., Zhang, M., Liu, B., Xu, Y. and Liu, X., "Insights into the influence of support and potassium or sulfur promoter on iron-based Fischer-Tropsch synthesis: understanding the control of catalytic activity, selectivity to lower olefins, and catalyst deactivation", Catal. Sci. Technol. 7, 1245-1265 (2017).

[58] Raupp, G. B. and Delgass, W. N., "Mössbauer Investigation of Supported Fe and FeNi Catalysts II. Carbides Formed by Fischer-Tropsch Synthesis", J. Catal. 58, 348-360 (1979).

[59] Tau, L. M., Borcar, S., Bianchi, D. and Bennett, C. O., "Investigation of supported iron Fischer-Tropsch synthesis catalysts by Mössbauer spectroscopy", J. Catal. 87, 36-54 (1984).

[60] du Plessis, H. E., de Villiers, J. P. R., Kruger, G. J., Steuwer, A. and Brunelli, M., "Rietveld and pair distribution function study of Hägg carbide using synchrotron X-ray diffraction", J. Synchrotron Radiat. 18, 266-271 (2011).

[61] Hofer, L. J. E., "Nature of the carbides of iron", U.S. Bureau of Mines Bulletin 631 (1966). Available at: http://www.onemine.org/document/abstract.cfm?docid=186163\&title=Nature-Of-The-CarbidesOf-Iron (Accessed: February 2014).

[62] Rhodes, C., Hutchings, G. J. and Ward, A. M., "Water-gas shift reaction: finding the mechanistic boundary", Catal. Today 23, 43-58 (1995).

[63] Dry, M. E. and Ferreira, L. C., "The distribution of promoters in magnetite catalysts", J. Catal. 7, 352358 (1967).

[64] Hofer, L. J. E., Cohn, E. M. and Peebles, W. C., "The Modifications of the Carbide, $\mathrm{Fe}_{2} \mathrm{C}$; Their Properties and Identification", J. Am. Chem. Soc. 71, 189-195 (1949).

[65] Hirotsu, Y. and Nagakura, S., "Crystal structure and morphology of the carbide precipitated from martensitic high carbon steel during the first stage of tempering", Acta Metall. 20, 645-655 (1972).

[66] Nagakura, S., Hirotsu, Y., Kusunoki, M., Suzuki, T. and Nakamura, Y., "Crystallographic study of the tempering of martensitic carbon steel by electron microscopy and diffraction", Metall. Trans. A 14, 1025-1031 (1983).

[67] Barton, G. H. and Gale, B., "The structure of a pseudo-hexagonal iron carbide", Acta Crystallogr. 17, 1460-1462 (1964).

[68] Hirotsu, Y. and Nagakura, S., "Electron Microscopy and Diffraction Study of the Carbide Precipitated 
at the First Stage of Tempering of Martensitic Medium Carbon Steel", Trans. Japan Inst. Met. 15, 129134 (1974).

[69] Matyi, R. J., Schwartz, L. H. and Butt, J. B., "Particle Size, Particle Size Distribution, and Related Measurements of Supported Metal Catalysts", Catal. Rev. 29, 41-99 (1987).

[70] Liu, X., Zhang, C., Li, Y., Niemantsverdriet, J. W., Wagner, J. B. and Hansen, T. W., "Environmental Transmission Electron Microscopy (ETEM) Studies of Single Iron Nanoparticle Carburization in Synthesis Gas", ACS Catal. 7, 4867-4875 (2017).

[71] Pendyala, V. R. R., Graham, U. M., Jacobs, G., Hamdeh, H. H. and Davis, B. H., "Fischer-Tropsch Synthesis: Morphology, Phase Transformation, and Carbon-Layer Growth of Iron-Based Catalysts", ChemCatChem 6, 1952-1960 (2014).

[72] Balzar, D., Audebrand, N., Daymond, M. R., Fitch, A., Hewat, A., Langford, J. I., Le Bail, A., Louër, D., Masson, O., McCowan, C. N., Popa, N. C., Stephens, P. W. and Toby, B. H., "Size-strain line-broadening analysis of the ceria round-robin sample", J. Appl. Crystallogr. 37, 911-924 (2004).

[73] Kril, C. E. and Birringer, R., "Estimating grain-size distributions in nanocrystalline materials from Xray diffraction profile analysis", Philos. Mag. A 77, 621-640 (1998).

[74] Anderson, R. B., Karn, F. S. and Shultz, J. F., "Factors in sulfur poisoning of iron catalysts in FischerTropsch synthesis", J. Catal. 4, 56-63 (1965).

[75] Torres Galvis, H. M., Koeken, A. C. J., Bitter, J. H., Davidian, T., Ruitenbeek, M., Dugulan, A. I. and De Jong, K. P., "Effect of precursor on the catalytic performance of supported iron catalysts for the FischerTropsch synthesis of lower olefins", Catal. Today 215, 95-102 (2013).

[76] Dry, M. E., "The Fischer-Tropsch process: 1950-2000", Catal. Today 71, 227-241 (2002).

[77] van der Laan, G. P. and Beenackers, A. A. C. M., "Kinetics and Selectivity of the Fischer-Tropsch Synthesis: A Literature Review", Catal. Rev. 41, 255-318 (1999).

[78] Ratnasamy, C. and Wagner, J. P., "Water Gas Shift Catalysis", Catal. Rev. 51, 325-440 (2009).

[79] Chianese, S., Loipersböck, J., Malits, M., Rauch, R., Hofbauer, H., Molino, A. and Musmarra, D., "Hydrogen from the high temperature water gas shift reaction with an industrial $\mathrm{Fe} / \mathrm{Cr}$ catalyst using biomass gasification tar rich synthesis gas", Fuel Process. Technol. 132, 39-48 (2015).

[80] Lywood, W. J. and Twigg, M. V., "Hydrogen production including a shift reaction process", Patent EP0361648A1 (1990).

[81] Amenomiya, Y. and Pleizier, G., "Alkali-promoted Alumina Catalysts II. Water-Gas Shift Reaction", J. Catal. 76, 345-353 (1982).

[82] Krupay, B. W. and Amenomiya, Y., "Alkali-promoted Alumina Catalysts I. Chemisorption and oxygen exchange of carbon monoxide and carbon dioxide on potassium-promoted alumina catalysts", J. Catal. 67, 362-370 (1981).

[83] Zhang, H.-B. and Schrader, G. L., "Characterization of a fused iron catalyst for Fischer-Tropsch synthesis by in situ laser Raman spectroscopy", J. Catal. 95, 325-332 (1985).

[84] Wang, P., Chen, W., Chiang, F., Dugulan, A. I., Song, Y., Pestman, R., Zhang, K., Yao, J., Feng, B., Miao, P., $\mathrm{Xu}, \mathrm{W}$. and Hensen, E. J. M., "Synthesis of stable and low- $\mathrm{CO}_{2}$ selective $\varepsilon$-iron carbide Fischer-Tropsch catalysts", Sci. Adv. 4, eaau2947 (2018).

[85] Krishnamoorthy, S., Li, A. and Iglesia, E., "Pathways for $\mathrm{CO}_{2}$ Formation and Conversion During FischerTropsch Synthesis on Iron-Based Catalysts", Catal. Lett. 80, 77-86 (2002).

[86] Hwang, K.-R., Lee, C.-B. and Park, J.-S., "Advanced nickel metal catalyst for water-gas shift reaction", J. Power Sources 196, 1349-1352 (2011).

[87] Arakawa, H. and Bell, A. T., "Effects of potassium promotion on the activity and selectivity of iron Fischer-Tropsch catalysts", Ind. Eng. Chem. Process Des. Dev. 22, 97-103 (1983). 
[88] Wezendonk, T. A., Santos, V. P., Nasalevich, M. A., Warringa, Q. S. E., Dugulan, A. I., Chojecki, A., Koeken, A. C. J., Ruitenbeek, M., Meima, G., Islam, H.-U., Sankar, G., Makkee, M., Kapteijn, F. and Gascon, J., "Elucidating the Nature of Fe Species during Pyrolysis of the Fe-BTC MOF into Highly Active and Stable Fischer-Tropsch Catalysts", ACS Catal. 6, 3236-3247 (2016).

[89] Davidian, T., Ruitenbeek, M., Koeken, A. C. J., Vos, M. and Wielemaker, M. F., "Novel Iron-based Catalysts and Treatment Process therefor for Use in Fischer-Tropsch Reactions", Patent W02014210089A1 (2014). 


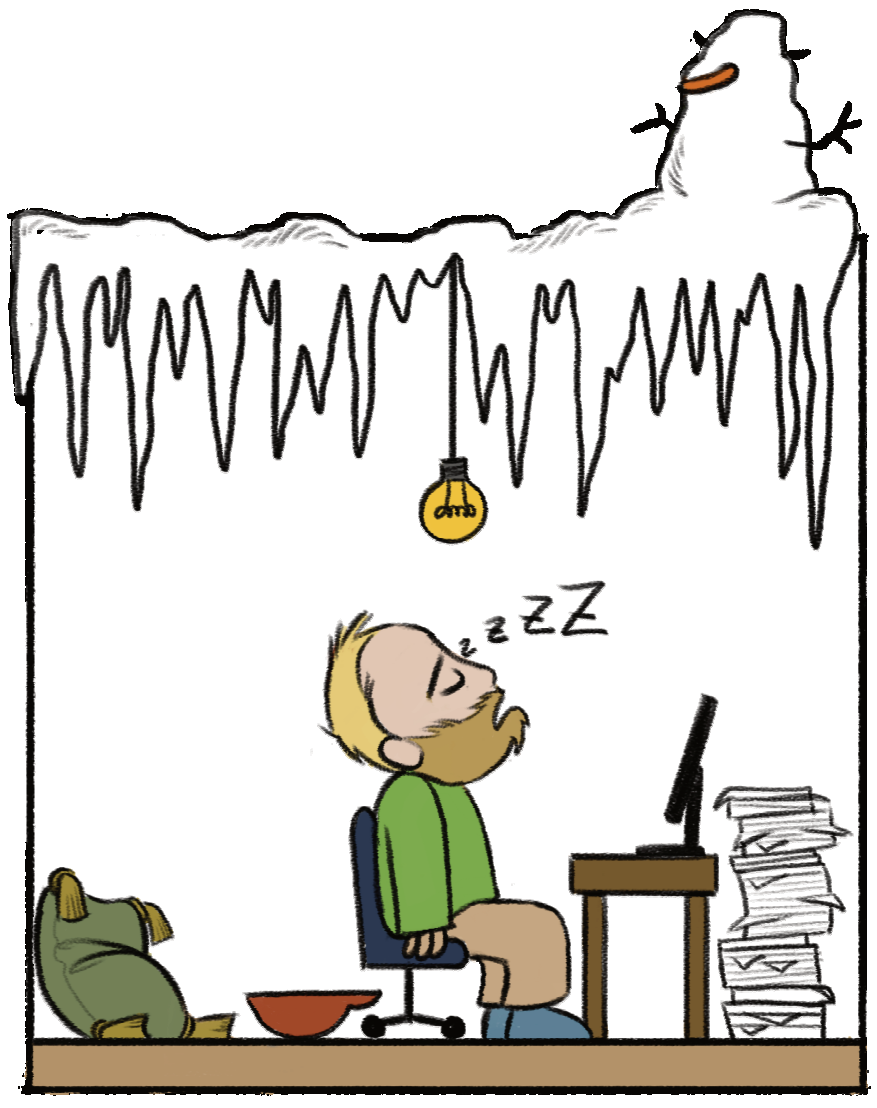




\section{Chapter 5}

\section{On the Effect of the Reaction Pressure in Fischer-Tropsch Synthesis with $\mathrm{Fe}(-\mathrm{Na}-$ $\mathrm{S}) / \alpha-\mathrm{Al}_{2} \mathrm{O}_{3}$ Catalysts}

Fe-based catalysts catalyze Fischer-Tropsch Synthesis (FTS) reaction producing hydrocarbons from an input $\mathrm{H}_{2}$ : $\mathrm{CO}$ gas mixture. The catalysts' FTS performance is modifiable by varying e.g. reaction conditions, catalyst pretreatment and added chemical promoters. We follow the combined effects of the FTS reaction pressure, catalyst $\mathrm{H}_{2}$ pre-reduction and Na-S promotion on the catalysts' FTS performance. Especially, formation of Fe carbides and their subtypes defined by distint lattice parameters, are followed. Supported, Na-S promoted and unpromoted Fe(-Na-S) $/ \alpha-$ $\mathrm{Al}_{2} \mathrm{O}_{3}$ catalysts were tested at 3,10 and 20 bar FTS reaction at $340^{\circ} \mathrm{C}$. Catalysts were either $\mathrm{H}_{2}$ pre-reduced or directly exposed to $\mathrm{H}_{2}$ : $\mathrm{CO}$ in the FTS reaction. X-ray Powder Diffractometry (XRPD) with Rietveld Quantitative Phase Analysis (R-QPA) was applied for Fe phase quantification and Transmission Electron Microscopy (TEM) for determining catalyst morphology. Na-S promotion induced a lowered 10 bar reaction pressure threshold for boosted catalyst activity and also decreased both Usage Ratio $\mathrm{H}_{2}: \mathrm{CO}$ and $\mathrm{CH}_{4}$ selectivity. 20 bar reaction pressure was required for unpromoted catalyst for similar effects. Regardless of Na-S promotion, catalysts directly exposed to $\mathrm{H}_{2}$ : $\mathrm{CO}$ showed increased $\mathrm{C}_{2} \mathrm{H}_{4} / \mathrm{C}_{2} \mathrm{H}_{6}$ ratio, which was attributed to the presence of $\chi-\mathrm{Fe}_{5} \mathrm{C}_{2}$ Type III. The results contribute towards understanding $\mathrm{Na}-\mathrm{S}$ promotion reaction pressure dependency, especially in relation to the associated Fe phase/carbide subtype changes. 


\subsection{INTRODUCTION}

With Fischer-Tropsch Synthesis (FTS) reaction a $\mathrm{H}_{2}$ :CO gas mixture (i.e. syngas) can be catalytically converted into hydrocarbon products. A wide variety of different hydrocarbons can be produced via the FTS reaction. ${ }^{1-5}$ With the use of Fe-based catalyst materials in the FTS reaction, increased amount of olefins (i.e. unsaturated hydrocarbons) as the synthesis hydrocarbon products can be attained.1,3,4

Fe-based catalyst materials can operate over a varied range of FTS reaction conditions. ${ }^{1,3,4,6}$ By altering the exact FTS reaction conditions, e.g. reaction pressure, ${ }^{5-8}$ temperature, ${ }^{4,5,7,9-11}$ or input $\mathrm{H}_{2}: \mathrm{CO}$ ratio, ${ }^{4,5,7,10}$ the hydrocarbon product slate can be steered towards the desired products. Besides the FTS reaction conditions, the hydrocarbon product selectivities can also be adjusted with use of chemical promoters added on the FTS catalyst materials. ${ }^{4,5,12-17}$ An added element from the alkali metal group, commonly $\mathrm{Na}$ or $\mathrm{K}$, is the most common chemical catalyst promoter applied in the Fe-based FTS reaction. The alkali alters the hydrocarbon product selectivities towards longer chained hydrocarbons with increased olefinicity.4,5,11,12,15-18 Furthermore, combining an alkali element with $\mathrm{S}$ is especially well-suited for steering the FTS reaction hydrocarbon products towards increased olefinicity, while simultaneously suppressing undesired $\mathrm{CH}_{4}$ selectivity. ${ }^{19-25}$

The active phases for the Fe-based FTS reaction are Fe carbides. Fe carbides with their crystal lattices encountered in FTS research are (Chapter 2): $\varepsilon-\mathrm{Fe}_{3} \mathrm{C} P 6_{3} 22$ (182), $\eta-\mathrm{Fe}_{2} \mathrm{C}$ Pnnm (58), $\chi-\mathrm{Fe}_{5} \mathrm{C}_{2} \mathrm{C} 2 / \mathrm{c}$ (15), $\theta-\mathrm{Fe}_{3} \mathrm{C}$ Pnma (62) and $\theta-\mathrm{Fe}_{7} \mathrm{C}_{3}$ Pnma (62). The $\eta-$ $\mathrm{Fe}_{2} \mathrm{C}$ and $\chi-\mathrm{Fe}_{5} \mathrm{C}_{2}$ phases can further be divided into subtypes after differences in their crystal lattice parameters, as induced by different formation conditions (Chapter 4). The Fe carbide subtype formation was observed to depend on the presence of Na-S promotion, the initial Fe phase used for carburization $\left(\alpha-\mathrm{Fe}_{2} \mathrm{O}_{3}\right.$ or $\left.\alpha-\mathrm{Fe}\right)$ and whether a Fe carbide was exposed to FTS reaction conditions $\left(340^{\circ} \mathrm{C}, 10\right.$ bar, $\left.\mathrm{H}_{2}: \mathrm{CO} 2: 1\right)$ or only $\mathrm{CO}$ carburized. The Fe carbide subtype divisions can be used to explain the various lattice parameters, ${ }^{26-29}$ and a distorted form, ${ }^{30}$ of the $\chi-\mathrm{Fe}_{5} \mathrm{C}_{2}$ phase observed in the literature.

The presence of any specific Fe carbide phase does not directly predict the FTS reaction performance of the applied catalyst. ${ }^{16,31-33}$ Nevertheless, without a known or direct correlation to any specific Fe carbide phase(s), the Fe-based catalysts FTS reaction performance can be altered with an applied catalyst pretreatment. That is, by altering the Fe phase(s) present in the catalyst material at the initiation of the FTS reaction. ${ }^{34-38}$ As examples, exposing a Fe-based FTS reaction catalyst material in its oxidic form directly to $\mathrm{CO}$ or $\mathrm{H}_{2}$ : $\mathrm{CO}$ without a prior $\mathrm{H}_{2}$ reduction of the catalyst, can induce an $\approx+10 \%$ increase in the achieved $\mathrm{C}_{2}-\mathrm{C}_{4}$ olefin hydrocarbon product fraction, 35 


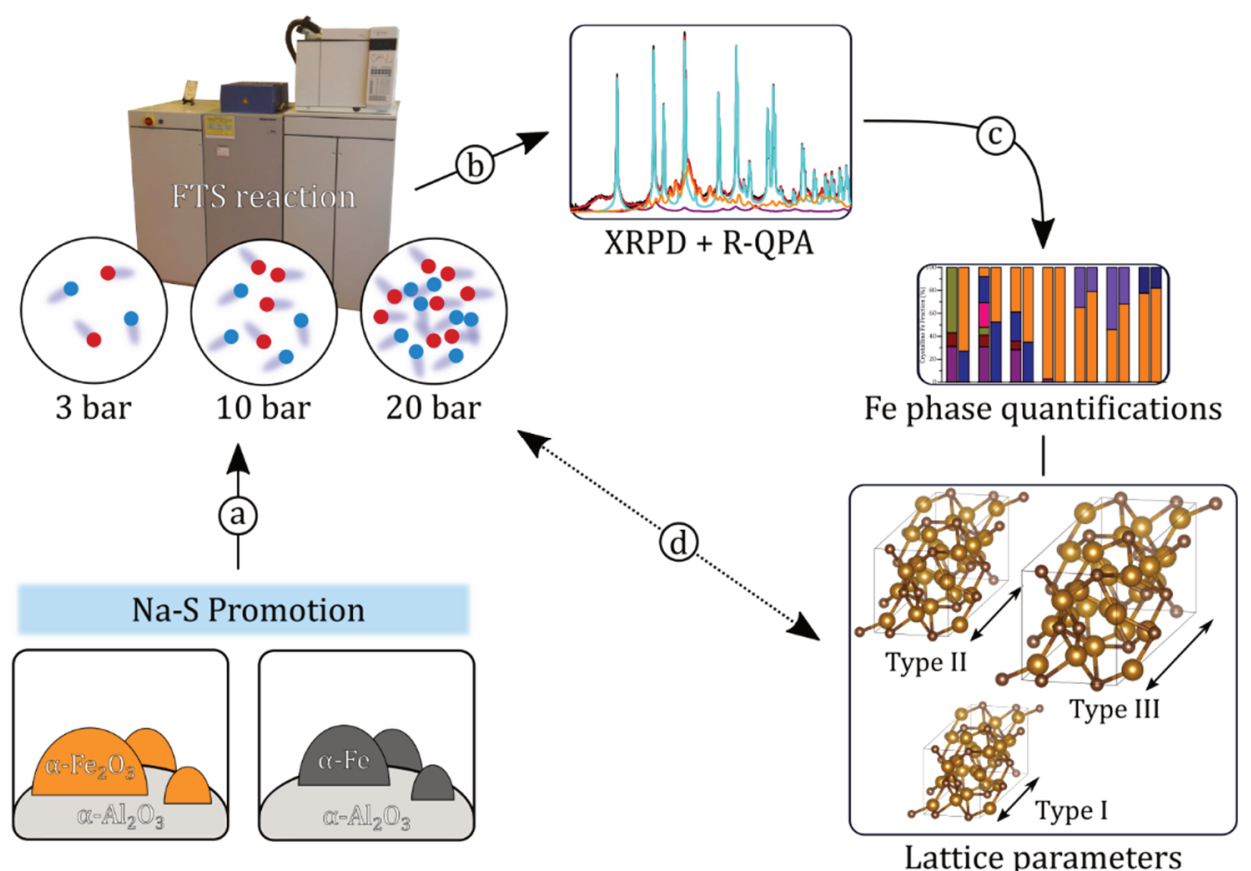

Figure 5.1

A schematic of the approach applied in this Chapter. Na-S promoted and unpromoted $\mathrm{Fe}(-\mathrm{Na}-\mathrm{S}) / \alpha-\mathrm{Al}_{2} \mathrm{O}_{3}$ catalyst materials are either $\mathrm{H}_{2}$ reduced prior $(\alpha-\mathrm{Fe})$ or exposed directly as calcined only $\left(\alpha-\mathrm{Fe}_{2} \mathrm{O}_{3}\right)$ catalysts to (a) Fischer-Tropsch Synthesis (FTS) reaction at reaction pressures of 3,10 or 20 bar at $340^{\circ} \mathrm{C}$. The catalyst materials are (b) subsequently characterized after the FTS reaction with ex-situ X-ray Powder Diffractometry (XRPD). Rietveld Quantitative Phase Analysis (R-QPA) is applied to the XRPD patterns in order to (c) quantify the Fe carbide phases and lattice parameters in the catalyst materials. Special attention is given on the Fe carbide subtypes, which are defined by their lattice parameters. The quantification results are then (d) compared to the catalyst materials FTS reaction catalysis performances.

or decrease the catalyst's hydrogenation ability. ${ }^{38}$ Origins of this effect, however, are not fundamentally understood. Especially, whether a connection between the $\mathrm{Fe}$ carbide subtypes and Fe-based FTS reaction catalysts' catalytic performance exists is unknown. The precise Fe carbide crystal lattice parameters, defining the Fe carbide subtypes, are commonly overlooked in the literature. This, despite the wide application of the X-ray Powder Diffractometry (XRPD) technique within the research field of Febased FTS reaction. 10,30,32,34,36,39-44

Within this Chapter, with use of supported $\mathrm{Fe}(-\mathrm{Na}-\mathrm{S}) / \alpha-\mathrm{Al}_{2} \mathrm{O}_{3}$ catalyst materials with and without Na-S promotion, we probe the combined effects of i) Na-S promotion, ii) FTS reaction pressure and iii) $\alpha-\mathrm{Fe}_{2} \mathrm{O}_{3}$ (hematite) or $\mathrm{H}_{2}$ pre-reduced $\alpha$-Fe phases as the FTS reaction's starting Fe phases. The observations are correlated to the catalyst 


\section{Table 5.1}

Inductively Coupled Plasma - Optical Emission Spectrophotometry (ICP-OES) results of elemental analysis on the used $\mathrm{Fe}(\mathrm{III})$ nitrate nonahydrate precursor $\mathrm{Fe}\left(\mathrm{NO}_{3}\right)_{3} \cdot 9 \mathrm{H}_{2} \mathrm{O}$ (Acros, 423710030) and the unpromoted (UP) and Na-S promoted (Na-S) catalysts materials after calcining (Calcin.). The nitrate precursor was measured at Mikrolab Kolbe and the UP and Na-S Calcin. materials at Universiteit Utrecht (UU) GeoLab.

\begin{tabular}{cccccccccc}
\hline & \multicolumn{9}{c}{ Elemental Analysis (weight-ppm) } \\
\cline { 2 - 10 } & $\mathrm{Co}$ & $\mathrm{Cr}$ & $\mathrm{Mn}$ & $\mathrm{Fe}$ & $\mathrm{Ca}$ & $\mathrm{K}$ & $\mathrm{Na}$ & $\mathrm{S}$ \\
\hline $\mathrm{Fe}\left(\mathrm{NO}_{3}\right)_{3} \cdot 9 \mathrm{H}_{2} \mathrm{O}$ & - & - & - & 132200 & - & 42 & 126 & 145 \\
\hline$(\mathrm{UP}) \mathrm{Fe} / \alpha-\mathrm{Al}_{2} \mathrm{O}_{3}$ & 2 & 457 & 16 & 130105 & 188 & $<\mathrm{dl}$ & $<\mathrm{dl}$ & 392 \\
$(\mathrm{Na}-\mathrm{S}) \mathrm{Fe}-\mathrm{Na}-\mathrm{S} / \alpha-\mathrm{Al}_{2} \mathrm{O}_{3}$ & 2 & 446 & 22 & 137297 & 203 & $<\mathrm{dl}$ & 5268 & 1184 \\
\hline$<\mathrm{dl}-$ Below detection limit. & & & & & & &
\end{tabular}

materials' subsequent FTS reaction performance (Figure 5.1). The Fe phases present in the catalyst materials before and after the FTS reaction are quantified by applying Rietveld Quantitative Phase Analysis (R-QPA) on the ex-situ measured XRPD patterns on the catalysts. The reaction performances of the catalysts under study are then correlated to the quantified Fe phases, and especially to the Fe carbide subtypes defined by their crystal lattice parameters. Additionally, Fe phases' crystallite diameters are also determined with the R-QPA. Transmission Electron Microscopy (TEM) is applied to obtain the Fe particle mean diameters and catalyst morphology for the studied catalyst materials. Quasi-In-situ Transmission Mössbauer Absorption Spectroscopy (MAS) is also applied as a complementary characterization technique for verifying some of the R-QPA-based Fe phase identification results on the studied catalyst materials.

\subsection{EXPERIMENTAL METHODS}

\subsubsection{Catalyst Preparation}

\subsubsection{Catalyst Synthesis}

Supported catalyst materials with a nominal 15 weight percent (wt-\%) Fe loading, with and without added Na-S promotion, were prepared using Incipient Wetness Impregnation (IWI) method on alpha-alumina $\left(\alpha-\mathrm{Al}_{2} \mathrm{O}_{3}\right)$ support material (BASF, $7 \mathrm{~m}^{2} / \mathrm{g}, 212-425 \mu \mathrm{m}$ particle size). The impregnation solution was prepared using Fe(III) nitrate nonahydrate (Acros, 423710030) as Fe precursor, Fe(II) sulfate heptahydrate (Merck, 103965) as S precursor and Na tribasic citrate dihydrate (SigmaAldrich, S4641) as $\mathrm{Na}$ precursor. Inductively Coupled Plasma Optical Emission Spectrophotometry (ICP-OES) was used for elemental analysis of the Fe precursor Fe(III) nitrate nonahydrate (Table 5.1). 
The precursor solution used for the preparation of the Na-S promoted catalyst material was prepared to a glass vial by individually solvating $3.823 \mathrm{~g}$ of Fe, $0.272 \mathrm{~g}$ of $\mathrm{S}$ and $0.941 \mathrm{~g}$ of Na precursors to $0.8 \mathrm{ml}$ of demineralized water. The precursor solution for the unpromoted catalyst material was prepared only with $3.833 \mathrm{~g}$ of the $\mathrm{Fe}$ precursor. Any insoluble crystals were dissolved by heating the closed precursor solution containing glass vial at $60^{\circ} \mathrm{C}$ for few minutes. The catalyst materials nominal weight loadings for the added $\mathrm{Na}$ and $\mathrm{S}$ promoters were $0.62 \mathrm{wt}-\% \mathrm{Na}$ and $0.09 \mathrm{wt}-\% \mathrm{~S}$.

$3.00 \mathrm{~g}$ of the $\alpha-\mathrm{Al}_{2} \mathrm{O}_{3}$ support material was placed to a round-bottom boiling flask with a magnetic stirring rod. In three sequential impregnation steps, $\approx 1.0 \mathrm{ml}, \approx 0.8 \mathrm{ml}$ and $\approx 0.7 \mathrm{ml}$ of the precursor solution was dropwise added on the $\alpha-\mathrm{Al}_{2} \mathrm{O}_{3}$ support with a syringe. The $\alpha-\mathrm{Al}_{2} \mathrm{O}_{3}$ support material was simultaneously stirred with the magnetic rod. Each impregnation step was followed by drying the impregnated $\alpha-\mathrm{Al}_{2} \mathrm{O}_{3}$ support at $120^{\circ} \mathrm{C}$, for at least $4 \mathrm{~h}$. The precursor solution containing glass vial was washed with $\approx 0.1 \mathrm{ml}$ of demineralized water within the last impregnation step. After each drying step, any catalyst particle aggregates formed were gently broken down with a mortar. In case of any precursor recrystallization and precipitation taking place in between the impregnation steps, the precursor solution was heated to $60^{\circ} \mathrm{C}$ for few minutes for dissolving the appeared crystals. The impregnation procedure was individually repeated twice for both unpromoted and Na-S promoted catalyst materials. After their final drying steps, the prepared unpromoted and Na-S promoted catalyst batches were respectively combined.

\subsubsection{Catalyst Calcining}

Catalyst material calcining was done under flowing air in an open quartz vessel by heating $\left(10^{\circ} \mathrm{C} / \mathrm{min}\right)$ the dried catalyst materials from room temperature to $500^{\circ} \mathrm{C}$, and holding the temperature for $6 \mathrm{~h}$. In order to avoid any gas diffusion limitations, the catalyst materials were calcined in batches of $\$ 3.0 \mathrm{~g}$. Each calcining batch lost $\approx 3-4 \%$ of its weight during the calcining step. The calcined catalyst materials were recombined within the unpromoted and Na-S promoted batches and re-sieved to a catalyst particle fraction of 212-425 $\mu \mathrm{m}$ in order to obtain the ready, calcined catalyst materials. Results for the elemental ICP-OES analysis on the calcined catalyst materials are given in

\section{Table 5.1.}

\subsubsection{Catalyst Reduction}

Reduction of the calcined catalyst materials was performed with use of a plug flow quartz reactor. For each reduction treatment, $1.0 \mathrm{~g}$ of calcined catalyst material was mixed with $5.0 \mathrm{~g}$ of $\mathrm{SiC}$ and the catalyst material/SiC mixture was loaded into the quartz reactor. The reactor was heated up $\left(10^{\circ} \mathrm{C} / \mathrm{min}\right)$ from room temperature to $350^{\circ} \mathrm{C}$ under $\mathrm{N}_{2}: \mathrm{H}_{2}$ flow (85:165 ml/min, at atmospheric pressure) and held at $350^{\circ} \mathrm{C}$ for $17 \mathrm{~h}$. After 
the reduction, the reactor was cooled down to room temperature under $\mathrm{N}_{2}: \mathrm{H}_{2}$ flow and subsequently flushed with $\mathrm{N}_{2}$ for couple of hours to passivate the catalysts. The reduced catalyst material was removed from reactor and exposed to air. The catalyst material and $\mathrm{SiC}$ diluent were separated using a magnet. Both the $\mathrm{H}_{2}$ and $\mathrm{N}_{2}$ gas purities were Grade 5.0 (99.999\% pure) and were supplied by Linde Gas Benelux.

\subsubsection{Catalyst Testing}

A high-throughput Avantium Flowrence catalyst testing set-up with 16 parallel fixed bed reactors was used for testing the calcined only and the calcined and subsequently $\mathrm{H}_{2}$ reduced catalyst materials in the FTS reactions.

The catalyst materials $(\approx 25 \mathrm{mg}, 212-425 \mu \mathrm{m})$ were diluted with $\mathrm{SiC}(\approx 125 \mathrm{mg}, 212$ $425 \mu \mathrm{m})$. The resulting catalyst material/SiC mixtures were loaded into stainless steel reactor tubes with an inner diameter of $2 \mathrm{~mm}$, giving catalyst beds with heights within $\approx 31-33 \mathrm{~mm}$. Each catalyst material/SiC mixture was loaded to two reactors for testing the data reproducibility. Two reactor tubes were loaded as blanks, one with the $\alpha-\mathrm{Al}_{2} \mathrm{O}_{3}$ support material (212-425 $\mu \mathrm{m})$ and the other one with the SiC $(212-425 \mu \mathrm{m})$ diluent.

The FTS reactions were performed at $340^{\circ} \mathrm{C}$, in separate $72 \mathrm{~h}$ runs with 3,10 and 20 bar $\mathrm{H}_{2}: \mathrm{CO}: \mathrm{He}$ (1.0:1.0:0.2 volume ratio) total reaction pressure. Nominal input gas flows for each reactor were $4.0 \mathrm{ml} / \mathrm{min}_{2}, 4.0 \mathrm{ml} / \mathrm{min} \mathrm{CO}$ and $0.8 \mathrm{ml} / \mathrm{min}$ He giving gas hourly space velocity (GHSV, as total input gas flow per total catalyst bed volume) of $\approx 5500 \mathrm{~h}^{-1}$. The loaded reactors were heated up $\left(2^{\circ} \mathrm{C} / \mathrm{min}\right)$ under pure He flow from room temperature to $270^{\circ} \mathrm{C}$. At $270^{\circ} \mathrm{C}$, the reactor was pressurized with the $\mathrm{H}_{2}: \mathrm{CO}: \mathrm{He}$ gas mixture to the desired, 3, 10 or 20 bar FTS reaction total pressure. Upon reaching the reaction pressure, the reactor temperature was increased from $270^{\circ} \mathrm{C}$ to $340^{\circ} \mathrm{C}$ $\left(2^{\circ} \mathrm{C} / \mathrm{min}\right)$ to start the FTS reaction test run. After $72 \mathrm{~h}$ of the FTS reaction, the input gas flow was switched to pure $\mathrm{He}$ and the reactor temperature and pressure were lowered to ambient conditions. The spent catalyst material/SiC mixtures were removed from the reactor tubes and exposed to air. The catalyst materials were separated from the $\mathrm{SiC}$ diluent using a magnet. The used $\mathrm{H}_{2}$ and He gas purities were grade 5.0 (99.999\% pure) and for CO grade 2.5 (99.5\% pure) gas was used.

On-line gas chromatography (GC) with Thermal Conductivity (TCD) and Flame Ionization Detector (FID) were used for analyzing the reactor outlet gas compositions. The on-line GC-based catalytic performance data analysis was performed in an identical manner to the analysis described in Chapter 4.

\subsubsection{Catalyst Characterization}

The catalyst materials collected after the $72 \mathrm{~h}$ FTS reaction were analyzed ex-situ with bright field TEM and XRPD. The term X-ray Powder Diffractometry (XRPD) is used to 
refer to X-ray diffraction phenomena based characterization experiments that are done under powder diffraction conditions. ${ }^{45}$ R-QPA was applied on the obtained XRPD patterns for crystalline Fe phase quantifications, i.e. for determining phase quantities, lattice parameters and crystallite diameters for each Fe phase. Fe particles counted from the TEM images were used for determining the Fe particle size distributions. The details for application of TEM, XRPD and R-QPA have been given earlier in Chapter 3.

With R-QPA, in addition to the technique application details in Chapter 3, residual SiC (\#00-029-1129 and \#00-029-1131) diffraction was fitted using the crystal structures from International Centre for Diffraction Data Powder Diffraction File-4+ 2016 database (ICDD-PDF-4+). The residual amounts of large SiC crystallites did not fully comfort to the random diffraction requirements for powder diffraction. That is, some SiC diffraction peaks were missing, were systematically shifted and/or appeared with enhanced intensity (i.e. "rocks-in-the-dust"). ${ }^{46}$ Thus, preferential orientation correction (March-Dollase) was applied with the SiC structures and SiC lattice parameters were refined in an arbitrary manner to match the shifted $\mathrm{SiC}$ diffraction peak positions. Furthermore, additional, individual Lorentzian peaks were used to fit the SiC diffraction when needed. In addition, in some of the studied catalyst materials, diffraction peaks from crystallized hydrocarbons were identified with crystal lattice of n-heptacosane (\#00-057-1555). The crystallized hydrocarbons were fitted with individual Lorentzian peaks, if needed.

\subsubsection{Quasi-In-situ Mössbauer Absorption Spectroscopy}

Quasi-In-situ Transmission ${ }^{57} \mathrm{Fe}$ MAS was performed with the Mössbauer spectra collected at liquid He temperature (4.2 K). A sinusoidal velocity spectrometer using a ${ }^{57} \mathrm{Co}(\mathrm{Rh})$ source was used. Velocity calibration was carried out using an $\alpha$-Fe foil, at room temperature. The source and the absorbing catalyst materials were kept at the same temperature during the MAS measurements. The Mössbauer spectra were fitted using the Mosswinn 4.0 software. ${ }^{47}$

In the Quasi-In-situ MAS experiments, all of the catalyst materials were reduced under $\mathrm{H}_{2}: \mathrm{Ar} 2: 1$ flow at $350^{\circ} \mathrm{C}$ for $12 \mathrm{~h}$. The applied FTS reaction conditions were $340^{\circ}$, 3 or $20 \mathrm{bar}, \mathrm{H}_{2}: \mathrm{CO} 1: 1$ for $48 \mathrm{~h}$. After the FTS reaction, the catalyst materials were cooled down from the reaction conditions to the liquid He temperature for collecting the Mössbauer spectra.

\subsection{RESULTS AND DISCUSSION}

Ex-situ XRPD has been applied for collecting X-ray diffraction patterns on Fe-based catalyst materials before and after their catalyst testing in the FTS reaction. The FTS 


\section{Table 5.2}

Overview of the used shorthand notation within this Chapter. Nominal elemental loadings used for the catalyst materials are $15 \mathrm{wt}-\%$ of Fe and when added, $0.62 \mathrm{wt}-\%$ of $\mathrm{Na}$ and $0.09 \mathrm{wt}-\%$ of S promoters.

\begin{tabular}{cl}
\hline Shorthand Notation & \multicolumn{1}{c}{ Catalyst Material Description } \\
\hline Calcin. & Calcined in air at $500^{\circ} \mathrm{C}$ for $6 \mathrm{~h}$ \\
$\mathrm{H}_{2}$ & $\mathrm{H}_{2}$ reduced at $350^{\circ} \mathrm{C}$ for $17 \mathrm{~h}$, after the calcining step \\
$\mathrm{UP}$ & Unpromoted $\mathrm{Fe} / \alpha-\mathrm{Al}_{2} \mathrm{O}_{3}$ catalyst material \\
$\mathrm{Na}-\mathrm{S}$ & Na-S promoted Fe- $\mathrm{Na}-\mathrm{S} / \alpha-\mathrm{Al}_{2} \mathrm{O}_{3}$ catalyst material \\
Fresh & Catalyst material before the FTS reaction ("i.e. fresh catalyst") \\
$3,10,20$ bar & Catalyst material after the $72 \mathrm{~h} 340^{\circ} \mathrm{C}$ FTS reaction with the indicated reaction \\
& pressure ("i.e. spent catalyst") \\
\hline
\end{tabular}

reactions were performed at different reaction pressures of 3,10 or 20 bar $\left(340^{\circ} \mathrm{C}\right.$, $\mathrm{H}_{2}$ :CO:He 1.0:1.0:0.2, GHSV $5500 \mathrm{~h}^{-1}$ ). R-QPA was employed on the XRPD patterns for quantifying the Fe phases present in the catalyst materials. This approach has been applied on both unpromoted $\mathrm{Fe} / \alpha-\mathrm{Al}_{2} \mathrm{O}_{3}$ (shorthand $U P$ ) and the $\mathrm{Na}-\mathrm{S}$ promoted Fe-Na$\mathrm{S} / \alpha-\mathrm{Al}_{2} \mathrm{O}_{3}(\mathrm{Na}-\mathrm{S})$ catalyst materials. These catalyst materials have been either only calcined (Calcin.) or also $\mathrm{H}_{2}$ reduced $\left(\mathrm{H}_{2}\right)$ prior to the FTS reactions. The catalyst materials FTS reaction performance has been correlated to the characterization results. This has been done, by systematically comparing the catalysts' FTS reaction performance on the changes in the Fe carbide subtypes, which are defined by their distinct crystal lattice parameters.

For catalyst material characterizations done before the FTS reaction, shorthand notation Fresh is used. The shorthand notation 3, 10 or 20 bar is used for indicating a
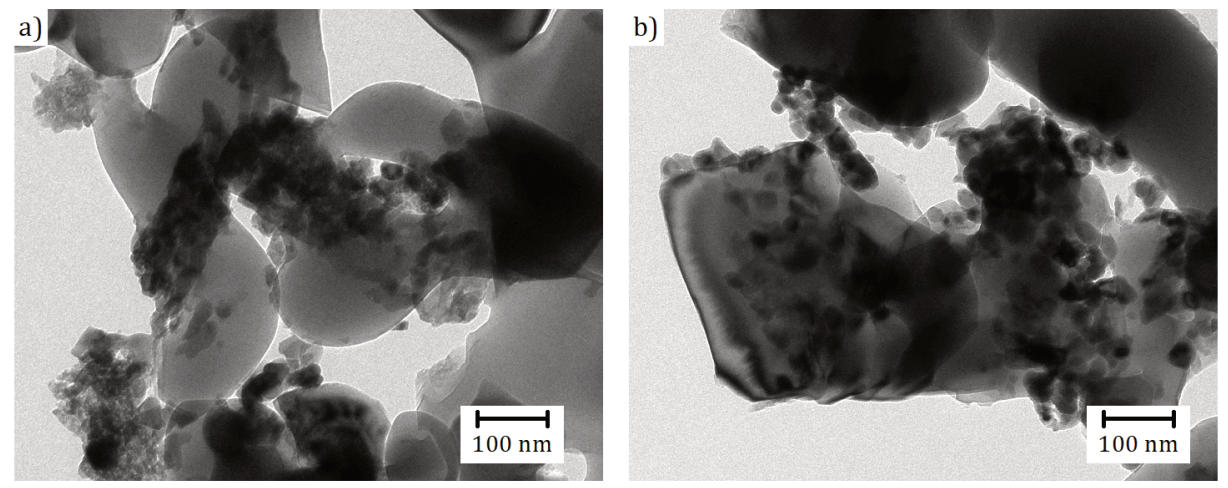

\section{Figure 5.2}

Transmission Electron Microscopy (TEM) images for the Na-S (Fe-Na-S $\left./ \alpha-\mathrm{Al}_{2} \mathrm{O}_{3}\right)$ catalyst materials before the Fischer-Tropsch Synthesis (FTS) reaction (Fresh). Images after a) calcining (Calcin.) and b) $\mathrm{H}_{2}$ reduction $\left(\mathrm{H}_{2}\right)$ treatments for the catalyst materials. 
On the Effect of the Reaction Pressure in Fischer-Tropsch Synthesis with Fe(-Na-S)/ $\alpha-\mathrm{Al} 203$ Catalysts
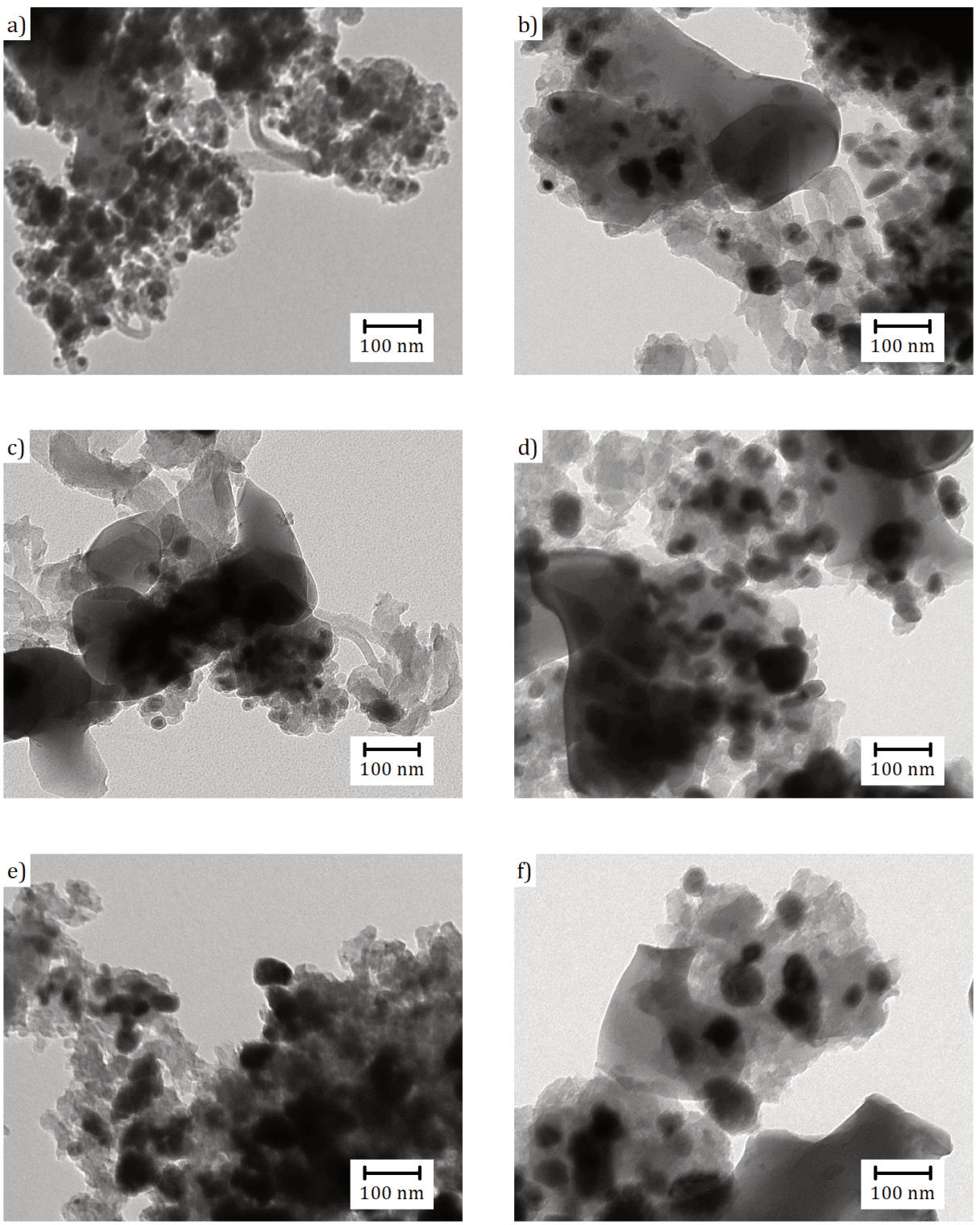

\section{Figure 5.3}

Transmission Electron Microscopy (TEM) images for the $\mathrm{Na}-\mathrm{S}\left(\mathrm{Fe}-\mathrm{Na}-\mathrm{S} / \alpha-\mathrm{Al}_{2} \mathrm{O}_{3}\right.$ ) catalyst materials after $72 \mathrm{~h}$ Fischer-Tropsch Synthesis (FTS) reactions. Images for calcined only (Calcin.) catalyst materials after a) 3 bar, c) 10 bar and e) 20 bar FTS reaction. Images for $\mathrm{H}_{2}$ reduced $\left(\mathrm{H}_{2}\right)$ catalysts after b) 3 bar, d) 10 bar and f) 20 bar FTS reaction. 

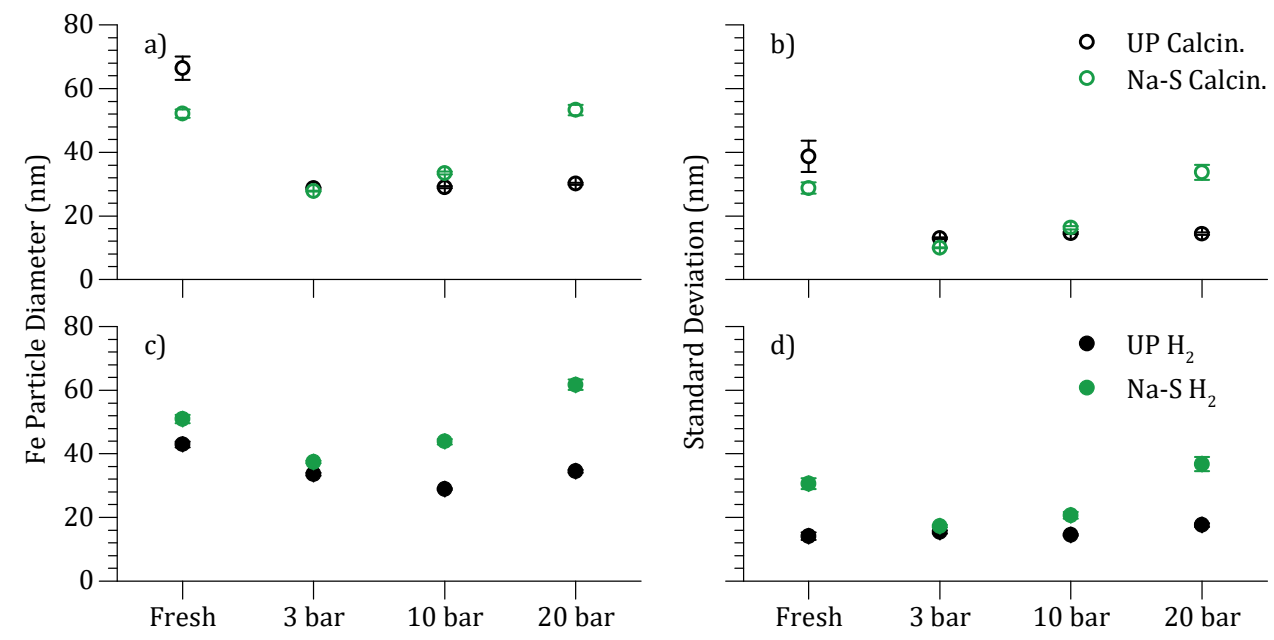

Figure 5.4

Transmission Electron Microscopy (TEM)-based, Fe particle size distribution derived values for UP and Na-S $\left(\mathrm{Fe}(-\mathrm{Na}-\mathrm{S}) / \alpha-\mathrm{Al}_{2} \mathrm{O}_{3}\right)$ catalyst materials. Fe particle (number-weighted) mean diameters for a) calcined only (Calcin.) and c) $\mathrm{H}_{2}$ reduced catalysts $\left(\mathrm{H}_{2}\right)$, before (Fresh) and after the $72 \mathrm{~h}$ Fischer-Tropsch Synthesis (FTS) reactions $\left(3,10,20\right.$ bar). Corresponding size distribution Standard Deviations (STDs) for b) Calcin. and d) $\mathrm{H}_{2}$ catalyst materials.

FTS reaction spent catalyst material and the applied reaction pressure. The overview of the shorthand notations used in this Chapter is given in the Table 5.2.

The catalysts used in this Chapter have been prepared using a Fe nitrate precursor, in comparison to the use of Fe citrate precursor in other Chapters. Differences in the FTS reaction performance of catalyst materials prepared with the nitrate and citrate precursors by impregnating the $\alpha-\mathrm{Al}_{2} \mathrm{O}_{3}$ support have been studied earlier by Torres Galvis et al..48

\subsubsection{Morphological Changes Probed with Transmission Electron Microscopy}

TEM images of the Na-S Calcin. and $\mathrm{H}_{2}$ Fresh catalyst materials before FTS reaction are given in Figure 5.2. The TEM images taken after the Na-S Calcin. and $\mathrm{H}_{2}$ catalyst materials' use in the $72 \mathrm{~h}$ FTS reaction at 3, 10 or 20 bar are in turn given in Figure 5.3. The TEM images for the UP catalyst materials are not shown, as they did not differ in their morphology from their respective Na-S catalyst material counterparts. The TEMbased particle size distribution derived Fe particle number-weighted mean diameters with their respective size distribution Standard Deviations (STDs) for the UP and Na-S catalyst materials before (Fresh) and after $(3,10$ or 20 bar) FTS reaction are given in Figure 5.4. 
The Fe particles in the Na-S (and UP) catalyst materials before the FTS reaction are as clusters of $\mathrm{Fe}$ particles rather than as individual particles on the $\alpha-\mathrm{Al}_{2} \mathrm{O}_{3}$ support material (Figure 5.2). With regard to the catalyst morphologies observed with TEM after the 3,10 or 20 bar FTS reaction: in all of the Na-S (and UP) catalyst materials the Fe particles capsulated in carbon and have grown off from the $\alpha-\mathrm{Al}_{2} \mathrm{O}_{3}$ support (Figure 5.3). These TEM results are analogous with observations in Chapter 4, and with observations in literature for the used catalyst type. ${ }^{49}$

With increasing reaction pressure, for Na-S catalyst materials the Fe particle mean diameters and size distribution STDs increase in comparison to the respective UP catalysts (Figure 5.4). This behavior takes place irrespective to the catalyst materials prior Calcin. or $\mathrm{H}_{2}$ treatment. Similarly, after $4 \mathrm{~h}$ FTS reaction $\left(340^{\circ} \mathrm{C}, 20\right.$ bar, $\mathrm{H}_{2}$ :CO (1:1) Na-S promotion induced Fe particle growth has been observed in the literature.50 The precise reasons for this possible Na-S promotion induced Fe particle growth are not clear. Nevertheless, the particle growth does have a reaction pressure dependency (Figure 5.4).

\subsubsection{Phase Quantification with X-ray Powder Diffractometry/Rietveld Quantitative Phase Analysis}

The background corrected, ex-situ measured XRPD and Rietveld method calculated diffraction and difference patterns are given in Figure 5.5 for the UP and Na-S, Calcin. and $\mathrm{H}_{2}$ Fresh catalyst materials. The diffraction patterns for the catalyst materials measured after their application in the $72 \mathrm{~h}$ FTS reaction at reaction pressures of 3,10 or 20 bar, are given in Figure 5.6 for the UP, and in Figure 5.7 for the Na-S catalyst materials. Only the diffraction pattern range of $13-29^{\circ} 2 \theta\left(\mathrm{Mo} \mathrm{K}_{\alpha}\right)$ is visualized but the R-QPA fittings were done over the full XRPD measurement range of 6-39 20 .

The XRPD diffraction pattern derived R-QPA Fe phase quantification results for the crystalline Fe phases and the R-QPA derived elemental Fe loadings are given in Figure 5.10. The $\mathrm{R}_{\mathrm{wp}}$-values for all of the R-QPA fits are given in Figure 5.8. ${ }^{51} \mathrm{R}-\mathrm{QPA}$ determined Fe phase (volume-weighted) crystallite mean diameters are given in Figure 5.9. A detailed discussion on the Fe phase crystallite diameters is omitted in this Chapter.

The Rietveld method calculated diffraction patterns represent the measured XRPD patterns in overall well, for all of the studied catalyst materials, both before (Figure 5.5) and after the FTS reactions for all of the UP (Figure 5.6) and Na-S catalyst materials (Figure 5.7). That is, it is unlikely that any significant Fe phases are missing from the RQPA Fe phase quantifications in Figure 5.10.

However, for all of the UP and Na-S Calcin. catalyst materials, systematic misfits in the calculated relative diffraction peak intensities for the $\chi-\mathrm{Fe}_{5} \mathrm{C}_{2}$ phase could be 

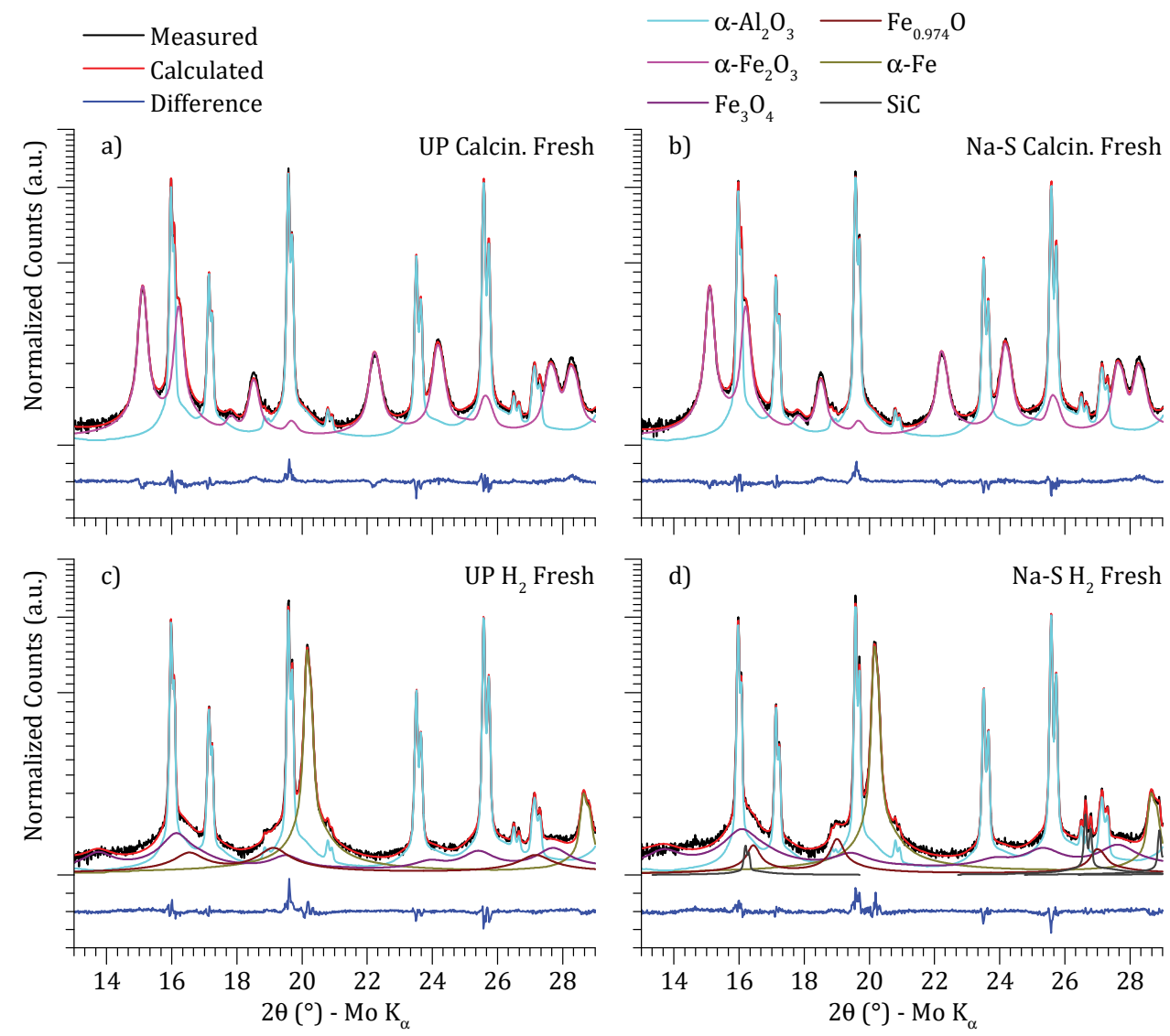

Figure 5.5

Background corrected, ex-situ measured X-ray Powder Diffractometry (XRPD) and Rietveld method calculated diffraction patterns over a range $13-29^{\circ} 2 \theta$ for UP and $\mathrm{Na}-\mathrm{S}\left(\mathrm{Fe}(-\mathrm{Na}-\mathrm{S}) / \alpha-\mathrm{Al}_{2} \mathrm{O}_{3}\right)$ catalyst materials before the Fischer-Tropsch Synthesis (FTS) reaction (Fresh). Diffraction patterns for calcined only (Calcin.) a) UP and b) Na-S, and for $\mathrm{H}_{2}$ reduced $\left(\mathrm{H}_{2}\right)$ c) UP and d) Na-S catalyst materials. Normalized counts on a square-root scale.

observed. Examples of these misfits are marked with $*$ for phases in UP (Figure 5.6a, $\boldsymbol{c}, \boldsymbol{e}$ ) and Na-S catalysts (Figure 5.7a, $\boldsymbol{c}, \boldsymbol{e}$ ). The misfits will be discussed more later on; when the $\chi-\mathrm{Fe}_{5} \mathrm{C}_{2}$ phase crystal lattice changes are discussed. Furthermore, imperfect fitting of large crystallite $\mathrm{SiC}$ residue phases caused large $\mathrm{R}_{\mathrm{wp}}-$ values for the UP Calcin. 3, 10 and 20 bar catalyst materials, as well as for the UP $\mathrm{H}_{2}$ 10 bar catalyst material (Figure 5.8).

Each of the FTS reaction used catalysts' duplicate reactors were also measured with XRPD and quantified with R-QPA. The results (data not shown) were well comparable to the Fe phase quantification results in Figure 5.10. 

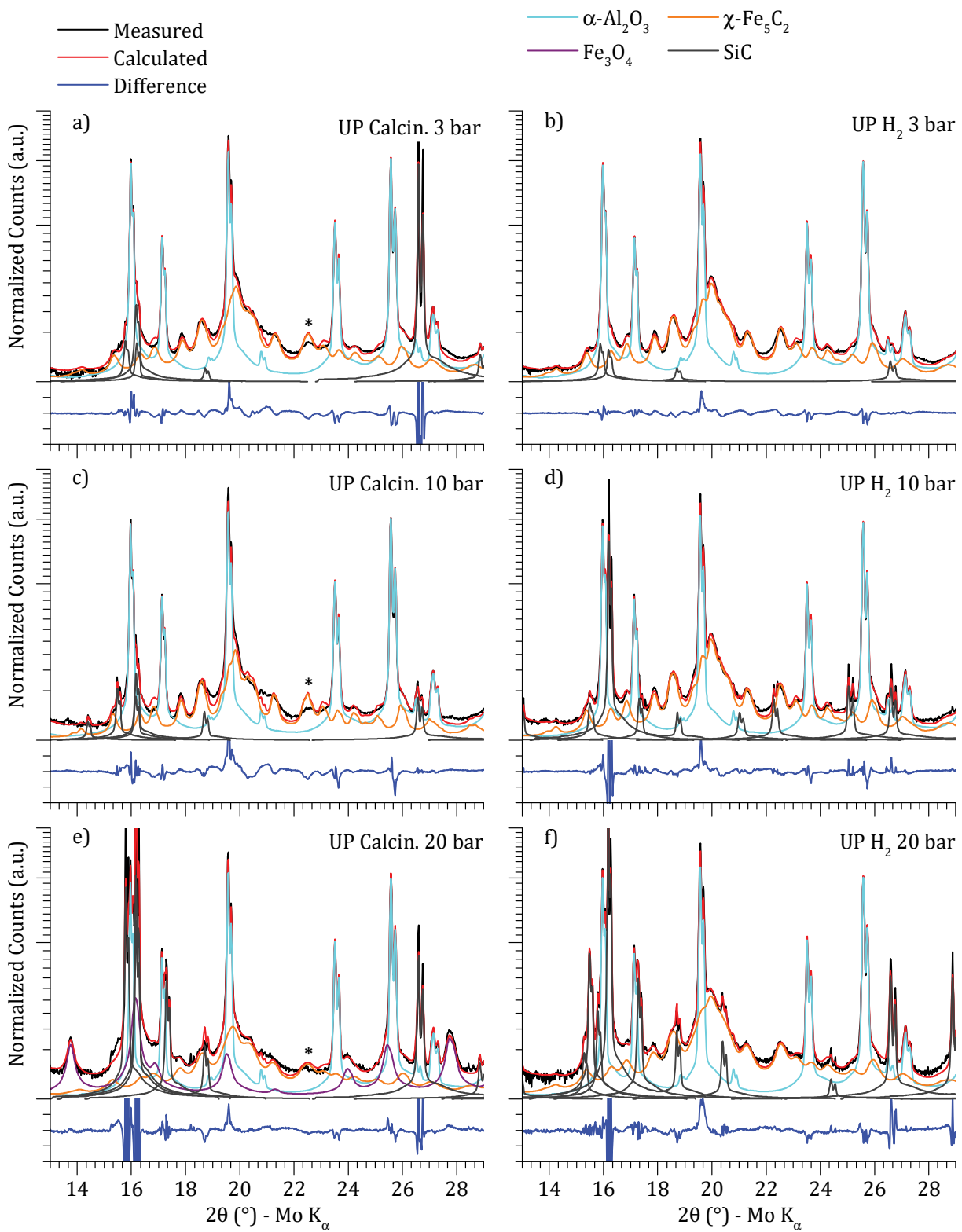

Figure 5.6

Background corrected, ex-situ measured X-ray Powder Diffractometry (XRPD) and Rietveld method calculated diffraction patterns over a range $13-29^{\circ} 2 \theta$ for UP $\left(\mathrm{Fe} / \alpha-\mathrm{Al}_{2} \mathrm{O}_{3}\right)$ catalysts after $72 \mathrm{~h}$ FischerTropsch Synthesis (FTS) reaction (3, 10, 20 bar). Calcined catalysts (Calcin.) after a) 3 bar, c) 10 bar and e) 20 bar, and $\mathrm{H}_{2}$ reduced $\left(\mathrm{H}_{2}\right)$ after b) 3 bar, d) 10 bar and f) 20 bar FTS reaction. Peak intensity misfit $\left.{ }^{*}\right)$ for $\chi$ $\mathrm{Fe}_{5} \mathrm{C}_{2}$ phase. Normalized counts on a square-root scale. 

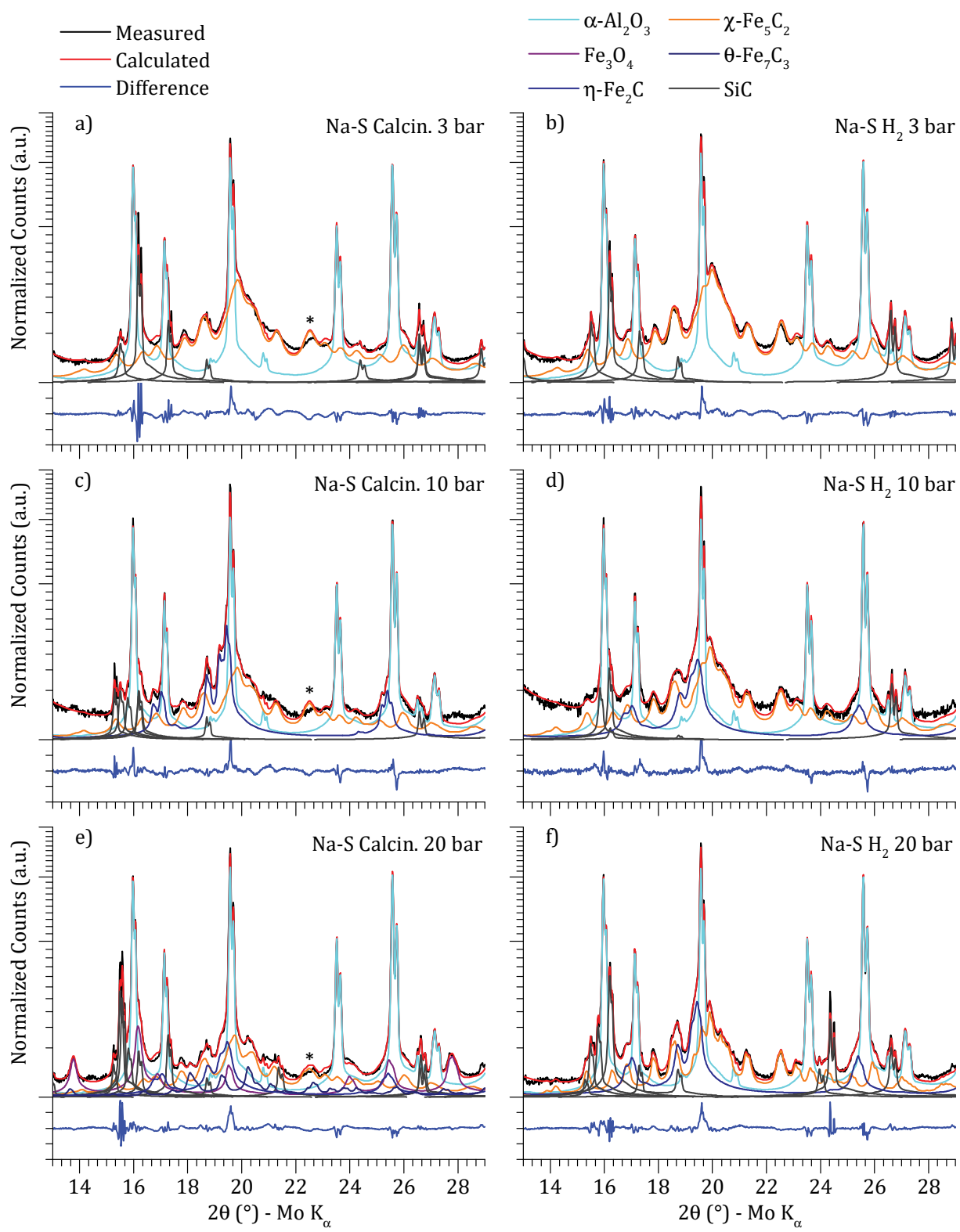

\section{Figure 5.7}

Background corrected, ex-situ measured X-ray Powder Diffractometry (XRPD) and Rietveld method calculated diffraction patterns over a range $13-29^{\circ} 2 \theta$ for $\mathrm{Na}-\mathrm{S}\left(\mathrm{Fe}-\mathrm{Na}-\mathrm{S} / \alpha-\mathrm{Al}_{2} \mathrm{O}_{3}\right)$ materials after $72 \mathrm{~h}$ Fischer-Tropsch Synthesis (FTS) reaction (3, 10, 20 bar). Calcined catalysts (Calcin.) after a) 3 bar, c) 10 bar and e) 20 bar and $\mathrm{H}_{2}$ reduced $\left(\mathrm{H}_{2}\right)$ after b) 3 bar, d) 10 bar and f) 20 bar FTS reaction. Peak intensity misfit $\left({ }^{*}\right)$ for $\chi-\mathrm{Fe}_{5} \mathrm{C}_{2}$ phase. Normalized counts on a square-root scale. 

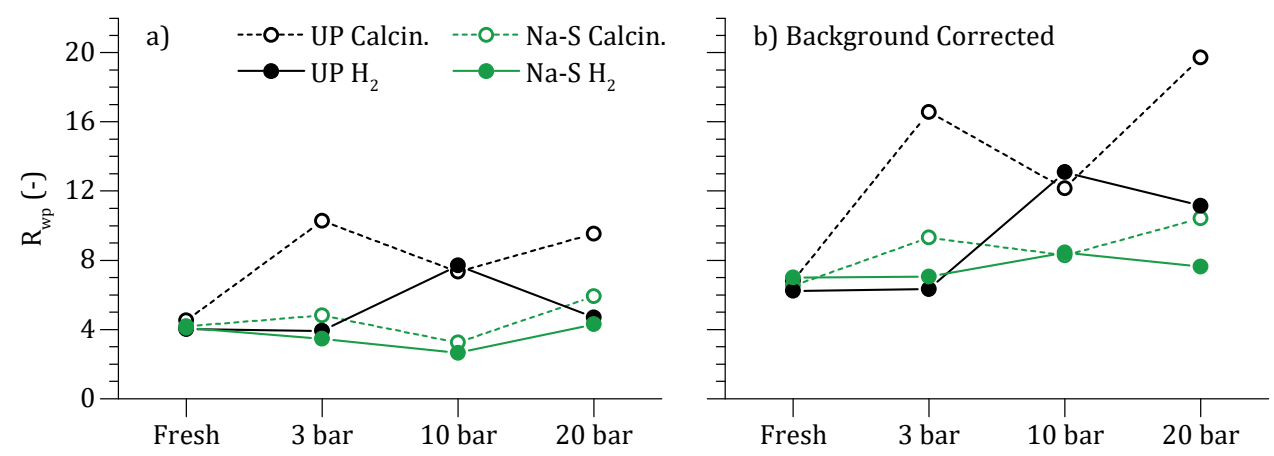

Figure 5.8

$\mathrm{R}_{\mathrm{wp}}$-values for the differences between the X-ray Powder Diffractometry (XRPD) measured and Rietveld method calculated diffractograms, for calcined only (Calcin.) and $\mathrm{H}_{2}$ reduced catalysts $\left(\mathrm{H}_{2}\right)$, before (Fresh) and after the $72 \mathrm{~h}$ Fischer-Tropsch Synthesis (FTS) reactions $(3,10,20 \mathrm{bar})$. $\mathrm{R}_{\mathrm{wp}}$-values for UP and Na-S (Fe($\mathrm{Na}-\mathrm{S}) / \alpha-\mathrm{Al}_{2} \mathrm{O}_{3}$ ) catalyst materials a) without and b) with diffraction pattern background correction.

The R-QPA crystalline Fe phase quantification derived elemental Fe wt-\%s vary somewhat: the average Fe wt-\%s with STDs are $13.2 \pm 2.1 \mathrm{wt}-\%$ over all of the UP catalysts and $14.2 \pm 2.4$ wt-\% over all of the Na-S catalysts materials (Figure 5.10c, $f$ ).

The high STDs for the elemental Fe wt-\%s can be taken as an indication of some inaccuracy in the R-QPA Fe phase quantifications. This is, likely, mainly due to the interference from the residual $\mathrm{SiC}$ content and a rather complex diffraction pattern background.

However, the identified Fe phases present in the R-QPA quantifications are correct as the diffraction peaks were well accounted for in the quantified XRPD patterns. Whether any elemental Fe was lost from any of the catalyst materials during the FTS reactions, cannot reliably be deduced from the R-QPA quantification results for the studied catalyst materials.

\subsubsection{Crystalline Fe Phase Quantifications}

For the UP and Na-S Calcin. Fresh catalyst materials, the only Fe phase present is $\alpha$ $\mathrm{Fe}_{2} \mathrm{O}_{3}$ (hematite), as would be expected after a calcining treatment (Figure 5.10a-b). Both, the UP and Na-S $\mathrm{H}_{2}$ Fresh catalyst materials exhibit $\alpha$-Fe as the major phase (64$68 \%$ ), with some oxidic $\mathrm{Fe}$ as $\mathrm{Fe}_{3} \mathrm{O}_{4}$ (magnetite) and $\mathrm{Fe}_{0.974} \mathrm{O}$ (wüstite) also present (Figure 5.10d-e). Alkali stabilizes the $\mathrm{Fe}_{0.974} \mathrm{O}$ phase, ${ }^{52}$ explaining its presence in the $\mathrm{Na}-\mathrm{S} \mathrm{H}_{2}$ Fresh catalyst material. The presence of $\mathrm{Fe}_{0.974} \mathrm{O}$ in the UP $\mathrm{H}_{2}$ Fresh catalyst material might indicate occurrence of some impurities, e.g. the alkali or Si,52,53 stabilizing the $\mathrm{Fe}_{0.974} \mathrm{O}$ phase.

With regard to the crystalline Fe phases in the catalysts' Fe phase compositions after the FTS reactions, three Fe carbide phases can be quantified: $\eta-\mathrm{Fe}_{2} \mathrm{C}$ Pnnm (58), $\chi-\mathrm{Fe}_{5} \mathrm{C}_{2}$ 

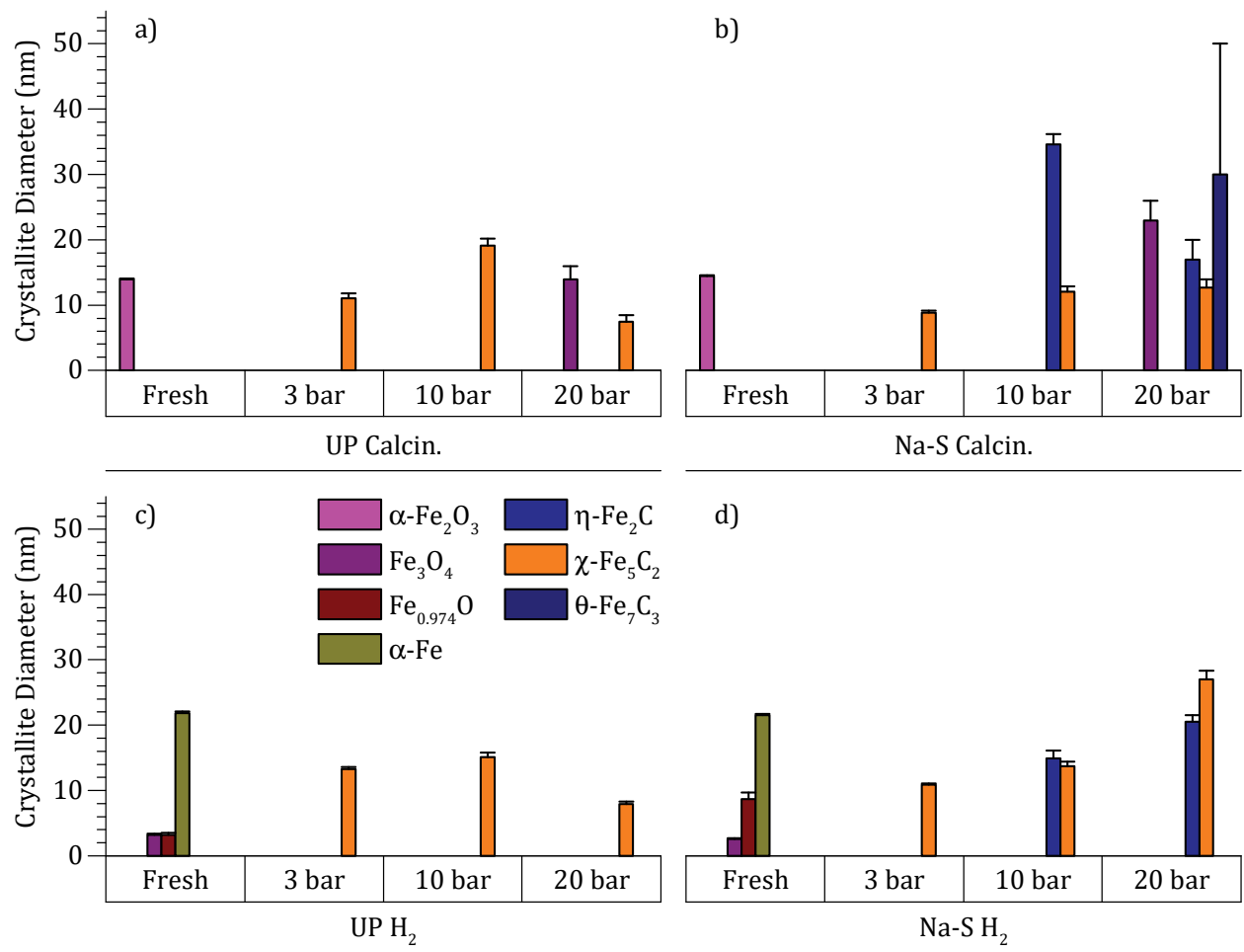

d)

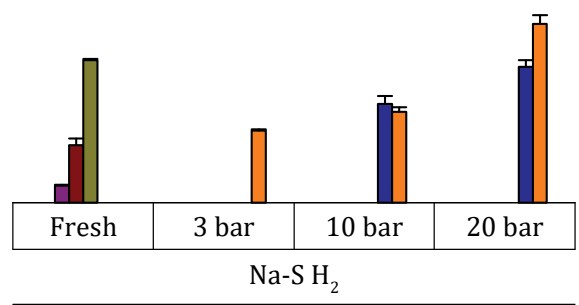

\section{Figure 5.9}

Rietveld Quantitative Phase Analysis (R-QPA) determined Fe phase (volume-weighted) crystallite mean diameters before (Fresh) and after $72 \mathrm{~h}$ Fischer-Tropsch Synthesis (FTS) reactions (3, 10, 20 bar). Crystallite diameters for UP ( $\left.\mathrm{Fe} / \alpha-\mathrm{Al}_{2} \mathrm{O}_{3}\right)$ catalyst materials with a) calcining only (Calcin.) and c) $\mathrm{H}_{2}$ reduction $\left(\mathrm{H}_{2}\right)$, and for Na-S (Fe-Na-S $/ \alpha-\mathrm{Al}_{2} \mathrm{O}_{3}$ ) materials after b) Calcin. and d) $\mathrm{H}_{2}$ treatments.

$C 2 / c$ (15) (the Hägg carbide) and $\theta-\mathrm{Fe}_{7} \mathrm{C}_{3} P$ Pna (62) (the Eckström-Adcock carbide) (Figure 5.10a-b, d-e). The identification and nomenclature of the Fe carbide phases is discussed in detail in Chapter 2.

After the 3 bar FTS reaction, only the $\chi-\mathrm{Fe}_{5} \mathrm{C}_{2}$ phase is detected as a Fe carbide and as the sole $\mathrm{Fe}$ phase regardless of the $\mathrm{Na}-\mathrm{S}$ promotion or the Calcin. or $\mathrm{H}_{2}$ catalyst treatments (Figure 5.10a-b, d-e). After 10 bar FTS reaction, the addition of Na-S promotion induces the appearance of the $\eta-\mathrm{Fe}_{2} \mathrm{C}$ alongside the $\chi-\mathrm{Fe}_{5} \mathrm{C}_{2}$ phase. The $\mathrm{Na}(-$ S) promotion's $\eta-\mathrm{Fe}_{2} \mathrm{C}$ phase stabilizing effect in the FTS reaction is in agreement to " $\varepsilon$ carbide" (i.e. $\eta-\mathrm{Fe}_{2} \mathrm{C}$ ) stabilizing alkali effect observations in literature. ${ }^{16,32,54}$ The expected maximum thermal stability for the $\eta-\mathrm{Fe}_{2} \mathrm{C}$ phase is $\lesssim 300^{\circ} \mathrm{C}$ (Chapter 3), but with the applied Na-S promotion, the stability is increased, at least, to the used FTS reaction temperature of $340^{\circ} \mathrm{C}$.

In the Na-S Calcin. 10 bar catalyst material the $\eta-\mathrm{Fe}_{2} \mathrm{C}$ phase contribution is higher 

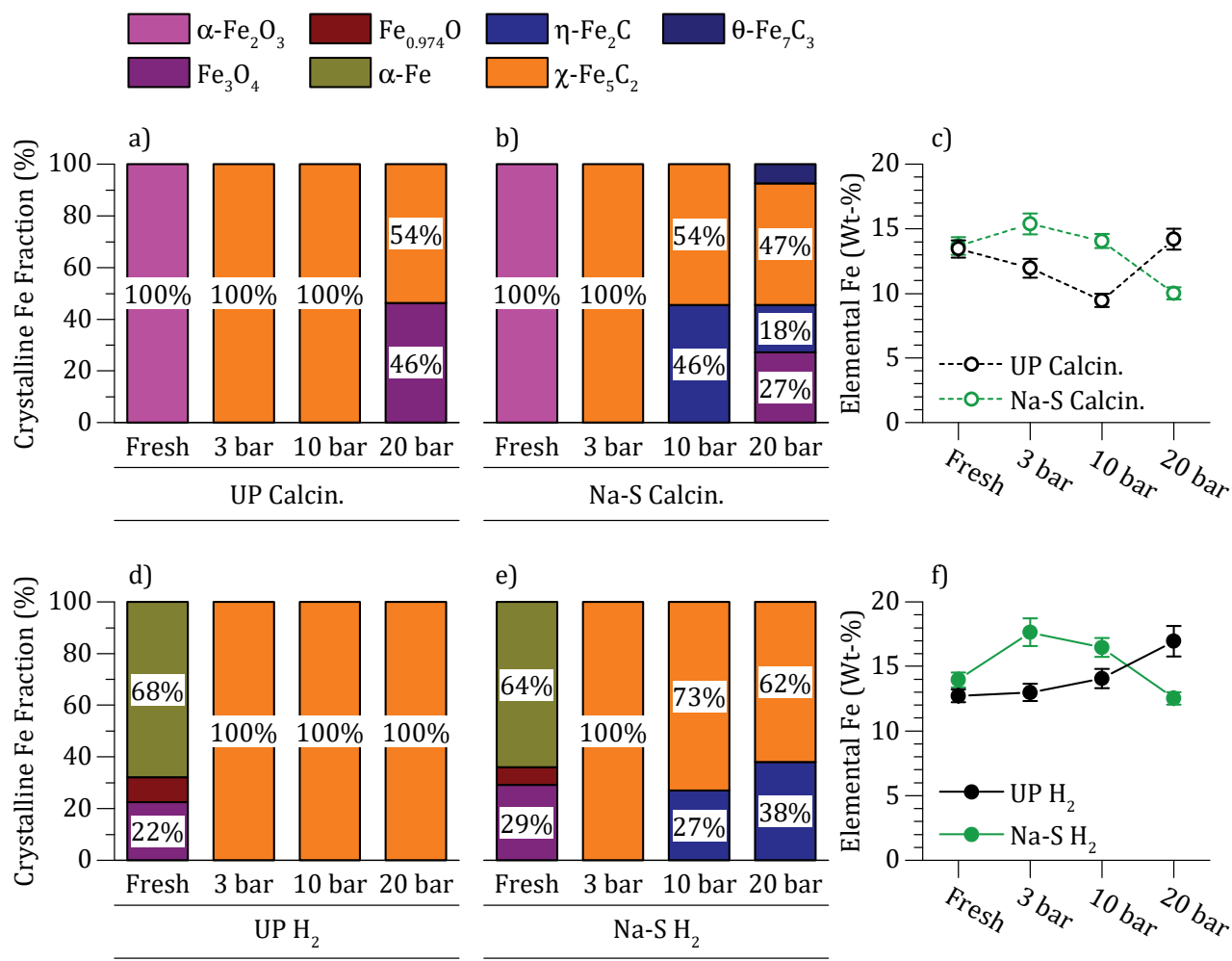

Figure 5.10

Rietveld Quantitative Phase Analysis (R-QPA) Fe phase quantifications before (Fresh) and after 72 h FischerTropsch Synthesis (FTS) reactions $\left(3,10,20\right.$ bar) for calcined only (Calcin.) and $\mathrm{H}_{2}$ reduced $\left(\mathrm{H}_{2}\right)$ catalyst materials. Crystalline Fe phase fractions for UP $\left(\mathrm{Fe} / \alpha-\mathrm{Al}_{2} \mathrm{O}_{3}\right)$ a) Calcin. and d) $\mathrm{H}_{2}$, and for Na-S (Fe-Na-S/ $\alpha-$ $\mathrm{Al}_{2} \mathrm{O}_{3}$ ) b) Calcin. and e) $\mathrm{H}_{2}$ catalyst materials. The elemental $\mathrm{Fe}$ wt-\% loadings on basis of the crystalline $\mathrm{Fe}$ phases for c) Calcin. and f) $\mathrm{H}_{2}$ materials. Percentage labels cut-off is set to $<10 \%$.

(46\%) than in the Na-S $\mathrm{H}_{2} 10$ bar catalyst (27\%). That is, $\alpha-\mathrm{Fe}_{2} \mathrm{O}_{3}$ as the FTS reaction and carburization starting material favors the formation or the FTS reaction stability of the $\eta-\mathrm{Fe}_{2} \mathrm{C}$ phase. As no $\eta-\mathrm{Fe}_{2} \mathrm{C}$ stabilizing effect with the Na-S addition is observed for the 3 bar FTS catalyst materials (Figure 5.10a-b, $\boldsymbol{d}-\boldsymbol{e}$ ): a >3 bar FTS reaction pressure is needed, for an unknown reason, for observing the $\eta-\mathrm{Fe}_{2} \mathrm{C}$ phase stabilization effect by $\mathrm{Na}-\mathrm{S}$ promotion addition. The $\eta-\mathrm{Fe}_{2} \mathrm{C}$ phase's stability dependency on the FTS reaction pressure and Na-S promotion was also observed with R-QPA independent Quasi-In-situ MAS measurements (Table 5.3).

It is worthy to emphasize the perfect fit of the Rietveld method calculated $\eta-\mathrm{Fe}_{2} \mathrm{C}$ phase in the XRPD patterns of the Na-S Calcin. 10 bar catalyst material (Figure 5.7c). Thus, the crystal structure solution by Hirotsu and Nagakura is a very good one for the 


\section{Table 5.3}

Quasi-In-situ Mössbauer Absorption Spectroscopy (MAS) fitted parameters for the UP and Na-S (Fe-(Na$\left.\mathrm{S}) / \alpha-\mathrm{Al}_{2} \mathrm{O}_{3}\right)$ catalyst materials. Catalyst reduction $\left(\mathrm{H}_{2}\right)$ was done at $350^{\circ} \mathrm{C}$ for $12 \mathrm{~h}$ under $\mathrm{H}_{2}: \mathrm{Ar} 2: 1$. FischerTropsch Synthesis (FTS) reaction conditions of $340^{\circ} \mathrm{C}, 3$ or 20 bar, $\mathrm{H}_{2}: \mathrm{CO}$ 1:1 for $48 \mathrm{~h}$, were used. MAS measurements were done at the liquid He temperature ( $4.2 \mathrm{~K})$.

\begin{tabular}{|c|c|c|c|c|c|c|}
\hline \multirow[t]{2}{*}{ Treatment } & Isomer Shift & $\begin{array}{l}\text { Quadrupole } \\
\text { Splitting }\end{array}$ & $\begin{array}{l}\text { Hyperfine } \\
\text { Field }\end{array}$ & Line Width & \multirow[t]{2}{*}{ Phase } & $\begin{array}{c}\text { Spectral } \\
\text { Contribution }\end{array}$ \\
\hline & $\left(\mathrm{mm} \mathrm{s}^{-1}\right)$ & $\left(\mathrm{mm} \mathrm{s}^{-1}\right)$ & $(\mathrm{T})$ & $\left(\mathrm{mm} \mathrm{s}^{-1}\right)$ & & $(\%)$ \\
\hline \multirow[t]{5}{*}{$\mathrm{UP} \mathrm{H}_{2}$} & 0.24 & -0.03 & 50.8 & 0.41 & $\mathrm{Fe}_{3} \mathrm{O}_{4}(\mathrm{I})$ & 36 \\
\hline & 0.54 & 0.04 & 51.7 & 0.60 & $\mathrm{Fe}_{3} \mathrm{O}_{4}$ (II) & 31 \\
\hline & 0.84 & -0.74 & 48.0 & 0.60 & $\mathrm{Fe}_{3} \mathrm{O}_{4}$ (III) & 16 \\
\hline & 0.77 & 0.84 & 41.1 & 0.60 & $\mathrm{Fe}_{3} \mathrm{O}_{4}(\mathrm{IV})$ & 8 \\
\hline & 1.04 & -0.01 & 46.7 & 0.60 & $\mathrm{Fe}_{3} \mathrm{O}_{4}(\mathrm{~V})$ & 9 \\
\hline UP $\mathrm{H}_{2}$ & 0.26 & - & 25.6 & 0.39 & $\chi-\mathrm{Fe}_{5} \mathrm{C}_{2}(\mathrm{I})$ & 41 \\
\hline \multirow[t]{2}{*}{3 bar } & 0.16 & - & 21.5 & 0.50 & $\chi-\mathrm{Fe}_{5} \mathrm{C}_{2}$ (II) & 37 \\
\hline & 0.20 & - & 12.9 & 0.48 & $\chi-\mathrm{Fe}_{5} \mathrm{C}_{2}$ (III) & 22 \\
\hline UP $\mathrm{H}_{2}$ & 0.24 & "'-' & 25.4 & 0.39 & $\chi-\mathrm{Fe}_{5} \mathrm{C}_{2}(\mathrm{I})$ & 40 \\
\hline \multirow[t]{2}{*}{20 bar } & 0.18 & - & 22.0 & 0.52 & $\chi-\mathrm{Fe}_{5} \mathrm{C}_{2}$ (II) & 38 \\
\hline & 0.19 & - & 13.5 & 0.56 & $\chi-\mathrm{Fe}_{5} \mathrm{C}_{2}$ (III) & 22 \\
\hline $\mathrm{Na}-\mathrm{S} \mathrm{H} 2$ & 0.00 & - & 34.2 & 0.34 & $\mathrm{Fe}^{0}$ & 100 \\
\hline $\mathrm{Na}-\mathrm{S} \mathrm{H}{ }_{2}$ & 0.24 & - & 25.4 & 0.61 & $\chi-\mathrm{Fe}_{5} \mathrm{C}_{2}(\mathrm{I})$ & 44 \\
\hline \multirow[t]{2}{*}{3 bar } & 0.17 & - & 21.0 & 0.61 & $\chi-\mathrm{Fe}_{5} \mathrm{C}_{2}$ (II) & 33 \\
\hline & 0.13 & - & 12.8 & 0.61 & $\chi-\mathrm{Fe}_{5} \mathrm{C}_{2}$ (III) & 23 \\
\hline $\mathrm{Na}-\mathrm{S} \mathrm{H}{ }_{2}$ & 0.26 & "'-"' & 18.3 & 0.35 & $\eta-\mathrm{Fe}_{2} \mathrm{C}$ & 60 \\
\hline \multirow[t]{3}{*}{20 bar } & 0.27 & - & 25.7 & 0.43 & $\chi-\mathrm{Fe}_{5} \mathrm{C}_{2}(\mathrm{I})$ & 17 \\
\hline & 0.19 & - & 20.7 & 0.44 & $\chi-\mathrm{Fe}_{5} \mathrm{C}_{2}$ (II) & 13 \\
\hline & 0.22 & - & 10.1 & 0.41 & $\chi-\mathrm{Fe}_{5} \mathrm{C}_{2}$ (III) & 10 \\
\hline
\end{tabular}

Experimental uncertainties: Isomer Shift $\pm 0.02 \mathrm{~mm} \mathrm{~s}^{-1}$, Quadrupole Splitting $\pm 0.02 \mathrm{~mm} \mathrm{~s}^{-1}$, Line Width $\pm 0.03 \mathrm{~mm} \mathrm{~s}^{-1}$, Hyperfine Field $\pm 0.1 \mathrm{~T}$, Spectral Contribution $\pm 3 \%$.

carbide. ${ }^{55,56}$ The $\eta-\mathrm{Fe}_{2} \mathrm{C}$ phase corresponds to the "pseudo-hexagonal Fe carbide" observed by Barton and Gale in a Fe-based catalyst material after the FTS reaction. ${ }^{57,58}$

After the 20 bar FTS reaction, the $\eta-\mathrm{Fe}_{2} \mathrm{C}$ is present with the $\chi-\mathrm{Fe}_{5} \mathrm{C}_{2}$ phase for both $\mathrm{Na}-\mathrm{S}$ Calcin. and $\mathrm{Na}-\mathrm{S} \mathrm{H}_{2}$ catalyst materials. Thus, the $\eta-\mathrm{Fe}_{2} \mathrm{C}$ phase's presence after the FTS reaction again requires the added Na-S promotion (Figure 5.10a-b, d-e). Intriguingly, in the Na-S Calcin. 20 bar catalyst material; a small amount (8\%) of the rare $\theta-\mathrm{Fe}_{7} \mathrm{C}_{3}$ phase is quantified (Figure 5.10b). This observation is interesting, as the $\theta-\mathrm{Fe}_{7} \mathrm{C}_{3}$ phase only appears with the Na-S promoted catalyst material but not with the respective unpromoted UP Calcin. 20 bar catalyst.

In Chapter 3, with CO carburization of an initial $\alpha-\mathrm{Fe}_{2} \mathrm{O}_{3}\left(/ \alpha-\mathrm{Al}_{2} \mathrm{O}_{3}\right) \mathrm{Fe}$ phase at $25 \mathrm{bar}$, the $\mathrm{Na}-\mathrm{S}$ promotion was not required for the formation of the $\theta-\mathrm{Fe}_{7} \mathrm{C}_{3}$ phase. The $\theta-\mathrm{Fe}_{7} \mathrm{C}_{3}$ phase formed from the oxide precursor regardless of the Na-S promotion. In a comparable manner to the Na-S addition requirement, Tajima and Hirano observed 
formation of $\theta-\mathrm{Fe}_{7} \mathrm{C}_{3}$ with $\mathrm{CO}$ carburization of $\mathrm{Ba}$ promoted $\mathrm{Fe}_{3} \mathrm{O}_{4}$. However, without $\mathrm{Ba}$, no $\theta-\mathrm{Fe}_{7} \mathrm{C}_{3}$ phase was formed. ${ }^{59}$ As a similarity to the results in Chapter 3, only the $\mathrm{Na}-\mathrm{S}$ Calcin. catalyst material forms any of the $\theta-\mathrm{Fe}_{7} \mathrm{C}_{3}$ phase. That is, a Fe oxide precursor is required for the $\theta-\mathrm{Fe}_{7} \mathrm{C}_{3}$ phase formation also in this Chapter. The topic of the $\theta-\mathrm{Fe}_{7} \mathrm{C}_{3}$ phase formation is more thoroughly discussed in Chapter 3. Nevertheless, the rare $\theta-\mathrm{Fe}_{7} \mathrm{C}_{3}$ phase may form under the relatively mild 20 bar FTS reaction conditions applied in this Chapter, and use of an industrial scale FTS reactor is not required. ${ }^{4}$

Moreover, after the 20 bar FTS reaction, the UP and Na-S Calcin. catalyst materials exhibit significant amounts of $\mathrm{Fe}_{3} \mathrm{O}_{4}$. In contrary, the $\mathrm{Fe}_{3} \mathrm{O}_{4}$ phase is absent from the $\mathrm{H}_{2}$ reduced, UP and Na-S $\mathrm{H}_{2} 20$ bar catalyst materials (Figure 5.10a-b, $\boldsymbol{d}-\boldsymbol{e}$ ). In the 20 bar FTS reaction, the initial $\alpha-\mathrm{Fe}_{2} \mathrm{O}_{3}\left(/ \alpha-\mathrm{Al}_{2} \mathrm{O}_{3}\right)$ phase may not be fully reduced to zerovalent $\alpha$-Fe but converts to $\mathrm{Fe}_{3} \mathrm{O}_{4}$ at the FTS reaction temperature, and thus is not subsequently fully carburized. A possible explanation could be in mass transport limitations. With the 20 bar FTS reaction pressure and the used $\mathrm{H}_{2}: \mathrm{CO}$ 1:1 ratio, the likely high initial inactive carbon deposition for the catalyst, ${ }^{49}$ might prevent the diffusion of the reducing $\mathrm{H}_{2}: \mathrm{CO}$ atmosphere into the inner parts of the catalyst particles. Alternatively, carbon deposition might create preferential flows to the catalyst bed preventing parts of it being reduced.

Fe carbide reoxidation by the oxidative $\mathrm{H}_{2} \mathrm{O}$ from the FTS reaction or by $\mathrm{CO}_{2}$ from the Water-gas Shift (WGS) reaction,4,16,36,54,60-65 is not a plausible explanation for the $\mathrm{Fe}_{3} \mathrm{O}_{4}$ formation in the UP and Na-S Calcin. 20 bar catalyst materials. The UP and Na-S $\mathrm{H}_{2} 20$ bar catalyst materials have higher or equal Fe Time Yields (FTYs) (Figure 5.13), Usage Ratios $\mathrm{H}_{2}$ : $\mathrm{CO}$ (Figure 5.14b) and $\mathrm{CO}_{2}$ selectivities (Figure 5.15e) in comparison to the UP and Na-S Calcin. 20 bar catalysts. Thus, all of the $\mathrm{H}_{2}$ and Calcin. 20 bar catalysts have comparable $\mathrm{H}_{2} \mathrm{O}$ and $\mathrm{CO}_{2}$ concentrations in the reactor gas atmosphere. Despite this, the $\mathrm{H}_{2} 20$ bar catalyst material does not show any signs of $\mathrm{Fe}_{3} \mathrm{O}_{4}$ as a carbide

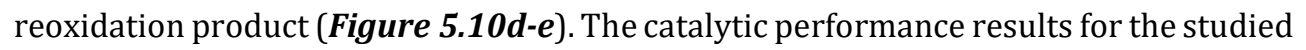
catalyst materials will be discussed more in a later section.

The addition of elements from the alkali metal group, e.g. as in Na-S promotion, increases the Fe oxides reducibility by $\mathrm{CO}$ at low temperatures $\left(\approx 270^{\circ} \mathrm{C}\right) .{ }^{66}$ In the Na-S Calcin. 20 bar catalyst (Figure 5.10a-b): a lesser amount of $\mathrm{Fe}_{3} \mathrm{O}_{4}(27 \%)$ observed in comparison to $\mathrm{Fe}_{3} \mathrm{O}_{4}(46 \%)$ in the UP Calcin. 20 bar catalyst material. This can be explained by more efficient $\alpha-\mathrm{Fe}_{2} \mathrm{O}_{3}\left(/ \mathrm{Fe}_{3} \mathrm{O}_{4}\right)$ low temperature reduction upon the catalyst exposure to the reducing $\mathrm{H}_{2}: \mathrm{CO}$ mixture at $270^{\circ} \mathrm{C}$, when the reactor is pressurized for the FTS reaction (Experimental methods). When the reactor temperature is ramped to $\gtrsim 300^{\circ} \mathrm{C}$, inactive carbon deposition becomes significant, $4,30,67,68$ and possible mass transportation limitations for the $\mathrm{H}_{2}$ :CO gas 
mixture might begin to appear, therefore hindering Fe phases reduction.

Before moving on, the discussion on the Fe phase quantifications so far can be summarized as: i) Na-S promotion stabilizes the $\eta-\mathrm{Fe}_{2} \mathrm{C}$ phase when FTS reaction pressure $\geq 10$ bar is applied, while after 3 bar FTS reaction, no $\eta-\mathrm{Fe}_{2} \mathrm{C}$ phase was present regardless of the Na-S promotion; ii) the $\theta-\mathrm{Fe}_{7} \mathrm{C}_{3}$ phase could only be observed in the Na-S Calcin. 20 bar catalyst material; and iii) only in UP and Na-S Calcin. 20 bar catalyst materials could $\mathrm{Fe}_{3} \mathrm{O}_{4}$ be observed. The presence of Na-S promotion reduced the amount of the quantified $\mathrm{Fe}_{3} \mathrm{O}_{4}$ phase in the Calcin. 20 bar catalysts.

\subsubsection{Fe Phase Lattice Changes}

As discussed in Chapter 4, both of the $\eta-\mathrm{Fe}_{2} \mathrm{C}$ and $\chi-\mathrm{Fe}_{5} \mathrm{C}_{2}$ phases can be categorized by their crystal lattice parameter $a$ 's into different carbide subtypes. The lattice parameters used for the R-QPA fits for the $\eta-\mathrm{Fe}_{2} \mathrm{C}$ and $\chi-\mathrm{Fe}_{5} \mathrm{C}_{2}$ phases within the current Chapter are given in Figure 5.11 (for $\eta-\mathrm{Fe}_{2} \mathrm{C}$ ) and Figure 5.12 (for $\chi-\mathrm{Fe}_{5} \mathrm{C}_{2}$ ). Markers for the carbide subtype defining lattice parameter $a$ 's are included in the figures. For the single observation of the $\theta-\mathrm{Fe}_{7} \mathrm{C}_{3}$ phase in the Na-S Calcin. 20 bar catalyst material, lattice parameters of $a$ : $4.537 \pm 0.007 \AA$, $b: 6.900 \pm 0.016 \AA$ and $c: 11.910 \pm 0.030 \AA$ were used, where the value after \pm is the R-QPA fit error estimate.

For the $\eta-\mathrm{Fe}_{2} \mathrm{C}$ phase, which was only observed in the presence of the added Na-S promotion, the lattice parameters $a$ are close to the $\eta-\mathrm{Fe}_{2} \mathrm{C}$ Type II for both the Calcin. and $\mathrm{H}_{2}$ catalyst materials (Figure 5.11a). The $\eta-\mathrm{Fe}_{2} \mathrm{C}$ Type II (a: $4.86 \pm 0.01 \AA$, $b$ : $4.35 \pm 0.00 \AA, c: 2.76 \pm 0.01 \AA$, with STDs) is expected to form in the FTS reaction with NaS promotion, while the Type I ( $a$ : $4.84 \pm 0.01 \AA, b: 4.35 \pm 0.00 \AA, c: 2.76 \pm 0.00 \AA$ ) is expected to form under $\mathrm{CO}$ carburization regardless of the Na-S promotion (Chapter 4). Thus, as would be expected, only a $\eta-\mathrm{Fe}_{2} \mathrm{C}$ Type II carbide is observed after the FTS reactions (Figure 5.11a). The lattice parameters for the $\eta-\mathrm{Fe}_{2} \mathrm{C}$ Type II correspond to the "pseudo-hexagonal Fe carbide" observed by Barton and Gale,57 with lattice parameters defined by Hirotsu and Nagakura (a: $4.862 \AA$, $b: 4.360 \AA, c: 2.755 \AA$ )..$^{56}$

Although, after the 10 bar FTS reaction for both Na-S Calcin. and Na-S $\mathrm{H}_{2}$ catalyst materials, the lattice parameter $a$ is larger ( $a$ : 4.88 $\pm 0.00 \AA$, with STD) than what would be expected for the $\eta-\mathrm{Fe}_{2} \mathrm{C}$ Type II (Figure 5.11a). As mentioned before, the Rietveld method calculated $\eta-\mathrm{Fe}_{2} \mathrm{C}$ phase pattern is especially well fitting to the XRPD pattern for the Na-S Calcin. 10 bar material (Figure 5.7c). The larger lattice parameter $a$ is therefore not due to inaccurate peak position fit. We shall take the $\eta-\mathrm{Fe}_{2} \mathrm{C}$ phase with the larger lattice parameter $a$ of $4.88 \pm 0.00 \AA$ as a member of the subtype Type II i.e. the $\eta-\mathrm{Fe}_{2} \mathrm{C}$ subtype forming in the FTS reaction in the presence of Na-S promotion.

The $\chi-\mathrm{Fe}_{5} \mathrm{C}_{2}$ phase lattice parameter $a$ changes largely follow the carbide phase's subtype expectations set in Chapter 4 (Figure 5.12a). Type I (a: 11.56 $\pm 0.01 \AA$, $b$ : 

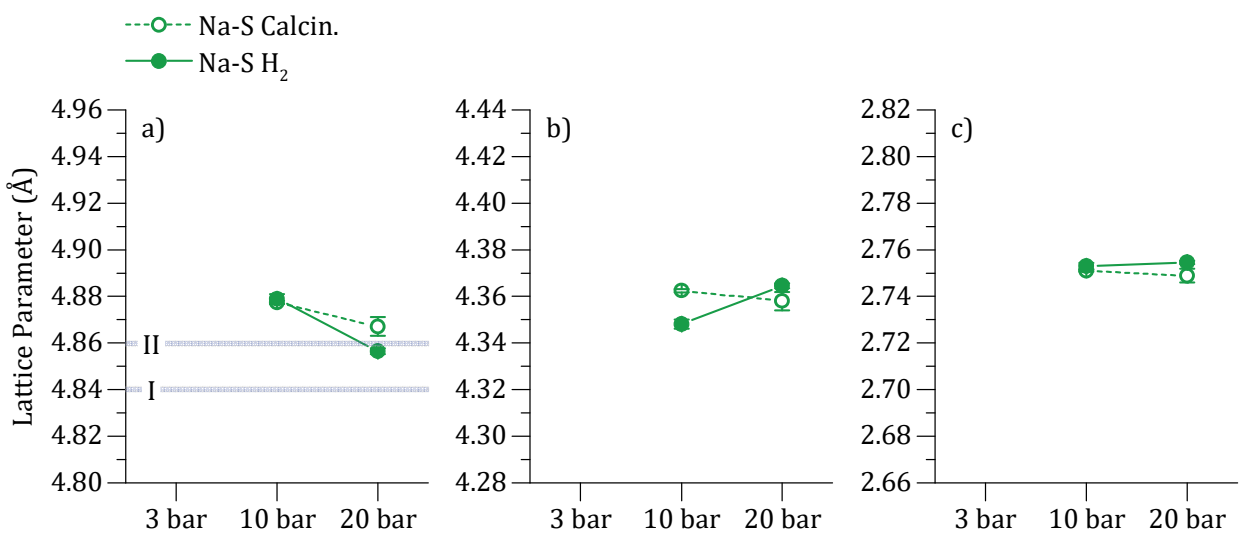

Figure 5.11

The lattice parameters used in Rietveld Quantitative Phase Analysis (R-QPA) for the $\eta-\mathrm{Fe}_{2} \mathrm{C}$ phase compared for Na-S (Fe-Na-S $/ \alpha-\mathrm{Al}_{2} \mathrm{O}_{3}$ ), calcined only (Calcin.) and $\mathrm{H}_{2}$ reduced $\left(\mathrm{H}_{2}\right)$, catalyst materials after $72 \mathrm{~h}$ FischerTropsch Synthesis (FTS) reactions (3, 10, 20 bar). Lattice parameters $a, b$, and $c$ in a), b) and c), respectively. Lattice parameters $a$ for the $\eta-\mathrm{Fe}_{2} \mathrm{C}$ subtypes I-II, as defined in Chapter 4, are also marked in a). No $\eta-\mathrm{Fe}_{2} \mathrm{C}$ formed during the FTS reactions without Na-S promotion.

$4.57 \pm 0.01 \AA, c: 5.06 \pm 0.00 \AA$ ) forms in the FTS reaction (or via CO carburization) without Na-S promotion. Type II ( $a: 11.58 \pm 0.00 \AA$, $b: 4.58 \pm 0.00 \AA$, $c: 5.05 \pm 0.00 \AA$ ) forms in the FTS reaction with Na-S promotion. Moreover, Type III ( $a: 11.61 \pm 0.01 \AA, b: 4.58 \pm 0.00 \AA$, c: $5.05 \pm 0.01 \AA$ ) forms in the FTS reaction (or via CO carburization) if a Fe oxide ( $\alpha$ $\mathrm{Fe}_{2} \mathrm{O}_{3}$ ) is used as the initial carburizing $\mathrm{Fe}$ phase regardless of the Na-S promotion. As observable discrepancies on the $\chi-\mathrm{Fe}_{5} \mathrm{C}_{2}$ subtype formation (Figure 5.12): i) with catalyst $\mathrm{Na}-\mathrm{S}_{2} 3$ bar, $\chi-\mathrm{Fe}_{5} \mathrm{C}_{2}$ Type I is formed instead of the expected Type II and ii) $\chi-\mathrm{Fe}_{5} \mathrm{C}_{2}$ Type III lattice distortion increases with increasing FTS reaction pressure with the UP and Na-S Calcin. catalyst materials.

First, as was the case for the Na-S promotion induced formation of the $\eta-\mathrm{Fe}_{2} \mathrm{C}$ phase - the phase was not observed after 3 bar FTS reaction (Figure 5.10) - the Na-S promotion requiring $\chi-\mathrm{Fe}_{5} \mathrm{C}_{2}$ Type II also requires higher than 3 bar FTS reaction pressure to form (Figure 5.12a). After 3 bar FTS reaction, only the $\chi-\mathrm{Fe}_{5} \mathrm{C}_{2}$ Type I is observed, regardless of the Na-S promotion. This is rather interesting, as will be discussed more in the next section when the catalytic performance data is treated, but shortly: Na-S $\mathrm{H}_{2} 3$ bar catalyst is only meagerly active in the FTS reaction while the Na$\mathrm{S} \mathrm{H}_{2} 10$ bar catalyst shows a significantly higher FTY. The FTS activity increase coincides with $\chi-\mathrm{Fe}_{5} \mathrm{C}_{2}$ subtype transition from Type I to Type II (Figure 5.12a). The crystal lattice change results point towards that Na-S promotion induced formation of $\chi-\mathrm{Fe}_{5} \mathrm{C}_{2}$ Type II is a FTS reaction pressure dependent phenomena, akin to the stabilization and appearance of the $\eta-\mathrm{Fe}_{2} \mathrm{C}$ phase. 

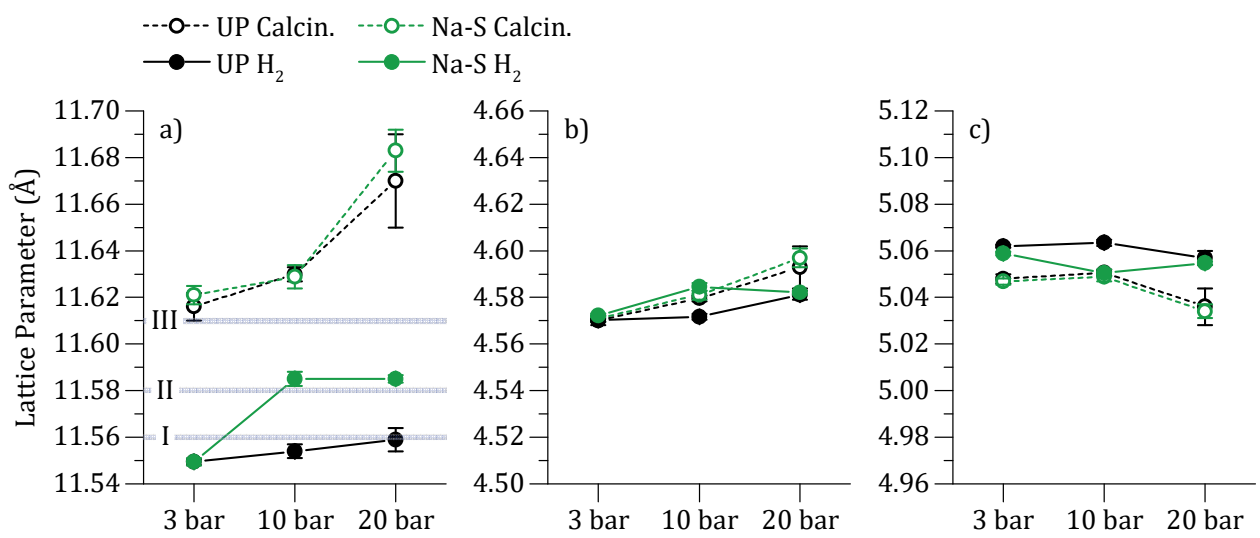

Figure 5.12

The lattice parameters used in Rietveld Quantitative Phase Analysis (R-QPA) for the $\chi-\mathrm{Fe}_{5} \mathrm{C}_{2}$ phase compared for UP and Na-S (Fe(-Na-S) $\left./ \alpha-\mathrm{Al}_{2} \mathrm{O}_{3}\right)$, calcined only (Calcin.) and $\mathrm{H}_{2}$ reduced $\left(\mathrm{H}_{2}\right)$, catalyst materials after $72 \mathrm{~h}$ Fischer-Tropsch Synthesis (FTS) reactions (3, 10, 20 bar). Lattice parameters $a, b$, and $c$ in a), b) and c), respectively. Lattice parameters $a$ for $\chi-\mathrm{Fe}_{5} \mathrm{C}_{2}$ subtypes I-III, as defined in Chapter 4, are also marked in a).

The formation of $\chi-\mathrm{Fe}_{5} \mathrm{C}_{2}$ Type III by carburizing a Fe oxide has been explained, possibly, to be due to the retained $\mathrm{O}$ atoms in the $\chi-\mathrm{Fe}_{5} \mathrm{C}_{2}$ lattice causing lattice distortion. ${ }^{30}$ The $\chi-\mathrm{Fe}_{5} \mathrm{C}_{2}$ Type III forms for all of the Calcin. catalyst materials tested in the FTS reactions, regardless of the Na-S promotion or the applied FTS reaction pressure (Figure 5.12a). The systematic shift in the most intense diffraction peak position for the $\chi-\mathrm{Fe}_{5} \mathrm{C}_{2}$ phase in Calcin., in comparison to $\mathrm{H}_{2}, 3,10$ and 20 bar catalyst materials is clear (Figure 5.6 and Figure 5.7). This peak shift is quantified as the change, especially, in the crystal lattice parameter $a$ 's for the $\chi-\mathrm{Fe}_{5} \mathrm{C}_{2}$ phase (Figure 5.12a).

The lattice parameter $a$ increases for the $\chi-\mathrm{Fe}_{5} \mathrm{C}_{2}$ phase in the Calcin. catalyst materials, in a nearly linear fashion, with the applied FTS reaction pressure (Figure 5.12a). In the earlier section, mass transfer limitations due to inactive carbon formation were offered as a possible explanation for the appearance of the $\mathrm{Fe}_{3} \mathrm{O}_{4}$ phase with the UP and Na-S Calcin. 20 bar catalysts (Figure 5.10a-b). Thus, perhaps, the fundamental origins of the increasing $\chi-\mathrm{Fe}_{5} \mathrm{C}_{2}$ Type III lattice parameter $a$ and the appearance of $\mathrm{Fe}_{3} \mathrm{O}_{4}$ with the 20 bar FTS reaction are the same. Increasing carbon deposition with increasing reaction pressure ${ }^{49}$ inhibits the removal of the 0 atoms from the initial $\mathrm{Fe}$ oxide $\left(\alpha-\mathrm{Fe}_{2} \mathrm{O}_{3} / \alpha-\mathrm{Al}_{2} \mathrm{O}_{3}\right)$ lattice in the Calcin. catalyst materials. Thus possibly, the increasingly retained $\mathrm{O}$ atoms in the $\chi-\mathrm{Fe}_{5} \mathrm{C}_{2}$ Type III lattice during its formation resulting in the progressively increasing lattice parameter $a$.

Besides the rather large lattice parameter $a$ for the $\chi-\mathrm{Fe}_{5} \mathrm{C}_{2}$ Type III in relation to the $\chi-\mathrm{Fe}_{5} \mathrm{C}_{2}$ Types I and II, also the $\chi-\mathrm{Fe}_{5} \mathrm{C}_{2}$ Type III crystal structure is systematically 
distorted in comparison to the crystal structures matching the $\chi-\mathrm{Fe}_{5} \mathrm{C}_{2}$ Types I and II. The $\chi-\mathrm{Fe}_{5} \mathrm{C}_{2}$ crystal structure (monoclinic $C 2 / c, 15$ ) used in the R-QPA fitting was solved by Retief, ${ }^{27}$ whose structure solution corresponds to the $\chi-\mathrm{Fe}_{5} \mathrm{C}_{2}$ Type II (Chapter 4). By refining the lattice parameters during the R-QPA fitting: the $\chi-\mathrm{Fe}_{5} \mathrm{C}_{2}$ Type III peak positions are correctly fitted (i.e. monoclinic space group $C 2 / c, 15$, is correct). However, the relative $\chi-\mathrm{Fe}_{5} \mathrm{C}_{2}$ Type III diffraction peak intensities are systematically changed (i.e. the crystal structure is systematically distorted). As an example, in Figure 5.6a, $\boldsymbol{c}, \boldsymbol{e}$ and in Figure 5.7a, $\boldsymbol{c}, \boldsymbol{e}$, systematic diffraction peak intensity misfits for the $\chi-\mathrm{Fe}_{5} \mathrm{C}_{2}$ (Type III) phase plane $\left(\begin{array}{lll}3 & 1 & 2\end{array}\right)\left(\approx 22.52^{\circ} \mathrm{Mo} \mathrm{K}_{\alpha 1}\right)$ are marked with * for all of the Calcin. catalyst materials. For all of the $\mathrm{H}_{2}$ catalyst materials, no clear $\chi-\mathrm{Fe}_{5} \mathrm{C}_{2}$ (Types I and II) phase diffraction peak intensity misfits are observable (Figure 5.6b, $\boldsymbol{d}, \boldsymbol{f}$ and Figure 5.7b, $d, f$ ). Further work for solving a more accurate crystal structure for the $\chi$ $\mathrm{Fe}_{5} \mathrm{C}_{2}$ Type III should be performed. Diffraction patterns obtained with monochromatic synchrotron radiation or electron diffraction should preferably be used for the work.

The Fe carbide lattice changes and the associated carbide subtype FTS reaction formation conditions can be summarized, in a following manner: i) $\eta-\mathrm{Fe}_{2} \mathrm{C}$ Type II forms in the FTS reaction with reaction pressure $\geq 10$ bar and added Na-S promotion, in both Calcin. and $\mathrm{H}_{2}$ catalysts; ii) $\chi-\mathrm{Fe}_{5} \mathrm{C}_{2}$ Type I forms in the FTS reaction with 3 bar pressure regardless of $\mathrm{Na}-\mathrm{S}$ promotion in $\mathrm{H}_{2}$ catalysts; iii) $\chi-\mathrm{Fe}_{5} \mathrm{C}_{2}$ Type II requires $\geq 10$ bar FTS reaction pressure with $\mathrm{Na}-\mathrm{S}$ promotion for transition from $\chi-\mathrm{Fe}_{5} \mathrm{C}_{2}$ Type I to Type II in $\mathrm{H}_{2}$ catalysts; iv) $\chi-\mathrm{Fe}_{5} \mathrm{C}_{2}$ Type III forms on all of the Calcin. catalysts in the FTS reaction regardless of Na-S promotion or the FTS reaction pressure. Moreover, iv) with increasing FTS reaction pressure ( 3 -> 20 bar) the $\chi-\mathrm{Fe}_{5} \mathrm{C}_{2}$ Type III lattice parameter $a$ increases progressively in the Calcin. catalysts regardless of Na-S promotion. This observation is speculated to be due to increasingly retained $\mathrm{O}$ atoms in the $\chi-\mathrm{Fe}_{5} \mathrm{C}_{2}$ Type III lattice from the initially carburizing $\alpha-\mathrm{Fe}_{2} \mathrm{O}_{3}\left(/ \alpha-\mathrm{Al}_{2} \mathrm{O}_{3}\right)$ phase.

\subsubsection{Catalytic Performance}

The catalytic performance results for the UP and Na-S, for both Calcin. and $\mathrm{H}_{2}$ catalyst materials at the reaction pressures of 3,10 or 20 bar are given in terms of FTY profiles in Figure 5.13 over the $72 \mathrm{~h}$ FTS reaction runs. Median values for the FTYs, Usage Ratios $\mathrm{H}_{2}$ :CO and estimates for total $\mathrm{CO}$ to hydrocarbons (HC) conversion ratios are plotted in Figure $\mathbf{5 . 1 4}$ for each of the studied catalyst for each applied FTS reaction pressure. The median $\mathrm{CO}_{2}$ and $\mathrm{CO}_{2}$-free hydrocarbon selectivities and $\mathrm{C}_{2} \mathrm{H}_{4} / \mathrm{C}_{2} \mathrm{H}_{6}$ (ethene/ethane) selectivity ratios for the catalyst materials at different reaction pressures are given in the Figure 5.15. Median Anderson-Schulz-Flory (ASF) distribution $\alpha$-values are summarized in Figure 5.16. All of the catalytic performance median values are taken over Time-on-Stream (ToS) of 33-72 h, and plotted with their 

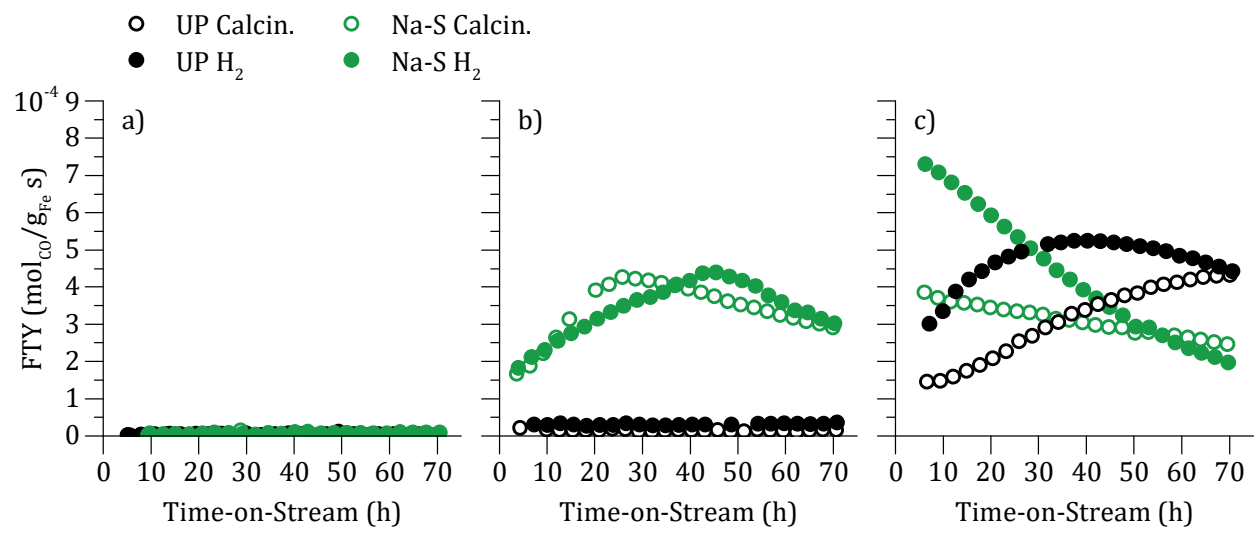

Figure 5.13

Fe Time Yield (FTY) profiles for the UP and Na-S (Fe(-Na-S) $/ \alpha-\mathrm{Al}_{2} \mathrm{O}_{3}$ ), calcined only (Calcin.) and $\mathrm{H}_{2}$ reduced $\left(\mathrm{H}_{2}\right)$ catalyst materials over the $72 \mathrm{~h}$ Fischer-Tropsch Synthesis (FTS) reactions. The FTS reaction FTY profiles with a) 3 bar, b) 10 bar and c) 20 bar reaction pressure. FTS conditions: $340^{\circ} \mathrm{C}, 3,10$ or 20 bar, $\mathrm{H}_{2}: \mathrm{CO}: \mathrm{He}$ 1.0:1.0:0.2 v/v, $\approx 5500 \mathrm{~h}^{-1}$.

STDs as the error bars. The median values are also tabulated in Table 5.4 for the FTS activity and in Table 5.5 for FTS reaction selectivity results.

In the following, the FTS reaction activity results are first discussed with reflections on the characterization results. The activity dialogue is then followed with likewise remarks on the obtained FTS reaction selectivity data.

Discussion on the $\theta-\mathrm{Fe}_{7} \mathrm{C}_{3}$ phase is omitted, as only minor quantity ( $8 \%$ ) of the phase was formed with the Na-S Calcin. 20 bar catalyst material (Figure 5.10b). This amount is unlikely sufficient for playing any relevant role in the catalyst's FTS reaction performance.

\subsubsection{Catalyst Activity}

Assigning precise causality for the FTY profiles representing the catalytic activity over the course of the FTS reactions is a complex task. Such processes e.g. as $\gtrsim 300^{\circ} \mathrm{C}$ inactive carbon deposition, 4,30,49,67,68 possible $\mathrm{Fe}$ particle diameter changes changing the available Fe surface area for the FTS reaction catalysis, ${ }^{50,69,70}$ and/or Fe phases carburization/reoxidation, 4,16,36,54,60,63-65,69 can all affect the observed FTY profiles simultaneously. Also, depending on how the FTS reaction is initiated, the FTY profiles can be significantly affected. . $5,37^{37}$ Thus, a comprehensive discussion on the FTY profile differences is challenging and not attempted in this Chapter in exhaustive detail. Nevertheless, few observations on the FTY profiles are worth a discussion.

The FTY profile developments of interest are (Figure 5.13): i) $\geq 10$ bar FTS reaction pressure is required for a significant activity gain from the $\mathrm{Na}-\mathrm{S}$ promotion, irrespective 

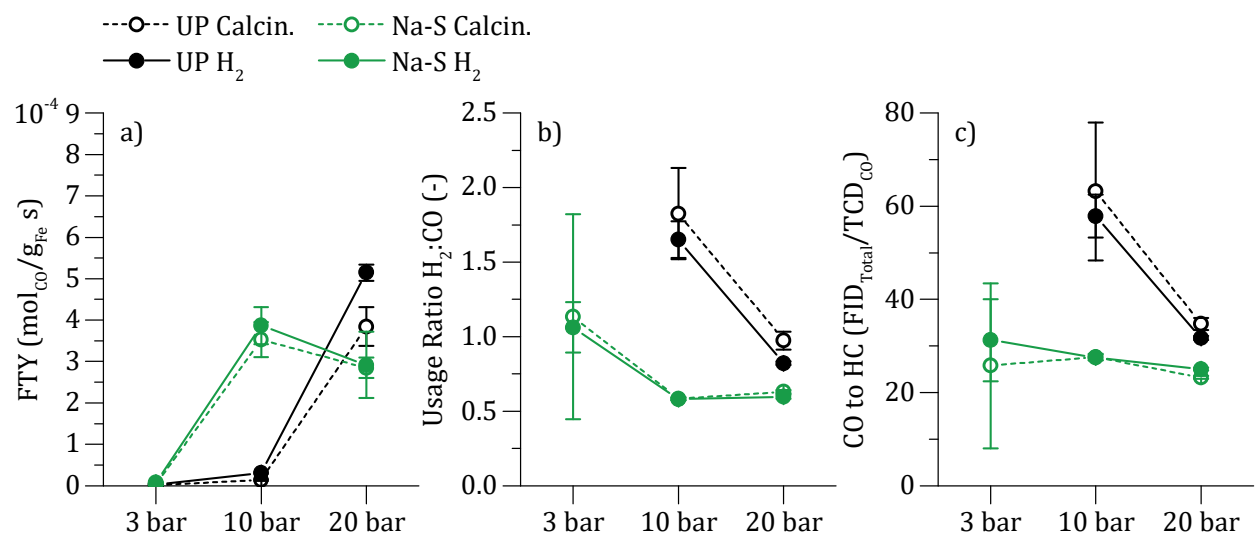

Figure 5.14

Fischer-Tropsch Synthesis (FTS) activity values for UP and Na-S (Fe(-Na-S)/ $\alpha-\mathrm{Al}_{2} \mathrm{O}_{3}$ ) calcined only (Calcin.) and $\mathrm{H}_{2}$ reduced $\left(\mathrm{H}_{2}\right)$ catalyst materials with 3,10 and 20 bar FTS reaction pressures. Median values with their Standard Deviations (STDs) over Time-on-Stream (ToS) 33-72 h for: a) Fe Time Yield (FTY), b) Usage Ratio $\mathrm{H}_{2}$ :CO and c) $\mathrm{CO}$ to hydrocarbons (HC) values. Missing values for UP 3 bar catalysts are omitted due to high STDs.

to the Calcin. and $\mathrm{H}_{2}$ catalyst materials, ii) with 20 bar FTS reaction pressure, the UP catalysts gain a significant boost in their FTYs, irrespective to the Calcin. or $\mathrm{H}_{2}$ treatments, iii) Na-S $\mathrm{H}_{2}$ catalyst deactivates very fast with 20 bar FTS reaction pressure and iv) with 20 bar FTS reaction pressure, the $\mathrm{H}_{2}$ catalysts give higher initial FTYs than the Calcin. catalyst materials, regardless of the Na-S promotion.

With the increase of the applied FTS reaction pressure from 3 to $10 \mathrm{bar}$, the Na-S Calcin. and $\mathrm{H}_{2}$ catalyst materials gain significantly in their FTYs (Figure 5.13a-b). For the Na-S $\mathrm{H}_{2}$ catalyst, the FTY boost coincidences with transition from $\chi-\mathrm{Fe}_{5} \mathrm{C}_{2}$ Type I to Type II (Figure 5.12a). For the Na-S Calcin. catalyst material, the $\chi-\mathrm{Fe}_{5} \mathrm{C}_{2}$ Type III can also be associated to increased FTY when the FTS reaction pressure is at least 10 bar (Figure 5.12a). When the FTS reaction pressure is increased to 20 bar (Figure 5.13c): the $\chi-\mathrm{Fe}_{5} \mathrm{C}_{2}$ Type I (UP $\mathrm{H}_{2}$ ) and $\chi-\mathrm{Fe}_{5} \mathrm{C}_{2}$ Type III (UP Calcin.), without Na-S promotion, can also be associated as active phases correlating with the increased overall FTYs for the catalysts. The Na-S promotion also results in stabilization of the $\eta-\mathrm{Fe}_{2} \mathrm{C}$ Type II with $\geq 10$ bar reaction pressure (Figure $5.10 b, e$ ). However, the presence of the $\eta-\mathrm{Fe}_{2} \mathrm{C}$ Type II in the catalyst material does not correlate with FTYs: the $\eta-\mathrm{Fe}_{2} \mathrm{C}$ (Type II) phase content is $46 \%$ for Na-S Calcin. and $27 \%$ for $\mathrm{Na}-\mathrm{S} \mathrm{H}_{2}$ catalyst, after the 10 bar FTS reaction (Figure 5.10b, $\boldsymbol{e}$ ). Despite this, the FTYs are virtually identical for the catalyst materials at the end of the FTS reaction runs (Figure 5.13b).

Thus, in simplified and succinct terms: the addition of Na-S promotion shifts the pressure threshold for increased FTY attainable in the FTS reaction to a lower reaction 
pressure. Possibly, Na-S promotion i) might sensitize the $\chi-\mathrm{Fe}_{5} \mathrm{C}_{2}$ Type III towards lower reaction pressure (20 -> 10 bar) FTS reactivity and ii) aids in transition for $\chi-\mathrm{Fe}_{5} \mathrm{C}_{2}$ Type I to Type II, where the Type II might possess properties for lower pressure (20 -> 10 bar) FTS reaction activity. Without Na-S promotion, $\chi-\mathrm{Fe}_{5} \mathrm{C}_{2}$ Type I and $\chi-\mathrm{Fe}_{5} \mathrm{C}_{2}$ Type III require 20 bar FTS reaction pressure for significant catalytic activity in the FTS reaction. The presence of $\eta-\mathrm{Fe}_{2} \mathrm{C}$ Type II phase does predict FTS reaction activity at any reaction pressure.

The increased FTYs for the UP catalyst materials with 20 bar FTS reaction pressure do not come for free. When the FTS reaction pressure is increased from 10 to 20 bar, the increase in the FTY values with the UP catalysts is also associated to both: decreases in the Usage Ratio $\mathrm{H}_{2}: \mathrm{CO}$ and in $\mathrm{CO}$ to $\mathrm{HC}$ conversion estimate (Figure 5.14). The drop in Usage Ratio $\mathrm{H}_{2}: \mathrm{CO}$ from $\approx 2$ signifies $\mathrm{CO}$ consuming activity also from other reactions than the hydrocarbon producing FTS.7,8 This is agreed by $\mathrm{CO}$ to HC conversion estimate. That is, less $\mathrm{CO}$ is directed towards the FTS hydrocarbon production, decreasing the FTS reaction's C efficiency towards hydrocarbon production with UP catalysts at 20 bar FTS reaction.

Likewise, but already with lower 10 bar reaction pressure: the Na-S promotion decreases the Usage Ratio $\mathrm{H}_{2}$ : $\mathrm{CO}$ and thus $\mathrm{CO}$ to $\mathrm{HC}$ conversion (Figure 5.14). $\mathrm{Na}(-\mathrm{S}$ )

\section{Table 5.4}

Median Fischer-Tropsch Synthesis (FTS) activity data over Time-on-Stream (ToS) 33-72 h for UP and Na-S (Fe(-Na-S) $\left./ \alpha-\mathrm{Al}_{2} \mathrm{O}_{3}\right)$ ) calcined only (Calcin.) and $\mathrm{H}_{2}$ reduced $\left(\mathrm{H}_{2}\right)$ catalyst materials. Values after $( \pm)$ are the Standard Deviations (STDs) of the median values.

\begin{tabular}{|c|c|c|c|c|c|}
\hline \multirow[t]{2}{*}{$\begin{array}{l}\text { Reaction } \\
\text { Pressure }\end{array}$} & \multirow[t]{2}{*}{ Catalyst } & CO Conversion & $\begin{array}{c}\text { Fe Time Yield } \\
\text { (FTY) }\end{array}$ & Usage Ratio $\mathrm{H}_{2}$ :CO & $\begin{array}{c}\text { CO to Hydrocarbons } \\
\text { Na-S versus UPa) }\end{array}$ \\
\hline & & $(\%)$ & $\left(10^{-4} \mathrm{~mol} \mathrm{Co} / \mathrm{g}_{\mathrm{Fe}} \mathrm{S}\right)$ & $(-)$ & $(-)$ \\
\hline \multirow[t]{4}{*}{3 bar } & UP Calcin. & $(0 \pm 0)$ & $(0.02 \pm 0.03)$ & $(1.6 \pm 2.5)$ & $\mathrm{N} / \mathrm{A}$ \\
\hline & UP $\mathrm{H}_{2}$ & $0 \pm 0$ & $0.03 \pm 0.01$ & $(1.4 \pm 0.9)$ & $\mathrm{N} / \mathrm{A}$ \\
\hline & Na-S Calcin. & $0 \pm 0$ & $0.04 \pm 0.02$ & $1.1 \pm 0.7$ & $(0.61 \pm 2.60)$ \\
\hline & $\mathrm{Na}-\mathrm{S} \mathrm{H}_{2}$ & $1 \pm 0$ & $0.08 \pm 0.02$ & $1.1 \pm 0.2$ & $(0.68 \pm 0.80)$ \\
\hline \multirow[t]{4}{*}{10 bar } & UP Calcin. & $2 \pm 0$ & $0.1 \pm 0.0$ & $1.8 \pm 0.3$ & $\mathrm{~N} / \mathrm{A}$ \\
\hline & $\mathrm{UP}_{2}$ & $4 \pm 0$ & $0.3 \pm 0.0$ & $1.7 \pm 0.1$ & $\mathrm{~N} / \mathrm{A}$ \\
\hline & Na-S Calcin. & $45 \pm 5$ & $3.5 \pm 0.4$ & $0.6 \pm 0.0$ & $0.44 \pm 0.24$ \\
\hline & $\mathrm{Na}-\mathrm{S} \mathrm{H}_{2}$ & $49 \pm 6$ & $3.9 \pm 0.5$ & $0.6 \pm 0.0$ & $0.47 \pm 0.08$ \\
\hline \multirow[t]{4}{*}{20 bar } & UP Calcin. & $50 \pm 6$ & $3.8 \pm 0.5$ & $1.0 \pm 0.1$ & $\mathrm{~N} / \mathrm{A}$ \\
\hline & $\mathrm{UP} \mathrm{H}_{2}$ & $67 \pm 3$ & $5.1 \pm 0.2$ & $0.8 \pm 0.0$ & $\mathrm{~N} / \mathrm{A}$ \\
\hline & Na-S Calcin. & $36 \pm 3$ & $2.8 \pm 0.3$ & $0.6 \pm 0.0$ & $0.67 \pm 0.04$ \\
\hline & $\mathrm{Na}-\mathrm{S} \mathrm{H}_{2}$ & $37 \pm 10$ & $2.9 \pm 0.8$ & $0.6 \pm 0.0$ & $0.79 \pm 0.02$ \\
\hline
\end{tabular}

FTS conditions: $340^{\circ} \mathrm{C}, 3,10$ or 20 bar, $\mathrm{H}_{2}: \mathrm{CO}: \mathrm{He} 1.0: 1.0: 0.2 \mathrm{v} / \mathrm{v}, \approx 5500 \mathrm{~h}^{-1}$.

() - Values in parentheses are rough estimates due to high associated STDs.

a) Change in fraction of $\mathrm{CO}$ converted to hydrocarbons with addition of Na-S promotion (Na-S) versus the respective unpromoted (UP) catalyst materials. 
promotion offers directly active sites for the WGS reaction, ${ }^{18,71,72}$ and/or increases the CO conversion to carbon deposits via the Boudouard reaction. ${ }^{4}$ All of the UP catalyst materials regardless of the reaction pressure convert larger fraction of CO to hydrocarbons than do the Na-S promoted catalysts (Table 5.4 and Figure 5.14c).

The discussed changes in the Usage Ratios $\mathrm{H}_{2}: \mathrm{CO}$ and in $\mathrm{CO}$ to $\mathrm{HC}$ conversions are accompanied by increased $\mathrm{CO}_{2}$ selectivities for the Na-S 10 bar and 20 bar, and UP 20 bar catalyst materials (Figure 5.15e). The increased amount of consumed CO is converted to $\mathrm{CO}_{2}$, and possibly to inactive carbon deposits on the catalysts, rather than to desired FTS hydrocarbon products.

$\mathrm{No} \mathrm{Fe}_{3} \mathrm{O}_{4},{ }^{61,62,73,74}$ or significant amounts of alkali (Table 5.1), ${ }^{12,18,71,72}$ is present with the UP $\mathrm{H}_{2} 20$ bar catalyst offering WGS active sites. $\mathrm{CO}_{2}$ then predominantly likely comes from the increased Boudouard reaction for the catalyst material.4,30,49,67,68 For both UP Calcin. and $\mathrm{H}_{2} 20$ bar catalyst materials, the origins of the $\mathrm{CO}_{2}$ are likely the same as the Usage Ratios $\mathrm{H}_{2}: \mathrm{CO}, \mathrm{CO}$ to $\mathrm{HC}$ conversion values (Figure 5.14b-c) and $\mathrm{CO}_{2}$ selectivities are very similar between the catalysts (Figure 5.15e). This despite the presence of, but yet seemingly inaccessible as discussed earlier, WGS active $\mathrm{Fe}_{3} \mathrm{O}_{4}$ in the UP Calcin. 20 bar catalyst (Figure 5.10a).

The fast deactivation of the Na-S $\mathrm{H}_{2} 20$ bar catalyst has reminiscence to results by van Dijk et al. and Zhou et al., where fast initial drop in catalysts' FTS activity was remedied by added $S .{ }^{75,76}$ The remedying effect by $S$ can be interpreted to be via a $S$ poisoning effect, decreasing CO conversion and the initial catalyst bed heating and therefore the inactive carbon deposition. ${ }^{77}$ For the Na-S $\mathrm{H}_{2} 20$ bar catalyst materials, thus, the initially high FTY possibly causes excessive reactor bed heating, deactivating the catalyst rather fast. The FTS initiation procedure for the Na-S $\mathrm{H}_{2} 20$ bar catalyst needs adjustment for attaining a more sustained FTS reaction activity.

As a final comment on the measured FTS reaction activity indicators, only with the 20 bar FTS reaction does the application of Calcin., in comparison to the $\mathrm{H}_{2}$ catalysts, make a difference in case of both the Na-S and UP catalyst materials (Figure 5.13). In the 3 and 10 bar FTS reaction runs, the UP and Na-S Calcin. and $\mathrm{H}_{2}$ catalyst materials behave FTS reaction activity-wise essentially the same within their respective promotion groups. In turn, when the $\mathrm{H}_{2}$ catalysts are applied in the 20 bar FTS reaction run, both UP and Na-S catalysts show higher initial FTY values in comparison to the respective Calcin. catalyst materials applied in the 20 bar FTS reaction (Figure 5.13c). This can be associated to the presence of the inaccessible $\mathrm{Fe}_{3} \mathrm{O}_{4}$ in the Na-S and UP Calcin. 20 bar catalysts (Figure 5.10a-b, d-e), as commented earlier. Due to the presence of $\mathrm{Fe}_{3} \mathrm{O}_{4}$, less $\mathrm{CO}$ dissociating near-zerovalent $\mathrm{Fe}$ carbides are available for the FTS reaction in the Calcin. than in the $\mathrm{H}_{2} 20$ bar catalyst materials. This explains the difference in the initial FTY profiles. 


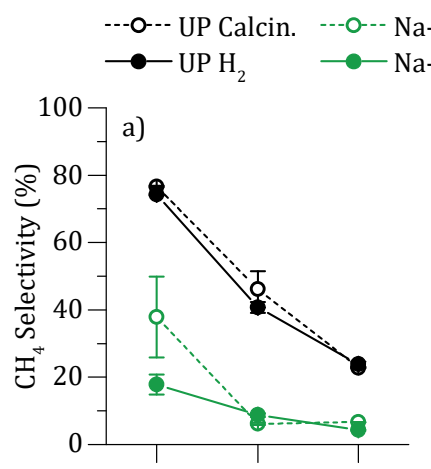

a-S Calcin.
a-S $\mathrm{H}_{2}$
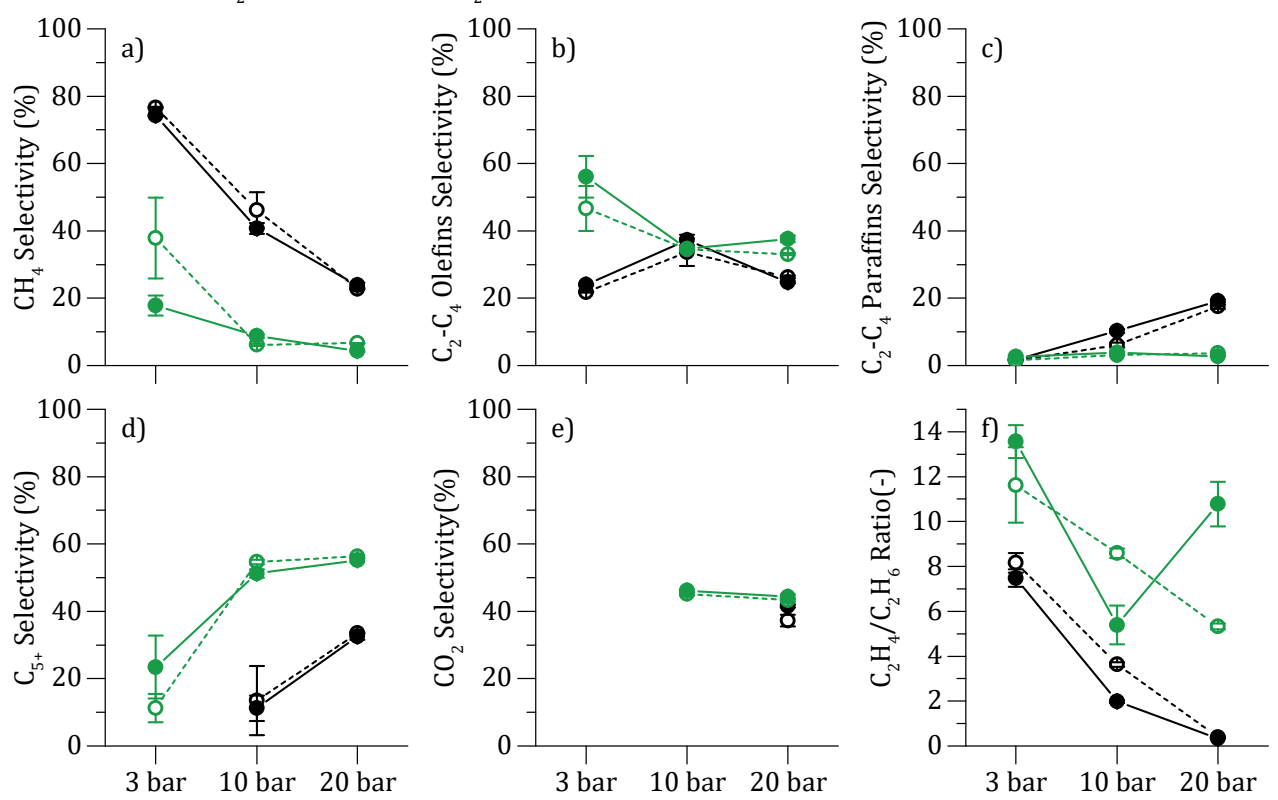

Figure 5.15

Fischer-Tropsch Synthesis (FTS) selectivity results for UP and Na-S $\left(\mathrm{Fe}(-\mathrm{Na}-\mathrm{S}) / \alpha-\mathrm{Al}_{2} \mathrm{O}_{3}\right)$ calcined only (Calcin.) and $\mathrm{H}_{2}$ reduced $\left(\mathrm{H}_{2}\right)$ catalyst materials for 3,10 and 20 bar FTS reaction pressures. Median selectivity values with their Standard Deviations (STDs) over Time-on-Stream (ToS) 33-72 h for a) $\mathrm{CH}_{4}$, b) $\mathrm{C}_{2}-\mathrm{C}_{4}$ olefin, c) $\mathrm{C}_{2}-\mathrm{C}_{4}$ paraffin, d) $\mathrm{C}_{5}$ and e) $\mathrm{CO}_{2}$. In f) $\mathrm{C}_{2} \mathrm{H}_{4} / \mathrm{C}_{2} \mathrm{H}_{6}$ ratio is compared. Missing values for the catalyst materials are below detection limit.

Fe particle growth has been proposed to be responsible for the deactivation of the $\mathrm{Na}-\mathrm{S}$ promoted FTS catalysts. ${ }^{50}$ However, no clear connection between the catalysts' morphology, Fe particle diameter (Figure 5.4) and FTS reaction activity (Figure 5.13) can be derived from the results in this Chapter.

\subsubsection{Catalyst Selectivity}

The added $\mathrm{Na}$-S promotion has the overall reaction pressure independent effects on the catalysts' FTS reaction hydrocarbon selectivities of (Figure 5.15): i) decreased $\mathrm{CH}_{4}$, ii) in general increased $\mathrm{C}_{2}-\mathrm{C}_{4}$ olefin and decreased $\mathrm{C}_{2}-\mathrm{C}_{4}$ paraffin, iii) increased $\mathrm{C}_{5+}$ selectivity, and iv) increased $\mathrm{C}_{2} \mathrm{H}_{4} / \mathrm{C}_{2} \mathrm{H}_{6}$ ratio. As would be expected from the changes in the hydrocarbon chain-length selectivities, with the Na-S promotion, the ASF distribution $\alpha$-value is in overall increased in comparison to the UP catalyst materials for all of the tested FTS reaction pressures (Figure 5.16). All of the mentioned selectivity effects are akin to alkali only promotion effects. . $, 5,11,12,15-18$ 
Furthermore, as a specific property with the combined Na-S promotion, the $\mathrm{CH}_{4}$ selectivity is especially suppressed to values below the expectations from the ASF distribution. ${ }^{19,20,25}$

This can be also observed in the results for the Na-S promoted catalyst materials (Figure 5.15a and Table 5.5). Both, the Calcin. and $\mathrm{H}_{2}$ catalyst materials show the same $\mathrm{Na}-\mathrm{S}$ promotion dependent trends. An exception, however, is being the $\mathrm{C}_{2} \mathrm{H}_{4} / \mathrm{C}_{2} \mathrm{H}_{6}$ ratio that shows a possible Calcin. versus $\mathrm{H}_{2}$ catalyst treatment dependency for catalyst materials with $\geq 10$ bar FTS reaction pressure (Figure 5.15f). The $\mathrm{C}_{2} \mathrm{H}_{4} / \mathrm{C}_{2} \mathrm{H}_{6}$ ratio Calcin. versus $\mathrm{H}_{2}$ catalyst dependency will be discussed in more detail shortly.

As a reference point, for the Na-S Calcin. and $\mathrm{H}_{2} 20$ bar catalyst materials, the FTS reaction hydrocarbon selectivities compare well with the results by Koeken et al. under similar FTS reaction conditions $\left(340^{\circ} \mathrm{C}, 20\right.$ bar, $\left.\mathrm{H}_{2}: \mathrm{CO} 1: 1,4200 \mathrm{~h}^{-1}\right)$ with a Fe-Na-S $/ \alpha-$ $\mathrm{Al}_{2} \mathrm{O}_{3}$ catalyst. 49

Also, as a general note, FTS reaction hydrocarbon selectivities are not directly affected by the CO conversion levels within range $\approx 10-80 \% .7,12$ Thus, despite the different FTYs for the studied catalyst materials, the selectivity results can be compared.

For High-temperature Fischer-Tropsch (HTFT, $320-375^{\circ} \mathrm{C}$ ), the $\mathrm{CH}_{4}$ selectivity correlates with average partial pressures $p$ in the reactor after Equation 5.1:5,8

$$
\mathrm{F}_{\mathrm{CH}_{4}}=\frac{\mathrm{p}_{\mathrm{H}_{2}}^{0.25}}{\mathrm{p}_{\mathrm{CO}}+0.7 \mathrm{p}_{\mathrm{CO}_{2}}+0.6 \mathrm{p}_{\mathrm{H}_{2} \mathrm{O}}} \quad \mathrm{CH}_{4} \text { Correlation in HTFT } \quad \text { (Eq. 5.1) }
$$

where a decreasing factor $\mathrm{F}_{\mathrm{CH}_{4}}$ correlates with decreasing $\mathrm{CH}_{4}$ selectivity. This is in agreement with the observed results of decreasing $\mathrm{CH}_{4}$ correlating with increasing $\mathrm{CO}_{2}$ selectivities (Figure 5.15a,e). More fundamentally, $\mathrm{CO}_{2}$ and $\mathrm{H}_{2} \mathrm{O}$ are thought to compete the FTS reaction reactants $\mathrm{H}_{2}$ and $\mathrm{CO}$ for the catalyst's Fe surface adsorption sites, effectively decreasing the $\mathrm{Fe}$ surface $\mathrm{H}: \mathrm{C}$ ratio and thus decreasing $\mathrm{CH}_{4}$ selectivity. ${ }^{5,8}$ The rest of the FTS hydrocarbon chain-length selectivities follow the implications from the $\mathrm{CH}_{4}$ selectivity. 5,8 That is, when $\mathrm{CH}_{4}$ decreases, total $\mathrm{C}_{2}-\mathrm{C}_{4}$ and $\mathrm{C}_{5+}$ selectivities generally increase (Figure 5.15a-d) due to the requirements from the ASF distribution. ${ }^{3,78}$

With the FTS reaction pressure increase from 10 to 20 bar (Figure 5.15a, $\boldsymbol{e}$ and Table 5.5): for the Na-S Calcin. and $\mathrm{H}_{2}$ catalyst materials the $\mathrm{CO}_{2}$ selectivity remains constant (43-46 \%) as does the $\mathrm{CH}_{4}$ selectivity (4-9\%); while for the UP Calcin. and $\mathrm{H}_{2}$ catalysts the $\mathrm{CO}_{2}$ selectivity increases from below detection limit to $37-42 \%$ and $\mathrm{CH}_{4}$ selectivity decreases from $41-46 \%$ to $23-24 \%$. That is, with total FTS reaction pressure within 10-20 bar: the Na-S catalysts are independent of the reaction pressure and the 
UP catalyst materials pressure dependent, in regard to the hydrocarbon $\mathrm{CH}_{4}, \mathrm{C}_{2}-\mathrm{C}_{4}$ (olefin and paraffin), $\mathrm{C}_{5+}$ and $\mathrm{CO}_{2}$ selectivities (Figure 5.15a-e). With 3 bar FTS reaction pressure, the $\mathrm{CO}_{2}$ selectivities are below detection limit for both UP and Na-S catalyst materials and increased $\mathrm{CH}_{4}$ selectivities are observed (Figure 5.15a, e). This is in-line with expectations of increased $\mathrm{CH}_{4}$ selectivity for low FTS reaction pressure ( $<6$ bar). ${ }^{6}$

The $\mathrm{CH}_{4}$ selectivity independency of the alkali promoted catalysts from the total FTS reaction pressure has also been observed previously with bulk Rührchemie (Fe:Cu:K:SiO${ }_{2}$ ) catalyst $\left(260^{\circ} \mathrm{C}, 15-25\right.$ bar, $\left.\mathrm{H}_{2}: \mathrm{CO} 2: 3\right),{ }^{7}$ and with K-promoted Fe-based catalyst material $\left(240^{\circ} \mathrm{C}, \approx 6-25\right.$ bar, $\left.\mathrm{H}_{2}: \mathrm{CO} \approx 2: 1\right),{ }^{6}$ for Low-temperature FischerTropsch (LTFT, $\left.200-250^{\circ} \mathrm{C}\right)$. With testing done on a Fe-Na-S $/ \alpha-\mathrm{Al}_{2} \mathrm{O}_{3}$ catalyst $(\approx 5$ 20 bar, $350^{\circ} \mathrm{C}$ ), the threshold pressure for $\mathrm{CH}_{4}$ selectivity independency can also be observed to be depended on the applied $\mathrm{H}_{2}: \mathrm{CO}$ ratio (see Fig. S16, in the cited article). ${ }^{49}$

Alkali (as $\mathrm{Na}$ or $\mathrm{K}$ ) intrinsically increases the $\mathrm{CO}_{2}$ releasing, inactive carbon depositing Boudouard reaction, 4,67,79 and the WGS activity converting $\mathrm{CO}+\mathrm{H}_{2} \mathrm{O}$ to $\mathrm{H}_{2}+$ $\mathrm{CO}_{2} \cdot{ }^{12,14,17,18}$ Both reactions affect the presence of $\mathrm{CO}_{2}$, and the WGS reaction also affects the $\mathrm{H}_{2} \mathrm{O}$ concentration in the FTS reaction and according to the Equation 5.1 alter the (hydrocarbon and) $\mathrm{CH}_{4}$ selectivity. The different total FTS reaction pressure responses with the Na-S and UP catalysts on the $\mathrm{CH}_{4}$ selectivity are then most likely related to the WGS and Boudouard reaction promotion ability of the $\mathrm{Na}(-\mathrm{S})$ addition.

The threshold pressure for the $\mathrm{CH}_{4}$ selectivity independency, with regard to the $\chi$ $\mathrm{Fe}_{5} \mathrm{C}_{2}$ subtypes, follows the discussion on the FTY pressure dependency. The Na-S promotion induced transition of $\chi-\mathrm{Fe}_{5} \mathrm{C}_{2}$ Type I to Type II and addition of Na-S promotion to $\chi-\mathrm{Fe}_{5} \mathrm{C}_{2}$ Type III (Figure 5.12a) correlate with lowered, 10 bar threshold for the $\mathrm{CH}_{4}$ selectivity independency (Figure 5.15a-d), as well as with the related FTY boost discussed earlier (Figure 5.13). Without Na-S promotion, $\chi-\mathrm{Fe}_{5} \mathrm{C}_{2}$ Type I and Type III do not show comparable behavior in either the FTYs or FTS reaction selectivities. The quantity of $\eta-\mathrm{Fe}_{2} \mathrm{C}$ (Type II) phase (Figure 5.10a-b, d-e ) does not correlate with the FTS reaction hydrocarbon selectivities (Figure 5.15). Whether the different $\chi-\mathrm{Fe}_{5} \mathrm{C}_{2}$ subtypes favor e.g. different lattice/surface positions for the Na-S promoters, possibly explaining the observations FTS performance differences, would be worthy of future research interest.

So far in the discussion, whether either the Calcin. or $\mathrm{H}_{2}$ catalyst material was applied in the FTS reaction, did not make any difference selectivity-wise versus the FTS reaction pressure. The Na-S promotion has been the catalysts' FTS reaction selectivity defining feature. The exception to this rule, however, comes with the $\mathrm{C}_{2} \mathrm{H}_{4} / \mathrm{C}_{2} \mathrm{H}_{6}$ selectivity ratio (Figure 5.15f) $\mathrm{C}_{2} \mathrm{H}_{4}$ is reactive, and may be hydrogenated to $\mathrm{C}_{2} \mathrm{H}_{6}$ via secondary reactions i.e. via its readsorption on the Fe surface which is followed by hydrogenation or oligomerization reaction., 4,10,78 In general it can be observed that 
increasing FTS reaction pressure decreases the $\mathrm{C}_{2} \mathrm{H}_{4} / \mathrm{C}_{2} \mathrm{H}_{6}$ ratio for all of the studied catalyst materials (Figure 5.15f). This, while minding a to-be discussed exemption with the $\mathrm{Na}-\mathrm{S} \mathrm{H}_{2} 20$ bar catalyst material (Figure 5.15f).

For all of the studied Na-S catalyst materials, the ASF $\alpha$-value (Figure 5.16), and the $\mathrm{C}_{2} \mathrm{H}_{4} / \mathrm{C}_{2} \mathrm{H}_{6}$ ratio is increased over the tested pressure range in comparison to the UP catalysts (Figure 5.15f). This is what would be expected to be the case with $\mathrm{Na}(-\mathrm{S})$ promotion.4,5,10-12,17,18 The more interesting point, however, is the increase in the $\mathrm{C}_{2} \mathrm{H}_{4} / \mathrm{C}_{2} \mathrm{H}_{6}$ ratio with the Calcin. catalysts in comparison to $\mathrm{H}_{2}$ catalyst materials at the FTS reaction pressure of 10 bar regardless of the Na-S promotion (Figure 5.15f). The duplicate reactors tested for the data reproducibility gave comparable results for the ToS resolved $\mathrm{C}_{2} \mathrm{H}_{4} / \mathrm{C}_{2} \mathrm{H}_{6}$ ratio results in Figure 5.17. The observation, therefore, is not due a "random" occurrence.

A result akin to the increased $\mathrm{C}_{2} \mathrm{H}_{4} / \mathrm{C}_{2} \mathrm{H}_{6}$ ratio with Calcin. 10 bar catalyst materials has been previously observed by Bukur et al.. ${ }^{35}$ By treating their bulk Rührchemie (Fe:Cu:K:SiO 2 ) FTS catalyst with $\mathrm{CO}$ (at $280^{\circ} \mathrm{C}$ ) or $\mathrm{H}_{2}: \mathrm{CO}$ (at $310^{\circ} \mathrm{C}$ ) prior to the FTS reaction $\left(250^{\circ} \mathrm{C}, 14.8\right.$ bar, $\left.\mathrm{H}_{2}: \mathrm{CO} 2: 3\right)$, a $\approx+10 \%$ increase in $\mathrm{C}_{2}-\mathrm{C}_{4}$ fraction olefin content could be achieved. This in comparison to the same catalysts reduced with $\mathrm{H}_{2}$ prior to the FTS reaction. The result by Bukur et al. is not perfectly the same as in this study: for their catalyst, the $\mathrm{C}_{2}-\mathrm{C}_{4}$ olefin fraction increased, while for the Calcin. 10 bar catalysts the $\mathrm{C}_{2} \mathrm{H}_{4} / \mathrm{C}_{2} \mathrm{H}_{6}$ ratio is increased (Figure 5.15f) without a marked increase in the total $\mathrm{C}_{2}-\mathrm{C}_{4}$ range olefin selectivity (Figure 5.15b). Furthermore, Wezendonk et al. have observed decreased hydrogenation ability for their catalyst material, when an initial $\gamma-\mathrm{Fe}_{2} \mathrm{O}_{3} @ \mathrm{C}$ catalyst was directly carburized $\left(340^{\circ} \mathrm{C}\right.$, $\mathrm{H}_{2}$ :CO 1:1) in comparison to a prior $\mathrm{H}_{2}$ reduction before the FTS reaction $\left(230^{\circ} \mathrm{C}\right.$, 20 bar, $\mathrm{H}_{2}$ :CO 1:1). ${ }^{38}$

Together with the literature observations, the results for the Calcin. catalyst materials with 10 bar FTS reaction are intriguing. Recalling from the discussion in R-QPA results section that regardless of the Na-S promotion, the $\chi$ $\mathrm{Fe}_{5} \mathrm{C}_{2}$ Type III subtype is formed with all of the Calcin. catalyst materials. With all of the studied $\mathrm{H}_{2}$ catalyst materials, either $\chi-\mathrm{Fe}_{5} \mathrm{C}_{2}$

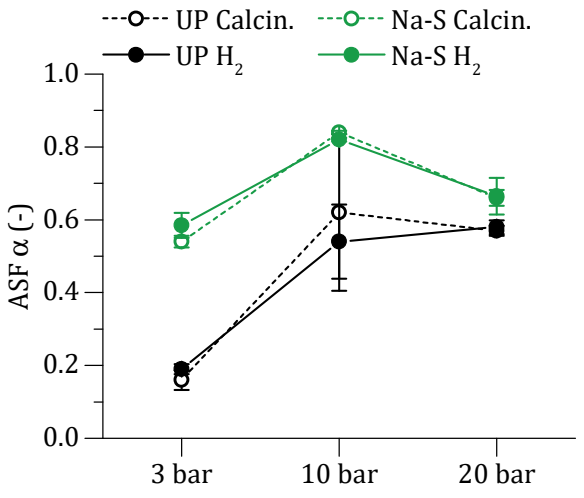

Figure 5.16

Median Anderson-Schulz-Flory (ASF) distribution $\alpha$-values with their Standard Deviations (STDs) over Time-on-Stream (ToS) 33-72 h compared for UP and $\mathrm{Na}-\mathrm{S}\left(\mathrm{Fe}(-\mathrm{Na}-\mathrm{S}) / \alpha-\mathrm{Al}_{2} \mathrm{O}_{3}\right)$ calcined only (Calcin.) and $\mathrm{H}_{2}$ reduced $\left(\mathrm{H}_{2}\right)$ catalyst materials for 3, 10 and 20 bar Fischer-Tropsch Synthesis (FTS) reaction pressures. 


\section{Table 5.5}

Median Fischer-Tropsch Synthesis (FTS) selectivity data over Time-on-Stream (ToS) 33-72 h for UP and Na$\mathrm{S}\left(\mathrm{Fe}(-\mathrm{Na}-\mathrm{S}) / \alpha-\mathrm{Al}_{2} \mathrm{O}_{3}\right)$ calcined only (Calcin.) and $\mathrm{H}_{2}$ reduced $\left(\mathrm{H}_{2}\right)$ catalyst materials. Values after $( \pm)$ are the Standard Deviations (STDs) of the median values.

\begin{tabular}{|c|c|c|c|c|c|c|c|c|}
\hline \multirow[t]{2}{*}{ Pressure } & Catalyst $^{\text {a) }}$ & $\mathrm{CO}_{2}$ & $\mathrm{CH}_{4}$ & $\begin{array}{l}\mathrm{C}_{2}-\mathrm{C}_{4} \\
\text { Olefin }\end{array}$ & $\begin{array}{c}\mathrm{C}_{2}-\mathrm{C}_{4} \\
\text { Paraffin }\end{array}$ & $\mathrm{C}_{5+}$ & $\begin{array}{c}\mathrm{C}_{2} \mathrm{H}_{4} / \\
\mathrm{C}_{2} \mathrm{H}_{6}\end{array}$ & $\begin{array}{c}\text { ASF } \\
\alpha \text {-value }\end{array}$ \\
\hline & & $(\%)$ & $(\%)$ & $(\%)$ & $(\%)$ & $(\%)$ & $(-)$ & (a.u) \\
\hline \multirow[t]{4}{*}{3 bar } & UP Calcin. & $<\mathrm{dl}$ & $77 \pm 0$ & $22 \pm 0$ & $2 \pm 0$ & - & $8.2 \pm 0.4$ & $0.16 \pm 0.03$ \\
\hline & UP $\mathrm{H}_{2}$ & $<\mathrm{dl}$ & $74 \pm 0$ & $24 \pm 0$ & $2 \pm 0$ & - & $7.5 \pm 0.4$ & $0.19 \pm 0.01$ \\
\hline & Na-S Calcin. & $<\mathrm{dl}$ & $38 \pm 12$ & $47 \pm 7$ & $2 \pm 0$ & $11 \pm 4$ & $11.6 \pm 1.7$ & $0.54 \pm 0.02$ \\
\hline & Na-S H 2 & $<\mathrm{dl}$ & $18 \pm 3$ & $56 \pm 6$ & $3 \pm 0$ & $23 \pm 9$ & $13.6 \pm 0.7$ & $0.59 \pm 0.03$ \\
\hline \multirow[t]{4}{*}{10 bar } & UP Calcin. & $<\mathrm{dl}$ & $46 \pm 5$ & $34 \pm 4$ & $6 \pm 1$ & $13 \pm 10$ & $3.6 \pm 0.1$ & $0.62 \pm 0.22$ \\
\hline & UP $\mathrm{H}_{2}$ & $<\mathrm{dl}$ & $41 \pm 2$ & $37 \pm 2$ & $10 \pm 1$ & $11 \pm 4$ & $2.0 \pm 0.1$ & $0.54 \pm 0.10$ \\
\hline & Na-S Calcin. & $45 \pm 0$ & $6 \pm 0$ & $35 \pm 0$ & $3 \pm 0$ & $55 \pm 1$ & $8.6 \pm 0.2$ & $0.84 \pm 0.00$ \\
\hline & $\mathrm{Na}-\mathrm{S} \mathrm{H}_{2}$ & $46 \pm 0$ & $9 \pm 1$ & $35 \pm 1$ & $4 \pm 0$ & $51 \pm 1$ & $5.4 \pm 0.9$ & $0.82 \pm 0.01$ \\
\hline \multirow[t]{4}{*}{20 bar } & UP Calcin. & $37 \pm 2$ & $23 \pm 0$ & $26 \pm 0$ & $18 \pm 1$ & $34 \pm 0$ & $0.4 \pm 0.0$ & $0.57 \pm 0.01$ \\
\hline & $\mathrm{UP}_{2}$ & $42 \pm 0$ & $24 \pm 1$ & $25 \pm 0$ & $19 \pm 0$ & $33 \pm 1$ & $0.3 \pm 0.0$ & $0.58 \pm 0.02$ \\
\hline & Na-S Calcin. & $43 \pm 0$ & $7 \pm 0$ & $33 \pm 0$ & $4 \pm 0$ & $56 \pm 0$ & $5.3 \pm 0.1$ & $0.66 \pm 0.02$ \\
\hline & $\mathrm{Na}-\mathrm{S} \mathrm{H} \mathrm{H}_{2}$ & $44 \pm 1$ & $4 \pm 0$ & $38 \pm 1$ & $3 \pm 0$ & $55 \pm 1$ & $10.8 \pm 1.0$ & $0.67 \pm 0.05$ \\
\hline
\end{tabular}

FTS conditions: $340^{\circ} \mathrm{C}, 3,10$ or 20 bar, $\mathrm{H}_{2}: \mathrm{CO}: \mathrm{He} 1.0: 1.0: 0.2 \mathrm{v} / \mathrm{v}, \approx 5500 \mathrm{~h}^{-1}$.

$<\mathrm{dl}$ - Below detection limit.

a) 10 bar: $\mathrm{MeOH}$ (methanol) selectivity was $1.3-1.4 \%$ for Na-S Calcin. and $\mathrm{H}_{2}$, and $0.3-0.4 \%$ for UP Calcin. and $\mathrm{H}_{2}$ catalysts. 3 and 20 bar: $\mathrm{MeOH}$ selectivity was $\lesssim 0.1 \%$ for all catalyst materials.

Type I or Type II forms but no Type III (Figure 5.12a). From the characterization results by Bukur et al. or Wezendonk et al., it cannot be deduced which $\chi-\mathrm{Fe}_{5} \mathrm{C}_{2}$ subtypes may have formed in their studies with the applied $\mathrm{H}_{2}$, $\mathrm{CO}$ or $\mathrm{H}_{2}$ : $\mathrm{CO}$ treatments. ${ }^{34,38}$

With the $\mathrm{CO}$ or $\mathrm{H}_{2}: \mathrm{CO}$ treatments applied before the FTS reaction, Bukur et al. mainly detected $\chi-\mathrm{Fe}_{5} \mathrm{C}_{2}$ and $\mathrm{Fe}_{3} \mathrm{O}_{4}$ phases, while with $\mathrm{H}_{2}$ treatments a " $\mathcal{C}^{\prime}-\mathrm{Fe}_{2.2} \mathrm{C}$ " phase (i.e. $\eta$ $\mathrm{Fe}_{2} \mathrm{C}$ ) was detected as the major Fe carbide phase after the FTS reaction. ${ }^{34}$ With the applied carburizing $\mathrm{H}_{2}$ :CO treatment, Wezendonk et al. characterized the $\chi-\mathrm{Fe}_{5} \mathrm{C}_{2}$ as being the major $\mathrm{Fe}$ carbide phase, with a minor contribution from " $\varepsilon^{\prime}-\mathrm{Fe}_{2.2} \mathrm{C}$ ". Thus, despite the enriched presence of the $\eta-\mathrm{Fe}_{2} \mathrm{C}$ phase in the Na-S Calcin. 10 bar catalyst it unlikely plays a role in the enhanced $\mathrm{C}_{2} \mathrm{H}_{4} / \mathrm{C}_{2} \mathrm{H}_{6}$ ratio. The $\eta-\mathrm{Fe}_{2} \mathrm{C}$ phase is also missing from the UP Calcin. 10 bar catalyst material after the FTS reaction (Figure 5.10a-b) but enhanced $\mathrm{C}_{2} \mathrm{H}_{4} / \mathrm{C}_{2} \mathrm{H}_{6}$ ratio is still observed (Figure 5.17b).

We, therefore, assign the increased $\mathrm{C}_{2} \mathrm{H}_{4} / \mathrm{C}_{2} \mathrm{H}_{6}$ ratio as an intrinsic property, i.e. being independent of the $\mathrm{Na}-\mathrm{S}$ promotion, to the $\chi-\mathrm{Fe}_{5} \mathrm{C}_{2}$ Type III phase when the FTS reaction pressure is around 10 bar (Figure 5.15f). This would also explain the literature observations: when a Fe oxide is directly exposed to carburizing atmosphere, $\chi-\mathrm{Fe}_{5} \mathrm{C}_{2}$ Type III phase forms, resulting in the catalyst material's decreased hydrogenation ability. ${ }^{35,38}$ 

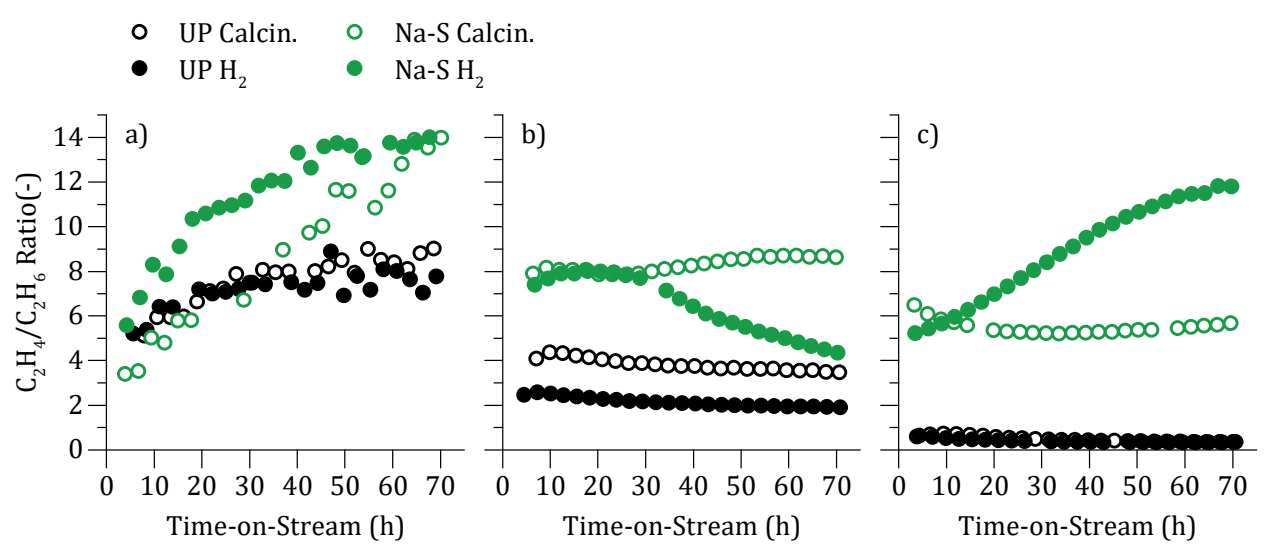

Figure 5.17

$\mathrm{C}_{2} \mathrm{H}_{4} / \mathrm{C}_{2} \mathrm{H}_{6}$ ratio Time-on-Stream (ToS) profiles for the UP and Na-S (Fe(-Na-S) $/ \alpha-\mathrm{Al}_{2} \mathrm{O}_{3}$ ), calcined only (Calcin.) and $\mathrm{H}_{2}$ reduced $\left(\mathrm{H}_{2}\right)$ catalysts over the $72 \mathrm{~h}$ Fischer-Tropsch Synthesis (FTS) reactions. FTS reaction $\mathrm{C}_{2} \mathrm{H}_{4} / \mathrm{C}_{2} \mathrm{H}_{6}$ ratio profiles for a) 3 bar, b) 10 bar and c) 20 bar FTS reaction pressures.

With 3 bar results for the UP and Na-S Calcin. catalyst materials, no positive effect towards olefin formation can be associated to the $\chi-\mathrm{Fe}_{5} \mathrm{C}_{2}$ Type III phase (Figure 5.15f). An explanation for the $\mathrm{C}_{2} \mathrm{H}_{4} / \mathrm{C}_{2} \mathrm{H}_{6}$ ratio trend breaking $\mathrm{Na}-\mathrm{S} \mathrm{H}_{2} 20$ bar catalyst (Figure 5.15f) could be suggested when contrasting the Na-S $\mathrm{H}_{2} 20$ bar catalyst's $\mathrm{C}_{2} \mathrm{H}_{4} / \mathrm{C}_{2} \mathrm{H}_{6}$ ratio ToS profile (Figure 5.17c) to the catalyst's corresponding FTY profile (Figure 5.13c). After the ToS resolved results for the catalyst material, the increasing $\mathrm{C}_{2} \mathrm{H}_{4} / \mathrm{C}_{2} \mathrm{H}_{6}$ ratio has an inverse relation with the decreasing FTY profile. Rather fast decrease in the FTY profile might indicate inactive carbon deposition blocking the catalyst surface, when FTS reaction conditions of 20 bar $\mathrm{H}_{2}: \mathrm{CO} 1: 1$ are applied. ${ }^{49}$ Carbon deposition could decrease the available Fe sites for the $\mathrm{C}_{2} \mathrm{H}_{4}$ readsorption and thus decrease its secondary reactions. ${ }^{10,75}$ This would result in the increasing the $\mathrm{C}_{2} \mathrm{H}_{4} / \mathrm{C}_{2} \mathrm{H}_{6}$ ratio (Figure 5.17c). Thus, whether the Na-S Calcin. 20 bar catalyst exhibits olefin favoring $\mathrm{C}_{2} \mathrm{H}_{4} / \mathrm{C}_{2} \mathrm{H}_{6}$ ratio in comparison to the $\mathrm{Na}-\mathrm{S} \mathrm{H}_{2} 20$ bar catalyst material cannot be judged due to lack of reference point. For the UP Calcin. 20 bar catalyst material; the overall 20 bar FTS reaction pressure suppresses the $\mathrm{C}_{2} \mathrm{H}_{4} / \mathrm{C}_{2} \mathrm{H}_{6}$ ratio (Figure 5.17).

In total, the favorable, Na-S promotion independent, properties towards $\mathrm{C}_{2} \mathrm{H}_{4}$ formation with the $\chi-\mathrm{Fe}_{5} \mathrm{C}_{2}$ Type III phase seem to be FTS reaction pressure dependent. The response of the $\chi-\mathrm{Fe}_{5} \mathrm{C}_{2}$ Type III phase to the reaction conditions, and to $\mathrm{C}_{2} \mathrm{H}_{4}$ and overall olefin production is well worth further investigation in the future.

\subsection{CONCLUSIONS}

Supported $\mathrm{Fe}(-\mathrm{Na}-\mathrm{S}) / \alpha-\mathrm{Al}_{2} \mathrm{O}_{3}$ catalyst materials, with and without Na-S promotion, 
were prepared, characterized and tested. The catalysts were either calcined only, or subsequently to calcining also $\mathrm{H}_{2}$ reduced, prior to the catalytic performance testing in the FTS reaction. The FTS reactions were separately ran for $72 \mathrm{~h}$ at either 3,10 or 20 bar total reaction pressure at otherwise identical reaction conditions $\left(340^{\circ} \mathrm{C}, \mathrm{H}_{2}: \mathrm{CO}: \mathrm{He}\right.$ 1.0:1.0:0.2 v/v, GHSV $\approx 5500 \mathrm{~h}^{-1}$ ). XRPD patterns of each of the studied catalyst materials were collected ex-situ after the FTS reaction runs and Fe phases present were quantified from the XRPD patterns using R-QPA.

Before performing the FTS reactions, both the unpromoted and Na-S promoted, calcined only catalyst materials only contained $\alpha-\mathrm{Fe}_{2} \mathrm{O}_{3}$ as the sole Fe phase. The subsequently $\mathrm{H}_{2}$ reduced catalysts consisted of $\alpha$-Fe as the major Fe phase with both $\mathrm{Fe}_{3} \mathrm{O}_{4}$ and $\mathrm{Fe}_{0.974} \mathrm{O}$ also present. After the FTS reactions, the Fe carbide phases identified in the catalyst materials were $\eta-\mathrm{Fe}_{2} \mathrm{C} \mathrm{Pnnm} \mathrm{(58),} \chi-\mathrm{Fe}_{5} \mathrm{C}_{2} \mathrm{C} 2 / c$ (15) and/or $\theta-\mathrm{Fe}_{7} \mathrm{C}_{3} \mathrm{Pnma}$ (62).

On all of the applied catalyst materials after the FTS reactions, the $\chi-\mathrm{Fe}_{5} \mathrm{C}_{2}$ phase was quantified as the major Fe carbide phase. Na-S promotion altered the Fe carbide formation by i) inducing appearance of the $\eta-\mathrm{Fe}_{2} \mathrm{C}$ phase in 10 and 20 bar FTS reaction runs but not with the 3 bar FTS run, and ii) with 10 bar FTS reaction pressure the Na-S promoted and calcined only catalyst materials showed more tendency towards the $\eta$ $\mathrm{Fe}_{2} \mathrm{C}$ formation than the $\mathrm{Na}-\mathrm{S}$ promoted and $\mathrm{H}_{2}$ reduced catalysts did. Also, iii) combining the Na-S promotion with the calcined only catalyst with 20 bar FTS reaction pressure induced formation of a minor quantity $(8 \%)$ of the $\theta-\mathrm{Fe}_{7} \mathrm{C}_{3}$ phase.

In Chapter 4, the $\eta-\mathrm{Fe}_{2} \mathrm{C}$ and $\chi-\mathrm{Fe}_{5} \mathrm{C}_{2}$ phase subtypes were defined by their crystal lattice parameters. Within this Chapter, the carbide subtype formation largely follows the set expectations. With respect to the FTS reaction pressure, the Fe carbide subtype formation takes place as follows:

$\chi-\mathrm{Fe}_{5} \mathrm{C}_{2}$

Type I Without Na-S promotion regardless of reaction pressure, or with 3 bar FTS reaction pressure regardless of Na-S promotion,

Type II With Na-S promotion, and $\geq 10$ bar FTS reaction pressure,

Type III Fe oxide $\left(\alpha-\mathrm{Fe}_{2} \mathrm{O}_{3}\right)$ as the FTS starting material regardless of the $\mathrm{Na}-\mathrm{S}$ promotion or the FTS reaction pressure,

$\eta-\mathrm{Fe}_{2} \mathrm{C}$

Type II With Na-S promotion, and $\geq 10$ bar FTS reaction pressure.

Increasing the FTS reaction pressure increased lattice parameter $a$ distortion for the $\chi-\mathrm{Fe}_{5} \mathrm{C}_{2}$ Type III. The $\chi-\mathrm{Fe}_{5} \mathrm{C}_{2}$ Type III distortion is conceivably due to increasingly retained $\mathrm{O}$ atoms in the $\chi-\mathrm{Fe}_{5} \mathrm{C}_{2}$ Type III crystal lattice. In addition to the phase's lattice 
parameter distortion, the crystal structure of the $\chi-\mathrm{Fe}_{5} \mathrm{C}_{2}$ Type III was systematically altered in comparison to the crystal structures of $\chi-\mathrm{Fe}_{5} \mathrm{C}_{2}$ Type I and Type II.

The catalysts' FTS performance dependency on the reaction pressure was mainly altered by the Na-S promotion. Whether the catalyst was only calcined or also $\mathrm{H}_{2}$ reduced, had a lesser influence on its catalytic performance. As reaction pressure independent, Na-S promotion effects: $\mathrm{CH}_{4}$ selectivity was suppressed, and ASF $\alpha$-value and overall hydrocarbon product slate olefin content both increased, in comparison to all of the unpromoted catalyst materials.

The Na-S promotion combined with 10 bar FTS reaction pressure threshold, had the effects of that: i) catalysts showed a significant boost in the FTYs, ii) Usage Ratio $\mathrm{H}_{2}: \mathrm{CO}$, $\mathrm{CO}$ converted to hydrocarbons and hydrocarbon selectivities became reaction pressure independent, and iii) $\mathrm{CO}_{2}$ selectivity was increased significantly. These FTS performance changes correlate with $\mathrm{Na}-\mathrm{S}$ induced transition of $\chi-\mathrm{Fe}_{5} \mathrm{C}_{2}$ Type I to Type II, and to the presence of $\chi-\mathrm{Fe}_{5} \mathrm{C}_{2}$ Type III with Na-S promoters. For unpromoted catalysts, 20 bar FTS reaction pressure threshold was required for attaining similar FTS catalysis effects as were observed with the $\mathrm{Na}-\mathrm{S}$ promoted catalyst materials with $\geq 10$ bar reaction pressure. For these changes, $\chi-\mathrm{Fe}_{5} \mathrm{C}_{2}$ Type I and unpromoted Type III were present as the $\chi-\mathrm{Fe}_{5} \mathrm{C}_{2}$ phase subtypes. With 3 bar reaction pressure, none of the studied catalyst materials showed significant FTYs regardless of the Na-S promotion.

For all of the catalyst materials under study: the $\mathrm{CH}_{4}$ selectivity inversely correlated with $\mathrm{CO}_{2}$ selectivity, as was expected from literature. The presence of $\eta-\mathrm{Fe}_{2} \mathrm{C}$ Type II did not correlate with any of the FTS catalytic performance - activity or selectivity indicators.

For both, Na-S promoted and unpromoted catalyst materials, the observed reaction pressure dependent FTY boosts were also accompanied by decreased Usage Ratios $\mathrm{H}_{2}$ : $\mathrm{CO}$ and $\mathrm{CO}$ conversion to hydrocarbons. That is, less $\mathrm{CO}$ was converted towards the desired FTS reaction produced hydrocarbons but was instead released as unwanted $\mathrm{CO}_{2}$, as an adverse effect to the boosted FTYs.

Regardless of the Na-S promotion, when employing calcined only catalyst materials in the FTS reaction: i) increased $\mathrm{C}_{2} \mathrm{H}_{4} / \mathrm{C}_{2} \mathrm{H}_{6}$ ratio with 10 bar FTS reaction pressure and ii) decreased overall FTYs with 20 bar reaction pressure in comparison to the $\mathrm{H}_{2}$ reduced catalysts were observed. The increased $\mathrm{C}_{2} \mathrm{H}_{4} / \mathrm{C}_{2} \mathrm{H}_{6}$ ratio can be independently of the Na-S promotion associated to the presence of $\chi-\mathrm{Fe}_{5} \mathrm{C}_{2}$ Type III with $10 \mathrm{bar}$ reaction pressure. That is, the $\chi-\mathrm{Fe}_{5} \mathrm{C}_{2}$ Type III correlates with catalyst material's decreased hydrogenation ability. Decreased FTYs in turn correlate with the presence of $\mathrm{Fe}_{3} \mathrm{O}_{4}$, in the calcined only, 20 bar FTS catalysts. The presence of the $\mathrm{Fe}_{3} \mathrm{O}_{4}$ phase possibly originates from mass transfer limited reduction of the initial $\alpha-\mathrm{Fe}_{2} \mathrm{O}_{3}\left(/ \alpha-\mathrm{Al}_{2} \mathrm{O}_{3}\right)$ phase. Na-S promotion combined with $\mathrm{H}_{2}$ reduction and 20 bar FTS reaction pressure 
resulted in high initial FTY with, however, FTY decreasing fast over the course of the FTS reaction.

For future research, the $\chi-\mathrm{Fe}_{5} \mathrm{C}_{2}$ Type III should be investigated in more detail for: i) an improved crystal structure solution, and ii) the carbide subtype's association to the increased $\mathrm{C}_{2} \mathrm{H}_{4} / \mathrm{C}_{2} \mathrm{H}_{6}$ ratio. The general relation e.g. whether Na-S promoters occupy different surface or crystal lattice positions in the $\chi-\mathrm{Fe}_{5} \mathrm{C}_{2}$ subtypes, is a further topic of interest. The observed fast deactivation of the $\mathrm{H}_{2}$ reduced, Na-S promoted catalysts in the 20 bar FTS reaction perhaps could be prevented with altered reaction initiation, which is another subject for a further study.

\subsection{ACKNOWLEDGEMENTS}

H. C. de Waard and C. Mulder (GeoLab at Universiteit Utrecht, UU) are acknowledged for the ICP-OES measurements. J. D. (Hans) Meeldijk and C. W. T. M. Schneijdenberg (UU) are acknowledged for their guidance in taking the TEM images.

\subsection{REFERENCES}

[1] Torres Galvis, H. M. and de Jong, K. P., "Catalysts for Production of Lower Olefins from Synthesis Gas: A Review", ACS Catal. 3, 2130-2149 (2013).

[2] Dry, M. E., "High quality diesel via the Fischer-Tropsch process - a review",J. Chem. Technol. Biotechnol. 77, 43-50 (2001).

[3] van der Laan, G. P. and Beenackers, A. A. C. M., "Kinetics and Selectivity of the Fischer-Tropsch Synthesis: A Literature Review", Catal. Rev. 41, 255-318 (1999).

[4] Dry, M. E., "The Fischer-Tropsch Synthesis" in Catalysis - Science and Technology (eds. Anderson, J. R. \& Boudart, M.) 1, 159-256 (Springer-Verlag, Berlin, 1981).

[5] Dry, M. E., "Chemical concepts used for engineering purposes" in Studies in Surface Science and Catalysis - Fischer-Tropsch Technology (eds. Steynberg, A. P. \& Dry, M. E.) 152, 196-257 (Elsevier B.V., Amsterdam, 2004).

[6] Botes, G. F., Niemantsverdriet, J. W. and van de Loosdrecht, J., "A comparison of cobalt and iron based slurry phase Fischer-Tropsch synthesis", Catal. Today 215, 112-120 (2013).

[7] Todic, B., Nowicki, L., Nikacevic, N. and Bukur, D. B., "Fischer-Tropsch synthesis product selectivity over an industrial iron-based catalyst: Effect of process conditions", Catal. Today 261, 28-39 (2016).

[8] Dry, M. E., "The Fischer-Tropsch process: 1950-2000", Catal. Today 71, 227-241 (2002).

[9] Dry, M. E., "Commercial conversion of carbon monoxide to fuels and chemicals", J. Organomet. Chem. 372, 117-127 (1989).

[10] Dictor, R. and Bell, A. T., "Fischer-Tropsch synthesis over reduced and unreduced iron oxide catalysts", J. Catal. 97, 121-136 (1986).

[11] Jiang, F., Zhang, M., Liu, B., Xu, Y. and Liu, X., "Insights into the influence of support and potassium or sulfur promoter on iron-based Fischer-Tropsch synthesis: understanding the control of catalytic activity, selectivity to lower olefins, and catalyst deactivation", Catal. Sci. Technol. 7, 1245-1265 (2017). 
[12] Ngantsoue-Hoc, W., Zhang, Y., O’Brien, R. J., Luo, M. and Davis, B. H., "Fischer-Tropsch synthesis: activity and selectivity for Group I alkali promoted iron-based catalysts", Appl. Catal. A Gen. 236, 7789 (2002).

[13] Dry, M. E. and Oosthuizen, G. J., "The correlation between catalyst surface basicity and hydrocarbon selectivity in the Fischer-Tropsch synthesis", J. Catal. 11, 18-24 (1968).

[14] Luo, M. and Davis, B. H., "Fischer-Tropsch synthesis: Group II alkali-earth metal promoted catalysts", Appl. Catal. A Gen. 246, 171-181 (2003).

[15] Pendyala, V. R. R., Graham, U. M., Jacobs, G., Hamdeh, H. H. and Davis, B. H., "Fischer-Tropsch Synthesis: Morphology, Phase Transformation, and Carbon-Layer Growth of Iron-Based Catalysts", ChemCatChem 6, 1952-1960 (2014).

[16] Pendyala, V. R. R., Graham, U. M., Jacobs, G., Hamdeh, H. H. and Davis, B. H., "Fischer-Tropsch Synthesis: Deactivation as a Function of Potassium Promoter Loading for Precipitated Iron Catalyst", Catal. Lett. 144, 1704-1716 (2014).

[17] Bukur, D. B., Mukesh, D. and Patel, S. A., "Promoter effects on precipitated iron catalysts for FischerTropsch synthesis", Ind. Eng. Chem. Res. 29, 194-204 (1990).

[18] Arakawa, H. and Bell, A. T., "Effects of potassium promotion on the activity and selectivity of iron Fischer-Tropsch catalysts", Ind. Eng. Chem. Process Des. Dev. 22, 97-103 (1983).

[19] Botes, G. F., Bromfield, T. C., Coetzer, R. L. J., Crous, R., Gibson, P. and Ferreira, A. C., "Development of a chemical selective iron Fischer Tropsch catalyst", Catal. Today 275, 40-48 (2016).

[20] Torres Galvis, H. M., Bitter, J. H., Khare, C. B., Ruitenbeek, M., Dugulan, A. I. and de Jong, K. P., "Supported Iron Nanoparticles as Catalysts for Sustainable Production of Lower Olefins", Science 335, 835-838 (2012).

[21] Torres Galvis, H. M., Koeken, A. C. J., Bitter, J. H., Davidian, T., Ruitenbeek, M., Dugulan, A. I. and de Jong, K. P., "Effects of sodium and sulfur on catalytic performance of supported iron catalysts for the Fischer-Tropsch synthesis of lower olefins", J. Catal. 303, 22-30 (2013).

[22] Johnson, J. Y., "Improvements in the Manufacture and Production of Unsaturated Hydrocarbons of Low Boiling Point", Patent GB322284A (1929).

[23] Crous, R., Bromfield, T. C. and Booyens, S., "Olefin Selective FT Catalyst Composition and Preparation Thereof", Patent W02010066386A1 (2010).

[24] Myddleton, W. W., "Improvements Relating to the Catalytic Synthesis of Hydrocarbon Oils from Gaseous Mixtures of Carbon Monoxide and Hydrogen", Patent GB509325A (1939).

[25] Xie, J., Yang, J., Dugulan, A. I., Holmen, A., Chen, D., De Jong, K. P. and Louwerse, M. J., "Size and Promoter Effects in Supported Iron Fischer-Tropsch Catalysts: Insights from Experiment and Theory", ACS Catal. 6, 3147-3157 (2016).

[26] Leineweber, A., Shang, S., Liu, Z.-K., Widenmeyer, M. and Niewa, R., "Crystal structure determination of Hägg carbide, $\chi-\mathrm{Fe}_{5} \mathrm{C}_{2}$ by first-principles calculations and Rietveld refinement", Zeitschrift für Krist. 227, 207-220 (2012).

[27] Retief, J. J., "Powder diffraction data and Rietveld refinement of Hägg-carbide, $\chi$-Fe ${ }_{5} \mathrm{C}_{2}$ ", Powder Diffr. 14, 130-132 (1999).

[28] Duggin, M. J. and Hofer, L. J. E., "Nature of $\chi$-Iron Carbide", Nature 212, 248-248 (1966).

[29] Jack, K. H. and Wild, S., "Nature of $\chi$-Carbide and Its Possible Occurrence in Steels", Nature 212, 248250 (1966).

[30] Cohn, E. M., Bean, E. H., Mentser, M., Hofer, L. J. E., Pontello, A., Peebles, W. C. and Jack, K. H., "The carburization of iron oxide with carbon monoxide: Modifications of Hägg iron carbide", J. Appl. Chem. 5, 418-425 (1955). 
[31] Sirimanothan, N., Hamdeh, H. H., Zhang, Y. and Davis, B. H., "Fischer-Trospch Synthesis; Changes in Phase and Activity during Use", Catal. Lett. 82, 181-191 (2002).

[32] O’Brien, R. J., Xu, L., Milburn, D. R., Li, Y.-X., Klabunde, K. J. and Davis, B. H., "Fischer-Tropsch synthesis: Impact of potassium and zirconium promoters on the activity and structure of an ultrafine iron oxide catalyst", Top. Catal. 2, 1-15 (1995).

[33] Rao, K. R. P. M., Huggins, F. E., Huffman, G. P., Gormley, R. J., O’Brien, R. J. and Davis, B. H., "Mössbauer Study of Iron Fischer-Tropsch Catalysts during Activation and Synthesis", Energy \& Fuels 10, 546-551 (1996).

[34] Bukur, D. B., Okabe, K., Rosynek, M. P., Li, C. P., Wang, D. J., Rao, K. R. P. M. and Huffman, G. P., "Activation Studies with a Precipitated Iron Catalyst for Fischer-Tropsch Synthesis. I. Characterization Studies", Journal of Catalysis 155, 353-365 (1995).

[35] Bukur, D. B., Nowicki, L., Manne, R. K. and Lang, X. S., "Activation Studies with a Precipitated Iron Catalyst for Fischer-Tropsch Synthesis II. Reaction Studies", J. Catal. 155, 366-375 (1995).

[36] Shultz, J. F., Hall, W. K., Seligman, B. and Anderson, R. B., "Studies of the Fischer-Tropsch Synthesis. XIV. Hägg Carbide as Catalysts", J. Am. Chem. Soc. 77, 213-221 (1955).

[37] Davidian, T., Ruitenbeek, M., Koeken, A. C. J., Vos, M. and Wielemaker, M. F., "Novel Iron-based Catalysts and Treatment Process therefor for Use in Fischer-Tropsch Reactions", Patent W02014210089A1 (2014).

[38] Wezendonk, T. A., Sun, X., Dugulan, A. I., van Hoof, A. J. F., Hensen, E. J. M., Kapteijn, F. and Gascon, J., "Controlled formation of iron carbides and their performance in Fischer-Tropsch synthesis", J. Catal. 362, 106-117 (2018).

[39] de Smit, E., Cinquini, F., Beale, A. M., Safonova, O. V., van Beek, W., Sautet, P. and Weckhuysen, B. M., "Stability and Reactivity of $\epsilon-\chi-\theta$ Iron Carbide Catalyst Phases in Fischer-Tropsch Synthesis: Controlling $\mu_{c}$ ", J. Am. Chem. Soc. 132, 14928-14941 (2010).

[40] de Smit, E., Beale, A. M., Nikitenko, S. and Weckhuysen, B. M., "Local and long range order in promoted iron-based Fischer-Tropsch catalysts: A combined in situ X-ray absorption spectroscopy/wide angle Xray scattering study", J. Catal. 262, 244-256 (2009).

[41] Datye, A. K., Jin, Y., Mansker, L. D., Motjope, R. T., Dlamini, T. H. and Coville, N. J., "The nature of the active phase in iron Fischer-Tropsch catalysts" in Studies in Surface Science and Catalysis 130, 11391144 (2000).

[42] Mansker, L. D., Jin, Y., Bukur, D. B. and Datye, A. K., "Characterization of slurry phase iron catalysts for Fischer-Tropsch synthesis", Appl. Catal. A Gen. 186, 277-296 (1999).

[43] Jung, H. and Thomson, W. J., "Dynamic X-ray diffraction study of an unsupported iron catalyst in Fischer-Tropsch synthesis", J. Catal. 134, 654-667 (1992).

[44] McCartney, J. T., Hofer, L. J. E., Seligman, B., Lecky, J. A., Peebles, W. C. and Anderson, R. B., "Electron and X-Ray Diffraction Studies of Iron Fischer-Tropsch Catalysts", J. Phys. Chem. 57, 730-736 (1953).

[45] Jenkins, R. and Snyder, R. L., "Introduction to X-ray Powder Diffractometry", Introduction to X-ray Powder Diffractometry 138 (John Wiley \& Sons, Inc., Hoboken, NJ, USA, 1996).

[46] De La Torre, A. G., Bruque, S. and Aranda, M. A. G., "Rietveld quantitative amorphous content analysis", J. Appl. Crystallogr. 34, 196-202 (2001).

[47] Klencsár, Z., Kuzmann, E. and Vértes, A., "User-friendly software for Mössbauer spectrum analysis", J. Radioanal. Nucl. Chem. Artic. 210, 105-118 (1996).

[48] Torres Galvis, H. M., Koeken, A. C. J., Bitter, J. H., Davidian, T., Ruitenbeek, M., Dugulan, A. I. and De Jong, K. P., "Effect of precursor on the catalytic performance of supported iron catalysts for the FischerTropsch synthesis of lower olefins", Catal. Today 215, 95-102 (2013). 
[49] Koeken, A. C. J., Torres Galvis, H. M., Davidian, T., Ruitenbeek, M. and de Jong, K. P., "Suppression of Carbon Deposition in the Iron-Catalyzed Production of Lower Olefins from Synthesis Gas", Angew. Chem., Int. Ed. 51, 7190-7193 (2012).

[50] Xie, J., Torres Galvis, H. M., Koeken, A. C. J., Kirilin, A., Dugulan, A. I., Ruitenbeek, M. and de Jong, K. P., "Size and Promoter Effects on Stability of Carbon-Nanofiber-Supported Iron-Based Fischer-Tropsch Catalysts", ACS Catal. 6, 4017-4024 (2016).

[51] Toby, B. H., "R factors in Rietveld analysis: How good is good enough?", Powder Diffr. 21, 67-70 (2006).

[52] Jozwiak, W. K., Kaczmarek, E., Maniecki, T. P., Ignaczak, W. and Maniukiewicz, W., "Reduction behavior of iron oxides in hydrogen and carbon monoxide atmospheres", Appl. Catal. A Gen. 326, 1727 (2007).

[53] Mogorosi, R. P., Fischer, N., Claeys, M. and van Steen, E., "Strong-metal-support interaction by molecular design: Fe-silicate interactions in Fischer-Tropsch catalysts", J. Catal. 289, 140-150 (2012).

[54] An, X., Wu, B., Wan, H.-J., Li, T.-Z., Tao, Z.-C., Xiang, H.-W. and Li, Y.-W., "Comparative study of ironbased Fischer-Tropsch synthesis catalyst promoted with potassium or sodium", Catal. Commun. 8 , 1957-1962 (2007).

[55] Hirotsu, Y. and Nagakura, S., "Crystal structure and morphology of the carbide precipitated from martensitic high carbon steel during the first stage of tempering", Acta Metall. 20, 645-655 (1972).

[56] Hirotsu, Y. and Nagakura, S., "Electron Microscopy and Diffraction Study of the Carbide Precipitated at the First Stage of Tempering of Martensitic Medium Carbon Steel", Trans. Japan Inst. Met. 15, 129134 (1974).

[57] Barton, G. H. and Gale, B., "The structure of a pseudo-hexagonal iron carbide", Acta Crystallogr. 17, 1460-1462 (1964).

[58] Niemantsverdriet, J. W., van der Kraan, A. M., van Dijk, W. L. and van der Baan, H. S., "Behavior of Metallic Iron Catalysts during Fischer-Tropsch Synthesis Studied with Mössbauer Spectroscopy, X-ray Diffraction, Carbon Content Determination, and Reaction Kinetic Measurements", J. Phys. Chem. 84, 3363-3370 (1980).

[59] Tajima, S. and Hirano, S., "Synthesis and Magnetic Properties of $\mathrm{Fe}_{7} \mathrm{C}_{3}$ Particles with High Saturation Magnetization", Jpn. J. Appl. Phys. 29, 662-668 (1990).

[60] O’Brien, R. J., Xu, L., Spicer, R. L. and Davis, B. H., "Activation Study of Precipitated Iron Fischer-Tropsch Catalysts", Energy \& Fuels 10, 921-926 (1996).

[61] Ratnasamy, C. and Wagner, J. P., "Water Gas Shift Catalysis", Catal. Rev. 51, 325-440 (2009).

[62] Rhodes, C., Hutchings, G. J. and Ward, A. M., "Water-gas shift reaction: finding the mechanistic boundary", Catal. Today 23, 43-58 (1995).

[63] Shultz, J. F., Abelson, M., Stein, K. C. and Anderson, R. B., "Studies of the Fischer-Tropsch Synthesis. XVIII. Influence of Catalyst Geometry on Synthesis on Iron Catalysts", J. Phys. Chem. 63, 496-500 (1959).

[64] Anderson, R. B., Hofer, L. J. E., Cohn, E. M. and Seligman, B., "Studies of the Fischer-Tropsch Synthesis. IX. Phase Changes of Iron Catalysts in the Synthesis", J. Am. Chem. Soc. 73, 944-946 (1951).

[65] Ning, W., Koizumi, N., Chang, H., Mochizuki, T., Itoh, T. and Yamada, M., "Phase transformation of unpromoted and promoted Fe catalysts and the formation of carbonaceous compounds during Fischer-Tropsch synthesis reaction", Appl. Catal. A Gen. 312, 35-44 (2006).

[66] Berry, F. J. and Smith, M. R., "A Mössbauer investigation of iron-containing catalysts prepared at low temperatures and active for carbon monoxide hydrogenation", J. Chem. Soc. Faraday Trans. 1 Phys. Chem. Condens. Phases 85, 467-477 (1989). 
[67] Dry, M. E., Shingles, T. and van H. Botha, C. S., "Factors influencing the formation of carbon on iron Fischer-Tropsch catalysts I. The influence of promoters", J. Catal. 17, 341-346 (1970).

[68] Dry, M. E., Shingles, T., Boshoff, L. J. and van H. Botha, C. S., "Factors influencing the formation of carbon on iron Fischer-Tropsch catalysts. II. The effect of temperature and of gases and vapors present during Fischer-Tropsch synthesis", J. Catal. 17, 347-354 (1970).

[69] Sarkar, A., Seth, D., Dozier, A. K., Neathery, J. K., Hamdeh, H. H. and Davis, B. H., "Fischer-Tropsch Synthesis: Morphology, Phase Transformation and Particle Size Growth of Nano-scale Particles", Catal. Lett. 117, 1-17 (2007).

[70] Steynberg, A. P., Espinoza, R. L., Jager, B. and Vosloo, A. C., "High temperature Fischer-Tropsch synthesis in commercial practice", Appl. Catal. A Gen. 186, 41-54 (1999).

[71] Amenomiya, Y. and Pleizier, G., "Alkali-promoted Alumina Catalysts II. Water-Gas Shift Reaction", J. Catal. 76, 345-353 (1982).

[72] Krupay, B. W. and Amenomiya, Y., "Alkali-promoted Alumina Catalysts I. Chemisorption and oxygen exchange of carbon monoxide and carbon dioxide on potassium-promoted alumina catalysts", J. Catal. 67, 362-370 (1981).

[73] Chianese, S., Loipersböck, J., Malits, M., Rauch, R., Hofbauer, H., Molino, A. and Musmarra, D., "Hydrogen from the high temperature water gas shift reaction with an industrial Fe/Cr catalyst using biomass gasification tar rich synthesis gas", Fuel Process. Technol. 132, 39-48 (2015).

[74] Lywood, W. J. and Twigg, M. V., "Hydrogen production including a shift reaction process", Patent EP0361648A1 (1990).

[75] van Dijk, W. L., Niemantsverdriet, J. W., van dar Kraan, A. M. and van der Baan, H. S., "Effects of Manganese Oxide and Sulphate on the Olefin Selectivity of Iron Catalysts in the Fischer Tropsch Reaction", Appl. Catal. 2, 273-288 (1982).

[76] Zhou, X., Ji, J., Wang, D., Duan, X., Qian, G., Chen, D. and Zhou, X., "Hierarchical structured $\alpha$-Al2O3 supported S-promoted Fe catalysts for direct conversion of syngas to lower olefins", Chem. Commun. 51, 8853-8856 (2015).

[77] Madon, R. J. and Shaw, H., "Effect of Sulfur on the Fischer-Tropsch Synthesis", Catal. Rev. 15, 69-106 (1977).

[78] Förtsch, D., Pabst, K. and Groß-Hardt, E., "The product distribution in Fischer-Tropsch synthesis: An extension of the ASF model to describe common deviations", Chem. Eng. Sci. 138, 333-346 (2015).

[79] Berry, T. F., Ames, R. N. and Snow, R. B., "Influence of Impurities and Role of Iron Carbides in Deposition of Carbon from Carbon Monoxide", J. Am. Ceram. Soc. 39, 308-318 (1956). 
This page is intentionally left blank 


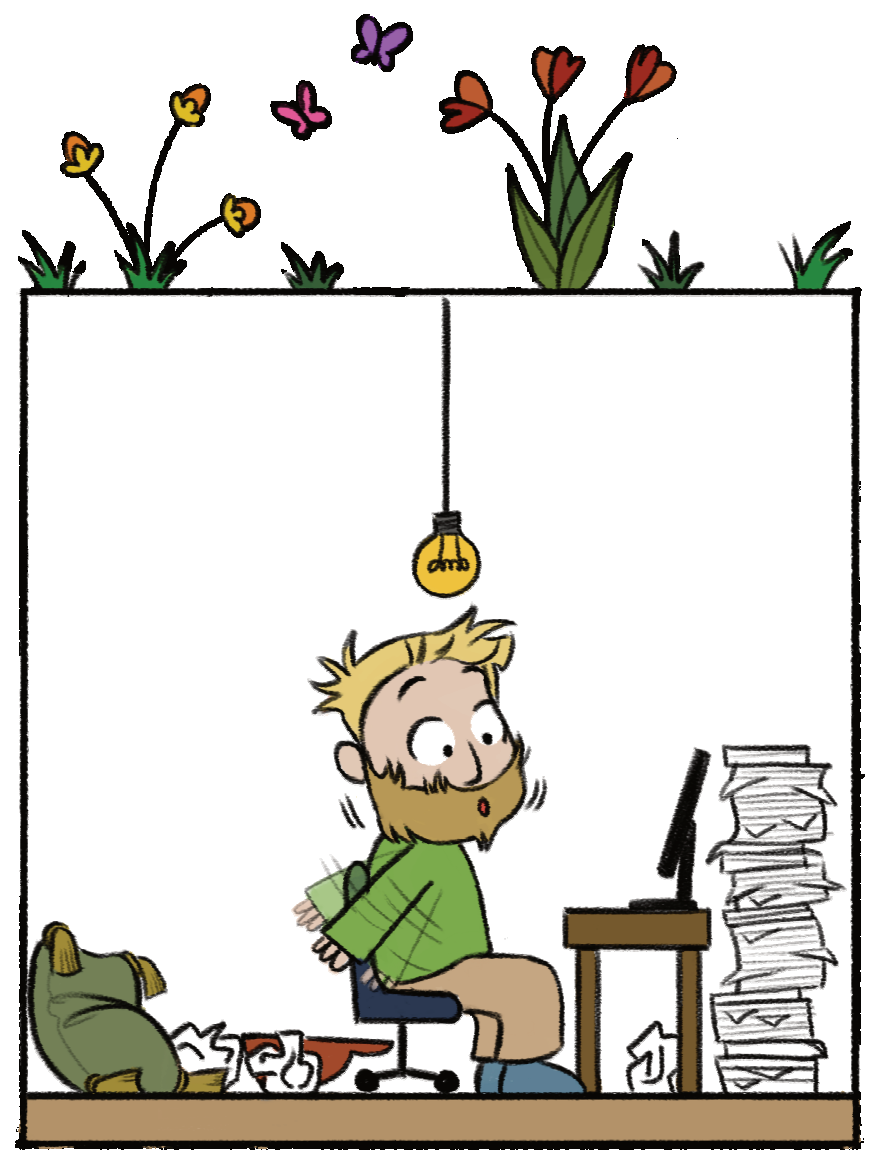




\section{Chapter 6}

\section{Combined In-situ X-Ray Powder}

Diffractometry/Raman Spectroscopy of Iron Carbide and Carbon Species Evolution in $\mathrm{Fe}$ (-NaS) $/ \alpha-\mathrm{Al}_{2} \mathrm{O}_{3}$ Catalysts during Fischer-Tropsch Synthesis

A Na-S promoted Fe-based catalyst converts a $\mathrm{H}_{2}$ : $\mathrm{CO}$ gas mixture into hydrocarbons with enriched $\mathrm{C}_{2}-\mathrm{C}_{4}$ olefin content in high-temperature $\left(320-375^{\circ} \mathrm{C}\right)$ Fischer-Tropsch Synthesis (FTS) reaction. Above $300^{\circ} \mathrm{C}$, the carbon depositing Boudouard reaction competes with the FTS reaction for the reactant CO. With a combined in-situ X-Ray Powder Diffractometry (XRPD)/Raman Spectroscopy set-up the simultaneous evolution of $\mathrm{Fe}_{\mathrm{x}} \mathrm{O}_{\mathrm{y}} / \alpha-\mathrm{Fe} / \mathrm{Fe}_{\mathrm{x}} \mathrm{C}$ and carbon species has been monitored during $340^{\circ} \mathrm{C}$, 10 bar FTS reaction. CO carburized, Na-S promoted and unpromoted $\mathrm{Fe}(-\mathrm{Na}-\mathrm{S}) / \alpha-$ $\mathrm{Al}_{2} \mathrm{O}_{3}$ catalysts were employed. Fe phases were quantified with Rietveld Quantitative Phase Analysis (R-QPA) from the XRPD patterns. The Raman D- and G-bands were analyzed for their relative intensities, bandwidths, and positions, and compared to reference carbon materials. Amorphous carbon with $\mathrm{C} \mathrm{sp}{ }^{3}$ and $\mathrm{C} \mathrm{sp}{ }^{2}$ in chain-like ordering evolved towards Carbon Nanofiber (CNF)-type structures during FTS reaction. Na-S promotion and initial $\mathrm{CO}$ carburization $\geq 340^{\circ} \mathrm{C}$ led to increased cyclic 6-fold $\mathrm{C} \mathrm{sp}{ }^{2}$ species. Preliminary carbon deposits on catalysts decreased the initial fast Raman band intensity increase, while Na-S promotion increased steady period band intensity growth. The carbon species evolution was unaffected by specific Fe carbides or carbide-to-carbide transitions. Na-S promotion aided in reduction of $\mathrm{Fe}_{3} \mathrm{O}_{4}$ by $\left(\mathrm{H}_{2}\right.$ :) CO to carbon depositing Fe carbides. The results add to understanding on Fe and carbon species evolution during high-temperature FTS reaction.

This PhD chapter is based on: Pasi P. Paalanen, Sophie H. van Vreeswijk and Bert M. Weckhuysen, "Combined In-situ X-Ray Powder Diffractometry/Raman Spectroscopy of Iron Carbide and Carbon Species Evolution in $\mathrm{Fe}(-\mathrm{Na}-\mathrm{S}) / \alpha-\mathrm{Al}_{2} \mathrm{O}_{3}$ Catalysts during Fischer-Tropsch Synthesis", ACS Catalysis, submitted. 


\subsection{INTRODUCTION}

With Fischer-Tropsch Synthesis (FTS) reaction, a $\mathrm{H}_{2}$ :CO gas mixture can be converted into valuable hydrocarbons. ${ }^{1,2,11,3-10}$ With the combination of high reaction temperature $\left(320-375^{\circ} \mathrm{C}\right)$ and $\mathrm{Na}-\mathrm{S}$ chemical promotion, the synthesized hydrocarbon product slate can be directed towards the formation of industrially valuable, short-chained $\left(C_{2}-C_{4}\right)$, olefinic hydrocarbons. ${ }^{12-22}$

However, for FTS reaction temperatures $\gtrsim 300^{\circ} \mathrm{C}^{2,23-25}$ the Boudouard reaction (i.e. $\mathrm{CO}$ disproportionation of $\mathrm{CO}$ into $\mathrm{C}$ and $\mathrm{CO}_{2}$ ) also becomes active depositing catalyst deactivating inactive carbon on the catalyst material. The Boudouard therefore competes with the FTS reaction directly for the reactant $\mathrm{CO}^{26,27}$ Regeneration and removal of the carbon deposits would be possible via catalyst hydrogenation $\geq 350^{\circ} \mathrm{C} .{ }^{2}$ However, because of catalyst material's fragmentation associated to the carbon deposition, especially with bulk Fe catalysts, the catalyst's lost mechanical stability cannot be remedied with simple hydrogenation treatments. ${ }^{2,28-30}$

Under FTS reaction conditions, the Fe-based catalyst material forms various (near)zerovalent $\mathrm{Fe}$ carbides. The $\mathrm{Fe}$ carbides are the $\mathrm{CO}$ dissociating active phases necessary for both the competing FTS and Boudouard reactions. 19,27,31,32 The deposition of inactive carbon has been proposed to be associated to the presence of $\theta-\mathrm{Fe}_{3} \mathrm{C}$ (cementite), ${ }^{33-35}$ and/or to the transition of $\mathrm{C}$-rich " $\varepsilon$-carbides" (i.e. $\varepsilon$ - $\mathrm{Fe}_{3} \mathrm{C}$ and/or $\eta$ $\mathrm{Fe}_{2} \mathrm{C}$, Chapter 2) to lesser C-containing $\chi-\mathrm{Fe}_{5} \mathrm{C}_{2}$ (the Hägg carbide) or $\theta-\mathrm{Fe}_{3} \mathrm{C} .{ }^{36-38}$

Elements from alkali metal group, most often as $\mathrm{Na}$ or $\mathrm{K}$, are commonly added chemical promoters to Fe-based FTS reaction catalyst materials. These alkali promoters intrinsically increase the carbon deposition rate. ${ }^{2,23}$ Individually added $\mathrm{S}$ as a promoter, is commonly observed to decrease the overall carbon deposition on Fe-based catalyst materials. ${ }^{39-43}$ However, the addition of $\mathrm{S}$ to the FTS reaction catalyst also results in an overall decreased CO conversion, therefore necessarily decreasing the carbon deposition. ${ }^{44}$ Decreased $C O$ conversion can also lead to removal of reactor bed hotspots which can lead to observations of decreased carbon deposition. ${ }^{45-48}$ That is, individual $\mathrm{S}$ addition does not necessarily alter the $\mathrm{C}$ selectivity between the FTS and Boudouard reactions, ${ }^{48}$ unlike the alkali promoters. When $\mathrm{Na}$ and $\mathrm{S}$ are added together as promoters, the combined Na-S promotion behaves "alkali-like" and the carbon deposition is effectively increased in the high-temperature FTS reaction in comparison to unpromoted catalyst materials. ${ }^{17,20}$

The type of carbon formed, deactivating the FTS reaction catalyst material, is often described with loosely defined terms as "graphitic-like".34,38,49,50 or as an amorphous carbonaceous overlayer. ${ }^{50-52}$ Furthermore, the formation of fibrous carbon species is possible under the FTS reaction conditions, in the presence of $\mathrm{H}_{2}$ or other $\mathrm{H}$ containing 
chemical species. ${ }^{37,53-55}$ The formation of carbon species has been proposed to be related to the transition of C-rich (" $\varepsilon$ ") into C-poor $(\chi, \theta)$ Fe carbide phases. The Fe carbide lattice ejected $\mathrm{C}$-atoms have been proposed to be providing nucleation centers for the deactivating carbon formation. ${ }^{36-38}$ The carbon species are thought to evolve from initial polymeric carbon towards more ordered carbon structures. ${ }^{38,50}$

$\mathrm{X}$-Ray Powder Diffractometry (XRPD) is a suitable tool for characterizing the Fe carbide phases and is widely applied within the FTS reaction research field.4,25,29,33,34,36,56-60 Raman Spectroscopy is a technique capable of differentiating between "graphitic-like", amorphous and fibrous carbon species.61-65 Both characterization methods can in principle also be applied under in-situ or operando conditions, thereby allowing probing of the changes in the Fe phases and carbon deposits formation during the FTS reaction. Previously, in-situ/operando Raman Spectroscopy approach has been applied e.g. to follow carbon deposit formation in propane dehydrogenation, ${ }^{66,67}$ and Co-based FTS reaction. ${ }^{68}$

In this Chapter, we combine XRPD and Raman Spectroscopy in one experimental
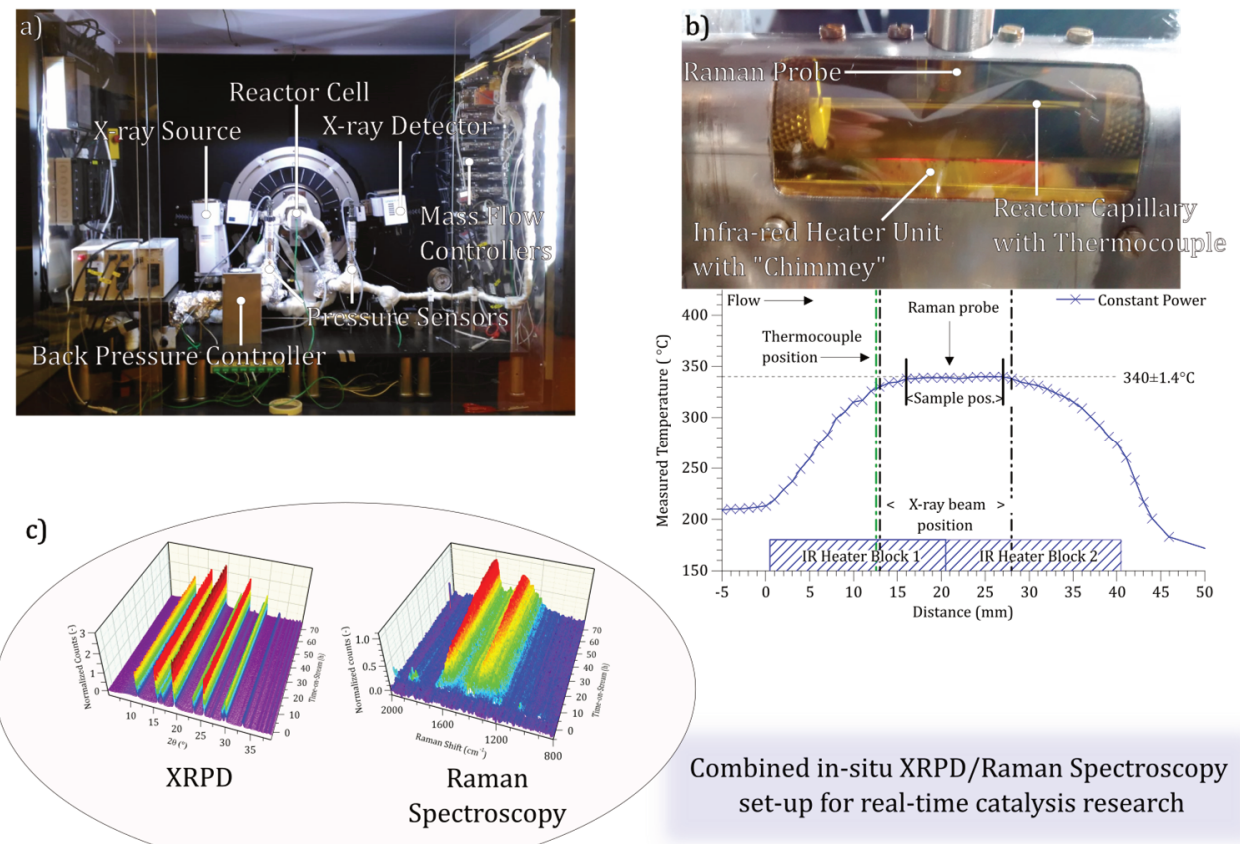

Figure 6.1

Illustration and photos of the scientific approach applied in this Chapter. Photos of the used a) combined insitu X-ray Powder Diffractometry (XRPD)/Raman Spectroscopy set-up, b) close-up on the reactor cell (top panel) with the measured reactor isothermal zone (bottom panel). With the set-up, both c) XRPD and Raman Spectroscopy can be performed in real-time, at reaction conditions relevant to catalysis research. 
set-up for studying the transformation of the different Fe phases as well as the evolution of carbon species in-situ (i.e. as measured at the reaction conditions) (Figure 6.1). This is done with a set-up recently developed in our research group. ${ }^{68}$ The catalyst materials under study are characterized under FTS reaction conditions of $340^{\circ} \mathrm{C}$ at 10 bar in a $\mathrm{H}_{2}$ : CO 2:1 gas mixture. The studied catalyst materials are supported $\mathrm{Fe}(-\mathrm{Na}-\mathrm{S}) / \alpha-\mathrm{Al}_{2} \mathrm{O}_{3}$ catalysts, prepared with and without $\mathrm{Na}-\mathrm{S}$ promotion. The catalysts have been prepared to contain preformed $\mathrm{Fe}$ carbides (i.e. $\varepsilon-\mathrm{Fe}_{3} \mathrm{C}, \eta-\mathrm{Fe}{ }_{2} \mathrm{C}, \chi-\mathrm{Fe}_{5} \mathrm{C}_{2}$ and/or $\theta-\mathrm{Fe}_{3} \mathrm{C}$ ) via a $\mathrm{CO}$ carburization step or to contain $\alpha-\mathrm{Fe} / \mathrm{Fe}_{\mathrm{x}} \mathrm{O}_{\mathrm{y}}$ phases via a $\mathrm{H}_{2}$ reduction step. The details on the catalyst preparation and related characterization data of these catalyst materials can be found in Chapter 3. Rietveld Quantitative Phase Analysis (R-QPA) is used to quantify the collected XRPD data. The R-QPA derived Fe phase content changes and the Fe phases' crystallite diameters are qualitatively compared to simultaneous changes observed in the collected Raman spectra.

\subsection{EXPERIMENTAL METHODS}

\subsubsection{Catalyst Preparation}

The details on the catalyst synthesis and the applied catalyst treatments of CO carburization and $\mathrm{H}_{2}$ reduction are described in detail in Chapter 3. In short, nominal 7 weight percent (wt-\%) of Fe on $\alpha$-alumina supported (Fe-(Na-S) $/ \alpha-\mathrm{Al}_{2} \mathrm{O}_{3}$ ) catalyst materials, with or without Na-S promotion, were synthesized with the use of a Fe citrate precursor. Nominal promoter loadings of $0.29 \mathrm{wt}-\%$ for $\mathrm{Na}$ and $0.04 \mathrm{wt}-\%$ for S were used. The synthesized catalyst materials were calcined $\left(500^{\circ} \mathrm{C}, 6 \mathrm{~h}\right)$, and reduced under $\mathrm{H}_{2}\left(400^{\circ} \mathrm{C}, 5 \mathrm{~h}\right)$. The $\mathrm{H}_{2}$ reduction step was followed by a CO carburization step at either $240^{\circ} \mathrm{C}, 340^{\circ} \mathrm{C}$ or $440^{\circ} \mathrm{C}$, in order to prepare preformed Fe carbides in the catalyst materials. For comparison, only $\mathrm{H}_{2}$ reduced catalyst materials were also prepared.

\subsubsection{Catalyst Characterization}

\subsubsection{Details of the In-situ Reactor Cell}

The (ex-situ) prepared catalyst materials were characterized under FTS reaction conditions using a modified in-situ XRPD plug flow capillary reactor cell (iKey). ${ }^{69-71}$ The cell was combined with high-temperature Raman Spectroscopy probe. ${ }^{68}$

The iKey reactor cell unit employs two infrared (IR) heater blocks for heating the reactor capillary up to the reaction temperature (Figure 6.1b). The reaction temperature is measured with a thermocouple inserted inside the reactor capillary. The original reactor cell was modified by reversing the inlet for reactant gas flow in order to avoid hydrocarbon condensation on the thermocouple inserted inside the reactor 
Combined In-situ X-Ray Powder Diffractometry/Raman Spectroscopy of Iron Carbide and Carbon Species Evolution in Fe(-Na-S)/ $\alpha-\mathrm{Al} 203$ Catalysts during Fischer-Tropsch Synthesis

capillary. In addition, the IR heaters were covered with a custom-made stainless steel "chimney" in order to decrease the thermal radiance from the IR heater blocks measured with the Raman probe. Furthermore, the IR heater blocks were resistance matched in order to acquire a uniform isothermal heating zone. Reactor cell temperature calibration was performed under nominal input flow of $1 \mathrm{ml} / \mathrm{min} \mathrm{Ar}$ and $1 \mathrm{ml} / \mathrm{min}$ He by moving the thermocouple inside the reactor capillary while keeping the power fed to the heater at set constant. The reactor isothermal zone was measured to be $340 \pm 1.4^{\circ} \mathrm{C}$ over a distance of $\approx 11.0 \mathrm{~mm}$. Further details on the used reactor cell type and the laboratory-based XRPD/Raman Spectroscopy set-up can be found from the cited references. ${ }^{68-71}$

\subsubsection{Catalytic Reaction}

The catalyst materials were inserted to $1000 \mu \mathrm{m}(10 \mu \mathrm{m}$ wall thickness) borosilicate capillaries. An undiluted catalyst bed of $\approx 11 \mathrm{~mm}$ (6.4-7.2 mg of catalyst material, 212$425 \mu \mathrm{m}$ catalyst particle size), pinned in between quartz wool plugs, was used for each separate in-situ FTS reaction run. Prior to each run, the experimental set-up was leak tested at $100^{\circ} \mathrm{C}$ under $1: 1 \mathrm{Ar}: \mathrm{He}(2 \mathrm{ml} / \mathrm{min})$ flow with the loaded sample capillary in place. After the leak test, the capillary reactor pressure was set to $0.5 \mathrm{bar}(\mathrm{g})$.

The FTS reactions were performed at $340^{\circ} \mathrm{C}$, at 10 bar $\mathrm{H}_{2}$ : $\mathrm{CO}: \mathrm{He}$ (2.0:1.0:0.33 volume ratio) total pressure. The nominal input gas flows to the in-situ reactor were $0.6 \mathrm{ml} / \mathrm{min} \mathrm{H}_{2}, 0.3 \mathrm{ml} / \mathrm{min} \mathrm{CO}$ and $0.1 \mathrm{ml} / \mathrm{min}$ He giving a gas hourly space velocity (GHSV, as total input gas flow per total reactor bed volume) of $\approx 7200 \mathrm{~h}^{-1}$. After the initial leak testing at $100^{\circ} \mathrm{C}$, the reactor capillary was heated to $120^{\circ} \mathrm{C}\left(10^{\circ} \mathrm{C} / \mathrm{min}\right)$ and pressurized at $120^{\circ} \mathrm{C}$ to 10 bar total reaction pressure. The pressurizing was done with $5 \mathrm{x}$ the FTS reaction gas flows, within $1 \mathrm{~h}$. After reaching the 10 bar reaction pressure, the reactor was heated to the reaction temperature of $340^{\circ} \mathrm{C}\left(2^{\circ} \mathrm{C} / \mathrm{min}\right)$. The $0 \mathrm{~h}$ Timeon-Stream (ToS) was taken as the moment when $340^{\circ} \mathrm{C}$ was reached and the FTS reaction was then subsequently ran for $72 \mathrm{~h}$. After the $72 \mathrm{~h}$ FTS reaction, the reactor was cooled down to $100^{\circ} \mathrm{C}$ within $1 \mathrm{~h}$ under the set $\mathrm{H}_{2}$ : $\mathrm{CO}$ : He flow while simultaneously decreasing the reaction pressure to $0.5 \mathrm{bar}(\mathrm{g})(0.5 \mathrm{bar} / \mathrm{min})$. At $100^{\circ} \mathrm{C}$, the input gas flow was switched to 1:1 Ar:He (2 $\mathrm{ml} / \mathrm{min})$ and the reactor was flushed under the inert gases. $\mathrm{H}_{2}$, Ar and He gas purities were grade 5.0 (99.999\% pure) and for CO grade 2.5 (99.5\% pure), as supplied by Linde Gas Benelux.

During the FTS reactions, up- and downstream pressure gauges and a mass flow meter upstream of the reactor capillary were used to follow possible mass flow limitations in the reactor bed. No mass flow limitations were observed in any of the performed FTS reaction runs. 


\subsubsection{In-situ X-ray Powder Diffractometry}

XRPD patterns were collected in-situ during the FTS reaction $\left(340^{\circ} \mathrm{C}, 10 \mathrm{bar}, \mathrm{Hz}: \mathrm{CO}: \mathrm{He}\right)$ as well as in quasi-in-situ (at $100^{\circ} \mathrm{C}, 0.5 \mathrm{bar}, \mathrm{Ar}$ :He, i.e. "spent catalyst") after the respective $72 \mathrm{~h}$ FTS reaction run. The initial "fresh catalyst" was measured also under $100^{\circ} \mathrm{C}(0.5 \mathrm{bar}, \mathrm{Ar}: \mathrm{He})$ after the initial leak testing step. For collecting the in-situ XRPD patterns, a Bruker D8 Discover instrument in Debye-Scherrer transmission (capillary) geometry with a Mo $\left(K_{\alpha 1} 0.709 \AA\right)$ source was used, as in previous Chapters. At the beginning of each in-situ reaction run, the capillary was focused onto the X-ray beam (beam: $\approx 600 \times 15000 \mu \mathrm{m}$, height $\mathrm{x}$ width) for maximum diffraction. The diffraction patterns were collected over a $2 \theta$ range of $5-39^{\circ}$ with scan step size of $0.015^{\circ}$. For collecting the XRPD patterns before and after the FTS reaction (quasi-in-situ), $6 \mathrm{~h}$ total measurement time was used. For the in-situ measurements during the FTS reaction at $340^{\circ} \mathrm{C}$ and during the pressurizing and temperature ramp steps, $0.5 \mathrm{~h}$ total measurement time was used. The term X-ray Powder Diffractometry (XRPD) is used to refer to X-ray diffraction phenomena based characterization experiments that are done under powder diffraction conditions. ${ }^{72}$

The R-QPA method was employed for quantifying the crystalline Fe phases in the collected XRPD patterns. The application of R-QPA fitting has been described in detail in Chapter 3 and is applied within this Chapter in a similar manner, with few modifications in the applied fitting model. As the primary modification in the R-QPA fitting model, the lattice parameters for the support material $\alpha-\mathrm{Al}_{2} \mathrm{O}_{3}$ were also refined simultaneously - unlike in the previous Chapters - with the rest of the fitted phases. This was done in order to accommodate the thermal expansion of the materials lattices during the in-situ measurements at elevated temperature. The effects of crystal lattice thermal expansion, ${ }^{73}$ reactor capillary deformation/displacement and sample material density changes in the capillary, ${ }^{74}$ all affect the phases' diffraction peak positions and thus the lattice parameters observed in the performed in-situ XRPD measurements. The changes to the lattice parameters for the fitted phases could not be decoupled from the mentioned experimental effects in the in-situ XRPD runs and the intrinsic phase lattice parameters could not be solved for the fitted phases. All refined lattice parameters were constrained to an arbitrary range with constrains from $-0.01 \AA$ to $+0.05 \AA$ from the literature values (for Fe carbides, Chapter 3). The R-QPA fitting models and constrain values were adjusted until the measured XRPD diffraction peak positions were satisfactorily accounted for. The literature and database sources for the crystal structures used in the R-QPA fitting models are given in Chapter 3.

As further differences to the applied fitting model, all measured XRPD pattern backgrounds were modelled with a $3^{\text {rd }}$ order Chebyshev polynomial. Three independent Lorentzian peaks were used for fitting the amorphous content scattering 
Combined In-situ X-Ray Powder Diffractometry/Raman Spectroscopy of Iron Carbide and Carbon Species Evolution in Fe(-Na-S)/ $\alpha$-Al203 Catalysts during Fischer-Tropsch Synthesis

and/or diffraction contribution within the range $\approx 8-12^{\circ} 2 \theta$.

Predetermined R-QPA fitting models were applied on the measured quasi- and insitu measured XRPD patterns over the full XRPD measurement range using a batch mode with the Bruker TOPAS v5.0 software. The phases with $<0.4$ wt- $\%$ phase content and/or relative wt- $\%$ fitting errors $\geq 30 \%$ were removed from the fitting models. The measured, background subtracted, XRPD patterns were aligned and normalized to the $\alpha-\mathrm{Al}_{2} \mathrm{O}_{3}$ peak $\left(\begin{array}{ll}0 & 2\end{array}\right)$ at its nominal position of $23.515^{\circ} 2 \theta$ (at Mo $\mathrm{K}_{\alpha 1}$ ) at room temperature, for presenting the diffraction pattern heatmaps versus the FTS reaction ToS.

The R-QPA derived values from the in-situ XRPD runs are reported as 5-point median values of the data points obtained every $0.5 \mathrm{~h}$ and expressed with median's Standard Deviation (STD) as an error estimate. R-QPA derived values for the "fresh catalysts" and quasi-in-situ measured "spent catalysts" after the FTS reaction run are reported for each single $(6 \mathrm{~h})$ measurement with their respective R-QPA fitting errors as the error estimates.

\subsubsection{In-situ Raman Spectroscopy}

Simultaneously to the in-situ XRPD measurements, in-situ Raman Spectroscopy measurements were performed in order to follow the formation and further development of carbon species on the catalyst materials during the FTS reactions. A thermoelectrically cooled AvaRaman (Avantes) spectroscopy system with $50 \mathrm{~mW}$ $532 \mathrm{~nm}$ excitation laser, coupled with a high-temperature InPhotonics Raman probe with $\approx 200 \mu \mathrm{m}$ measurement spot size at focus, was used. The spectrometer's spectral resolution was $6 \mathrm{~cm}^{-1}$. At the start of each FTS reaction run, the Raman probe was focused on the catalyst bed using the $\alpha-\mathrm{Al}_{2} \mathrm{O}_{3}$ peak at $\approx 4389 \mathrm{~cm}^{-1}$ (see e.g. RRUFF database \#R040096 for reference). The dark spectrum corrected, in-situ Raman spectra were collected over a spectral range of $\approx 174-5685 \mathrm{~cm}^{-1}$. A single Raman spectrum was recorded every 10 min by averaging 600 scans with $1 \mathrm{~s}$ integration time.

Carbon Nanofibers (CNF, Pyrograf, PR-24-XT-LHT), graphite (Aldrich, 282863), Activated Carbon (AC, Alfa Aesar, Norit ROW 0.8 mm, L16334) and Carbon Nanotubes (CNT, Baytubes, C150 HP) were measured as reference carbon materials. The reference materials were measured at room temperature (600 scans with a $1 \mathrm{~s}$ integration time) using a stand-alone LaserQuantum MPC $6000532 \mathrm{~nm}$ laser operated at $200 \mathrm{~mW}$ power. The same AvaRaman spectrometer and the high-temperature InPhotonics Raman probe were used to collect the carbon reference spectra as was used for the in-situ Raman Spectroscopy measurements.

The in-situ collected Raman spectra were corrected for (fluorescence) background with the use of the Asymmetric Least Squares Smoothing (ALSS) algorithm. The so- 


\section{Table 6.1}

Overview of the used shorthand notation for catalyst materials used in this Chapter. The catalyst materials after the applied $\mathrm{H}_{2}$ reduction or $\mathrm{CO}$ carburization catalyst treatments are discussed in more detail in Chapter 3.

\begin{tabular}{cl}
\hline Shorthand Notation & \multicolumn{1}{c}{ Catalyst Material Description } \\
\hline $\mathrm{H}_{2}$ & $\mathrm{H}_{2}$ reduction at $400^{\circ} \mathrm{C}$ for $5 \mathrm{~h}$, after the calcining step \\
$240,340,440$ & $\mathrm{CO}$ carburization $(\mathrm{at} 1 \mathrm{~atm})$ temperature in ${ }^{\circ} \mathrm{C}$, after the reduction step \\
$\mathrm{UP}$ & Unpromoted $\mathrm{Fe} / \alpha-\mathrm{Al}_{2} \mathrm{O}_{3}$ catalyst \\
$\mathrm{Na}-\mathrm{S}$ & $\mathrm{Na-S}$ promoted $\mathrm{Fe}-\mathrm{Na}-\mathrm{S} / \alpha-\mathrm{Al}_{2} \mathrm{O}_{3}$ catalyst \\
Carb. & Catalyst material after $\mathrm{H}_{2}$ reduction or CO carburization ("fresh catalyst") \\
Q-FTS & Catalyst material after $72 \mathrm{~h} 340^{\circ} \mathrm{C}, 10$ bar in-situ FTS run, measured quasi- \\
& in-situ at $100^{\circ} \mathrm{C}(0.5$ bar, $1: 1 \mathrm{Ar}: \mathrm{He})($ "spent catalyst"). \\
\hline
\end{tabular}

called D $\left(\approx 1350 \mathrm{~cm}^{-1}\right)$ and G $\left(\approx 1600 \mathrm{~cm}^{-1}\right)$ Raman bands were fitted with four peak shapes. Iterative peak fitting (Levenberg-Marquardt) algorithm was used to find the best fit to the measured spectra.

D-band was fitted with Lorentzian peak $\mathrm{D}_{1}$ at $\approx 1320 \mathrm{~cm}^{-1}$ and Gaussian peaks $\mathrm{D}_{3}$ at $\approx 1500 \mathrm{~cm}^{-1}$ and $\mathrm{D}_{4}$ at $\approx 1220 \mathrm{~cm}^{-1}$. G-band was fitted with a single Gaussian peak shape. The data points that had $\geq 40 \%$ relative error in the fitted peak area or width were filtered out. Peaks fitted for the $\mathrm{D}_{3}$ and $\mathrm{D}_{4}$ positions were constrained near their nominal locations during the iterative peak fitting process. This was done in order to avoid the tendency of the $\mathrm{D}_{3}$ and $\mathrm{D}_{4}$ peaks from shifting and nearly or fully overlapping with the $\mathrm{D}_{1}$ - and G-band positions. Thus, the $\mathrm{D}_{3}$ peak was constrained to a range of $1465-1525 \mathrm{~cm}^{-1}$ and $\mathrm{D}_{4}$ to a range of $1100-1230 \mathrm{~cm}^{-1}$ during the fitting process.

The obtained fitted peak positions were used to follow the D- and G-band peak position shifts during the FTS reaction. The peak intensities $I(D)$ (D-band) and $I(G)$ (Gband) in the measured in-situ Raman spectra were obtained from the locations defined by the fitted peak positions as 3-point median values. The fitted peak widths were used to estimate the measured D- and G-band widths as peak Full Width at Half Maximums (FWHMs). The collected data points obtained every 10 mins were averaged to 12-point median values and are presented with their corresponding STDs as the error estimates.

For each in-situ Raman measurement run, the obtained Raman spectra were normalized to the, background corrected, maximum peak intensity of the Raman Gband within 0-72 $\mathrm{h}$ ToS. The measured carbon reference materials were normalized to the, background corrected, maximum of their respective Raman G-bands, and fitted with four individual peaks akin to the measured data.

\subsection{RESULTS AND DISCUSSION}

A combined in-situ XRD/Raman Spectroscopy set-up has been used to monitor the 
Combined In-situ X-Ray Powder Diffractometry/Raman Spectroscopy of Iron Carbide and Carbon Species Evolution in Fe(-Na-S)/ $\alpha$-Al203 Catalysts during Fischer-Tropsch Synthesis

changes taking place in the carbon species and Fe carbide phases formed during the FTS reaction $\left(340^{\circ} \mathrm{C}, 10\right.$ bar, $\mathrm{H}_{2}$ : CO:He 2.0:1.0:0.33, GHSV $\left.7200 \mathrm{~h}^{-1}\right)$. This methodology has been applied on both unpromoted $\mathrm{Fe} / \alpha-\mathrm{Al}_{2} \mathrm{O}_{3}$ (shorthand UP) and the Na-S promoted $\mathrm{Fe}-\mathrm{Na}-\mathrm{S} / \alpha-\mathrm{Al}_{2} \mathrm{O}_{3}(\mathrm{Na}-\mathrm{S})$ catalyst materials.

By applying a $\mathrm{H}_{2}$ reduction and $\mathrm{CO}$ carburization treatments, the relative amounts and types of preformed $\mathrm{Fe}$ carbides and/or $\alpha-\mathrm{Fe} / \mathrm{Fe}_{\mathrm{x}} \mathrm{O}_{\mathrm{y}}$ phases were varied in the starting catalyst materials. The shorthand notation of 240,340 or 440 is used to refer to the applied $\mathrm{CO}$ carburization temperature (in ${ }^{\circ} \mathrm{C}$ ). Shorthand notation $\mathrm{H}_{2}$ is used for the only $\mathrm{H}_{2}$ reduced catalyst materials. The shorthand notation of Carb. (i.e. "fresh catalyst")
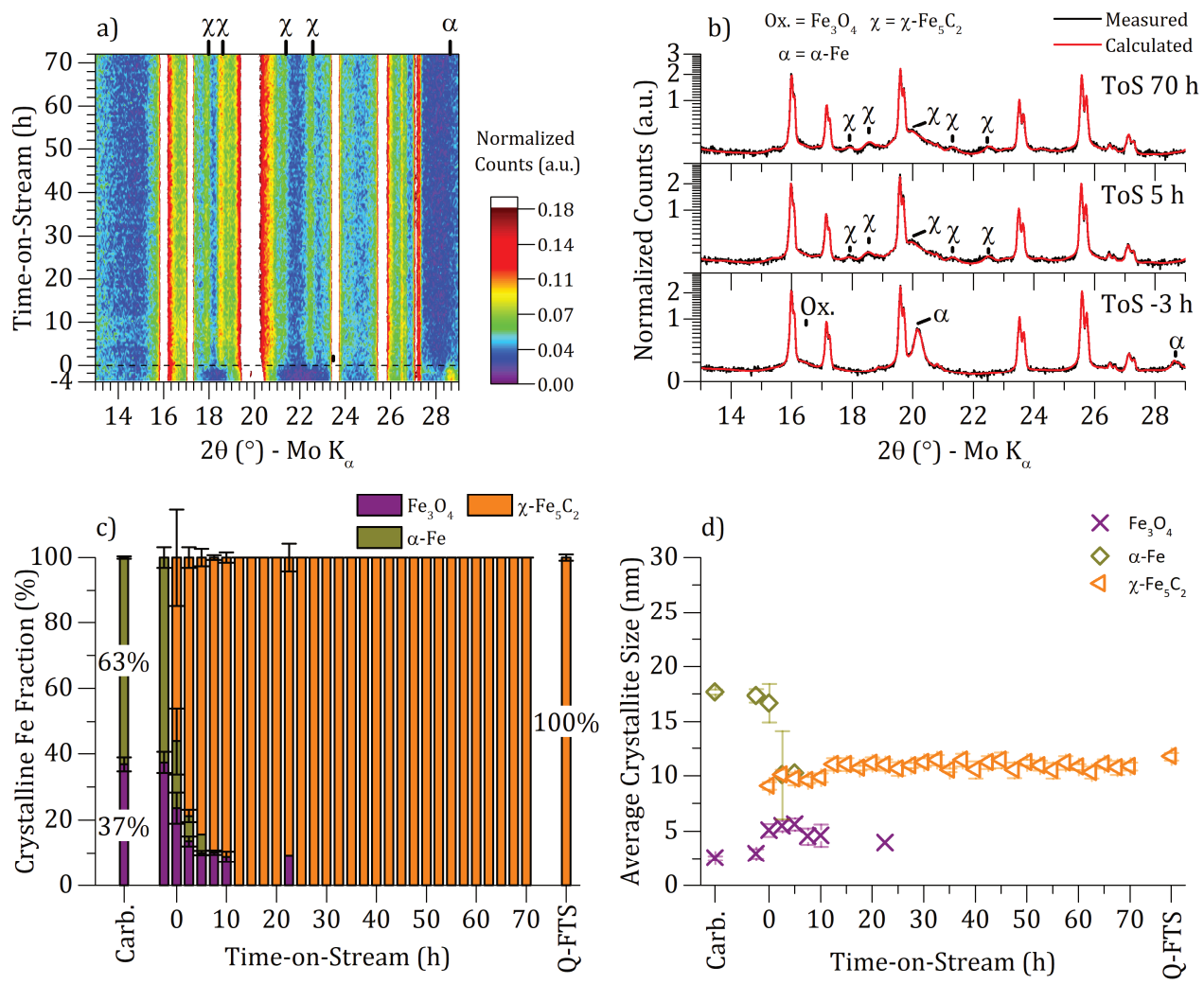

Figure 6.2

X-ray Powder Diffractometry (XRPD) results for UP $\mathrm{H}_{2}\left(\mathrm{Fe} / \alpha-\mathrm{Al}_{2} \mathrm{O}_{3}\right)$ catalyst material during $72 \mathrm{~h}$ FischerTropsch Synthesis (FTS) reaction. A) Heatmap of aligned and background corrected, in-situ collected XRPD patterns. B) Measured and Rietveld method calculated XRPD patterns at $-3 \mathrm{~h}, 5 \mathrm{~h}$ and $70 \mathrm{~h}$ Time-on-Stream (ToS). Some characteristic peak positions for the Fe phases are indicated with markers. C) The crystalline Fe phase composition of catalyst and D) Fe phases average crystallite sizes during the $72 \mathrm{~h}$ FTS reaction. The measurements before (Carb.) and quasi-in-situ (Q-FTS) after the FTS reaction run are included for comparison. FTS conditions: $340^{\circ} \mathrm{C}, 10$ bar, $\mathrm{H}_{2}: \mathrm{CO}: \mathrm{He} 2.0: 1.0: 0.33 \mathrm{v} / \mathrm{v}, \approx 7200 \mathrm{~h}^{-1}$. 
is used collectively for the catalyst characterizations done before the FTS reaction runs. $Q$-FTS notation is used for characterizations done after the FTS reaction runs (i.e. "spent catalyst"), as measured quasi-in-situ. The used shorthand notation, within the Chapter, for the catalyst materials is summarized in Table 6.1. Details of the catalyst material preparation and the related characterization data can be found in Chapter 3, while their catalytic performance in the FTS reaction is described in Chapter 4.

\subsubsection{In-situ X-ray Powder Diffractometry}

The results for different in-situ XRPD reaction runs are given in Figures 6.2-6.9 for the
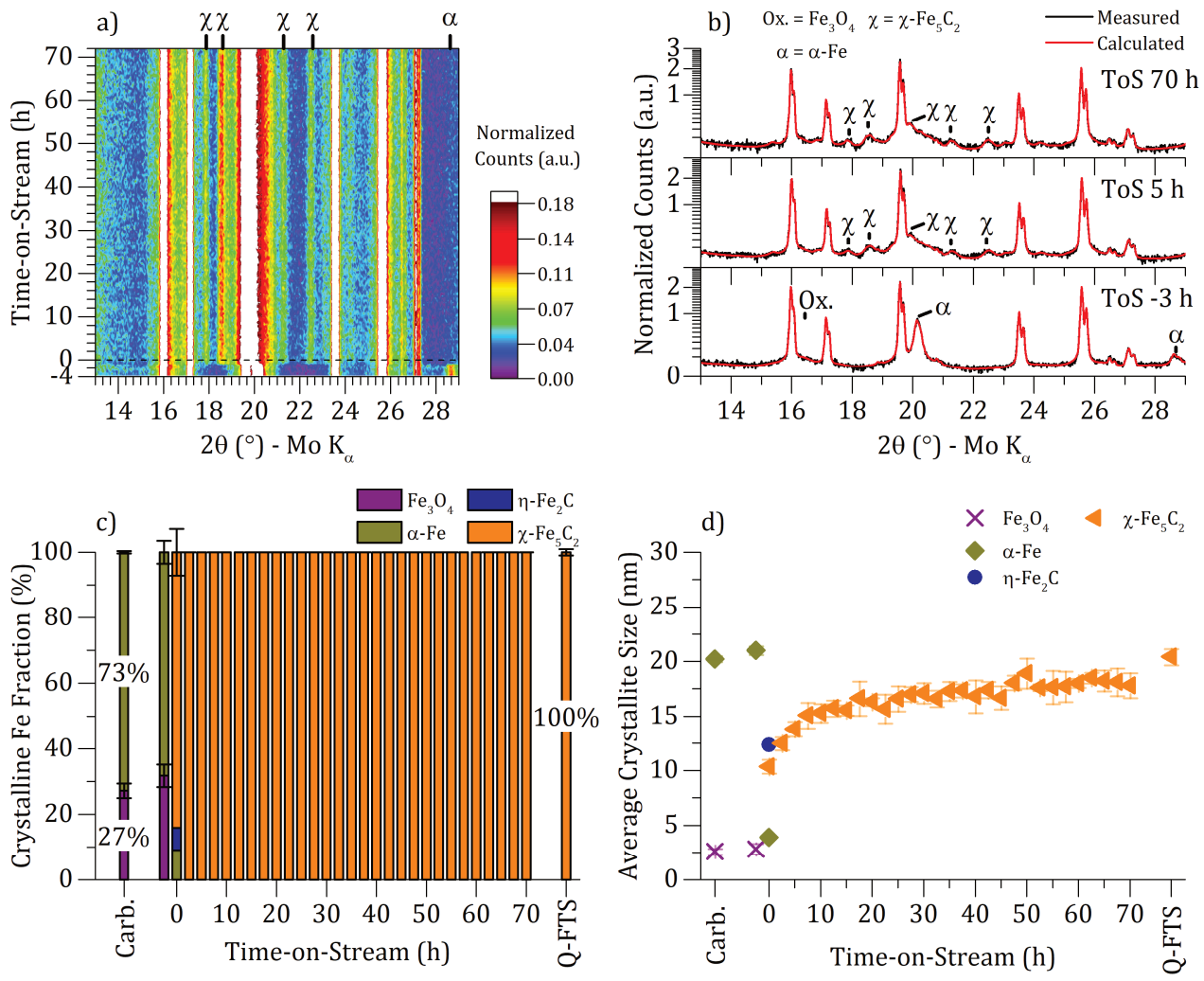

Figure 6.3

X-ray Powder Diffractometry (XRPD) results for Na-S $\mathrm{H}_{2}\left(\mathrm{Fe}-\mathrm{Na}-\mathrm{S} / \alpha-\mathrm{Al}_{2} \mathrm{O}_{3}\right.$ ) catalyst material during $72 \mathrm{~h}$ Fischer-Tropsch Synthesis (FTS) reaction. A) Heatmap of aligned and background corrected, in-situ collected XRPD patterns. B) Measured and Rietveld method calculated XRPD patterns at $-3 \mathrm{~h}, 5 \mathrm{~h}$ and $70 \mathrm{~h}$ Time-onStream (ToS). Some characteristic peak positions for the Fe phases are indicated with markers. C) The crystalline Fe phase composition of catalyst and D) Fe phases average crystallite sizes during the $72 \mathrm{~h}$ FTS reaction. The measurements before (Carb.) and quasi-in-situ (Q-FTS) after the FTS reaction run are included for comparison. FTS conditions: $340^{\circ} \mathrm{C}, 10 \mathrm{bar}, \mathrm{H}_{2}: \mathrm{CO}: \mathrm{He} 2.0: 1.0: 0.33 \mathrm{v} / \mathrm{v}, \approx 7200 \mathrm{~h}^{-1}$. 
Combined In-situ X-Ray Powder Diffractometry/Raman Spectroscopy of Iron Carbide and Carbon Species Evolution in Fe(-Na-S)/ $\alpha$-Al203 Catalysts during Fischer-Tropsch Synthesis
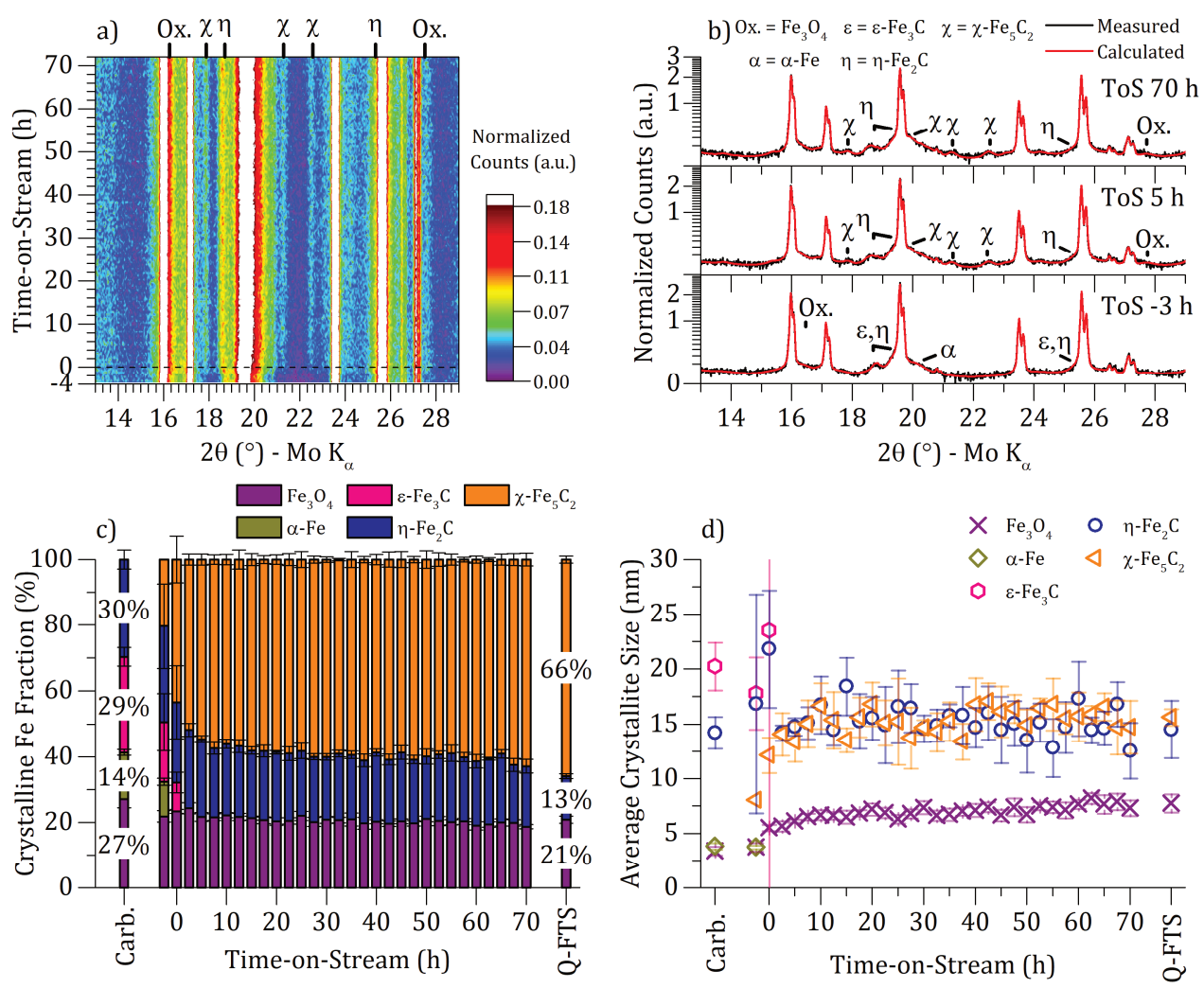

\section{Figure 6.4}

X-ray Powder Diffractometry (XRPD) results for UP $240\left(\mathrm{Fe} / \alpha-\mathrm{Al}_{2} \mathrm{O}_{3}\right)$ catalyst material during $72 \mathrm{~h}$ FischerTropsch Synthesis (FTS) reaction. A) Heatmap of aligned and background corrected, in-situ collected XRPD patterns. B) Measured and Rietveld method calculated XRPD patterns at $-3 \mathrm{~h}, 5 \mathrm{~h}$ and $70 \mathrm{~h}$ Time-on-Stream (ToS). Some characteristic peak positions for the Fe phases are indicated with markers. C) The crystalline Fe phase composition of catalyst and D) Fe phases average crystallite sizes during the $72 \mathrm{~h}$ FTS reaction. The measurements before (Carb.) and quasi-in-situ (Q-FTS) after the FTS reaction run are included for comparison. FTS conditions: $340^{\circ} \mathrm{C}, 10$ bar, $\mathrm{H}_{2}: \mathrm{CO}: \mathrm{He} 2.0: 1.0: 0.33 \mathrm{v} / \mathrm{v}, \approx 7200 \mathrm{~h}^{-1}$.

studied UP and Na-S catalyst materials. In the figures, the aligned and background corrected in-situ collected XRPD patterns during the FTS reactions are given as heatmaps (Figures 6.2a-6.9a) as well as individual diffraction patterns at start of the FTS reaction at $-3 \mathrm{~h}\left(120^{\circ} \mathrm{C}, \mathrm{H}_{2}: \mathrm{CO}\right), 5 \mathrm{~h}$ and $70 \mathrm{~h}$ ToS $\left(340^{\circ} \mathrm{C}, \mathrm{H}_{2}: \mathrm{CO}\right)$ (Figures 6.2b6.9b). Markers on characteristic Fe phase diffraction positions in have been added to the diffraction pattern containing figures.

For each of the individual diffraction patterns, the corresponding Rietveld method calculated patterns are also included in the figures (Figures 6.2b-6.9b). The most intense and sharp diffraction peaks in the individual diffraction patterns in the figures 

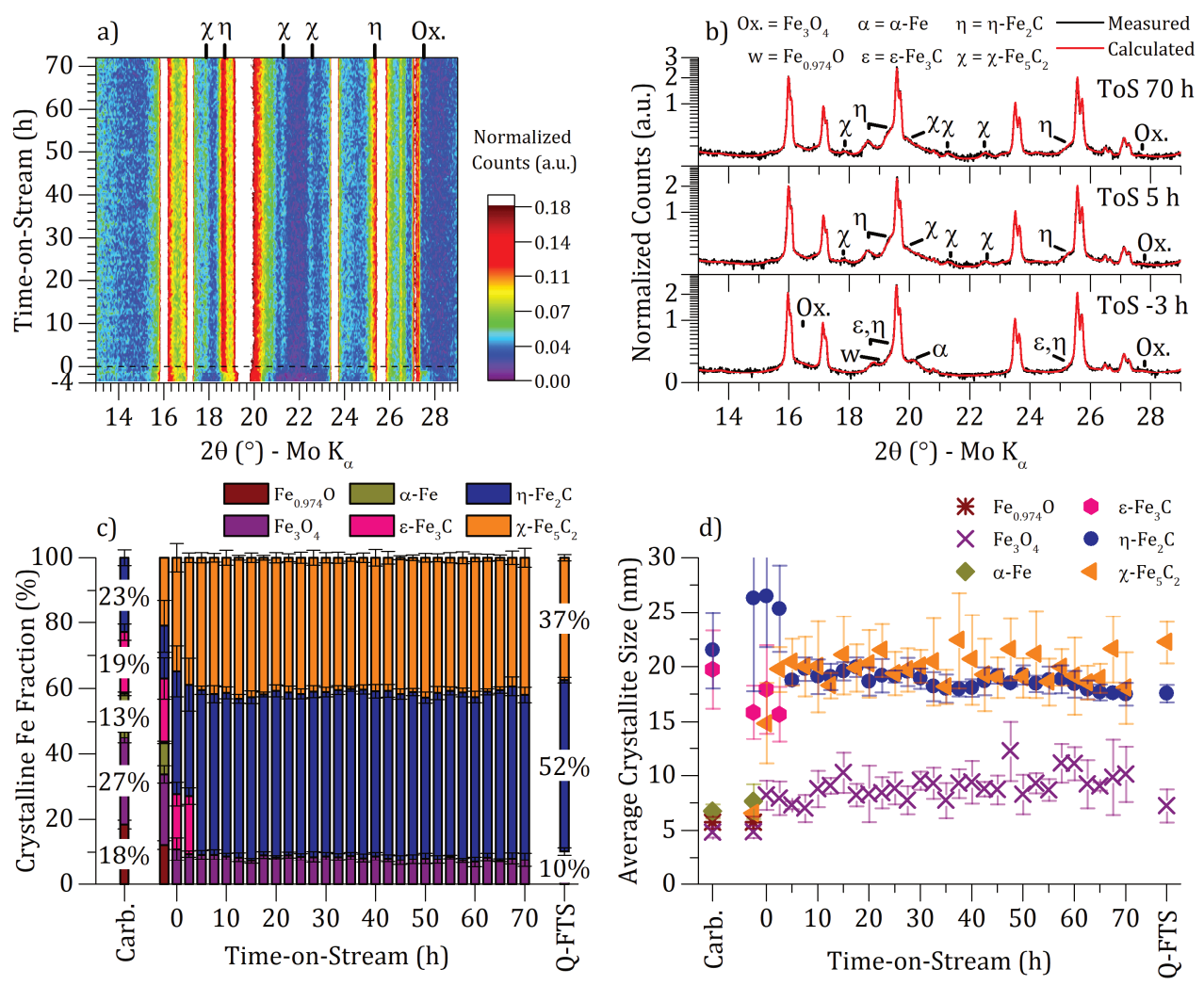

\section{Figure 6.5}

X-ray Powder Diffractometry (XRPD) results for Na-S 240 (Fe-Na-S/ $\alpha-\mathrm{Al}_{2} \mathrm{O}_{3}$ ) catalyst material during $72 \mathrm{~h}$ Fischer-Tropsch Synthesis (FTS) reaction. A) Heatmap of aligned and background corrected, in-situ collected XRPD patterns. B) Measured and Rietveld method calculated XRPD patterns at $-3 \mathrm{~h}, 5 \mathrm{~h}$ and $70 \mathrm{~h}$ Time-onStream (ToS). Some characteristic peak positions for the Fe phases are indicated with markers. C) The crystalline Fe phase composition of catalyst and D) Fe phases average crystallite sizes during the $72 \mathrm{~h}$ FTS reaction. The measurements before (Carb.) and quasi-in-situ (Q-FTS) after the FTS reaction run are included for comparison. FTS conditions: $340^{\circ} \mathrm{C}, 10$ bar, $\mathrm{H}_{2}: \mathrm{CO}: \mathrm{He} 2.0: 1.0: 0.33 \mathrm{v} / \mathrm{v}, \approx 7200 \mathrm{~h}^{-1}$.

(e.g. at $19.58^{\circ}$ and $27.13^{\circ} 2 \theta$ ) originate from the support $\alpha-\mathrm{Al}_{2} \mathrm{O}_{3}$, a phase of which diffraction markers are excluded for convenience.

The Fe carbide phases observed in the FTS reaction related research have been identified as $\varepsilon-\mathrm{Fe}_{3} \mathrm{C} P 6_{3} 22$ (182), $\eta-\mathrm{Fe}_{2} \mathrm{C}$ Pnnm (58), $\chi-\mathrm{Fe}_{5} \mathrm{C}_{2} C 2 / c$ (15) (the Hägg carbide), $\theta-\mathrm{Fe}_{3} \mathrm{C}$ Pnma (62) (cementite) and $\theta-\mathrm{Fe}_{7} \mathrm{C}_{3}$ Pnma (62) (Eckström-Adcock Carbide) on basis of the literature in Chapter 2. For all of the performed Rietveld method fittings (i.e. R-QPA) on the quasi- and in-situ measured XRPD patterns, the previously identified Fe carbide crystal structures give very good calculated fits (Figures 6.2b-6.9b). 
Combined In-situ X-Ray Powder Diffractometry/Raman Spectroscopy of Iron Carbide and Carbon Species Evolution in Fe(-Na-S)/ $\alpha$-Al203 Catalysts during Fischer-Tropsch Synthesis

Despite the $\mathrm{Fe}$ oxide phase being fitted with a $\mathrm{Fe}_{3} \mathrm{O}_{4}$ crystal structure, similar overall R-QPA fits could have been archived with ferrihydrite crystal structures. ${ }^{75}$ The Rietveld method calculated Fe phase patterns have been given and discussed in detail in the previous Chapters and are omitted here.

Background corrected R-values $\left(\mathrm{R}_{\mathrm{wp}}\right.$ ) representing the difference between the Rietveld calculated and measured XRPD patterns are given in Figure 6.10.76 The R-QPA derived elemental Fe contents from the in-situ XRPD patterns stay close, yet are somewhat higher ( $\approx 6.8-8.0$ wt-\%) (Figure 6.11), than the expected $\approx 6.5-6.6 \mathrm{wt}-\%$ of elemental Fe for each catalyst material (Chapter 3).
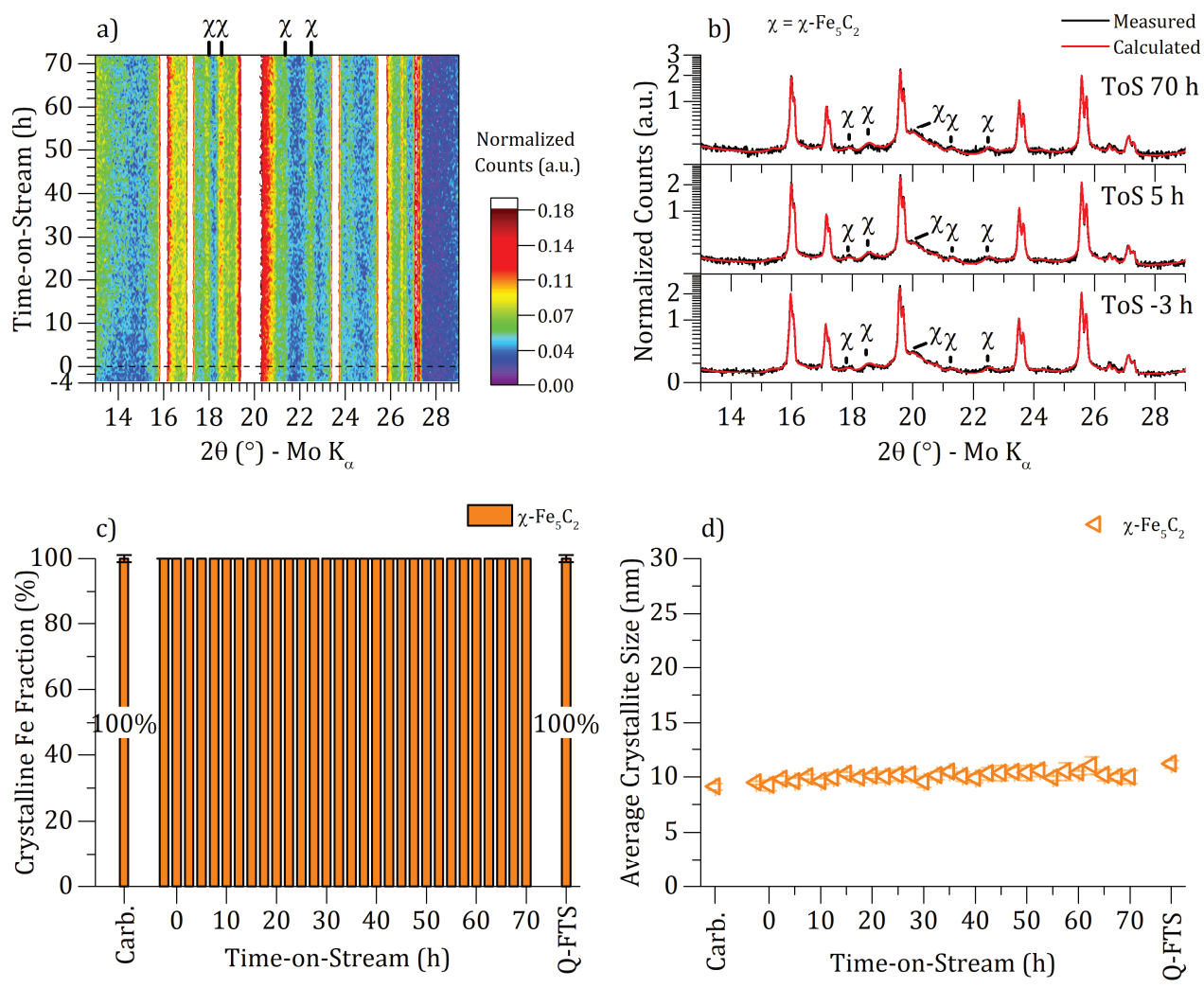

Figure 6.6

X-ray Powder Diffractometry (XRPD) results for UP $340\left(\mathrm{Fe} / \alpha-\mathrm{Al}_{2} \mathrm{O}_{3}\right)$ catalyst material during $72 \mathrm{~h}$ FischerTropsch Synthesis (FTS) reaction. A) Heatmap of aligned and background corrected, in-situ collected XRPD patterns. B) Measured and Rietveld method calculated XRPD patterns at $-3 \mathrm{~h}, 5 \mathrm{~h}$ and $70 \mathrm{~h}$ Time-on-Stream (ToS). Some characteristic peak positions for the Fe phases are indicated with markers. C) The crystalline Fe phase composition of catalyst and D) Fe phases average crystallite sizes during the $72 \mathrm{~h}$ FTS reaction. The measurements before (Carb.) and quasi-in-situ (Q-FTS) after the FTS reaction run are included for comparison. FTS conditions: $340^{\circ} \mathrm{C}, 10$ bar, $\mathrm{H}_{2}: \mathrm{CO}: \mathrm{He} 2.0: 1.0: 0.33 \mathrm{v} / \mathrm{v}, \approx 7200 \mathrm{~h}^{-1}$. 
The main contribution on the varying elemental Fe content originates, likely, from the varying accuracy of the fitted background polynomial. This alters the absolute quantity of the fitted crystalline material area and thus the derived elemental Fe wt-\%s. Both, the R-values and the R-QPA derived elemental Fe contents, indicate very good and consistent R-QPA results in between the performed in-situ reaction runs.

\subsubsection{Fe Phase Rietveld Quantitative Phase Analysis Quantification Changes}

For both UP and Na-S $\mathrm{H}_{2}$ catalyst materials, upon reaching the $0 \mathrm{~h}$ ToS when the FTS
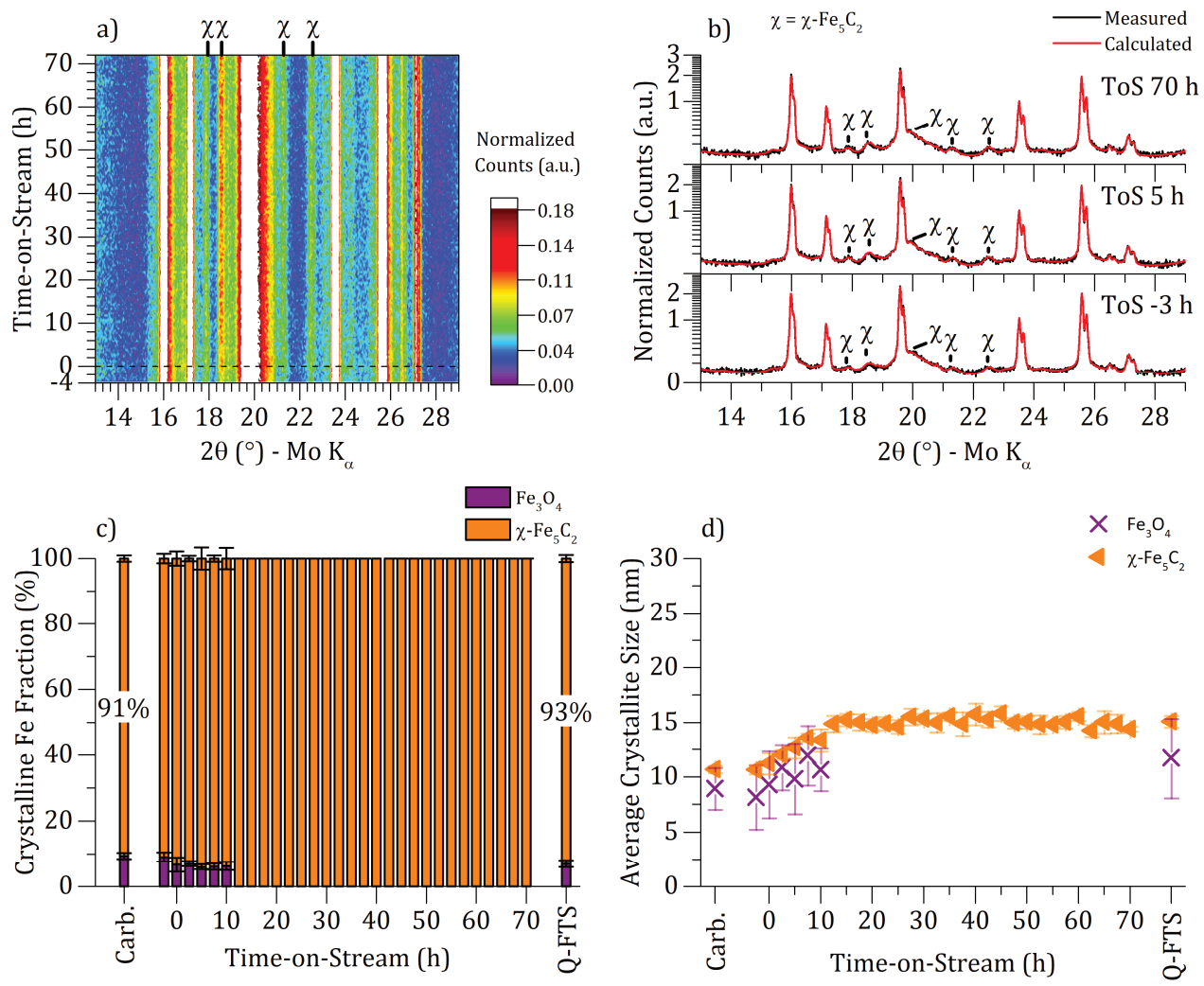

Figure 6.7

X-ray Powder Diffractometry (XRPD) results for Na-S 340 (Fe-Na-S/ $\alpha-\mathrm{Al}_{2} \mathrm{O}_{3}$ ) catalyst material during $72 \mathrm{~h}$ Fischer-Tropsch Synthesis (FTS) reaction. A) Heatmap of aligned and background corrected, in-situ collected XRPD patterns. B) Measured and Rietveld method calculated XRPD patterns at $-3 \mathrm{~h}, 5 \mathrm{~h}$ and $70 \mathrm{~h}$ Time-onStream (ToS). Some characteristic peak positions for the Fe phases are indicated with markers. C) The crystalline Fe phase composition of catalyst and D) Fe phases average crystallite sizes during the $72 \mathrm{~h}$ FTS reaction. The measurements before (Carb.) and quasi-in-situ (Q-FTS) after the FTS reaction run are included for comparison. FTS conditions: $340^{\circ} \mathrm{C}, 10 \mathrm{bar}, \mathrm{H}_{2}: \mathrm{CO}: \mathrm{He} 2.0: 1.0: 0.33 \mathrm{v} / \mathrm{v}, \approx 7200 \mathrm{~h}^{-1}$. 

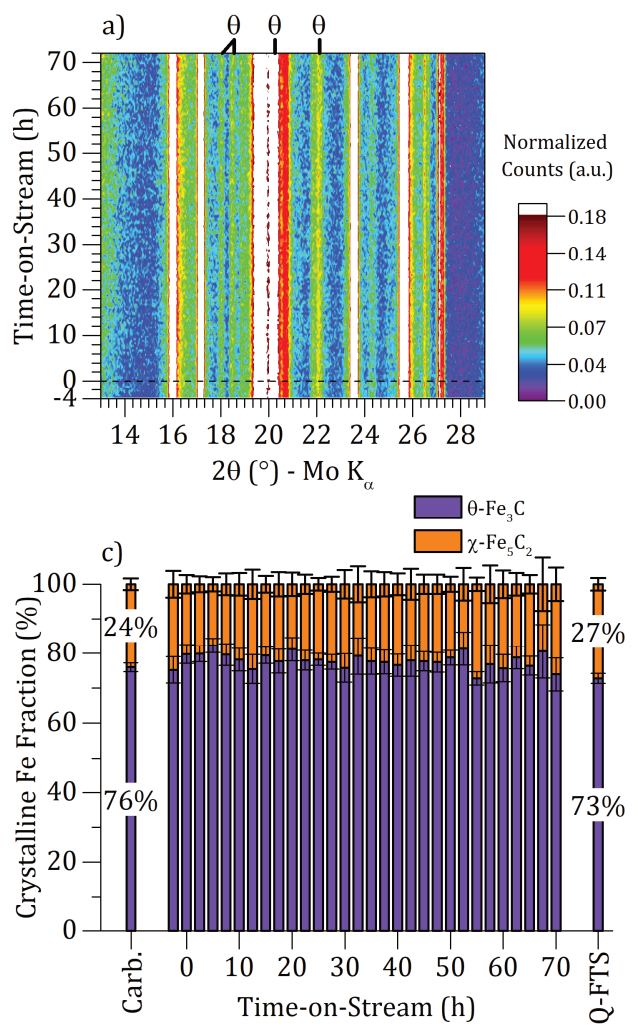

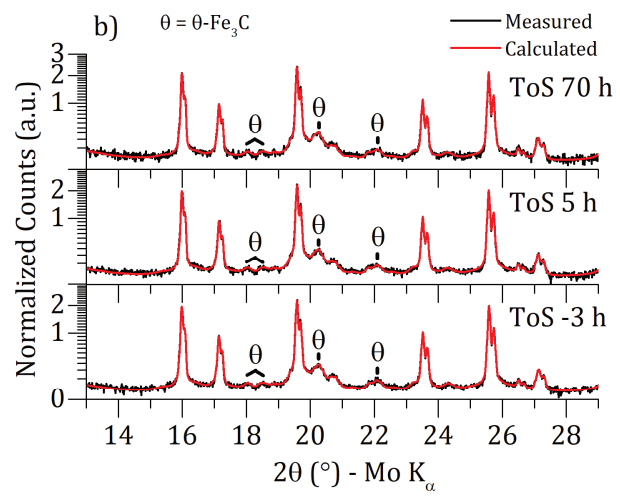

d)

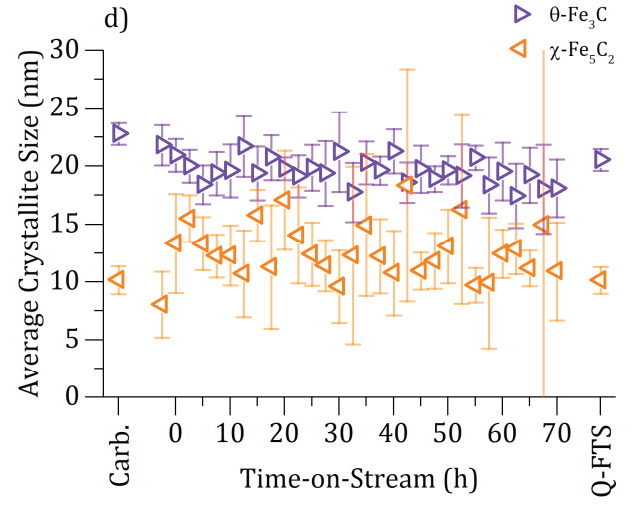

Figure 6.8

X-ray Powder Diffractometry (XRPD) results for UP $440\left(\mathrm{Fe} / \alpha-\mathrm{Al}_{2} \mathrm{O}_{3}\right)$ catalyst material during $72 \mathrm{~h}$ FischerTropsch Synthesis (FTS) reaction. A) Heatmap of aligned and background corrected, in-situ collected XRPD patterns. B) Measured and Rietveld method calculated XRPD patterns at $-3 \mathrm{~h}, 5 \mathrm{~h}$ and $70 \mathrm{~h}$ Time-on-Stream (ToS). Some characteristic peak positions for the Fe phases are indicated with markers. C) The crystalline $\mathrm{Fe}$ phase composition of catalyst and D) Fe phases average crystallite sizes during the $72 \mathrm{~h}$ FTS reaction. The measurements before (Carb.) and quasi-in-situ (Q-FTS) after the FTS reaction run are included for comparison. FTS conditions: $340^{\circ} \mathrm{C}, 10$ bar, $\mathrm{H}_{2}: \mathrm{CO}: \mathrm{He} 2.0: 1.0: 0.33 \mathrm{v} / \mathrm{v}, \approx 7200 \mathrm{~h}^{-1}$.

reaction temperature of $340^{\circ} \mathrm{C}$ is reached, the majority of the initially present $\alpha$ $\mathrm{Fe} / \mathrm{Fe}_{\mathrm{x}} \mathrm{O}_{\mathrm{y}}$ phases transforms into the temperature's most stable Fe carbide phase of $\chi$ $\mathrm{Fe}_{5} \mathrm{C}_{2}$ (Figures 6.2c and 6.3c). ${ }^{29,36,77-84}$ Without the added Na-S promotion, the transition of $\alpha-\mathrm{Fe} / \mathrm{Fe}_{\mathrm{x}} \mathrm{O}_{\mathrm{y}}$ phases to $\chi-\mathrm{Fe}_{5} \mathrm{C}_{2}$ takes longer in comparison to the unpromoted catalyst materials. Indeed, at $0 \mathrm{~h}$ ToS: for the $\mathrm{Na}-\mathrm{S} \mathrm{H}_{2}$ catalyst material the $\chi-\mathrm{Fe}_{5} \mathrm{C}_{2}$ phase already accounts for $\approx 81 \pm 7 \%$ of the Fe phases (Figure 6.3c), while for the $\mathrm{UP} \mathrm{H}_{2}$ catalyst material only $\approx 56 \pm 15 \%$ of the $\chi-\mathrm{Fe}_{5} \mathrm{C}_{2}$ phase is present (Figure 6.2c).

This can be explained by an observed alkali effect, i.e. $\mathrm{Na}(-\mathrm{S})$ aiding in the reduction of Fe oxides at $\lesssim 300^{\circ} \mathrm{C}$ by $\mathrm{CO} .^{56,85,86}$ In the Na-S $\mathrm{H}_{2}$ catalyst materials the Fe oxidation 

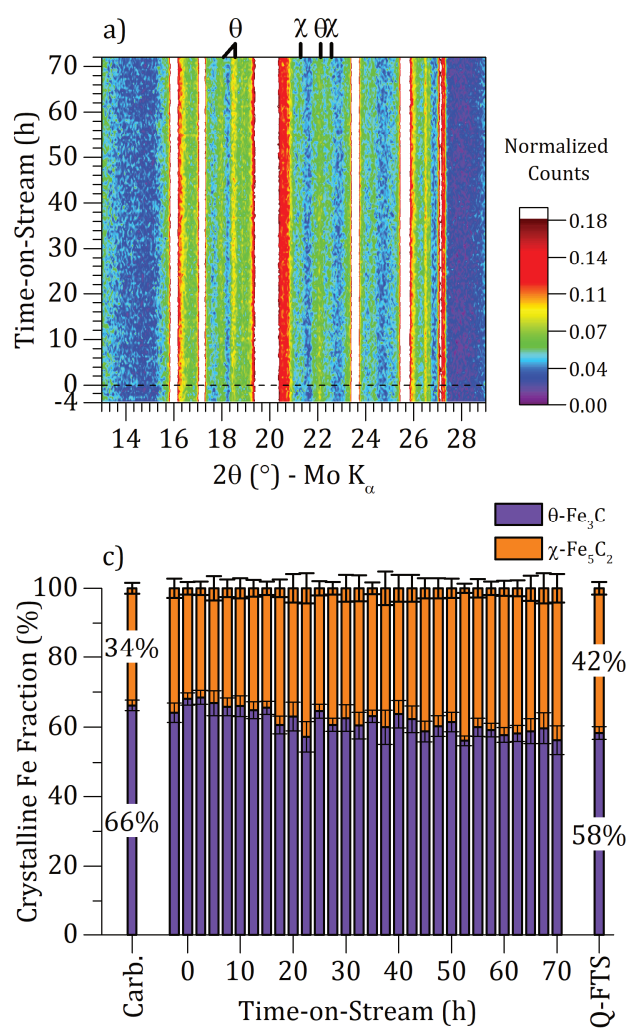

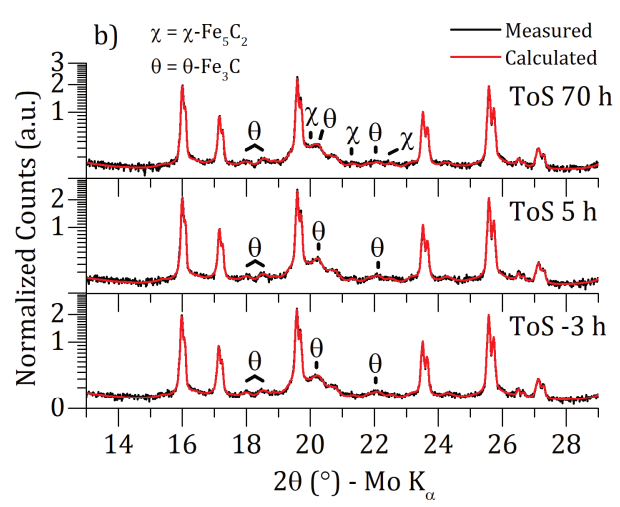

d)

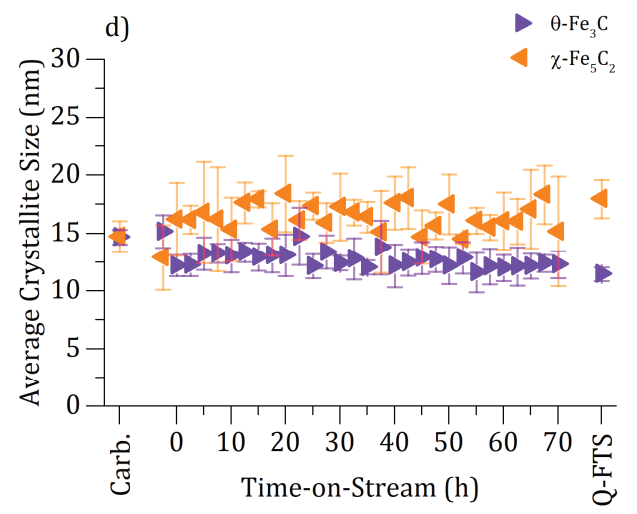

Figure 6.9

X-ray Powder Diffractometry (XRPD) results for Na-S $440\left(\mathrm{Fe}-\mathrm{Na}-\mathrm{S} / \alpha-\mathrm{Al}_{2} \mathrm{O}_{3}\right)$ catalyst material during $72 \mathrm{~h}$ Fischer-Tropsch Synthesis (FTS) reaction. A) Heatmap of aligned and background corrected, in-situ collected XRPD patterns. B) Measured and Rietveld method calculated XRPD patterns at $-3 \mathrm{~h}, 5 \mathrm{~h}$ and $70 \mathrm{~h}$ Time-onStream (ToS). Some characteristic peak positions for the Fe phases are indicated with markers. C) The crystalline Fe phase composition of catalyst and D) Fe phases average crystallite sizes during the $72 \mathrm{~h}$ FTS reaction. The measurements before (Carb.) and quasi-in-situ (Q-FTS) after the FTS reaction run are included for comparison. FTS conditions: $340^{\circ} \mathrm{C}, 10 \mathrm{bar}, \mathrm{H}_{2}: \mathrm{CO}: \mathrm{He} 2.0: 1.0: 0.33 \mathrm{v} / \mathrm{v}, \approx 7200 \mathrm{~h}^{-1}$.

shell is removed more efficiently by $\mathrm{CO}$ in the presence of $\mathrm{Na}(-\mathrm{S})$ promotion during the temperature ramp ( $\approx-2-0 \mathrm{~h} \mathrm{ToS})$ to $340^{\circ} \mathrm{C}$, than with the unpromoted $\mathrm{UP}_{2}$ catalyst materials. The Fe oxides, which are present as Fe particle shell and/or core, are reduced into $\alpha$-Fe and subsequently converted into the $\chi-\mathrm{Fe}_{5} \mathrm{C}_{2}$ phase by CO. Regardless of the $\mathrm{Na}-\mathrm{S}$ promotion, the transition of $\alpha$-Fe to Fe carbides in the presence of $\mathrm{CO}$ with temperatures $\gtrsim 200-250^{\circ} \mathrm{C}$ is very fast. ${ }^{36,83,87-89}$ and not the limiting step in the $\mathrm{Fe}$ carbide formation. ${ }^{90}$

As a discrepancy to the previous results described in Chapter 4, during the in-situ XRPD run within this Chapter: no $\eta-\mathrm{Fe}_{2} \mathrm{C}$ phase is observed for the $\mathrm{Na}-\mathrm{S} \mathrm{H}_{2}$ catalyst 

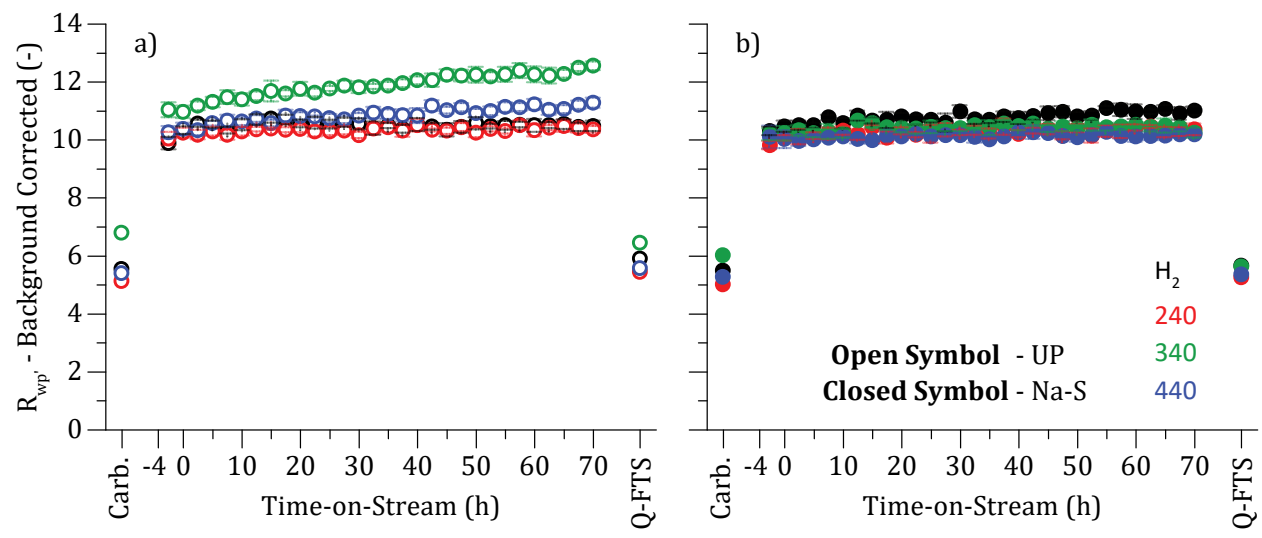

Figure 6.10

Background corrected R-values ( $\left(\mathrm{R}_{\mathrm{wp}}\right.$ ) for a) UP ( $\left.\mathrm{Fe} / \alpha-\mathrm{Al}_{2} \mathrm{O}_{3}\right)$ and b) Na-S (Fe-Na-S $\left./ \alpha-\mathrm{Al}_{2} \mathrm{O}_{3}\right) \mathrm{H}_{2}, 240,340$ and 440 catalyst materials. Values during the in-situ Fischer-Tropsch Synthesis (FTS) runs versus Time-onStream (ToS) and for the catalysts measured before (Carb.) and quasi-in-situ after the reaction run (Q-FTS).

material. Previously, after the $72 \mathrm{~h}$ FTS $\left(340^{\circ} \mathrm{C}, \mathrm{H}_{2}: \mathrm{CO}: \mathrm{He} 2.0: 1.0: 0.33, \mathrm{GHSV} \approx 5000 \mathrm{~h}^{-}\right.$ 1) reaction run in Chapter 4, $\approx 27 \% \quad \eta-\mathrm{Fe}_{2} \mathrm{C}$ phase contribution in the R-QPA quantifications was observed for the $\mathrm{Na}-\mathrm{S} \mathrm{H}_{2}$ (FTS) catalyst. The presence of " $\varepsilon$ carbides" (i.e. $\varepsilon-\mathrm{Fe}_{3} \mathrm{C} / \eta-\mathrm{Fe}_{2} \mathrm{C}$ phases) is generally increased in Fe-based FTS catalyst materials after the FTS reaction with added alkali elements as K or Na.4,56,91,92

As discussed in more detail in Chapter 3, the $\eta-\mathrm{Fe}_{2} \mathrm{C}$ phase does not form $\gtrsim 300^{\circ} \mathrm{C}$ but can be stable up to $\approx 340^{\circ} \mathrm{C}$ if it is formed and present prior to the FTS reaction. When comparing the UP and Na-S 240 catalyst materials with the initial presence of Fe carbides as the $\varepsilon-\mathrm{Fe}_{3} \mathrm{C}$ and $\eta-\mathrm{Fe}_{2} \mathrm{C}$ phases in the Carb. catalyst materials, the presence of $\mathrm{Na}-\mathrm{S}$ promotion further stabilizes the $\eta-\mathrm{Fe}_{2} \mathrm{C}$ phase during the FTS reaction run. For the Na-S 240 Q-FTS measurement, $50 \pm 1 \%$ of the $\eta-\mathrm{Fe}_{2} \mathrm{C}$ phase is quantified (Figure 6.4c) after the FTS reaction run in comparison to $13 \pm 1 \%$ of the phase in UP 240 Q-FTS catalyst material (Figure 6.5c).

Thus, altogether, the results for the $\mathrm{H}_{2}$ and 240 catalyst materials suggest that for an observable $\mathrm{Na}(-\mathrm{S})$ stabilization effect of the $\eta-\mathrm{Fe}_{2} \mathrm{C}$ phase in the FTS reaction: the $\varepsilon$ $\mathrm{Fe}_{3} \mathrm{C} / \eta-\mathrm{Fe}_{2} \mathrm{C}$ phases need to be present prior to the start of the FTS reaction. Without $\mathrm{Na}(-\mathrm{S})$ promotion, significantly lesser amount of the initial $\varepsilon-\mathrm{Fe}{ }_{3} \mathrm{C} / \eta-\mathrm{Fe}_{2} \mathrm{C}$ phases are stabilized as the $\eta-\mathrm{Fe}_{2} \mathrm{C}$ phase during the high-temperature FTS reaction.

Rather interestingly, the requirement for a priori presence of the $\varepsilon-\mathrm{Fe}_{3} \mathrm{C} / \eta-\mathrm{Fe}_{2} \mathrm{C}$ phases for the $\eta-\mathrm{Fe}_{2} \mathrm{C}$ phase stability in the FTS reaction is comparable to the stability behavior of " $\varepsilon$-iron (carbo)nitrides". ${ }^{93}$ These Fe (carbo-)nitrides have crystal structures closely related to the $\varepsilon-\mathrm{Fe}_{3} \mathrm{C} / \eta-\mathrm{Fe}_{2} \mathrm{C}$ phases. ${ }^{94-99}$ The precise, fundamental reasons for 

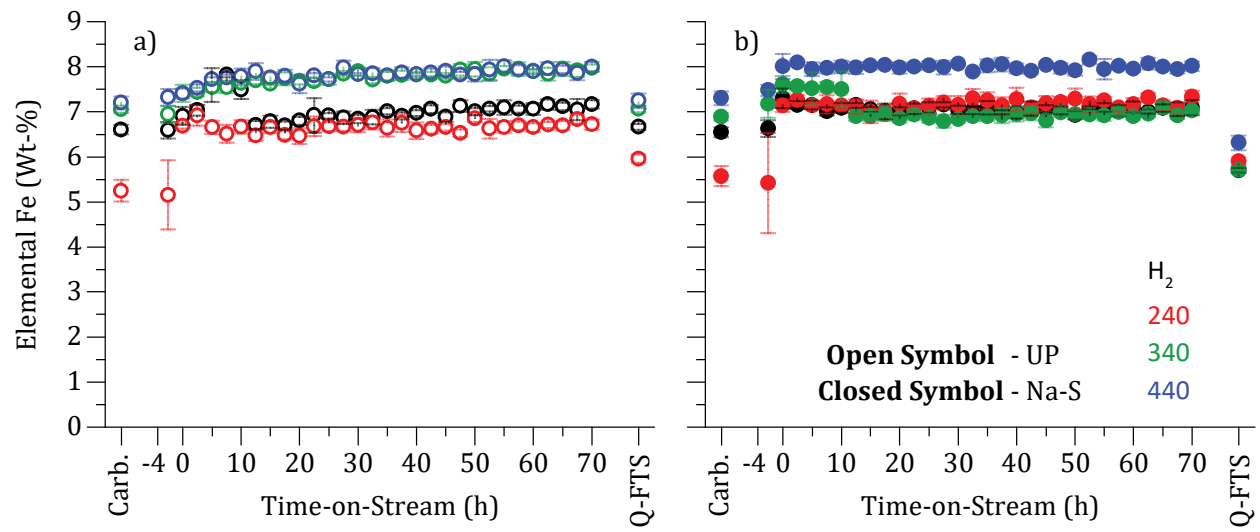

Figure 6.11

The Rietveld Quantitative Phase Analysis (R-QPA) derived elemental Fe wt-\%'s for a) UP $\left(\mathrm{Fe} / \alpha-\mathrm{Al}_{2} \mathrm{O}_{3}\right)$ and b) $\mathrm{Na}-\mathrm{S}\left(\mathrm{Fe}-\mathrm{Na}-\mathrm{S} / \alpha-\mathrm{Al}_{2} \mathrm{O}_{3}\right) \mathrm{H}_{2}, 240,340$ and 440 catalyst materials. Values during the in-situ Fischer-Tropsch Synthesis (FTS) runs versus Time-on-Stream (ToS) and for the catalysts measured before (Carb.) and quasiin-situ after the reaction run (Q-FTS).

the increased stability behavior with the added $\mathrm{Na}(-\mathrm{S})$ promotion in the FTS reaction with crystal structures corresponding to the $\eta-\mathrm{Fe}_{2} \mathrm{C}$ and " $\varepsilon$-iron (carbo)nitrides" phases would make an interesting topic for a further study. The $\varepsilon-\mathrm{Fe}_{3} \mathrm{C}$ phase was not stable in the FTS reaction (Figures $6.4 \boldsymbol{c}$ and $6.5 c$ ), regardless of the Na-S promotion.

Besides the stabilization of the $\eta-\mathrm{Fe}_{2} \mathrm{C}$ phase by the Na-S promotion addition in the $\mathrm{Na}-\mathrm{S} 240$ catalyst materials, the catalysts $\mathrm{Fe}_{3} \mathrm{O}_{4}$ content is also reduced during the FTS reaction. A steady $\mathrm{Fe}_{3} \mathrm{O}_{4}$ fraction of $\approx 20 \%$ is quantified for the UP 240 catalyst (Figure 6.4c) while for the $\mathrm{Na}-\mathrm{S} 240$ catalyst material (Figure 6.5c) only $\approx 8 \%$ of $\mathrm{Fe}_{3} \mathrm{O}_{4}$ is observed after the start of the FTS reaction. This difference in the Fe oxide content is visible (at $\approx 27.5^{\circ} 2 \theta$ ) in the in-situ collected XRPD patterns (Figures 6.4a and 6.5a). The added alkali has been observed to reduce the Fe oxide content in Fe-based catalyst materials during the FTS reaction also in the literature, ${ }^{91,92}$ in agreement with the results for the Na-S 240 catalyst material.

Rather counter-intuitively, Na-S promotion increases the Fe oxide content in the NaS 240 Carb. catalyst materials after the CO carburization (i.e. in the fresh catalysts), as is evident by comparing the R-QPA quantifications in Figures $6.4 c$ and $6.5 c$. This effect presumably originates from the $\mathrm{Na}-\mathrm{S}$ promotion induced increased Fe phase affinity towards $\mathrm{O}$ atoms from the $\mathrm{CO}$ dissociation during the $\mathrm{CO}$ carburization step with short treatment time (Chapter 3).

Despite the Na-S 240 catalyst behaving as expected, i.e. Na-S promotion decreasing $\mathrm{Fe}_{3} \mathrm{O}_{4}$ content during the FTS reaction, why both UP and Na-S 240 catalyst materials show the presence of $\mathrm{Fe}_{3} \mathrm{O}_{4}$ at all during the in-situ XRPD runs, is odd. Any of the UP $\mathrm{H}_{2}$, 
Combined In-situ X-Ray Powder Diffractometry/Raman Spectroscopy of Iron Carbide and Carbon Species Evolution in Fe(-Na-S)/ $\alpha$-Al203 Catalysts during Fischer-Tropsch Synthesis

340 or 440 (Figures 6.2c, 6.6c and 6.8c), or $\mathrm{Na}-\mathrm{S} \mathrm{H}_{2}, 340$ or 440 (Figures 6.3c, 6.7c and 6.9c) catalysts do not show a significant presence or stabilization of the $\mathrm{Fe}_{3} \mathrm{O}_{4}$ phase. Furthermore, in Chapter 4, neither of the UP or Na-S 240 (FTS) catalyst materials characterized ex-situ after their FTS reaction runs showed any presence of the $\mathrm{Fe}_{3} \mathrm{O}_{4}$ phase. Perhaps, the $\varepsilon-\mathrm{Fe}_{3} \mathrm{C} / \eta-\mathrm{Fe}_{2} \mathrm{C}$ phases might be more susceptible towards $\mathrm{Fe}$ carbide reoxidation by $\mathrm{CO}_{2}$ and $\mathrm{H}_{2} \mathrm{O}, 2,29,100-102$ than the other Fe phases. Furthermore, undiluted reactor beds were used with the in-situ XRPD runs in contrast to the SiC diluted reactor beds used in Chapter 4 during the FTS reaction runs. Possibly, the catalyst bed dilution with inert $\mathrm{SiC}$, allows easier removal of the $\mathrm{CO}_{2}$ and $\mathrm{H}_{2} \mathrm{O}$, in that way preventing the Fe phases oxidation.

With respect to the phase changes in the UP and Na-S 340 and 440 catalyst materials during their respective FTS reaction runs, only minor changes take place in contrast to the initial Carb. catalyst materials (Figures 6.6c-6.9c). Small amount of $\mathrm{Fe}_{3} \mathrm{O}_{4}(\approx 7 \%)$ is present in the Na-S 340 catalyst material during the first $10 \mathrm{~h}$ ToS of the FTS reaction. For the UP 340 catalyst material, no Fe oxide is quantified. For Na-S 240 catalyst material, the Na-S promotion aided in the $\mathrm{Fe}_{3} \mathrm{O}_{4}$ reduction during the FTS reaction. However, when comparing the UP 340 and Na-S 340 catalysts materials, only the Na-S 340 catalyst shows the presence of the Fe oxides (Figures $6.6 c$ and $6.7 c$ ). Thus, giving the opposite result as would be expected based on the discussion in earlier paragraphs.

A possible explanation could be the initially higher carbon deposition with the Na-S 340 than with the UP 340 catalyst materials during the initial CO carburization to prepare the catalyst (Chapter 3). The deposited carbon could be responsible for mass transfer limitations on the Na-S 340 catalyst material inhibiting the reduction of the initial $\mathrm{Fe}_{3} \mathrm{O}_{4}$ during the FTS reaction. Furthermore, Na-S 340 catalyst material gave significantly higher CO conversion than the UP 340 catalyst during the FTS reaction (Chapter 4). Therefore, Fe phases' reoxidation by $\mathrm{H}_{2} \mathrm{O}$ may also play a role resulting in the presence of $\mathrm{Fe}_{3} \mathrm{O}_{4}$ in the $\mathrm{Na}-\mathrm{S} 340$ catalyst material, together with the undiluted reactor bed used for the in-situ XRPD runs.

For the UP and Na-S 440 catalyst materials, the effect of Na-S promotion is to aid the transformation of the $\theta-\mathrm{Fe}_{3} \mathrm{C}$ phase into $\chi-\mathrm{Fe}_{5} \mathrm{C}_{2}$, which is the most stable Fe carbide at $340^{\circ} \mathrm{C}$, during the FTS reaction (Figures 6.8c and 6.9c). With the Na-S 440 catalyst material, the $\theta-\mathrm{Fe}_{3} \mathrm{C}$ phase content steadily decreases with increasing $\chi-\mathrm{Fe}_{5} \mathrm{C}_{2}$ contribution (Figure 6.9c).

\subsubsection{Changes in Fe Phases' Crystallite Diameters}

The in-situ XRPD observations for the R-QPA derived Fe phases' crystallite (volumeweighted) mean diameters follow the Fe phase quantities in the FTS reaction. The increasing phase quantity in general translates to increasing Fe phase crystallite 
diameters, as more of the phase is present, thus allowing formation of larger crystallites. The Fe crystallite diameter evolutions are plotted in Figures 6.2d-6.9d for the studied catalyst materials.

Additionally, the presence of $\mathrm{Na}-\mathrm{S}$ promotion favors the formation of larger $\mathrm{Fe}$ carbide crystallites for the $\mathrm{H}_{2}, 240$ and 340 catalyst materials, while for the 440 catalyst materials the situation is mixed. The Na-S H2, 240 and 340 catalyst materials show Fe carbide crystallite growth within the first $\approx 10 \mathrm{~h}$ ToS in comparison to their UP catalyst counterparts (Figures 6.2d-6.7d). This could be associated to Fe particle growth, which has been observed to take place within the first hours of the FTS reaction when Na-S promotion is added to the catalyst. ${ }^{20}$ In Chapter 4, Transmission Electron Microscopy (TEM) results derived Fe particle growth could be observed for the Na-S 240 and Na-S 340 catalyst materials after the FTS reaction run in comparison to the respective UP catalyst materials, but not for the $\mathrm{Na}-\mathrm{S} \mathrm{H}_{2}$ catalyst material.

However, as discussed in more detail in Chapter 4, when comparing ex-situ obtained results for the R-QPA and TEM, the Fe phase crystallite diameters did not conclusively follow the TEM-based Fe particle trends after the FTS reaction. In general, Fe crystallite growth does not necessitate Fe particle growth, as the measurables are different. ${ }^{103}$ Thus, definitive conclusions whether Fe particle growth takes place cannot be made solely on the basis of the in-situ XRPD derived R-QPA crystallite diameter results. Whatever the fundamental origins are, the Na-S promotion causes increased Fe carbide crystallite mean diameters and the increase takes place within the first $\approx 10 \mathrm{~h}$ ToS of the FTS reaction.

The peculiar effect of $\mathrm{Na}-\mathrm{S}$ promotion decreasing the $\theta-\mathrm{Fe}_{3} \mathrm{C}$ phase stability is also observed in the changes of the $\theta-\mathrm{Fe}_{3} \mathrm{C}$ phase's crystallite diameters between the UP and Na-S 440 catalyst materials (Figures 6.8d and 6.9d). The added Na-S promotion induces slowly decreasing $\theta-\mathrm{Fe}_{3} \mathrm{C}$ crystallite mean diameters while increasing both, the $\chi-\mathrm{Fe}_{5} \mathrm{C}_{2}$ content (Figure 6.9c), and its crystallite mean diameter (Figure 6.9d).

The large crystallite mean diameters with large STDs for the $\varepsilon-\mathrm{Fe}_{3} \mathrm{C}$ and $\eta-\mathrm{Fe}_{2} \mathrm{C}$ phases at around $\approx 0 \mathrm{~h}$ ToS are due to correlations between the $\varepsilon-\mathrm{Fe}_{3} \mathrm{C}, \eta-\mathrm{Fe}_{2} \mathrm{C}$ and $\alpha$ $\mathrm{Al}_{2} \mathrm{O}_{3}$ lattice parameters. During the temperature ramp $\lesssim 0 \mathrm{~h}$ ToS, the $\varepsilon-\mathrm{Fe}_{3} \mathrm{C}$ and $\eta-\mathrm{Fe}_{2} \mathrm{C}$ crystal structures tend to fit the sharp $\alpha-\mathrm{Al}_{2} \mathrm{O}_{3}$ diffraction peak $(113)$ at $\approx 19.58^{\circ} 2 \theta$, giving rise to very large crystallite mean diameters with large STDs. After the steady FTS reaction temperature is reached and/or the $\varepsilon-\mathrm{Fe}_{3} \mathrm{C}$ phase consumed $\gtrsim 3 \mathrm{~h}$ ToS, no correlation exists anymore with the $\eta-\mathrm{Fe}_{2} \mathrm{C}$ and $\alpha-\mathrm{Al}_{2} \mathrm{O}_{3}$ phases and $\eta-\mathrm{Fe}_{2} \mathrm{C}$ crystallite mean diameters become consistent again (Figures 6.4 and 6.5).

Summarizing the results so far, as based on the R-QPA results derived from the insitu XRPD runs, the Na-S promotion has the effects of: 
i) To stabilize the $\eta-\mathrm{Fe}_{2} \mathrm{C}$ phase if $\varepsilon$ - $\mathrm{Fe}_{3} \mathrm{C}$ and/or $\eta-\mathrm{Fe}_{2} \mathrm{C}$ phases are present in the initial catalyst at the start of the FTS reaction ( $\mathrm{Na}-\mathrm{S} \mathrm{H}_{2}$ versus $\mathrm{Na}-\mathrm{S} 240$ );

ii) to decrease $\mathrm{Fe}_{3} \mathrm{O}_{4}$ content (UP 240 versus Na-S 240);

iii) to destabilize $\theta-\mathrm{Fe}_{3} \mathrm{C}$ phase during the FTS reaction (UP 440 versus Na-S 440); and also

iv) Fe carbide crystallite mean diameters grow larger within the first $10 \mathrm{~h}$ ToS (excluding the $\theta-\mathrm{Fe}_{3} \mathrm{C}$ in $\mathrm{Na}-\mathrm{S} 440$ catalyst material) in comparison to the respective UP catalysts.

\subsubsection{In-situ Raman Spectroscopy}

Simultaneously to the in-situ XRPD data collection, in-situ Raman Spectroscopy
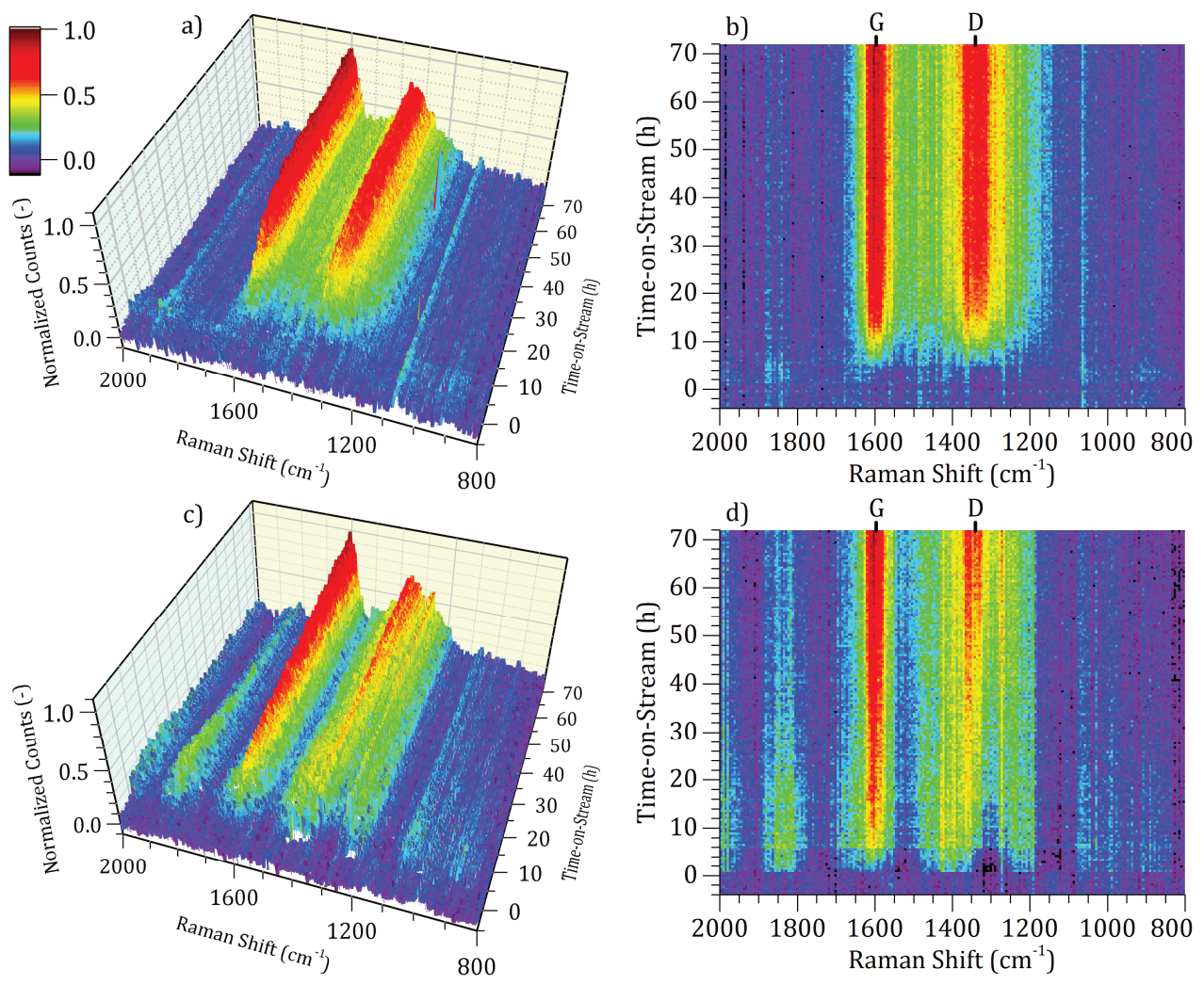

Figure 6.12

In-situ Raman Spectroscopy of $\mathrm{H}_{2}$ catalysts during the $72 \mathrm{~h}$ Fischer-Tropsch Synthesis (FTS) reaction. 3D plot of background corrected and normalized Raman spectra a) for UP $\mathrm{H}_{2}$ and c) for Na-S $\mathrm{H}_{2}\left(\mathrm{Fe}(-\mathrm{Na}-\mathrm{S}) / \alpha-\mathrm{Al}_{2} \mathrm{O}_{3}\right)$ catalysts. Corresponding heatmaps of the Raman spectra b) for UP $\mathrm{H}_{2}$ and d) Na-S H . D- $\left(\approx 1350 \mathrm{~cm}^{-1}\right)$ and Gbands $\left(\approx 1600 \mathrm{~cm}^{-1}\right)$ are marked to the heatmaps (D, G). FTS conditions: $340^{\circ} \mathrm{C}, 10$ bar, $\mathrm{H}_{2}: \mathrm{CO}: \mathrm{He}$ 2.0:1.0:0.33 $\mathrm{v} / \mathrm{v}, \approx 7200 \mathrm{~h}^{-1}$. 

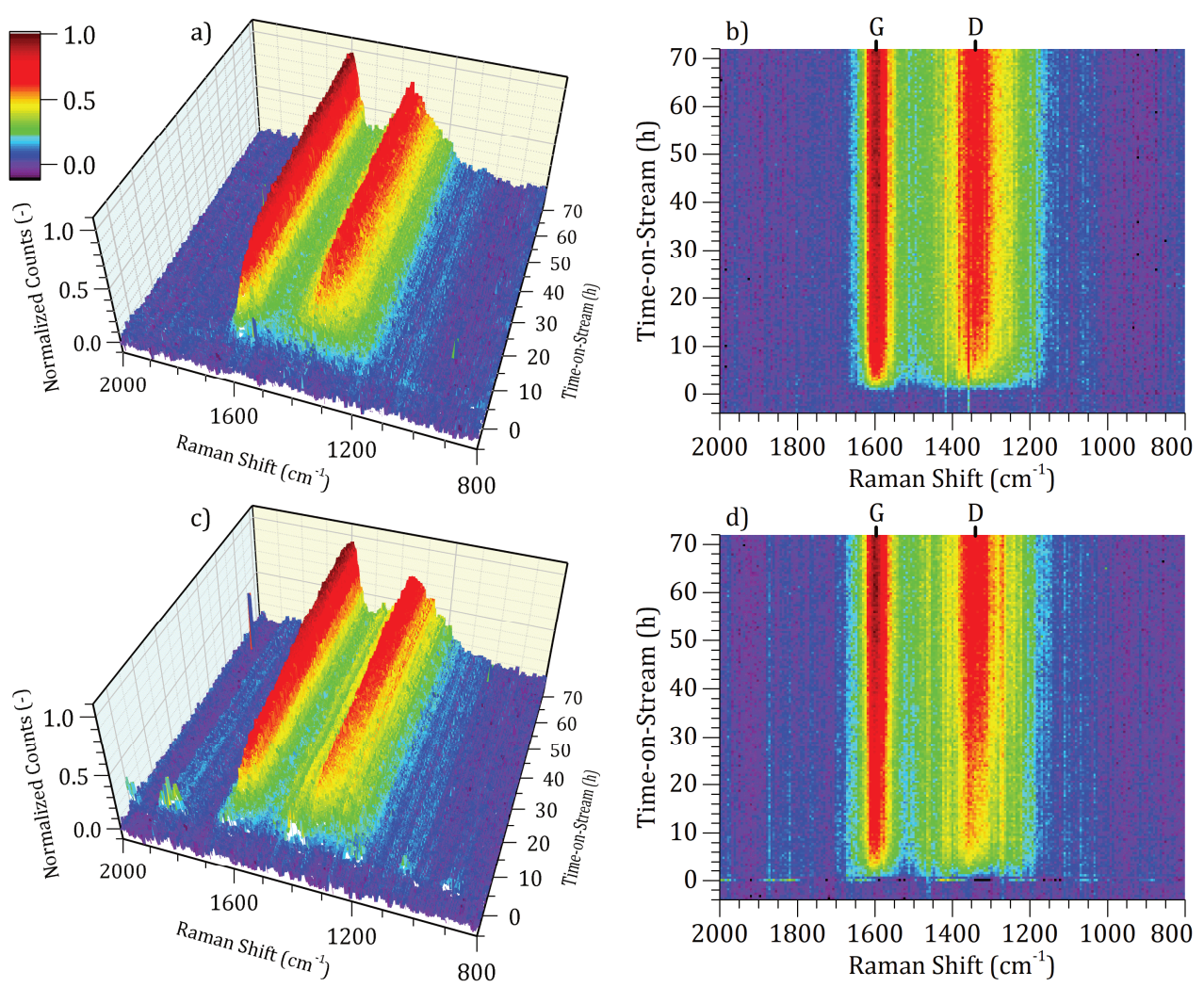

Figure 6.13

In-situ Raman Spectroscopy of 240 catalyst materials during the $72 \mathrm{~h}$ Fischer-Tropsch Synthesis (FTS) reaction. 3D plot of background corrected and normalized Raman spectra a) for UP 240 and c) for Na-S 240 (Fe(-Na-S) $/ \alpha-\mathrm{Al}_{2} \mathrm{O}_{3}$ ) catalysts. Corresponding heatmaps of the Raman spectra b) for UP 240 and d) Na-S 240. D- $\left(\approx 1350 \mathrm{~cm}^{-1}\right)$ and G-bands $\left(\approx 1600 \mathrm{~cm}^{-1}\right)$ are marked to the heatmaps (D, G). FTS conditions: $340^{\circ} \mathrm{C}, 10 \mathrm{bar}$, $\mathrm{H}_{2}: \mathrm{CO}: \mathrm{He} 2.0: 1.0: 0.33 \mathrm{v} / \mathrm{v}, \approx 7200 \mathrm{~h}^{-1}$.

measurements were performed over the duration of the $72 \mathrm{~h}$ FTS reactions. With FTS reaction temperatures above $\approx 300^{\circ} \mathrm{C}$, the Boudouard reaction deposits carbon from CO on the FTS reaction catalyst materials. ${ }^{2,23-25}$ In the spectra, bands with Raman shifts of $\approx 1350 \mathrm{~cm}^{-1}$ (D-band) and $\approx 1600 \mathrm{~cm}^{-1}$ (G-band) originate from carbon species.61-65,104 These bands can be used to follow the carbon deposition process during the course of the FTS reaction.

The in-situ collected, background corrected and normalized Raman spectra of the studied catalyst materials are given as 3D plots and heatmaps in Figures 6.12-6.15. The in-situ Raman spectra are shown over the Raman shift range 800-2 $000 \mathrm{~cm}^{-1}$ showing the evolution of the D- and G-bands during the $72 \mathrm{~h}$ ToS in the FTS reaction.

The deviations in the in-situ collected Raman spectra in Figure 6.12c-d with the Na- 

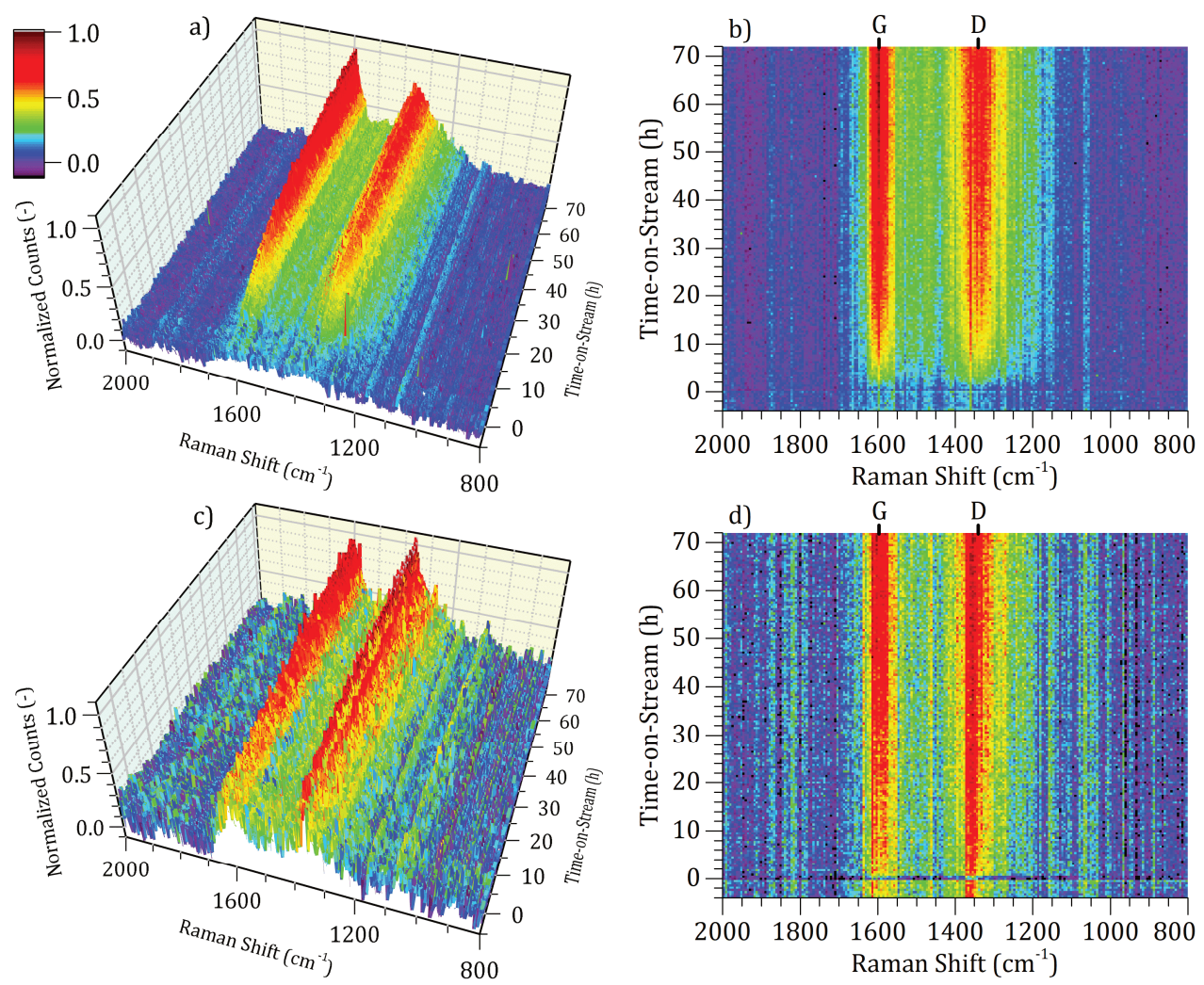

Figure 6.14

In-situ Raman Spectroscopy of 340 catalyst materials during the $72 \mathrm{~h}$ Fischer-Tropsch Synthesis (FTS) reaction. 3D plot of background corrected and normalized Raman spectra a) for UP 340 and c) for Na-S 340 (Fe(-Na-S $/ \alpha-\mathrm{Al}_{2} \mathrm{O}_{3}$ ) catalysts. Corresponding heatmaps of the Raman spectra b) for UP 340 and d) Na-S 340. D- $\left(\approx 1350 \mathrm{~cm}^{-1}\right)$ and G-bands $\left(\approx 1600 \mathrm{~cm}^{-1}\right)$ are marked to the heatmaps (D, G). FTS conditions: $340^{\circ} \mathrm{C}, 10 \mathrm{bar}$, $\mathrm{H}_{2}:$ CO:He 2.0:1.0:0.33 v/v, $\approx 7200 \mathrm{~h}^{-1}$.

$\mathrm{S} \mathrm{H}_{2}$ catalyst material originate from a very high fluorescence background during the FTS reaction run. From the FTS reaction $\approx 0 \mathrm{~h}$ ToS onwards until $\approx 60 \mathrm{~h}$ ToS, high spectral background caused very high collected counts in the Raman spectrometer. This caused formation of artifacts with spectral period of $\approx 198 \pm 6 \mathrm{~cm}^{-1}$ together with the demanding background correction. Origins of the high spectral background for the run are unclear, but were only repeated in a significantly lesser degree with the run Na-S 240 (Figure 6.13c-d), and not with the other FTS reaction runs.

The in-situ collected Raman Spectroscopy data is not quantitative without a proper use of an internal standard. Inherently, the intensity of the Raman spectra is dependent on the focus of the incident excitation laser on the catalyst material, thereby causing variations in the absolute amount of collected Raman scattered photons. Thus, the 

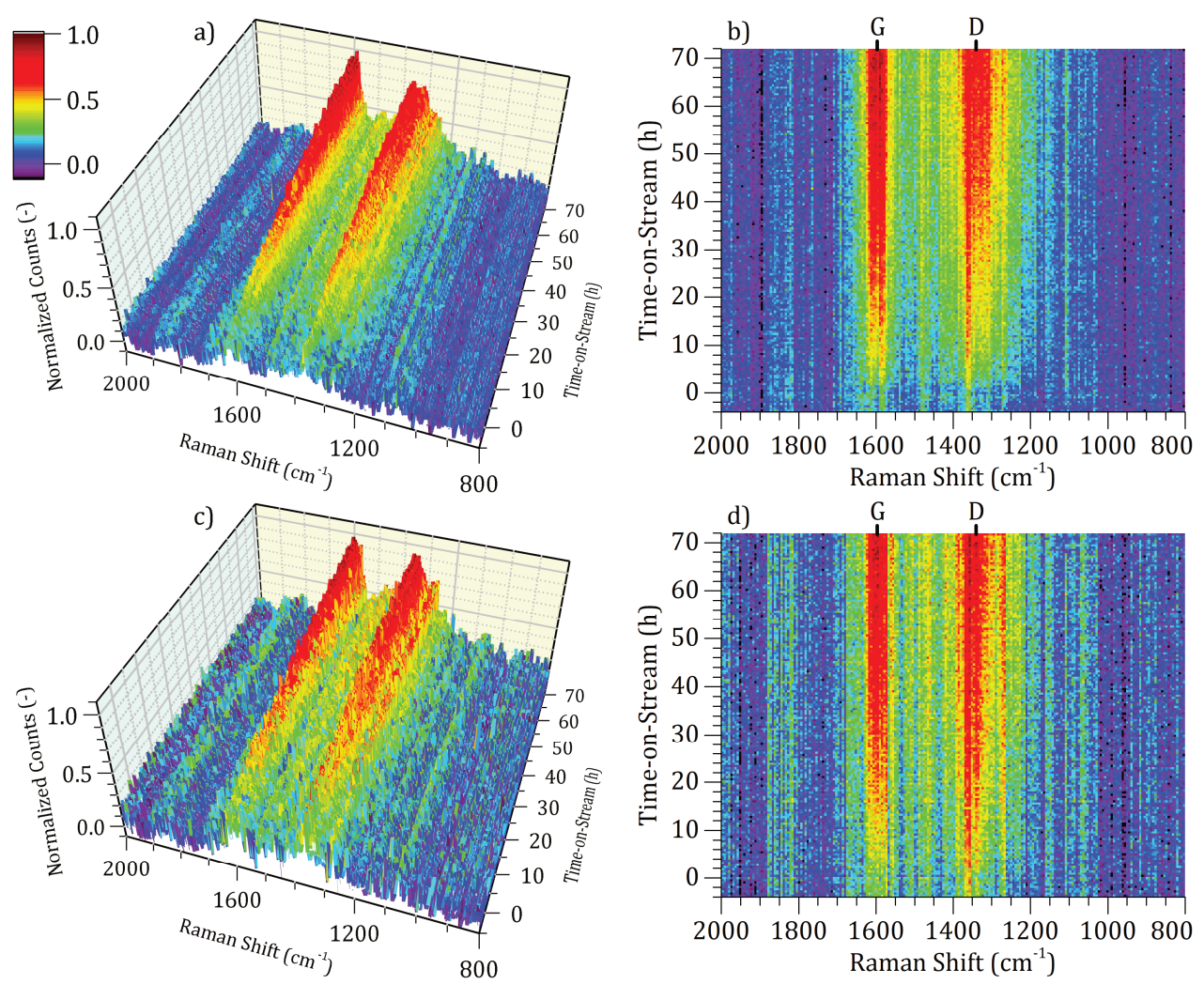

Figure 6.15

In-situ Raman Spectroscopy of 440 catalyst materials during the $72 \mathrm{~h}$ Fischer-Tropsch Synthesis (FTS) reaction. 3D plot of background corrected and normalized Raman spectra a) for UP 440 and c) for Na-S 440 (Fe(-Na-S) $/ \alpha-\mathrm{Al}_{2} \mathrm{O}_{3}$ ) catalysts. Corresponding heatmaps of the Raman spectra b) for UP 440 and d) Na-S 440. D- $\left(\approx 1350 \mathrm{~cm}^{-1}\right)$ and G-bands $\left(\approx 1600 \mathrm{~cm}^{-1}\right)$ are marked to the heatmaps (D, G). FTS conditions: $340^{\circ} \mathrm{C}, 10 \mathrm{bar}$, $\mathrm{H}_{2}: \mathrm{CO}: \mathrm{He} 2.0: 1.0: 0.33 \mathrm{v} / \mathrm{v}, \approx 7200 \mathrm{~h}^{-1}$.

quantity of deposited carbon is not comparable between the different FTS reaction runs with the applied experimental approach.

In addition, any carbon species present in the Carb. catalyst materials at the beginning of the FTS reaction runs decreased the collected Raman D- and G-band intensities. During the catalyst preparation, Na-S 340 Carb. and UP and Na-S 440 Carb. catalyst materials deposited carbon during the CO carburization step (Chapter 3). This can be observed in overall decreased non-normalized Raman band intensities (data not shown), and thus as decreased signal-to-noise ratios in the normalized Raman spectra in Figures 6.14c-d and 6.15. On the Counts per Second (CPS) scale with the background corrected data, the highest G-band intensity was collected for the Na-S 240 $(\approx 0.055 \mathrm{CPS})$ and lowest for the Na-S $440(\approx 0.023 \mathrm{CPS})$ catalyst material. 
Combined In-situ X-Ray Powder Diffractometry/Raman Spectroscopy of Iron Carbide and Carbon Species Evolution in Fe(-Na-S)/ $\alpha-\mathrm{Al} 203$ Catalysts during Fischer-Tropsch Synthesis

Notwithstanding the above considerations, a qualitative discussion on the in-situ Raman spectra is possible by normalizing the observed Raman intensities to the maximum of (e.g.) the G-band within each respective FTS reaction run. By following the relative band intensity ratio $I(D) / I(G)$ and the spectral positions for the D-and G-bands, information on the type and ToS dependent evolution of the carbon species can be followed, ${ }^{64}$ over the course of the FTS reaction.

The observable Raman shifts with the visible wavelength (i.e. $532 \mathrm{~nm}$ ) laser excitation originate from differences in the polarizability of the $\pi$-bonding in the carbon-containing compounds. Long range polarizable $\mathrm{C} \mathrm{sp}{ }^{2} \pi$-bonds give rise to the Raman D- and G-bands while $\mathrm{C} \mathrm{sp}^{3} \sigma$-bonds polarize poorly, being weakly or nonRaman active with a visible light wavelength excitation laser. ${ }^{64}$ The G-band is due to cyclic and/or chain structures with $\mathrm{C} \mathrm{sp}{ }^{2}$ while the $\mathrm{D}$-band originates from the presence of disorder/defects in the cyclic $C \mathrm{sp}^{2}$ arrangements. ${ }^{61,63-65}$ The differences in $\mathrm{C} \mathrm{sp}^{2}$ and $\mathrm{C} \mathrm{sp}^{3}$ bond polarization and thus in observed Raman activity is a another reason why quantitative carbon deposition is hard to determine with Raman Spectroscopy.

Both the D- and G-bands are present in all of the in-situ Raman spectra in Figures 6.12-6.15. This points towards formation of $\mathrm{C} \mathrm{sp} \mathrm{sp}^{2}$ containing carbon species with disordered structures, ${ }^{65}$ within the FTS reaction runs.

\subsubsection{Temporal Changes in the D-and G-band Intensities and Positions}

In the Figures 6.16-6.19 are given the evolution of D- and G-band intensities as determined from the measured in-situ Raman spectra versus the FTS reaction ToS. The corresponding, measured $I(D) / I(G)$ band intensity ratios are also included in the figures.

\section{A. $\quad D$ - and $G$-band Intensities and $I(D) / I(G)$ Ratios}

First, an interesting yet expected difference can be observed between the UP and Na-S $\mathrm{H}_{2}$ catalyst materials on how their D- and G-band intensities develop as a function of the FTS reaction ToS. With UP $\mathrm{H}_{2}$ catalyst material, the initial D- and G-bands only appear after an onset period of $\approx 5-7 \mathrm{~h}$ ToS, while with the Na-S $\mathrm{H}_{2}$ catalyst material the G-band appears at $\approx 0 \mathrm{~h}$ ToS (Figure 6.16c-d). This behavior matches with the in-situ XRPD results.

As the carbon depositing Boudouard reaction requires $\mathrm{CO}$ dissociation (i.e. $\mathrm{CO}$ disproportionation to $\mathrm{C}$ and $\left.\mathrm{CO}_{2}\right),{ }^{26,27}$ first the $\mathrm{Fe}_{3} \mathrm{O}_{4}$ phase need to be converted into (near-)zerovalent Fe carbides capable of dissociating CO. ${ }^{19,31,105}$ Without $\mathrm{Na}-\mathrm{S}$, the $\mathrm{Fe}_{3} \mathrm{O}_{4}$ to $\chi-\mathrm{Fe}_{5} \mathrm{C}_{2}$ conversion is slower under $\mathrm{H}_{2}$ :CO (Figures 6.2c and 6.3c), resulting in the onset period for the D- and G-band formation with the UP $\mathrm{H}_{2}$ catalyst material (Figure 6.16c). This effect also explains literature observations of lower initial deposition of carbon with unpromoted Fe-based catalyst materials with respect to Na- 

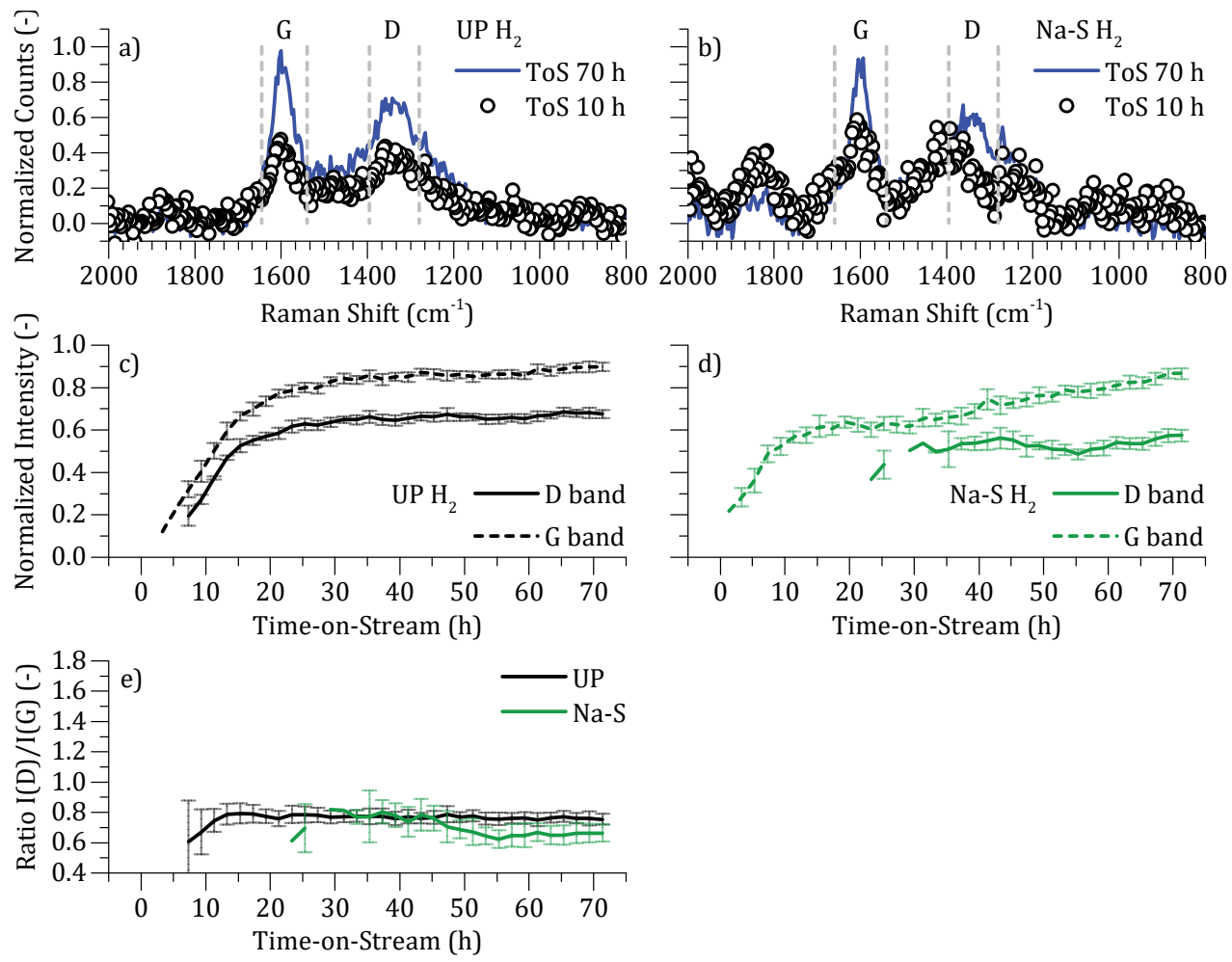

Figure 6.16

Analysis of changes in D- and G-band intensities as derived from in-situ collected Raman spectra in $\mathrm{H}_{2}$ catalyst materials during the $72 \mathrm{~h}$ Fischer-Tropsch Synthesis (FTS) reactions. Comparison of background corrected and normalized Raman spectra at $10 \mathrm{~h}$ and $70 \mathrm{~h}$ Time-on-Stream (ToS) for a) $\mathrm{UP} \mathrm{H}_{2}$ and b) Na-S $\mathrm{H}_{2}$ (Fe(-Na$\left.\mathrm{S}) / \alpha-\mathrm{Al}_{2} \mathrm{O}_{3}\right)$ catalyst materials. Gray vertical dashed lines mark the Raman D- $\left(\approx 1350 \mathrm{~cm}^{-1}\right)$ and G-band $\left(\approx 1600 \mathrm{~cm}^{-1}\right.$ ) regions. Changes in the intensity of the G- and D-bands for c) $\mathrm{UP} \mathrm{H}_{2}$ and d) Na-S $\mathrm{H}_{2}$, and the bands' intensity ratio I(D)/I(G) for e) UP and $\mathrm{Na}-\mathrm{S}_{2}$ catalysts during $72 \mathrm{~h}$ FTS reaction.

S promoted ones. ${ }^{20}$ However, it is worth to keep in mind the earlier mentioned fluorescence induced spectral deviations in the Raman spectra of the $\mathrm{Na}-\mathrm{S} \mathrm{H}_{2}$ catalyst material which may skew the results. This especially at the early FTS reaction ToS.

For the UP and Na-S 240 and 340, and UP 440 catalyst materials, no differences in the G- and D-band appearance onset times are observed (Figures 6.17c-d - 6.19c-d), as these catalysts' majority phases are Fe carbides at the start of the FTS reactions (Figures 6.4c-6.9c).

With respect to the different $\mathrm{Fe}$ crystalline phases: the $\mathrm{C}$ atoms ejected during the transition of C-rich " $\varepsilon$-carbides" (i.e. $\varepsilon$ - $\mathrm{Fe}_{3} \mathrm{C}$ and/or $\eta-\mathrm{Fe}_{2} \mathrm{C}$ ), to the lesser $\mathrm{C}$ containing $\mathrm{Fe}$ carbides $(\chi, \theta)$ have been proposed to act as nucleation sites for carbon formation. ${ }^{36-38}$ As is experimentally shown for Fe carbides observable in the FTS reaction in Chapter 3, 

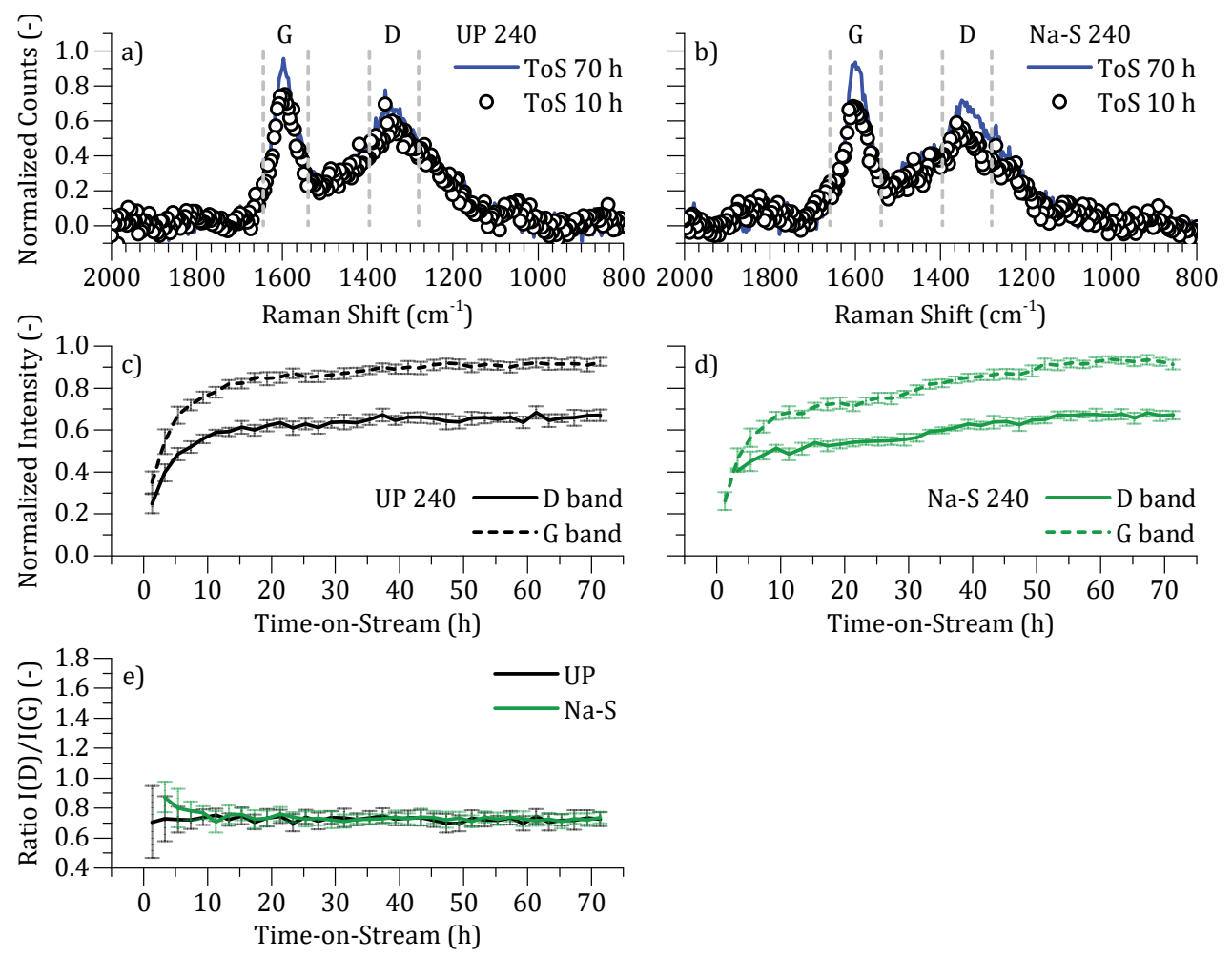

Figure 6.17

Analysis of changes in D- and G-band intensities as derived from in-situ collected Raman spectra in 240 catalyst materials during the $72 \mathrm{~h}$ Fischer-Tropsch Synthesis (FTS) reactions. Comparison of background corrected and normalized Raman spectra at $10 \mathrm{~h}$ and $70 \mathrm{~h}$ Time-on-Stream (ToS) for a) UP 240 and b) Na-S 240 (Fe(-Na-S) $\left./ \alpha-\mathrm{Al}_{2} \mathrm{O}_{3}\right)$ catalyst materials. Gray vertical dashed lines mark the Raman D- $\left(\approx 1350 \mathrm{~cm}^{-1}\right)$ and G-band ( $\approx 1600 \mathrm{~cm}^{-1}$ ) regions. Changes in the intensity of the G- and D-bands for c) UP 240 and d) Na-S 240 , and the bands' intensity ratio I(D)/I(G) for e) UP and Na-S $\mathrm{H}_{2} 240$ catalysts during $72 \mathrm{~h}$ FTS reaction.

the $\eta-\mathrm{Fe}_{2} \mathrm{C}$ phase is $\mathrm{C}$-rich while the $\varepsilon$ - $\mathrm{Fe}_{3} \mathrm{C}$ phase contains the least $\mathrm{C}$ atoms in the carbide's crystal lattice. Thus, transition of the $\eta-\mathrm{Fe}_{2} \mathrm{C}$ phase to $\chi-\mathrm{Fe}_{5} \mathrm{C}_{2}$ or $\theta-\mathrm{Fe}_{3} \mathrm{C}$ phases should correlate to changes in the Raman spectra. However, no direct correlation exists between any of the Fe carbide phases or carbide-to-carbide transitions observed in the in-situ XRPD (Figures 6.2c-6.9c) and the simultaneous in-situ Raman D- and G-band intensity evolutions (Figures 6.12c-d - 6.15c-d). This conclusion while excluding the mentioned $\mathrm{Fe}_{3} \mathrm{O}_{4}$ to $\mathrm{Fe}$ carbide transition.

The changes in the D- and G-band intensities derived from the in-situ collected Raman spectra have two regions of band intensity growth for the UP and $\mathrm{Na}-\mathrm{S} \mathrm{H}_{2}$ and 240 catalyst materials (Figures $6.16 \boldsymbol{c}$-d and $\mathbf{6 . 1 7} \boldsymbol{c}$ - $\boldsymbol{d}$ ). These can be divided to: i) within $\approx 10-15 \mathrm{~h}$ ToS from the initial appearance of the D- and G-bands the intensity growth is 

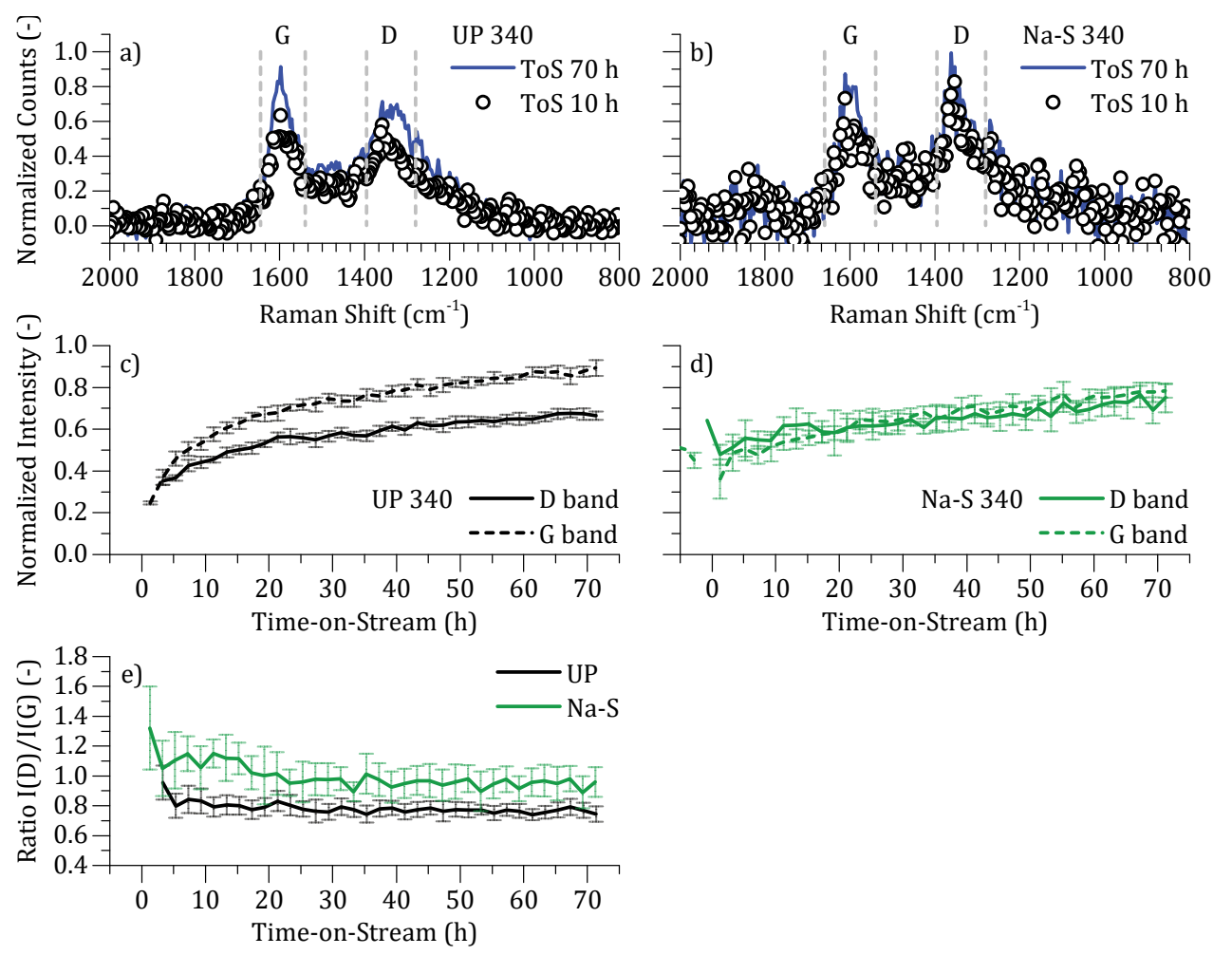

Figure 6.18

Analysis of changes in D- and G-band intensities as derived from in-situ collected Raman spectra in 340 catalyst materials during the $72 \mathrm{~h}$ Fischer-Tropsch Synthesis (FTS) reactions. Comparison of background corrected and normalized Raman spectra at $10 \mathrm{~h}$ and $70 \mathrm{~h}$ Time-on-Stream (ToS) for a) UP 340 and b) Na-S 340 (Fe(-Na-S) $\left./ \alpha-\mathrm{Al}_{2} \mathrm{O}_{3}\right)$ catalyst materials. Gray vertical dashed lines mark the Raman D- $\left(\approx 1350 \mathrm{~cm}^{-1}\right)$ and G-band ( $\approx 1600 \mathrm{~cm}^{-1}$ ) regions. Changes in the intensity of the G- and D-bands for c) UP 340 and d) Na-S 340, and the bands' intensity ratio I(D)/I(G) for e) UP and Na-S $\mathrm{H}_{2} 340$ catalysts during $72 \mathrm{~h}$ FTS reaction.

fast, and ii) after $\geq 20 \mathrm{~h}$ ToS the D- and G-bands intensities settle to a near steady values or to a lower rate of intensity growth. With the Na-S $\mathrm{H}_{2}$ catalyst materials, the initial Dand G-band intensity growth is observed to be lesser which is, possibly, due to data analysis problems instigated by the high fluorescence background.

However, with Na-S promotion, the D- and G-band intensities continue growing after the initial fast growth period ( $\$ 15 \mathrm{~h}$ ToS), unlike with UP $\mathrm{H}_{2}$ and 240 catalyst materials. The normalized, median G-band intensity increases with a rate of $\approx 4.7 \pm 0.3 \times 10^{-3} \mathrm{~h}^{-1}$ for the Na-S H 2 and 240 , and $\approx 1.9 \pm 0.7 \times 10^{-3} \mathrm{~h}^{-1}$ for the UP $\mathrm{H}_{2}$ and 240 catalyst materials over the $\approx 21-70 \mathrm{~h}$ ToS period (Figures $6.16 c-d$ and $6.17 c-d$ ). That is, G-band intensities grow $\approx 2.5 \pm 0.9$ faster for the Na-S than for the respective UP $\mathrm{H}_{2}$ and 240 catalyst materials. 

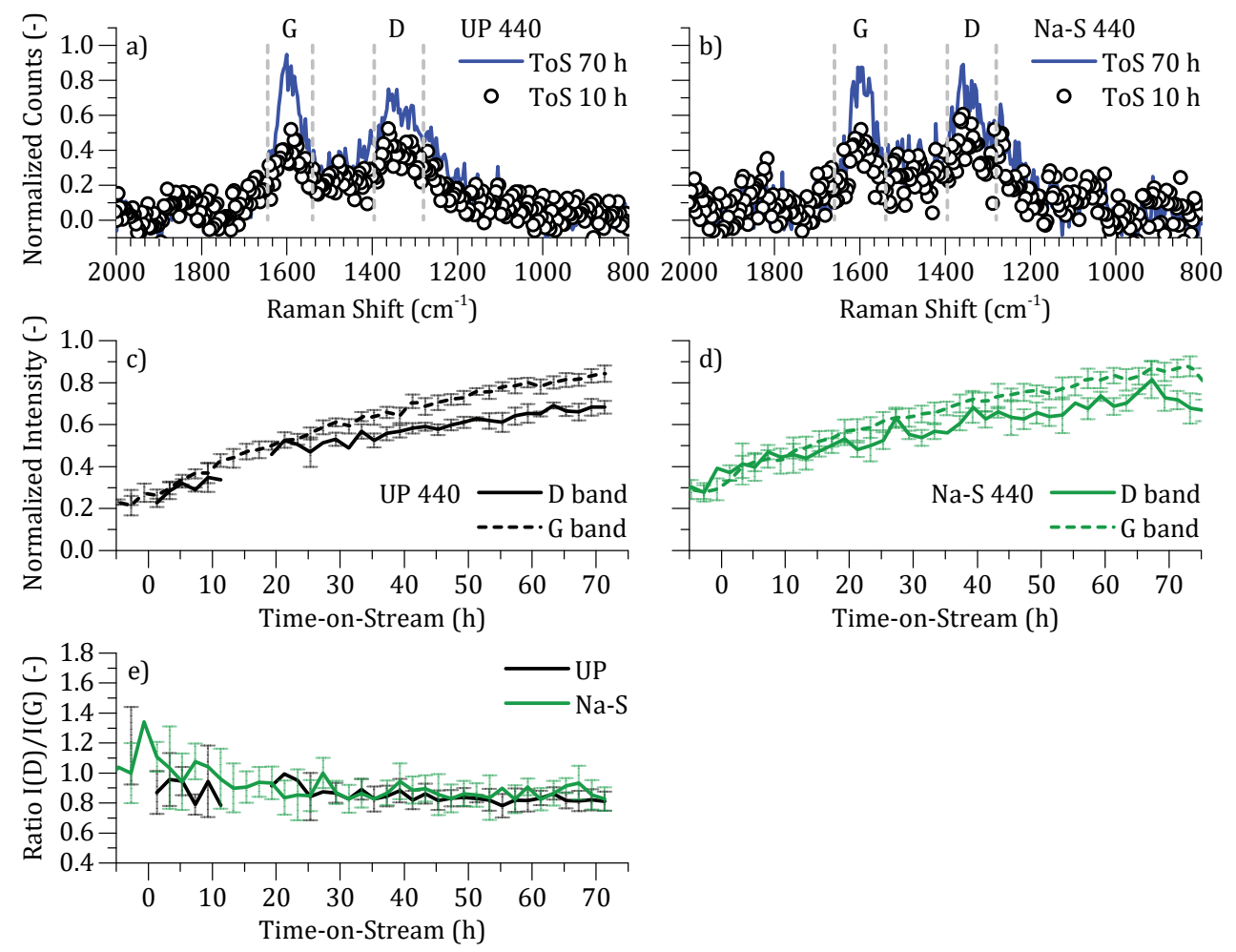

Figure 6.19

Analysis of changes in D- and G-band intensities as derived from in-situ collected Raman spectra in 440 catalyst materials during the $72 \mathrm{~h}$ Fischer-Tropsch Synthesis (FTS) reactions. Comparison of background corrected and normalized Raman spectra at $10 \mathrm{~h}$ and $70 \mathrm{~h}$ Time-on-Stream (ToS) for a) UP 440 and b) Na-S 440 (Fe(-Na-S) $\left./ \alpha-\mathrm{Al}_{2} \mathrm{O}_{3}\right)$ catalyst materials. Gray vertical dashed lines mark the Raman D- $\left(\approx 1350 \mathrm{~cm}^{-1}\right)$ and G-band ( $\approx 1600 \mathrm{~cm}^{-1}$ ) regions. Changes in the intensity of the G- and D-bands for c) UP 440 and d) Na-S 440 , and the bands' intensity ratio I(D)/I(G) for e) UP and Na-S $\mathrm{H}_{2} 440$ catalysts during $72 \mathrm{~h}$ FTS reaction.

This would be expected as the Na-S catalyst materials have in overall significantly higher CO conversions than the UP catalyst materials have under the FTS reaction conditions applied here (Chapter 4). This leads to a higher possible rate of the Boudouard reaction. ${ }^{26}$ The added alkali $\mathrm{Na}(-\mathrm{S})$ promotion also intrinsically increases the carbon deposition rate for the Fe-based catalyst materials. ${ }^{2}$

Nevertheless, the initial fast period $(\leqslant 15 \mathrm{~h}$ ToS) of the D- and G-band intensity growth is present for both the UP and Na-S 240 catalyst materials regardless of the Na$\mathrm{S}$ promotion. This suggests that non-Na-S promotion related phenomena are operative, perhaps such as the reactor bed heating by the exothermic FTS reaction. ${ }^{106} \mathrm{~A} 10^{\circ} \mathrm{C}$ increase in the reactor bed temperature in the FTS reaction, increases the carbon deposition by $\approx 50 \% .^{2}$ 

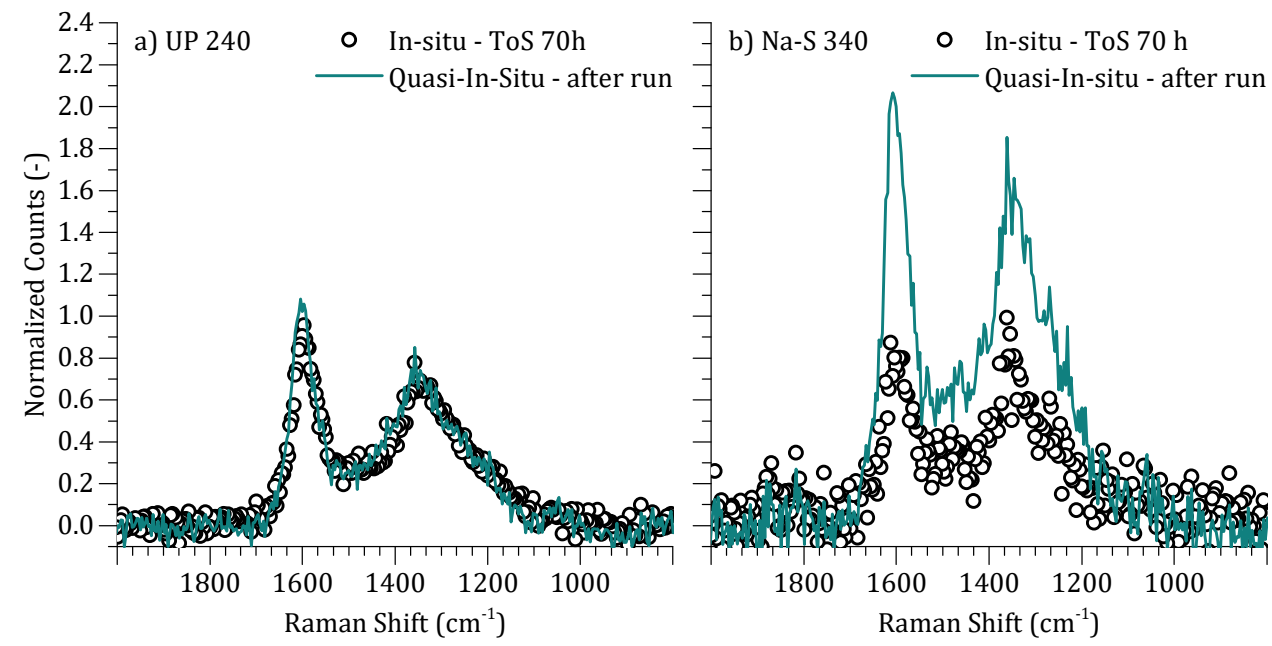

Figure 6.20

Comparison of in-situ $\left(340^{\circ} \mathrm{C}, \mathrm{H}_{2}: \mathrm{CO}\right)$ at $\approx 70 \mathrm{~h} \mathrm{ToS}$, and quasi-in-situ $\left(100^{\circ} \mathrm{C}\right.$, Ar:He) collected Raman spectra after the $72 \mathrm{~h}$ Fischer-Tropsch Synthesis (FTS) reaction. The spectra are compared for FTS reaction runs with a) UP 240 and b) $\mathrm{Na}-\mathrm{S} 340\left(\mathrm{Fe}(-\mathrm{Na}-\mathrm{S}) / \alpha-\mathrm{Al}_{2} \mathrm{O}_{3}\right)$ catalyst materials.

Furthermore, similarly to the results in Figures 6.16c-d and 6.17c-d, two-phase carbon deposition periods have been observed by Sancier, Isakson and Wise. ${ }^{107}$ The initial fast rate growth deposition period was proposed to be due to the initial $\mathrm{Fe}$ carburization, which however is not supported by our results. The e.g. UP $\mathrm{H}_{2}$ (as $\alpha$ $\mathrm{Fe} / \mathrm{Fe}_{\mathrm{x}} \mathrm{O}_{\mathrm{y}}$ ) and $\mathrm{Na}-\mathrm{S} 240$ (as $\approx 89 \% \mathrm{Fe}$ carbides at $\approx 0 \mathrm{~h}$ ToS) catalyst materials (Figures 6.2c and 6.5c), behave in similar manner with regard to their D- and G-band intensities (Figures 6.16c and 6.17d), regardless of their initial level of $\mathrm{Fe}$ carburization.

For the UP and Na-S 340 and 440 catalyst materials, with initial carbon deposits present from the $\mathrm{CO}$ carburizations applied in the catalyst preparation, the initial fast step ( $\$ 15 \mathrm{~h}$ ToS) of rapid D- and G-band intensity growth is absent (Figures 6.18c-d and $6.19 c-d$ ). The UP 340 catalyst, with the least amount of initially deposited carbon within the group of UP and Na-S 340 and 440 catalyst materials, shows slight yet subdued initial fast Raman signal increase. Thus, either the initial carbon deposits i) dilute the catalyst bed with FTS inert carbon, allowing a more isothermal bed operation, ii) cover and deactivate the supported Fe particles i.e. decrease the CO conversion and/or iii) hide the Fe surface, where the Boudouard reaction takes place, from the Raman probe. That is, the Raman inactive carbon species formed during CO carburization should be different from Raman active carbon species formed during the FTS reaction. This will be discussed more later on. 
Combined In-situ X-Ray Powder Diffractometry/Raman Spectroscopy of Iron Carbide and Carbon Species Evolution in Fe(-Na-S)/ $\alpha-\mathrm{Al} 203$ Catalysts during Fischer-Tropsch Synthesis

All of the above listed phenomena would effectively decrease the observable Raman signal from the carbon deposition during the FTS reaction. Which explanation is the most correct, cannot be deduced from the results.

For all of the FTS reaction runs, the $I(D) / I(G)$ band intensity ratios are within range $\approx 1.0-0.7$ (Figures 6.16e - 6.19e). As exceptions, the Na-S 340 and 440 catalyst materials show initially higher $>1.0 I(D) / I(G)$ ratios (Figures 6.18e and 6.19e), due to poor data quality (Figures 6.14c-d and 6.15c-d). At a different measurement spot, quasi-in-situ measured Raman spectrum and the $I(D) / I(G)$ ratio of the Na-S 340 catalyst material was comparable to the other FTS reaction runs (Figure 6.20).

Due to a hysteresis phenomenon, when carbon species evolve from tetrahedral $\mathrm{C} \mathrm{sp}^{3}$ towards $\mathrm{C} \mathrm{sp}^{2}$ carbon species with more order, the interpretation of the $I(D) / I(G)$ band intensity ratios is not straight-forward. ${ }^{64}$ Nevertheless, the measured Raman spectra with $I(D) / I(G)<1.0$ can be assigned to carbon species with little crystal order and/or disordered structures, with diameters $<7 \mathrm{~nm} .{ }^{65}$ Decrease in the $I(D)$ intensity (i.e. $I(D) / I(G)<1.0)$, and increase in the D-bands width as will be discussed later, are signs of the presence of cyclic $\mathrm{C} \mathrm{sp}^{2}$ carbon species which have cyclic carbon different from cyclic 6-fold carbon structures. Increasing $I(D) / I(G) \gtrsim 1.0$ ratio in turn signifies the increasing presence of clustered 6-fold cyclic $\mathrm{C} \mathrm{sp}{ }^{2}$ (i.e. aromatic) carbon structures, ${ }^{64}$ in disordered carbon species. ${ }^{64,65}$

\section{B. D- and G-band Deconvolution, Peak Positions and Widths}

The measured Raman D-band can be divided into sub-bands. Following the approach by Sadezky et al., ${ }^{104}$ the Raman D-band can further be divided into bands such as $\mathrm{D}_{1}$ centered at $\approx 1320 \mathrm{~cm}^{-1}, \mathrm{D}_{2}$ at $\approx 1620 \mathrm{~cm}^{-1}, \mathrm{D}_{3}$ at $\approx 1500 \mathrm{~cm}^{-1}$ and $\mathrm{D}_{4}$ at $\approx 1220 \mathrm{~cm}^{-1}$.

The G-band at $\approx 1590 \mathrm{~cm}^{-1}$ is thought to correspond to the ring vibration of defectfree graphitic lattice ( $E_{2 g}$-symmetry). The $D_{1}$ position is believed to arise from disordered graphite lattice with $A_{1 g}$-symmetry (e.g. in graphene layer edges). $D_{2}$ position in turn is assignable e.g. to graphite disorder with $\mathrm{E}_{2 \mathrm{~g}}$-symmetry in graphene surface layers. $\mathrm{D}_{3}$ position is often associated to presence of amorphous carbon. Finally, $\mathrm{D}_{4}$ position signals presence of disordered graphitic lattice with A1g-symmetry (e.g. polyenes or ionic impurities). . $^{104}$

Thus, by fitting the separate $\mathrm{D}_{1-4}$ - (and G-) band positions with individual peaks, additional information should in principle be obtainable from the collected in-situ Raman spectra. Our group has applied this approach earlier in operando experiments to study the formation of carbon species. ${ }^{66-68}$

For each of the in-situ measured Raman spectra multiple peaks were fitted. One peak for G-band at $\approx 1590 \mathrm{~cm}^{-1}$ and three peaks to correspond the $\mathrm{D}_{1}\left(\approx 1320 \mathrm{~cm}^{-1}\right), \mathrm{D}_{3}$ $\left(\approx 1500 \mathrm{~cm}^{-1}\right)$ and $\mathrm{D}_{4}\left(\approx 1220 \mathrm{~cm}^{-1}\right)$ positions. It was not possible to fit a peak for the $\mathrm{D}_{2}$ $\left(\approx 1620 \mathrm{~cm}^{-1}\right)$ position. Lack of sub-band peak for the $\mathrm{D}_{2}$ position can be taken as 

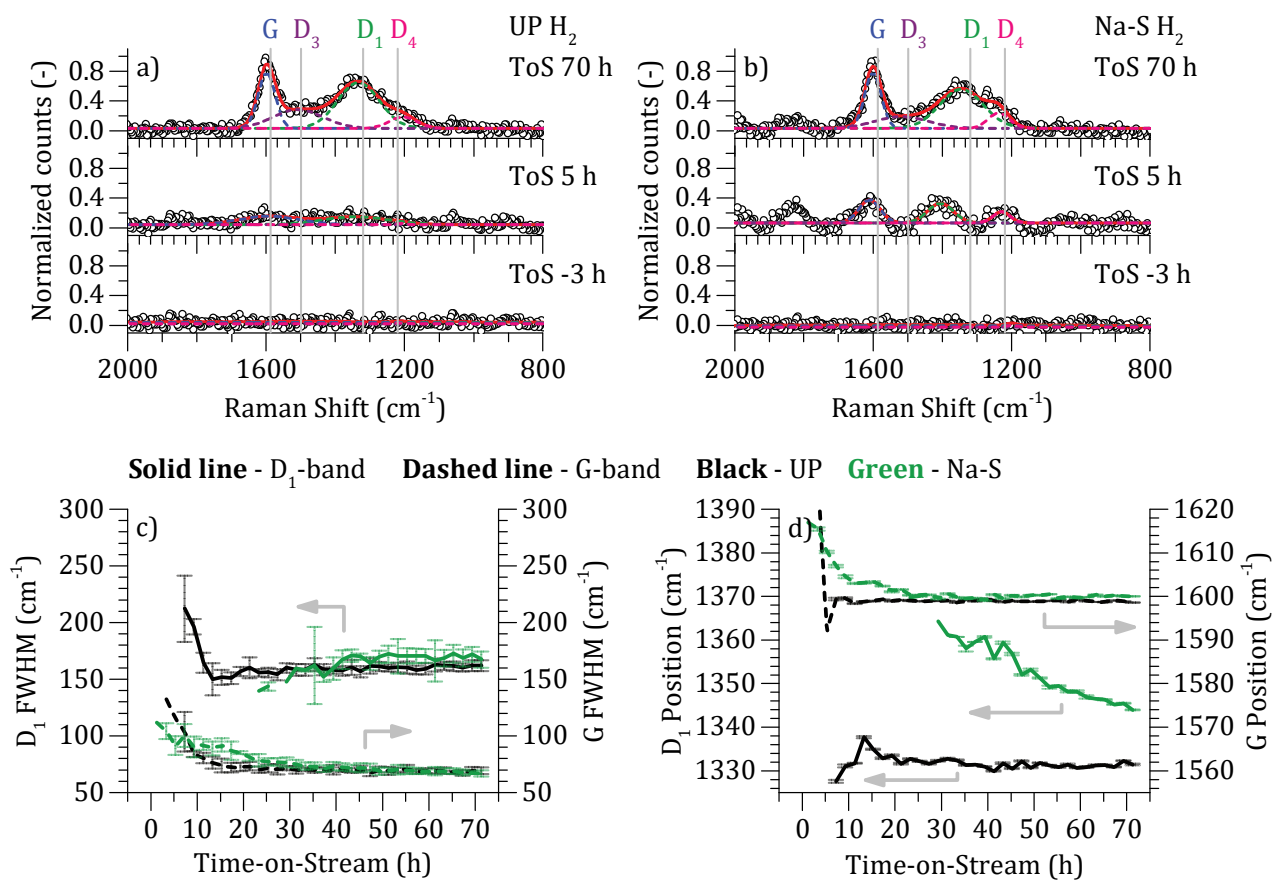

Figure 6.21

Peak fitting of the in-situ collected Raman spectra during the $72 \mathrm{~h}$ Fischer-Tropsch Synthesis (FTS) reaction with the $\mathrm{H}_{2}$ catalysts. The measured Raman spectra at $-3 \mathrm{~h}, 5 \mathrm{~h}$ and $70 \mathrm{~h}$ Time-on-Stream (ToS) with fitted G, $\mathrm{D}_{1}, \mathrm{D}_{3}$ and $\mathrm{D}_{4}$ peaks for a) UP $\mathrm{H}_{2}$ and b) Na-S $\mathrm{H}_{2}$ (Fe(-Na-S) $/ \alpha-\mathrm{Al}_{2} \mathrm{O}_{3}$ ) catalysts. Vertical gray lines mark the corresponding nominal Raman band positions, fitted peaks are indicated by their colored letters and the cumulative fit is in red. The peak $\mathrm{D}_{1}$ - and G-band widths over $72 \mathrm{~h}$ ToS in e) and positions in d) for UP and Na$\mathrm{S} \mathrm{H}_{2}$ catalysts.

indication of poorly ordered graphite structure. ${ }^{68}$

Moreover, the fitted peak relative area or width errors for the $\mathrm{D}_{3}$ and $\mathrm{D}_{4}$ peaks were only occasionally below the set filtering condition of $40 \%$. Therefore, no usable quantitative data could be collected for the $\mathrm{D}_{3}$ or $\mathrm{D}_{4}$ peaks. Therefore, in the following, the discussion is focused on the information collected from the fitted $\mathrm{D}_{1}$ - and G-bands, akin to what has been done earlier by our group. ${ }^{66,68}$ In principle, $\mathrm{D}_{1}$ - and G-bands provide sufficient information to identify the carbon species. ${ }^{104}$

The general trends with shifts in the $\mathrm{D}_{1}$ - and $\mathrm{G}$-band peak positions show similarities between the different FTS reaction runs (Figures 6.21-6.24). That is, i) G-band positions start $(\approx 0 \mathrm{~h} \mathrm{ToS})$ at $>1600 \mathrm{~cm}^{-1}$ and shift $\$ 1600 \mathrm{~cm}^{-1}$, ii) the $\mathrm{D}_{1}$-band positions start at $\gtrsim 1350 \mathrm{~cm}^{-1}$ while shifting towards $\approx 1330 \mathrm{~cm}^{-1}$ by the time when $\approx 70 \mathrm{~h}$ ToS in the FTS reaction is reached. The Na-S promotion tends to iii) increase the initial $(\approx 0 \mathrm{~h}$ ToS) G-band positions towards $\gtrsim 1600 \mathrm{~cm}^{-1}$. 

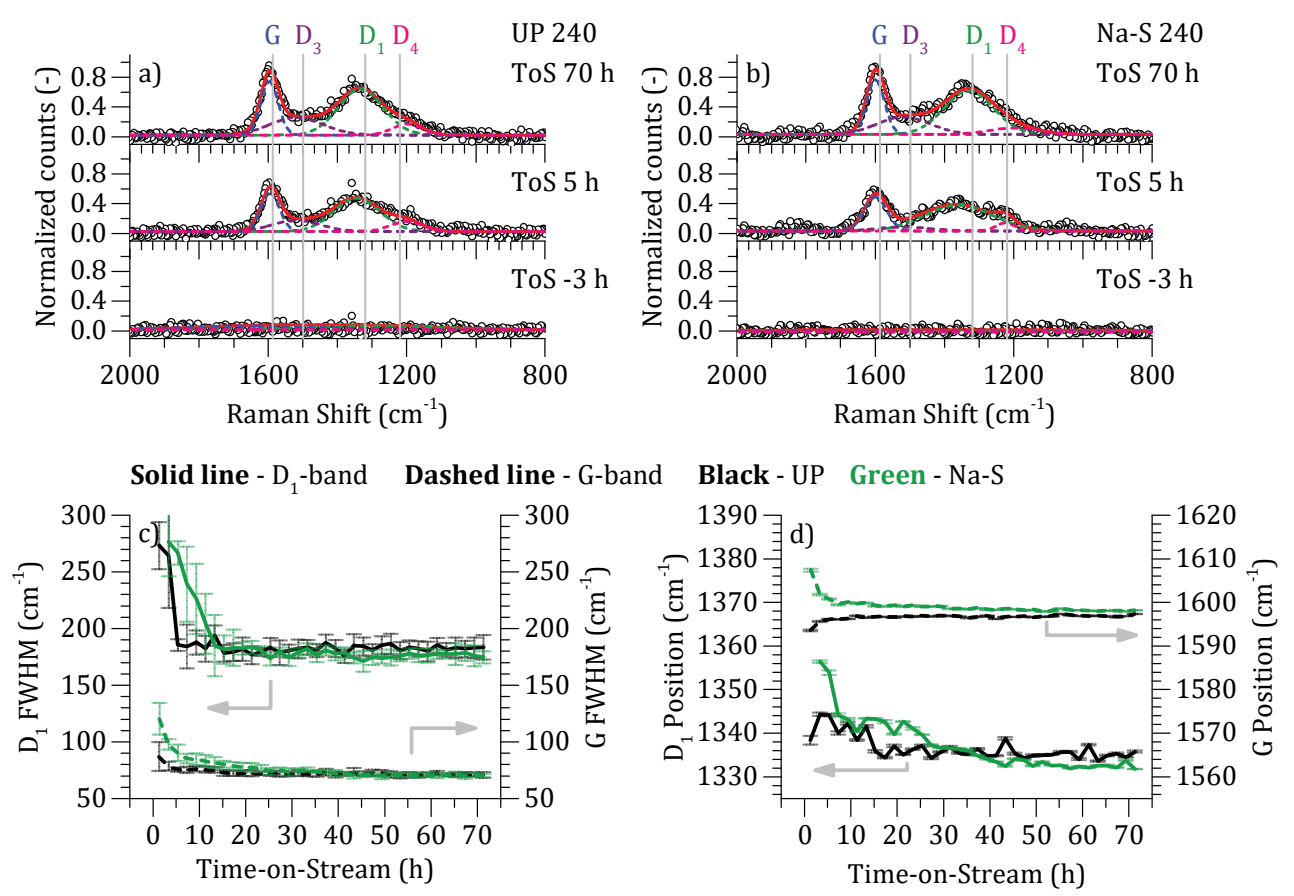

Figure 6.22

Peak fitting of the in-situ collected Raman spectra during the $72 \mathrm{~h}$ Fischer-Tropsch Synthesis (FTS) reaction with the 240 catalysts. The measured Raman spectra at $-3 \mathrm{~h}, 5 \mathrm{~h}$ and $70 \mathrm{~h}$ Time-on-Stream (ToS) with fitted $\mathrm{G}, \mathrm{D}_{1}, \mathrm{D}_{3}$ and $\mathrm{D}_{4}$ peaks for a) UP 240 and b) Na-S 240 (Fe(-Na-S)/ $\alpha-\mathrm{Al}_{2} \mathrm{O}_{3}$ ) catalysts. Vertical gray lines mark the corresponding nominal Raman band positions, fitted peaks are indicated by their colored letters and the cumulative fit is in red. The peak $\mathrm{D}_{1}$ - and G-band widths over $72 \mathrm{~h}$ ToS in e) and positions in d) for UP and NaS 240 catalysts.

The initial, high (>1600 $\mathrm{cm}^{-1}$ ) G-band Raman shifts are due to olefinic, chain-like carbon species with $\mathrm{C} \mathrm{sp}{ }^{2}$ and (tetrahedral) $\mathrm{C} \mathrm{sp}{ }^{3}$ amorphous carbon structures. ${ }^{64,108}$ The high $\left(\gtrsim 1350 \mathrm{~cm}^{-1}\right.$ ) initial position for the $\mathrm{D}_{1}$-band originates from the presence of amorphous carbon. ${ }^{63}$ For both the $\mathrm{D}_{1}$ - and G-bands, the shifts towards lower wavenumbers during the FTS reactions (i.e. $\mathrm{D}_{1}$-band $->\approx 1330 \mathrm{~cm}^{-1}$ and G-band -> $\approx 1600 \mathrm{~cm}^{-1}$ ), signify a transition towards an increasing $\mathrm{C} \mathrm{sp}{ }^{2}$ content in relation to $\mathrm{C}$ $\mathrm{sp}^{3}$. Also, more cyclic, rather than chain-like, carbon species form. ${ }^{64}$

The general trends in the $\mathrm{D}_{1}$ - and G-band peak widths (FWHM) evolution also suggest increasing carbon species ordering processes during the FTS reaction (Figures 6.21c-6.24c). Decreasing $\mathrm{D}_{1}$ and G-band peak widths have been associated to increasing graphitization and ordering of the carbon species. ${ }^{66,68}$

The G-band widths decrease from initial $>100 \mathrm{~cm}^{-1}$ at $\approx 0-10 \mathrm{~h}$ ToS towards 70 $80 \mathrm{~cm}^{-1}$ at $\approx 70 \mathrm{~h} \mathrm{ToS}$ for all of the studied catalyst materials (Figures 6.21c $-6.24 \mathrm{c}$ ). 

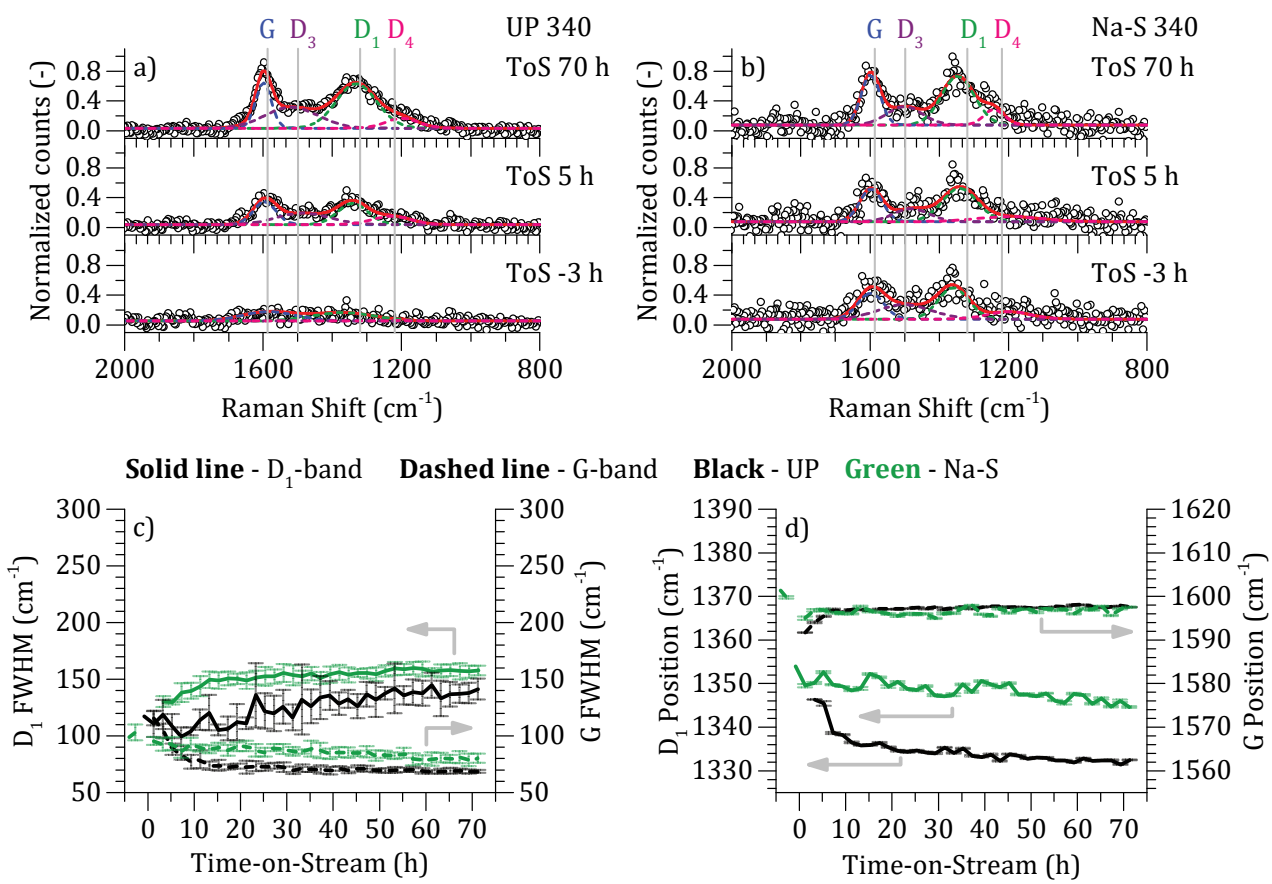

Figure 6.23

Peak fitting of the in-situ collected Raman spectra during the $72 \mathrm{~h}$ Fischer-Tropsch Synthesis (FTS) reaction with the 340 catalysts. The measured Raman spectra at $-3 \mathrm{~h}, 5 \mathrm{~h}$ and $70 \mathrm{~h}$ Time-on-Stream (ToS) with fitted $\mathrm{G}, \mathrm{D}_{1}, \mathrm{D}_{3}$ and $\mathrm{D}_{4}$ peaks for a) UP 340 and b) Na-S 340 ( $\mathrm{Fe}(-\mathrm{Na}-\mathrm{S}) / \alpha-\mathrm{Al}_{2} \mathrm{O}_{3}$ ) catalysts. Vertical gray lines mark the corresponding nominal Raman band positions, fitted peaks are indicated by their colored letters and the cumulative fit is in red. The peak $\mathrm{D}_{1}$ - and G-band widths over $72 \mathrm{~h}$ ToS in e) and positions in d) for UP and NaS 340 catalysts.

This G-band width decrease thus can be associated to a carbon species ordering processes during the FTS reaction ToS.

For the $\mathrm{D}_{1}$-band width evolution the situation is mixed. With UP and $\mathrm{Na}-\mathrm{S}_{2}$ and 240 catalysts the $\mathrm{D}_{1}$-band decreases (from $\gtrsim 200 \mathrm{~cm}^{-1}$ to $\approx 160-180 \mathrm{~cm}^{-1}$ ), while for the UP and Na-S 340 and 440 catalysts the $\mathrm{D}_{1}$-band increases (from $\approx 100 \mathrm{~cm}^{-1}$ to $\approx 140$ $160 \mathrm{~cm}^{-1}$ ) during the FTS reaction (Figures 6.23c and 6.24c). The $\mathrm{D}_{1}$-band width decrease is associated to the carbon species ordering process.66,68 The UP and Na-S 340 and 440 catalysts have initial carbon deposits from the $\mathrm{CO}$ carburization. Also, for these catalysts the initial $I(D) / I(G)$ ratios are higher is a sign of increased initial presence of $C$ $\mathrm{sp}^{2}$ in cyclic 6-fold ordered carbon rings, as was discussed earlier. Thus, the $\mathrm{D}_{1}$-band width increase for the UP and Na-S 340 and 440 catalyst materials can be explained by formation of cyclic $\mathrm{C} \mathrm{sp}^{2}$, non-6-fold cyclic carbon structures during the FTS reaction. ${ }^{64}$ This is in-line with the previous discussion on the $I(D) / I(G)$ ratios for the UP and Na-S 

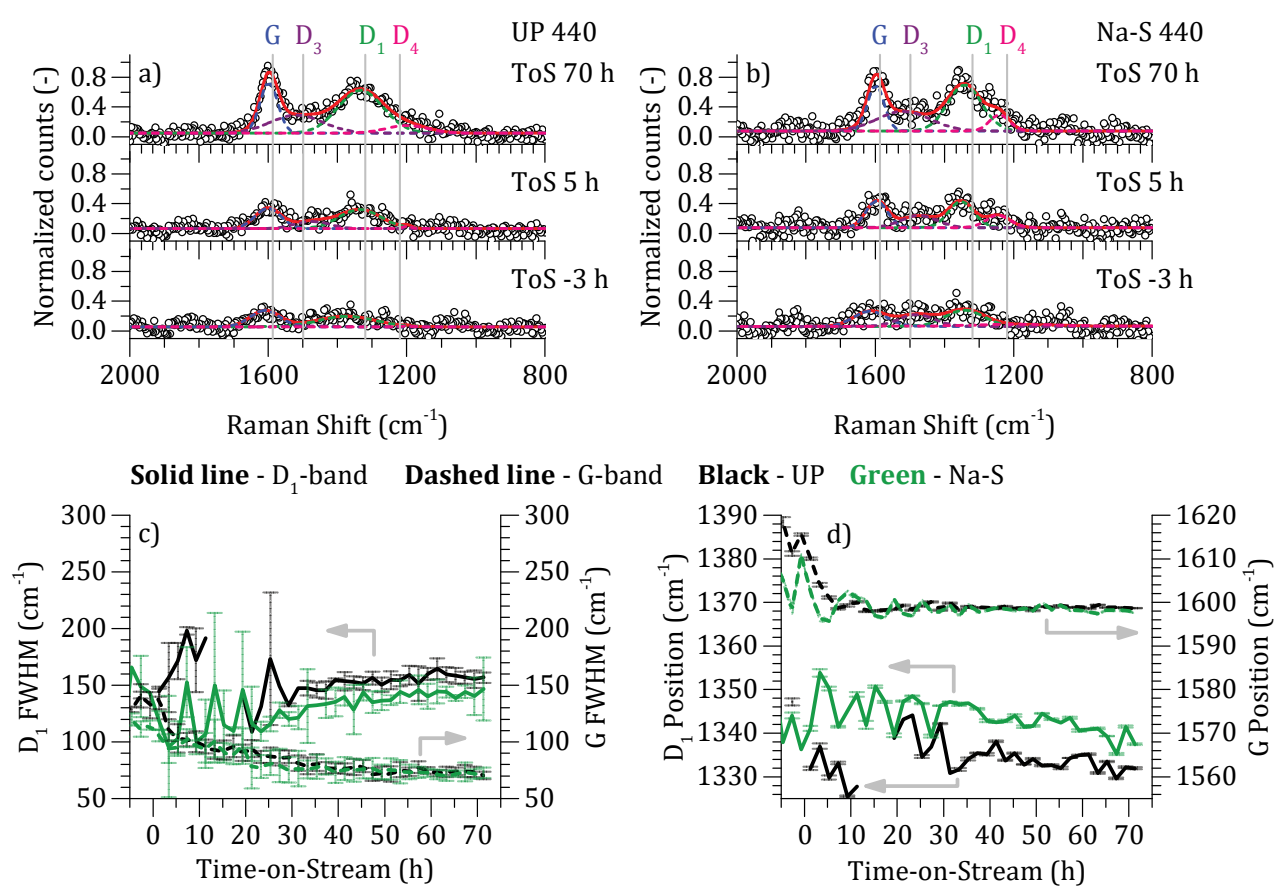

Figure 6.24

Peak fitting of the in-situ collected Raman spectra during the $72 \mathrm{~h}$ Fischer-Tropsch Synthesis (FTS) reaction with the 440 catalysts. The measured Raman spectra at $-3 \mathrm{~h}, 5 \mathrm{~h}$ and $70 \mathrm{~h}$ Time-on-Stream (ToS) with fitted $\mathrm{G}, \mathrm{D}_{1}, \mathrm{D}_{3}$ and $\mathrm{D}_{4}$ peaks for a) UP 440 and b) Na-S 440 (Fe(-Na-S)/ $\alpha-\mathrm{Al}_{2} \mathrm{O}_{3}$ ) catalysts. Vertical gray lines mark the corresponding nominal Raman band positions, fitted peaks are indicated by their colored letters and the cumulative fit is in red. The peak $\mathrm{D}_{1}$ - and G-band widths over $72 \mathrm{~h}$ ToS in e) and positions in d) for UP and NaS 440 catalysts.

\section{0 and 440 catalyst.}

Summarizing the general trends for the carbon species evolution for the catalyst materials under study during the FTS reaction runs: the carbon species evolve from, tetrahedral C $\mathrm{sp}^{3}$ with $\mathrm{C} \mathrm{sp}{ }^{2}$ in chain-like structured amorphous carbon species towards carbon materials with varying cyclic - but non- 6 - fold order with features $<7 \mathrm{~nm}$ in diameter. The trend of increasing ordering of the carbon species is in-line with previous studies for carbon deposits formation during the FTS reaction. . $^{0}$

\subsubsection{Comparison to Carbon Reference Materials}

Further identification of the carbon species formed during the FTS reactions can be done by comparing the in-situ collected Raman spectra at $\approx 70 \mathrm{~h}$ ToS to (ex-situ) measured carbon reference materials of graphite, Carbon Nanotubes (CNT), Carbon Nanofibers (CNF) and Activated Carbon (AC). 

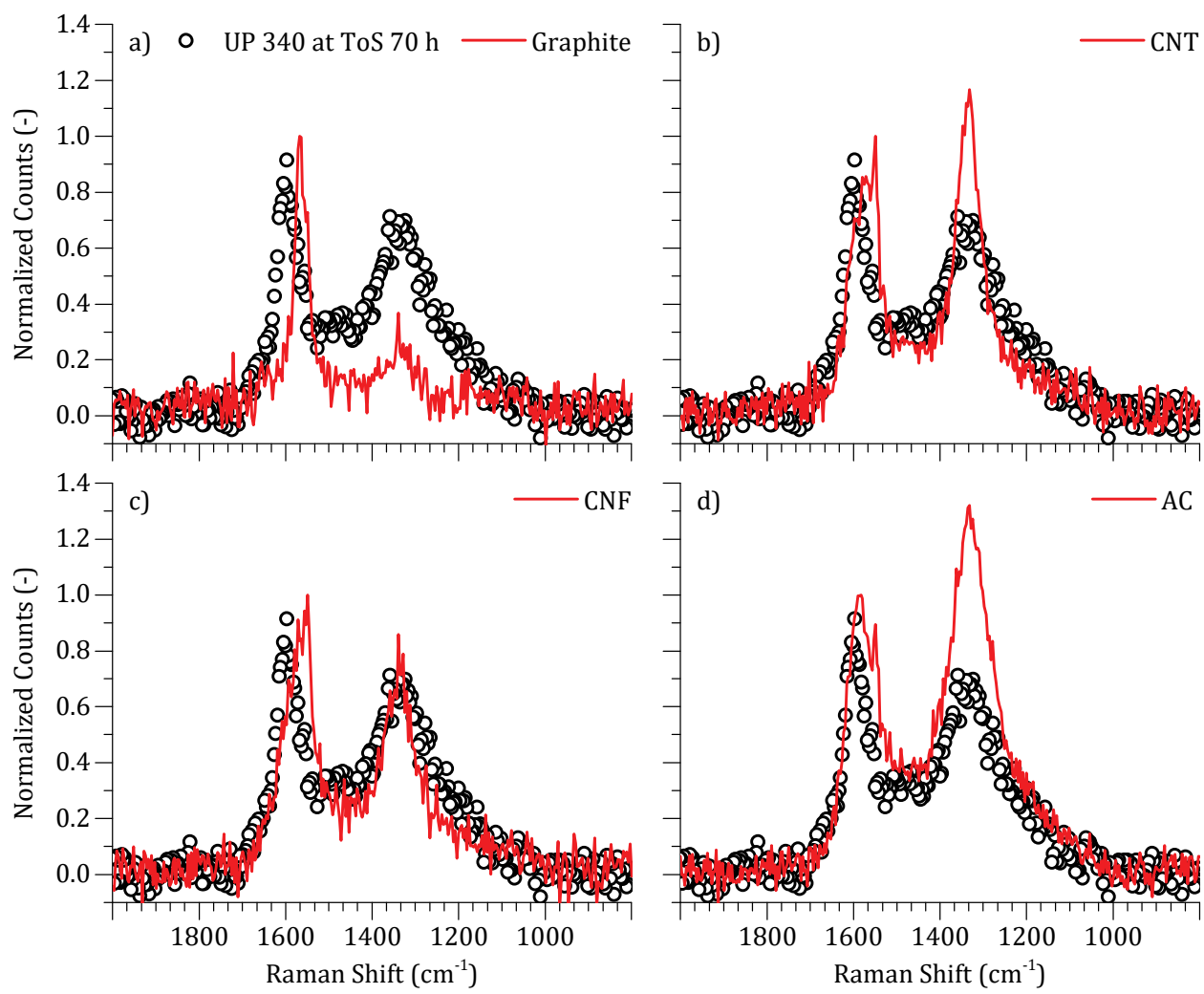

Figure 6.25

Comparison of in-situ collected Raman spectrum of UP $340\left(\mathrm{Fe} / \alpha-\mathrm{Al}_{2} \mathrm{O}_{3}\right)$ catalyst material at $\approx 70 \mathrm{~h}$ Time-onStream (ToS) of Fischer-Tropsch Synthesis (FTS) reaction with ex-situ measured carbon reference materials. Comparison of the Raman spectrum obtained on the catalyst material to a) graphite, b) Carbon Nanotube (CNT), c) Carbon Nanofiber (CNF) and d) Activated Carbon (AC) carbon reference materials.

Saturated and unsaturated long-chained hydrocarbons i.e. waxes, can also give observable Raman spectra, ${ }^{108}$ and can be produced in the FTS reaction. However, the collected Raman spectra do not show any particular resemblance to Raman spectra from wax samples. ${ }^{108}$ With the used $(532 \mathrm{~nm})$ visible wavelength excitation laser the structural majority $\mathrm{C} \mathrm{sp} \mathrm{s}^{3} \sigma$-bonds in hydrocarbons polarize poorly and do not give strong Raman signal, ${ }^{64}$ even if present. Thus, it is unlikely that any hydrocarbon wax species contribute in any significant manner to the collected Raman spectra.

As examples, in Figure 6.25 the ex-situ collected Raman spectra for the carbon reference materials are compared to the in-situ collected Raman spectrum for the UP 340 catalyst material at $\approx 70 \mathrm{~h}$ ToS. The other catalyst materials gave comparable Raman spectra to the UP 340 catalyst at $\approx 70 \mathrm{~h}$ ToS (Figures 6.21a-b - 6.24a-b).

The in-situ measured median values, at $\approx 70 \mathrm{~h}$ ToS, for $\mathrm{D}_{1}$ - and G-band peak 
Combined In-situ X-Ray Powder Diffractometry/Raman Spectroscopy of Iron Carbide and Carbon Species Evolution in Fe(-Na-S)/ $\alpha$-Al203 Catalysts during Fischer-Tropsch Synthesis

\section{Table 6.2}

In-situ Raman Spectroscopy derived median $\mathrm{D}_{1}$ - and G-band values at $\approx 70 \mathrm{~h}$ Time-on-Stream (ToS) of the Fischer-Tropsch Synthesis (FTS) reaction runs for the studied UP and $\mathrm{Na}-\mathrm{S}\left(\mathrm{Fe}(-\mathrm{Na}-\mathrm{S}) / \alpha-\mathrm{Al}_{2} \mathrm{O}_{3}\right)$ catalyst materials. Reference carbon materials measured ex-situ at room temperature.

\begin{tabular}{|c|c|c|c|c|c|c|}
\hline \multicolumn{2}{|c|}{ Catalyst } & \multicolumn{2}{|c|}{$\mathrm{D}_{1}$-band } & \multicolumn{2}{|c|}{ G-band } & \multirow[b]{2}{*}{$\begin{array}{c}\mathrm{I}(\mathrm{D}) / \mathrm{I}(\mathrm{G}) \\
(-)\end{array}$} \\
\hline & & $\begin{array}{c}\text { Position } \\
\left(\mathrm{cm}^{-1}\right)\end{array}$ & $\begin{array}{c}\text { FWHM } \\
\left(\mathrm{cm}^{-1}\right)\end{array}$ & $\begin{array}{c}\text { Position } \\
\left(\mathrm{cm}^{-1}\right)\end{array}$ & $\begin{array}{l}\text { FWHM } \\
\left(\mathrm{cm}^{-1}\right)\end{array}$ & \\
\hline \multirow[t]{2}{*}{$\mathrm{H}_{2}$} & UP & $1332 \pm 1$ & $162 \pm 5$ & $1599 \pm 0$ & $69 \pm 3$ & $0.76 \pm 0.05$ \\
\hline & $\mathrm{Na}-\mathrm{S}$ & $1346 \pm 2$ & $172 \pm 7$ & $1600 \pm 0$ & $67 \pm 1$ & $0.66 \pm 0.06$ \\
\hline \multirow[t]{2}{*}{240} & UP & $1334 \pm 0$ & $183 \pm 9$ & $1597 \pm 0$ & $71 \pm 2$ & $0.73 \pm 0.05$ \\
\hline & $\mathrm{Na}-\mathrm{S}$ & $1334 \pm 0$ & $176 \pm 9$ & $1598 \pm 0$ & $71 \pm 2$ & $0.72 \pm 0.04$ \\
\hline \multirow[t]{2}{*}{340} & UP & $1331 \pm 0$ & $157 \pm 5$ & $1598 \pm 0$ & $68 \pm 2$ & $0.77 \pm 0.05$ \\
\hline & $\mathrm{Na}-\mathrm{S}$ & $1346 \pm 0$ & $137 \pm 9$ & $1597 \pm 0$ & $79 \pm 6$ & $0.89 \pm 0.11$ \\
\hline \multirow[t]{2}{*}{440} & UP & $1332 \pm 0$ & $155 \pm 8$ & $1599 \pm 0$ & $73 \pm 5$ & $0.82 \pm 0.07$ \\
\hline & $\mathrm{Na}-\mathrm{S}$ & $1342 \pm 0$ & $140 \pm 17$ & $1599 \pm 0$ & $71 \pm 4$ & $0.85 \pm 0.10$ \\
\hline \multirow{4}{*}{ 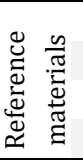 } & Graphite & 1334 & 113 & 1565 & 39 & 0.37 \\
\hline & $\mathrm{CNF}$ & 1338 & 111 & 1571 & 89 & 0.86 \\
\hline & $\mathrm{AC}$ & 1328 & 143 & 1584 & 97 & 1.31 \\
\hline & CNT & 1333 & 86 & 1576 & 91 & 1.36 \\
\hline
\end{tabular}

FWHM = Full Width at Half Maximum (of fitted peak), CNF = Carbon Nanotubes, AC = Activated Carbon, CNT $=$ Carbon Nanotubes.

positions, related peak widths as FWHMs and $I(D) / I(G)$ ratios are given in Table 6.2 with the corresponding values for the carbon reference materials.

By comparing the in-situ collected Raman spectrum for the UP 340 catalyst at $\approx 70 \mathrm{~h}$ ToS, the most comparable carbon reference spectrum is the one of CNF (Figure 6.25). Both the $\mathrm{D}_{1}$-band position and $I(D) / I(G)$ ratio are very similar to the reference $\mathrm{CNF}$ material. However, the G-band position is at a higher wavenumber $\left(\approx 1598 \mathrm{~cm}^{-1}\right.$ versus $\approx 1571 \mathrm{~cm}^{-1}$ ) and the $\mathrm{D}_{1}$-band width is also wider in the measured spectrum for the catalyst material than for the reference CNF material. The G-band position is more akin to the G-band position with that of the AC reference carbon material (Figure 6.25).

The higher width of the measured $\mathrm{D}_{1}$-band can be explained by a wider variety of carbon ring sizes whilst the G-band shift towards higher wavenumber would suggest the increased presence of $\mathrm{C} \mathrm{sp}^{2}$ in chain-like carbon species or clustering, ${ }^{64}$ than is present in the reference CNF material.

For the measured catalyst materials, the Na-S 340 and Na-S 440 catalysts show in addition an increase in their $\mathrm{D}_{1}$-band intensities in comparison to the rest of the catalyst materials at $\approx 70 \mathrm{~h} \mathrm{ToS}$ (Figures 6.18e and 6.19e). The $\mathrm{D}_{1}$-band intensity increase i.e. increase in the $I(D) / I(G)$ ratio, is due to the increased presence of clustered cyclic 6-fold $\mathrm{C}$ sp$^{2}$ carbon species. ${ }^{64}$

Summarizing the discussion on carbon species evolution: during the FTS reaction 
under the applied reaction conditions, the carbon species formed resemble most closely the CNF reference carbon material. With added Na-S promotion in comparison to the respective UP catalyst materials: i) for $\mathrm{H}_{2}$ and 240 catalyst materials, the carbon species evolution starts with more $\mathrm{C} \mathrm{sp}{ }^{2}$ in chain-like species; ii) more of the clustered 6 -fold cyclic carbon species for 340 and 440 catalyst materials are present; and iii) the $\mathrm{Fe}_{3} \mathrm{O}_{4}$ phases are reduced more efficiently by $\mathrm{CO}$, removing the onset time for appearance of the carbon species between the $\mathrm{Na}-\mathrm{S}_{2}$ and UP $\mathrm{H}_{2}$ catalyst materials.

It is noteworthy to mention that the CNF materials contain "graphite-like" planes in their fiber structure. ${ }^{37,53}$ Thus assigning a Fe-based FTS reaction catalyst's deactivation to be due to "graphitic" carbon overlayers over the active Fe carbides should be done with care, and with clear distinction to a possible presence of CNF materials. CNF materials grow away from the catalyst surface, ${ }^{35,109}$ which is a significantly different phenomena than a carbon overlayer formation over the Fe surface. 109

Finally, the carbon species formed under $\mathrm{CO}$ carburization and under $\mathrm{H}_{2}$ : $\mathrm{CO}$ during the FTS reaction are different. No significant or only weak Raman D- and G-bands were observed for the initial carbon containing UP and Na-S 340 and 440 catalyst materials at the beginning of the FTS reactions. Rather, the $\mathrm{D}_{1}$ - and G-bands appear and grow during the FTS reaction (Figures 6.14 and 6.15). As based on the observations in literature, under pure $\mathrm{CO}$ encapsulating carbon species form while in the presence of $\mathrm{H}_{2}$ :CO, growth of filamentous carbon takes place. $35,37,54,55,109,110$ This is in agreement with the results, i.e. the initial carbon deposits due to the CO carburization are formed with $\mathrm{C} \mathrm{sp}{ }^{3}$ structures which are Raman inactive with the used $(532 \mathrm{~nm})$ visible wavelength excitation laser. ${ }^{64}$ The formation of CNF-like carbon species with Raman active $\mathrm{C} \mathrm{sp} \mathrm{s}^{2}$ structures takes place under the applied FTS conditions in the presence of $\mathrm{H}_{2}: \mathrm{CO}_{3}, 37$ explaining the rise of the in-situ collected Raman spectra for the studied catalyst materials (Figures 6.12-6.15).

\subsection{CONCLUSIONS}

We have studied supported $\mathrm{Fe}(-\mathrm{Na}-\mathrm{S}) / \alpha-\mathrm{Al}_{2} \mathrm{O}_{3}$ catalyst materials, with and without Na$\mathrm{S}$ promotion. Ex-situ CO carburizations at various temperatures $\left(240-440^{\circ} \mathrm{C}\right)$ were applied to prepare preformed Fe carbides, or only $\mathrm{H}_{2}$ reduction was applied for preparing $\alpha-\mathrm{Fe} / \mathrm{Fe}$ oxide mixtures on the catalysts. The catalyst materials were characterized in-situ during $72 \mathrm{~h}$ FTS reaction $\left(340^{\circ} \mathrm{C}, 10\right.$ bar $\mathrm{H}_{2}: \mathrm{CO}: \mathrm{He}$ 2.0:1.0:0.33 v/v, GHSV $\approx 7200 \mathrm{~h}^{-1}$ ). The in-situ characterization was performed using a combined XRPD/Raman Spectroscopy set-up. The collected XRPD patterns were quantified with the use of R-QPA.

Out of the prepared Fe carbides, namely: $\varepsilon-\mathrm{Fe}_{3} \mathrm{C} P 6_{3} 22$ (182), $\eta-\mathrm{Fe}_{2} \mathrm{C}$ Pnnm (58), $\chi-$ 
Combined In-situ X-Ray Powder Diffractometry/Raman Spectroscopy of Iron Carbide and Carbon Species Evolution in Fe(-Na-S)/ $\alpha$-Al203 Catalysts during Fischer-Tropsch Synthesis

$\mathrm{Fe}_{5} \mathrm{C}_{2} C 2 / c$ (15) (the Hägg carbide) and, $\theta-\mathrm{Fe}_{3} \mathrm{C}$ Pnma (62) (cementite), the $\chi-\mathrm{Fe}_{5} \mathrm{C}_{2}$ phase was the most stable under the used FTS reaction temperature. Hence, the $\chi-\mathrm{Fe}_{5} \mathrm{C}_{2}$ phase formed preferentially. Furthermore, the Na-S promotion altered the Fe oxides and carbide phases' properties by:

i) stabilizing the $\eta-\mathrm{Fe}_{2} \mathrm{C}$ phase, but only if either $\varepsilon-\mathrm{Fe}_{3} \mathrm{C}$ and/or $\eta-\mathrm{Fe}_{2} \mathrm{C}$ phase was present in the initial catalyst,

ii) destabilizing the $\theta-\mathrm{Fe}_{3} \mathrm{C}$ phase and aiding in its transition to $\chi-\mathrm{Fe}_{5} \mathrm{C}_{2}$,

iii) increasing the reducibility of $\mathrm{Fe}_{3} \mathrm{O}_{4}$ phase by $\left(\mathrm{H}_{2}\right.$ :) CO,

iv) decreasing the $\mathrm{Fe}_{3} \mathrm{O}_{4}$ content during the FTS reaction for catalyst with initial Fe carbide phases as $\varepsilon-\mathrm{Fe}_{3} \mathrm{C}$ and/or $\eta-\mathrm{Fe}_{2} \mathrm{C}$; and also by

v) inducing Fe crystallite growth for the Fe carbides within the $\lesssim 15 \mathrm{~h} \mathrm{ToS}$, with the exception of the $\theta-\mathrm{Fe}_{3} \mathrm{C}$ phase, for which the crystallite diameters decreased.

Irrespective to the Na-S promotion, no significant presence of the $\mathrm{Fe}_{3} \mathrm{O}_{4}$ phase for catalyst materials with $\alpha-\mathrm{Fe} / \mathrm{Fe}_{\mathrm{x}} \mathrm{O}_{\mathrm{y}}, \chi-\mathrm{Fe}_{5} \mathrm{C}_{2}$ or $\theta-\mathrm{Fe}_{3} \mathrm{C}$ as the reaction starting materials were observed during the FTS reactions.

Carbon deposition via the Boudouard reaction was observed via in-situ Raman Spectroscopy. The carbon deposition did not correlate with any specific Fe carbide phase or carbide-to-carbide transition. However, with $\mathrm{H}_{2}$ reduced catalysts, the initial carbon deposition did not start before the $\mathrm{Fe}_{3} \mathrm{O}_{4}$ phase, a phase incapable of $\mathrm{CO}$ dissociation, was reduced to Fe carbides.

For the catalyst materials with insignificant initial carbon deposits i.e. $\mathrm{H}_{2}$ reduced or $<300^{\circ} \mathrm{C} \mathrm{CO}$ carburized catalysts, the Raman D- and G-band intensity growth during the FTS reaction could be divided into two periods: i) an initial fast ( $\$ 10-15 \mathrm{~h}$ ToS), and ii) a steady or lesser ( $\gtrsim 15 \mathrm{~h}$ ToS) intensity growth period. The initial fast growth period was present regardless of the Na-S promotion. However, after reaching the steady growth period Na-S promotion induced a higher rate of the Raman D- and G-band intensity growth (for the G-band $\approx 2.5 \pm 0.9$ faster) in comparison to the unpromoted catalyst materials. For catalysts with carbon deposits from CO carburization $\geq 340^{\circ} \mathrm{C}$, the initial fast growth period was absent, regardless of the Na-S promotion. Possibly, an initial non-isothermal bed operation could explain the initial fast D- and G-band intensity gain within $\lesssim 10-15 \mathrm{~h}$ ToS.

The carbon species formed during catalyst preparation CO carburizations were weakly/non-Raman active with the used (532 nm) visible wavelength excitation laser i.e. mainly made of structures with $\mathrm{C} \mathrm{sp}^{3}$. Under $\mathrm{H}_{2}$ : $\mathrm{CO}$ during the FTS reaction more ordered CNF-like phases with $\mathrm{C} \mathrm{sp}^{2}$ formed resulting in increased Raman signal 
intensity. During the FTS reactions, the carbon species evolved from amorphous carbon species, with structures containing both tetrahedral $\mathrm{C} \mathrm{sp}^{3}$ and $\mathrm{C} \mathrm{sp}^{2}$ in chain-like ordering, towards more ordered carbon materials with their structures containing $\mathrm{C}$ $\mathrm{sp}^{2}$ in varying non-6-fold cyclic order. The formed carbon materials had feature sizes $<7 \mathrm{~nm}$ in diameter.

In comparison to the measured carbon reference materials, the catalysts' Raman spectra at $\approx 70 \mathrm{~h}$ ToS compared best to a CNF reference material. The catalyst materials contained higher amounts of $\mathrm{C} \mathrm{sp}{ }^{2}$ in chain-like structures and a wider variety of non6-fold carbon rings than were present in the measured reference CNF material.

Na-S promotion affected the FTS reaction formed carbon species by: i) increasing the initial $\mathrm{C} \mathrm{sp}{ }^{2}$ content in chain-like carbon structures, and ii) increasing 6-fold cyclic carbon species for catalyst materials with the initial CO carburization $\geq 340^{\circ} \mathrm{C}$.

As most of the changes in the Fe phases and Raman spectra take place within the first $\lesssim 15 \mathrm{~h}$ ToS with the used Fe-based catalyst materials, the initial catalyst changes would be an interesting further research topic for the continued application of the combined in-situ XRPD/Raman Spectroscopy approach.

\subsection{ACKNOWLEDGEMENTS}

J. P. Falkenhagen (Universiteit Utrecht, UU) is thanked for his technical expertise and for providing design solutions for the in-situ XRPD/Raman Spectroscopy set-up. 0. Kerkenaar, P. Wijten, A. van der Eerden, H. Schaink and R. van Zwienen (UU) are acknowledged for the practical realization of the in-situ XRPD/Raman Spectroscopy set-up.

\subsection{REFERENCES}

[1] Mittasch, A. and Schneider, C., "Producing Compounds Containing Carbon and Hydrogen", Patent US1201850A (1916).

[2] Dry, M. E., "The Fischer-Tropsch Synthesis" in Catalysis - Science and Technology (eds. Anderson, J. R. \& Boudart, M.) 1, 159-256 (Springer-Verlag, Berlin, 1981).

[3] Dry, M. E., "FT catalysts" in Fischer-Tropsch Technology (eds. Steynberg, A. P. \& Dry, M. E.) 152, 533600 (Elsevier, Amsterdam, 2004).

[4] Dictor, R. and Bell, A. T., "Fischer-Tropsch synthesis over reduced and unreduced iron oxide catalysts", J. Catal. 97, 121-136 (1986).

[5] Todic, B., Nowicki, L., Nikacevic, N. and Bukur, D. B., "Fischer-Tropsch synthesis product selectivity over an industrial iron-based catalyst: Effect of process conditions", Catal. Today 261, 28-39 (2016).

[6] van der Laan, G. P. and Beenackers, A. A. C. M., "Kinetics and Selectivity of the Fischer-Tropsch Synthesis: A Literature Review", Catal. Rev. 41, 255-318 (1999).

[7] dos Santos, R. G. and Alencar, A. C., "Biomass-derived syngas production via gasification process and 
Combined In-situ X-Ray Powder Diffractometry/Raman Spectroscopy of Iron Carbide and Carbon Species Evolution in Fe(-Na-S)/ $\alpha$-Al203 Catalysts during Fischer-Tropsch Synthesis

its catalytic conversion into fuels by Fischer Tropsch synthesis: A review", Int. J. Hydrogen Energy (2019). DOI:10.1016/j.ijhydene.2019.07.133

[8] Kirchner, J., Baysal, Z. and Kureti, S., "Activity and Structural Changes of Fe-based Catalysts during $\mathrm{CO}_{2}$ Hydrogenation towards $\mathrm{CH}_{4}-\mathrm{A}$ Mini Review", ChemCatChem 12, 981-988 (2020).

[9] Mahmoudi, H., Mahmoudi, M., Doustdar, O., Jahangiri, H., Tsolakis, A., Gu, S. and Lech Wyszynski, M., "A review of Fischer Tropsch synthesis process, mechanism, surface chemistry and catalyst formulation", Biofuels Eng. 2, 11-31 (2017).

[10] Molino, A., Larocca, V., Chianese, S. and Musmarra, D., "Biofuels Production by Biomass Gasification: A Review", Energies 11, 811 (2018).

[11] Muleja, A. A., Gorimbo, J. and Masuku, C. M., "Effect of Co-Feeding Inorganic and Organic Molecules in the Fe and Co Catalyzed Fischer-Tropsch Synthesis: A Review", Catalysts 9, 746 (2019).

[12] Torres Galvis, H. M. and de Jong, K. P., "Catalysts for Production of Lower Olefins from Synthesis Gas: A Review", ACS Catal. 3, 2130-2149 (2013).

[13] Torres Galvis, H. M., Bitter, J. H., Khare, C. B., Ruitenbeek, M., Dugulan, A. I. and de Jong, K. P., "Supported Iron Nanoparticles as Catalysts for Sustainable Production of Lower Olefins", Science 335, 835-838 (2012).

[14] Myddleton, W. W., "Improvements Relating to the Catalytic Synthesis of Hydrocarbon Oils from Gaseous Mixtures of Carbon Monoxide and Hydrogen", Patent GB509325A (1939).

[15] Torres Galvis, H. M., Bitter, J. H., Davidian, T., Ruitenbeek, M., Dugulan, A. I. and de Jong, K. P., "Iron Particle Size Effects for Direct Production of Lower Olefins from Synthesis Gas", J. Am. Chem. Soc. 134, 16207-16215 (2012).

[16] Torres Galvis, H. M., Koeken, A. C. J., Bitter, J. H., Davidian, T., Ruitenbeek, M., Dugulan, A. I. and De Jong, K. P., "Effect of precursor on the catalytic performance of supported iron catalysts for the FischerTropsch synthesis of lower olefins", Catal. Today 215, 95-102 (2013).

[17] Torres Galvis, H. M., Koeken, A. C. J., Bitter, J. H., Davidian, T., Ruitenbeek, M., Dugulan, A. I. and de Jong, K. P., "Effects of sodium and sulfur on catalytic performance of supported iron catalysts for the Fischer-Tropsch synthesis of lower olefins", J. Catal. 303, 22-30 (2013).

[18] Botes, G. F., Bromfield, T. C., Coetzer, R. L. J., Crous, R., Gibson, P. and Ferreira, A. C., "Development of a chemical selective iron Fischer Tropsch catalyst", Catal. Today 275, 40-48 (2016).

[19] Xie, J., Yang, J., Dugulan, A. I., Holmen, A., Chen, D., De Jong, K. P. and Louwerse, M. J., "Size and Promoter Effects in Supported Iron Fischer-Tropsch Catalysts: Insights from Experiment and Theory", ACS Catal. 6, 3147-3157 (2016).

[20] Xie, J., Torres Galvis, H. M., Koeken, A. C. J., Kirilin, A., Dugulan, A. I., Ruitenbeek, M. and de Jong, K. P., "Size and Promoter Effects on Stability of Carbon-Nanofiber-Supported Iron-Based Fischer-Tropsch Catalysts", ACS Catal. 6, 4017-4024 (2016).

[21] Crous, R., Bromfield, T. C. and Booyens, S., "Olefin Selective FT Catalyst Composition and Preparation Thereof", Patent W02010066386A1 (2010).

[22] Johnson, J. Y., "Improvements in the Manufacture and Production of Unsaturated Hydrocarbons of Low Boiling Point", Patent GB322284A (1929).

[23] Dry, M. E., Shingles, T. and van H. Botha, C. S., "Factors influencing the formation of carbon on iron Fischer-Tropsch catalysts I. The influence of promoters", J. Catal. 17, 341-346 (1970).

[24] Dry, M. E., Shingles, T., Boshoff, L. J. and van H. Botha, C. S., "Factors influencing the formation of carbon on iron Fischer-Tropsch catalysts. II. The effect of temperature and of gases and vapors present during Fischer-Tropsch synthesis", J. Catal. 17, 347-354 (1970).

[25] Cohn, E. M., Bean, E. H., Mentser, M., Hofer, L. J. E., Pontello, A., Peebles, W. C. and Jack, K. H., "The 
carburization of iron oxide with carbon monoxide: Modifications of Hägg iron carbide", J. Appl. Chem. 5, 418-425 (1955).

[26] Bartholomew, C. H., "Mechanisms of catalyst deactivation", Appl. Catal. A Gen. 212, 17-60 (2001).

[27] Niemantsverdriet, J. W. and van der Kraan, A. M., "On the time-dependent behavior of iron catalysts in Fischer-Tropsch synthesis", J. Catal. 72, 385-388 (1981).

[28] Dry, M. E., "The Fischer-Tropsch process: 1950-2000", Catal. Today 71, 227-241 (2002).

[29] Shultz, J. F., Hall, W. K., Seligman, B. and Anderson, R. B., "Studies of the Fischer-Tropsch Synthesis. XIV. Hägg Carbide as Catalysts", J. Am. Chem. Soc. 77, 213-221 (1955).

[30] Steynberg, A. P., Espinoza, R. L., Jager, B. and Vosloo, A. C., "High temperature Fischer-Tropsch synthesis in commercial practice", Appl. Catal. A Gen. 186, 41-54 (1999).

[31] Benziger, J. and Madix, R. J., "The effects of carbon, oxygen, sulfur and potassium adlayers on CO and $\mathrm{H}_{2}$ adsorption on $\mathrm{Fe}(100) "$ ", Surf. Sci. 94, 119-153 (1980).

[32] Le Caër, G., Dubois, J. M., Pijolat, M., Perrichon, V. and Bussière, P., "Characterization by Moessbauer Spectroscopy of Iron Carbides Formed by Fischer-Tropsch Synthesis", J. Phys. Chem. 86, 4799-4808 (1982).

[33] de Smit, E., Beale, A. M., Nikitenko, S. and Weckhuysen, B. M., "Local and long range order in promoted iron-based Fischer-Tropsch catalysts: A combined in situ X-ray absorption spectroscopy/wide angle Xray scattering study", J. Catal. 262, 244-256 (2009).

[34] de Smit, E., Cinquini, F., Beale, A. M., Safonova, O. V., van Beek, W., Sautet, P. and Weckhuysen, B. M., "Stability and Reactivity of $\epsilon-\chi-\theta$ Iron Carbide Catalyst Phases in Fischer-Tropsch Synthesis: Controlling $\mu c$ ", J. Am. Chem. Soc. 132, 14928-14941 (2010).

[35] Mazzucco, S., Wang, Y., Tanase, M., Picher, M., Li, K., Wu, Z., Irle, S. and Sharma, R., "Direct evidence of active and inactive phases of Fe catalyst nanoparticles for carbon nanotube formation", J. Catal. 319, 54-60 (2014).

[36] Jung, H. and Thomson, W. J., "Dynamic X-ray diffraction study of an unsupported iron catalyst in Fischer-Tropsch synthesis", J. Catal. 134, 654-667 (1992).

[37] Kock, A. J. H. M., de Bokx, P. K., Boellaard, E., Klop, W. and Geus, J. W., "The formation of filamentous carbon on iron and nickel catalysts II. Mechanism", J. Catal. 96, 468-480 (1985).

[38] Eliason, S. A. and Bartholomew, C. H., "Reaction and deactivation kinetics for Fischer-Tropsch synthesis on unpromoted and potassium-promoted iron catalysts", Appl. Catal. A Gen. 186, 229-243 (1999).

[39] Schneider, A. and Inden, G., "Carbon diffusion in cementite ( $\left.\mathrm{Fe}_{3} \mathrm{C}\right)$ and $\mathrm{Hägg}$ carbide ( $\left.\mathrm{Fe}_{5} \mathrm{C}_{2}\right) "$, Calphad 31, 141-147 (2007).

[40] Moszyński, D., Grabke, H. J. and Schneider, A., "Effect of sulphur on the formation of graphite at the surface of carburized iron", Surf. Interface Anal. 34, 380-383 (2002).

[41] Olsson, R. G. and Turkdogan, E. T., "Catalytic effect of iron on decomposition of carbon monoxide: II. Effect of additions of $\mathrm{H}_{2}, \mathrm{H}_{2} \mathrm{O}, \mathrm{CO}_{2}, \mathrm{SO}_{2}$ and $\mathrm{H}_{2} \mathrm{~S}^{\prime \prime}$, Metall. Trans. 5, 21-26 (1974).

[42] Arabczyk, W., Moszyński, D., Narkiewicz, U., Pelka, R. and Podsiadły, M., "Poisoning of iron catalyst by sulfur", Catal. Today 124, 43-48 (2007).

[43] Berry, T. F., Ames, R. N. and Snow, R. B., "Influence of Impurities and Role of Iron Carbides in Deposition of Carbon from Carbon Monoxide", J. Am. Ceram. Soc. 39, 308-318 (1956).

[44] Xu, J.-D., Chang, Z.-Y., Zhu, K.-T., Weng, X.-F., Weng, W.-Z., Zheng, Y.-P., Huang, C.-J. and Wan, H.-L., "Effect of sulfur on $\alpha-\mathrm{Al}_{2} \mathrm{O}_{3}$-supported iron catalyst for Fischer-Tropsch synthesis", Appl. Catal. A Gen. 514, 103-113 (2016). 
Combined In-situ X-Ray Powder Diffractometry/Raman Spectroscopy of Iron Carbide and Carbon Species Evolution in Fe(-Na-S)/ $\alpha$-Al203 Catalysts during Fischer-Tropsch Synthesis

[45] Zhou, X., Ji, J., Wang, D., Duan, X., Qian, G., Chen, D. and Zhou, X., "Hierarchical structured $\alpha$-Al2O3 supported S-promoted Fe catalysts for direct conversion of syngas to lower olefins", Chem. Commun. 51, 8853-8856 (2015).

[46] Dalla Betta, R. A., Piken, A. G. and Shelef, M., "Heterogeneous methanation: Steady-state rate of CO hydrogenation on supported ruthenium, nickel and rhenium", J. Catal. 40, 173-183 (1975).

[47] van Dijk, W. L., Niemantsverdriet, J. W., van dar Kraan, A. M. and van der Baan, H. S., "Effects of Manganese Oxide and Sulphate on the Olefin Selectivity of Iron Catalysts in the Fischer Tropsch Reaction", Appl. Catal. 2, 273-288 (1982).

[48] Madon, R. J. and Shaw, H., "Effect of Sulfur on the Fischer-Tropsch Synthesis", Catal. Rev. 15, 69-106 (1977).

[49] Herranz, T., Rojas, S., Pérez-Alonso, F. J., Ojeda, M., Terreros, P. and Fierro, J. L. G., "Genesis of iron carbides and their role in the synthesis of hydrocarbons from synthesis gas", J. Catal. 243, 199-211 (2006).

[50] Xu, J. and Bartholomew, C. H., "Temperature-Programmed Hydrogenation (TPH) and in Situ Mössbauer Spectroscopy Studies of Carbonaceous Species on Silica-Supported Iron Fischer-Tropsch Catalysts", J. Phys. Chem. B 109, 2392-2403 (2005).

[51] Hamilton, N. G., Warringham, R., Silverwood, I. P., Kapitán, J., Hecht, L., Webb, P. B., Tooze, R. P., Zhou W., Frost, C. D., Parker, S. F. and Lennon, D., "The application of inelastic neutron scattering to investigate CO hydrogenation over an iron Fischer-Tropsch synthesis catalyst", J. Catal. 312, 221-231 (2014).

[52] Ning, W., Koizumi, N., Chang, H., Mochizuki, T., Itoh, T. and Yamada, M., "Phase transformation of unpromoted and promoted Fe catalysts and the formation of carbonaceous compounds during Fischer-Tropsch synthesis reaction", Appl. Catal. A Gen. 312, 35-44 (2006).

[53] Nolan, P. E., Lynch, D. C. and Cutler, A. H., "Carbon Deposition and Hydrocarbon Formation on Group VIII Metal Catalysts", J. Phys. Chem. B 102, 4165-4175 (1998).

[54] Boellaard, E., de Bokx, P. K., Kock, A. J. H. M. and Geus, J. W., "The formation of filamentous carbon on iron and nickel catalysts III. Morphology", J. Catal. 96, 481-490 (1985).

[55] de Bokx, P. K., Kock, A. J. H. M., Boellaard, E., Klop, W. and Geus, J. W., "The formation of filamentous carbon on iron and nickel catalysts. I. Thermodynamics", J. Catal. 96, 454-467 (1985).

[56] O’Brien, R. J., Xu, L., Milburn, D. R., Li, Y.-X., Klabunde, K. J. and Davis, B. H., "Fischer-Tropsch synthesis: Impact of potassium and zirconium promoters on the activity and structure of an ultrafine iron oxide catalyst", Top. Catal. 2, 1-15 (1995).

[57] Bukur, D. B., Okabe, K., Rosynek, M. P., Li, C. P., Wang, D. J., Rao, K. R. P. M. and Huffman, G. P., "Activation Studies with a Precipitated Iron Catalyst for Fischer-Tropsch Synthesis. I. Characterization Studies", Journal of Catalysis 155, 353-365 (1995).

[58] Datye, A. K., Jin, Y., Mansker, L. D., Motjope, R. T., Dlamini, T. H. and Coville, N. J., "The nature of the active phase in iron Fischer-Tropsch catalysts" in Studies in Surface Science and Catalysis 130,11391144 (2000).

[59] Mansker, L. D., Jin, Y., Bukur, D. B. and Datye, A. K., "Characterization of slurry phase iron catalysts for Fischer-Tropsch synthesis", Appl. Catal. A Gen. 186, 277-296 (1999).

[60] McCartney, J. T., Hofer, L. J. E., Seligman, B., Lecky, J. A., Peebles, W. C. and Anderson, R. B., "Electron and X-Ray Diffraction Studies of Iron Fischer-Tropsch Catalysts", J. Phys. Chem. 57, 730-736 (1953).

[61] Wang, Y., Alsmeyer, D. C. and McCreery, R. L., "Raman spectroscopy of carbon materials: structural basis of observed spectra", Chem. Mater. 2, 557-563 (1990).

[62] Wang, Y., Serrano, S. and Santiago-Avilés, J. J., "Raman characterization of carbon nanofibers prepared using electrospinning", 138, 423-427 (2003). 
[63] Shimodaira, N. and Masui, A., "Raman spectroscopic investigations of activated carbon materials", J. Appl. Phys. 92, 902-909 (2002).

[64] Ferrari, A. C. and Robertson, J., "Interpretation of Raman spectra of disordered and amorphous carbon", Phys. Rev. B 61, 14095-14107 (2000).

[65] Escribano, R., Sloan, J. J., Siddique, N., Sze, N. and Dudev, T., "Raman spectroscopy of carboncontaining particles", Vib. Spectrosc. 26, 179-186 (2001).

[66] Sattler, J. J. H. B., Beale, A. M. and Weckhuysen, B. M., "Operando Raman Spectroscopy Study on the Deactivation of $\mathrm{Pt} / \mathrm{Al}_{2} \mathrm{O}_{3}$ and $\mathrm{Pt}-\mathrm{Sn} / \mathrm{Al}_{2} \mathrm{O}_{3}$ Propane Dehydrogenation Catalysts", Phys. Chem. Chem. Phys. 15, 12095 (2013).

[67] Sattler, J. J. H. B., Mens, A. M. and Weckhuysen, B. M., "Real-Time Quantitative Operando Raman Spectroscopy of a $\mathrm{CrO}_{x} / \mathrm{Al}_{2} \mathrm{O}_{3}$ Propane Dehydrogenation Catalyst in a Pilot-Scale Reactor", ChemCatChem 6, 3139-3145 (2014).

[68] Cats, K. H. and Weckhuysen, B. M., "Combined Operando X-ray Diffraction/Raman Spectroscopy of Catalytic Solids in the Laboratory: The Co/TiO 2 Fischer-Tropsch Synthesis Catalyst Showcase", ChemCatChem 8, 1531-1542 (2016).

[69] Fischer, N. and Claeys, M., "Phase changes studied under in situ conditions - A novel cell", Catal. Today 275, 149-154 (2016).

[70] Centre for Catalysis Research - University of Cape Town, "iKey in-situ XRD Cell", Available at: http://www.catcentre.uct.ac.za/cat/ikey-products/xrd-cell (Accessed: March 2020).

[71] Bare, S. R., Yang, N., Kelly, S. D., Mickelson, G. E. and Modica, F. S., "Design and operation of a high pressure reaction cell for in situ X-ray absorption spectroscopy", Catal. Today 126, 18-26 (2007).

[72] Jenkins, R. and Snyder, R. L., "Introduction to X-ray Powder Diffractometry", Introduction to X-ray Powder Diffractometry 138 (John Wiley \& Sons, Inc., Hoboken, NJ, USA, 1996).

[73] Wood, I. G., Vočadlo, L., Knight, K. S., Dobson, D. P., Marshall, W. G., Price, G. D. and Brodholt, J., "Thermal expansion and crystal structure of cementite, $\mathrm{Fe}_{3} \mathrm{C}$, between 4 and $600 \mathrm{~K}$ determined by timeof-flight neutron powder diffraction", J. Appl. Crystallogr. 37, 82-90 (2004).

[74] Sabine, T. M., Hunter, B. A., Sabine, W. R. and Ball, C. J., "Analytical Expressions for the Transmission Factor and Peak Shift in Absorbing Cylindrical Specimens", J. Appl. Crystallogr. 31, 47-51 (1998).

[75] Michel, F. M., Ehm, L., Antao, S. M., Lee, P. L., Chupas, P. J., Liu, G., Strongin, D. R., Schoonen, M. A. A., Phillips, B. L. and Parise, J. B., "The Structure of Ferrihydrite, a Nanocrystalline Material", Science 316, 1726-1729 (2007).

[76] Toby, B. H., "R factors in Rietveld analysis: How good is good enough?", Powder Diffr. 21, 67-70 (2006).

[77] Nagakura, S., "Study of Metallic Carbides by Electron Diffraction Part III. Iron Carbides", J. Phys. Soc. Japan 14, 186-195 (1959).

[78] Amelse, J. A., Grynkewich, G., Butt, J. B. and Schwartz, L. H., "Moessbauer spectroscopic study of passivated small particles of iron and iron carbide", J. Phys. Chem. 85, 2484-2488 (1981).

[79] Jiang, F., Zhang, M., Liu, B., Xu, Y. and Liu, X., "Insights into the influence of support and potassium or sulfur promoter on iron-based Fischer-Tropsch synthesis: understanding the control of catalytic activity, selectivity to lower olefins, and catalyst deactivation", Catal. Sci. Technol. 7, 1245-1265 (2017).

[80] Raupp, G. B. and Delgass, W. N., "Mössbauer Investigation of Supported Fe and FeNi Catalysts II. Carbides Formed by Fischer-Tropsch Synthesis", J. Catal. 58, 348-360 (1979).

[81] Tau, L. M., Borcar, S., Bianchi, D. and Bennett, C. O., "Investigation of supported iron Fischer-Tropsch synthesis catalysts by Mössbauer spectroscopy", J. Catal. 87, 36-54 (1984). 
Combined In-situ X-Ray Powder Diffractometry/Raman Spectroscopy of Iron Carbide and Carbon Species Evolution in Fe(-Na-S)/ $\alpha$-Al203 Catalysts during Fischer-Tropsch Synthesis

[82] du Plessis, H. E., de Villiers, J. P. R., Kruger, G. J., Steuwer, A. and Brunelli, M., "Rietveld and pair distribution function study of Hägg carbide using synchrotron X-ray diffraction", J. Synchrotron Radiat. 18, 266-271 (2011).

[83] Hofer, L. J. E., "Nature of the carbides of iron", U.S. Bureau of Mines Bulletin 631 (1966). Available at: http://www.onemine.org/document/abstract.cfm?docid=186163\&title=Nature-Of-The-CarbidesOf-Iron (Accessed: February 2014).

[84] Riedel, T., Schulz, H., Schaub, G., Jun, K.-W., Hwang, J.-S. and Lee, K.-W., "Fischer-Tropsch on Iron with $\mathrm{H}_{2} / \mathrm{CO}$ and $\mathrm{H}_{2} / \mathrm{CO}_{2}$ as Synthesis Gases: The Episodes of Formation of the Fischer-Tropsch Regime and Construction of the Catalyst", Top. Catal. 26, 41-54 (2003).

[85] Berry, F. J. and Smith, M. R., "A Mössbauer investigation of iron-containing catalysts prepared at low temperatures and active for carbon monoxide hydrogenation", J. Chem. Soc. Faraday Trans. 1 Phys. Chem. Condens. Phases 85, 467-477 (1989).

[86] Ribeiro, M. C., Jacobs, G., Davis, B. H., Cronauer, D. C., Kropf, A. J. and Marshall, C. L., "Fischer-Tropsch Synthesis: An In-Situ TPR-EXAFS/XANES Investigation of the Influence of Group I Alkali Promoters on the Local Atomic and Electronic Structure of Carburized Iron/Silica Catalysts", J. Phys. Chem. C 114, 7895-7903 (2010).

[87] Manes, M., Damick, A. D., Mentser, M., Cohn, E. M. and Hofer, L. J. E., "Hexagonal Iron Carbide as an Intermediate in the Carbiding of Iron Fischer-Tropsch Catalysts", J. Am. Chem. Soc. 74, 6207-6209 (1952).

[88] Boellaard, E., van der Kraan, A. M. and Geus, J. W., "Behaviour of a cyanide-derived $\mathrm{Fe} / \mathrm{Al}_{2} \mathrm{O}_{3}$ catalyst during Fischer-Tropsch synthesis", Appl. Catal. A Gen. 147, 229-245 (1996).

[89] Pijolat, M., Perrichon, V. and Bussière, P., "Study of the carburization of an iron catalyst during the Fischer-Tropsch synthesis: Influence on its catalytic activity", J. Catal. 107, 82-91 (1987).

[90] Butt, J. B., "Carbide phases on iron-based Fischer-Tropsch synthesis catalysts part II: Some reaction studies", Catal. Lett. 7, 83-106 (1990).

[91] Pendyala, V. R. R., Graham, U. M., Jacobs, G., Hamdeh, H. H. and Davis, B. H., "Fischer-Tropsch Synthesis: Deactivation as a Function of Potassium Promoter Loading for Precipitated Iron Catalyst", Catal. Lett. 144, 1704-1716 (2014).

[92] An, X., Wu, B., Wan, H.-J., Li, T.-Z., Tao, Z.-C., Xiang, H.-W. and Li, Y.-W., "Comparative study of ironbased Fischer-Tropsch synthesis catalyst promoted with potassium or sodium", Catal. Commun. 8, 1957-1962 (2007).

[93] Shultz, J. F., Abelson, M., Shaw, L. and Anderson, R. B., "Fischer-Tropsch Synthesis. Nitrides and Carbonitrides of Iron as Catalysts", Ind. Eng. Chem. 49, 2055-2060 (1957).

[94] Niewa, R., Rau, D., Wosylus, A., Meier, K., Hanfland, M., Wessel, M., Dronskowski, R., Dzivenko, D. A., Riedel, R. and Schwarz, U., "High-Pressure, High-Temperature Single-Crystal Growth, Ab initio Electronic Structure Calculations, and Equation of State of $\varepsilon-F e_{3} N_{1+x} "$, Chem. Mater. 21, 392-398 (2009).

[95] Leineweber, A., Shang, S., Liu, Z.-K., Widenmeyer, M. and Niewa, R., "Crystal structure determination of Hägg carbide, $\chi-\mathrm{Fe}_{5} \mathrm{C}_{2}$ by first-principles calculations and Rietveld refinement", Zeitschrift für Krist. 227, 207-220 (2012).

[96] Jack, K. H., "The iron-nitrogen system: the crystal structures of $\varepsilon$-phase iron nitrides", Acta Crystallogr. 5, 404-411 (1952).

[97] Rechenbach, D. and Jacobs, H., "Structure determination of $\zeta-\mathrm{Fe}_{2} \mathrm{~N}$ by neutron and synchrotron powder diffraction", J. Alloys Compd. 235, 15-22 (1996).

[98] Jacobs, H., Rechenbach, D. and Zachwieja, U., "Structure determination of $\gamma^{\prime}-\mathrm{Fe}_{4} \mathrm{~N}$ and $\epsilon-\mathrm{Fe}_{3} \mathrm{~N}^{\text {", J. Alloys }}$ Compd. 227, 10-17 (1995). 
[99] Nagakura, S. and Tanehashi, K., "Electronic Structure of Iron Nitrides Studied by Electron Diffraction. II. $\varepsilon-\mathrm{Fe}_{2} \mathrm{~N}$ and $\zeta-\mathrm{Fe}_{2} \mathrm{~N}^{\prime \prime}$, J. Phys. Soc. Japan 25, 840-846 (1968).

[100] O’Brien, R. J., Xu, L., Spicer, R. L. and Davis, B. H., "Activation Study of Precipitated Iron Fischer-Tropsch Catalysts", Energy \& Fuels 10, 921-926 (1996).

[101] Shultz, J. F., Abelson, M., Stein, K. C. and Anderson, R. B., "Studies of the Fischer-Tropsch Synthesis. XVIII. Influence of Catalyst Geometry on Synthesis on Iron Catalysts", J. Phys. Chem. 63, 496-500 (1959).

[102] Anderson, R. B., Hofer, L. J. E., Cohn, E. M. and Seligman, B., "Studies of the Fischer-Tropsch Synthesis. IX. Phase Changes of Iron Catalysts in the Synthesis", J. Am. Chem. Soc. 73, 944-946 (1951).

[103] Matyi, R. J., Schwartz, L. H. and Butt, J. B., "Particle Size, Particle Size Distribution, and Related Measurements of Supported Metal Catalysts", Catal. Rev. 29, 41-99 (1987).

[104] Sadezky, A., Muckenhuber, H., Grothe, H., Niessner, R. and Pöschl, U., "Raman Microspectroscopy of Soot and Related Carbonaceous Materials: Spectral Analysis and Structural Information", Carbon 43, 1731-1742 (2005).

[105] Ratnasamy, C. and Wagner, J. P., "Water Gas Shift Catalysis", Catal. Rev. 51, 325-440 (2009).

[106] Podgurski, H. H., Kummer, J. T., DeWitt, T. W. and Emmett, P. H., "Preparation, Stability and Adsorptive Properties of the Carbides of Iron", J. Am. Chem. Soc. 72, 5382-5388 (1950).

[107] Sancier, K. M., Isakson, W. E. and Wise, H., "Carburization Studies of Iron Fischer-Tropsch Catalysts" in Hydrocarbon Synthesis from Carbon Monoxide and Hydrogen (eds. Kugler, E. L. \& Steffgen, F. W.) 178, 129-145 (American Chemical Society, Washington, 1979).

[108] Edwards, H. G. M. and Falk, M. J. P., "Fourier-transform Raman spectroscopic study of unsaturated and saturated waxes", Spectrochim. Acta Part A Mol. Biomol. Spectrosc. 53, 2685-2694 (1997).

[109] Nolan, P. E., Lynch, D. C. and Cutler, A. H., "Catalytic disproportionation of CO in the absence of hydrogen: Encapsulating shell carbon formation", Carbon 32, 477-483 (1994).

[110] Alstrup, I., Tavares, M. T., Bernardo, C. A., Sørensen, O. and Rostrup-Nielsen, J. R., "Carbon formation on nickel and nickel-copper alloy catalysts", Mater. Corros. 49, 367-372 (1998). 
This page is intentionally left blank 


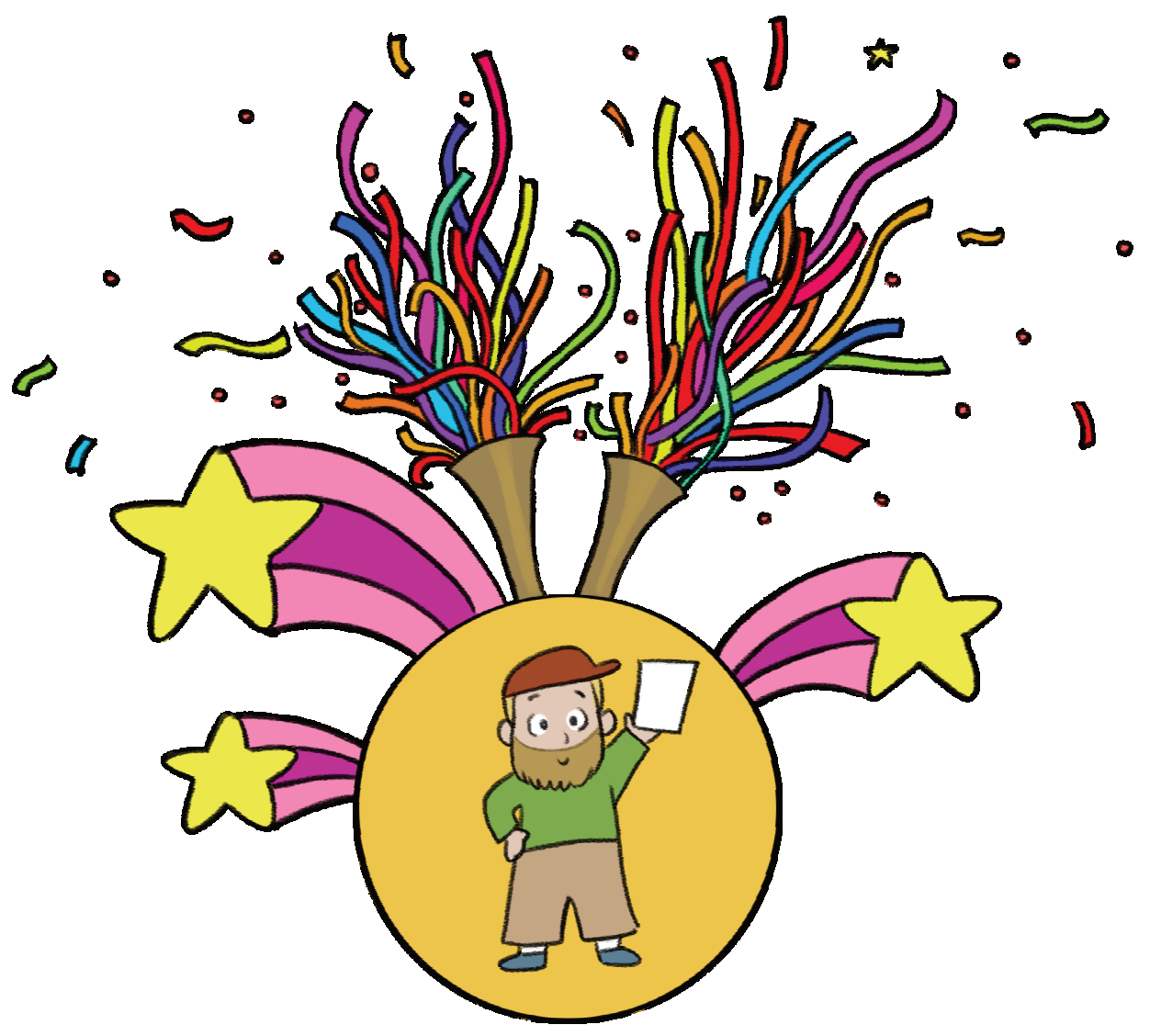




\section{Chapter 7}

\section{Summary and Future Perspectives}

Currently, hydrocarbons are used in the vast majority of modern industrial processes leading to the production of transportation fuels, plastics, coatings and other materials. In the foreseeable future the current primary source for these hydrocarbons, namely crude oil from Earth's crust, will be depleted and new alternatives as a source for hydrocarbons will be required. The industrial FischerTropsch Process (FTP) is capable of converting a carbon-containing feedstock, such as natural gas, coal, biomass or municipal waste, into a variety of product hydrocarbons. The heterogeneously catalyzed Fischer-Tropsch Synthesis (FTS) reaction is employed in the FTP for converting a mixture of carbon monoxide (CO) and hydrogen $\left(\mathrm{H}_{2}\right)$, better known as synthesis gas or syngas, into synthetic crude, which is further refined to the hydrocarbon products. In this $\mathrm{PhD}$ Dissertation, we have studied the FTS reaction using iron (Fe)-based, sodium (Na)-sulphur (S) promoted or unpromoted supported $\mathrm{Fe}(-\mathrm{Na}-\mathrm{S}) / \alpha-\mathrm{Al}_{2} \mathrm{O}_{3}$ catalyst materials. The main focus of this Dissertation has been on the characterization of the active phases of the catalyst materials catalyzing the FTS reaction, namely the in-depth characterization of Fe carbides. X-ray Diffraction (XRD) was applied as the primary characterization technique and the collected data were quantified with Rietveld Quantitative Phase Analysis (R-QPA). Fe carbide identification, formation conditions and their subtypes, as defined by carbide's lattice parameters, were investigated. This PhD Dissertation defines the effects that the promotion of $\mathrm{Na}$ and $\mathrm{S}$ has on the Fe carbide formation and the carbides properties in the FTS reaction. 


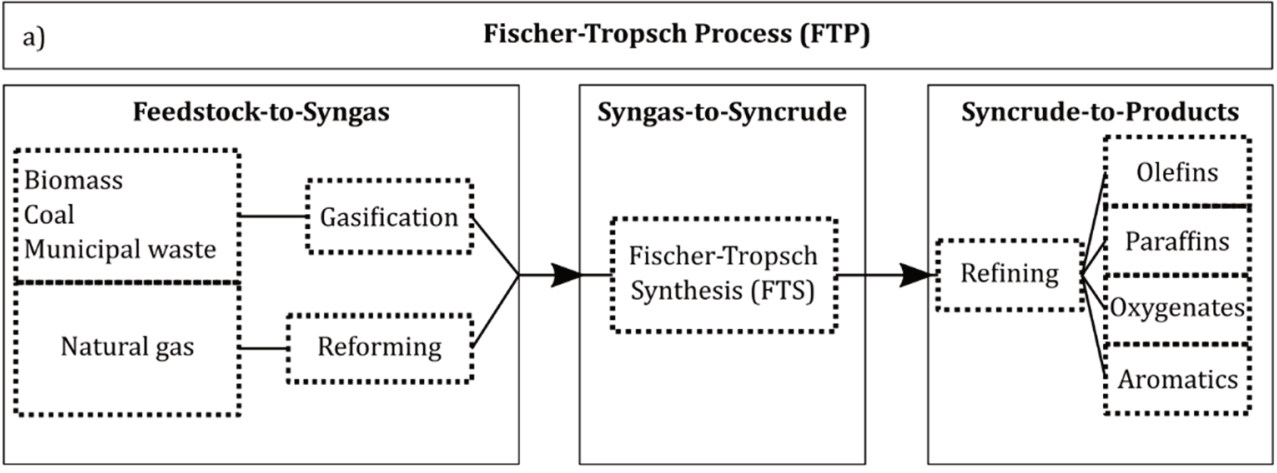

b)

\begin{tabular}{|cccc|}
\hline Feedstock & \multicolumn{2}{c}{$\mathrm{H}: \mathrm{C}$} & $\mathrm{CO}_{2}$ footprint \\
& \multicolumn{2}{c}{ (elemental ratio) } & $\mathrm{CO}_{2}: \mathrm{HC}$ ) \\
\cline { 2 - 4 } & Nominal & Effective & \\
\hline Natural gas & 4.00 & 4.00 & 0.00 \\
Waste & 1.83 & 0.65 & 3.96 \\
Coal & 0.80 & 0.40 & 4.80 \\
Biomass & 1.45 & 0.12 & 5.75 \\
\hline
\end{tabular}

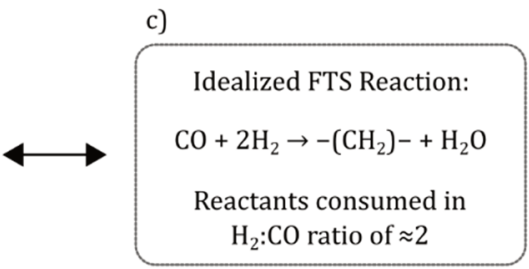

\section{Figure 7.1}

Overview of the overall Fischer-Tropsch Process (FTP) applying the Fischer-Tropsch Synthesis (FTS) reaction in the industrial process of converting carbon-containing feedstock into refined hydrocarbon products. The general FTP steps are a) Feedstock-to-Syngas, Syngas-to-Syncrude and Syncrude-to-Products. The input feedstock b) largely defines the $\mathrm{CO}_{2}$ footprint of the overall FTP, as the c) FTS reaction consumes the reaction reactant syngas $\mathrm{H}_{2}: \mathrm{CO}$ in ratio of $\approx 2$ in the Syngas-to-Syncrude FTP step. With feedstocks other than natural gas, the effective syngas $\mathrm{H}_{2}$ : $\mathrm{CO}$ ratio is $<2$ and $\mathrm{H}_{2}$ needs to be produced and added to the FTP, raising the total $\mathrm{CO}_{2}$ footprint. Waste $=$ Municipal waste, Coal $=$ Average of different coal types, Biomass $=$ Switchgrass, $\mathrm{HC}=$ hydrocarbons.

\subsection{SUMMARY}

Despite that the catalytic conversion of a mixture of $\mathrm{H}_{2}$ and $\mathrm{CO}$, also known as synthesis gas or syngas, into hydrocarbons is already known for more than 100 years, a lot remains to be learned. This catalytic reaction is called the Fischer-Tropsch Synthesis (FTS). At the heart of the FTS reaction with Iron (Fe)-based catalyst materials are the Fe carbides, which are considered to be the active phases in this reaction. The main motivation for conducting the research work in this PhD Dissertation has been to learn more on the behavior and formation of the different Fe carbide phases and how they are affected by adding Sodium (Na) and Sulphur (S) as chemical promoters. In the following, the work conducted within this PhD Dissertation is summarized, highlighting the main conclusions from this research work. 


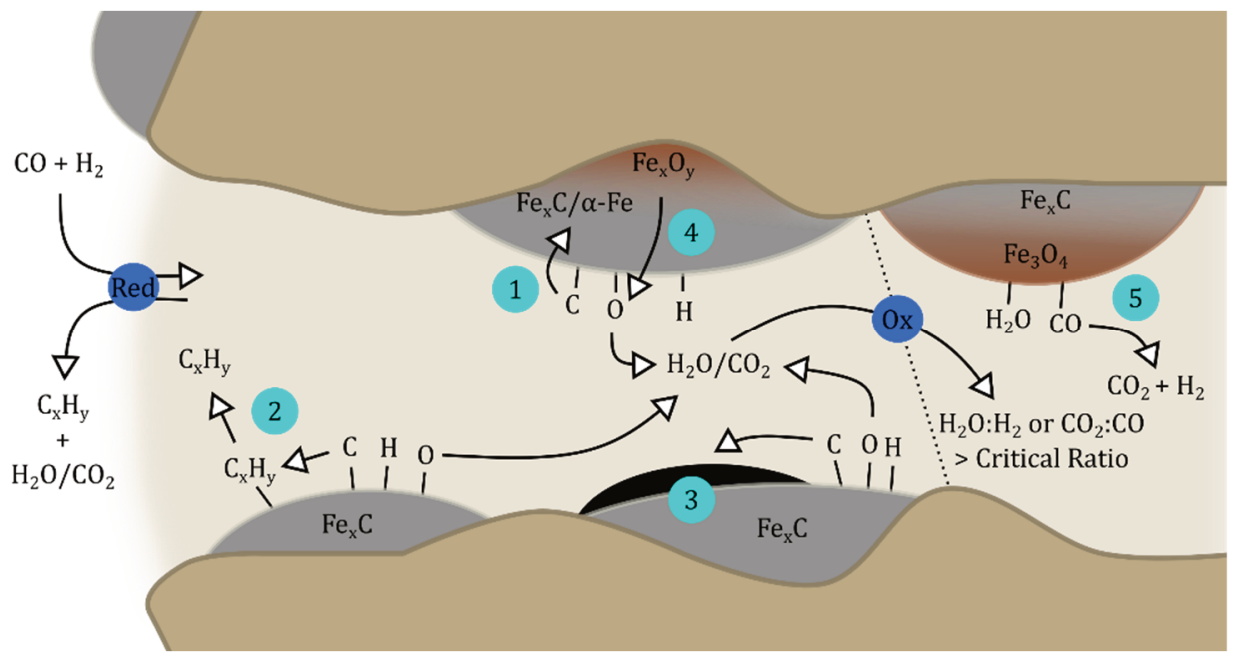

\section{Figure 7.2}

A simplified summary of the reaction pathways for $\mathrm{C}$ from input $\mathrm{CO}$ in a Fe-based Fischer-Tropsch Synthesis (FTS) catalyst particle. The input $\mathrm{CO}+\mathrm{H}_{2}$ gives originally a reductive environment (Red). For $C$ from $\mathrm{CO}$ dissociation the pathways to react are: (1) with $\alpha$-Fe to form $\mathrm{Fe}_{\mathrm{x}} \mathrm{C}$, (2) with $\mathrm{H}$ to form hydrocarbons, (3) with $\mathrm{C}$ to form inactive carbon. Without $\mathrm{CO}$ dissociation: The $\mathrm{C}$ pathway (4) may reduce $\mathrm{Fe}_{\mathrm{x}} \mathrm{O}_{\mathrm{y}}$ to $\alpha$-Fe, while releasing $\mathrm{CO}_{2}$. Removal of $\mathrm{O}$ atoms from $\mathrm{CO}$ as $\mathrm{H}_{2} \mathrm{O}$ or $\mathrm{CO}_{2}$ gradually leads to an oxidative environment $(\mathrm{Ox})$ towards the core of the catalyst particle, and the outlet of a fixed bed reactor. If the FTS active $\mathrm{Fe}_{\mathrm{x}} \mathrm{C}$ phase is oxidized to $\mathrm{Fe}_{3} \mathrm{O}_{4}$, pathway (5) may convert $\mathrm{H}_{2} \mathrm{O}+\mathrm{CO}$ to $\mathrm{CO}_{2}+\mathrm{H}_{2}$ via the Water-gas Shift (WGS) reaction.

Chapter 1 - The history and motivations on the application of the overall industrial Fischer-Tropsch Process (FTP) was briefly introduced. In the FTP, a carbon-containing input feedstock is converted into refined hydrocarbon products. The FTP steps discussed were (Figure 7.1): Feedstock-to-Syngas, Syngas-to-Syncrude and Syncrude-toProducts. The catalyzed FTS reaction itself is employed in the Syngas-to-Syncrude process step for converting syngas into a mixture of various hydrocarbons and their derivatives (syncrude).

The $\mathrm{CO}_{2}$ footprint of the overall FTP depends in a large part on the input feedstock. The $\mathrm{CO}_{2}$ footprint increases in the following order: natural gas $<$ communal waste $<$ coal $<$ biomass. Due to its lack of impurities, natural gas can be directly converted into readyto-use syngas with $\mathrm{H}_{2}$ : $\mathrm{CO}$ ratio $\approx 2$, satisfying the required FTS reaction stoichiometry. With the other feedstocks, which contain heteroatoms (mainly Nitrogen $(\mathrm{N})$, Oxygen (O) and Sulphur (S)) as impurities, the effective attainable $\mathrm{H}_{2}$ : $\mathrm{CO}$ ratio is $<2$. Therefore, cheap and environmentally sourced $\mathrm{H}_{2}$ needs to be added to the FTP for these feedstocks to be able to compete with the natural gas-based FTP environmentally. The used FTP feedstock is also important from an economic perspective. Producing the purified syngas for use in the FTS reaction takes $\approx 0-70 \%$ of the total capital costs in a 
coal-based FTP, while the corresponding costs for natural gas-based FTP are $\approx 30-50 \%$.

Chapter 2 - The heterogeneous catalysis of the FTS reaction with Fe-based catalyst materials was reviewed. Topics, such as the common catalyst compositions, the various chemical reactions taking place and the possible reaction pathways for $\mathrm{C}$ from input $\mathrm{CO}$ in a Fe-based FTS reaction catalyst, were discussed. The $\mathrm{C}$ possible pathways in the FTS reaction are summarized in Figure 7.2. Furthermore, the effects of the various FTS reaction condition parameters on the hydrocarbon and Carbon (C) pathway selectivities were reviewed. The effects of parameters, such as the reaction temperature, input $\mathrm{H}_{2}$ : $\mathrm{CO}$ ratio, gas space velocity and reaction total pressure, were discussed. As the specific topics of this PhD Dissertation: the effects arising from the added chemical Na-S promotion in the Fe-based FTS reaction and the identification of the Fe carbide phases, encountered in the field of FTS catalysis, were discussed.

The Na-S promotion in the FTS reaction has the effects of i) decreasing the selectivity towards methane $\left(\mathrm{CH}_{4}\right)$ to a lower value than predicted by the Anderson-Schulz-Flory (ASF) distribution, ii) a lesser increase in the hydrocarbon chain length (i.e. $\alpha$-value) in comparison if only alkaline metal (i.e., $\mathrm{Na}$ ) is added, iii) an increased selectivity towards olefins, and iv) an overall increasing CO conversion. These selectivity effects resemble alkaline metals (e.g., $\mathrm{K}$ or $\mathrm{Na}$ ) only promotion effects in the FTS reaction. However, the unique feature of the promotion by $\mathrm{Na}$ and $\mathrm{S}$ is its ability to decrease the $\mathrm{CH}_{4}$ selectivity to a value lower than predicted by the ASF-distribution. This special feature is not attainable without the added S.

The Fe carbides, observable in the field of FTS catalysis, with their corresponding crystal structures are:

- hexagonal $\varepsilon-\mathrm{Fe}_{3} \mathrm{C} \mathrm{P}_{3} 22$ (182),

- orthorhombic $\eta-\mathrm{Fe}_{2} \mathrm{C}$ Pnnm (58),

- monoclinic $\chi-\mathrm{Fe}_{5} \mathrm{C}_{2} \mathrm{C} 2 / c$ (15) (the Hägg carbide),

- orthorhombic $\theta-\mathrm{Fe}_{3} \mathrm{C}$ Pnma (62) (cementite), and

- orthorhombic $\theta-\mathrm{Fe}_{7} \mathrm{C}_{3}$ Pnma (62) (the Eckström-Adcock carbide).

The formation of the $\mathrm{Fe}$ carbides is dependent on the temperature where the zerovalent $\alpha-\mathrm{Fe}$ is exposed to a $\mathrm{C}$ atom source. The expected thermal stability and formation temperature ranges for the $\mathrm{Fe}$ carbides are $\varepsilon-\mathrm{Fe}_{3} \mathrm{C}\left(100-265^{\circ} \mathrm{C}\right)<\eta-\mathrm{Fe}_{2} \mathrm{C}$ $\left(160-340^{\circ} \mathrm{C}\right)<\chi-\mathrm{Fe}_{5} \mathrm{C}_{2}\left(240-350^{\circ} \mathrm{C}\right)<\theta-\mathrm{Fe}_{3} \mathrm{C}\left(310-600^{\circ} \mathrm{C}\right)$ as based on the literature data.

The formation of the $\theta-\mathrm{Fe}_{7} \mathrm{C}_{3}$ phase does not follow the same route as for the rest of the Fe carbides. Although, despite rather extreme reaction conditions have often been cited as requirements for the formation of the $\theta-\mathrm{Fe}_{7} \mathrm{C}_{3}$ phase, it may also form under 


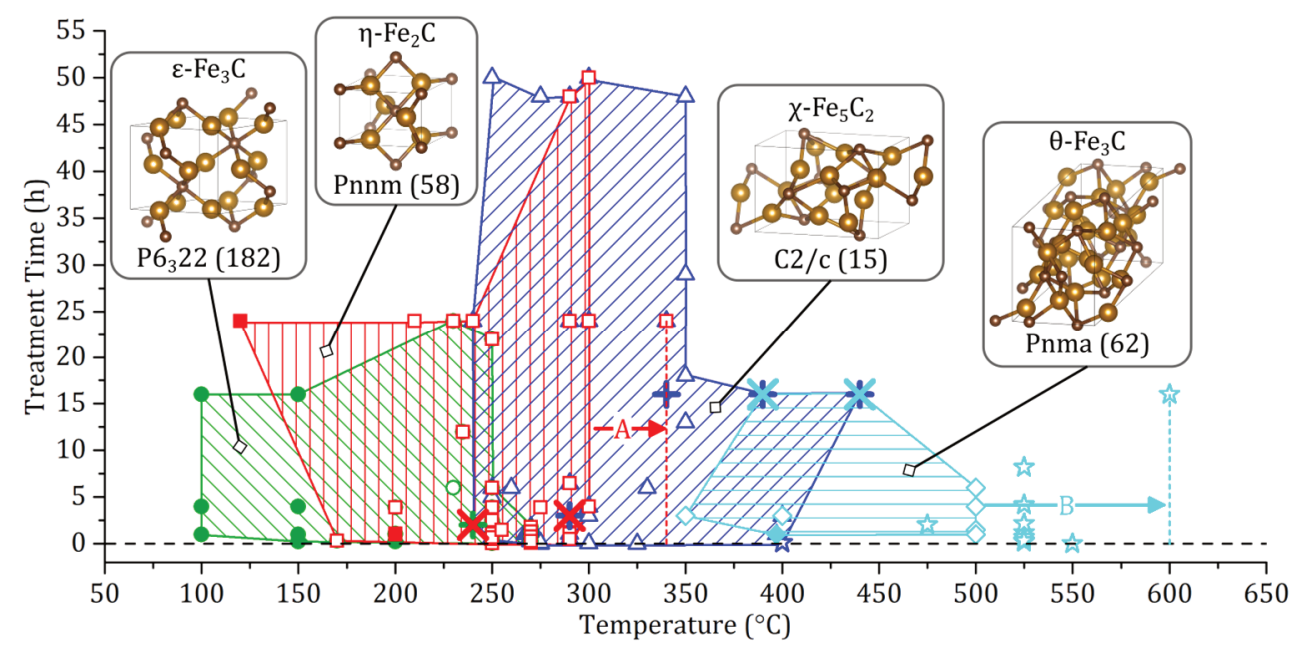

Figure 7.3

Fe carbide formation regions (hatched fill) as temperature versus treatment exposure time as based on the available experimental literature data (non-cross) and this PhD Dissertation (cross). Arrows: Maximum thermal stability with preformed $\mathrm{Fe}$ carbides for $(\mathrm{A}) \eta-\mathrm{Fe}_{2} \mathrm{C}$ and $(\mathrm{B}) \theta-\mathrm{Fe}_{3} \mathrm{C}$. The $\theta-\mathrm{Fe}_{7} \mathrm{C}_{3}$ Pnma (62) formation temperatures are excluded as its formation follows a different, not solely temperature dependent, route. Each of the Fe carbides were assigned with their precise crystal structures, on the basis of literature.

relatively mild FTS reaction conditions. Observations of the $\theta-\mathrm{Fe}_{7} \mathrm{C}_{3}$ phase forming at $\approx 340^{\circ} \mathrm{C}$ under mild reaction conditions have been made in the literature. It was speculated that the $\theta-\mathrm{Fe}_{7} \mathrm{C}_{3}$ phase formation could be related to the presence of $\mathrm{CO}_{2}$ under Fe carburizing conditions.

Chapter 3 - In this first experimental research Chapter of this PhD Dissertation, the identification and formation temperatures of the Fe carbides in the field of FTS catalysis were experimentally probed. Especially, the effect of the Na-S promotion on the $\mathrm{Fe}$ carbide formation process was studied. The study was performed by carburizing $\mathrm{H}_{2}$ pre-reduced supported $\mathrm{Fe}(-\mathrm{Na}-\mathrm{S}) / \alpha-\mathrm{Al}_{2} \mathrm{O}_{3}$ catalyst materials, with and without the added Na-S promotion, with $\mathrm{CO}$ within carburization temperatures of $240-440^{\circ} \mathrm{C}$. In this way prepared Fe carbides were then characterized by using ex-situ X-ray Diffraction (XRD) with Rietveld Quantitative Phase Analysis (R-QPA). The obtained RQPA results were compared to the corresponding data, obtained with ex-situ Mössbauer Absorption Spectroscopy (MAS), on the same, carburized, catalyst materials.

With this characterization methods at hand, it was possible to confirm the identification of the Fe carbides, their crystal structures and the unified nomenclature for the XRD and MAS techniques, as assigned in Chapter 2. Relative C atom contents, non-equivalent Fe position ratios and their corresponding MAS hyperfine fields were 
assigned for the Fe carbides from the experimental data.

Together with the obtained experimental results and literature, the Fe carbide thermal stability ranges were refined to be at $5 \mathrm{~h}$ treatment time (Figure 7.3):

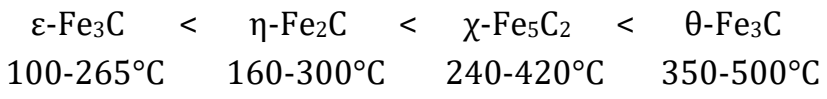

The $\eta-\mathrm{Fe}_{2} \mathrm{C}$ phase may, however, be stable up to $340^{\circ} \mathrm{C}$ in case if it is first formed at a lower temperature $\left(\$ 300^{\circ} \mathrm{C}\right)$ and after that it has been exposed to a higher temperature. This was explained by comparing the experimental results to the literature observations. The $\theta-\mathrm{Fe}_{3} \mathrm{C}$ phase decomposition into $\mathrm{C}$ and $\alpha$-Fe may require a temperature as high as $600^{\circ} \mathrm{C}$ if the carbide phase is covered by an oxide shell, as was also explained on the basis of literature observations.

Some amounts of the $\theta-\mathrm{Fe}_{7} \mathrm{C}_{3}$ phase were formed if a $\mathrm{Fe}(-\mathrm{Na}-\mathrm{S}) / \alpha-\mathrm{Al}_{2} \mathrm{O}_{3}$ catalyst material - with or without Na-S promotion - was carburized at 25 bar CO pressure at $340^{\circ} \mathrm{C}$, directly after a catalyst calcining step without first reducing it from $\alpha-\mathrm{Fe}_{2} \mathrm{O}_{3}$ to $\alpha$-Fe. In addition, with direct carburization of the $\alpha-\mathrm{Fe}_{2} \mathrm{O}_{3}$ phase, a distinctively distorted form of the $\chi-\mathrm{Fe}_{5} \mathrm{C}_{2}$ phase was formed. Its distorted crystal lattice parameters deviated clearly from the $\chi-\mathrm{Fe}_{5} \mathrm{C}_{2}$ phase formed from the $\mathrm{H}_{2}$ pre-reduced catalysts with $\alpha$-Fe as the carburization precursor.

The Na-S promotion did not affect the formation of the Fe carbides from the $\alpha$-Fe phase in the $\mathrm{H}_{2}$ pre-reduced catalyst materials. However, as explained with comparisons to the literature, alkaline promoters increase the reducibility of Fe oxides by $\mathrm{CO}$ at low reduction temperatures $\left(\approx 270^{\circ} \mathrm{C}\right)$. Thus, the presence of alkaline metal increases the availability of the $\alpha$-Fe phase and its subsequent carburization by $\mathrm{CO}$ with Fe oxide carburization starting materials. This alkaline effect accounts for the occasionally observed increase in the Fe-based catalysts' carburization levels in literature, when the carburization process is started with a Fe oxide.

Some Na-S promotion effects in the $\mathrm{CO}$ carburization of the reduced $\alpha-\mathrm{Fe} / \alpha-\mathrm{Al}_{2} \mathrm{O}_{3}$ catalyst materials were also observed. The observations are: i) increase in Fe oxidation, presumably by $\mathrm{O}$ from the $\mathrm{CO}$ dissociation acting as oxidation agent, with short, low temperature CO carburization treatments $\left(\leq 290^{\circ} \mathrm{C}, \leq 3 \mathrm{~h}\right)$, ii) decreased crystallite diameter of the $\theta-\mathrm{Fe}_{3} \mathrm{C}$ phase formed after $\mathrm{CO}$ treatment at $440^{\circ} \mathrm{C}$, and iii) increased rate of carbon deposition from $\mathrm{CO}$ at $\geq 340^{\circ} \mathrm{C}$ via the Boudouard reaction. However, the increased carbon deposition rate diminished at $390^{\circ} \mathrm{C}$ and disappeared when reaching the $\mathrm{CO}$ carburization temperature of $440^{\circ} \mathrm{C}$, in comparison to the carbon deposition on the respective unpromoted $\mathrm{Fe} / \alpha-\mathrm{Al}_{2} \mathrm{O}_{3}$ catalyst materials.

Chapter 4 - The effect of added Na-S promotion on the performance and stability of 
the various Fe carbides in the FTS reaction was studied. The supported Fe(-Na-S)/ $\alpha-$ $\mathrm{Al}_{2} \mathrm{O}_{3}$ catalyst materials, prepared in Chapter 3, with preformed Fe carbides or only $\mathrm{H}_{2}$ reduced catalysts, with or without Na-S promotion, were used for this purpose. The catalyst materials were tested in a $72 \mathrm{~h}$ FTS reaction $\left(340^{\circ} \mathrm{C}, 10\right.$ bar $\mathrm{H}_{2}: \mathrm{CO}: \mathrm{He}$ 2.0:1.0:0.33 v/v, GHSV $\approx 5000 \mathrm{~h}^{-1}$ ) to evaluate their catalytic performances. The catalysts were characterized after the FTS reaction by using XRD with R-QPA. The obtained results were compared to the R-QPA results from Chapter 3 before the FTS reaction.

The Fe carbide phases of $\eta-\mathrm{Fe}_{2} \mathrm{C}$ Pnnm (58), $\chi-\mathrm{Fe}_{5} \mathrm{C}_{2} C 2 / c$ (15), $\theta-\mathrm{Fe}_{3} \mathrm{C}$ Pnma (62) and $\theta-\mathrm{Fe}_{7} \mathrm{C}_{3}$ Pnma (62) were observed in the catalysts. The $\varepsilon-\mathrm{Fe}_{3} \mathrm{C} P 6_{3} 22$ (182) phase was not stable in the FTS reaction and was not present in any of the studied catalyst materials after the FTS reaction. By comparing the lattice parameters of the Fe carbides, the $\eta-\mathrm{Fe}_{2} \mathrm{C}$ and $\chi-\mathrm{Fe}_{5} \mathrm{C}_{2}$ phases were further divided into carbide subtypes with their typical formation conditions as:

$\begin{array}{ll}\chi-\mathrm{Fe}_{5} \mathrm{C}_{2} & \\ \text { Type I } & \text { After CO carburization, or FTS without Na-S promotion, } \\ \text { Type II } & \text { After FTS with Na-S promotion, } \\ \text { Type III } & \begin{array}{l}\text { After CO carburization of Fe oxide }\left(\alpha-\mathrm{Fe}_{2} \mathrm{O}_{3}\right), \text { the subtyp } \\ \text { stable in FTS regardless of the Na-S promotion, }\end{array} \\ \eta-\mathrm{Fe}_{2} \mathrm{C} & \\ \begin{array}{l}\text { Type I } \\ \text { Type II }\end{array} & \begin{array}{l}\text { After CO carburization, or FTS without Na-S promotion, } \\ \text { Type III }\end{array} \\ & \text { After martensite tempering at } 120^{\circ} \mathrm{C} \text {. Not observed in FTS. }\end{array}$

Most interestingly, the distinctively distorted $\chi-\mathrm{Fe}_{5} \mathrm{C}_{2}$ phase (i.e. Type III) prevailed unchanged in the FTS reaction. No Fe carbide subtypes could be assigned for the $\theta-\mathrm{Fe}_{3} \mathrm{C}$ or $\theta-\mathrm{Fe}_{7} \mathrm{C}_{3}$ phases.

The addition of the Na-S promotion affected the Fe carbides in the FTS reaction by i) causing a maximum of $\approx 0.02 \AA$ anisotropic changes in the crystal lattice parameters for the $\eta-\mathrm{Fe}_{2} \mathrm{C}$ and $\chi-\mathrm{Fe}_{5} \mathrm{C}_{2}$ phases (i.e. subtypes Type II), ii) the $\eta-\mathrm{Fe}_{2} \mathrm{C}$ phase was stabilized, while iii) the $\theta-\mathrm{Fe}_{3} \mathrm{C}$ phase was destabilized, in comparison to the respective phases in the unpromoted catalyst materials. The stability of the $\theta-\mathrm{Fe}_{7} \mathrm{C}_{3}$ phase in the FTS reaction was not affected by the Na-S promotion (Figure 7.4).

With respect to the catalysts' FTS reaction performance, the Na-S promotion had the general effects of i) increased $\mathrm{CO}$ conversion, ii) $\approx+10 \%$ increased $\mathrm{C}_{2}-\mathrm{C}_{4}$ selectivity with significantly suppressed $\mathrm{CH}_{4}$ selectivity below the ASF expectations, iii) increased olefin content and iv) increased $\alpha$-value, in comparison to the respective unpromoted catalyst 


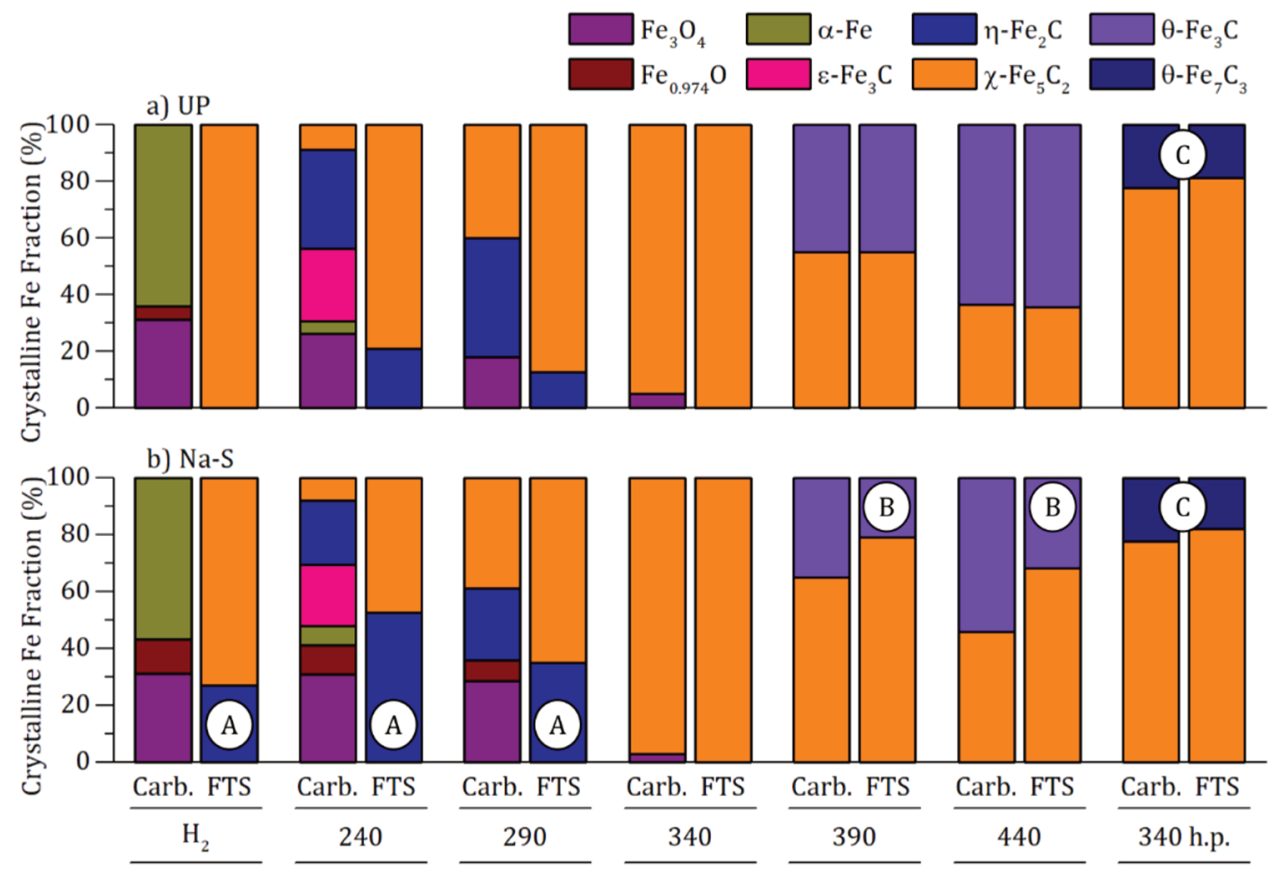

Figure 7.4

Overview of Na-S promotion dependent Fe carbide phase stabilities, as compared before (Carb.) and after 72 h Fischer-Tropsch Synthesis (FTS) reaction. Rietveld Quantitative Phase Analysis (R-QPA)-based Fe phase fractions for a) unpromoted (UP) and b) Na-S promoted (Na-S) $\mathrm{Fe}(-\mathrm{Na}-\mathrm{S}) / \alpha-\mathrm{Al}_{2} \mathrm{O}_{3}$ catalyst materials. Na-S promotion $\mathbf{A}$ stabilizes $\eta-\mathrm{Fe}_{2} \mathrm{C}$, $\mathbf{B}$ destabilizes $\theta-\mathrm{Fe}_{3} \mathrm{C}$ and $\mathbf{C}$ does not affect the stability of the $\theta-\mathrm{Fe}_{7} \mathrm{C}_{3}$ phase in the FTS reaction. FTS conditions: $340^{\circ} \mathrm{C}, 10$ bar, $\mathrm{H}_{2}: \mathrm{CO}: \mathrm{He} 2.0: 1.0: 0.33 \mathrm{v} / \mathrm{v}, \approx 5000 \mathrm{~h}^{-1}$.

materials. However, as adverse FTS reaction effects, with the Na-S promotion i) $\mathrm{CO}_{2}$ selectivity increased significantly from $\leqslant 16 \%$ to $\approx 38-43 \%$ and ii) the fraction of CO converted to FTS reaction produced hydrocarbons dropped to $\approx 0.42-0.47$ with respect to the unpromoted catalyst materials.

Furthermore, the Usage Ratios $\mathrm{H}_{2}$ :CO decreased from $\approx 1.8-2.3$ to $\approx 0.7-0.8$ with the added $\mathrm{Na}-\mathrm{S}$ promotion as a sign of increased, $\mathrm{CO}_{2}$ releasing, Boudouard and/or Watergas Shift (WGS) reactions consuming the input CO. No WGS reaction active $\mathrm{Fe}_{3} \mathrm{O}_{4}$ was present in any of the catalyst materials after the FTS reaction. Therefore, the $\mathrm{Na}(-\mathrm{S})$ promotion was discussed to provide the directly active reaction sites for the possible WGS reaction.

Nevertheless, despite the lost $\mathrm{C}$ pathway selectivity towards $\mathrm{CO}$ conversion to hydrocarbons: $\approx 4.4$ times more hydrocarbons were still converted from $\mathrm{CO}$ per unit of time with the most active Na-S promoted catalyst in comparison to the most active unpromoted catalyst material. 
When the FTS reaction activity results were contrasted to the R-QPA quantification results, no direct correlation between the FTS reaction activity and any of the bulk Fe carbide phases could be made. That is, any specific Fe carbide was not more active in the FTS reaction than any other of the studied carbides. However, in terms of hydrocarbon product selectivities, some cautious correlations could be assigned. It was observed that i) the $\theta-\mathrm{Fe}_{3} \mathrm{C}$ phase mildly ( $\approx+5 \%$ ) increased the hydrocarbon product slate paraffin content. In combination with the $\mathrm{Na}-\mathrm{S}$ promotion: ii) the $\eta-\mathrm{Fe}_{2} \mathrm{C}$ Type II and $\chi-\mathrm{Fe}_{5} \mathrm{C}_{2}$ Type II were present in the high olefin and low $\mathrm{CH}_{4}$ selective FTS catalyst materials, and iii) the $\chi-\mathrm{Fe}_{5} \mathrm{C}_{2}$ Type III might increase the olefin selectivity more than the $\chi-\mathrm{Fe}_{5} \mathrm{C}_{2}$ Type II does, when combined with the Na-S promotion.

Any conclusions on the role of the $\theta-\mathrm{Fe}_{7} \mathrm{C}_{3}$ phase in the FTS reaction could not be drawn, as the phase was present in too minor phase quantities in the studied FTS reaction catalyst materials.

Chapter 5 - The combined effects of the total FTS reaction pressure, Na-S promotion and the catalysts' $\mathrm{H}_{2}$ reduction prior to the FTS reaction were studied. The main aim of the work was to follow the effects of the aforementioned parameters on the formation of Fe carbides and their subtypes, and to correlate this information on $\mathrm{Fe}$ carbide phases to the FTS reaction performance of the catalyst materials. Thus, supported $\mathrm{Fe}(-\mathrm{Na}-\mathrm{S}) / \alpha-\mathrm{Al}_{2} \mathrm{O}_{3}$ catalyst materials, with and without $\mathrm{Na}-\mathrm{S}$ promotion, were prepared for this study. The catalysts were either calcined only or subsequently to calcining also $\mathrm{H}_{2}$ reduced, before the catalytic performance testing in the FTS reaction. The FTS reactions were separately ran for $72 \mathrm{~h}$ at either 3,10 or 20 bar total reaction pressure $\left(340^{\circ} \mathrm{C}, \mathrm{H}_{2}: \mathrm{CO}: \mathrm{He} 1.0: 1.0: 0.2 \mathrm{v} / \mathrm{v}, \mathrm{GHSV} \approx 5500 \mathrm{~h}^{-1}\right)$. XRD patterns were collected ex-situ for each of the studied catalyst materials after the FTS reaction runs and were quantified using R-QPA.

After the FTS reactions, the Fe carbide phases identified in the catalyst materials were $\eta-\mathrm{Fe}_{2} \mathrm{C}$ Pnnm (58), $\chi-\mathrm{Fe}_{5} \mathrm{C}_{2} \mathrm{C} 2 / c$ (15) and/or $\theta-\mathrm{Fe}_{7} \mathrm{C}_{3}$ Pnma (62). For all of the studied catalyst materials the $\chi-\mathrm{Fe}_{5} \mathrm{C}_{2}$ phase was quantified as the most abundant. The $\mathrm{Na}-\mathrm{S}$ promotion had the FTS reaction pressure dependent effects on the Fe carbide formation of $i$ ) inducing the appearance of the $\eta-\mathrm{Fe}_{2} \mathrm{C}$ phase with $\geq 10$ bar FTS reaction pressure, ii) whereas the calcined only catalyst materials favored the $\eta-\mathrm{Fe}_{2} \mathrm{C}$ phase formation more than the $\mathrm{H}_{2}$ reduced catalyst, and iii) also inducing formation of minor amounts of the $\theta-\mathrm{Fe}_{7} \mathrm{C}_{3}$ phase in the calcined only catalyst after the 20 bar FTS reaction.

In relation to the FTS reaction pressure and the added $\mathrm{Na}-\mathrm{S}$ promotion, the carbide subtypes form as follows:

Type I Without Na-S promotion regardless of reaction pressure, or 
Type II

with 3 bar FTS reaction pressure regardless of Na-S promotion,

Type III Fe oxide $\left(\alpha-\mathrm{Fe}_{2} \mathrm{O}_{3}\right)$ i.e. calcined only catalyst as the FTS starting material regardless of the Na-S promotion or the FTS reaction pressure,

$\eta-\mathrm{Fe}_{2} \mathrm{C}$

Type II With Na-S promotion, and $\geq 10$ bar FTS reaction pressure.

The performance of the catalyst materials depended on the reaction pressure, which could be altered by the presence of $\mathrm{Na}$ and $\mathrm{S}$ as promotors. Whether the catalyst was only calcined or also $\mathrm{H}_{2}$ reduced, had a lesser influence on the catalytic performance.
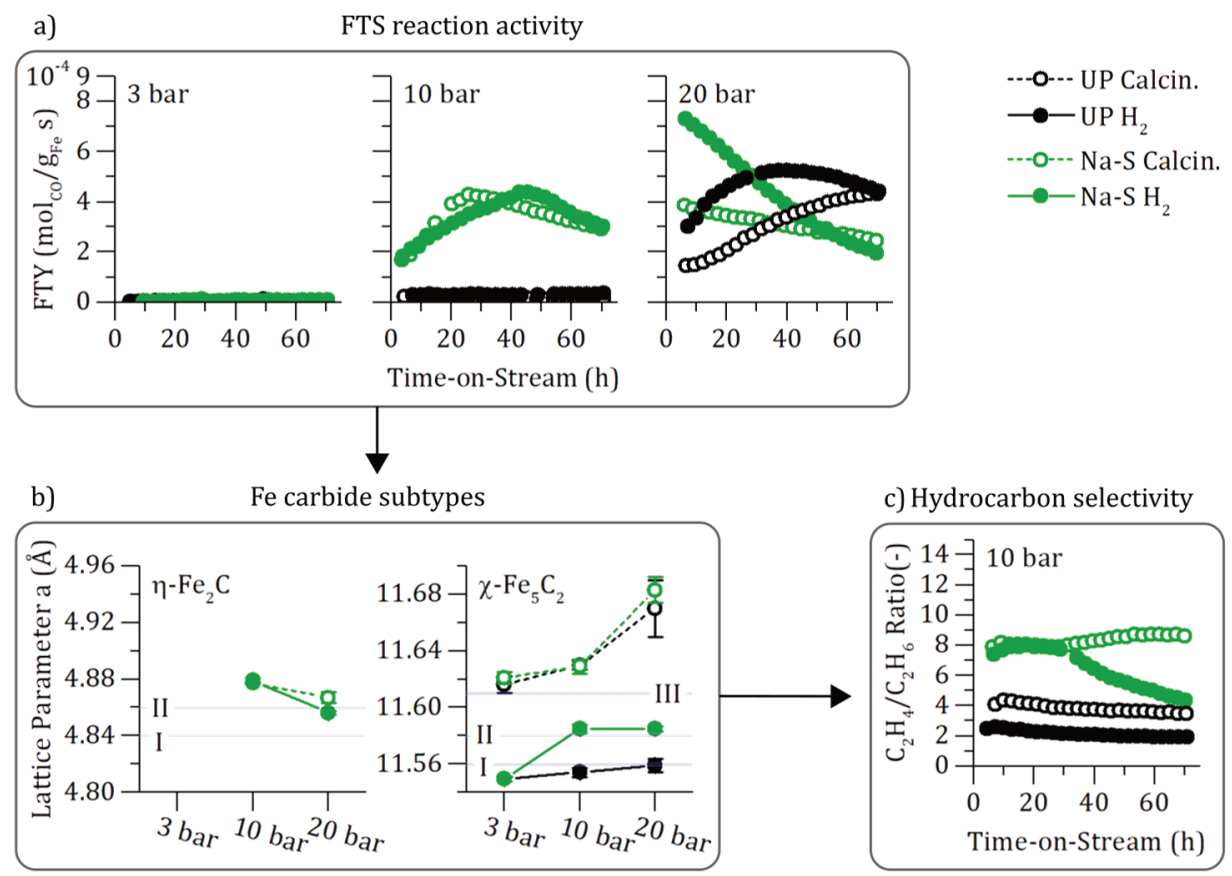

\section{Figure 7.5}

Overview of the effects of Fischer-Tropsch Synthesis (FTS) reaction pressure, Na-S promotion and catalyst $\mathrm{H}_{2}$ pre-reduction on unpromoted (UP) and Na-S promoted (Na-S) Fe(-Na-S) $/ \alpha-\mathrm{Al}_{2} \mathrm{O}_{3}$ catalyst materials in a $72 \mathrm{~h}$ FTS reaction for different pressures $(3,10,20$ bar). The catalyst materials were exposed to the FTS reaction directly after calcining (Calcin.) or $\mathrm{H}_{2}$ reduced prior to the reaction $\left(\mathrm{H}_{2}\right.$ ). The FTS activity as a) Fe Time Yields (FTYs) show 10 bar pressure threshold for Na-S and 20 bar threshold for UP catalyst materials for boosted FTY. The FTY boost b) correlates to the Fe carbide subtypes (I, II, III) with transition of $\chi$ - $\mathrm{Fe}_{5} \mathrm{C}_{2}$ Type I to II and the presence of $\chi-\mathrm{Fe}_{5} \mathrm{C}_{2}$ Type III with Na-S promotion. At 10 bar FTS reaction pressure c) the $\mathrm{C}_{2} \mathrm{H}_{4} / \mathrm{C}_{2} \mathrm{H}_{6}$ ratio is increased with the Calcin. catalysts, regardless of the Na-S promotion. This correlates with the presence of $\chi-\mathrm{Fe}_{5} \mathrm{C}_{2}$ Type III. FTS conditions: $340^{\circ} \mathrm{C}, 3,10$ or 20 bar, $\mathrm{H}_{2}: \mathrm{CO}: \mathrm{He}$ 1.0:1.0:0.2 v/v, $\approx 500 \mathrm{~h}^{-1}$. 
The Na-S promotion combined with a 10 bar FTS reaction pressure threshold, had the effects that: i) catalysts showed a significant boost in the Fe Time Yields (FTYs), ii) Usage Ratio $\mathrm{H}_{2}: \mathrm{CO}, \mathrm{CO}$ converted to hydrocarbons and hydrocarbon selectivities became reaction pressure independent, and iii) the selectivity towards $\mathrm{CO}_{2}$ was increased significantly. These FTS reaction performance changes correlated with Na-S induced transition of $\chi-\mathrm{Fe}_{5} \mathrm{C}_{2}$ Type I to Type II, and to the presence of $\chi-\mathrm{Fe}_{5} \mathrm{C}_{2}$ Type III with Na-S promotion. For unpromoted catalysts, a 20 bar FTS reaction pressure threshold was required for attaining similar FTS reaction catalysis effects as were observed with the Na-S promoted catalyst materials with $\geq 10$ bar reaction pressure. For these changes in the unpromoted catalysts, $\chi-\mathrm{Fe}_{5} \mathrm{C}_{2}$ Type I and unpromoted Type III were present as the $\chi-\mathrm{Fe}_{5} \mathrm{C}_{2}$ phase subtypes.

With 3 bar reaction pressure, none of the studied catalyst materials showed significant FTYs regardless of the Na-S promotion. The presence of $\eta-\mathrm{Fe}_{2} \mathrm{C}$ Type II did not correlate with any of the FTS reactions' catalytic performance - activity or selectivity - indicators, as was deduced on the basis of the R-QPA quantified Fe phase fractions.

Regardless of the Na-S promotion, when calcined only catalyst materials were used in the FTS reaction: i) the $\mathrm{C}_{2} \mathrm{H}_{4} / \mathrm{C}_{2} \mathrm{H}_{6}$ ratio was increased with 10 bar FTS reaction pressure and ii) the overall FTYs were decreased with 20 bar reaction pressure in comparison to the $\mathrm{H}_{2}$ reduced catalysts. The increased $\mathrm{C}_{2} \mathrm{H}_{4} / \mathrm{C}_{2} \mathrm{H}_{6}$ ratio was associated to the presence of $\chi-\mathrm{Fe}_{5} \mathrm{C}_{2}$ Type III, independently of the Na-S promotion, with $10 \mathrm{bar}$ reaction pressure. In other words, the $\chi-\mathrm{Fe}_{5} \mathrm{C}_{2}$ Type III phase correlated with a decreased hydrogenation ability of the catalyst material. Decreased FTYs in turn correlated with the presence of the $\mathrm{Fe}_{3} \mathrm{O}_{4}$ phase, in the calcined only, 20 bar FTS catalysts. The $\mathrm{Fe}_{3} \mathrm{O}_{4}$ likely originated from carbon deposition induced mass transfer limitations inhibiting the reduction of the initial $\alpha-\mathrm{Fe}_{2} \mathrm{O}_{3}\left(/ \alpha-\mathrm{Al}_{2} \mathrm{O}_{3}\right)$ phase by $\mathrm{H}_{2}: \mathrm{CO}$ during the FTS reaction. An overview of the discussed FTS reaction performance effects is given in Figure 7.5.

Chapter 6 - In this final research Chapter, the main aim was to study the effect of the Na-S promotion on the simultaneous Fe carbide phase changes and carbon deposition during the FTS reaction. This was done by using in-situ characterization methods. More specifically, a combined XRD/Raman spectroscopy set-up was employed. The supported $\mathrm{Fe}(-\mathrm{Na}-\mathrm{S}) / \alpha-\mathrm{Al}_{2} \mathrm{O}_{3}$ catalyst materials, prepared in Chapter 3, with preformed $\mathrm{Fe}$ carbides or only $\mathrm{H}_{2}$ reduced catalysts, with or without Na-S promotion, were used. The catalysts were characterized in-situ with XRD/Raman spectroscopy during a $72 \mathrm{~h}$ FTS reaction $\left(340^{\circ} \mathrm{C}, 10\right.$ bar $\mathrm{H}_{2}: \mathrm{CO}: \mathrm{He}$ 2.0:1.0:0.33 v/v, GHSV $\approx 7200 \mathrm{~h}^{-1}$ ). The in-situ collected XRD patterns were quantified with the use of RQPA. The Raman spectra were evaluated for the carbon species by following the 
a) Raman signal intensity

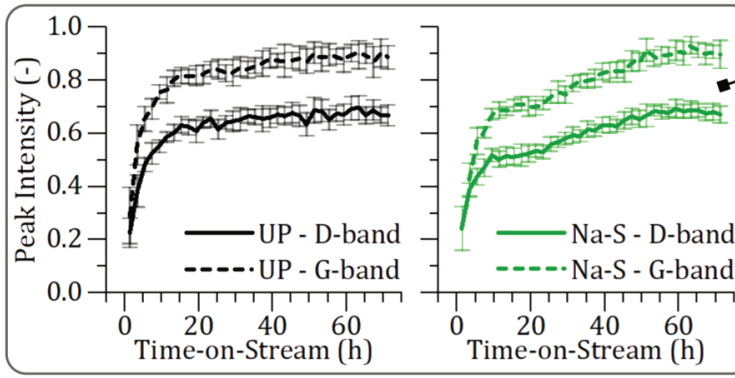

b) R-QPA

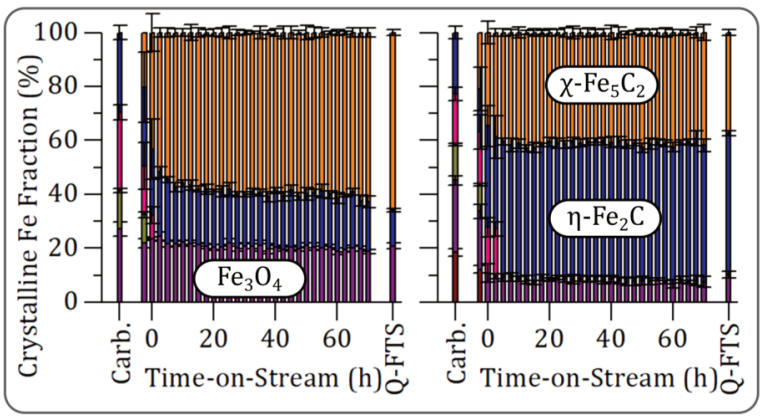

c) Comparison to reference

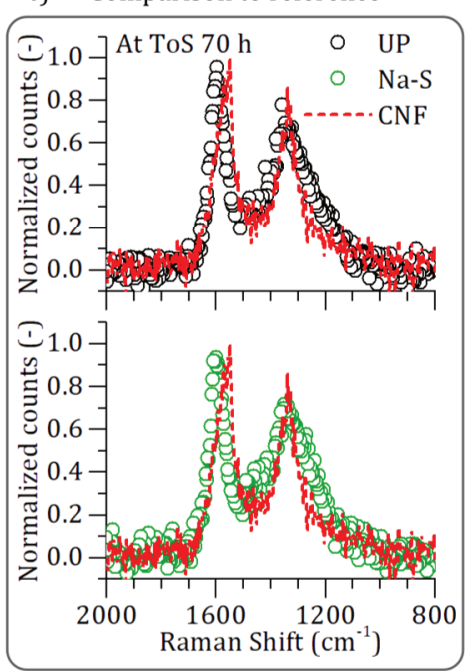

\section{Figure 7.6}

Summary of simultaneous in-situ X-ray Powder Diffractometry (XRPD)/Raman spectroscopy results during the Fischer-Tropsch Synthesis (FTS) reaction on unpromoted (UP) and Na-S promoted (Na-S) Fe-(Na-S)/ $\alpha$ $\mathrm{Al}_{2} \mathrm{O}_{3}$ catalyst materials without initial carbon deposits. The intensities of the $\mathrm{D}$ and $\mathrm{G}$ Raman bands a) initially grow fast within Time-on-Stream (ToS) $\lesssim 15 \mathrm{~h}$, and for ToS $\gtrsim 15 \mathrm{~h}$ ToS the growth remains faster with the Na$\mathrm{S}$ catalysts. Rietveld Quantitative Phase Analysis (R-QPA)-based Fe phase fractions change b) as the $\chi-\mathrm{Fe}_{5} \mathrm{C}_{2}$ phase preferentially forms during the FTS reaction at $340^{\circ} \mathrm{C}$, but the Na-S promotion significantly stabilizes the $\eta-\mathrm{Fe}_{2} \mathrm{C}$ phase. If present, Na-S promotion destabilizes the $\theta-\mathrm{Fe}_{3} \mathrm{C}$ and $\mathrm{Fe}_{3} \mathrm{O}_{4}$ phases. The formed carbon species c) compare closely to Carbon Nanofiber (CNF) reference carbon material at ToS $70 \mathrm{~h}$, for both UP and Na-S catalysts. FTS conditions: $340^{\circ} \mathrm{C}, 10$ bar, $\mathrm{H}_{2}: \mathrm{CO}: \mathrm{He} 2.0: 1.0: 0.33 \mathrm{v} / \mathrm{v}, \approx 7200 \mathrm{~h}^{-1}$.

intensities of the $D\left(\approx 1350 \mathrm{~cm}^{-1}\right)$ and $\mathrm{G}\left(\approx 1600 \mathrm{~cm}^{-1}\right)$ Raman bands. An overview of the most significant results fron this study is given in Figure 7.6.

The Na-S promotion altered the Fe oxide and carbide phases' properties by:

i) stabilizing the $\eta-\mathrm{Fe}_{2} \mathrm{C}$ phase, but only if either $\varepsilon-\mathrm{Fe}_{3} \mathrm{C}$ and/or $\eta-\mathrm{Fe}_{2} \mathrm{C}$ was present in the initial catalyst,

ii) destabilizing the $\theta-\mathrm{Fe}_{3} \mathrm{C}$ phase and aiding in its transition to $\chi-\mathrm{Fe}_{5} \mathrm{C}_{2}$,

iii) increasing the reducibility of $\mathrm{Fe}_{3} \mathrm{O}_{4}$ phase by $\left(\mathrm{H}_{2}\right.$ :)CO,

iv) decreasing the $\mathrm{Fe}_{3} \mathrm{O}_{4}$ content during the FTS reaction on the catalysts; and also by

v) inducing Fe crystallite growth for the Fe carbides within the $\lesssim 15 \mathrm{~h}$ Time-on- 
Stream (ToS) period. The $\theta-\mathrm{Fe}_{3} \mathrm{C}$ phase turned out to be an exception to this, as for the phase the crystallite diameters decreased with Na-S promotion.

The carbon deposition on the catalyst materials did not correlate with any specific Fe carbide phase or carbide-to-carbide transition. With $\mathrm{H}_{2}$ reduced catalysts, the carbon deposition did not start before the $\mathrm{Fe}_{3} \mathrm{O}_{4}$ phase, a phase incapable for $\mathrm{CO}$ dissociation, was reduced.

With catalyst materials with insignificant initial carbon deposits i.e. with $\mathrm{H}_{2}$ reduced or $<300^{\circ} \mathrm{C} \mathrm{CO}$ carburized catalysts, the increase of the D and G Raman bands during the FTS reaction could be divided into two periods: i) an initial fast ( $\$ 10-15 \mathrm{~h}$ ToS), and ii) a steady or lesser ( $\gtrsim 15 \mathrm{~h} \mathrm{ToS}$ ) intensity growth period. The initial fast growth period was present regardless of the Na-S promotion. However, after reaching the steady growth period Na-S promotion induced a higher rate of intensity growth of the $\mathrm{D}$ and $\mathrm{G}$ Raman bands (for the G-band $\approx 2.7 \pm 0.2$ faster) in comparison to the unpromoted catalyst materials. For catalysts with initial carbon deposits from CO carburization $\geq 340^{\circ} \mathrm{C}$, the initial fast growth period was absent, regardless of the Na-S promotion. Possibly, an initial non-isothermal bed operation could have explained by the initial fast D- and G-band intensity gains within $\lesssim 10-15 \mathrm{~h}$ ToS for the catalyst materials without initial carbon deposits.

The carbon species formed during catalyst preparation CO carburizations were weakly Raman active with the used (532 nm) visible wavelength excitation laser. That is, they were mainly made of carbon structures with $\mathrm{C} \mathrm{sp}{ }^{3}$ with a band gap above the energy available from the visible wavelength excitation laser. Under $\mathrm{H}_{2}$ : $\mathrm{CO}$ during the FTS reaction, more ordered CNF-like phases with $\mathrm{C} \mathrm{sp}^{2}$ formed, which led to an increased Raman signal intensity. During the FTS reactions, the carbon species evolved from amorphous carbon species, with structures containing both tetrahedral $\mathrm{C} \mathrm{sp} \mathrm{s}^{3}$ and $\mathrm{C} \mathrm{sp}^{2}$ in chain-like ordering, towards more ordered carbon materials with their structures containing $\mathrm{C} \mathrm{sp}{ }^{2}$ in varying non-6-fold cyclic order with feature sizes $<7 \mathrm{~nm}$ in diameter.

In comparison to the measured carbon reference materials, the catalysts' Raman spectra at $\approx 70 \mathrm{~h}$ ToS compared best to a Carbon Nanofiber (CNF) reference material. However, the catalyst materials contained possibly a higher amount of $\mathrm{C} \mathrm{sp} \mathrm{sp}^{2}$ in chainlike structures (G-band blue shift to higher $\mathrm{cm}^{-1}$ ) and a wider variety of non-6-fold carbon rings (wider D-band peak) than in the reference CNF material (Figure 7.6c).

$\mathrm{Na}-\mathrm{S}$ promotion affected the FTS reaction formed carbon species by: i) increasing the initial $\mathrm{C} \mathrm{sp}{ }^{2}$ content in chain-like carbon structures, and ii) increasing 6-fold cyclic carbon species for catalyst materials with the initial CO carburization temperature $\geq 340^{\circ} \mathrm{C}$. 


\subsection{FUTURE PERSPECTIVES}

As invariably tends to happen with any research, new scientific questions arise at the same pace as the old ones are answered. In the following, few of the most interesting future research topics arising from the work conducted in this PhD Dissertation are briefly discussed.

Comparison of $\varepsilon-\mathrm{Fe}_{3} \mathrm{C}$ and $\boldsymbol{\eta}-\mathrm{Fe}_{2} \mathrm{C}$ to $\boldsymbol{\varepsilon}-\mathrm{Fe}_{3} \mathrm{~N}$ and $\boldsymbol{\zeta}-\mathrm{Fe}_{2} \mathrm{~N}$. As was highlighted in the Chapter 3, the $\mathrm{Fe}$ carbide $\varepsilon-\mathrm{Fe}_{3} \mathrm{C}$ and $\eta-\mathrm{Fe}_{2} \mathrm{C}$ phases' crystal structures are closely related to the $\varepsilon-\mathrm{Fe}_{3} \mathrm{~N}$ and $\zeta-\mathrm{Fe}_{2} \mathrm{~N}$ Fe nitrides' structures, respectively. A comparative study on the behavior and the FTS reaction performance of the $\varepsilon-\mathrm{Fe}_{3} \mathrm{C}$ and $\eta-\mathrm{Fe}_{2} \mathrm{C}$ in comparison to the $\mathrm{Fe}_{3} \mathrm{~N}$ and $\zeta-\mathrm{Fe}_{2} \mathrm{~N}$ phases, with and without the $\mathrm{Na}-\mathrm{S}$ promotion, would make an interesting study. Especially, it would be worth finding out whether the $\zeta-\mathrm{Fe}_{2} \mathrm{~N}$ phase can be stabilized in the FTS reaction with Na-S promotion in the same way as was observed for the $\eta-\mathrm{Fe}_{2} \mathrm{C}$ phase. The Fe nitride phases are usually not stable under carburizing conditions but rather convert into Fe carbonitrides. ${ }^{1-3}$ Fe nitride-based catalyst materials have been observed to be selective toward olefin production in the FTS reaction. ${ }^{3}$

Formation of the $\boldsymbol{\theta}-\mathrm{Fe}_{7} C_{3}$ Phase. Although some minor amounts of the $\theta-\mathrm{Fe}_{7} \mathrm{C}_{3}$ phase was formed in the studies, described in the research Chapters of this Dissertation, more effort is still needed on studying the phase's formation behavior under the FTS reaction relevant conditions. As was speculated in Chapter 3, the $\theta-\mathrm{Fe}_{7} \mathrm{C}_{3}$ phase's formation under carburizing conditions might be dependent on the presence of $\mathrm{CO}_{2}$ during the carburization process. A systematic probing on the effect of carburizing $\mathrm{CO}_{2}$ : $\mathrm{CO}$ gas mixtures on the $\theta-\mathrm{Fe}_{7} \mathrm{C}_{3}$ phase's formation from Fe-based materials should be conducted in the future. This would aid in determining the FTS reaction role and properties of this still vastly understudied Fe carbide.

Fe Carbide Subtypes' Crystal Structures. In Chapter 4 he $\eta-\mathrm{Fe}_{2} \mathrm{C}$ and the $\chi-\mathrm{Fe}_{5} \mathrm{C}_{2}$ phases were divided into distinct subtypes based on their distinctively different lattice parameter $a$. However, the precise structural origins of the crystal lattice changes for the resulting carbide subtypes could not be answered within the scope of this $\mathrm{PhD}$ Dissertation. In the future, more detailed probing of the crystal structure differences in the Fe carbide subtypes should be performed with the use of a monochromatic X-ray source. In addition, probing of whether the Na-S promotion induced subtype formations are truly due to the $\mathrm{Na}(-\mathrm{S})$ promoters entering the Fe carbide lattices or something else, could be investigated using Neutron Diffraction (ND).

Furthermore, in Chapter 5, the presence of the $\chi-\mathrm{Fe}_{5} \mathrm{C}_{2}$ Type III phase was correlated with a decreased hydrogenation ability in the FTS reaction. That is, the $\chi$ $\mathrm{Fe}_{5} \mathrm{C}_{2}$ Type III increased the catalyst material's FTS hydrocarbon selectivity towards the 
production of olefins. The presence of $\chi-\mathrm{Fe}_{5} \mathrm{C}_{2}$ Type III would also explain similar selectivity results obtained in literature.4,5 This effect, neither, was not explained with any fundamental insight in the Chapter. How and why the $\chi-\mathrm{Fe}_{5} \mathrm{C}_{2}$ Type III favors the olefin formation in comparison to the other Fe carbide subtypes would make an interesting study by peforming Density Functional Theory (DFT) calculations.

\subsection{REFERENCES}

[1] Jack, K. H., "Binary and Ternary Interstitial Alloys III. The Iron-Carbon System: The Characterization of a New Iron Carbide", Proc. R. Soc. A Math. Phys. Eng. Sci. 195, 56-61 (1948).

[2] Jack, K. H., "Binary and Ternary Interstitial Alloys. II. The Iron-Carbon-Nitrogen System", Proc. R. Soc. A Math. Phys. Eng. Sci. 195, 41-55 (1948).

[3] Butt, J. B., "Carbide phases on iron-based Fischer-Tropsch synthesis catalysts part II: Some reaction studies", Catal. Lett. 7, 83-106 (1990).

[4] Bukur, D. B., Nowicki, L., Manne, R. K. and Lang, X. S., "Activation Studies with a Precipitated Iron Catalyst for Fischer-Tropsch Synthesis II. Reaction Studies", J. Catal. 155, 366-375 (1995).

[5] Wezendonk, T. A. et al., "Controlled formation of iron carbides and their performance in FischerTropsch synthesis", J. Catal. 362, 106-117 (2018). 


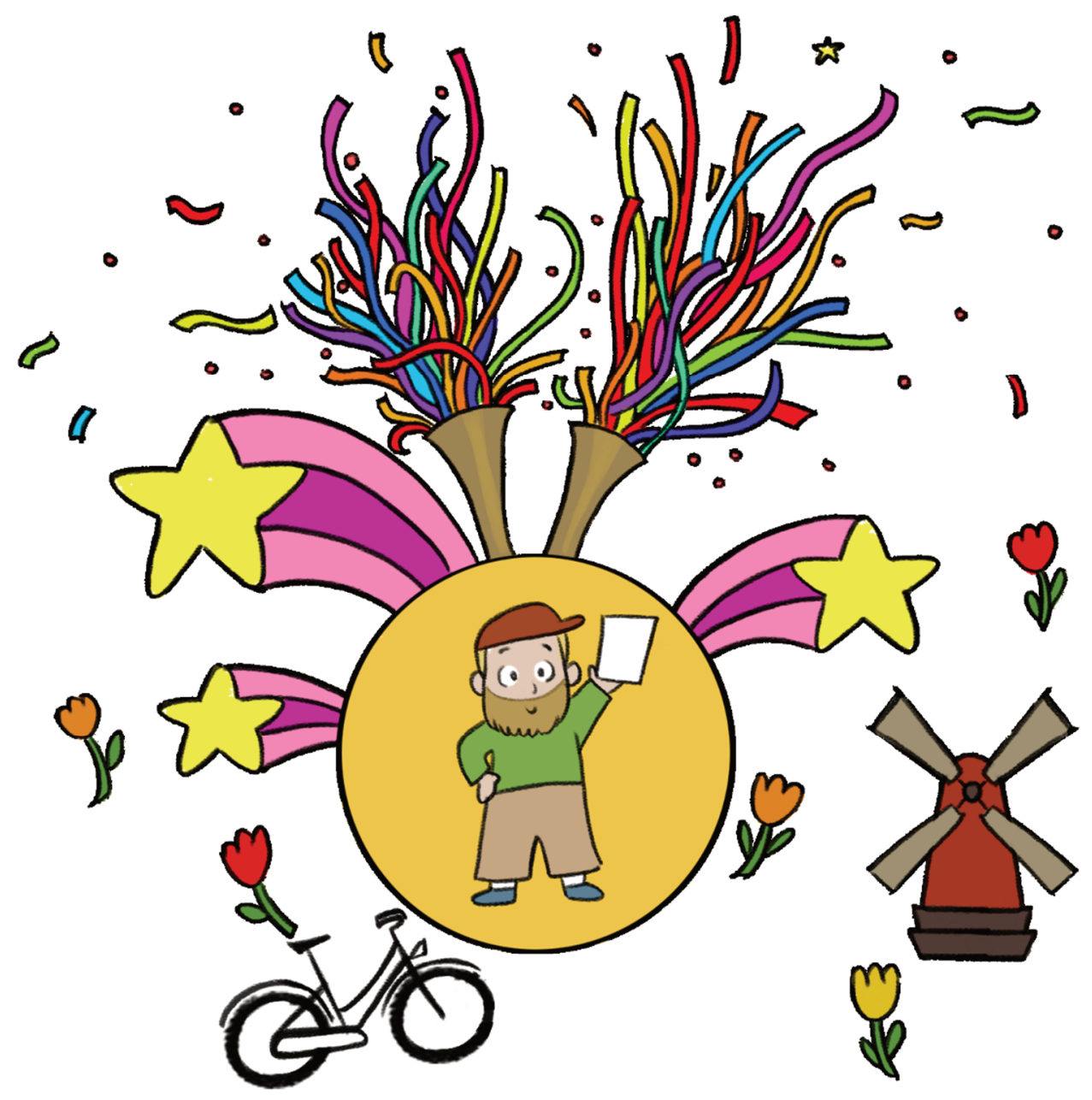




\section{Chapter 8}

\section{Nederlandse Samenvatting en Toekomstperspectieven}

Momenteel worden koolwaterstoffen gebruikt in het overgrote deel van moderne industriële processen, die leiden tot de productie van brandstoffen, kunststoffen, coatings en andere materialen. In de nabije toekomst zal de huidige primaire bron voor deze koolwaterstoffen, namelijk ruwe olie uit de aardkorst, opraken en zullen nieuwe alternatieven als bron voor koolwaterstoffen nodig zijn. Het industriële Fischer-Tropsch-proces (FTP) is in staat om een koolstofhoudende grondstof, zoals aardgas, steenkool, biomassa of gemeentelijk afval, om te zetten in een verscheidenheid aan koolwaterstofproducten. De heterogeen gekatalyseerde Fischer-Tropsch synthese (FTS)-reactie wordt gebruikt in het FTP voor het omzetten van een synthesegasmengsel van $\mathrm{CO}+\mathrm{H}_{2}$ in synthetische ruwe olie, die verder wordt gezuiverd tot de uiteindelijke koolwaterstofproducten. In dit proefschrift hebben we de FTS-reactie bestudeerd met behulp van Fe-gebaseerde, wel of niet met Na-S gepromote, ondersteunde $\mathrm{Fe}(-\mathrm{Na}-\mathrm{S}) / \alpha-\mathrm{Al}_{2} \mathrm{O}_{3}$-katalysatormaterialen. De focus van deze dissertatie lag voornamelijk op de karakterisering van de actieve fasen van de FTS-reactie katalysatoren: de Fe-carbiden. Röntgenpoederdiffractometrie (XRPD) werd toegepast als de primaire karakteriseringstechniek en de verzamelde gegevens werden gekwantificeerd met Rietveld kwantitatieve fase-analyse (R-QPA). Identificatie van Fe-carbide, vormingsomstandigheden en hun subtypen, zoals gedefinieerd door de roosterparameters van carbide, werden onderzocht. Dit proefschrift specificeert de effecten, die Na-S-promotor heeft op de Fecarbidevorming en de carbide-eigenschappen in de FTS-reactie. 
a) Fischer-Tropsch-proces (FTP)

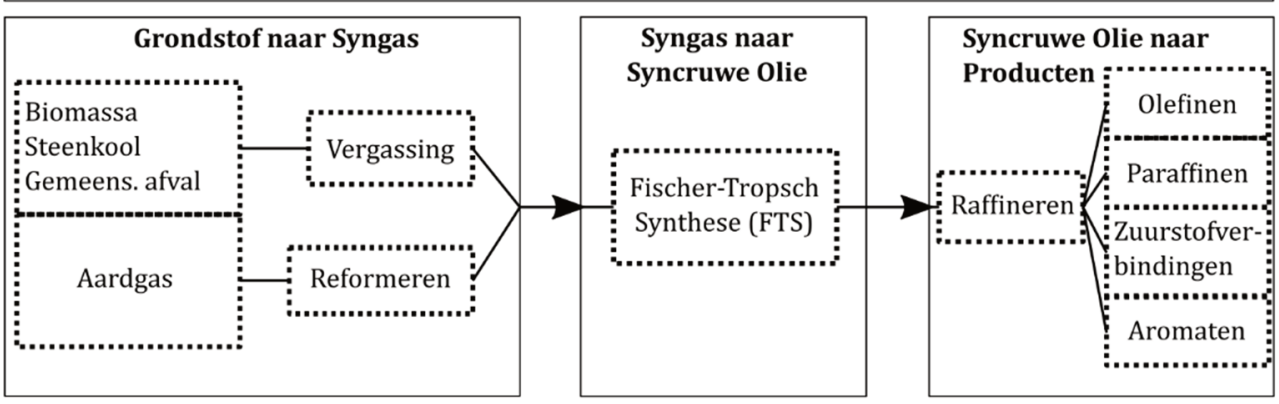

b)

\begin{tabular}{|cccc|}
\hline Grondstof & \multicolumn{2}{c}{$\mathrm{H}: \mathrm{C}$} & $\mathrm{CO}_{2}$ voetspoor \\
& $\begin{array}{c}\text { (elementaire verhouding) } \\
\left(\mathrm{CO}_{2}: \mathrm{HC}\right)\end{array}$ \\
\cline { 2 - 4 } & Nominaal & Effectief & \\
\hline Aardgas & 4.00 & 4.00 & 0.00 \\
Afval & 1.83 & 0.65 & 3.96 \\
Steenkool & 0.80 & 0.40 & 4.80 \\
Biomassa & 1.45 & 0.12 & 5.75 \\
\hline
\end{tabular}

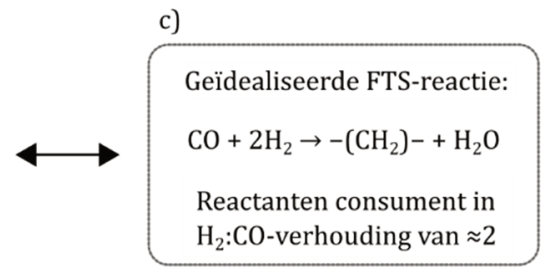

\section{Figuur 8.1}

Overzicht van het volledige Fischer-Tropsch-proces (FTP), die de Fischer-Tropsch synthese (FTS)-reactie toepast in het industriële proces van de omzetting van koolstofhoudende grondstoffen in geraffineerde koolwaterstofproducten. De algemene FTP-stappen zijn a) Grondstof naar Syngas, Syngas naar Synruwe Olie en Synruwe Olie naar Producten. De grondstof b) bepaalt grotendeels de $\mathrm{CO}_{2}$-voetafdruk van de algehele FTP, aangezien de c) FTS-reactie een syngas $\mathrm{H}_{2}$ : $\mathrm{CO}$ in een verhouding van $\approx 2$ in de Syngas-naar-Synruwe Olie FTPstap verbruikt. Met andere grondstoffen dan aardgas moet de effectieve syngas $\mathrm{H}_{2}$ :CO-verhouding $<2$ en $\mathrm{H}_{2}$ worden geproduceerd en toegevoegd aan de FTP, waardoor de totale $\mathrm{CO}_{2}$-voetafdruk toeneemt. Afval = Gemeentelijk afval, Steenkool = Gemiddelde van verschillende kolentypen, Biomassa = vingergras, $\mathrm{HC}=$ koolwaterstoffen.

\subsection{SAMENVATTING}

Ondanks dat de katalytische omzetting van een $\mathrm{H}_{2}+\mathrm{CO}$ mengsel in koolwaterstoffen met de Fischer-Tropsch synthese-reactie (FTS-reactie) al meer dan 100 jaar bekend is, valt er nog veel te leren. De Fe-carbiden, de actieve fasen in de reactie, vormen de basis van de FTS-reactie met Fe-gebaseerde katalysatormaterialen. De belangrijkste motivatie voor het uitvoeren van het onderzoek in dit proefschrift was om meer te leren over het gedrag en de vorming van de verschillende Fe-carbide fasen en hoe ze worden beïnvloed door de toegevoegde chemische Na-S-promotor. Hieronder volgt een samenvatting van het werk dat is uitgevoerd voor dit proefschrift, waarbij de belangrijkste conclusies worden belicht. 
Hoofdstuk 1 - De geschiedenis over én motivaties voor de toepassing van het algehele industriële Fischer-Tropsch-proces (FTP) werden kort geïntroduceerd. In de FTP wordt een koolstofhoudende grondstof omgezet in geraffineerde koolwaterstofproducten. De FTP-stappen die werden besproken zijn (Figuur 8.1): Grondstof naar Syngas, Syngas naar Synruwe Olie en Synruwe olie naar Producten. De gekatalyseerde FTS-reactie wordt zelf gebruikt in de Syngas naar Synruwe Olieprocesstap voor het omzetten van $\mathrm{H}_{2}+\mathrm{CO}$ mengsel (syngas) naar een mengsel van verschillende koolwaterstoffen en hun derivaten (synruwe olie).

De $\mathrm{CO}_{2}$-voetafdruk van het algehele FTP hangt voor een groot deel af van de grondstof in de volgende volgorde: aardgas < gemeenschappelijk afval<steenkool<biomassa. Vanwege de zuiverheid kan aardgas direct worden omgezet in gebruiksklaar syngas met een $\mathrm{H}_{2}$ :CO-verhouding $\approx 2$, dat voldoet aan de vereiste stoichiometrie van de FTS-reactie. Met de andere grondstoffen, die heteroatomen (voornamelijk $\mathrm{N}, \mathrm{O}$ en $\mathrm{S}$ ) als onzuiverheden bevatten, is de effectief haalbare $\mathrm{H}_{2}$ :CO-verhouding minder dan 2. Daarom moet voor deze grondstoffen goedkoop en duurzaam geproduceerd $\mathrm{H}_{2}$ aan de FTP worden toegevoegd om met aardgas gebaseerde FTP te kunnen concurreren. De gebruikte FTP-grondstof is ook belangrijk vanuit economisch perspectief. Het produceren van het gezuiverde syngas voor gebruik in de FTS-reactie bedraagt 60-70 \% van de totale kapitaalkosten in een op steenkool gebaseerde FTP, terwijl dat voor de op aardgas gebaseerde FTP slechts 30$50 \%$ is.

Hoofdstuk 2 - De heterogene katalyse van de FTS-reactie met Fe-gebaseerde katalysatormaterialen is besproken. Onderwerpen zoals de gangbare katalysatorsamenstellingen, de verschillende chemische reacties die plaatsvinden en de mogelijke reactietrajecten voor $\mathrm{C}$ van CO-input in een Fe-gebaseerde FTSreactiekatalysator zijn de revue gepasseerd. De mogelijke C-routes in de FTS-reactie zijn samengevat in Figuur 8.2. Verder werden de effecten van de verschillende FTSreactie-conditieparameters op de koolwaterstof- en C-route-selectiviteiten bekeken. De effecten van parameters, zoals reactietemperatuur, input $\mathrm{H}_{2}$ :CO-verhouding, gasruimte-snelheid en totale reactiedruk, werden besproken. De specifieke onderwerpen van deze dissertatie zijn: i) de effecten die voortkomen uit de toegevoegde chemische Na-S-promotie in de Fe-gebaseerde FTS-reactie en ii) de identificatie van de Fe-carbidefasen die werden aangetroffen in het FTSonderzoeksdomein.

De Na-S-promotie in de FTS-reactie heeft als effect i) het verlagen van de $\mathrm{CH}_{4}$ selectiviteit tot een lagere waarde dan voorspeld door de Anderson-Shulz-Floryverdeling (ASF-verdeling), ii) minder toename in de koolwaterstofketenlengte (d.w.z., $\alpha$-waarde) in vergelijking met wanneer alleen alkali wordt toegevoegd, iii) verhoogde 


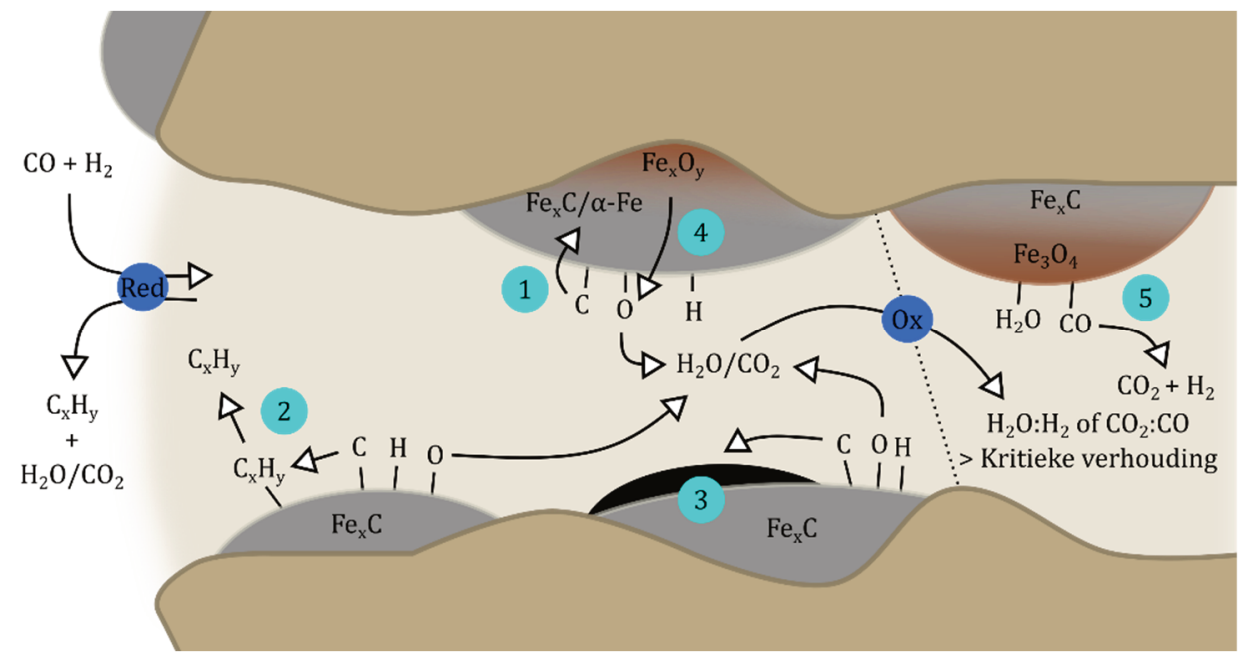

\section{Figuur 8.2}

Een vereenvoudigde samenvatting van de reactiepaden voor $\mathrm{C}$ uit $\mathrm{CO}$-invoer in een katalysatordeeltje voor de Fe-gebaseerd Fischer-Tropsch synthese (FTS)-reactie. De invoer $\mathrm{CO}+\mathrm{H}_{2}$ geeft oorspronkelijk een reductieve omgeving (rood). Voor $C$ van CO-dissociatie zijn de reactiepaden: (1) met $\alpha$-Fe om $\mathrm{Fe}_{\mathrm{x}} \mathrm{C}$ te vormen, (2) met $\mathrm{H}$ om koolwaterstoffen te vormen, (3) met $\mathrm{C}$ om inactieve koolstof te vormen. Zonder CO-dissociatie: C-pad (4) kan $\mathrm{Fe}_{\mathrm{x}} \mathrm{O}_{\mathrm{y}}$ reduceren tot $\alpha$-Fe terwijl $\mathrm{CO}_{2}$ vrijkomt. Verwijdering van 0 -atomen uit $\mathrm{CO}$ naar $\mathrm{H}_{2} \mathrm{O}$ of $\mathrm{CO}_{2}$ leidt geleidelijk tot een oxiderende omgeving $(\mathrm{Ox})$ in de richting van de kern van het katalysatordeeltje en de uitlaat van een reactor met een vast bed. Als de actieve FTS-reactie $\mathrm{Fe}_{\mathrm{x}} \mathrm{C}$ geoxideerd is tot $\mathrm{Fe}_{3} \mathrm{O}_{4}$, $\mathrm{kan}$ route (5) $\mathrm{H}_{2} \mathrm{O}+\mathrm{CO}$ omzetten in $\mathrm{CO}_{2}+\mathrm{H}_{2}$ via de water-gasverschuivingsreactie (WGS).

selectiviteit van alkenen, en iv) een algeheel verhoogde CO-omzetting. Deze selectiviteitseffecten lijken op promotie-effecten van alleen alkali (als $\mathrm{K}$ of $\mathrm{Na}$ ) in de FTS-reactie. De unieke eigenschap van de Na-S-promotie is echter het vermogen om de $\mathrm{CH}_{4}$-selectiviteit te verlagen tot een lagere waarde dan voorspeld door de ASFverdeling. Deze prestatie is niet haalbaar zonder de toegevoegde S.

De Fe-carbiden die waarneembaar zijn in het FTS-reactieonderzoeksveld met hun kristalstructuren zijn:

- hexagonal $\varepsilon-\mathrm{Fe}_{3} \mathrm{C} \mathrm{P}_{3} 22$ (182),

- orthorhombic $\eta-\mathrm{Fe}_{2} \mathrm{C}$ Pnnm (58),

- monoclinic $\chi-\mathrm{Fe}_{5} \mathrm{C}_{2} C 2 / c(15)$ (the Hägg carbide),

- orthorhombic $\theta-\mathrm{Fe}_{3} \mathrm{C}$ Pnma (62) (cementite), and

- orthorhombic $\theta-\mathrm{Fe}_{7} \mathrm{C}_{3}$ Pnma (62) (the Eckström-Adcock carbide).

De totstandkoming van de Fe-carbiden is afhankelijk van de temperatuur waarbij het nulwaardige $\alpha$-Fe wordt blootgesteld aan een $\mathrm{C}$-atoombron. De verwachte thermische 
stabiliteit en vormingstemperatuur voor de Fe-carbiden zijn op basis van de literatuurgegevens: $\varepsilon-\mathrm{Fe}_{3} \mathrm{C}\left(100-265^{\circ} \mathrm{C}\right)<\eta-\mathrm{Fe}_{2} \mathrm{C}\left(160-340^{\circ} \mathrm{C}\right)<\chi-\mathrm{Fe}_{5} \mathrm{C}_{2}\left(240-350^{\circ} \mathrm{C}\right)<$ $\theta-\mathrm{Fe}_{3} \mathrm{C}\left(310-600^{\circ} \mathrm{C}\right)$.

De totstandkoming van de $\theta-\mathrm{Fe}_{7} \mathrm{C}_{3}$-fase verloopt niet op dezelfde manier als voor de rest van de carbiden van Fe. Ondanks dat nogal extreme reactieomstandigheden vaak worden genoemd als vereisten voor de vorming van de $\theta-\mathrm{Fe}_{7} \mathrm{C}_{3}$-fase, kan deze zich ook vormen onder relatief milde FTS-reactieomstandigheden. Waarnemingen van de totstandkoming van de $\theta-\mathrm{Fe}_{7} \mathrm{C}_{3}$-fase $\mathrm{bij} \approx 340^{\circ} \mathrm{C}$ onder milde reactieomstandigheden zijn gemaakt in de literatuur. Er is gespeculeerd dat de totstandkoming van de $\theta-\mathrm{Fe}_{7} \mathrm{C}_{3}-$ fase gerelateerd zou kunnen zijn aan de aanwezigheid van $\mathrm{CO}_{2}$ onder Fecarburiseringsomstandigheden.

Hoofdstuk 3 - In dit eerste experimentele hoofdstuk werden de identificatie- en vormingstemperaturen van de Fe-carbiden in het FTS-onderzoeksdomein onderzocht. Vooral het effect van de Na-S-promotie op het Fe-carbidevormingsproces werd bestudeerd. De studie werd uitgevoerd door het carboneren van $\mathrm{H}_{2}$-voorgereduceerde gedragen $\mathrm{Fe}(-\mathrm{Na}-\mathrm{S}) / \alpha-\mathrm{Al}_{2} \mathrm{O}_{3}$-katalysatormaterialen, met en zonder de toegevoegde $\mathrm{Na}$ $\mathrm{S}$-promotie, met $\mathrm{CO}$ binnen carburisatietemperaturen van $240-440^{\circ} \mathrm{C}$. Op deze manier bereide Fe-carbiden werden vervolgens gekarakteriseerd door ex-situ Röntgenpoederdiffractometrie (XRPD) te gebruiken met Rietveld kwantitatieve faseanalyse (R-QPA). De verkregen R-QPA-resultaten werden vergeleken met respectievelijke ex-situ Mössbauer absorptie spectroscopie (MAS)karakterisatieresultaten op dezelfde, gecarburiseerde katalysatormaterialen.

Met deze benadering was het mogelijk om de identificatie van de Fe-carbiden, hun kristalstructuren en de uniforme nomenclatuur voor de XRPD- en MAS-technieken te bevestigen, zoals toegewezen in Hoofdstuk 2. Relatieve C-atomen, niet-equivalente Fepositieverhoudingen en hun overeenkomstige MAS-hyperfijnvelden werden toegewezen voor de Fe-carbiden uit de experimentele gegevens.

Samen met de verkregen experimentele resultaten en literatuur werden de thermische stabiliteits-bereiken van de Fe-carbiden verfijnd om tot een behandelingstijd van 5 uur te komen (Figuur 8.3):

$$
\begin{aligned}
& \varepsilon-\mathrm{Fe}_{3} \mathrm{C}<\eta-\mathrm{Fe}_{2} \mathrm{C}<\chi-\mathrm{Fe}_{5} \mathrm{C}_{2}<\theta-\mathrm{Fe}_{3} \mathrm{C} \\
& 100-265^{\circ} \mathrm{C} \quad 160-300^{\circ} \mathrm{C} \quad 240-420^{\circ} \mathrm{C} \quad 350-500^{\circ} \mathrm{C}
\end{aligned}
$$

De $\eta-\mathrm{Fe}_{2} \mathrm{C}$ fase kan echter stabiel zijn tot $340^{\circ} \mathrm{C}$ in het geval dat het eerst wordt gevormd bij een lagere temperatuur $\left(\leqslant 30^{\circ} \mathrm{C}\right)$ en daarna wordt blootgesteld aan een hogere temperatuur. Door de experimentele resultaten te vergelijken met de literatuurobservaties kwamen we tot een verklaring. De ontleding van de $\theta-\mathrm{Fe}_{3} \mathrm{C}$-fase 


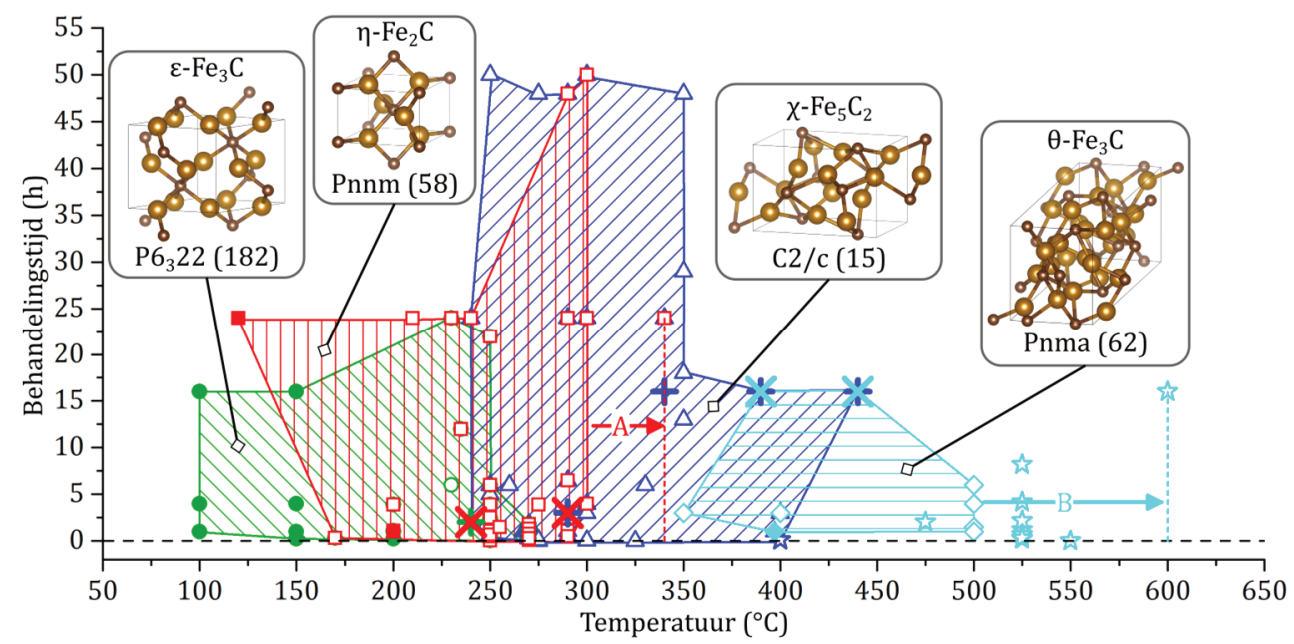

\section{Figuur 8.3}

Fe-carbide-vormingsgebieden (gearceerd gedeelte) voor temperatuur versus behandelingsblootstellingstijd op basis van de beschikbare literatuurgegevens over experimenteel onderzoek (niet-aangekruist) en deze PhD-dissertatie (aangekruist). Pijlen: maximale thermische stabiliteit met voorgevormde Fe-carbiden voor (A) $\eta-\mathrm{Fe}_{2} \mathrm{C}$ en (B) $\theta-\mathrm{Fe}_{3} \mathrm{C}$. De $\theta-\mathrm{Fe}_{7} \mathrm{C}_{3}$ Pnma (62) vormingstemperaturen zijn uitgesloten omdat de formatie ervan een andere, niet alleen temperatuurafhankelijke, route volgt. Elk van de Fe-carbiden kreeg, op basis van literatuur, hun exacte kristalstructuren toegewezen.

tot $\mathrm{C}$ en $\alpha$-Fe kan een temperatuur van wel $600^{\circ} \mathrm{C}$ vereisen als de carbidefase wordt bedekt door een oxidatieschaal, zoals ook werd verklaard aan de hand van literatuurobservaties.

Sommige hoeveelheden van de $\theta-\mathrm{Fe}_{7} \mathrm{C}_{3}$-fase werden gevormd wanneer een $\mathrm{Fe}(-\mathrm{Na}$ $\mathrm{S}) / \alpha-\mathrm{Al}_{2} \mathrm{O}_{3}$-katalysatormateriaal - met of zonder Na-S-promotie - werd gecarburiseerd bij 25 bar $\mathrm{CO}$-druk bij $340^{\circ} \mathrm{C}$, direct ná een katalysator-calcinatiestap zonder deze eerst te reduceren van $\alpha-\mathrm{Fe}_{2} \mathrm{O}_{3}$ tot a $\alpha$-Fe. Met directe carburisatie van de $\alpha-\mathrm{Fe}_{2} \mathrm{O}_{3}$-fase, werd een duidelijk vervormde vorm van de $\chi$ - $\mathrm{Fe}_{5} \mathrm{C}_{2}$-fase gevormd. De vervormde kristalroosterparameters weken duidelijk af van de $\chi$ - $\mathrm{Fe}_{5} \mathrm{C}_{2}$-fase gevormd uit de $\mathrm{H}_{2}$ voorgereduceerde katalysatoren met $\alpha$-Fe als de carburisatie-precursor.

De Na-S-promotie had geen invloed op de vorming van de Fe-carbiden uit de $\alpha$-Fefase in de $\mathrm{H}_{2}$-voorgereduceerde katalysatormaterialen. Echter, zoals is uitgelegd middels vergelijkingen met de literatuur, verhogen alkali-promoters de reduceerbaarheid van Fe-oxiden door $\mathrm{CO}$ bij lage reductietemperaturen $\left(\approx 270^{\circ} \mathrm{C}\right)$. Oftewel, alkali verhoogt de beschikbaarheid van de $\alpha$-Fe-fase en de daaropvolgende carburisering door CO met Fe-oxide carboneringsuitgangsmaterialen. Dit alkalische effect verklaart de van tijd tot tijd waargenomen toename van de carburiseringsniveaus van de op basis van Fe-gebaseerde katalysatoren in de literatuur, wanneer het 
carburisatieproces wordt gestart met een Fe-oxide.

Sommige Na-S-promotie-effecten bij de CO-carburisatie van de gereduceerde $\alpha$ $\mathrm{Fe} / \alpha-\mathrm{Al}_{2} \mathrm{O}_{3}$-katalysatormaterialen werden ook waargenomen. De waarnemingen zijn: i) toename van de Fe-oxidatie, vermoedelijk door 0 van de CO-dissociatie die werkt als oxidatiemiddel, met korte CO-carburatiebehandelingen bij lage temperatuur $\left(\leq 290^{\circ} \mathrm{C}\right.$, $\leq 3$ uur), ii) verminderde kristallietdiameter van de $\theta$-Fe3 $\mathrm{C}$-fase gevormd na CObehandeling bij $440^{\circ} \mathrm{C}$, en iii) verhoogde snelheid van koolstofafzetting uit $\mathrm{CO}$ bij $\geq 340^{\circ} \mathrm{C}$ via de Boudouard-reactie. De verhoogde koolstofafzettingssnelheid nam echter af bij $390^{\circ} \mathrm{C}$ en verdween bij het bereiken van de CO-carburisatietemperatuur van $440^{\circ} \mathrm{C}$, in vergelijking met de koolstofafzetting op de respectievelijke niet-gepromote $\mathrm{Fe} / \alpha-\mathrm{Al}_{2} \mathrm{O}_{3}$-katalysatormaterialen.

Hoofdstuk 4 - Het effect van toegevoegde Na-S-promotie op de prestaties en stabiliteit van de verschillende Fe-carbiden in de FTS-reactie werd bestudeerd. De ondersteunde $\mathrm{Fe}(-\mathrm{Na}-\mathrm{S}) / \alpha-\mathrm{Al}_{2} \mathrm{O}_{3}$-katalysatormaterialen bereid in Hoofdstuk 3 met voorgevormde $\mathrm{Fe}$-carbiden of alleen met $\mathrm{H}_{2}$-gereduceerde katalysatoren, met of zonder Na-S-promotie, werden hiervoor gebruikt. De katalysatormaterialen werden getest in een 72 uur FTS-reactie $\left(340^{\circ} \mathrm{C}, 10\right.$ bar $\mathrm{H}_{2}: \mathrm{CO}: \mathrm{He} 2.0: 1.0: 0.33 \mathrm{v} / \mathrm{v}$, GHSV $\left.\approx 5000 \mathrm{~h}^{-1}\right)$ vanwege hun katalytische prestatie. De katalysatoren werden gekarakteriseerd ná de FTS-reactie door XRPD met R-QPA te gebruiken. De verkregen resultaten werden vergeleken met de R-QPA-resultaten vóór de FTS-reactie uit Hoofdstuk 3.

De $\mathrm{Fe}$ carbide-fasen van $\eta-\mathrm{Fe}_{2} \mathrm{C} P n n m$ (58), $\chi-\mathrm{Fe}_{5} \mathrm{C}_{2} \mathrm{C2} / c$ (15), $\theta-\mathrm{Fe}_{3} \mathrm{C}$ Pnma (62) en $\theta-\mathrm{Fe}_{7} \mathrm{C}_{3} \mathrm{Pnma}$ (62) werden waargenomen in de katalysatoren. De $\varepsilon-\mathrm{Fe}_{3} \mathrm{C} P{ }_{6} 22$ (182)fase was niet stabiel in de FTS-reactie en was na de FTS-reactie in geen enkele van de bestudeerde katalysatormaterialen aanwezig.

Door de roosterparameters van de Fe-carbiden te vergelijken, werden $\mathrm{de} \eta-\mathrm{Fe} 2 \mathrm{C}$ - en de $\chi-\mathrm{Fe}_{5} \mathrm{C}_{2}$-fase verder onderverdeeld in carbide-subtypen met hun typische formaties zoals:

\section{$\chi-\mathrm{Fe}_{5} \mathrm{C}_{2}$}

Type I Na CO-carburisatie, of FTS zónder Na-S promotie,

Type II Na FTS mét Na-S promotie,

Type III Na CO-carburisatie of $\mathrm{Fe}$ oxide $\left(\alpha-\mathrm{Fe}_{2} \mathrm{O}_{3}\right)$, het subtype is stabiel in FTS óngeacht de Na-S promotie,

$\eta-\mathrm{Fe}_{2} \mathrm{C}$

Type I Na CO-carburisatie, of FTS zónder Na-S promotie,

Type II Na FTS mét Na-S promotie,

Type III Na martensiet vermenging bij $120^{\circ} \mathrm{C}$. Niet geobserveerd in FTS. 

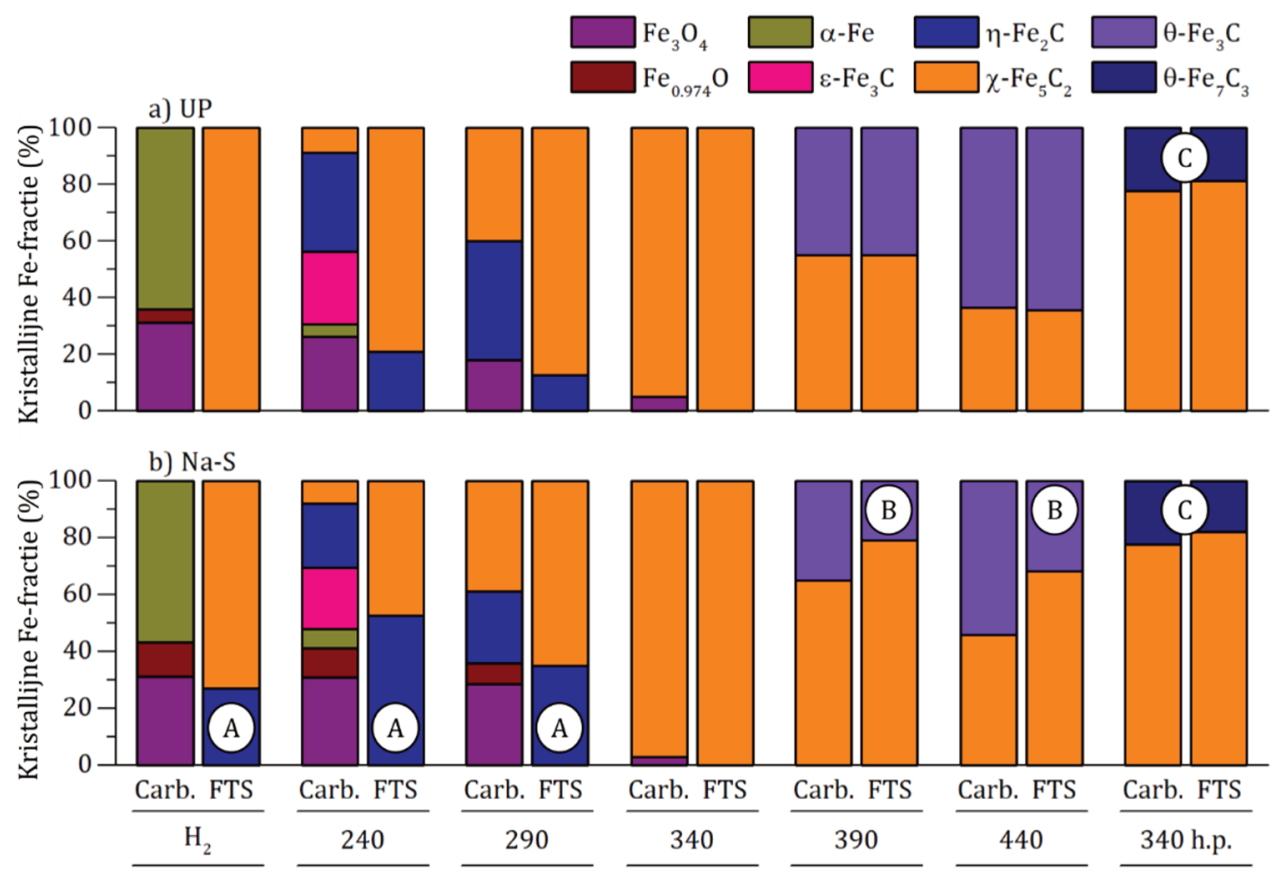

Figuur 8.4

Overzicht van Na-S-promotie-afhankelijke Fe-carbide-fasestabiliteit, zoals vergeleken vóór (Carb.) en na 72 u ur Fischer-Tropsch synthese (FTS)-reactie. Rietveld kwantitatieve fase-analyse (R-QPA) gebaseerde Fefase fracties voor a) niet-gepromote (unpromoted, UP) en b) Na-S-gepromoot (Na-S) $\mathrm{Fe}(-\mathrm{Na}-\mathrm{S}) / \alpha-\mathrm{Al}_{2} \mathrm{O}_{3}$ katalysatormaterialen. Na-S-promotie A stabiliseert $\eta-\mathrm{Fe}_{2} \mathrm{C}, \mathbf{B}$ destabiliseert $\theta-\mathrm{Fe}_{3} \mathrm{C}$ en $\mathbf{C}$ heeft geen effect op de stabiliteit van de $\theta-\mathrm{Fe}_{7} \mathrm{C}_{3}$-fase in de FTS-reactie. FTS-omstandigheden: $340^{\circ} \mathrm{C}, 10 \mathrm{bar}, \mathrm{H}_{2}: \mathrm{CO}: \mathrm{He}$ 2.0:1.0:0.33 v/v, $\approx 5000 \mathrm{~h}^{-1}$.

Het meest interessante is dat de kenmerkend vervormde $\chi$ - $\mathrm{Fe}_{5} \mathrm{C}_{2}$-fase (d.w.z., Type III) onveranderd bleef in de FTS-reactie. Er konden geen Fe-carbide-subtypen worden toegewezen voor de $\theta-\mathrm{Fe}_{3} \mathrm{C}$ - of $\theta-\mathrm{Fe}_{7} \mathrm{C}_{3}$-fasen.

De toevoeging van de Na-S-promotie had invloed op de Fe-carbiden in de FTSreactie door i) het veroorzaakte anisotropische veranderingen met een maximum van $\approx 0,02 \AA$ in de kristalroosterparameters voor de $\eta-\mathrm{Fe}_{2} \mathrm{C}$ - en $\chi-\mathrm{Fe}_{5} \mathrm{C}_{2}$-fasen (d.w.z., subtypen Type II), ii) de $\eta-\mathrm{Fe}_{2} \mathrm{C}$-fase was gestabiliseerd, terwijl iii) de $\theta$-Fe3C-fase was gedestabiliseerd, in vergelijking met de respectievelijke fasen in de niet-gepromote katalysatormaterialen. De stabiliteit van de $\theta-\mathrm{Fe}_{7} \mathrm{C}_{3}$-fase in de FTS-reactie werd niet beïnvloed door de Na-S-promotie (Figuur 8.4).

Met betrekking tot de FTS-reactieprestaties van de katalysator had de Na-Spromotie de algemene effecten van i) verhoogde CO-omzetting, ii) $\approx+10 \%$ verhoogde $\mathrm{C}_{2}$ - $\mathrm{C}_{4}$-selectiviteit met aanzienlijk onderdrukte $\mathrm{CH}_{4}$-selectiviteit onder de ASF- 
verwachtingen, iii) toegenomen alkeengehalte en iv) verhoogde $\alpha$-waarde in vergelijking met de respectieve niet-gepromote katalysatormaterialen. Als nadelige FTS-reactie-effecten nam met de $\mathrm{Na}$-S-promotie i) de $\mathrm{CO}_{2}$-selectiviteit significant toe van $\lesssim 16 \%$ tot $\approx 38-43 \%$ en ii) de hoeveelheid CO geconverteerd tot FTS-reactiekoolwaterstoffen daalde tot $\approx 0,42-0,47$ in relatie tot de niet-gepromote katalysatormaterialen.

Verder namen de gebruiksratio's $\mathrm{H}_{2}: \mathrm{CO}$ af van $\approx 1.8-2.3$ tot $\approx 0.7-0.8$ met de toegevoegde Na-S-promotie. Dit is een indicatie van $\mathrm{CO}_{2}$-vrijmakende, Boudouarden/of Water-Gas Shift-reacties (WGS-reacties) die de ingevoerde CO verbruiken. Omdat er na de FTS-reactie in geen enkel van de katalysatormaterialen WGS-reactie-actieve $\mathrm{Fe}_{3} \mathrm{O}_{4}$ aanwezig was, werd de $\mathrm{Na}(-\mathrm{S})$-promotie besproken om de direct actieve reactieplaatsen voor de mogelijke WGS-reactie te verschaffen.

Desalniettemin, ondanks de verloren C-route was er selectiviteit naar CO-omzetting in koolwaterstoffen: $\approx 4.4$ keer meer koolwaterstoffen werden nog steeds omgezet van CO per tijdseenheid met de meest actieve Na-S gepromote katalysator in vergelijking met het meest actieve niet-gepromote katalysatormateriaal.

Toen de resultaten van de FTS-reactieactiviteit werden gecontrasteerd met de RQPA-kwantificeringsresultaten, kon geen directe correlatie tussen de FTSreactieactiviteit en een van de bulk Fe-carbidefasen worden gemaakt. Dat wil zeggen dat geen enkele Fe-carbide in de FTS-reactie actiever was dan elk andere bestudeerde Fe-carbide. In termen van selectiviteit van koolwaterstofproducten konden echter enkele voorzichtige correlaties worden toegewezen. Er werd waargenomen dat i) de $\theta$ $\mathrm{Fe}_{3} \mathrm{C}$-fase $(\approx+5 \%)$ het paraffine-gehalte van het koolwaterstofproducttablet enigszins verhoogde. In combinatie met de Na-S-promotie: ii) de $\eta$ - $\mathrm{Fe}_{2} \mathrm{C}$ Type II en $\chi$ - $\mathrm{Fe}_{5} \mathrm{C}_{2}$ Type II waren aanwezig in de hoge alkeen- en lage $\mathrm{CH}_{4}$-selectieve FTS-katalysatormaterialen en iii) het $\chi-\mathrm{Fe}_{5} \mathrm{C}_{2}$ Type III zou, meer dan de $\chi-\mathrm{Fe}_{5} \mathrm{C}_{2}$ Type II, de alkeenselectiviteit kunnen verhogen wanneer het gecombineerd wordt met de Na-S-promotie.

Over de rol van de $\theta-\mathrm{Fe}_{7} \mathrm{C}_{3}$-fase in de FTS-reactie konden geen conclusies getrokken worden, omdat de fase aanwezig was in te kleine fasehoeveelheden in de bestudeerde FTS-reactiekatalysatormaterialen.

Hoofdstuk 5 - De gecombineerde effecten van de totale FTS-reactiedruk, Na-Spromotie en de $\mathrm{H}_{2}$-reductie van de katalysator voorafgaand aan de FTS-reactie werden bestudeerd. Het hoofddoel was voornamelijk om de effecten van de bovengenoemde parameters op de Fe-carbiden en hun subtypevorming te volgen en deze informatie te correleren met de FTS-reactieprestaties van de katalysatormaterialen. Hiervoor werden ondersteunde $\mathrm{Fe}(-\mathrm{Na}-\mathrm{S}) / \alpha-\mathrm{Al}_{2} \mathrm{O}_{3}$ katalysatormaterialen, met en zonder Na-Spromotie, voor het onderzoek bereid. De katalysatoren werden ofwel alleen gecalcineerd of na calcinatie ook gereduceerd in $\mathrm{H}_{2}$, voorafgaand aan de prestatietesten 
voor de katalysatoren in de FTS-reactie. De FTS-reacties werden afzonderlijk gedurende 72 uur uitgevoerd bij een totale reactiedruk van 3,10 of 20 bar $\left(340^{\circ} \mathrm{C}\right.$, $\mathrm{H}_{2}$ :CO:He 1.0:1.0:0.2 v/v, GHSV $\approx 5500 \mathrm{~h}^{-1}$ ). XRPD-patronen werden ex-situ verzameld voor elk van de bestudeerde katalysatormaterialen nadat de FTS-reactie werd uitgevoerd en werden gekwantificeerd met behulp van R-QPA.

$\mathrm{Na}$ de FTS-reacties waren de Fe-carbidefasen, die in de katalysatormaterialen werden geïdentificeerd, $\eta-\mathrm{Fe}_{2} \mathrm{C}$ Pnnm (58), $\chi-\mathrm{Fe}_{5} \mathrm{C}_{2} C 2 / c$ (15) en/of $\theta-\mathrm{Fe}_{7} \mathrm{C}_{3}$ Pnma (62). Voor alle bestudeerde katalysatormaterialen werd de $\chi-\mathrm{Fe}_{5} \mathrm{C}_{2}$-fase als de meest voorkomende gekwantificeerd. De Na-S-promotie had de volgende FTS-reactiedrukafhankelijke effecten op de Fe-carbidevorming: i) het induceren van het verschijnen van de $\eta$ - $\mathrm{Fe}_{2} \mathrm{C}$-fase bij $\geq 10$ bar FTS-reactiedruk, terwijl de katalysatormaterialen die alleen gecalcineerd waren de $\eta$ - $\mathrm{Fe}_{2} \mathrm{C}$-fasevorming meer begunstigden dan de $\mathrm{H}_{2}$-gereduceerde katalysator, en ii) de vorming van minieme hoeveelheden van de $\theta-\mathrm{Fe}_{7} \mathrm{C}_{3}$-fase na de FTS-reactie onder 20 bar in de katalysator die alleen gecalcineerd was.

Met betrekking tot de FTS-reactiedruk en de toegevoegde Na-S-promotie, vormen de carbide-subtypen zich als volgt:

$$
\chi-\mathrm{Fe}_{5} \mathrm{C}_{2}
$$

Type I Zónder Na-S-promotie óngeacht reactiedruk of met 3 bar FTSreactiedruk óngeacht $\mathrm{Na}-\mathrm{S}$-promotie,

Type II Mét Na-S-promotie en $\geq 10$ bar FTS-reactiedruk,

Type III Fe-oxide $\left(\alpha-\mathrm{Fe}_{2} \mathrm{O}_{3}\right)$ d.w.z. alleen gecalcineerde katalysator als startmateriaal voor de FTS óngeacht de Na-S-promotie of de FTS-reactiedruk,

$\eta-\mathrm{Fe}_{2} \mathrm{C}$

Type II Mét Na-S-promotie en $\geq 10$ bar FTS-reactiedruk.

De prestatieafhankelijkheid van de katalysator van de FTS-reactie op de reactiedruk werd voornamelijk veranderd door de Na-S-promotie. Het alleen calcineren van de katalysator en ook reduceren in $\mathrm{H}_{2}$ hadden een kleinere invloed op de katalytische prestatie van het katalysatormateriaal.

De Na-S-promotie in combinatie met een FTS-reactiedrukdrempel van 10 bar had als effecten dat i) de katalysatoren een significante toename in de Fe-tijdsopbrengsten (FTY) vertoonden en ii) de gebruiksverhouding $\mathrm{H}_{2}$ :CO, $\mathrm{CO}$ omgezet in koolwaterstoffen en koolwaterstof selectiviteiten reactiedruk-onafhankelijk werden en iii) de $\mathrm{CO}_{2}$ selectiviteit aanzienlijk verhoogd werd. Deze veranderingen in de FTS-reactieprestatie correleerden met Na-S-geïnduceerde overgang van $\chi-\mathrm{Fe}_{5} \mathrm{C}_{2}$ Type I naar Type II, en met de aanwezigheid van $\chi-\mathrm{Fe}_{5} \mathrm{C}_{2}$ Type III met Na-S-promotie. Voor niet-gepromote 


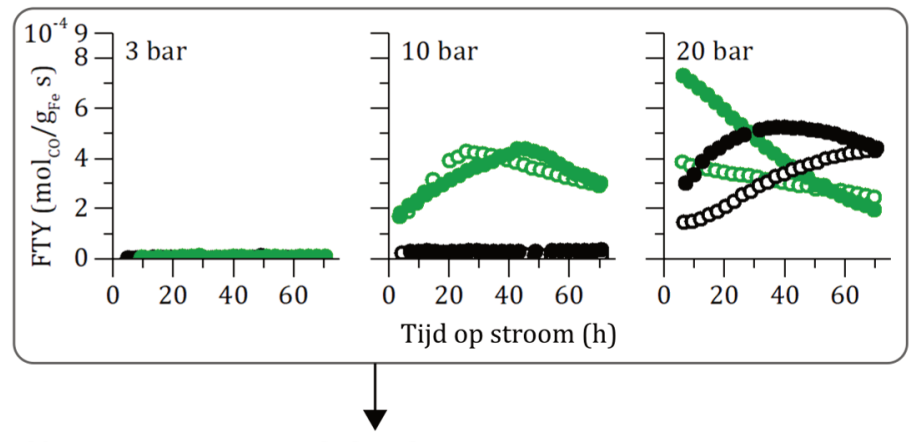

FTS-reactieactiviteit

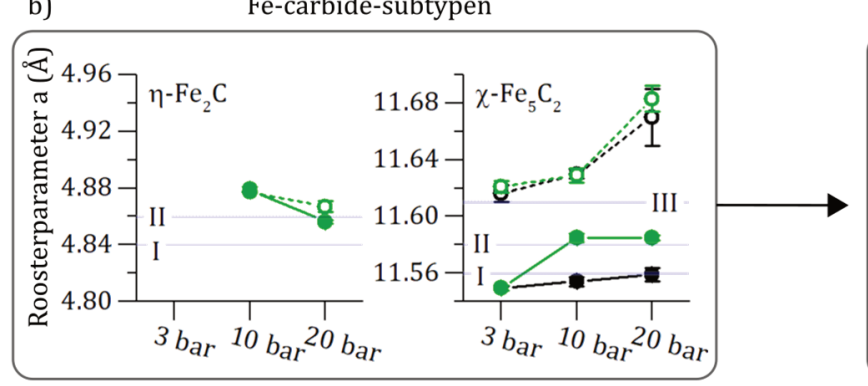

c) Koolwaterstofselectiviteit

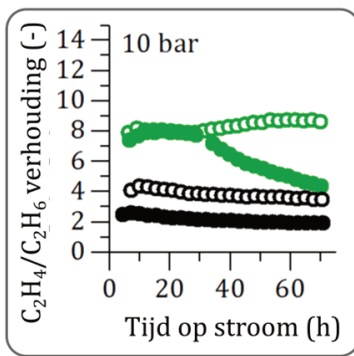

\section{Figuur 8.5}

Belangrijkste observaties van de effecten van Fischer-Tropsch synthese (FTS)-reactiedruk, Na-S-promotie en katalysator $\mathrm{H}_{2}$-pre-reductie op niet-gepromote (UP) en Na-S-gepromote (Na-S) $\mathrm{Fe}(-\mathrm{Na}-\mathrm{S}) / \alpha-\mathrm{Al}_{2} \mathrm{O}_{3}$ katalysatormaterialen in een 72 uur durende FTS-reactie $(3,10,20$ bar). De katalysatormaterialen werden direct na calcineren (Calcin.) aan de FTS-reactie blootgesteld of werden in $\mathrm{H}_{2}$ gereduceerd voorafgaand aan de reactie $\left(\mathrm{H}_{2}\right)$. De FTS-reactieactiviteit van de katalysatormaterialen als a) Fe-tijdsopbrengsten (FTY's) tonen een drempel van 10 bar voor Na-S en een drempel van 20 bar voor UP-katalysatormaterialen voor versterkte FTY. De FTY-verhoging b) correleert met de Fe-carbide-subtypen (I, II, III) met de overgang van $\chi$ $\mathrm{Fe}_{5} \mathrm{C}_{2}$ Type I naar II en de aanwezigheid van $\chi-\mathrm{Fe}_{5} \mathrm{C}_{2}$ Type III met Na-S-promotie. Bij een FTS-reactiedruk van 10 bar c) de $\mathrm{C}_{2} \mathrm{H}_{4} / \mathrm{C}_{2} \mathrm{H}_{6}$-verhouding wordt verhoogd met de Calcin. katalysatoren, ongeacht de Na-S-promotie. Dit komt overeen met de aanwezigheid van $\chi-\mathrm{Fe}_{5} \mathrm{C}_{2}$ Type III. FTS-omstandigheden: $340^{\circ} \mathrm{C}, 3,10$ or 20 bar, $\mathrm{H}_{2}: \mathrm{CO}: \mathrm{He} 1.0: 1.0: 0.2 \mathrm{v} / \mathrm{v}, \approx 5500 \mathrm{~h}^{-1}$.

katalysatoren was een reactiedrukdrempel van 20 bar FTS vereist voor het bereiken van vergelijkbare FTS-reactie-katalyse-effecten, zoals is waargenomen met de Na-Sgepromote katalysatormaterialen met $\geq 10$ bar reactiedruk. Voor deze veranderingen in de niet-gepromote katalysatoren waren $\chi-\mathrm{Fe}_{5} \mathrm{C}_{2}$ Type I en niet-gepromote Type III aanwezig als de $\chi$ - $\mathrm{Fe}_{5} \mathrm{C}_{2}$-fase-subtypen.

Met een reactiedruk van 3 bar vertoonde geen van de bestudeerde katalysatormaterialen significante FTY's ongeacht de Na-S-promotie. De aanwezigheid van $\eta-\mathrm{Fe}_{2} \mathrm{C}$ Type II correleerde niet met de katalytische prestatie-indicatoren - activiteit of selectiviteit - van de FTS-reacties, zoals werd afgeleid van de door R-QPA 
gekwantificeerde Fe-fasefracties.

Wanneer alleen gecalcineerde katalysatormaterialen gebruikt werden in de FTSreactie, ongeacht de Na-S-promotie: i) werd de $\mathrm{C}_{2} \mathrm{H}_{4} / \mathrm{C}_{2} \mathrm{H}_{6}$-verhouding verhoogd met 10 bar FTS reactiedruk en ii) werden de totale FTY's verlaagd bij 20 bar reactiedruk in vergelijking met de $\mathrm{H}_{2}$ gereduceerde katalysatoren. De verhoogde $\mathrm{C}_{2} \mathrm{H}_{4} / \mathrm{C}_{2} \mathrm{H}_{6}$ verhouding wordt geassocieerd met de aanwezigheid van $\chi$ - $\mathrm{Fe}_{5} \mathrm{C}_{2}$ Type III, onafhankelijk van de Na-S-promotie, bij een reactiedruk van 10 bar. Dat wil zeggen, $\chi$ $\mathrm{Fe}_{5} \mathrm{C}_{2}$ Type III correleerde met het verminderde hydrogenatievermogen van katalysatormaterialen. Verminderde FTY's op hun beurt correleerden met de aanwezigheid van $\mathrm{Fe}_{3} \mathrm{O}_{4}$ in de alleen gecalcineerde, 20 bar FTS-katalysatoren. Het $\mathrm{Fe}_{3} \mathrm{O}_{4}$ is waarschijnlijk afkomstig van door koolstofdepositie geïnduceerde stoftransportbeperkingen die de reductie remmen van de initiële $\alpha-\mathrm{Fe}_{2} \mathrm{O}_{3}\left(/ \alpha-\mathrm{Al}_{2} \mathrm{O}_{3}\right)$ fase door $\mathrm{H}_{2}$ :CO gedurende de FTS-reactie. Het overzicht van de besproken effecten van de FTS-reactieresultaten wordt gegeven in Figuur $\mathbf{8 . 5}$.

Hoofdstuk 6 - In dit hoofdstuk was het voornaamste doel om het effect van de NaS-promotie op de gelijktijdige Fe-carbide faseveranderingen en koolstofdepositie tijdens de FTS-reactie, d.w.z. met in-situ karakteriseringsmethoden, te bestuderen. Hiertoe werd een gecombineerde XRPD/Raman-spectroscopie-opstelling gebruikt. De ondersteunde $\mathrm{Fe}(-\mathrm{Na}-\mathrm{S}) / \alpha-\mathrm{Al}_{2} \mathrm{O}_{3}$-katalysatormaterialen bereid in Hoofdstuk 3 met voorgevormde $\mathrm{Fe}$-carbiden of alleen met $\mathrm{H}_{2}$ gereduceerde katalysatoren, met of zonder Na-S-promotie, werden gebruikt. De katalysatoren werden in situ gekarakteriseerd met de XRPD/Raman-spectroscopiebenadering tijdens een FTS-reactie van 72 uur $\left(340^{\circ} \mathrm{C}\right.$, 10 bar $\mathrm{H}_{2}$ :CO:He 2.0:1.0:0.33 v/v, GHSV $\approx 7200 \mathrm{~h}^{-1}$ ). De in-situ verzamelde XRPDpatronen werden gekwantificeerd met behulp van R-QPA. De Raman-spectra werden beoordeeld op de koolstofsoorten door het volgen van de intensiteit van de D$\left(\approx 1350 \mathrm{~cm}^{-1}\right)$ en G- $\left(\approx 1600 \mathrm{~cm}^{-1}\right)$ Raman-banden. Een overzicht van de belangrijkste resultaten wordt gegeven in Figuur 8.6.

De Na-S-promotie veranderde de eigenschappen van Fe-oxide en carbidefasen door:

i) de $\eta$ - $\mathrm{Fe}_{2} \mathrm{C}$-fase te stabiliseren, maar alléén als $\varepsilon$ - $\mathrm{Fe}_{3} \mathrm{C}$ en/of $\eta-\mathrm{Fe}_{2} \mathrm{C}$ in de initiële katalysator aanwezig was,

ii) destabilisatie van de $\theta-\mathrm{Fe}_{3} \mathrm{C}$-fase en het helpen ervan bij de overgang naar $\chi$ $\mathrm{Fe}_{5} \mathrm{C}_{2}$,

iii) verhoging van de reduceerbaarheid van $\mathrm{Fe}_{3} \mathrm{O}_{4}$-fase door $\left(\mathrm{H}_{2}\right.$ :)CO,

iv) verlaging van het $\mathrm{Fe}_{3} \mathrm{O}_{4}$-gehalte tijdens de FTS-reactie op de katalysatoren; en ook door

v) het induceren van Fe-kristallietgroei voor de Fe-carbiden binnen de $\lesssim 15$ uur tijd-op-stroom (ToS). De $\theta-\mathrm{Fe}_{3} \mathrm{C}$-fase was hierop een uitzondering, aangezien 
a) Raman-signaalintensiteit

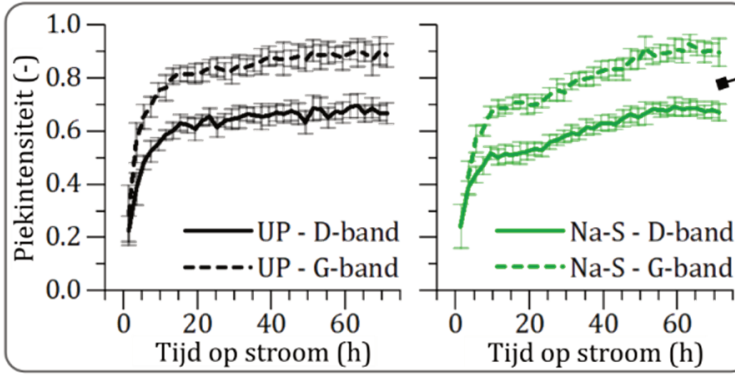

b)

R-QPA

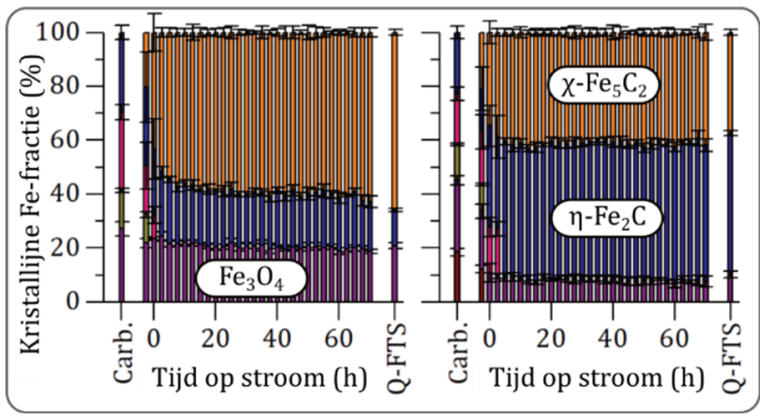

c) Vergelijking met referentie

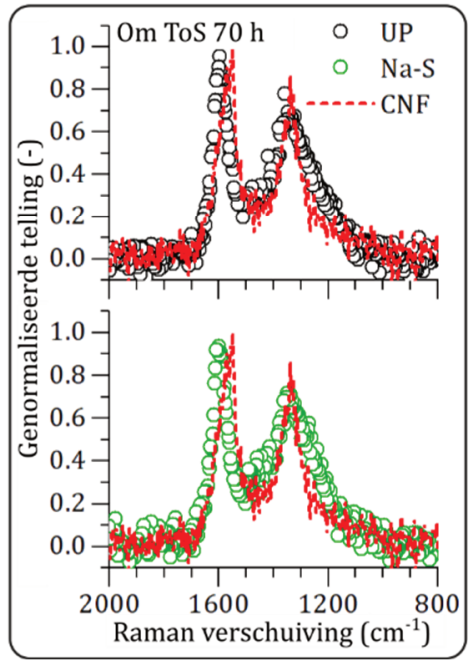

\section{Figuur 8.6}

Een samenvatting van simultane in-situ Röntgenpoederdiffractometrie (XRPD)/Ramanspectroscopieresultaten tijdens Fischer-Tropsch synthese (FTS)-reactie op niet-gepromote (UP) en Na-Sgepromote (Na-S) Fe-(Na-S)/ $\alpha-\mathrm{Al}_{2} \mathrm{O}_{3}$-katalysatormaterialen zonder initiële koolstofafzettingen. Ramanspectra D- en G-band intensiteiten a) groeien aanvankelijk snel binnen tijd-op-stroom (ToS) $\lesssim 15$ uur en $\gtrsim 15$ uur ToS-band intensiteitsgroei blijft sneller met de Na-S-katalysatoren. Op Rietveld kwantitatieve faseanalyse (R-QPA) gebaseerde Fe-fasefracties veranderen b) aangezien de $\chi-\mathrm{Fe}_{5} \mathrm{C}_{2}$-fase bij voorkeur wordt gevormd tijdens de FTS-reactie bij $340^{\circ} \mathrm{C}$, maar de $\mathrm{Na}$-S-promotie stabiliseert de $\eta$ - $\mathrm{Fe}_{2} \mathrm{C}$-fase significant. Indien aanwezig, destabiliseert Na-S-promotie de $\theta-\mathrm{Fe}_{3} \mathrm{C}$ - en $\mathrm{Fe}_{3} \mathrm{O}_{4}$-fasen. De gevormde koolstofsoorten c) lijken erg op een koolstof nanofiber (CNF) referentiekoolstofmateriaal bij ToS 70 uur, voor zowel UP- als NaS-katalysatoren. FTS-omstandigheden: $340^{\circ} \mathrm{C}, 10$ bar, $\mathrm{H}_{2}: \mathrm{CO}: \mathrm{He} 2.0: 1.0: 0.33 \mathrm{v} / \mathrm{v}, \approx 7200 \mathrm{~h}^{-1}$.

voor de fase de kristallietdiameters afnamen met Na-S-promotie.

De koolstofafzetting op de katalysatormaterialen correleerde met geen enkele Fecarbidefase of carbide-naar-carbide-overgang. Met $\mathrm{H}_{2}$-gereduceerde katalysatoren startte de koolstofdepositie pas als de $\mathrm{Fe}_{3} \mathrm{O}_{4}$-fase, een fase die niet in staat is om COdissociatie uit te voeren, was gereduceerd.

Bij katalysatormaterialen met verwaarloosbare initiële koolstofafzettingen, d.w.z. met $\mathrm{H}_{2}$-gereduceerde of $<300^{\circ} \mathrm{C}$ CO-gecarburiseerde katalysatoren, kon de intensiteitsgroei van de D- en G-banden in de Raman-spectra tijdens de FTS-reactie worden opgeknipt in twee perioden: i) een initiële snelle ( $\$ 10-15$ uur ToS), en ii) een 
gestage of kleinere ( $\gtrsim 15$ uur ToS) intensiteitsgroeiperiode. De initiële snelle groeiperiode was aanwezig ongeacht de Na-S-promotie. Maar na het bereiken van de gestage groeiperiode wekte Na-S-promotie een hogere mate van groei op van de $\mathrm{D}$ - en G-bandintensiteit (voor de G-band $<2,7 \pm 0,2$ sneller) in vergelijking met de nietgepromote katalysatormaterialen. Voor katalysatoren met initiële koolstofafzettingen van CO-carburatie $\geq 340^{\circ} \mathrm{C}$ was de initiële snelle groeiperiode afwezig, ongeacht de NaS-promotie. Een mogelijke verklaring voor de initiële snelle D- en G-band intensiteitswinst binnen $\lesssim 10-15$ uur ToS voor de katalysatormaterialen zonder initiële koolstofafzettingen, zou een eerste niet-isotherme bedoperatie zijn.

De koolstofsoorten die gevormd zijn tijdens katalysatorbereiding-CO-carburaties waren licht Raman-actief met de gebruikte $(532 \mathrm{~nm})$ zichtbare golflengte laser. Met andere woorden, ze waren hoofdzakelijk gemaakt van koolstofstructuren met $\mathrm{C} \mathrm{sp}{ }^{3}$ met een bandafstand boven de energie die beschikbaar is van de zichtbare golflengte-laser. Onder $\mathrm{H}_{2}$ : $\mathrm{CO}$ tijdens de FTS-reactie vormden meer geordende $\mathrm{CNF}$-achtige fasen met $\mathrm{C}$ $\mathrm{sp}^{2}$, wat leidde tot verhoogde Raman-signaalintensiteit. Tijdens de FTS-reacties evolueerden de koolstofsoorten van amorfe koolstofsoorten, met structuren die zowel tetrahedrische $\mathrm{C} \mathrm{sp^{3 }}$ als $\mathrm{C} \mathrm{sp}{ }^{2}$ in ketenachtige ordening bevatten, naar meer geordende koolstofmaterialen met structuren die $\mathrm{C} \mathrm{sp}^{2}$ bevatten in variërende niet-zesvoudige cyclische volgorde met kenmerkende afmetingen $<7 \mathrm{~nm}$ in diameter.

In vergelijking met de gemeten koolstofreferentiematerialen waren de Ramanspectra van de katalysator bij $\approx 70$ uur ToS het best te vergelijken met deze van een koolstof nanovezel (CNF) referentiemateriaal. De katalysatormaterialen bevatten echter mogelijk een hogere hoeveelheid $\mathrm{C} \mathrm{sp}^{2}$ in ketenachtige structuren (G-band blauwe verschuiving naar hogere $\mathrm{cm}^{-1}$ ) en een grotere verscheidenheid aan nietzesvoudige koolstofringen (bredere D-bandpiek) dan in het referentie CNF-materiaal (Figuur 8.6c).

Na-S-promotie beïnvloedde de door FTS-reactie gevormde koolstofsoorten door: i) het aanvankelijke $\mathrm{C} \mathrm{sp}^{2}$-gehalte in ketenachtige koolstofstructuren te verhogen, en ii) het verhogen van zesvoudige cyclische koolstofsoorten voor katalysatormaterialen met de initiële CO-carburatie temperatuur $\geq 340^{\circ} \mathrm{C}$.

\subsection{TOEKOMSTPERSPECTIEVEN}

Zoals bij elk onderzoek het geval is ontstaan nieuwe wetenschappelijke vragen in hetzelfde tempo als dat de oude worden beantwoord. Enkele van de meest interessante toekomstige onderzoeksthema's, die voortkomen uit het werk van dit proefschrift, worden hierna kort besproken.

Vergelijking van $\varepsilon-\mathrm{Fe}_{3} \mathrm{C}$ en $\mathrm{\eta}-\mathrm{Fe}_{2} \mathrm{C}$ tot $\varepsilon-\mathrm{Fe}_{3} \mathrm{~N}$ en $\zeta-\mathrm{Fe}_{2} \mathrm{~N}$. Zoals kort in Hoofdstuk 3 
werd benadrukt, zijn de kristalstructuren van Fe-carbide $\varepsilon$ - $\mathrm{Fe}{ }_{3} \mathrm{C}$ en $\eta$ - $\mathrm{Fe}_{2} \mathrm{C}$-fasen nauw verwant aan respectievelijk de structuren $\varepsilon-\mathrm{Fe}_{3} \mathrm{~N}$ en $\zeta-\mathrm{Fe}_{2} \mathrm{~N}$ Fe-nitriden. Een vergelijkend onderzoek naar het gedrag en de FTS-reactieprestaties van $\varepsilon-\mathrm{Fe}_{3} \mathrm{C}$ en $\eta$ $\mathrm{Fe}_{2} \mathrm{C}$ in vergelijking met de $\mathrm{Fe}_{3} \mathrm{~N}$ - en $\zeta-\mathrm{Fe}_{2} \mathrm{~N}$-fasen, met en zonder de Na-S-promotie, zou een interessant onderzoek opleveren.

In het bijzonder zou het waardevol zijn om uit te vinden of de $\zeta-\mathrm{Fe}_{2} \mathrm{~N}$-fase kan worden gestabiliseerd in de FTS-reactie met Na-S-promotie op dezelfde manier als werd waargenomen voor de $\eta-\mathrm{Fe}_{2} \mathrm{C}$-fase. De Fe-nitridefasen zijn gewoonlijk niet stabiel onder carburiserende omstandigheden maar worden eerder omgezet in $\mathrm{Fe}$ carbonitriden. $^{1-3}$ Er is waargenomen dat op Fe-nitride gebaseerde katalysatormaterialen selectief zijn richting alkeenproductie in de FTS-reactie. ${ }^{3}$

Vorming van de $\boldsymbol{\theta}-\mathrm{Fe}_{7} \mathrm{C}_{3}$-fase. Hoewel enkele kleine hoeveelheden van de $\theta-\mathrm{Fe}_{7} \mathrm{C}_{3}-$ fase werden gevormd in het onderzoek van deze dissertatie, is er nog meer inspanning nodig om het vormingsgedrag van de fase te bestuderen onder de FTS-reactie-relevante omstandigheden. Zoals in Hoofdstuk 3 werd gespeculeerd, kan de vorming van de $\boldsymbol{\theta}$ $\mathrm{Fe}_{7} \mathrm{C}_{3}$-fase onder carburiserende omstandigheden afhankelijk zijn van de aanwezigheid van $\mathrm{CO}_{2}$ tijdens het carburisatieproces. Een systematisch onderzoek naar het effect van carburiserende $\mathrm{CO}_{2}$ : $\mathrm{CO}$-gasmengsels op de $\theta-\mathrm{Fe}_{7} \mathrm{C}_{3}$-fase-vorming van op Fe-gebaseerde materialen zou in de toekomst moeten worden uitgevoerd. Dit zou helpen bij het bepalen van de rol van de FTS-reactie en de eigenschappen van dit nog steeds veel te weinig bestudeerde Fe-carbide.

Kristalstructuren van Fe Carbide Subtypen. De $\eta$ - $\mathrm{Fe}_{2} \mathrm{C}$ - en de $\chi-\mathrm{Fe}_{5} \mathrm{C}_{2}$-fasen werden onderverdeeld in verschillende subtypen naar hun kenmerkend verschillende roosterparameter a's in Hoofdstuk 4. Echter, de precieze kristalstructuuroorsprong van de roosterwijzigingen voor de uiteindelijke carbide-subtypen kon niet worden achterhaald binnen de reikwijdte van deze dissertatie. In de toekomst moet meer gedetailleerd onderzoek gedaan worden naar de fundamentele verschillen in kristalstructuur in de Fe-carbide-subtypen met behulp van een monochromatische röntgenbron. Daarnaast kan neutronenpoederdiffractie (NPD) ingezet worden om te achterhalen of de door Na-S-promotie geïnduceerde subtype-formaties echt het gevolg zijn van de $\mathrm{Na}(-\mathrm{S})$-promotoren die de Fe-carbideroosters binnenkomen of van iets anders.

Verder werd in Hoofdstuk 5 de associatie gemaakt van het $\chi$ - $\mathrm{Fe}_{5} \mathrm{C}_{2}$ Type III met katalysatormaterialen met verminderd hydrogeneringsvermogen in de FTS-reactie. Dat wil zeggen dat het $\chi-\mathrm{Fe}_{5} \mathrm{C}_{2}$ Type III de FTS-koolwaterstofselectiviteit van het katalysatormateriaal naar de productie van alkenen verhoogde. De aanwezigheid van $\chi-\mathrm{Fe}_{5} \mathrm{C}_{2}$ Type III zou ook de vergelijkbare selectiviteitsresultaten in de literatuur kunnen verklaren. ${ }^{4,5}$ In het hoofdstuk wordt dit effect niet voorzien van fundamenteel 
inzicht. Hoe en waarom het $\chi-\mathrm{Fe}_{5} \mathrm{C}_{2}$ Type III de alkeenvorming ten goede komt in vergelijking met de andere Fe-carbidesubtypen, zou een interessant onderzoek opleveren. Hierbij zou dan gebruik kunnen gemaakt worden van de dichtheid functionele theorie (DFT) berekeningenbenadering.

\subsection{REFERENTIES}

[1] Jack, K. H., "Binary and Ternary Interstitial Alloys III. The Iron-Carbon System: The Characterization of a New Iron Carbide", Proc. R. Soc. A Math. Phys. Eng. Sci. 195, 56-61 (1948).

[2] Jack, K. H., "Binary and Ternary Interstitial Alloys. II. The Iron-Carbon-Nitrogen System", Proc. R. Soc. A Math. Phys. Eng. Sci. 195, 41-55 (1948).

[3] Butt, J. B., "Carbide phases on iron-based Fischer-Tropsch synthesis catalysts part II: Some reaction studies", Catal. Lett. 7, 83-106 (1990).

[4] Bukur, D. B., Nowicki, L., Manne, R. K. and Lang, X. S., "Activation Studies with a Precipitated Iron Catalyst for Fischer-Tropsch Synthesis II. Reaction Studies", J. Catal. 155, 366-375 (1995).

[5] Wezendonk, T. A., Sun, X., Dugulan, A. I., van Hoof, A. J. F., Hensen, E. J. M., Kapteijn, F. and Gascon, J., "Controlled formation of iron carbides and their performance in Fischer-Tropsch synthesis", J. Catal. 362, 106-117 (2018). 


\section{List of Abbreviations}

AC Activated Carbon

ALSS Asymmetric Least

Squares Smoothing

ASF Anderson-Schulz-Flory

BASF Badische Anilin \& Soda

Fabrik

BTL Biomass-to-Liquids

CNF Carbon Nanofiber

CNT Carbon Nanotube

COD Crystallography Open

Database

CPS Counts per Second

CTL Coal-to-Liquids

DFT Density Functional

Theory

ED Electron Diffraction

ETEM Environmental TEM

FEG Field Emission Gun

FID Flame Ionization

Detector

FPA Fundamental

Parameters Approach

FTP Fischer-Tropsch Process

FTS Fischer-Tropsch

Synthesis

FTY Fe Time Yield
FWHM Full Width at Half

Maximum

GC Gas Chromatography

GHSV Gas Hourly Space

Velocity

GTL Gas-to-Liquids

HC Hydrocarbons

HTFT High-temperature

Fischer-Tropsch

ICDD- International Centre for

PDF Diffraction Data Powder

Diffraction File

ICP-OES Inductively Coupled

Plasma - Optical

Emission

Spectrophotometry

IR Infrared

IWI Incipient Wetness

Impregnation

JM Johnson Matthey

LTFT Low-temperature

Fischer-Tropsch

MAS Mössbauer Absorption

Spectroscopy

MOF Metal Organic

Framework

MTH Methanol-to-

Hydrocarbons

MT0 Methanol-to-Olefins 


\begin{tabular}{|c|c|c|c|}
\hline $\begin{array}{l}\text { NBS- } \\
\text { SRM }\end{array}$ & $\begin{array}{l}\text { National Bureau of } \\
\text { Standards - Standard } \\
\text { Reference Material }\end{array}$ & $\begin{array}{l}\text { STD } \\
\text { TCD }\end{array}$ & $\begin{array}{l}\text { Standard Deviation } \\
\text { Thermal Conductivity } \\
\text { Detector }\end{array}$ \\
\hline NNPC & $\begin{array}{l}\text { Nigerian National } \\
\text { Petroleum Corporation }\end{array}$ & TEM & $\begin{array}{l}\text { Transmission Electron } \\
\text { Microscopy }\end{array}$ \\
\hline NPD & $\begin{array}{l}\text { Neutron Powder } \\
\text { Diffraction }\end{array}$ & TGA & $\begin{array}{l}\text { Thermogravimetric } \\
\text { Analysis }\end{array}$ \\
\hline NWO & $\begin{array}{l}\text { Nederlandse Organisatie } \\
\text { voor Wetenschappelijk } \\
\text { Onderzoek }\end{array}$ & TGA-MS & $\begin{array}{l}\text { Thermogravimetric } \\
\text { Analysis - Mass } \\
\text { Spectrometry }\end{array}$ \\
\hline OX-ZEO & Oxide-Zeolite & TM & Thermomagnetic \\
\hline PetroSA & $\begin{array}{l}\text { South African national } \\
\text { oil company }\end{array}$ & ToS & Time-on-Stream \\
\hline PSD & $\begin{array}{l}\text { Position Sensitive } \\
\text { Detector }\end{array}$ & TU Delft & $\begin{array}{l}\text { Technische Universiteit } \\
\text { Delft }\end{array}$ \\
\hline QP & Qatar Petroleum & $\mathbf{U U}$ & Universiteit Utrecht \\
\hline R-QPA & $\begin{array}{l}\text { Rietveld Quantitative } \\
\text { Phase Analysis }\end{array}$ & $\begin{array}{l}\text { WGS } \\
\text { Wt-\% }\end{array}$ & $\begin{array}{l}\text { Water-gas Shift } \\
\text { Weight percent }\end{array}$ \\
\hline $\mathbf{R}_{\mathbf{w p}}$ & R-Weighted Pattern & WTL & Waste-to-Liquids \\
\hline Sasol & Suid Afrikaanse & XRF & X-ray Fluorescence \\
\hline & Steenkool en Olie & XRPD & X-ray Powder \\
\hline SMR & Steam Methane & & Diffractometry \\
\hline & Reforming & XTL & Anything-to-Liquids \\
\hline SPM & Superparamagnetic & & \\
\hline
\end{tabular}


This page is intentionally left blank 

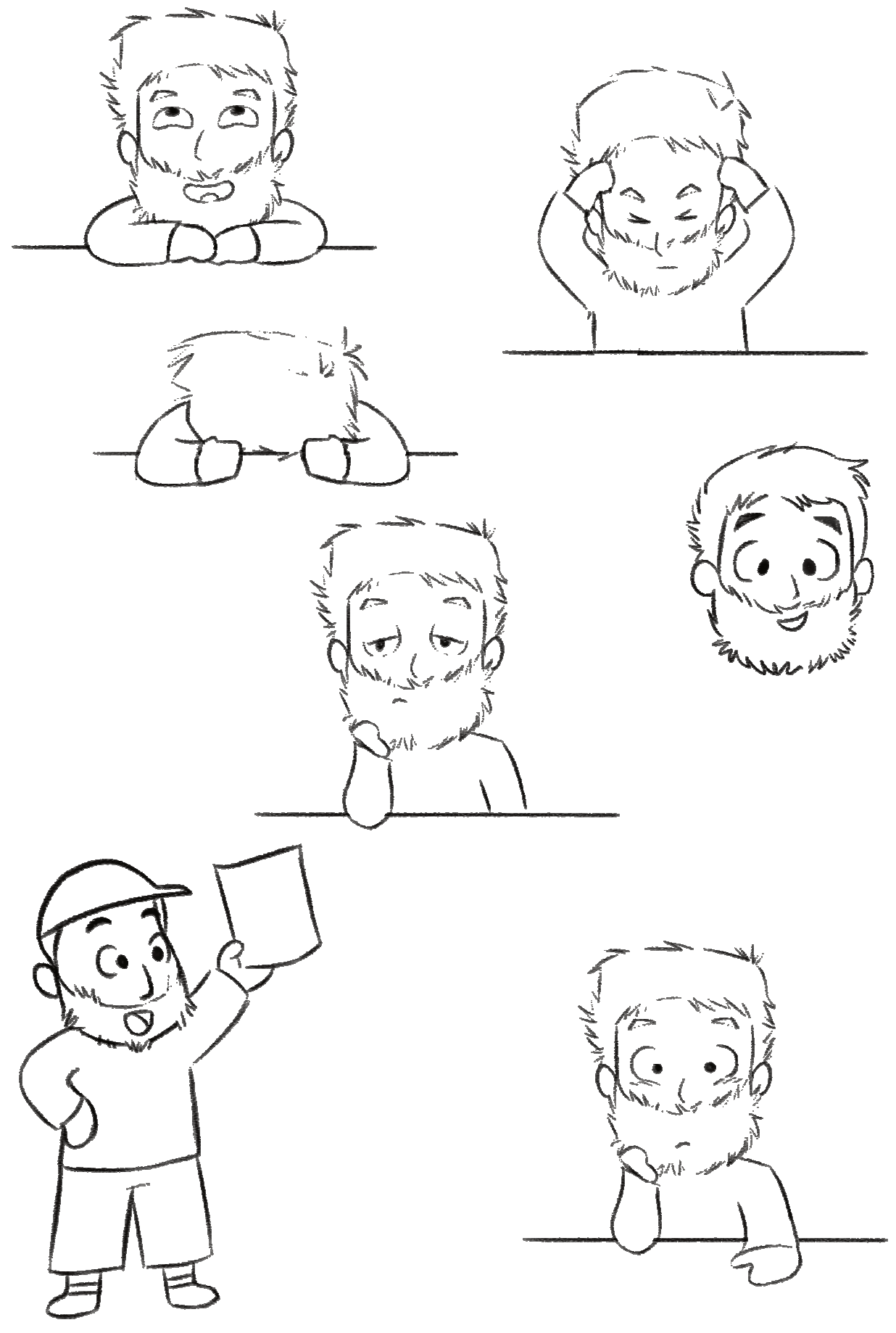


\section{Acknowledgements}

I am grateful to a large group of persons who have helped and participated in the research related to this Dissertation. None of this research could have been accomplished without the expertise and dedication of all the talented individuals working in the Inorganic Chemistry and Catalysis group of Universiteit Utrecht, and in The Dow Chemical Company and Johnson Matthey.

On a more individual level, I would like to thank my promotor Prof. dr. ir. Bert Weckhuysen for enabling the possibility to perform this work in his research group. The unique freedom to pursue various research directions in the group is a great asset allowing creative and innovative research output. You manage to provide an amplitude of opportunities for your group members through hours of your own hard work in the background. For this alone, acknowledgement is due, I think you deserve it.

As participants and collaborators on the same TASC research project, I am also grateful for the Fischer-Tropsch catalysis understanding and advices of Prof. dr. ir. Krijn de Jong and Dr. Jingxiu Xie (JX). Any of the catalysis experiments within this Dissertation could not have been performed without you. Thus, this Dissertation, and my work, is greatly indebted to your efforts. Prof. dr. ir. de Jong is also thanked for commentary during preparation of this Dissertation.

Dr. Matthijs Ruitenbeek and Dr. Ard Koeken from The Dow Chemical Company, and Dr. Mike Watson and Dr. Leon van de Water from Johnson Matthey as well as Dr. Iulian Dugulan from Technische Universiteit Delft are gratefully thanked for all the discussions, comments and questions in the meetings over the past years. Your comments and questions have inspired me to look deeper in to the details of my results and the underlying data. Dr. Ruitenbeek and Dr. Watson are kindly thanked for the provided comments during the preparation of this Dissertation.

Dr. Florian Meirer, thank you for reading, helping in correcting and grading the reports of my students Geert, Manon and Sophie, and for your help in guiding them. Thank you for the overall guidance and discussions on various issues during my $\mathrm{PhD}$.

Geert, Manon and Sophie, you can be proud of your work as students doing your own research in relation to my PhD Dissertation! You did a very good job. I hope I have been a good guide for you. Manon and Sophie, good luck with your chosen paths of doing your own PhD Dissertations!

Oscar, Pascal, Herrick, AdM, AdE, Marjan, Rien and Jan Willem; thank you for all of your hard work on keeping the equipment and laboratories running, thus enabling the research work done in this Dissertation. AdM, Rien and Marjan, I hope you are enjoying your retirement days! Oscar, AdE, Sander, Pascal, Marjan, Rien and Herrick, your work on the XRPD/Raman Spectroscopy set-up is especially appreciated. Dymph, Iris and 
Monique; thank you very much for keeping the administration of the group running and always helping with whatever silly issues I might have needed assistance with.

During my PhD years, I have had the privilege to travel around the World to various beamlines to experiment with a large set of different synchrotron-based techniques. These trips have left me with great memories to cherish. Thanks Sam for bringing (S)TXM, Menlo Park, Saskatoon and Tim Hortons to my life. I cannot remember much of the scientific work done but I do remember the Mad Caddies. Zoran, I truly enjoyed our time and discussions at SLS. Thank you all: Korneel, Özgün, Jan, Matthias, AdE, Herrick, Ilse, Marjolein, Ramon and Tom (and anyone I might have forgotten to mention) for your time and assistance with TXM experiments. Thanks AdE for sharing your lifewisdom during the various beamtimes we attended together. Thanks Florian for all of your efforts on teaching me on analysis of X-ray absorption based data. Doug van Campen and Yijin Liu, your help and hard work at SSRL is greatly appreciated.

$J X$, thanks for all the help regarding Fischer-Tropsch Synthesis catalysis, gossips, and after meeting coffee moments. It was a joy to work in the same project with you. Jan, in a similar manner; thank you for all of your Fischer-Tropsch Synthesis enthusiasm and help with various technical issues that I could not have solved without your naturalborn engineering skills.

There are so many interesting people I have come to know during this $\mathrm{PhD}$ period. Özgün and Ivan, it was a pleasure to share the adjacent desks with you. Thanks to you Ivan, I now know more about the hydrogen future than I ever wanted to. I tried to think of some witty science joke for you too Özgün but I think we spent more time interacting outside than inside the science environment, thanks for that. I truly enjoyed the late night New York pizzas with you Sang-Ho, but just do not teach your newborn this habit. Jose, Ru-Pan, Sandra, Heba, Fang and Antonio, thank you all for making our small office corner a very enjoyable place. Frank, thanks for the beers and checking the Dutch translation. The poker group: Egor, Lennart, Rolf, Miguel and the rest, thanks the one time for your money and rest of the times for the memories. Zafer, thanks for all the discussions we have had. Abhishek, thanks for often reminding me to improve myself and my decision-making, I shall improve! Matthias, well, you know, thanks for your unique and exquisite sense of humor. Pierre, thanks for having a funny name enabling some cheap jokes and for the Six Nations at O'Leary's. For Wiesja, thank you for the wonderful cover drawings, the Dutch summary translation and for being the wonderful and lovable person you are.

A collective thank you for all of you in the Inorganic Chemistry and Catalysis group and in other affiliations with whom I have worked with directly or indirectly during this $\mathrm{PhD}$. There would be some many more to mention, than can possible be individually written here. Science is teamwork. 


\title{
List of Publications and Presentations
}

\author{
PUBLICATIONS
}

2020 Paalanen, P. P. and Weckhuysen B. M., "Carbon Pathways, SodiumSulfur Promotion and Identification of Iron Carbides in Iron-based Fischer-Tropsch Synthesis", ChemCatChem, accepted article (2020).

2019 Xie, J., Paalanen, P. P., van Deelen, T. W., Weckhuysen, B. M., Louwerse, M. J. and de Jong, K. P., "Promoted cobalt metal catalysts suitable for the production of lower olefins from natural gas", Nat. Commun. 10, 167 (2019).

2018 Falkenhagen, J. P., Maisonneuve, L., Paalanen, P. P., Coste, N., Malicki, N. and Weckhuysen, B. M., "Cobalt-Iron-Manganese Catalysts for the Conversion of End-of-Life-Tire-Derived Syngas into Light Terminal Olefins", Chem. - A Eur. J. 24, 4597-4606 (2018).

2017 Marquez, C., Rivera-Torrente, M., Paalanen, P. P., Weckhuysen, B. M., Cirujano, F. G., de Vos, D. and de Baerdemaeker, T., "Increasing the availability of active sites in $\mathrm{Zn}$-Co double metal cyanides by dispersion onto a SiO 2 support", J. Catal. 354, 92-99 (2017).

2016 Kalirai, S., Paalanen, P. P., Wang, J., Meirer, F. and Weckhuysen, B. M., "Visualizing Dealumination of a Single Zeolite Domain in a Real-Life Catalytic Cracking Particle", Angew. Chemie Int. Ed. 55, 11134-11138 (2016).

Whiting, G. T., Chowdhury, A. D., Oord, R., Paalanen, P. P. and Weckhuysen, B. M., "The curious case of zeolite-clay/binder interactions and their consequences for catalyst preparation", Faraday Discuss. 188, 369-386 (2016).

2014 Lezcano-Gonzalez, I., Deka, U., van der Bij, H. E., Paalanen, P. P., Arstad, B., Weckhuysen, B. M. and Beale, A. M., "Chemical deactivation of $\mathrm{Cu}$ - 
SSZ-13 ammonia selective catalytic reduction ( $\mathrm{NH}_{3}$-SCR) systems", Appl. Catal. B Environ. 154-155, 339-349 (2014).

2013 Paalanen, P. P., Weckhuysen, B. M. and Sankar, M., "Progress in controlling the size, composition and nanostructure of supported gold-palladium nanoparticles for catalytic applications", Catal. Sci. Technol. 3, 2869 (2013).

\section{PRESENTATIONS}

2017 Poster Presentation - Paalanen, P. P., Bertram M., Velthoen, E. Z. M., Meirer, F. and Weckhuysen, B. M., "Pyridine-IR Spectroscopy Investigation on $\mathrm{Na}, \mathrm{S}$ and $\mathrm{Na}-\mathrm{S}$ Promotion Effect on a Fe/ $\alpha-\mathrm{Al} 2 \mathrm{O} 3$ Catalyst for FTO", The Netherland's Catalysis and Chemistry Conference XVIII, Noordwijkerhout, the Netherlands, March 2017.

Poster Presentation - Paalanen, P. P., Xie, J., de Jong, K. P., Meirer, F., and Weckhuysen, B. M., "Na-S Promoter Effect on the Formation of Fe Carbides in the Fischer-Tropsch-to-Olefins Reaction", 25th North American Meeting (NAM25) of the Catalysis Society, Denver, Colorado, United States of America, June 2017. 


\section{Curriculum Vitae}

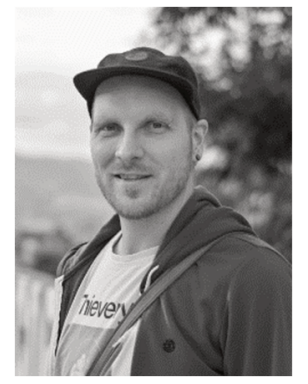

Pasi P. Paalanen was born in Otava, Mikkeli in Finland on February $27^{\text {th }} 1985$. Following the completion of the sports oriented high school in 2004 , he moved on to complete his obligatory military service as well as to spent time working, and also travelling in Europe.

In 2007 he continued pursuing higher education, and began university studies in University of Jyväskylä in Finland. This included a year spent in an Erasmus student exchange program at the Warsaw University of Technology in Poland. He graduated in 2011 with a Bachelor's degree in physical chemistry, with minors in physics and mathematics.

Following this, further continuing with higher education, he then moved to Utrecht, the Netherlands in 2011 and began studies in the Nanomaterials: Chemistry and Physics Master's program at Utrecht University. After finishing the Master's degree in 2013, he embarked with his scientific career towards a PhD in Utrecht University in the Inorganic Catalysis and Chemistry group under de supervision of Professor Bert Weckhuysen. This endeavor has led to the work described in this PhD Dissertation. 
This page is intentionally left blank 